SERVIÇO DE PÓS-GRADUAÇÃO DO ICMC-USP

Data de Depósito:

Assinatura:

\title{
Desenvolvimento de um método numérico implícito para a simulação de escoamentos viscoelásticos com superfícies livres
}

\section{Fernando Pacanelli Martins}

Orientador: Prof. Dr. José Alberto Cuminato

Dissertação apresentada ao Instituto de Ciências Matemáticas e de Computação - ICMC-USP, como parte dos requisitos para obtenção do título de Mestre em Ciências - Ciências de Computação e Matemática Computacional.

USP - São Carlos

Junho/2009 

Desenvolvimento de um método numérico implícito para a simulação de escoamentos viscoelásticos com superfícies livres

Fernando Pacanelli Martins 



\section{Conteúdo}

$\begin{array}{ll}\text { Conteúdo } & \text { iii }\end{array}$

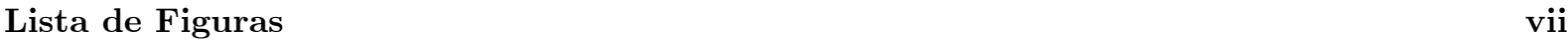

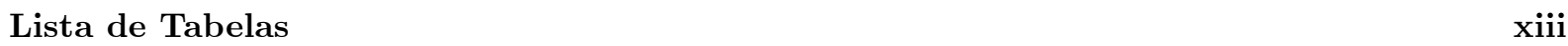

Resumo $\quad$ xix

$\begin{array}{ll}\text { Abstract } & \mathrm{xxi}\end{array}$

$\begin{array}{ll}\text { Prefácio } & \text { xxiii }\end{array}$

1 Formulação matemática $\quad 1$

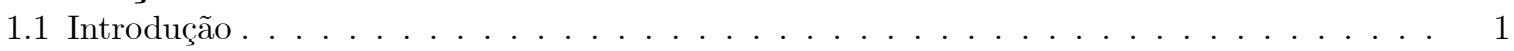

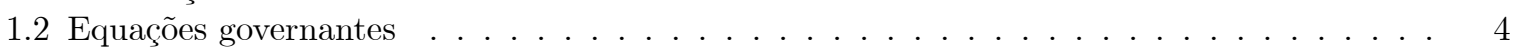

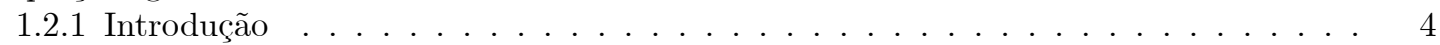

1.2 .2 Modelo Newtoniano . . . . . . . . . . . . . . . . . . . . . 5

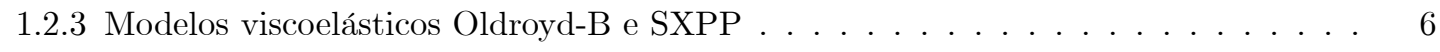

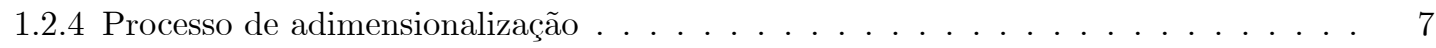

1.3 Condições auxiliares . . . . . . . . . . . . . . . . . . . . . . . 9

2 A metodologia GENSMAC $\quad 13$

2.1 Introdução . . . . . . . . . . . . . . . . . . . . . . . . . . . 13

2.2 Método de projeção . . . . . . . . . . . . . . . . . . . . . . . . 16

2.3 Formulações explícita e implícita . . . . . . . . . . . . . . . . . . . . 17

3 Modificações no GENSMAC 21

3.1 Introdução. . . . . . . . . . . . . . . . . . . . . . . . . . . 21

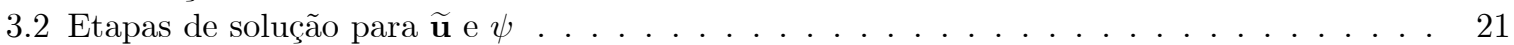

3.2 .1 Introdução . . . . . . . . . . . . . . . . . . . . . . . . . . . . . . . . . . . . . .

3.2.2 Métodos de solução dos sistemas lineares . . . . . . . . . . . . . . . . . . . . . . . . . . . . . . . . . . .

3.3 A massa de fluido computacional . . . . . . . . . . . . . . . . . . . . . . 27

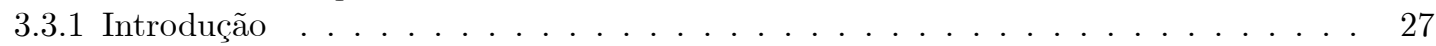

3.3.2 Movimento da superfície livre . . . . . . . . . . . . . . . . . . . . 28

4 Resultados: modificações no GENSMAC $\quad 31$

4.1 Introdução . . . . . . . . . . . . . . . . . . . . . . . . . . . . . . . . . . . . . . . . . . . . . .

4.2 Resolução dos sistemas lineares . . . . . . . . . . . . . . . . . . . . . . . . . . . . . . . . . . . . . . . . . . . . . .

4.2 .1 Introdução . . . . . . . . . . . . . . . . . . . . . . . . . . . . . . . . . . . . . 31

4.2.2 Problema 1: inchamento do extrudado . . . . . . . . . . . . . . . . 32 
4.2 .3 Problema 2 fountain flow . . . . . . . . . . . . . . . . . . . . . . . . . . . . . . . . . . . . . 35

4.2.4 Problema 3 : enchimento estável . . . . . . . . . . . . . . . . . . . . 38

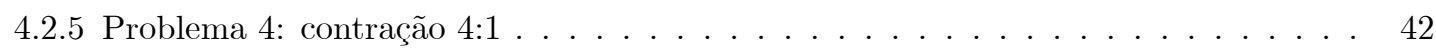

4.2 .6 Problema 5 : jet flow . . . . . . . . . . . . . . . . . . . . . . . 45 45

4.3 Esquemas de movimentação da superfície livre . . . . . . . . . . . . . . . . . . . . . 49

4.3 .1 Introdução . . . . . . . . . . . . . . . . . . . . . . . . 49

4.3.2 Problema 1: enchimento estável . . . . . . . . . . . . . . . . . . 49

4.3.3 Problema 2: enchimento de uma caixa contendo fluido . . . . . . . . . . . . . . 54

4.3.4 Problema 3 : gota em impacto . . . . . . . . . . . . . . . 56

4.3.5 Problema 4: inchamento do extrudado . . . . . . . . . . . . . . . 59

4.4 Conclusões . . . . . . . . . . . . . . . . . . . . . . . 63

$\begin{array}{ll}5 \text { O GENSMAC-SXPP } & \mathbf{6 7}\end{array}$

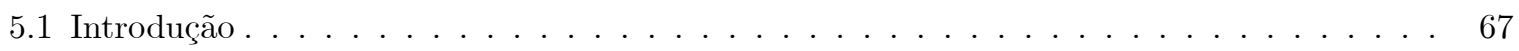

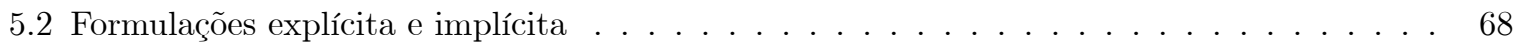

5.3 Discretização das equações governantes . . . . . . . . . . . . . . . . . . . . . . . . . 73

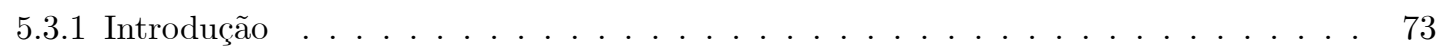

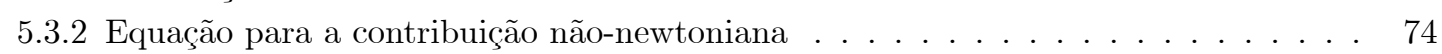

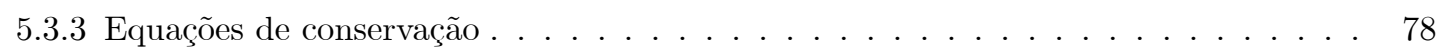

5.3.4 Condições de fronteira para a velocidade intermediária . . . . . . . . . . . . . . 79

5.3.5 Cálculo do campo de pressão e da velocidade solenoidal . . . . . . . . . . . . . . . 80

5.3.6 Equação de Poisson para o potencial auxiliar . . . . . . . . . . . . . . . . . 81

5.3 .7 Condições de fronteira na superfície livre . . . . . . . . . . . . . . . . . . . 83

5.3.8 Cálculo da pressão na superfície livre . . . . . . . . . . . . . . . . . . . . 84

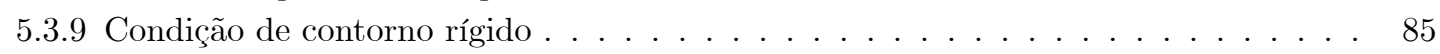

5.3 .10 Condição de contorno em injetores . . . . . . . . . . . . . . . . . . . . 86

5.3.11 Condição de contorno em ejetores continuados . . . . . . . . . . . . . . 87

6 Resultados: GENSMAC-SXPP $\quad 89$

6.1 Introdução . . . . . . . . . . . . . . . . . . . . . . . . . . 89

6.2 Escoamento em um canal . . . . . . . . . . . . . . . . . . . . . 90

6.2 .1 Introdução . . . . . . . . . . . . . . . . . . . . . . . . 90 90

6.2 .2 Verificação do método numérico: modelo Oldroyd-B . . . . . . . . . . . . . . . . . 91

6.2.3 Análise de convergência do método numérico: modelo SXPP . . . . . . . . . . . 95

$6.3 \mathrm{O}$ inchamento do extrudado . . . . . . . . . . . . . . . . . . . . 98

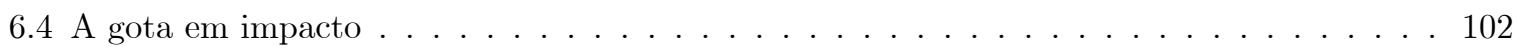

6.5 O jato oscilante . . . . . . . . . . . . . . . . . . 105

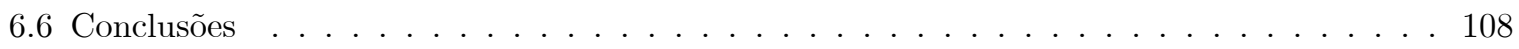

$\begin{array}{lr}7 \text { Considerações finais } & 109\end{array}$

A Análise do GENSMAC-SXPP: o escoamento de Hagen-Poiseuille 111

A.1 Introdução . . . . . . . . . . . . . . . . . . . . . . . . 111

A.2 Influência de $\alpha \ldots \ldots \ldots \ldots \ldots \ldots \ldots \ldots \ldots \ldots \ldots$

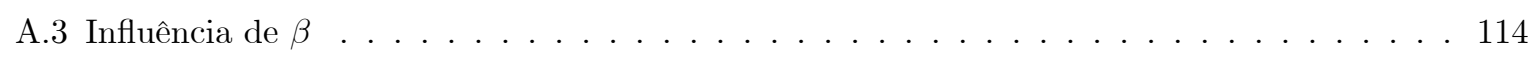

A.4 Influência de $\gamma \ldots \ldots \ldots \ldots \ldots \ldots \ldots \ldots \ldots$

A.5 Influência de $W e \ldots \ldots \ldots \ldots \ldots \ldots \ldots \ldots \ldots$

A.6 Influência de $Q \ldots \ldots \ldots \ldots \ldots \ldots \ldots \ldots$ 
B Análise do GENSMAC-SXPP: o inchamento do extrudado 123

B.1 Introdução. . . . . . . . . . . . . . . . . . . . . . . . . . . . . 123

B.2 Modelo Oldroyd-B . . . . . . . . . . . . . . . . . . . . . . . . . 123

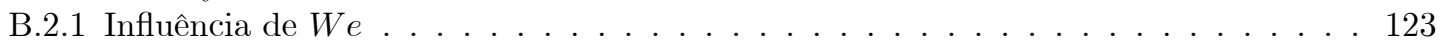

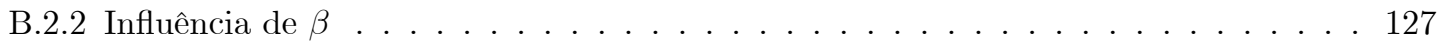

B.3 Modelo SXPP . . . . . . . . . . . . . . . . . . . . . 130

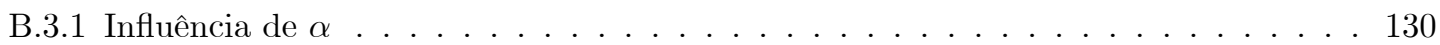

B.3.2 Influência de $\beta \ldots \ldots \ldots \ldots \ldots \ldots$

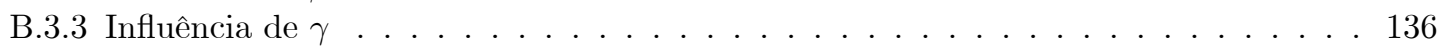

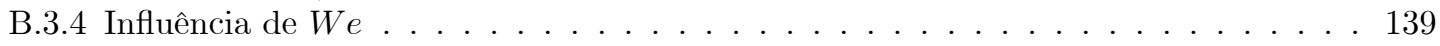

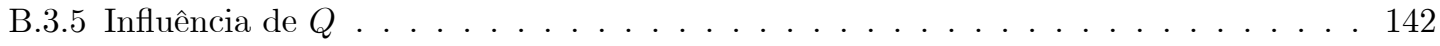

C Simulações numéricas para o problema da gota em impacto 145

C.1 Introdução. . . . . . . . . . . . . . . . . . . . . . . . . . . . 145

C.2 Modelo newtoniano . . . . . . . . . . . . . . . . . . . . . . . . . . 145

C.3 Modelos viscoelásticos (Oldroyd-B e SXPP) . . . . . . . . . . . . . . . . . . . . 147

$\begin{array}{lr}\text { Bibliografia } & 157\end{array}$ 
CONTEÚDO 


\section{Lista de Figuras}

1.1.1 Ilustração do efeito Barus em um fluido não-newtoniano. Na esquerda, repare que existe um aumento na largura da coluna de fluido que é expulso várias vezes maior que o diâmetro do injetor, caracterizando o efeito citado. Na direita, com um fluido newtoniano, o fenômeno é imperceptível, com a coluna de fluido extrudado mantendo um diâmetro aproximadamente igual ao do bico injetor. . . . . . . . . . . . . . . . . .

1.1.2 Esquema de uma molécula polimérica do tipo Pom-Pom. . . . . . . . . . . . . . .

2.1.1 Representação da célula $C_{i, j}$, tipo MAC, em $t=t_{n}$ : a velocidade é avaliada nas faces e, as demais grandezas (representada pela variável genérica $\phi$ ), no centro da célula. . . . . . . .

2.1.2 Classificação das células do domínio computacional (a) para a simulação do escoamento representado em (b), extraído de [TM99]. Em (a), as células em branco são do tipo E. . . 14

2.1.3 Aproximações da superfície livre em célula $\mathbf{S}$ : casos de $90^{\circ}$. . . . . . . . . . . . . . 16

2.1.4 Aproximações da superfície livre em célula S: casos de $45^{\circ}$. . . . . . . . . . . . . . . . . 16

3.2.1 Número médio de iterações na resolução dos sistemas lineares envolvendo $\widetilde{\mathbf{u}}(\mathrm{a})$ e $\psi(\mathrm{b})$ presentes nas FE-EE e FI-CN . . . . . . . . . . . . . . . . . . . .

3.2.2 Tempo de CPU médio gasto na resolução dos sistemas lineares envolvendo $\widetilde{\mathbf{u}}(\mathrm{a})$ e $\psi(\mathrm{b})$ presentes nas FE-EE e FI-CN. Em (a), a curva associada à FE-EE corresponde ao tempo de CPU médio associado ao cálculo explícito de $\widetilde{\mathbf{u}} \ldots$. . . . . . . . . . . . . .

3.2.3 Comportamento do número de condição, na norma $l_{2}$, na aplicação do pré-condicionador SOR aos sistemas lineares obtidos com as FE-EE e FI-CN, em $t=3.0$. Analisamos o

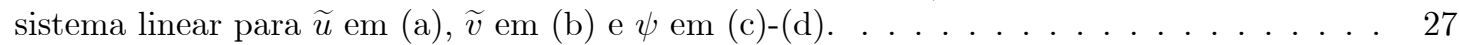

3.3.1 Partícula $P$ em uma célula $C_{i, j}$ (a) e esquema de interpolação das velocidades $u$ (b) e $v$ (c). 29

4.2.1 Análise comparativa, em função de $t$, do desempenho do pré-condicionador SOR (aplicado ao BiCGstab) com relação ao CG/BiCG-Jacobi, para a solução dos sistemas lineares obtidos pela FI-CN no inchamento do extrudado de um fluido newtoniano $(R e=0.1)$ : número médio de iterações na resolução dos sistemas lineares para $\widetilde{\mathbf{u}}$ (a) e $\psi$ (c), com tempo de

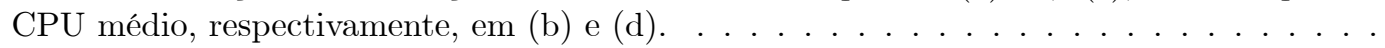

4.2.2 Solução numérica da FI-CN para $u$ (a) e $v$ (b) no inchamento do extrudado de um fluido newtoniano $(R e=0.1, t=45.0)$ utilizando o BiCGstab-SOR na resolução dos sistemas lineares obtidos na discretização das equações de momento (com $\omega=1.4)$ e de Poisson $(\operatorname{com} \omega=1.8) \ldots \ldots \ldots \ldots \ldots \ldots \ldots \ldots$

4.2.3 Análise comparativa, em função de $t$, do desempenho do pré-condicionador SOR (aplicado ao BiCGstab) com relação ao CG/BiCG-Jacobi, para a solução dos sistemas lineares obtidos pela FI-CN no fountain flow de um fluido newtoniano $(R e=0.1)$ : número médio de iterações na resolução dos sistemas lineares para $\widetilde{\mathbf{u}}(\mathrm{a})$ e $\psi(\mathrm{c})$, com tempo de CPU médio, respectivamente, em $(\mathrm{b})$ e $(\mathrm{d}) \ldots \ldots \ldots \ldots \ldots \ldots$ 
4.2.4 Solução numérica da FI-CN para $u$ (a) e $v$ (b) no fountain flow de um fluido newtoniano ( $R e=0.1, t=100.0)$ utilizando o BiCGstab-SOR na resolução dos sistemas lineares obtidos na discretização das equações de momento (com $\omega=1.6$ ) e de Poisson (com $\omega=1.8) \ldots \ldots \ldots \ldots \ldots \ldots \ldots \ldots \ldots \ldots$

4.2.5 Análise comparativa, em função de $t$, do desempenho do pré-condicionador SOR (aplicado ao BiCGstab) com relação ao CG/BiCG-Jacobi, para a solução dos sistemas lineares obtidos pela FI-CN no enchimento estável de um fluido newtoniano $(R e=0.8)$ : número médio de iterações na resolução dos sistemas lineares para $\widetilde{\mathbf{u}}(\mathrm{a})$ e $\psi$ (c), com tempo de CPU médio, respectivamente, em $(\mathrm{b})$ e $(\mathrm{d}) \ldots \ldots \ldots \ldots \ldots \ldots$

4.2.6 Solução numérica da FI-CN para $u$ (a) e $v$ (b) no enchimento estável de um fluido newtoniano $(R e=0.8, t=200.0)$ utilizando o BiCGstab-SOR na resolução dos sistemas lineares obtidos na discretização das equações de momento ( $\operatorname{com} \omega=1.0)$ e de Poisson $(\operatorname{com} \omega=1.8)$. 41

4.2.7 Análise comparativa, em função de $t$, do desempenho do pré-condicionador SOR (aplicado ao BiCGstab) com relação ao CG/BiCG-Jacobi, para a solução dos sistemas lineares obtidos pela FI-CN na contração 4:1 de um fluido newtoniano $(R e=0.05)$ : número médio de iterações na resolução dos sistemas lineares para $\widetilde{\mathbf{u}}(\mathrm{a})$ e $\psi(\mathrm{c})$, com tempo de CPU médio, respectivamente, em $(\mathrm{b})$ e $(\mathrm{d}) \ldots \ldots \ldots \ldots \ldots \ldots$

4.2.8 Solução numérica da FI-CN para $u$ (a) e $v$ (b) na contração 4:1 de um fluido newtoniano ( $R e=0.05, t=450.0)$ utilizando o BiCGstab-SOR na resolução dos sistemas lineares obtidos na discretização das equações de momento (com $\omega=1.4$ ) e de Poisson (com $\omega=1.8) \ldots \ldots \ldots \ldots \ldots \ldots \ldots \ldots \ldots \ldots \ldots$

4.2.9 Análise comparativa, em função de $t$, do desempenho do pré-condicionador SOR (aplicado ao BiCGstab) com relação ao CG/BiCG-Jacobi, para a solução dos sistemas lineares obtidos pela FI-CN no jet flow de um fluido newtoniano $(R e=0.2)$ : número médio de iterações na resolução dos sistemas lineares para $\widetilde{\mathbf{u}}(\mathrm{a})$ e $\psi(\mathrm{c})$, com tempo de CPU médio, respectivamente, em (b) e (d) . . . . . . . . . . . . . . . . .

4.2.10 Solução numérica da FI-CN para $u$ (a) e $v(\mathrm{~b})$ no jet flow de um fluido newtoniano $(R e=$ $0.2, t=70.0)$ utilizando o BiCGstab-SOR na resolução dos sistemas lineares obtidos na discretização das equações de momento $(\operatorname{com} \omega=1.4)$ e de Poisson $(\operatorname{com} \omega=1.8)$. . . . .

4.3.1 Erro na massa (a), tempo de CPU total (b) e função perda total (c), todos em função de $\delta t$, em $t=t_{F}$. Em (d) temos a evolução do erro na massa no tempo com os diferentes métodos usando o maior passo temporal admitido pela FE-EE $\left(F_{v i s c}=0.3872\right)$. . . . . .

4.3.2 Simulações numéricas do enchimento estável de um fluido newtoniano $(R e=0.1)$ utilizando EE para movimentação das partículas marcadoras com FI-CN (a), FI-EI (b) e FE-EE (c), usando $F_{\text {visc }}=0.121$. Na figura, estão representadas as curvas de nível para $u$ obtidas nos

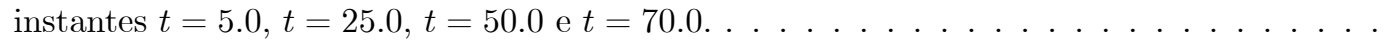

4.3.3 Simulações numéricas do enchimento estável de um fluido newtoniano $(R e=0.1)$ utilizando RK21 para movimentação das partículas marcadoras com FI-CN (a), FI-EI (b) e FE-EE (c), usando $F_{v i s c}=0.121$. Na figura, estão representadas as curvas de nível para $u$ obtidas

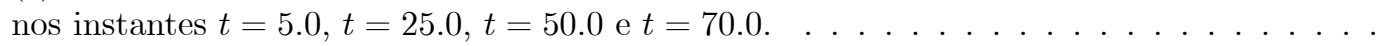

4.3.4 Simulações numéricas do enchimento de uma caixa contendo fluido, ambos newtonianos ( $R e=0.8)$, utilizando EE (a) e RK21 (b) para movimentação das partículas marcadoras com FI-CN, usando $F_{v i s c}=12.8$. Na figura, estão representadas as curvas de nível para $u$ obtidas nos instantes $t=7.0, t=21.0, t=63.0$ e $t=98.0 \ldots \ldots \ldots$. . . . . . . . .

4.3.5 Erro na massa (a), tempo de CPU total (b) e função perda total (c), todos em função de $\delta t$, em $t=t_{F}$. Em (d) temos a evolução do erro na massa no tempo com os diferentes métodos usando o maior passo temporal admitido pela $\mathrm{FE}-\mathrm{EE}\left(F_{v i s c}=0.3\right)$. . . . . . . .

4.3.6 Erro na massa (a), tempo de CPU total (b) e função perda total (c), todos em função de $\delta t$, em $t=t_{F}$. Em (d) temos a evolução do erro na massa no tempo com os diferentes métodos usando o maior passo temporal admitido pela FE-EE $\left(F_{v i s c}=0.32\right)$. . . . . . . .

4.3.7 Simulações numéricas da gota em impacto de um fluido newtoniano $(R e=0.5)$ utilizando EE (coluna esquerda) e RK21 (coluna direita) com: FI-CN (a), FI-EI (b) e FE-EE (c), em $t=1.5$. Para a FI foi usado $F_{v i s c}=51.2 \mathrm{e}$, para a FE, $F_{v i s c}=0.128 \ldots \ldots \ldots$ 
4.3.8 Erro na massa (a), tempo de CPU total (b) e função perda total (c), todos em função de $\delta t$, em $t=t_{F}$. Em (d) temos a evolução do erro na massa no tempo com os diferentes métodos usando o maior passo temporal admitido pela FE-EE $\left(F_{v i s c}=0.32\right)$. . . . . . .

4.3.9 Simulações numéricas do inchamento do extrudado de um fluido newtoniano $(R e=0.05)$ utilizando EE para movimentação das partículas marcadoras com FI-CN (a), FI-EI (b) e FE-EE (c), usando, respectivamente, $F_{\text {visc }}=160.0, F_{\text {visc }}=160.0$ e $F_{\text {visc }}=0.32$. Na figura, estão representadas as curvas de nível para $v$ obtidas nos instantes $t=12.0$ e $t=23.0$. 61

4.3.10 Simulações numéricas do inchamento do extrudado de um fluido newtoniano $(R e=0.05)$ utilizando RK21 para movimentação das partículas marcadoras com FI-CN (a), FI-EI (b) e FE-EE (c), usando, respectivamente, $F_{\text {visc }}=160.0, F_{v i s c}=160.0$ e $F_{v i s c}=0.32$. Na figura, estão representadas as curvas de nível para $v$ obtidas nos instantes $t=12.0$ e $t=23.0$. 62

5.3.1 Ilustração de fronteira rígida paralela ao eixo $y(\mathrm{a})$, paralela ao eixo $x$ (b) e com mudança brusca na orientação (c) . . . . . . . . . . . . . . . . . . 77

5.3.2 Ilustração de uma célula de ejetores continuados paralelos ao eixo $y$ (a) e ao eixo $x$ (b). $\quad$. 80

5.3.3 Superfície livre interceptando duas faces opostas (a) e adjacentes (b) de uma célula $C_{i, j}$ tipo S. Em (a), admitimos $\vec{n}$ paralelo ao eixo $y$ e, em (b), supomos que $\vec{n}$ forma um ângulo de $45^{\circ}$ com os eixos coordenados. . . . . . . . . . . . . . . . .

5.3.4 Ilustração de uma célula $C_{i, j}$ em fronteiras rígidas e impermeáveis paralelas ao eixo $y$ (a) e ao eixo $x(\mathrm{~b}) \ldots \ldots \ldots \ldots \ldots \ldots \ldots \ldots$

5.3.5 Ilustração da célula $C_{i, j}$ de injetores paralelos ao eixo $y$ (a) e ao eixo $x$ (b). . . . . . . .

5.3.6 Ilustração da célula $C_{i, j}$ de ejetores continuados paralelos ao eixo $y$ (a) e ao eixo $x$ (b). . . 87 88

6.2.1 Simulação numérica da FI-EI para o fountain flow de um fluido Oldroyd-B (Re $=0.01$, $W e=2.0, \beta=0.5$ ) em: $t=2.0(\mathrm{a}) ; t=5.5(\mathrm{~b}) ; t=6.5(\mathrm{c}) ; t=8.0(\mathrm{~d}) . \ldots . .$.

6.2.2 Comparação das soluções numéricas obtidas pela FI-CN com a solução analítica (coluna esquerda) e comportamento do erro (coluna direita) em cada malha empregada para simular o escoamento totalmente desenvolvido em um canal $(t=100.0)$ de um fluido Oldroyd-B $(R e=0.01, W e=2.0, \beta=0.5) \ldots \ldots \ldots \ldots \ldots \ldots \ldots$

6.2.3 Simulação numérica da FI-CN para o fountain flow de um fluido $\operatorname{SXPP}(R e=0.01$, $W e=2.0, \alpha=0.2, \beta=0.5, \gamma=0.5, Q=2.0) \mathrm{em}: t=2.0(\mathrm{a}) ; t=4.0(\mathrm{~b}) ; t=6.0$ (c); $t=8.0(\mathrm{~d}) \ldots \ldots \ldots \ldots \ldots \ldots \ldots \ldots \ldots \ldots \ldots \ldots \ldots \ldots \ldots \ldots \ldots \ldots \ldots \ldots \ldots \ldots \ldots \ldots$

6.2.4 Soluções numéricas obtidas pela FI-EI em cada malha empregada para simular o escoamento totalmente desenvolvido em um canal $(t=100.0)$ de um fluido $\operatorname{SXPP}(R e=0.1$, $W e=2.0, \alpha=0.2, \beta=0.5, \gamma=0.5, Q=2.0) \ldots \ldots \ldots \ldots$

6.2.5 Soluções numéricas obtidas pela FI-CN em cada malha empregada para simular o escoamento totalmente desenvolvido em um canal $(t=100.0)$ de um fluido $\operatorname{SXPP}(R e=0.01$, $W e=2.0, \alpha=0.2, \beta=0.5, \gamma=0.5, Q=2.0) \ldots \ldots \ldots \ldots$

6.3.1 Simulações numéricas para o inchamento do extrudado $(t=24.0)$ de um fluido Oldroyd-B $(R e=0.05, W e=2.0, \beta=0.5)$ com: FI-CN (a); FI-EI (b); FE-RK22 (c); FE-EE (d). Na coluna esquerda foi usado EE para movimentar as partículas marcadoras, enquanto que, na coluna direita, adotou-se o RK21. . . . . . . . . . . . . . . . .

6.3.2 Simulações numéricas para o inchamento do extrudado $(t=22.0)$ de um fluido SXPP $(R e=0.05, W e=20.0, \alpha=0.01, \beta=0.5, \gamma=0.8, Q=8.0)$ com: FI-CN (a); FI-EI (b); FE-RK22 (c); FE-EE (d). Na coluna esquerda foi usado EE para movimentar as partículas marcadoras, enquanto que, na coluna direita, adotou-se o RK21. . . . . . . . . . .

6.4.1 Solução numérica para $u$ no problema da gota em impacto $(t=7.0)$ de um fluido Oldroyd$\mathrm{B}(R e=5.0, W e=1.0, \beta=0.1)$ com: FI-CN (a); FI-EI (b); FE-RK22 (c); FE-EE (d). Na coluna esquerda foi usado EE para movimentar as partículas marcadoras, enquanto que, na coluna direita, adotou-se o RK21. . . . . . . . . . . . . . . 
6.4.2 Solução numérica para $u$ no problema da gota em impacto $(t=7.0)$ de um fluido SXPP $(R e=5.0, W e=1.0, \alpha=0.01, \beta=0.1, \gamma=0.8, Q=4.0) \operatorname{com}:$ FI-CN (a); FI-EI (b); FE-RK22 (c); FE-EE (d). Na coluna esquerda foi usado EE para movimentar as partículas marcadoras, enquanto que, na coluna direita, adotou-se o RK21.

6.5.1 Solução numérica para $v$ no jato oscilante de um fluido Oldroyd-B $(R e=0.01, W e=2.0$, $\beta=0.1$ ) com: FI-CN (a); FI-EI (b). Visualização nos instantes $t=18.0, t=19.0, t=23.0$, $t=30.0, t=50.0, t=75.0, t=100.0, t=150.0 \ldots \ldots \ldots \ldots \ldots \ldots$

6.5.2 Solução numérica para $v$ no jato oscilante de um fluido $\operatorname{SXPP}(R e=0.01, W e=2.0$, $\alpha=0.1, \beta=0.1, \gamma=0.8, Q=4.0)$ com: FI-CN (a); FI-EI (b). Visualização nos instantes $t=18.0, t=19.0, t=23.0, t=30.0, t=50.0, t=75.0, t=100.0, t=150.0 \ldots \ldots .$.

A.2.1 Simulações numéricas para o escoamento de Hagen-Poiseuille de um fluido $\mathrm{SXPP}(R e=$ $0.05, W e=3.0, \beta=0.12, \gamma=0.35, Q=2.0)$ : perfis de solução totalmente desenvolvida $(t=100.0)$ de $u$ e $\lambda$, em função de $\alpha$, obtidos com a FI-CN $(-)$ e com a FI-EI (x). . . . .

A.2.2 Simulações numéricas para o escoamento de Hagen-Poiseuille de um fluido SXPP $(R e=$ $0.05, W e=3.0, \beta=0.12, \gamma=0.35, Q=2.0)$ : perfis de solução totalmente desenvolvida $(t=100.0)$ de $T$, em função de $\alpha$, obtidos com a FI-CN $(-)$ e com a FI-EI (x). . . . . . .

A.3.1 Simulações numéricas para o escoamento de Hagen-Poiseuille de um fluido SXPP $(R e=$ $0.05, W e=3.0, \alpha=0.15, \gamma=0.35, Q=2.0)$ : perfis de solução totalmente desenvolvida $(t=100.0)$ de $u$ e $\lambda$, em função de $\beta$, obtidos com a FI-CN $(-)$ e com a FI-EI (x). . . . .

A.3.2 Simulações numéricas para o escoamento de Hagen-Poiseuille de um fluido SXPP $(R e=$ $0.05, W e=3.0, \alpha=0.15, \gamma=0.35, Q=2.0)$ : perfis de solução totalmente desenvolvida $(t=100.0)$ de $T$, em função de $\beta$, obtidos com a FI-CN $(-)$ e com a FI-EI (x). . . . . . .

A.4.1 Simulações numéricas para o escoamento de Hagen-Poiseuille de um fluido SXPP $(R e=$ $0.05, W e=3.0, \alpha=0.15, \beta=0.12, Q=2.0)$ : perfis de solução totalmente desenvolvida $(t=100.0)$ de $u$ e $\lambda$, em função de $\gamma$, obtidos com a FI-CN $(-)$ e com a FI-EI $(\mathrm{x})$. . . . .

A.4.2 Simulações numéricas para o escoamento de Hagen-Poiseuille de um fluido SXPP $(R e=$ $0.05, W e=3.0, \alpha=0.15, \beta=0.12, Q=2.0)$ : perfis de solução totalmente desenvolvida $(t=100.0)$ de $T$, em função de $\gamma$, obtidos com a FI-CN $(-)$ e com a FI-EI (x). . . . . . .

A.5.1 Simulações numéricas para o escoamento de Hagen-Poiseuille de um fluido SXPP $(R e=$ $0.05, \alpha=0.15, \beta=0.12, \gamma=0.35, Q=2.0)$ : perfis de solução totalmente desenvolvida $(t=100.0)$ de $u$ e $\lambda$, em função de $W e$, obtidos com a FI-CN (-) e com a FI-EI (x). . . .

A.5.2 Simulações numéricas para o escoamento de Hagen-Poiseuille de um fluido $\operatorname{SXPP}(R e=$ $0.05, \alpha=0.15, \beta=0.12, \gamma=0.35, Q=2.0)$ : perfis de solução totalmente desenvolvida $(t=100.0)$ de $T$, em função de $W e$, obtidos com a FI-CN $(-)$ e com a FI-EI (x). . . . . .

A.6.1 Simulações numéricas para o escoamento de Hagen-Poiseuille de um fluido SXPP $(R e=$ $0.05, W e=3.0, \alpha=0.15, \beta=0.12, \gamma=0.35)$ : perfis de solução totalmente desenvolvida $(t=100.0)$ de $u$ e $\lambda$, em função de $Q$, obtidos com a FI-CN $(-)$ e com a FI-EI (x). . . . .

A.6.2 Simulações numéricas para o escoamento de Hagen-Poiseuille de um fluido SXPP $(R e=$ $0.05, W e=3.0, \alpha=0.15, \beta=0.12, \gamma=0.35)$ : perfis de solução totalmente desenvolvida $(t=100.0)$ de $T$, em função de $Q$, obtidos com a FI-CN $(-)$ e com a FI-EI $(\mathrm{x})$. . . . . . .

B.2.1 Taxa de inchamento $S_{r}$ de um fluido Oldroyd-B $(R e=0.05, \beta=0.5)$ em função de $W e$ para as soluções numéricas obtidas com: FI-EI (a); FI-CN (b) . . . . . . . . . . . . . .

B.2.2 Soluções numéricas (curvas de nível para $u(\mathrm{a}), v(\mathrm{~b}), p(\mathrm{c}), T^{x x}$ (d), $T^{x y}$ (e) e $T^{y y}$ (f)) da FI-EI para o inchamento do extrudado de um fluido Oldroyd-B $(R e=0.05, \beta=0.5)$ com $W e=0.2$ (coluna esquerda) e $W e=1.8$ (coluna direita), respectivamente, nos instantes $t=15.0$ e $t=23.0 \ldots \ldots \ldots \ldots \ldots \ldots \ldots \ldots \ldots \ldots$

B.2.3 Simulações numéricas para o inchamento do extrudado de um fluido Oldroyd-B $(R e=$ $0.05, \beta=0.5)$ com $W e=0.4(\mathrm{a}), W e=0.8(\mathrm{~b}), W e=1.2(\mathrm{c}), W e=1.6$ (d) e $W e=2.0$ (e) obtidas com a FI-CN nos instantes $t=10.0, t=15.0$ e $t=30.0 \ldots \ldots$. . . . . . . 126

B.2.4 Taxa de inchamento $S_{r}$ de um fluido Oldroyd-B $(R e=1.0, W e=1.0)$ em função de $\beta$ para as soluções numéricas obtidas com: FI-EI (a); FI-CN (b). . . . . . . . . . . . . . 
B.2.5 Soluções numéricas (curvas de nível para $u$ (a), $v(\mathrm{~b}), p(\mathrm{c}), T^{x x}$ (d), $T^{x y}$ (e) e $T^{y y}$ (f)) da FI-CN para o inchamento do extrudado de um fluido Oldroyd-B $(R e=1.0, W e=1.0)$ com $\beta=0.1$ (coluna esquerda) e $\beta=0.9$ (coluna direita), respectivamente, nos instantes

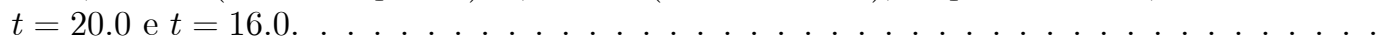

B.2.6 Simulações numéricas para o inchamento do extrudado de um fluido Oldroyd-B (Re $=1.0$, $W e=1.0$ ) com $\beta=0.1$ (a), $\beta=0.3$ (b), $\beta=0.5$ (c), $\beta=0.7$ (d) e $\beta=0.9$ (e) obtidas com a FI-EI nos instantes $t=10.0, t=15.0$ e $t=30.0 \ldots \ldots \ldots \ldots \ldots$

B.3.1 Taxa de inchamento $S_{r}$ de um fluido $\operatorname{SXPP}(R e=0.05, W e=10.0, \beta=0.5, \gamma=0.8$, $Q=8.0$ ) em função de $\alpha$ para as soluções numéricas obtidas com: FI-EI (a); FI-CN (b). . 130

B.3.2 Soluções numéricas (curvas de nível para $u$ (a), $v$ (b), $p$ (c), $T^{x x}$ (d), $T^{x y}$ (e), $T^{y y}$ (f) e $\lambda$ (g)) da FI-EI para o inchamento do extrudado de um fluido $\operatorname{SXPP}(R e=0.05, W e=10.0$, $\beta=0.5, \gamma=0.8, Q=8.0$ ) com $\alpha=0.0$ (coluna esquerda) e $\alpha=0.8$ (coluna direita),

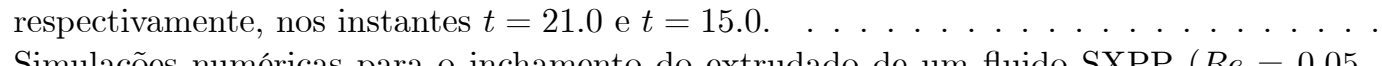

B.3.3 Simulações numéricas para o inchamento do extrudado de um fluido SXPP $(R e=0.05$, $W e=10.0, \beta=0.5, \gamma=0.8, Q=8.0) \operatorname{com} \alpha=0.0(\mathrm{a}), \alpha=0.1$ (b), $\alpha=0.2(\mathrm{c}), \alpha=0.4$ (d) e $\alpha=0.8$ (e) obtidas com a FI-CN nos instantes $t=10.0, t=15.0$ e $t=65.0 \ldots \ldots$.

B.3.4 Taxa de inchamento $S_{r}$ de um fluido $\operatorname{SXPP}(R e=0.05, W e=10.0, \alpha=0.01, \gamma=0.3$, $Q=8.0$ ) em função de $\beta$ para as soluções numéricas obtidas com: FI-EI (a); FI-CN (b). . 133

B.3.5 Soluções numéricas (curvas de nível para $u$ (a), $v$ (b), $p(\mathrm{c}), T^{x x}$ (d), $T^{x y}$ (e), $T^{y y}$ (f) e $\lambda$ (g)) da FI-CN para o inchamento do extrudado de um fluido SXPP $(R e=0.05, W e=10.0$, $\alpha=0.01, \gamma=0.3, Q=8.0$ ) com $\beta=0.2$ (coluna esquerda) e $\beta=0.9$ (coluna direita), respectivamente, nos instantes $t=19.0$ e $t=15.0$.

B.3.6 Simulações numéricas para o inchamento do extrudado de um fluido $\operatorname{SXPP}(R e=0.05$, $W e=10.0, \alpha=0.01, \gamma=0.3, Q=8.0) \operatorname{com} \beta=0.2$ (a), $\beta=0.4$ (b), $\beta=0.6$ (c), $\beta=0.8$ (d) e $\beta=0.9$ (e) obtidas com a FI-EI nos instantes $t=10.0, t=15.0$ e $t=30.0 . \quad \ldots \ldots$.

B.3.7 Taxa de inchamento $S_{r}$ de um fluido $\operatorname{SXPP}(R e=0.05, W e=10.0, \alpha=0.01, \beta=0.5$, $Q=8.0$ ) em função de $\gamma$ para as soluções numéricas obtidas com: FI-EI (a); FI-CN (b). . 136

B.3.8 Soluções numéricas (curvas de nível para $u$ (a), $v(\mathrm{~b}), p(\mathrm{c}), T^{x x}$ (d), $T^{x y}(\mathrm{e}), T^{y y}$ (f) e $\lambda$ (g)) da FI-EI para o inchamento do extrudado de um fluido SXPP $(R e=0.05, W e=10.0$, $\alpha=0.01, \beta=0.5, Q=8.0)$ com $\gamma=0.2$ (coluna esquerda) e $\gamma=0.9$ (coluna direita), respectivamente, nos instantes $t=17.0$ e $t=21.0 \ldots \ldots \ldots \ldots \ldots \ldots \ldots \ldots \ldots \ldots$

B.3.9 Simulações numéricas para o inchamento do extrudado de um fluido SXPP $(R e=0.05$, $W e=10.0, \alpha=0.01, \beta=0.5, Q=8.0) \operatorname{com} \gamma=0.2(\mathrm{a}), \gamma=0.25(\mathrm{~b}), \gamma=0.4(\mathrm{c}), \gamma=0.6$ (d) e $\gamma=0.8$ (e) obtidas com a FI-CN nos instantes $t=10.0, t=15.0$ e $t=30.0$. . . . .

B.3.10 Taxa de inchamento $S_{r}$ de um fluido $\operatorname{SXPP}(R e=0.05, \alpha=0.01, \beta=0.5, \gamma=0.8$, $Q=8.0$ ) em função de $W e$ para as soluções numéricas obtidas com: FI-EI (a); FI-CN (b). 139

B.3.11 Soluções numéricas (curvas de nível para $u$ (a), $v(\mathrm{~b}), p(\mathrm{c}), T^{x x}$ (d), $T^{x y}$ (e), $T^{y y}$ (f) e $\lambda$ (g)) da FI-EI para o inchamento do extrudado de um fluido $\operatorname{SXPP}(R e=0.05, \alpha=0.01$, $\beta=0.5, \gamma=0.8, Q=8.0)$ com $W e=0.5$ (coluna esquerda) e $W e=15.0$ (coluna direita), respectivamente, nos instantes $t=15.0$ e $t=21.0$.

B.3.12 Simulações numéricas para o inchamento do extrudado de um fluido SXPP $(R e=0.05$, $\alpha=0.01, \beta=0.5, \gamma=0.8, Q=8.0)$ com $W e=0.5$ (a), $W e=2.0(\mathrm{~b}), W e=7.0$ (c), $W e=11.0(\mathrm{~d})$ e $W e=15.0(\mathrm{e})$ obtidas com a FI-CN nos instantes $t=10.0, t=15.0 \mathrm{e}$

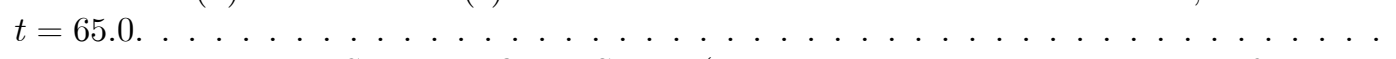

B.3.13 Taxa de inchamento $S_{r}$ de um fluido SXPP $(R e=0.05, W e=10.0, \alpha=0.01, \beta=0.5$, $\gamma=0.3$ ) em função de $Q$ para as soluções numéricas obtidas com: FI-EI (a); FI-CN (b). . 142

B.3.14 Soluções numéricas (curvas de nível para $u$ (a), $v$ (b), $p$ (c), $T^{x x}$ (d), $T^{x y}$ (e), $T^{y y}$ (f) e $\lambda$ (g)) da FI-CN para o inchamento do extrudado de um fluido SXPP $(R e=0.05, W e=10.0$, $\alpha=0.01, \beta=0.5, \gamma=0.3)$ com $Q=1.0$ (coluna esquerda) e $Q=20.0$ (coluna direita), respectivamente, nos instantes $t=16.0$ e $t=19.0 \ldots \ldots \ldots \ldots$
Simulacões numéricas para o inchamento do extrudado de um fluido $\operatorname{SXPP}(R e=0.05$.

B.3.15 Simulações numéricas para o inchamento do extrudado de um fluido $\operatorname{SXPP}(R e=0.05$,
$W e=10.0, \alpha=0.01, \beta=0.5, \gamma=0.3)$ com $Q=1.0$ (a), $Q=4.0$ (b), $Q=11.0$ (c), $Q=15.0$ (d) e $Q=20.0$ (e) obtidas com a FI-EI nos instantes $t=10.0, t=15.0$ e $t=65.0 .144$ 
C.2.1 Solução numérica para $u$ no problema da gota em impacto de um fluido newtoniano $(R e=$ 5.0) com a FI-EI em: $t=1.3$ (a); $t=1.4$ (b); $t=1.5$ (c); $t=1.7$ (d); $t=1.9$ (e); $t=2.1$ (f); $t=3.0(\mathrm{~g}) ; t=6.0(\mathrm{~h}) ; t=8.5(\mathrm{i}) ; t=12.5(\mathrm{j}) ; t=19.0(\mathrm{k}) ; t=30.0(\mathrm{l}) . \ldots \ldots .146$

C.3.1 Solução numérica para $u$ no problema da gota em impacto de um fluido Oldroyd-B $(R e=$ 5.0, $W e=1.0, \beta=0.1$ ) com a FI-CN em: $t=1.3(\mathrm{a}) ; t=1.5(\mathrm{~b}) ; t=2.0(\mathrm{c}) ; t=2.2$ (d); $t=2.4(\mathrm{e}) ; t=2.7(\mathrm{f}) ; t=3.7(\mathrm{~g}) ; t=3.9(\mathrm{~h}) ; t=4.0(\mathrm{i}) ; t=4.3(\mathrm{j}) ; t=5.9(\mathrm{k}) ; t=6.0$ $(\mathrm{l}) ; t=6.1(\mathrm{~m}) ; t=6.4(\mathrm{n}) ; t=6.5(\mathrm{o}) ; t=6.6(\mathrm{p}) ; t=6.8(\mathrm{q}) ; t=7.1(\mathrm{r}) ; t=7.3(\mathrm{~s}) ;$

$t=8.0(\mathrm{t}) ; t=9.6(\mathrm{u}) ; t=11.2(\mathrm{v}) ; t=23.3(\mathrm{x}) ; t=30.0(\mathrm{y}) \ldots \ldots \ldots \ldots \ldots$
C.3.2 Solução numérica para $u$ no problema da gota em impacto de um fluido Oldroyd-B $(R e=$ 5.0, $W e=2.5, \beta=0.1$ ) com a FI-EI em: $t=1.3$ (a); $t=1.5$ (b); $t=2.0$ (c); $t=2.2$ (d); $t=2.4(\mathrm{e}) ; t=2.7$ (f); $t=3.7(\mathrm{~g}) ; t=3.9(\mathrm{~h}) ; t=4.0(\mathrm{i}) ; t=4.3(\mathrm{j}) ; t=5.9(\mathrm{k}) ; t=6.0$ $(\mathrm{l}) ; t=6.1(\mathrm{~m}) ; t=6.4(\mathrm{n}) ; t=6.5(\mathrm{o}) ; t=6.6(\mathrm{p}) ; t=6.8(\mathrm{q}) ; t=7.1(\mathrm{r}) ; t=7.3(\mathrm{~s}) ;$ $t=8.0(\mathrm{t}) ; t=9.6(\mathrm{u}) ; t=11.2(\mathrm{v}) ; t=23.3(\mathrm{x}) ; t=30.0(\mathrm{y}) \ldots \ldots \ldots \ldots$

C.3.3 Solução numérica para $u$ no problema da gota em impacto de um fluido SXPP (Re $=$ $5.0, W e=1.0, \alpha=0.01, \beta=0.1, \gamma=0.8, Q=4.0)$ com a FI-CN em: $t=1.3$ (a); $t=1.5$ (b); $t=2.0$ (c); $t=2.2$ (d); $t=2.4$ (e); $t=2.5$ (f); $t=3.7$ (g); $t=4.0$ (h); $t=4.1$ (i); $t=4.3(\mathrm{j}) ; t=5.9(\mathrm{k}) ; t=6.0(\mathrm{l}) ; t=6.1(\mathrm{~m}) ; t=6.3(\mathrm{n}) ; t=6.5(\mathrm{o}) ; t=6.6(\mathrm{p}) ; t=6.8$ $(\mathrm{q}) ; t=7.1(\mathrm{r}) ; t=7.3(\mathrm{~s}) ; t=8.0(\mathrm{t}) ; t=9.2(\mathrm{u}) ; t=10.3(\mathrm{v}) ; t=20.5(\mathrm{x}) ; t=30.0(\mathrm{y})$.

C.3.4 Solução numérica para $u$ no problema da gota em impacto de um fluido SXPP (Re $=$ 5.0, $W e=2.5, \alpha=0.01, \beta=0.1, \gamma=0.8, Q=4.0)$ com a FI-EI em: $t=1.3$ (a); $t=1.5$ (b); $t=2.1$ (c); $t=2.4$ (d); $t=3.0(\mathrm{e}) ; t=3.4$ (f); $t=5.0(\mathrm{~g}) ; t=5.3(\mathrm{~h}) ; t=5.4$ (i); $t=5.7(\mathrm{j}) ; t=8.0(\mathrm{k}) ; t=8.2(\mathrm{l}) ; t=8.4(\mathrm{~m}) ; t=8.6(\mathrm{n}) ; t=8.8(\mathrm{o}) ; t=9.3(\mathrm{p}) ; t=9.5$ $(\mathrm{q}) ; t=9.7(\mathrm{r}) ; t=10.4(\mathrm{~s}) ; t=10.9(\mathrm{t}) ; t=14.0(\mathrm{u}) ; t=15.5(\mathrm{v}) ; t=18.2(\mathrm{x}) ; t=30.0$

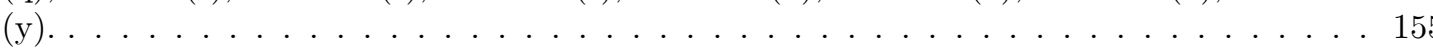




\section{Lista de Tabelas}

3.2.1 Comparação da resolução dos sistemas lineares nas FE-EE e FI-CN, em $t=20.0$. . . . . .

3.2.2 Número de condição na norma $l_{2}$ para os sistemas lineares envolvendo $\widetilde{u}, \widetilde{v}$ e $\psi$ obtidos com as FE-EE e FI-CN, em $t=3.0$. . . . . . . . . . . . . . . . . . . . . . .

3.2.3 Número de condição na norma $l_{2}$ para os sistemas lineares envolvendo $\widetilde{u}, \widetilde{v}$ e $\psi$ obtidos com as FE-EE e FI-CN, em $t=3.0$. Constam, também, os números de condição para tais sistemas com os pré-condicionadores Jacobi e Gauss-Seidel. . . . . . . . . . . . . . . . .

4.2.1 Valores de referência para a solução dos sistemas lineares obtidos pela FI-CN no inchamento do extrudado de um fluido newtoniano $(R e=0.1, t=45.0)$ através do par CG/BiCG-Jacobi. 33

4.2.2 Valores relativos obtidos pela comparação dos demais métodos com o CG/BiCG-Jacobi (valores absolutos na Tabela 4.2.1) . . . . . . . . . . . . . . . .

4.2.3 Valores de referência para a solução dos sistemas lineares obtidos pela FI-CN no fountain flow de um fluido newtoniano $(R e=0.1, t=100.0)$ através do par CG/BiCG-Jacobi. . . .

4.2.4 Valores relativos obtidos pela comparação dos demais métodos iterativos com o CG/BiCGJacobi (valores absolutos na Tabela 4.2.3) . . . . . . . . . . . . . . . . .

4.2.5 Valores de referência para a solução dos sistemas lineares obtidos pela FI-CN no enchimento estável de um fluido newtoniano $(R e=0.8, t=250.0)$ através do par CG/BiCG-Jacobi.

4.2.6 Valores relativos obtidos pela comparação dos demais métodos iterativos com o CG/BiCGJacobi (valores absolutos na Tabela 4.2.5) . . . . . . . . . . . . . . . . .

4.2.7 Valores de referência para a solução dos sistemas lineares obtidos pela FI-CN na contração 4:1 de um fluido newtoniano $(R e=0.05, t=450.0)$ através do par CG/BiCG-Jacobi. . . .

4.2.8 Valores relativos obtidos pela comparação dos demais métodos iterativos com o CG/BiCGJacobi (valores absolutos na Tabela 4.2.7) . . . . . . . . . . . . . . . . . . .

4.2.9 Valores de referência para a solução dos sistemas lineares obtidos pela FI-CN no jet flow de um fluido newtoniano $(R e=0.2, t=70.0)$ através do par CG/BiCG-Jacobi. . . . . . .

4.2.10 Valores relativos obtidos pela comparação dos demais métodos iterativos com o CG/BiCGJacobi (valores absolutos na Tabela 4.2.9) . . . . . . . . . . . . . . . . .

6.2.1 Resultados para o fountain flow de um fluido Oldroyd-B $(R e=0.1, W e=2.0, \beta=0.5) . \quad$. 92

6.2.2 Resultados para o fountain flow de um fluido Oldroyd-B $(R e=0.01, W e=2.0, \beta=0.5)$.

6.2.3 Taxa de convergência para o fountain flow de um fluido $\operatorname{SXPP}(W e=2.0, \beta=0.5, \gamma=0.5$, $\alpha=0.2, Q=2.0) \ldots \ldots \ldots \ldots \ldots \ldots \ldots$

6.2.4 Tempos de CPU para o fountain flow de um fluido $\operatorname{SXPP}(W e=2.0, \beta=0.5, \gamma=0.5$, $\alpha=0.2, Q=2.0)$. . . . . . . . . . . . . . . . . . 98

6.3.1 Comparação dos métodos usados nas simulações numéricas apresentadas na Figura 6.3.1. 101

6.3.2 Comparação dos métodos usados nas simulações numéricas apresentadas na Figura 6.3.2. 101

6.4.1 Comparação dos métodos usados nas simulações numéricas apresentadas na Figura 6.4.1. 105

6.4.2 Comparação dos métodos usados nas simulações numéricas apresentadas na Figura 6.4.2. 105 
B.2.1 Taxas de inchamento de um fluido Oldroyd-B $(R e=0.05, \beta=0.5)$ em função de $W e . \quad$. . 124

B.2.2 Taxas de inchamento de um fluido Oldroyd-B $(R e=1.0, W e=1.0)$ em função de $\beta . \quad$. . . 127

B.3.1 Taxas de inchamento de um fluido $\operatorname{SXPP}(R e=0.05, W e=10.0, \beta=0.5, \gamma=0.8$,

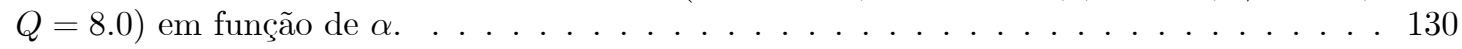

B.3.2 Taxas de inchamento de um fluido $\operatorname{SXPP}(R e=0.05, W e=10.0, \alpha=0.01, \gamma=0.3$, $Q=8.0$ ) em função de $\beta . \ldots \ldots \ldots \ldots \ldots \ldots \ldots \ldots$

B.3.3 Taxas de inchamento de um fluido $\operatorname{SXPP}(R e=0.05, W e=10.0, \alpha=0.01, \beta=0.5$,

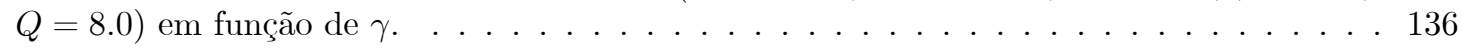

B.3.4 Taxas de inchamento de um fluido $\operatorname{SXPP}(R e=0.05, \alpha=0.01, \beta=0.5, \gamma=0.8, Q=8.0)$

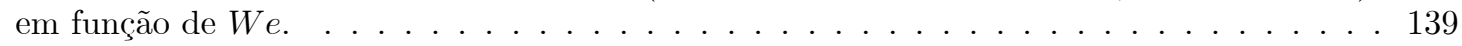

B.3.5 Taxas de inchamento de um fluido $\operatorname{SXPP}(R e=0.05, W e=10.0, \alpha=0.01, \beta=0.5$,

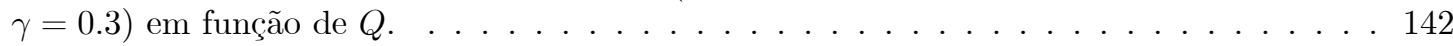


E pur si muove.

Galileu Galilei 


\section{Agradecimentos}

A Deus e Santa Maria, Sua Mãe e Senhora Nossa, pela companhia e proteção.

A meus pais, por tudo e, principalmente, por me aguentarem.

Ao professor José Alberto Cuminato, pela colaboração e paciência.

Aos familiares e amigos que me ajudaram quando precisei.

Aos colegas, funcionários e professores do ICMC-USP, pela convivência.

À FAPESP, pelo suporte financeiro.

A todos que participaram da realização deste trabalho.

Ao mosquito Aedes aegypti, pelas férias forçadas ano passado.

A você, .......................(preencha com seu nome), para não se magoar e pensar que sou mal-agradecido.

Por fim, às pedras do meio do caminho, pelo prazer da superação. 


\section{Resumo}

O tema deste trabalho é o estudo de métodos numéricos para a simulação de escoamentos incompressíveis com superfície livre a baixos Reynolds, por meio da metodologia GENSMAC e suas formulações explícita e implícita. Neste contexto, temos especial interesse na formulação implícita, objetivando o enfraquecimento da restrição de estabilidade parabólica no passo temporal em escoamentos com superfície livre.

O trabalho pode ser dividido em duas partes: na primeira, algumas modificações são discutidas, propostas e testadas com o objetivo de tornar a formulação implícita mais eficiente e precisa; em seguida, aproveitamos os resultados obtidos e generalizamos o método numérico existente para simular escoamentos viscoelásticos modelados pela equação constitutiva SXPP. Em ambos os casos, a formulação explícita também é usada para comparação e teste.

Resultados que demonstram a eficiência e robustez das técnicas desenvolvidas são apresentados por meio da simulação numérica de complexos problemas envolvendo superfície livre.

Palavras-chaves: escoamentos com superfície livre; métodos numéricos; reologia. 


\section{Abstract}

The purpose of this work is the study of numerical methods for low Reynolds number incompressible free surface flows, including the GENSMAC methodology and its explicit and implicit formulations. In this context, we have special interest in the implicit formulation, in order to circumvent the parabolic stability restriction in the time step for free surface flows.

The work can be divided into two parts: firstly, some modifications are proposed, discussed, and tested with the purpose of making the implicit formulation more efficient and accurate; secondly, we take advantage of the results derived in the first part to generalize the numerical method for simulating viscoelastic free surface flows modeled by the constitutive equation SXPP. In both cases, the explicit formulation is also used for comparison and tests.

Results that demonstrate the efficiency and robustness of the developed techniques are presented and illustrated by the numerical simulation of complex problems involving free surface flows.

Keywords: free surface flows; numerical methods; rheology. 


\section{Prefácio}

Certamente, um dos problemas mais interessantes em Mecânica dos Fluidos Computacional (MFC) é a simulação de escoamentos com superfície livre. Com efeito, apenas a resolução das equações de NavierStokes em domínios com fronteira bem definida desperta o respeito e o interesse de muitos pesquisadores. Já quando existe a presença de superfície livre (cuja configuração transiente é uma incógnita adicional ao problema) surgem, no mínimo, dois agravantes: a correta imposição de condições de fronteira na superfície livre e a estratégia de representação da mesma em nível numérico e computacional.

Neste contexto, podemos dizer que a primeira tentativa bem sucedida de desenvolvimento de uma metodologia numérica para a simulação de escoamentos (newtonianos) incompressíveis com superfície livre, via variáveis primitivas, surge com o método MAC (Marker-And-Cell) em [HW65]. Neste, o fluido é representado por uma nuvem de partículas virtuais (langrangeanas) que são convectadas (pelo campo de velocidade) sobre uma malha fixa (euleriana), onde as equações são discretizadas e resolvidas. Assim, célula com partícula possui fluido, enquanto que células vazias são as que não contêm nenhuma dessas partículas.

Quanto à sua formulação, o MAC pode ser interpretado como um método de projeção, muito embora sua formulação original não tenha considerado esse fato ([MI96]). Utilizando aproximações baseadas em diferenças finitas, o principal conceito introduzido pelo MAC foi, certamente, o de malha deslocada (staggered grid), que é um lugar comum para a maior parte dos métodos que surgem a partir de então.

Quase meio século depois de sua criação, as idéias principais do MAC ainda são aproveitadas atualmente. Obviamente, tal metodologia não estava isenta de dificuldades e limitações, as quais foram sendo superadas, ao longo do tempo, pelas técnicas mais recentes. Entre outras, podemos citar, neste contexto: o IMAC (Improved Marker-And-Cell), proposto em [HN71] e que modifica o tratamento das condições de contorno na superfície livre; o SOLA ([HC72]), que modifica o MAC para contornar o problema de impor condições de fronteira para a pressão, resultando em uma metodologia que proporciona extrema facilidade para tratamento de obstáculos no domínio computacional; o SOLA-VOF ([HN81]), que a partir do método SOLA apresenta uma nova formulação para a representação da superfície livre, que passa a ser atualizada através de uma equação de conveção.

Cronologicamente, a primeira evolução significativa na metodologia MAC se dá em [AH70], que apresenta o SMAC (Simplified Marker-And-Cell). Em uma formulação nitidamente influenciada pelas idéias dos métodos de projeção ([CH67], [CH68]), tal metodologia simplifica a aplicação das condições de fronteira, introduzindo um potencial auxiliar (então denominado pseudo-pressão) para a formulação da equação de Poisson.

Com [TM93] e [TM94] surge o GENSMAC (GENeralized Simplified Marker-And-Cell), que generaliza as idéis do SMAC para domínios arbitrários com fronteiras curvas. Além disso, o GENSMAC passa a empregar partículas virtuais para representar apenas a superfície livre do fluido. Proporcinando uma significativa redução no uso de memória, tal fato contrasta com o SMAC que, a exemplo do MAC, usa partículas em toda extensão do fluido no domínio computacional.

Inicialmente apresentado como uma metodologia para a simulação de escoamentos 2D incompressíveis e newtonianos, ao longo do tempo, novos aprimoramentos foram incorporados ao GENSMAC. Isto tem se dado, a grosso modo, em duas linhas: 
- Na primeira, com uma maior quantidade de trabalhos, vemos a adaptação desta técnica para problemas de natureza parecida, que mantêm o cerne do método original e o ampliam de forma que seja possível lidar com problemas cada vez mais complexos. Nesta linha podemos citar, entre outros, [CF99] (por considerar tensão superficial), [TM00] (por considerar escoamentos com simetria radial), [TM01] (por ampliar as técnicas 2D ao caso 3D), [FR01] (por tratar de escoamentos em regime turbulento) e [SO02] (por tratar de escoamentos multifásicos). Ainda podemos mencionar [TM96], [TM02A], [TM02B], [GF03], [DO03], [GR03], [SL05], [PL06] e [SV07], que ampliaram o GENSMAC para a simulação numérica de escoamentos não-newtonianos regidos por diversas equações constitutivas.

- Já na segunda linha, com menos trabalhos, temos a tentativa de modificar a técnica GENSMAC, mas, não para resolver novos problemas, porém, para tratar aqueles já resolvidos pelas técnicas existentes por meio de uma nova abordagem mais robusta. Um bom exemplo de trabalho nesta linha é [OC04], que pela modificação da metodologia GENSMAC original (via a implicitação da discretização da equação do momento e da condição de contorno na superfície livre) conseguiu uma relaxação significativa da restrição no passo temporal, permitindo reduzir consideravelmente o custo computacional na simulação de escoamentos em que $R e<<1$.

Escoamentos viscoelásticos, na sua maioria, transcorrem a baixos Reynolds $(R e<<1)$. No contexto de problemas transientes, metodologias explícitas de solução numérica das equações governantes apresentam restrições de estabilidade muito severas para a definição do passo temporal, acarretando um custo computacional relativamente alto. Sendo assim, é conveniente que a equação de conservação da quantidade de movimento seja aproximada utilizando métodos implícitos.

Para escoamentos confinados, a discretização semi-implícita da equação de conservação da quantidade de movimento não apresenta maiores problemas, sendo suficiente para eliminar a severa restrição de estabilidade imposta pelo seu termo de natureza parabólica ([OC06]).

Porém, no caso de escoamentos não confinados, existe o acoplamento adicional da velocidade com a pressão, imposto pela condição de fronteira na superfície livre. Assim, para que se possa marchar no tempo com passos maiores, não basta resolver a equação de conservação do momento por um método implícito: também é necessário tratar a condição de fronteira para a pressão na superfície livre implicitamente ([OC04], [OC06], [OC08B]), o que conduz a um novo acoplamento entre velocidade e pressão.

No contexto da solução numérica das equações de Navier-Stokes, resolver o problema acoplado é, geralmente, muito caro computacionalmente. Métodos de projeção podem ser utilizados para desacoplar velocidade e pressão. Para escoamentos confinados, isto se restringe a separar os processos de solução das equações de conservação, permitindo determinar os campos de velocidade e de pressão de forma segregada. Já nos escoamentos com superfície livre, resultados consequentes do processo de projeção são usados de forma bastante elegante, possibilitando eliminar, também, o acoplamento entre velocidade e pressão advindo do tratamento implícito da condição de contorno na superfície livre ([OC04], [OC06], [OC08B]).

Conforme já adiantamos, o caso de escoamentos newtonianos com superfície livre foi considerado em [OC04], [OC06] e [OC08B], onde é proposta, seguindo a metodologia GENSMAC, uma estratégia implícita, que resolve as equações desacopladas, eliminando a restrição de estabilidade parabólica ao passo temporal.

O GENSMAC, tal como proposto em [TM94], apresenta abordagem explícita tanto para a integração temporal da equação de conservação do momento quanto para a aproximação da condição de contorno para a pressão na superfície livre, constituindo o que chamamos de Formulação Explícita (FE) do GENSMAC. Já a estratégia proposta em [OC04] preserva as principais características do GENSMAC, exceto a forma semi-implícita como trata a equação de conservação do momento e a pressão na superfície livre, constituindo sua Formulação Implícita (FI).

Neste contexto, o foco deste trabalho é o estudo dos métodos implícitos para a simulação de escoamentos viscoelásticos com superfície livre a baixos Reynolds $(R e<<1)$ dentro da metodologia GENSMAC. Tal estudo pôde ser particionado em duas etapas consecutivas, que se enquadram, respectivamente, na segunda e na primeira linhas de expansões do GENSMAC, citadas inicialmente: 
- Embora bastante robusta se comparada à FE, a FI inicial ainda mostrava deficiências, sugerindo a necessidade de que certos aprimoramentos fossem realizados a fim de torná-la ainda mais precisa e eficiente. Alguns desses aperfeiçoamentos, como o tratamento das condições de contorno rígido na solução da equação do momento ([OC08A]), a adoção de uma equação para atualização do campo de pressão (baseada em [BR01]) coerente com a discretização semi-implícita da equação do momento e testes para a obtenção de métodos de projeção de segunda ordem no tempo para escoamentos com superfície livre já haviam sido tratados em [OC08B].

Buscando eliminar as deficiências remanescentes, dois pontos importantes foram analisados: a resolução dos sistemas lineares presentes na FI e a estratégia de movimentação da superfície livre. Tal análise teve sua motivação no fato desses dois processos serem diretamente afetados pela possibilidade (existente na FI) de aumento do passo temporal, bem como no compromisso entre eficiência computacional e precisão da solução numérica.

- Uma vez que as análises realizadas na etapa anterior proporcionaram modificações que contribuíram para tornar a FI do GENSMAC mais eficiente e precisa, foi, então, realizada sua extensão para o modelo viscoelástico Single eXtended Pom-Pom (SXPP), que apresenta como caso particular (obtido pela conveniente escolha de seus parâmetros) o modelo Oldroyd-B. Assim, foi desenvolvido o GENSMAC-SXPP, que apresenta duas versões, baseadas nas formulações explícita e implícita do GENSMAC.

Todas as técnicas e resultados produzidos neste trabalho foram implementadas no Sistema Freeflow 2D ([OL99], [CF00]), que é um pacote computacional desenvolvido pelo grupo de MFC do Laboratório de Computação de Alto Desempenho (LCAD) do ICMC-USP. Este software, implementado em C sobre o sistema operacional LINUX, é composto por três módulos, permitindo a modelagem, a simulação e a visualização de escoamentos transientes e incompressíveis com superfície livre.

Mais detalhes sobre os resultados obtidos e as técnicas desenvolvidas são apresentados no decorrer do texto, que se divide em:

- Revisão bibliográfica;

- Modificações no GENSMAC;

- O GENSMAC-SXPP;

- Considerações finais.

A primeira parte, com o levantamento bibliográfico, compreende os Capítulos 1 e 2 . No primeiro deles, os conceitos introdutórios e a formulação matemática das Equações a Derivadas Parciais (EDPs) e das condições auxiliares convenientes às mesmas em cada circunstância física (ou seja, a formulação teórica dos Problemas de Valor Inicial e de Fronteira (PVIFs) que modelam os escoamentos de interesse) são apresentados. Já no Capítulo 2 a idéia central dos métodos de projeção (através do Teorema da Decomposição de Helmholtz-Hodge) é esboçada, culminando na apresentação do GENSMAC como um método desta classe e dando, também, mais detalhes sobre esta metodologia. Embora tratados com mais profundidade no contexto do GENSMAC-SXPP, introduzimos, ainda, os principais aspectos das formulações explícita e implícita do GENSMAC. Isto porque, na segunda parte deste trabalho, são testadas modificações no GENSMAC com respeito à solução dos sistemas lineares da FI e sobre a estratégia de movimentação da superfície livre. Enquanto o Capítulo 3 justifica a necessidade dessas alterações e estabelece as ferramentas a serem empregadas, o Capítulo 4 apresenta uma série de resultados que comprovam a eficácia das modificações adotadas.

Na terceira parte, apresentamos o GENSMAC-SXPP no Capítulo 5 e exibimos uma série de simulações obtidas com esta metodologia (Capítulo 6 e apêndices). Aliás, nos apêndices, realizamos um estudo numérico (via a FI do GENSMAC-SXPP) relativo à influência dos parâmetros do modelo SXPP em dois problemas clássicos (escoamento de Hagen-Poiseuille e inchamento do extrudado).

Na quarta e última parte deste trabalho, abrangendo o Capítulo 7, as considerações finais são apresentadas, estabelecendo possíveis rumos a serem seguidos para dar continuidade a este projeto de pesquisa. 
Por fim, todas as simulações apresentadas neste trabalho foram executadas no cluster do LCAD, sempre em regime de uma simulação por processador, a fim de que os tempos de CPU obtidos fossem sempre os mais fiéis possíveis às configurações das máquinas empregadas. Assim, todas as simulações do Capítulo 6 e da Seção 4.3 foram executadas em máquinas $8 \times$ Intel Xeon E5345 de $2.33 \mathrm{GHz}$ com 16GB RAM. As simulações do Capítulo 3 e da Seção 4.2 foram realizadas em máquinas $2 \times$ AMD Opteron 232 de $1.60 \mathrm{GHz}$ com 2GB RAM, exceto as da Seção 4.2.6, que se utilizaram de máquinas $4 \times$ AMD Opteron 844 de $1.80 \mathrm{GHz}$ com 8GB RAM. Além disso, ao longo do trabalho, rotineiramente expressaremos um tempo de processamento usando a notação h:min:s, isto é, em horas, minutos e segundos, sendo que, sempre que um tempo de CPU for dado por um número simples, fica subentendido que a unidade de medida empregada é o segundo. 


\section{CAPÍTULO \\ Formulação matemática}

\subsection{Introdução}

A construção de modelos matemáticos possibilita a representação de uma vasta quantidade de situações, as quais vão de simples problemas do cotidiano até complexos fenômenos físicos. Uma vez que a construção de um modelo tenha sido feita de modo adequado, preservando as características do objeto em estudo, esperamos que sua solução matemática possa fornecer informações sobre propriedades importantes do processo em questão.

Em muitos casos relevantes, o modelo matemático que representa a situação é um PVIF envolvendo EDPs. Exceto quando tais equações apresentam uma estrutura extremamente simples ou quando são adicionadas hipóteses simplificadoras ao problema, são raras as vezes que soluções analíticas exatas podem ser encontradas.

Tal panorama é frequentemente encontrado na Mecânica dos Fluidos (MF), cujos principais fenômenos acabam sendo modelados por EDPs que, em geral, envolvem termos não-lineares. Tais termos dificultam e, muitas vezes, impossibilitam a obtenção de solução analítica exata. Aliás, em diversos casos, a teoria matemática sequer consegue garantir a existência e unicidade de solução para modelos que, fisicamente, aparentam ter uma resposta bem definida.

Por essa razão, é frequente em MF a realização de ensaios experimentais. Essa abordagem empírica, embora possa simular um fenômeno da forma mais realista possível (e não da forma descrita pelo modelo matemático, que se constitui uma representação ideal do sistema em questão), apresenta diversas desvantagens. Entre as inconveniências da realização de experimentos, podemos citar o alto custo para montagem do ensaio, bem como o tempo que se gasta para prepará-lo e, eventualmente, repará-lo ou substituí-lo para uma nova experimentação. Além disso, não é tão simples garantir que aparelhos de medição não interferirão na dinâmica do experimento, o que, juntamente com limitações de custo, tempo e equipamento, faz com que essas sejam realizadas apenas em determinadas regiões do sistema experimental. Outro problema que pode ser enfrentado neste tipo de abordagem diz respeito ao ajuste das várias escalas envolvidas, de forma a garantir que os resultados obtidos no experimento com aparatos em tamanho reduzido ou ampliado possam ser generalizados ao fenômeno em tamanho real. Aliás, não se pode deixar de lado a impossibilidade de reproduzir em laboratório certos fenômenos, como, por exemplo, a dinâmica atmosférica e a circulação do sangue no corpo de um animal.

Portanto, tendo em vista as desvantagens inerentes às técnicas teóricas e experimentais, uma terceira possibilidade ganhou força na segunda metade do século XX: o tratamento numérico. Impulsionada pelo avanço na computação, que tornou viável a solução numérica de PVIFs envolvendo EDPs "intratáveis" do ponto de vista analítico, o desenvolvimento de técnicas numéricas cada vez mais robustas proporcionou a consolidação da que é hoje conhecida como Mecânica dos Fluidos Computacional (MFC).

Assim, muitos fenômenos e modelos em MF que antes eram difíceis de ser tratados, tanto de modo 
experimental quanto analítico, passam a ser simulados computacionalmente, possibilitando resultados aproximados. Estes, apesar dos erros (sejam eles de arredondamento ou de truncamento) envolvidos em qualquer técnica numérica, podem ser tornados tão precisos quanto se deseje (desde que estejamos dispostos a bancar o custo computacional que isto acarreta). Desse modo, essa metodologia numérica possibilita diversas vantagens, como, entre outras, a flexibilidade de variação de parâmetros e a repetição do "experimento numérico" a custos adicionais praticamente nulos.

No entanto, deve ficar claro que, de modo algum, o emprego de simulações numéricas implica no descarte das técnicas analíticas e dos estudos experimentais em MF. Na verdade, essas três técnicas devem se complementar ([FT00]), com as desvantagens de uma sendo supridas pelas vantagens das demais.

Assim, resultados numéricos podem não só orientar a montagem de um experimento prático como também chamar a atenção para determinados detalhes de sua realização, visando diminuir o número de vezes que o mesmo precisará ser repetido. Por outro lado, novos modelos teóricos para MF podem ser resolvidos numericamente, com tais resultados sendo comparados a dados experimentais, confirmando ou não a validade desse novo modelo (processo de validação). Além disso, um método numérico e sua implementação têm a correção testada pela verificação se suas respostas são condizentes com dados experimentais ou com dados de problemas simples que possuem solução analítica exata (processo de verificação).

Com isso, existe uma relação de interdependência entre essas três vertentes da MF. Neste trabalho, nosso foco é a MFC e, de forma mais específica, a simulação numérica de escoamentos incompressíveis e isotérmicos. Estes são regidos por um modelo matemático fundamentado nas equações de Navier-Stokes incompressíveis e isotérmicas (equações de conservação da massa e do momento) e na equação constitutiva do fluido, bem como por um conjunto de condições auxiliares fisicamente plausíveis que definem o que ocorre no limiar da região de definição (espacial e temporal) do escoamento.

Entre as diversas configurações possíveis de escoamentos, uma que chama a atenção diz respeito à presença de uma fronteira livre do fluido em contato com uma atmosfera inerte. O tratamento da superfície livre não é uma das tarefas mais simples do ponto de vista numérico, já que grandezas sobre tal fronteira, bem como a posição da mesma, precisam ser determinadas como parte do processo de solução. Além disso, já visando a questão computacional, uma estratégia de representação da fronteira livre (a qual influenciará o modo de atualização da posição da superfície livre com o passar do tempo) precisa ser determinada e, embora existam diversas metodologias para esta finalidade descritas na literatura ([EM57], [GM88], [PI04], [OS01], [FD02], [LU05], [LI01], [YY05], [YT01]), nenhuma é isenta de limitações. Apesar dessas desafiantes dificuldades, tal configuração é extremamente interessante na dinâmica de um escoamento, apresentando diversas aplicações de cunho tecnológico, como no processo de injeção em moldes.

Já quanto ao fluido, podemos classificá-lo, basicamente, como ideal e real, sendo esta última categoria comumente dividida em fluidos newtonianos e não-newtonianos.

Os ideais, também chamados de invíscidos (sem viscosidade), desprezam as forças de resistência internas ao fluido, tendo sua dinâmica modelada pelas equações de Euler. Tais equações, na verdade, são um caso particular das equações de Navier-Stokes, obtidas quando as forças de contato internas ao fluido (oriundas de seu movimento) são desconsideradas. Apesar de ser um modelo bastante simplificado da realidade, a suposição de fluidos ideais é razoável em diversas aplicações, podendo produzir resultados bastante satisfatórios ([TO99], [LE02]).

Por outro lado, classificamos como fluido newtoniano aquele para o qual a taxa de deformação é proporcional à tensão de cisalhamento a que é submetido, sendo a constante de proporcionalidade a viscosidade dinâmica ou molecular do fluido. Certamente, tal modelo é uma boa aproximação para cerca de $99 \%$ do volume total de fluidos presentes na biosfera ([FI06]). Não é difícil notar que um fluido ideal é uma aproximação limite para um fluido newtoniano quando fazemos a viscosidade tender a zero.

A despeito do grande volume de fluidos newtonianos, existem outros para os quais a relação de proporcionalidade acima mencionada não é satisfeita, o que caracteriza os fluidos não-newtonianos. Embora estes possam ser considerados, em volume, uma pequena minoria, certamente superam as ocorrências de fluidos newtonianos no que diz respeito à variedade com que aparecem na biosfera, tanto de forma natural quanto artificial ([FI06]). Isso se reflete diretamente na existência de uma grande diversidade de categorias de fluidos não-newtonianos, o que, por sua vez, gera um grande leque de modelos matemáticos 
para tais fluidos.

Além de propriedades viscosas, alguns fluidos não-newtonianos possuem uma "dose" de elasticidade, que é a tendência em retornarem a uma configuração atingida em um passado próximo ([BH87]). Em outras palavras, os fluidos newtonianos são, basicamente, inelásticos, não sendo capazes de armazenar energia interna, por deformação ou arranjo de suas moléculas, para, posteriormente, a devolver ao escoamento, sendo, portanto, meramente dissipativos. Por outro lado, fluidos que apresentam a capacidade de reverter essa energia de volta ao escoamento, como se tivessem certa memória do que lhes aconteceu em um passado próximo, são ditos apresentarem elasticidade, e, por também possuírem propriedades viscosas intensas, são ditos, então, viscoelásticos. Estes, quando submetidos a uma tensão de cisalhamento qualquer, sofrem uma deformação e, logo que esta cessa, ocorre certa recuperação da deformação sofrida, de acordo com uma memória de passado recente. Nos vários modelos matemáticos de fluidos viscoelásticos, essa memória do fluido é "regulada" matematicamente pelas suas constantes temporais.

Por estabelecer uma resposta diferente às tensões neles aplicadas, os fluidos viscoelásticos apresentam alguns comportamentos muito diferentes quando comparados aos fluidos newtonianos, conforme ilustra, com um simples exemplo, a Figura 1.1.1. Tal comportamento não representa uma anomalia que algum fluido estranho exibe, mas, mostra um típico comportamento de fluidos com uma composição molecular distinta da dos fluidos newtonianos.

Basicamente, fluidos newtonianos fazem menção a composições de pequenas moléculas e com pequena massa molecular. Já os fluidos que exibem comportamento não-newtoniano são de natureza polimérica ou soluções de polímeros em solventes newtonianos. Desse modo, compostos de natureza não-newtoniana se caracterizam por apresentarem macromoléculas em sua constituição, as quais são as responsáveis pela alta massa molecular desses compostos e por uma estrutura que é responsável por propriedades que não ocorrem no caso de fluidos newtonianos ([BH87]).

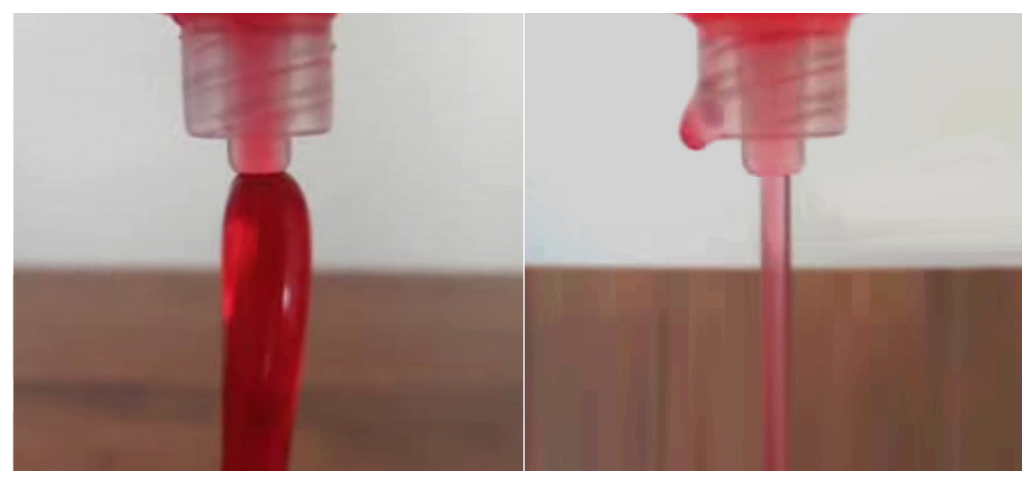

Figura 1.1.1: Ilustração do efeito Barus em um fluido não-newtoniano. Na esquerda, repare que existe um aumento na largura da coluna de fluido que é expulso várias vezes maior que o diâmetro do injetor, caracterizando o efeito citado. Na direita, com um fluido newtoniano, o fenômeno é imperceptível, com a coluna de fluido extrudado mantendo um diâmetro aproximadamente igual ao do bico injetor.

Uma grande quantidade de equações constitutivas descrevendo fluidos viscoelásticos (como os modelos PTT, Giesekus, K-BKZ, entre outros) pode ser encontrada em [BH87], todas, porém, não completamente satisfatórias na descrição não-linear do correto comportamento elongacional e de cisalhamento ([VB01]). Um grande passo, no sentido de superar tal falha, foi dado, recentemente, com o modelo Pom-Pom ([ML98]). Este se baseia na representação da molécula polimérica na forma de tubo, constituindo sua coluna vertebral, nos extremos da qual existe um dado número de ramificações (braços). Visando eliminar alguns inconvenientes do modelo original, é proposto em [VB01] o modelo XPP (eXtended Pom-Pom), capaz de prever quantitativamente a reologia de soluções comerciais LDPE e HDPE (Low/High Density Polyethylene).

Além do XPP, o modelo Pom-Pom apresenta diversas variantes ([VB01]), como o SIPP (Single Improved Pom-Pom), o DIPP (Double Improved Pom-Pom), o SXPP (Single eXtended Pom-Pom), o DXPP (Double eXtended Pom-Pom), o $\lambda^{2} \mathrm{XPP}$, o mXPP (modified eXtended Pom-Pom) e o Semi-Linear SXPP. 


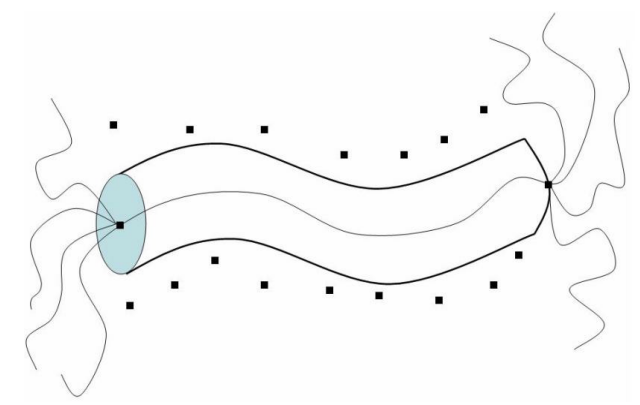

Figura 1.1.2: Esquema de uma molécula polimérica do tipo Pom-Pom.

Utilizamos, neste trabalho, a equação constitutiva newtoniana e a versão Single do modelo XPP. Um caso particular importante incluso no modelo SXPP é o Oldroyd-B ([BH87]), também conhecido como modelo convectivo contravariante de Jeffreys, modelando os chamados fluidos de Boger, que são, basicamente, soluções poliméricas diluídas em solventes newtonianos de elevada viscosidade ([FI06]).

Assim, o modelo Oldroyd-B supõe as moléculas do polímero suficientemente distantes entre si, de forma que suas interações são desprezadas na formulação do modelo. Por outro lado, a família PomPom busca modelar soluções poliméricas concentradas, onde as moléculas do polímero não mais estão dispersas, e, assim, suas interações não são desconsideradas (sendo exatamente esta a função dos braços nos extremos da molécula polimérica).

No restante do capítulo, são apresentadas as equações empregadas no decorrer do texto, bem como as condições auxiliares que devem ser impostas às mesmas nas situações de interesse.

\subsection{Equações governantes}

\subsubsection{Introdução}

Supondo que a hipótese do contínuo esteja satisfeita ([BT67], [FX01]), a dinâmica de um escoamento qualquer é descrita pelas equações de Navier-Stokes. Tais equações traduzem, para a linguagem matemática, os princípios físicos de conservação de massa, de momento e de energia.

Neste trabalho, estamos interessados em escoamentos incompressíveis e isotérmicos, e assim, ignoramos variações de densidade e de temperatura, respectivamente. Esta última suposição permite eliminar o princípio de conservação de energia, de forma que abordamos como equações de Navier-Stokes, neste trabalho, apenas os princípios de conservação de massa (ou equação da continuidade) e de momento (ou quantidade de movimento).

Considerando um sistema de coordenadas cartesianas $2 \mathrm{D}$, seja $\mathbf{x}=\left[\begin{array}{l}x \\ y\end{array}\right]$ o vetor posição e $\mathbf{u}=\left[\begin{array}{l}u \\ v\end{array}\right]$ o vetor velocidade, com $\mathbf{u}=\mathbf{u}(x, y, t)=\mathbf{u}(\mathbf{x}, t)$, cujas entradas são as componentes $u=u(x, y, t)=u(\mathbf{x}, t)$ e $v=v(x, y, t)=v(\mathbf{x}, t)$ da velocidade ao longo das direções $x$ e $y$, respectivamente, no instante de tempo $t \geqslant 0$. Seja, também, $\mathbf{g}=\left[\begin{array}{l}g^{x} \\ g^{y}\end{array}\right], \mathbf{g}=\mathbf{g}(x, y, t)=\mathbf{g}(\mathbf{x}, t)$, a aceleração da resultante das forças de campo e $\tau=\left[\begin{array}{ll}\tau^{x x} & \tau^{x y} \\ \tau^{y x} & \tau^{y y}\end{array}\right], \tau=\tau(x, y, t)=\tau(\mathbf{x}, t)$, o tensor tensão extra, que é simétrico ([BH87]), com $p=p(x, y, t)=p(\mathbf{x}, t)$ a pressão hidrostática, ou, simplesmente, pressão. Assim, definimos o tensor tensão total $\sigma=\sigma(x, y, t)=\sigma(\mathbf{x}, t)$ por

$$
\sigma=\tau-p \mathbf{I}=\left[\begin{array}{cc}
\tau^{x x}-p & \tau^{x y} \\
\tau^{x y} & \tau^{y y}-p
\end{array}\right],
$$

onde I é o tensor identidade.

Portanto, as equações de conservação do momento e da massa são dadas na forma vetorial, respecti- 
vamente, por

$$
\begin{aligned}
\frac{\partial \mathbf{u}}{\partial t}+\nabla \cdot(\mathbf{u u}) & =\nabla \cdot \sigma+\frac{1}{F r^{2}} \mathbf{g} \\
\nabla \cdot \mathbf{u} & =0
\end{aligned}
$$

ou, na forma cartesiana,

$$
\begin{aligned}
\frac{\partial u}{\partial t}+\frac{\partial\left(u^{2}\right)}{\partial x}+\frac{\partial(v u)}{\partial y} & =-\frac{\partial p}{\partial x}+\frac{\partial \tau^{x x}}{\partial x}+\frac{\partial \tau^{x y}}{\partial y}+\frac{1}{F r^{2}} g^{x} \\
\frac{\partial v}{\partial t}+\frac{\partial(u v)}{\partial x}+\frac{\partial\left(v^{2}\right)}{\partial y} & =-\frac{\partial p}{\partial y}+\frac{\partial \tau^{x y}}{\partial x}+\frac{\partial \tau^{y y}}{\partial y}+\frac{1}{F r^{2}} g^{y} \\
\frac{\partial u}{\partial x}+\frac{\partial v}{\partial y} & =0
\end{aligned}
$$

onde $\mathrm{Fr}$ é o número adimensional de Froude.

Nas equações de Navier-Stokes, a determinação do tensor tensão extra se dá via a equação constitutiva do fluido, expressando $\tau$ em função das quantidades cinemáticas do escoamento. No que segue, apresentaremos a equação constitutiva do modelo newtoniano e o modelo viscoelástico SXPP.

Antes, porém, definimos o tensor taxa de deformação $D$, com

$$
D=\frac{1}{2}\left(L+L^{\dagger}\right) \text {, }
$$

sendo $L$ o tensor gradiente de velocidade e $L^{\dagger}$ seu transposto, isto é,

$$
L=\nabla \mathbf{u}=\left[\begin{array}{ll}
\frac{\partial u}{\partial x} & \frac{\partial u}{\partial y} \\
\frac{\partial v}{\partial x} & \frac{\partial v}{\partial y}
\end{array}\right], L^{\dagger}=(\nabla \mathbf{u})^{\dagger}=\left[\begin{array}{cc}
\frac{\partial u}{\partial x} & \frac{\partial v}{\partial x} \\
\frac{\partial u}{\partial y} & \frac{\partial v}{\partial y}
\end{array}\right]
$$

Além disso, denotamos por $\operatorname{tr}(\Omega)$ o traço do tensor $\Omega$, sendo

$$
\stackrel{\nabla}{\Omega}=\frac{\partial \Omega}{\partial t}+\nabla \cdot(\mathbf{u} \Omega)-\left(L \cdot \Omega+\Omega \cdot L^{\dagger}\right)
$$

sua derivada convectiva contravariante, onde

$$
\begin{aligned}
\nabla \cdot(\mathbf{u} \Omega) & =\left[\begin{array}{ll}
\frac{\partial\left(u \Omega^{x x}\right)}{\partial x}+\frac{\partial\left(v \Omega^{x x}\right)}{\partial y} & \frac{\partial\left(u \Omega^{x y}\right)}{\partial x}+\frac{\partial\left(v \Omega^{x y}\right)}{\partial y} \\
\frac{\partial\left(u \Omega^{y x}\right)}{\partial x}+\frac{\partial\left(v \Omega^{y x}\right)}{\partial y} & \frac{\partial\left(u \Omega^{y y}\right)}{\partial x}+\frac{\partial\left(v \Omega^{y y}\right)}{\partial y}
\end{array}\right], \\
L \cdot \Omega & =\left[\begin{array}{ll}
\Omega^{x x} \frac{\partial u}{\partial x}+\Omega^{y x} \frac{\partial u}{\partial y} & \Omega^{x y} \frac{\partial u}{\partial x}+\Omega^{y y} \frac{\partial u}{\partial y} \\
\Omega^{x x} \frac{\partial v}{\partial x}+\Omega^{y x} \frac{\partial v}{\partial y} & \Omega^{x y} \frac{\partial v}{\partial x}+\Omega^{y y} \frac{\partial v}{\partial y}
\end{array}\right], \\
\Omega \cdot L^{\dagger} & =\left[\begin{array}{ll}
\Omega^{x x} \frac{\partial u}{\partial x}+\Omega^{x y} \frac{\partial u}{\partial y} & \Omega^{x x} \frac{\partial v}{\partial x}+\Omega^{x y} \frac{\partial v}{\partial y} \\
\Omega^{y x} \frac{\partial u}{\partial x}+\Omega^{y y} \frac{\partial u}{\partial y} & \Omega^{y x} \frac{\partial v}{\partial x}+\Omega^{y y} \frac{\partial v}{\partial y}
\end{array}\right] .
\end{aligned}
$$

\subsubsection{Modelo Newtoniano}

Para fluidos de natureza newtoniana, considera-se que a taxa de deformação é proporcional à tensão imposta ([BH87], [BT67], [FT00], [FX01]). Assim, supondo (1.2.3), a equação constitutiva para esse tipo de fluido é dada por

$$
\tau=\frac{2}{R e} D,
$$

onde $R e$ é o número adimensional de Reynolds.

Substituindo (1.2.13) nas equações (1.2.2) e (1.2.3), segue que

$$
\begin{aligned}
\frac{\partial \mathbf{u}}{\partial t}+\nabla \cdot(\mathbf{u u}) & =-\nabla p+\frac{1}{R e} \nabla^{2} \mathbf{u}+\frac{1}{F r^{2}} \mathbf{g}, \\
\nabla \cdot \mathbf{u} & =0 .
\end{aligned}
$$


Portanto, as equações de Navier-Stokes para um escoamento incompressível e isotérmico de um fluido newtoniano são dadas, em coordenadas cartesianas bidimensionais, por

$$
\begin{aligned}
\frac{\partial u}{\partial t}+\frac{\partial\left(u^{2}\right)}{\partial x}+\frac{\partial(v u)}{\partial y} & =-\frac{\partial p}{\partial x}+\frac{1}{R e}\left(\frac{\partial^{2} u}{\partial x^{2}}+\frac{\partial^{2} u}{\partial y^{2}}\right)+\frac{1}{F r^{2}} g^{x} \\
\frac{\partial v}{\partial t}+\frac{\partial(u v)}{\partial x}+\frac{\partial\left(v^{2}\right)}{\partial y} & =-\frac{\partial p}{\partial y}+\frac{1}{R e}\left(\frac{\partial^{2} v}{\partial x^{2}}+\frac{\partial^{2} v}{\partial y^{2}}\right)+\frac{1}{F r^{2}} g^{y} \\
\frac{\partial u}{\partial x}+\frac{\partial v}{\partial y} & =0 .
\end{aligned}
$$

\subsubsection{Modelos viscoelásticos Oldroyd-B e SXPP}

Para a definição da equação constitutiva de um fluido SXPP utilizamos a formulação EVSS (elasticviscous split stress), inicialmente apresentada em [RJ90] e utilizada por diversos trabalhos, como em [AB05].

Tal abordagem consiste em supor que o tensor tensão extra é dado pela soma de duas parcelas: a primeira representando a contribuição newtoniana (viscosa) e, a segunda, a contribuição não-newtoniana (elástica). Assim,

$$
\tau=\frac{2 \beta}{R e} D+T,
$$

$\operatorname{com} \beta \in] 0,1[$ um parâmetro adimensional.

Com isso, as equações de Navier-Stokes (1.2.2) e (1.2.3) passam a ser dadas por

$$
\begin{aligned}
\frac{\partial \mathbf{u}}{\partial t}+\nabla \cdot(\mathbf{u u}) & =-\nabla p+\frac{\beta}{R e} \nabla^{2} \mathbf{u}+\nabla \cdot T+\frac{1}{F r^{2}} \mathbf{g}, \\
\nabla \cdot \mathbf{u} & =0,
\end{aligned}
$$

isto é,

$$
\begin{aligned}
\frac{\partial u}{\partial t}+\frac{\partial\left(u^{2}\right)}{\partial x}+\frac{\partial(v u)}{\partial y} & =-\frac{\partial p}{\partial x}+\frac{\beta}{R e}\left(\frac{\partial^{2} u}{\partial x^{2}}+\frac{\partial^{2} u}{\partial y^{2}}\right)+\frac{\partial T^{x x}}{\partial x}+\frac{\partial T^{x y}}{\partial y}+\frac{1}{F r^{2}} g^{x}, \\
\frac{\partial v}{\partial t}+\frac{\partial(u v)}{\partial x}+\frac{\partial\left(v^{2}\right)}{\partial y} & =-\frac{\partial p}{\partial y}+\frac{\beta}{R e}\left(\frac{\partial^{2} v}{\partial x^{2}}+\frac{\partial^{2} v}{\partial y^{2}}\right)+\frac{\partial T^{x y}}{\partial x}+\frac{\partial T^{y y}}{\partial y}+\frac{1}{F r^{2}} g^{y}, \\
\frac{\partial u}{\partial x}+\frac{\partial v}{\partial y} & =0 .
\end{aligned}
$$

Para o modelo SXPP ([VB01]), a contribuição não-newtoniana $T$ é expressa por

$$
f(\lambda, T) T+W e \stackrel{\nabla}{T}+\frac{1-\beta}{R e W e}(f(\lambda, T)-1) \mathbf{I}+\alpha \frac{\operatorname{ReWe} e}{1-\beta} T \cdot T=2 \frac{1-\beta}{R e} D,
$$

onde

$$
\begin{aligned}
f(\lambda, T) & =\frac{2}{\gamma}\left(1-\frac{1}{\lambda}\right) e^{Q_{o}(\lambda-1)}+\frac{1}{\lambda^{2}}\left[1-\frac{\alpha}{3}\left(\frac{\operatorname{ReWe}}{1-\beta}\right)^{2} \operatorname{tr}(T \cdot T)\right], \\
\lambda & =\sqrt{1+\frac{1}{3}\left(\frac{\operatorname{ReWe}}{1-\beta}\right)|\operatorname{tr}(T)|}
\end{aligned}
$$

sendo $f=f(\lambda, T)$ uma variável auxiliar e $\lambda=\lambda(T)$ a extensão relativa da coluna vertebral da molécula polimérica. Já $W e$ é o número de Weissemberg, que, juntamente com $\alpha, \beta, \gamma$ e $Q_{o}$, são os parâmetros adimensionais do modelo SXPP.

Aliás, é válido observar que ignorando a equação (1.2.27), redefinindo (1.2.26) como $f=1$ e tomando $\alpha=0$, segue de (1.2.25) a equação que define a contribuição não-newtoniana $T$ para um fluido Oldroyd-B ([BH87]), dada por

$$
T+W e \stackrel{\nabla}{T}=2 \frac{1-\beta}{R e} D .
$$


A equação (1.2.25), em coordenadas cartesianas bidimensionais, resulta em

$$
\begin{aligned}
& \frac{\partial T^{x x}}{\partial t}+\frac{\partial\left(u T^{x x}\right)}{\partial x}+\frac{\partial\left(v T^{x x}\right)}{\partial y}=2\left(\frac{\partial u}{\partial x} T^{x x}+\frac{\partial u}{\partial y} T^{x y}\right)+\ldots \\
& \ldots+2 \xi \frac{\partial u}{\partial x}- \frac{1}{W e}\left\{f(\lambda, T) T^{x x}+\xi(f(\lambda, T)-1)+\frac{\alpha}{\xi}\left[\left(T^{x x}\right)^{2}+\left(T^{x y}\right)^{2}\right]\right\} \\
& \frac{\partial T^{y y}}{\partial t}+\frac{\partial\left(u T^{y y}\right)}{\partial x}+\frac{\partial\left(v T^{y y}\right)}{\partial y}=2\left(\frac{\partial v}{\partial x} T^{x y}+\frac{\partial v}{\partial y} T^{y y}\right)+\ldots \\
& \ldots+2 \xi \frac{\partial v}{\partial y}-\frac{1}{W e}\left\{f(\lambda, T) T^{y y}+\xi(f(\lambda, T)-1)+\frac{\alpha}{\xi}\left[\left(T^{y y}\right)^{2}+\left(T^{x y}\right)^{2}\right]\right\} \\
& \frac{\partial T^{x y}}{\partial t}+\frac{\partial\left(u T^{x y}\right)}{\partial x}+\frac{\partial\left(v T^{x y}\right)}{\partial y}=\left(\frac{\partial v}{\partial x} T^{x x}+\frac{\partial u}{\partial y} T^{y y}\right)+\ldots \\
& \ldots+\xi\left(\frac{\partial u}{\partial y}+\frac{\partial v}{\partial x}\right)-\frac{1}{W e}\left\{f(\lambda, T) T^{x y}+\frac{\alpha}{\xi}\left[T^{x y}\left(T^{x x}+T^{y y}\right)\right]\right\}
\end{aligned}
$$

com

$$
\xi=\frac{1-\beta}{R e W e}
$$

Portanto, as equações de Navier-Stokes incompressíveis e isotérmicas para um fluido SXPP, segundo a formulação EVSS, são dadas por (1.2.20) e (1.2.21), onde a contribuição não-newtoniana atua como um termo fonte, definido por (1.2.25), (1.2.26) e (1.2.27). Já para o modelo Oldroyd-B, a diferença está apenas na definição do termo fonte que, mediante conveniente ajuste dos parâmetros do modelo SXPP, é definido por (1.2.28). Em ambos os casos, porém, temos um sistema de EDPs de natureza mista, sendo parabólica a equação de conservação do momento, elíptica a equação da continuidade e hiperbólica a equação para a contribuição não-newtoniana, demonstrando a complexidade do problema.

\subsubsection{Processo de adimensionalização}

Todas as equações apresentadas ao longo desta seção estão em sua forma adimensional, assim como suas variáveis e parâmetros. Esta é uma abordagem mantida ao longo dos capítulos seguintes, pois, em MF, os problemas podem, em geral, ser representados por escalas de grandeza específicas, que passam a caracterizar o escoamento de forma biunívoca quando agrupadas em parâmetros adimensionais.

As escalas de grandeza comumente adotadas para caracterizar um escoamento são $L_{0}, U_{0}, \mu_{0}, \rho_{0}$, $g_{0}$, para representar, respectivamente, o comprimento, a velocidade, a viscosidade dinâmica, a densidade e a aceleração de campo. Como estamos considerando apenas escoamentos incompressíveis, podemos convencionar $\rho_{0}$ como a própria densidade constante do fluido.

Desse modo, as variáveis adimensionais apresentadas ao longo desta seção relacionam-se com as respectivas variáveis dimensionais (acrescidas do índice *) através das relações:

$$
\mathbf{x}=\frac{\mathbf{x}_{*}}{L_{0}}, \mathbf{u}=\frac{\mathbf{u}_{*}}{U_{0}}, t=\frac{t_{*} U_{0}}{L_{0}}, \mathbf{g}=\frac{\mathbf{g}_{*}}{g_{0}}, p=\frac{p_{*}}{\rho_{0} U_{0}^{2}}, \tau=\frac{\tau_{*}}{\rho_{0} U_{0}^{2}}, T=\frac{T_{*}}{\rho_{0} U_{0}^{2}} .
$$

Os parâmetros adimensionais variam de acordo com o modelo considerado, existindo alguns, porém, que merecem atenção especial por sua importância ([BH87]):

- Número de Reynolds (Re) - Dado por

$$
R e=\frac{\rho_{0} U_{0} L_{0}}{\mu_{0}}
$$

fornece a razão entre as forças inerciais (associadas ao termo convectivo) e as forças viscosas ou difusivas (associadas ao termo difusivo) que operam no escoamento, isto é, a razão entre os processos 
de convecção e de difusão ([FT00]). Portanto, quando $R e>1$, temos um escoamento onde o transporte convectivo é mais intenso que o difusivo, ao passo que, quando $R e<1$, o processo difusivo é mais intenso que o convectivo.

- Número de Froude (Fr) - Dado por

$$
F r=\frac{U_{0}}{\sqrt{g_{0} L_{0}}}
$$

estabelece a razão entre as forças inerciais e as forças de campo ([FT00]), sendo útil para caracterizar escoamentos nos quais forças de campo têm papel importante.

- Número de Strouhal (St) - Sendo $t_{0}$ uma escala de tempo característica de algum fenômeno, fornece a razão entre $t_{0}$ e uma escala de tempo característica do escoamento, isto é,

$$
S t=\frac{t_{0} U_{0}}{L_{0}}=\frac{t_{0}}{\left(\frac{L_{0}}{U_{0}}\right)}
$$

- Número de Weissemberg (We) - Para um fluido viscoelástico, sendo $\lambda_{1}$ uma de suas constantes temporais,

$$
W e=\frac{\lambda_{1} U_{0}}{L_{0}}=\frac{\lambda_{1}}{\left(\frac{L_{0}}{U_{0}}\right)}
$$

é a razão entre uma escala de tempo característica do fluido e uma escala de tempo característica do escoamento. Com isso, como $\lambda_{1}$ relaciona-se com a memória do fluido, a medida que $W e$ tende a zero nos aproximamos de um fluido newtoniano, inelástico, com a molécula polimérica relaxando muito rapidamente com relação à escala de tempo do escoamento. Já quando $W e$ tende ao infinito, a escala de tempo do escoamento é tão pequena que a molécula polimérica não tem tempo de relaxar, com o fluido apresentando o comportamento, a grosso modo, de um sólido com elasticidade. Em outras palavras, para um mesmo fluido em um dado tipo de escoamento, quanto maior $W e$, maior a viscoelasticidade.

Para cada modelo, no entanto, existem outros parâmetros específicos, que, juntamente com $W e$, também afetam a viscoelasticidade.

No caso do modelo SXPP, sendo $Q$ o número de braços em cada extremidade da molécula,

$$
Q_{o}=\frac{2}{Q}
$$

enquanto $\alpha$ é a anisotropia do material. Por outro lado,

$$
\gamma=\frac{\lambda_{2}}{\lambda_{1}}
$$

onde $\lambda_{1}$ e $\lambda_{2}$ são os tempos de relaxação do modelo. Sendo

$$
\mu=\mu_{S}+\mu_{P}
$$

isto é, a viscosidade total dada pela soma da viscosidade do solvente newtoniano $\left(\mu_{S}\right)$ com a viscosidade do polímero $\left(\mu_{P}\right)$ dissolvido, temos

$$
\beta=\frac{\mu_{S}}{\mu}
$$

As relações (1.2.39), (1.2.41) e (1.2.40) são igualmente válidas no caso do modelo Oldroyd-B, no qual

$$
\beta=\frac{\mu_{S}}{\mu}=\frac{\lambda_{2}}{\lambda_{1}}=\gamma
$$


Ainda no modelo SXPP, com $G_{0}$ o módulo da relaxação linear,

$$
\mu_{P}=G_{0} \lambda_{1} .
$$

Nos próximos capítulos, o modelo adotado para a simulação de um dado escoamento é responsável por definir as escalas $L_{0}, U_{0}$ e $g_{0}$, caracterizando o número de Froude, além de g, que deve ser especificado. Além disso, independentemente do tipo de fluido, a especificação de $\rho_{0}$ e $\mu_{0}$ permite definir o número de Reynolds. Para o caso newtoniano, os números de Reynolds e de Froude são os únicos parâmetros adimensionais importantes, conforme verificamos nas equações (1.2.14) e (1.2.14). Para o modelo SXPP faz-se necessário especificar, ainda, o parâmetro dimensional $\lambda_{1}$ (caracterizando o número de Weissemberg), que, juntamente com os parâmetros adimensionais $\alpha, \beta, \gamma$ e $Q$, caracterizam as equações (1.2.20), (1.2.21), (1.2.25), (1.2.26) e (1.2.27). Por fim, no caso de um fluido Oldroyd-B, além das simplificações que geraram (1.2.28), basta adotarmos o parâmetro dimensional $\lambda_{1}$ (caracterizando o número de Weissemberg) e o parâmetro adimensional $\beta$.

\subsection{Condições auxiliares}

De modo geral, uma EDP ou um sistema de EDPs deverá, isoladamente, apresentar uma família infinita de soluções. No entanto, é razoável esperar que uma equação (ou sistema de equações) representando um modelo bem formulado apresente solução única. Para que isso se faça possível, e possamos escolher a solução adequada dentro da família infinita de soluções, será necessária, adicionalmente, a prescrição de condições auxiliares, as quais conseguem caracterizar melhor a situação modelada. Existem, basicamente, dois tipos de condições auxiliares:

- Condições iniciais - Fornecem informações sobre o estado inicial do problema, informando a partir de onde e com qual valor a solução vai se propagar.

- Condições de fronteira - Especificam o que ocorre no limiar da região de definição do problema, isto é, informam sobre o estado da solução na fronteira espacial da região onde ela existe.

No restante desta seção, apresentamos as condições auxiliares mais comuns em MF, as quais são empregadas no decorrer deste trabalho. Assim, especificaremos as condições iniciais e de fronteira que podem ser agregadas às EDPs $(1.2 .20),(1.2 .21),(1.2 .25),(1.2 .26)$ e $(1.2 .27)$, de acordo com o problema de interesse, segundo a formulação do modelo SXPP. Para o modelo Oldroyd-B, as mesmas condições continuam válidas, respeitando as escolhas que resultaram em (1.2.28). Já o caso newtoniano fica subentendido, sendo obtido quando a equação que define a contribuição não-newtoniana ao tensor tensão extra é desprezada e a mesma é tomada identicamente nula na equação de conservação do momento.

A prescrição de um campo inicial de velocidade ao escoamento deve satisfazer, essencialmente, o estado de equilíbrio imposto pela equação da continuidade ([FT00]). Já a pressão e a contribuição não-newtoniana precisam ser escolhidas de forma compatível com o campo de velocidade, sendo, costumeiramente, consideradas nulas no instante inicial ([BT67], [FT00], [FX01], [BH87]). Adicionalmente, em escoamentos com superfície livre, esta deve ter sua posição inicial conhecida.

Condições iniciais que não são fisicamente relevantes, isto é, que não podem ser construídas na prática, possibilitam efeitos inesperados na solução numérica, a saber: marchando no tempo a partir de um estado inicial "absurdo" podemos tanto continuar obtendo soluções numéricas "absurdas" quanto ter dificuldades de convergência por parte do esquema numérico ([FL91]).

Para especificar o que ocorre no limiar da região espacial de definição do problema, ou seja, nos contornos do fluido no escoamento, dividimos a análise em partes condizentes com as situações físicas associadas a tais fronteiras:

- Fronteira impermeável - Entendemos por fronteira impermeável toda aquela, material ou não, ao longo da qual não poderá haver fluxo de massa em sua direção normal. Assim, sendo $\mathbf{u}_{\vec{n}}$ a componente normal de velocidade a esse tipo de fronteira, devemos ter

$$
\mathbf{u}_{\vec{n}}=0
$$

ao longo da mesma. Quanto à prescrição da velocidade tangencial $\mathbf{u}_{\vec{t}}$ ao longo desse tipo de fronteira, existem, basicamente, dois casos que podem ser escolhidos, conforme se faça conveniente: 
- Condição de aderência completa (no-slip) - Empregada, comumente, em superfícies sólidas ou paredes rígidas, esta condição parte do pressuposto que o fluido irá aderir à fronteira rígida considerada, ficando a ela "colado". Assim,

$$
\mathbf{u}_{\vec{t}}=u_{f}
$$

onde $u_{f}$ é a velocidade da fronteira. Assumindo que a fronteira está em repouso,

$$
u_{f}=0
$$

- Condição de aderência nula (free-slip) - Usada para impor condição de simetria, permite que o fluido deslize livremente sobre a fronteira, sem atrito. Assim, é conveniente impor que a taxa de variação da componente da velocidade paralela à fronteira seja nula ao longo da direção $\vec{n}$, isto é,

$$
\frac{\partial \mathbf{u}_{\vec{t}}}{\partial \vec{n}}=0
$$

- Fronteira de entrada de fluido (injetor ou inflow) - Este tipo de fronteira é responsável pela injeção de fluido no domínio. Assim, as componentes $\mathbf{u}_{\vec{n}}$ e $\mathbf{u}_{\vec{t}}$ da velocidade, respectivamente, nomal e tangencial a uma fronteira desse tipo devem ser tais que

$$
\begin{aligned}
& \mathbf{u}_{\vec{n}}=u_{\text {inflow }}, \\
& \mathbf{u}_{\vec{t}}=0,
\end{aligned}
$$

onde $u_{\text {inflow }}$ é especificado conforme a vazão desejada no injetor. Já para a contribuição nãonewtoniana $T$, pode ser feita a hipótese ([MD97]) de que o fluido prestes a entrar no domínio está livre de perturbações a montante do injetor, e com isso, neste tipo de fronteira

$$
T=0 .
$$

- Fronteira de saída de fluido (ejetor ou outflow) - Este tipo de fronteira é responsável pela saída de fluido do domínio onde ocorre o escoamento, podendo ser de dois tipos:

- Ejeção continuada - Pode ser interpretada como um "buraco" no domínio da solução que permite ao fluido sair do mesmo de forma espontânea. Tentando imitar esse fato, sendo $\vec{n}$ a direção normal ao mesmo, deve ser imposta a condição de Neumann homogênea para a velocidade e para a contribuição não-newtoniana, ou seja,

$$
\begin{gathered}
\frac{\partial \mathbf{u}_{\vec{t}}}{\partial \vec{n}}=0, \\
\frac{\partial \mathbf{u}_{\vec{n}}}{\partial \vec{n}}=0, \\
\frac{\partial T}{\partial \vec{n}}=0 .
\end{gathered}
$$

- Ejeção prescrita - Nada mais é que um inflow "ao contrário", que ao invés de injetar, suga o fluido do domínio computacional. Esse tipo de fronteira, não utilizado neste trabalho, em outras palavras, corresponde à colocação de uma bomba de sucção em determinada porção do domínio. Logo, sendo $\vec{n}$ a direção normal à fronteira, as condições impostas são

$$
\begin{gathered}
\mathbf{u}_{\vec{n}}=u_{\text {outflow }}, \\
\mathbf{u}_{\vec{t}}=0, \\
T=0,
\end{gathered}
$$

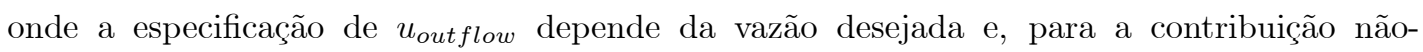
newtoniana, pode ser feita a suposição que o fluido que deixa o domínio está livre de perturbações a jusante do ejetor (generalizando o raciocínio empregado em [MD97]). 
- Fronteira ou superfície livre - Tal fronteira se relaciona com a superfície de contato do fluido com a atmosfera externa ao mesmo. Assim, ao longo desse tipo de fronteira, desprezando mudanças de estado de agregação da matéria e supondo uma atmosfera externa passiva (que não exerça tensões na superfície livre do fluido), existem duas condições básicas que precisam ser satisfeitas ([BT67] e [FZ97]):

- Condição de fronteira cinemática - Visa garantir que, de fato, a fronteira livre do fluido esteja bem definida, sendo uma superfície limítrofe (com fluido de um lado e com a atmosfera passiva do outro). Logo, devemos impor que a descarga resultante $\left(\dot{m}_{S L}\right)$ ao longo da superfície livre $(S L)$ seja nula, isto é,

$$
\dot{m}_{S L}=\int_{S L}\left(\rho_{0} \mathbf{u}\right) \cdot \vec{n} d S=0,
$$

impedindo que o fluido "vaze" por ela; em outras palavras, esta condição equivale a dizer que a componente da velocidade do fluido normal à superfície livre corresponde à própria velocidade com que a superfície livre "anda" nessa direção.

- Condição de fronteira dinâmica - É obtida impondo que as forças atuantes na superfície livre estejam em equilíbrio, ou seja, que a resultante das forças de contato que atuam em cada ponto da superfície livre seja nula. Como estamos considerando que a atmosfera externa é passiva, a resultante das forças que ela exerce na superfície livre é nula, portanto, devemos exigir que a componente do tensor tensão total $\sigma$ ao longo da superfície livre também seja nula. Segue de (1.2.1) que a componente de $\sigma$ ao longo da superfície livre é dada pelo vetor $\sigma \cdot \vec{n}$; logo, exigir que $\sigma \cdot \vec{n}$ seja nula ao longo da superfície livre corresponde impor que as componentes, normal e tangencial, de $\sigma \cdot \vec{n}$ ao longo dessa fronteira se anulem, isto é,

$$
\begin{aligned}
& \vec{n} \cdot(\sigma \cdot \vec{n})=0, \\
& \vec{t} \cdot(\sigma \cdot \vec{n})=0,
\end{aligned}
$$

onde

$$
\vec{n}=\left[\begin{array}{l}
n_{x} \\
n_{y}
\end{array}\right], \vec{t}=\left[\begin{array}{c}
-n_{y} \\
n_{x}
\end{array}\right]
$$

especificam, respectivamente, os versores normal e tangencial ao longo da superfície livre do fluido.

Além dessas, uma condição de Neumann homogênea para a contribuição não-newtoniana deve ser considerada na superfície livre do fluido ([TM02A], [TM02B]), isto é,

$$
\frac{\partial T}{\partial \vec{n}}=0
$$

Observação 1 Para obtermos uma condição de contorno para $T$ em fronteiras impermeáveis no-slip estacionárias (que é o único tipo de fronteira impermeável utilizada neste trabalho), existem duas situações a serem levadas em consideração.

Na primeira, seja uma parede rígida paralela à direção x. Assim, ao longo dessa fronteira, pela condição de impermeabilidade,

$$
v=0 \Rightarrow \frac{\partial v}{\partial x}=0
$$

ao passo que, pela condição de não deslizamento,

$$
u=0 \Rightarrow \frac{\partial u}{\partial x}=0
$$

mas, pela equação da continuidade,

$$
\frac{\partial u}{\partial x}=0 \Rightarrow \frac{\partial v}{\partial y}=0 .
$$


Portanto, por (1.2.25),

$$
\begin{aligned}
\frac{\partial T^{x x}}{\partial t} & =2 \frac{\partial u}{\partial y} T^{x y}-\frac{1}{W e}\left\{f(\lambda, T) T^{x x}+\xi(f(\lambda, T)-1)+\frac{\alpha}{\xi}\left[\left(T^{x x}\right)^{2}+\left(T^{x y}\right)^{2}\right]\right\}, \\
\frac{\partial T^{y y}}{\partial t} & =-\frac{1}{W e}\left\{f(\lambda, T) T^{y y}+\xi(f(\lambda, T)-1)+\frac{\alpha}{\xi}\left[\left(T^{y y}\right)^{2}+\left(T^{x y}\right)^{2}\right]\right\}, \\
\frac{\partial T^{x y}}{\partial t} & =\frac{\partial u}{\partial y}\left(T^{y y}+\xi\right)-\frac{1}{W e}\left\{f(\lambda, T) T^{x y}+\frac{\alpha}{\xi}\left[T^{x y}\left(T^{x x}+T^{y y}\right)\right]\right\} .
\end{aligned}
$$

Já no segundo caso, seja uma parede rígida paralela à direção y. Analogamente, pelas condições de impermeabilidade, não deslizamento e incompressibilidade temos, respectivamente,

$$
\begin{aligned}
u & =0 \Rightarrow \frac{\partial u}{\partial y}=0, \\
v & =0 \Rightarrow \frac{\partial v}{\partial y}=0, \\
\frac{\partial v}{\partial y} & =0 \Rightarrow \frac{\partial u}{\partial x}=0 .
\end{aligned}
$$

Portanto, por (1.2.25), temos

$$
\begin{aligned}
\frac{\partial T^{x x}}{\partial t} & =-\frac{1}{W e}\left\{f(\lambda, T) T^{x x}+\xi(f(\lambda, T)-1)+\frac{\alpha}{\xi}\left[\left(T^{x x}\right)^{2}+\left(T^{x y}\right)^{2}\right]\right\}, \\
\frac{\partial T^{y y}}{\partial t} & =2 \frac{\partial v}{\partial x} T^{x y}-\frac{1}{W e}\left\{f(\lambda, T) T^{y y}+\xi(f(\lambda, T)-1)+\frac{\alpha}{\xi}\left[\left(T^{y y}\right)^{2}+\left(T^{x y}\right)^{2}\right]\right\}, \\
\frac{\partial T^{x y}}{\partial t} & =\frac{\partial v}{\partial x}\left(T^{x x}+\xi\right)-\frac{1}{W e}\left\{f(\lambda, T) T^{x y}+\frac{\alpha}{\xi}\left[T^{x y}\left(T^{x x}+T^{y y}\right)\right]\right\} .
\end{aligned}
$$

Observação 2 Considere uma porção $V$ de fluido que escoa, de forma incompressível, tal que toda sua fronteira é livre. Sendo $\dot{m}_{S L}$ a descarga resultante ao longo da superfície livre, temos

$$
\dot{m}_{S L}=\int_{S L}\left(\rho_{0} \mathbf{u}\right) \cdot \vec{n} d S=\int_{V}\left[\frac{\partial\left(\rho_{0} u\right)}{\partial x}+\frac{\partial\left(\rho_{0} v\right)}{\partial y}\right] d V=\rho_{0} \int_{V}\left(\frac{\partial u}{\partial x}+\frac{\partial v}{\partial y}\right) d V=0
$$

graças, respectivamente, à definição de descarga, ao Teorema da Divergência de Gauss e à hipótese de incompressibilidade, garantindo que a condição de fronteira cinemática na superfície livre esteja satisfeita. Assim, ainda que a porção de fluido não esteja envolvida apenas por superfície livre no escoamento incompressivel considerado, este fato motiva o uso da equação da continuidade como condição de fronteira "adicional" ao longo da superfície livre, suficiente para que a condição de fronteira cinemática seja satisfeita.

Observação 3 No que diz respeito à condição de fronteira dinâmica na superfície livre, segue de (1.2.1) e de (1.2.19) que

$$
\sigma=\left[\begin{array}{cc}
\frac{2 \beta}{R e} \frac{\partial u}{\partial x}+T^{x x}-p & \frac{\beta}{R e}\left(\frac{\partial u}{\partial y}+\frac{\partial v}{\partial x}\right)+T^{x y} \\
\frac{\beta}{R e}\left(\frac{\partial u}{\partial y}+\frac{\partial v}{\partial x}\right)+T^{x y} & \frac{2 \beta}{R e} \frac{\partial v}{\partial y}+T^{y y}-p
\end{array}\right]
$$

logo, por (1.3.15), temos

$$
p=\frac{2 \beta}{R e}\left[n_{x}^{2} \frac{\partial u}{\partial x}+n_{x} n_{y}\left(\frac{\partial u}{\partial y}+\frac{\partial v}{\partial x}\right)+n_{y}^{2} \frac{\partial v}{\partial y}\right]+n_{x}^{2} T^{x x}+2 n_{x} n_{y} T^{x y}+n_{y}^{2} T^{y y},
$$

ao passo que, por (1.3.16),

$$
n_{x} n_{y}\left(T^{x x}-T^{y y}\right)+\left(n_{y}^{2}-n_{x}^{2}\right) T^{x y}=\frac{\beta}{R e}\left[2 n_{x} n_{y}\left(\frac{\partial v}{\partial y}-\frac{\partial u}{\partial x}\right)+\left(n_{x}^{2}-n_{y}^{2}\right)\left(\frac{\partial u}{\partial y}+\frac{\partial v}{\partial x}\right)\right] .
$$




\section{Capítulo}

\section{A metodologia GENSMAC}

\subsection{Introdução}

Estendendo a aplicabilidade do SMAC para o tratamento de domínios 2D arbitrários, surge com [TM93] o GENSMAC. Neste, partículas marcadoras são empregadas apenas na superfície livre, determinando uma aproximação linear por partes para a mesma. Em outras palavras, uma sequência de partículas marcadoras (formando uma poligonal orientada) constitui a aproximação numérica para a superfície livre do fluido.

Com isso, existe uma malha fixa (euleriana), onde as equações são discretizadas, e uma malha móvel (lagrangeana), que representa a superfície livre e é movimentada de acordo com o campo de velocidade calculado.

No GENSMAC, a malha fixa, onde as equações de Navier-Stokes são discretizadas por diferenças finitas, apresenta espaçamento uniforme $\delta m$, com

$$
\delta m=\delta x=\delta y
$$

e é composta por células deslocadas (staggered grid). Assim, de acordo com a Figura 2.1.1, a velocidade tem suas componentes avaliadas nas faces da célula a que é normal, enquanto as demais grandezas são tomadas em seu centro.

Por outro lado, a movimentação da superfície livre está ligada à convecção das partículas que a determinam. Isto é feito resolvendo, para cada uma delas, a equação

$$
\frac{d \mathbf{x}}{d t}=\mathbf{u}(\mathbf{x}, t),
$$

onde $\mathbf{x}$ é a posição da partícula na malha fixa.

Nesta etapa, as arestas que definem a superfície livre são modificadas, podendo ser aumentadas ou diminuídas, respectivamente, com o distanciamento ou com a aproximação de seus vértices. Para que este processo seja mantido sob controle ([OL99], [CF00]), preservando os detalhes da representação sem desperdiçar armazenamento, são adotados parâmetros de refinamento $c_{m i n}$ e $c_{m a ́ x}$ (expressos em função de $\delta m$ ) para a malha móvel. Assim, sempre que uma aresta excede um comprimento $c_{m a ́ x}$, ela é dividida pela inserção de uma nova partícula em seu ponto médio, originando duas novas arestas. Por outro lado, quando uma aresta (inteiramente contida em uma mesma célula) torna-se menor que um comprimento $c_{m i n}$, ela é removida e suas duas arestas adjacentes passam a ser ligadas por um vértice inserido no ponto médio da aresta eliminada.

Para a descrição do posicionamento da superfície livre no domínio, bem como dos demais tipos de fronteiras, o GENSMAC adota um esquema de classificação das células, conforme ilustra a Figura 2.1.2. Assim, temos: 


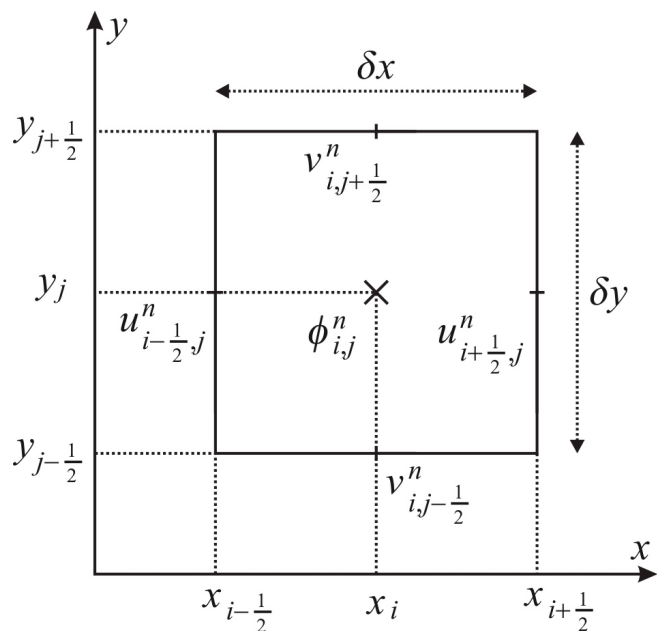

Figura 2.1.1: Representação da célula $C_{i, j}$, tipo MAC, em $t=t_{n}$ : a velocidade é avaliada nas faces e, as demais grandezas (representada pela variável genérica $\phi$ ), no centro da célula.

- Células vazias (E: empty) - são aquelas que não têm fluido em seu interior.

- Células cheias (F: full) - possuem fluido em seu interior e não apresentam interface em contato com célula $\mathbf{E}$.

- Células de superfície (S: surface) - possuem fluido em seu interior e apresentam interface em contato com célula $\mathbf{E}$.

- Células de fronteira (B: boundary) - determinam a configuração dos contornos rígidos.

- Células do injetor (I: inflow) - determinam a entrada de fluido no domínio.

- Células do ejetor (O: outflow) - empregadas para demarcar os ejetores continuados.

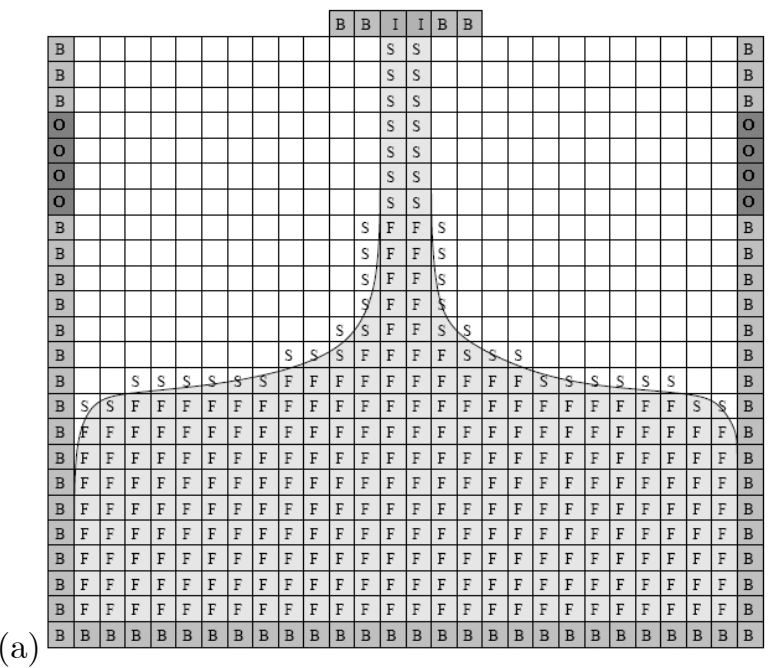

(b)

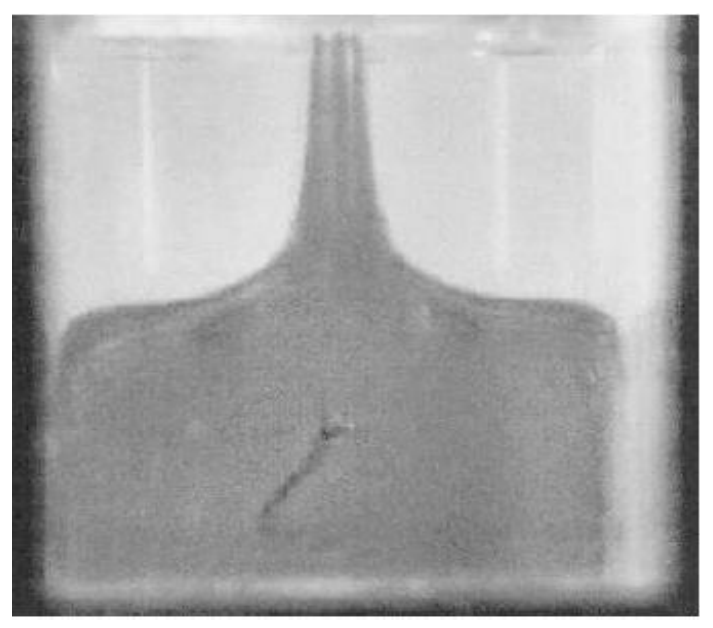

Figura 2.1.2: Classificação das células do domínio computacional (a) para a simulação do escoamento representado em (b), extraído de [TM99]. Em (a), as células em branco são do tipo E. 
Outro detalhe interessante empregado no GENSMAC é a utilização de um de passo temporal automático, isto é, o valor de $\delta t$ é calculado a cada ciclo ${ }^{1}$, podendo variar ao longo da simulação de acordo com a restrição de estabilidade apresentada na Seção 2.3 .

Aliás, a cada ciclo, a posição da superfície livre muda e, consequentemente, as células do domínio precisam ser novamente classificadas. Isto é feito por um algoritmo ([OL99], [CF00]) que leva em conta a classificação anterior das células e produz a nova através dos seguintes passos:

- Todas as células $\mathbf{E}$ e $\mathbf{F}$ que abrigam alguma partícula marcadora são alteradas para tipo $\mathbf{S}$.

- Células tipo $\mathbf{S}$ sem interface comum com célula tipo $\mathbf{E}$ se tornam tipo $\mathbf{F}$.

- Células tipo $\mathbf{S}$ sem partícula marcadora se tornam tipo $\mathbf{E}$; todas as grandezas que estavam definidas nestas novas células $\mathbf{E}$ passam a ser desconsideradas.

- Células tipo $\mathbf{S}$ sem interface comum com célula tipo $\mathbf{E}$ se tornam tipo $\mathbf{F}$.

- Células tipo $\mathbf{F}$ com interface comum com célula tipo $\mathbf{E}$ se tornam tipo $\mathbf{S}$.

Observação 4 A descrição do GENSMAC apresentada nesta seção considera não só as publicações originais [TM93] e [TM94] como também detalhes relevantes de sua implementação mais recente no Sistema Freeflow 2D ([OL99], [CF00]). Neste, não apenas o fluido, mas, todos os objetos geométricos (contêineres com superfícies rígidas, injetores e ejetores) têm sua fronteira aproximada por uma poligonal e são representados pela estrutura half-edge. Esta é um tipo de estrutura de dados B-Rep (Boundary Representation), a qual representa objetos geométricos por suas faces, arestas, vértices e relações topológicas $([M Y 88])$.

Um aspecto importante do GENSMAC diz respeito às aproximações realizadas para a superfície livre na aplicação das condições de contorno (1.3.15) e (1.3.16), que exigem um valor para o versor normal $\vec{n}$. Seguindo as idéias presentes em [TM93], isto se faz com base na vizinhança de células $\mathbf{E}$ de uma célula tipo S. Além disso, supomos que a malha esteja suficientemente refinada para que a superfície livre intercepte cada célula $\mathbf{S}$ uma única vez e em apenas dois lados. Conseguimos, então, duas situações principais.

Na primeira delas, a célula $\mathbf{S}$ possui uma única aresta comum com célula $\mathbf{E}$, sendo razoável aproximar $\vec{n}$ pela direção paralela a um dos eixos coordenados. Esta situação está ilustrada na Figura 2.1.3, onde, da esquerda para a direita,

$$
\vec{n}=\left[\begin{array}{l}
0 \\
1
\end{array}\right], \vec{n}=\left[\begin{array}{l}
1 \\
0
\end{array}\right], \vec{n}=\left[\begin{array}{c}
0 \\
-1
\end{array}\right], \vec{n}=\left[\begin{array}{c}
-1 \\
0
\end{array}\right]
$$

Na segunda situação, a célula $\mathbf{S}$ possui duas arestas adjacentes comum com células $\mathbf{E}$, sendo razoável aproximar $\vec{n}$ por um versor formando ângulo de $45^{\circ}$ com os eixos coordenados. Na Figura 2.1.4 ilustramos esta situação, onde, da esquerda para a direita,

$$
\vec{n}=\left[\begin{array}{c}
\frac{\sqrt{2}}{2} \\
\frac{\sqrt{2}}{2}
\end{array}\right], \vec{n}=\left[\begin{array}{c}
\frac{\sqrt{2}}{2} \\
-\frac{\sqrt{2}}{2}
\end{array}\right], \vec{n}=\left[\begin{array}{c}
-\frac{\sqrt{2}}{2} \\
-\frac{\sqrt{2}}{2}
\end{array}\right], \vec{n}=\left[\begin{array}{c}
-\frac{\sqrt{2}}{2} \\
\frac{\sqrt{2}}{2}
\end{array}\right]
$$

Para os demais tipos de vizinhança entre células $\mathbf{S}$ e $\mathbf{E}$, como uma aproximação plausível para $\vec{n}$ não pode ser definida, as equações (1.3.15) e (1.3.16) não são aplicadas, sendo o refinamento da malha uma possível solução para tal problema.

No restante deste capítulo apresentamos o GENSMAC como um método de projeção e, por fim, esboçamos as principais etapas dos algoritmos envolvidos em suas formulações explícita e implícita. Salientamos que mais detalhes sobre o GENSMAC serão dados no Capítulo 5, dentro do contexto do GENSMAC-SXPP.

\footnotetext{
${ }^{1}$ Entendemos por ciclo o conjunto de etapas que são executadas para marchar no tempo com a solução de $t=t_{n}$ a $t=t_{n+1}=t_{n}+\delta t$.
} 


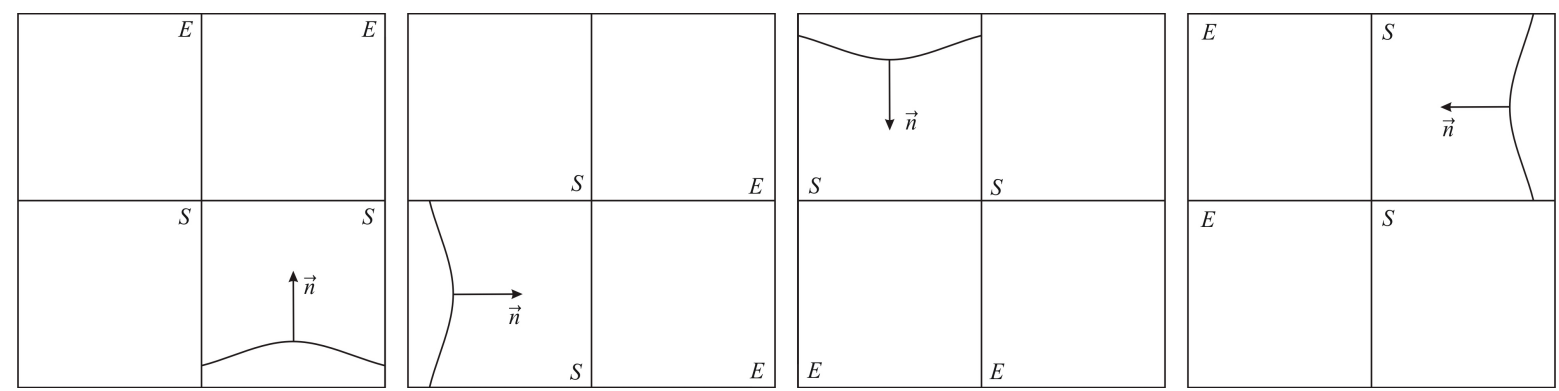

Figura 2.1.3: Aproximações da superfície livre em célula S: casos de $90^{\circ}$.
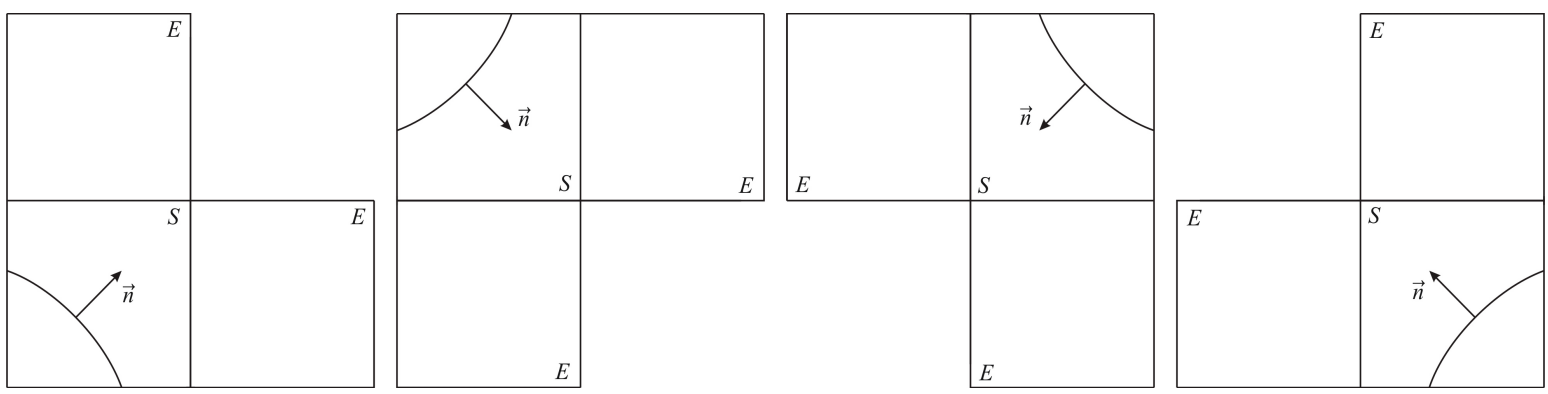

Figura 2.1.4: Aproximações da superfície livre em célula S: casos de $45^{\circ}$.

\subsection{Método de projeção}

As equações (1.2.14) e (1.2.15), rescritas abaixo,

$$
\begin{aligned}
\frac{\partial \mathbf{u}}{\partial t}+\nabla \cdot(\mathbf{u u}) & =-\nabla p+\frac{1}{R e} \nabla^{2} \mathbf{u}+\frac{1}{F r^{2}} \mathbf{g}, \\
\nabla \cdot \mathbf{u} & =0
\end{aligned}
$$

determinam um sistema de EDPs de natureza mista ([FL91] e [CM02]), pois, enquanto a equação de conservação da massa é de caráter elíptico (fazendo alusão a um estado de equilíbrio), a equação de conservação da quantidade de movimento determina um processo transiente, sendo, neste caso, parabólica.

Assim, temos a informação de como a velocidade $\mathbf{u}$ evolui com o tempo, mas, não sabemos como isso acontece com $p$. Como as duas equações devem ser simultaneamente satisfeitas, a ligação entre a equação da continuidade e a de conservação do momento se dá através da especificação do campo escalar de pressão. Em outras palavras, isto significa que a pressão em escoamentos incompressíveis tem o papel de garantir que a equação da continuidade esteja satisfeita a cada instante de tempo ([CH00]).

Dessa maneira, existe um acoplamento entre pressão e velocidade, que se reflete no estabelecimento de duas classes de métodos: acoplados e segregados.

Os métodos acoplados resolvem as equações (2.2.1) e (2.2.2) simultaneamente, calculando $\mathbf{u}$ e $p$ em um mesmo processo. Tal fato é bastante incomodo do ponto de vista computacional devido à complexidade do sistema obtido com a discretização das equações (2.2.1) e (2.2.2), o que se dá tanto pela extensão quanto pelas não-linearidades. Como exemplo, um método acoplado bastante conhecido é o SCGS (SymmetricCoupled Gauss-Seidel), proposto em [VK86].

Já em um método segregado, a solução das equações de Navier-Stokes incompressíveis se faz calculando $\mathbf{u}$ e $p$ de forma consecutiva e separada. Esse desacoplamento na solução de $\mathbf{u}$ e $p$ em um método segregado constitui-se uma estratégia ou método de projeção ([CH67], [CH68]), o qual se fundamenta no Teorema da Decomposição de Helmholtz-Hodge (TDHH), cuja demonstração pode ser consultada em [CH00]. 
Teorema 1 (Decomposição de Helmholtz-Hodge) Seja $\Omega$ uma região com fronteira suave $\partial \Omega$ e $\widetilde{\mathbf{u}}$ um campo vetorial definido em $\Omega$. Nestes termos, a decomposição de $\widetilde{\mathbf{u}}$ na forma

$$
\widetilde{\mathbf{u}}=\mathbf{u}+\nabla \psi
$$

existe e é única, sendo $\psi$ um campo escalar também definido em $\Omega$. Já o campo vetorial $\mathbf{u}$ é solenoidal e paralelo a $\partial \Omega$, isto é,

$$
\nabla \cdot \mathbf{u}=0
$$

e, ao longo de $\partial \Omega$,

$$
\mathbf{u} \cdot \vec{n}=0
$$

onde $\vec{n}$ é o vetor normal a $\partial \Omega$ e orientado "para fora" de $\Omega$.

Existe, na literatura, uma vasta quantidade de trabalhos abordando aspectos teóricos ([CH67], [CH68], [KN86], [MI96], [CH00], [DE03]), análises de precisão ([ST99], [BR01]) e estudo de condições de fronteira ([WL95], [LK01]) relativa aos métodos de projeção. Como exemplo clássico de um método segregado que adota a estratégia de projeção para resolver Navier-Stokes temos o SMAC, bem como, consequentemente, o próprio GENSMAC.

No GENSMAC, a idéia fundamental do método de projeção consiste em definir um campo de velocidade intermediária $\widetilde{\mathbf{u}}$ por

$$
\frac{\partial \widetilde{\mathbf{u}}}{\partial t}+\nabla \cdot(\mathbf{u u})=-\nabla \widetilde{p}+\frac{1}{R e}\left[(1-\nu) \nabla^{2} \mathbf{u}+\nu \nabla^{2} \widetilde{\mathbf{u}}\right]+\frac{1}{F r^{2}} \mathbf{g},
$$

com $\nu \in[0,1]$, sendo $\widetilde{p}$ o campo de pressão tentativo que, em geral, é tal que

$$
p \neq \widetilde{p}
$$

Consequentemente, como o papel da pressão em escoamentos incompressíveis é fazer com que o campo de velocidade calculado por (2.2.1) satisfaça (2.2.2), segue que $\widetilde{\mathbf{u}}$ não é necessariamente solenoidal.

Neste ponto aplica-se o TDHH, pelo qual $\widetilde{\mathbf{u}}$ admite a decomposição $(2.2 .3)$, sendo $\psi=\psi(\mathbf{x}, t)$ um potencial auxiliar que é determinado pela equação de Poisson

$$
\nabla^{2} \psi=\nabla \cdot \widetilde{\mathbf{u}}
$$

obtida substituindo (2.2.3) em (2.2.2). Segundo [TM93], as condições de contorno para $\psi$ em (2.2.8) são, em fronteiras rígidas e em injetores,

$$
\frac{\partial \psi}{\partial \vec{n}}=0
$$

enquanto que, em ejetores continuados,

$$
\psi=0 \text {. }
$$

Assim, do ponto de vista numérico, assumindo que o termo não-linear em Navier-Stokes seja aproximado explicitamente, a estratégia apresentada exige a resolução da equação (2.2.6) para a velocidade $\widetilde{\mathbf{u}}$ e da equação de Poisson (2.2.8) para $\psi$. Conhecido $\psi$, a velocidade u é obtida através de $(2.2 .3)$, sendo que esta mesma equação, juntamente com (2.2.6) e (2.2.1), permite definir uma fórmula de atualização ([BR01]) para expressar $p$ em termos de $\widetilde{p}$ e $\psi$, caracterizando a formulação do GENSMAC como um método de projeção. Temos, portanto, um método segregado cuja eficiência está intimamente ligada à resolução das equações (2.2.6) e, principalmente, (2.2.8).

\subsection{Formulações explícita e implícita}

A seção anterior mostrou o GENSMAC sendo formulado a partir de um método de projeção. Agora, estamos interessados em estabelecer como essa formulação se reflete em seu algoritmo numérico. Para isso, supondo que a velocidade $\mathbf{u}^{n}$ e a pressão $p^{n}$ são conhecidos em $t=t_{n}$, apresentamos os passos que formam um ciclo computacional, o qual permite o cálculo de $\mathbf{u}^{n+1}$ e $p^{n+1}$ em $t_{n+1}=t_{n}+\delta t$. 
Assim, seja

$$
\begin{aligned}
\frac{\mathbf{u}^{n+1}-\mathbf{u}^{n}}{\delta t}+\operatorname{conv}(\mathbf{u u})^{n+\zeta_{1}} & =-\nabla p^{n+1}+\frac{1}{R e}\left[(1-\nu) \nabla^{2} \mathbf{u}^{n}+\nu \nabla^{2} \mathbf{u}^{n+1}\right]+\frac{1}{F r^{2}} \mathbf{g}^{n+\zeta_{2}} \\
\nabla \cdot \mathbf{u}^{n+1} & =0
\end{aligned}
$$

uma aproximação discreta no tempo para (2.2.1) e (2.2.2), onde conv $(\mathbf{u u})^{n+\zeta_{1}}$ e $\mathbf{g}^{n+\zeta_{2}}$ são aproximações explícitas para $\nabla \cdot(\mathbf{u u})$ em $t=t_{n+\zeta_{1}}$ e $\mathbf{g}$ em $t=t_{n+\zeta_{2}}$, respectivamente. Neste trabalho, consideramos o termo convectivo não-linear de (2.3.1) discretizado no espaço pelo esquema convectivo CUBISTA ([AP03]).

Três casos para $\nu \in[0,1]$ são considerados:

- $\operatorname{FE} E E(\nu=0)$ - método de Euler Explícito (EE), no qual $\zeta_{1}=\zeta_{2}=0$.

- FI-EI $(\nu=1)$ - método de Euler Implícito $(\mathrm{EI})$, onde tomamos $\zeta_{1}=0$ e $\zeta_{2}=1$.

- FI-CN $(\nu=0.5)$ - método de Crank-Nicholson $(\mathrm{CN})$, onde $\zeta_{1}=\zeta_{2}=0.5$, sendo

$$
\operatorname{conv}(\mathbf{u u})^{n+\frac{1}{2}}=\frac{3}{2} \nabla \cdot(\mathbf{u u})^{n}-\frac{1}{2} \nabla \cdot(\mathbf{u u})^{n-1}
$$

uma aproximação obtida pelo método de Adams-Bashforth ([BU03]) inspirada em [AR97].

Seja, também,

$$
\frac{\widetilde{\mathbf{u}}^{n+1}-\widetilde{\mathbf{u}}^{n}}{\delta t}+\operatorname{conv}(\mathbf{u u})^{n+\zeta_{1}}=-\nabla \widetilde{p}^{n+1}+\frac{1}{R e}\left[(1-\nu) \nabla^{2} \widetilde{\mathbf{u}}^{n}+\nu \nabla^{2} \widetilde{\mathbf{u}}^{n+1}\right]+\frac{1}{F r^{2}} \mathbf{g}^{n+\zeta_{2}}
$$

uma aproximação discreta no tempo para (2.2.6) análoga à equação $(2.3 .1)$, com $\widetilde{\mathbf{u}}^{n}=\mathbf{u}^{n}$ e $\widetilde{p}^{n+1}$ uma aproximação para $p^{n+1}$ que, no GENSMAC, é escolhida como

$$
\widetilde{p}^{n+1}=p^{n}
$$

Por (2.2.3),

$$
\mathbf{u}^{n+1}=\widetilde{\mathbf{u}}^{n+1}-\nabla \psi^{n+1}
$$

que, juntamente com $(2.3 .2)$, resulta em

$$
\nabla^{2} \psi^{n+1}=\nabla \cdot \widetilde{\mathbf{u}}^{n+1}
$$

Uma vez calculado $\widetilde{\mathbf{u}}^{n+1}$ e $\psi^{n+1}$ resolvendo, respectivamente, (2.3.4) e (2.3.7), calculamos $\mathbf{u}^{n+1}$ por (2.3.6). Resta, porém, obtermos uma expressão para o cálculo de $p^{n+1}$, o que pode ser feito ([BR01]) substituindo (2.3.6) em (2.3.4) e comparando a expressão resultante com (2.3.1), de onde obtemos, então,

$$
p^{n+1}=\widetilde{p}^{n+1}+\frac{\psi^{n+1}}{\delta t}-\frac{\nu}{R e} \nabla^{2} \psi^{n+1} .
$$

Essa apresentação do GENSMAC foi feita de modo a condensar os procedimentos de suas formulações explícita e implícita, criadas, respectivamente, em [TM93] e em [OC04]. Originalmente, o algoritmo computacional da FE do GENSMAC é resumido nos seguintes passos:

Passo 1: cálculo de $\widetilde{\mathbf{u}}^{n+1}$ por (2.3.4) com $\nu=0$, isto é, via Euler Explícito.

Passo 2: solução da equação de Poisson (2.3.7) com a condição de contorno

$$
\psi^{n+1}=0
$$

ao longo da superfície livre, isto é, em células $\mathbf{S}$. 
Passo 3: cálculo da velocidade $\mathbf{u}^{n+1}$ usando (2.3.6) no interior do domínio e as equações (1.3.16) e (2.3.2) na superfície livre, isto é,

$$
\begin{aligned}
2 n_{x} n_{y}\left(\frac{\partial v^{n+1}}{\partial y}-\frac{\partial u^{n+1}}{\partial x}\right)+\left(n_{x}^{2}-n_{y}^{2}\right)\left(\frac{\partial u^{n+1}}{\partial y}+\frac{\partial v^{n+1}}{\partial x}\right) & =0 \\
\frac{\partial u^{n+1}}{\partial x}+\frac{\partial v^{n+1}}{\partial y} & =0 .
\end{aligned}
$$

Passo 4: cálculo da pressão $p^{n+1}$ usando (2.3.8) no interior do domínio, isto é,

$$
p^{n+1}=\widetilde{p}^{n+1}+\frac{\psi^{n+1}}{\delta t}
$$

em células $\mathbf{F}$, e a equação (1.3.15) na superfície livre, isto é, em células $\mathbf{S}$,

$$
p^{n+1}=\frac{2}{R e}\left[n_{x}^{2} \frac{\partial u^{n+1}}{\partial x}+n_{x} n_{y}\left(\frac{\partial u^{n+1}}{\partial y}+\frac{\partial v^{n+1}}{\partial x}\right)+n_{y}^{2} \frac{\partial v^{n+1}}{\partial y}\right] .
$$

Passo 5: cálculo da nova posição $\mathbf{x}^{n+1}$ das partículas que definem a superfície livre, resolvendo (2.1.2) por "Euler Explícito", isto é,

$$
\frac{\mathbf{x}^{n+1}-\mathbf{x}^{n}}{\delta t}=\mathbf{u}\left(\mathbf{x}^{n}, t_{n+1}\right)
$$

Conforme já adiantamos, o GENSMAC utiliza um passo temporal variável, de acordo com a restrição de estabilidade da FE, gerando duas limitações para $\delta t$.

A primeira, imposta pelo tratamento dispensado ao termo não-linear da equação de conservação do momento, resulta no chamado $\delta t_{C F L}$, dado por

$$
\delta t_{C F L}=\min \left\{\delta t_{C F L x}, \delta t_{C F L y}\right\},
$$

onde

$$
\begin{aligned}
& \frac{\delta t_{C F L x} \operatorname{má} x\left\{\left|u^{n}\right|\right\}}{\delta x} \leqslant 1 \\
& \frac{\delta t_{C F L y} m a ́ x\left\{\left|v^{n}\right|\right\}}{\delta y} \leqslant 1
\end{aligned}
$$

expressam a condição CFL ([FL91]) ao longo das direções $x$ e $y$, respectivamente. Por outro lado, o caráter explícito de integração temporal da equação do momento faz com que seu termo viscoso ocasione a chamada restrição de estabilidade parabólica, determinando $\delta t_{v i s c}$, dado por

$$
\delta t_{v i s c}=\frac{R e}{2}\left[\frac{1}{(\delta x)^{2}}+\frac{1}{(\delta y)^{2}}\right]^{-1}=\frac{R e}{4}(\delta m)^{2} .
$$

Dessa forma, o passo temporal da FE é definido como

$$
\delta t=F_{o} \min \left\{F_{v i s c} \delta t_{v i s c}, F_{C F L} \delta t_{C F L}\right\},
$$

onde $F_{o}, F_{v i s c}, F_{C F L}$ são fatores de controle do passo no tempo, tomados no intervalo ]0,1] para garantir estabilidade.

Pelas equações (2.3.15) e (2.3.18), para $R e<<1$ ou para uma malha muito refinada,

$$
\delta t_{v i s c}=O\left[\operatorname{Re}(\delta m)^{2}\right]<<\delta t_{C F L}=O(\delta m),
$$

fazendo com que a FE exija passo temporal muito pequeno, escolhido por questão de estabilidade e não de precisão. Com isso, é natural esperar um elevado número de ciclos computacionais da FE nessas situações, acarretando um elevado tempo de processamento. 
A FI do GENSMAC foi desenvolvida justamente com o objetivo de eliminar a restrição de estabilidade parabólica nos casos em que $R e<<1$. Neste contexto, a primeira mudança a ser pensada foi a alteração no esquema de integração temporal da equação do momento. Com isso, a primeira mudança que surge na FI é a alteração do Passo 1 para:

Passo 1-FI: cálculo de $\widetilde{\mathbf{u}}^{n+1}$ de forma semi-implícita (2.3.4), o que pode ser feito por Euler Implícito $(\nu=1)$ ou Crank-Nicholson $(\nu=0.5)$.

De fato, a modificação introduzida pelo Passo 1-FI removeu a restrição parabólica em escoamentos confinados. No entanto, na presença de superfície livre, o ganho em estabilidade permanecia pequeno, devido ao acoplamento entre pressão e velocidade na superfície livre ([OC06]). Uma evidência para isso é que quando impomos $p=0$ na superfície livre o ganho em estabilidade passa a ser igual ao dos casos confinados. Tal fato sugeriu que o cálculo da pressão ao longo da superfície livre também fosse alterado, passando a ser feito de forma implícita. Para isso, duas novas modificações na FE passam a ser feitas e incorporadas à FI:

Passo 2-FI: solução da equação de Poisson (2.3.7) com a condição de contorno para $\psi^{n+1}$ ao longo da superfície livre dada por (2.3.6), (1.3.15) e (2.3.8), isto é,

$$
\left\{\begin{array}{c}
\mathbf{u}^{n+1}=\widetilde{\mathbf{u}}^{n+1}-\nabla \psi^{n+1} \\
p^{n+1}=\frac{2}{R e}\left[n_{x}^{2} \frac{\partial u^{n+1}}{\partial x}+n_{x} n_{y}\left(\frac{\partial u^{n+1}}{\partial y}+\frac{\partial v^{n+1}}{\partial x}\right)+n_{y}^{2} \frac{\partial v^{n+1}}{\partial y}\right] \\
p^{n+1}=\widetilde{p}^{n+1}+\frac{\psi^{n+1}}{\delta t}-\frac{\nu}{R e} \nabla^{2} \psi^{n+1}
\end{array},\right.
$$

ou melhor,

$$
\begin{aligned}
& \tilde{p}^{n+1}+\frac{\psi^{n+1}}{\delta t}-\frac{\nu}{R e} \nabla^{2} \psi^{n+1}=\ldots \\
& \ldots=\frac{2}{R e}\left\{\frac{\partial}{\partial x}\left(\widetilde{u}^{n+1}-\frac{\partial \psi^{n+1}}{\partial x}\right) n_{x}^{2}+\frac{\partial}{\partial y}\left(\widetilde{v}^{n+1}-\frac{\partial \psi^{n+1}}{\partial y}\right) n_{y}^{2}+\ldots\right. \\
&\left.\ldots+\left[\frac{\partial}{\partial y}\left(\widetilde{u}^{n+1}-\frac{\partial \psi^{n+1}}{\partial x}\right)+\frac{\partial}{\partial x}\left(\widetilde{v}^{n+1}-\frac{\partial \psi^{n+1}}{\partial y}\right)\right] n_{x} n_{y}\right\} .
\end{aligned}
$$

Passo 4-FI: cálculo da pressão $p^{n+1}$ por (2.3.8) tanto no interior do domínio quanto na superfície livre, isto é, em células $\mathbf{F}$ e $\mathbf{S}$; em células $\mathbf{E}$ adotamos, como condição de contorno, $\psi^{n+1}=0$.

Repare que a FE calcula explicitamente a pressão na superfície livre por (2.3.13), usando (2.3.9) como condição de contorno para a solução da equação de Poisson (2.3.7). Já na FI, é a própria condição de contorno para a pressão na superfície livre que é usada como condição de contorno na solução da equação de Poisson, resultando na equação $(2.3 .22)$ para $\psi^{n+1}$ em células $\mathbf{S}$.

Dessa maneira, a FI do GENSMAC ([OC04], [OC06], [OC08B]), estabelecida nos Passos 1-FI, 2-FI, 3, 4-FI e 5, elimina a restrição de estabilidade parabólica em escoamentos com superfície livre, possibilitando um aumento considerável no passo temporal e uma redução significativa do custo computacional, especialmente em escoamentos a baixos Reynolds.

Assim, torna-se possível usar $F_{\text {visc }}>1$ em (2.3.19), restando, em termos de estabilidade, apenas a restrição ${ }^{2}$ CFL para a FI. Portanto, devido à boa condição de estabilidade, a escolha de $\delta t$ na FI deve ser guiada, principalmente, pelo compromisso entre precisão e custo computacional.

Detalhes sobre a discretização das equações apresentadas nesta seção podem ser encontrados nas referências originais citadas, bem como no Capítulo 5, onde as idéias aqui expressas são generalizadas e pormenorizadas no contexto do GENSMAC-SXPP.

\footnotetext{
${ }^{2} \mathrm{Na}$ verdade, a condição CFL é intransponível no GENSMAC, não só pelo tratamento dispensado para o termo convectivo não-linear em (2.3.4), mas, pela representação de superfície livre adotada. Isto porque durante o processo de movimentação das partículas, nenhuma delas pode percorrer distância superior ao lado de uma célula em um passo de tempo, sob pena de criar "buracos" no domínio computacional (que não poderão ser "preenchidos pela solução" quando da aplicação das condições de fronteira) e invalidar, entre outros, o algoritmo de reclassificação das células apresentado na Seção 2.1 .
} 


\section{CAPÍTUlo}

\section{Modificações no GENSMAC}

\subsection{Introdução}

Conforme já citamos, uma modificação importante no GENSMAC original ([TM93], [TM94]) foi a criação de sua FI ([OC04], [OC06]). Esta, por eliminar a restrição de estabilidade parabólica, contribuiu significativamente para a simulação de escoamentos incompressíveis a baixos Reynolds.

Apesar de ser precisa e eficiente desde sua origem, o passar do tempo tem proporcionado diversos aprimoramentos à FI, tornando-a cada vez mais robusta. Entre as modificações mais significativas, podemos citar: o estudo de estabilidade realizado [OC08A], o qual sugeriu um novo tratamento para as condições de contorno rígido para a velocidade; em [OC08B] adota-se uma nova equação para a atualização do campo de pressão, inspirada em [BR01], coerente com a discretização semi-implícita da equação do momento; ainda em [OC08B] é feita a expansão da FI para o caso 3D, resultando em [OC08C]. Assim, todas essas modificações, em linhas gerais, originaram a versão da FI apresentada no capítulo anterior, a qual serve de referência para os desenvolvimentos realizados durante este trabalho.

É importante citar que a FI torna-se fundamental se desejamos usar $\delta t$ maiores (com relação à FE) para simulações onde a restrição de estabilidade parabólica é proibitiva, como no caso de malhas muito refinadas ou a baixos Reynolds $(R e<<1)$, conforme deixa transparecer (2.3.18). Isto porque, ao eliminar a restrição imposta por $\delta t_{v i s c}$ no passo temporal, passa a ser possível tomar, em $(2.3 .19), F_{v i s c}>1$. Com isso, pela FI, torna-se realidade simular em algumas horas o que antes levava dias pela FE ([OC04]).

Desta forma, em função do possível aumento de $\delta t$, alguns detalhes relativos à FI precisavam ser estudados e aprimorados. Neste trabalho, foram investigados dois aspectos pertinentes a tal fato: a solução dos sistemas lineares e a questão do erro na quantidade de massa computacional. No restante do capítulo serão abordadas as investigações elaboradas nessas direções.

\subsection{Etapas de solução para $\widetilde{\mathbf{u}} \mathbf{e} \psi$}

\subsubsection{Introdução}

Na descrição do GENSMAC, apresentada na Seção 2.3, existem duas etapas que são decisivas para a eficiência do algoritmo: o cálculo da velocidade intermediária $\widetilde{\mathbf{u}}$ por (2.3.4) e, principalmente, a determinação de $\psi$ na solução da equação de Poisson (2.3.7).

Para a FE, o caráter crítico dessas duas etapas acaba sendo atenuado pela estratégia de solução. Com efeito, em termos de custo computacional, a estratégia de integração temporal para $\widetilde{\mathbf{u}}$ em (2.3.4) por Euler Explícito é a melhor possível. Por outro lado, ao adotar a condição de contorno (2.3.9) sobre a superfície livre, a discretização espacial de (2.3.7) resulta em um sistema linear simétrico, esparso e de grande porte, resolvido, desde [TM93], pelo método dos Gradientes Conjugados (CG), que é um método iterativo bastante robusto. 
Já na FI, o cálculo de $\widetilde{\mathbf{u}}$ e de $\psi$, relativamente à FE, é mais delicado. De fato, quando (2.3.4) é discretizada semi-implicitamente, cada componente de $\widetilde{\mathbf{u}}$ resulta em um sistema linear simétrico, esparso e de grande porte, resolvido pelo CG desde [OC04]. Embora o CG seja bastante eficiente, enquanto o cálculo de $\widetilde{\mathbf{u}}$ na FE é feito de forma direta, na FI, a grosso modo, o sistema linear para cada componente de $\widetilde{\mathbf{u}}$ precisa ser montado (praticamente igualando o custo de cálculo de $\widetilde{\mathbf{u}}$ na FE) e resolvido (gerando um gasto extra, exclusivo da FI). Por outro lado, o emprego da condição de contorno (2.3.22), sobre a superfície livre, na resolução da equação de Poisson (2.3.7), resulta em um sistema linear não-simétrico, esparso e de grande porte, resolvido, desde [OC04], pelo método dos Gradientes Bi-Conjugados com o pré-condicionador de Jacobi (BiCG-Jacobi). Em outras palavras, a perda de simetria no sistema linear obtido na discretização espacial da equação de Poisson da FI requer o emprego do BiCG-Jacobi, que, com relação ao CG, além de apresentar um maior custo por iteração, não possui nenhum resultado conhecido garantindo convergência. Em adição, o sistema linear para $\psi$ apresenta mais equações na FI (pois, emprega (2.3.22) em células $\mathbf{S}$ ) que na FE (onde se adota (2.3.9) nas células $\mathbf{S}$ ).

Dessa forma, sob as mesmas condições, podemos afirmar que o custo computacional de um ciclo da FI deverá ser maior que o da FE. Neste contexto, o leitor poderia perguntar: "Se o custo computacional por ciclo na FI é maior que na FE, como ela pode ser mais eficiente?".

Na verdade, a resposta para tal questão já foi dada: com a possibilidade de aumento no $\delta t$, a integração temporal pela FI até um dado instante final pode ser feita em um número menor de ciclos. Portanto, até então, a vantagem da FI sobre a FE não se dá na diminuição do custo de cada ciclo, mas, na diminuição do número de ciclos computacionais pelo aumento de $\delta t$. Assim, o interesse desta seção e o primeiro dos três objetivos principais deste trabalho é investigar como a possibilidade de aumento de $\delta t$ interfere na eficiência da FI no que diz respeito ao cálculo de $\widetilde{\mathbf{u}}$ e, principalmente, de $\psi$. Com isso, esperamos encontrar alternativas para tornar tais etapas mais eficientes, e, mediante a possibilidade de redução (já existente) do número de ciclos, imprimir à FI ainda mais eficiência.

Ao longo deste trabalho, assim como já é feito nas formulações explícita e implícita, assumimos que o "chute" ou condição inicial para a solução de um sistema linear por um método iterativo é a solução do sistema análogo obtido no instante de tempo anterior. Assim, na solução da equação de Poisson da FE, devido à restrição de estabilidade parabólica implicar um $\delta t$ bastante pequeno, o chute inicial para o método iterativo já é próximo da solução procurada, o que contribui para que a convergência do processo seja bastante rápida, tanto em número de iterações quanto em tempo de CPU.

Na FI, porém, a medida que aumentamos $\delta t$, é natural esperar que a solução numérica tende a ser, em geral, cada vez mais distinta entre dois instantes de tempo discretos consecutivos. Isto significa que o aumento do passo temporal estraga o chute inicial disponível, exigindo um maior número de iterações do método iterativo. Para o cálculo de $\widetilde{\mathbf{u}}$ isto gera um custo crescente que inexiste na FE, ao passo que, no cálculo de $\psi$, exigimos cada vez mais iterações do BiCG-Jacobi, cada uma delas mais caras que uma iteração do CG pela FE.

Chegamos, com isso, à primeira conclusão desta seção, que é relativamente óbvia: aumentando $\delta t$ deterioramos o chute inicial para o método iterativo, que passa a gastar mais iterações até atingir a convergência.

$\mathrm{Na}$ prática, porém, apesar do aumento do $\delta t$, observamos que as soluções da FI entre dois instantes de tempo consecutivos ainda são bastante próximas, pois, no limite da estabilidade, o passo temporal precisa satisfazer a condição CFL, pela qual, $\delta t=O\left(\delta t_{C F L}\right)=O(\delta m)$. Assim, não cabe atribuirmos apenas ao chute inicial o maior número de iterações necessário para os sistemas lineares da FI convergirem quando aumentamos $\delta t$, o que nos leva a suspeitar do condicionamento desses sistemas. Especialmente, quando substituímos a condição de contorno (2.3.9) por (2.3.22) na solução da equação de Poisson da FI, a matriz coeficiente do sistema linear em questão deixa de ser simétrica, o que aparenta resultar um pior condicionamento.

Chegamos, com isso, à segunda conclusão desta seção: o aumento de $\delta t$ aparenta piorar o condicionamento dos sistemas lineares presentes na FI.

Observação 5 Seja

$$
A \mathbf{z}=\mathbf{b}
$$

um sistema linear onde $A$ é a matriz coeficiente, $\mathbf{z}$ o vetor incógnita e $\mathbf{b}$ o termo independente. Se $\mathbf{z}^{(m)}$ 
é a aproximação para a solução produzida pelo método iterativo na m-ésima iteração, o citério da parada adotado neste trabalho é, com $\varepsilon>0$,

$$
\left\|\mathbf{b}-A \mathbf{z}^{(m)}\right\|_{\infty}<\varepsilon .
$$

Além disso, definimos o número de condição de A na norma $l_{2}$ por

$$
K_{2}(A)=\|A\|_{2}\left\|A^{-1}\right\|_{2} .
$$

Exemplo 1 Embora não tenha sido feito um estudo teórico formal para as duas conclusões acima apresentadas, elas são bastante claras empiricamente. Para comprovar isso, utilizamos algumas simulações realizadas para o inchamento do extrudado de um fluido newtoniano a $R e=0.05$, onde o modelo empregado corresponde ao descrito na Seção 4.3.5 e a versão do GENSMAC à descrita na Seção 2.3.

Assim, o problema foi resolvido pela FE-EE com $\delta$ t próximo do limite de estabilidade $\left(F_{\text {visc }}=0.32\right)$ e pela FI-CN em cinco situações com $\delta t$ crescente $\left(F_{\text {visc }} \in\{0.32,1.6,3.2,32.0,160.0\}\right)$. Em todas as seis simulações, $F_{o}=1.0, F_{C F L}=0.5$ e $\varepsilon=1.0 \times 10^{-10}$. A Tabela 3.2.1 apresenta alguns dados das simulações em $t=20.0$, instante no qual o fluido ainda não atingiu o ejetor continuado. Visualmente, as simulações produziram resultados muito parecidos e, por isso, omitidos.

Inicialmente, a partir do tempo de CPU total é possivel verificar que um pequeno aumento no $\delta t$ para a FI-CN $\left(F_{\text {visc }}=1.6\right)$ representa uma economia de, aproximadamente, $60 \%$ em tempo de CPU em comparação à FE-EE $\left(F_{\text {visc }}=0.32\right)$, confirmando sua eficiência. No entanto, para um mesmo $\delta t$ $\left(F_{v i s c}=0.32\right)$ e, consequentemente, um mesmo número de ciclos computacionais, a vantagem se inverte, e a FI-CN apresenta um tempo de CPU, aproximadamente, $60 \%$ maior que o gasto pela FE-EE. A causa para essa perda de desempenho está bem clara, sendo o tempo de CPU médio para o cálculo de $\widetilde{\mathbf{u}}$ e de $\psi$ na FI-CN muito maior que o gasto nas etapas análogas da FE-EE.

Outro detalhe importante é que o tempo de CPU médio gasto pela FE-EE na solução de $\widetilde{\mathbf{u}}$ é, conforme já argumentamos, significativamente menor que o gasto pela FI-CN. Além disso, podemos observar que, para $F_{\text {visc }}=0.32$, na solução de $\psi$, o BiCG-Jacobi converge em um número bastante próximo de iterações àquele do CG, mas, como as iterações do BiCG-Jaccobi são mais caras, o tempo de CPU médio gasto pela FI-CN nesta etapa é quase o dobro daquele gasto pela FE-EE.

Além disso, é muito importante observar que, à medida que se aumenta $\delta t$, as etapas de solução de $\widetilde{\mathbf{u}}$ e de $\psi$ pela FI-CN perdem muito desempenho em número de iterações (Figura 3.2.1) gastas pelo método iterativo e, consequentemente, em tempo de CPU médio por ciclo (Figura 3.2.2). Uma das causas para isso está na deterioração do chute inicial empregado pelo método iterativo. A outra está no crescimento do número de condição dos sistemas lineares resolvidos pela FI-CN, conforme ilustra a Tabela 3.2.2.

Com efeito, a Tabela 3.2.2 confirma duas expectativas inicialmente citadas: os sistemas lineares para $\widetilde{\mathbf{u}}$ e $\psi$ tornam-se piores condicionados à medida que aumentamos $\delta t$ e, para um mesmo $\delta t$, o sistema para $\psi$ da FI-CN é pior condicionado que o análogo da FE-EE.

\begin{tabular}{|c|c|c|c|c|c|c|c|}
\hline \multicolumn{2}{|c|}{ método } & $F E-E E$ & \multicolumn{5}{|c|}{$F I-C N$} \\
\hline \multirow{3}{*}{\multicolumn{2}{|c|}{$\begin{array}{c}F_{\text {visc }} \\
\text { ciclos } \\
\text { células }(\mathbf{F}: \mathbf{S})\end{array}$}} & 0.32 & 0.32 & 1.60 & 3.20 & 32.00 & 160.0 \\
\hline & & 2000000 & 2000000 & 400000 & 200000 & 20000 & 4000 \\
\hline & & $5390: 328$ & $5379: 328$ & $5377: 328$ & $5380: 330$ & $5280: 329$ & $5281: 329$ \\
\hline \multicolumn{2}{|c|}{ tempo de CPU total } & $25: 22: 46$ & $42: 17: 12$ & $10: 12: 30$ & $05: 41: 36$ & 01:04:33 & $00: 15: 19$ \\
\hline \multicolumn{2}{|c|}{$\begin{array}{c}\text { tempo de } \\
\text { CPU médio } \\
\text { por ciclo }\left(\times 10^{-2}\right)\end{array}$} & 4.5683 & 7.6133 & 9.1915 & 10.2530 & 19.4300 & 23.6500 \\
\hline média de & $\widetilde{\mathbf{u}}$ & - & 9.9073 & 17.7484 & 24.7151 & 88.4401 & 173.1878 \\
\hline iterações & $\psi$ & 83.0661 & 70.2515 & 94.3330 & 110.6052 & 247.9791 & 279.2783 \\
\hline tempo de & $\widetilde{\mathbf{u}}\left(\times 10^{-3}\right)$ & 2.5897 & 9.9030 & 11.8018 & 12.6277 & 24.9105 & 42.8680 \\
\hline CPU médio & $\psi\left(\times 10^{-2}\right)$ & 2.0917 & 4.3759 & 5.7551 & 6.8135 & 14.7566 & 16.4675 \\
\hline
\end{tabular}

Tabela 3.2.1: Comparação da resolução dos sistemas lineares nas FE-EE e FI-CN, em $t=20.0$. 
Tabela 3.2.2: Número de condição na norma $l_{2}$ para os sistemas lineares envolvendo $\widetilde{u}, \widetilde{v}$ e $\psi$ obtidos com as FE-EE e FI-CN, em $t=3.0$.

\begin{tabular}{|c|c|c|c|c|c|c|}
\hline método & $F E-E E$ & \multicolumn{5}{|c|}{$F I-C N$} \\
\hline \hline$K_{2}^{\psi}\left(\times 10^{3}\right)$ & 4.99308 & 5.03336 & 5.13958 & 6.24802 & 57.68031 & 295.41117 \\
$K_{2}^{\widetilde{u}}$ & - & 1.31819 & 2.58345 & 4.14834 & 29.47840 & 101.00523 \\
$K_{2}^{\widetilde{v}}$ & - & 1.31739 & 2.57949 & 4.14043 & 29.40228 & 100.68822 \\
\hline \hline$F_{\text {visc }}$ & 0.32 & 0.32 & 1.60 & 3.20 & 32.00 & 160.00 \\
\hline número de células $(\mathbf{F}: \mathbf{S})$ & $846: 28$ & $846: 28$ & $846: 28$ & $846: 28$ & $846: 28$ & $845: 28$ \\
\hline
\end{tabular}

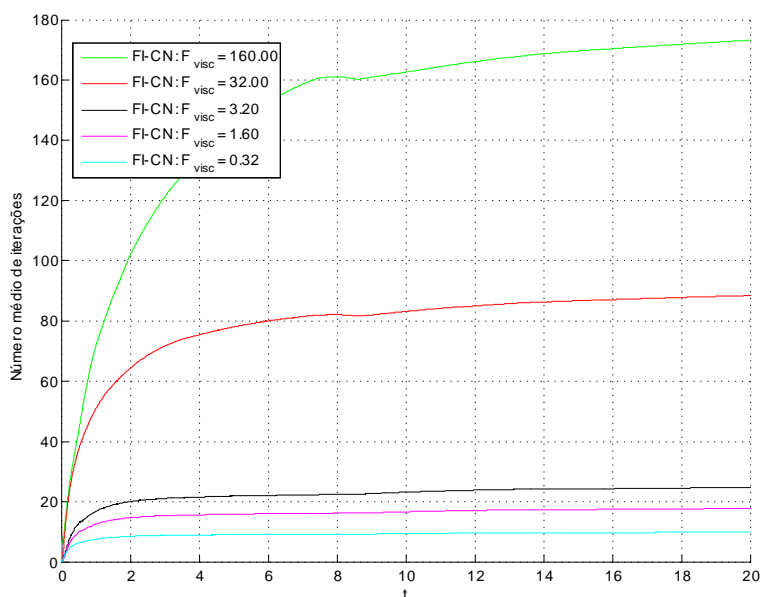

(a)

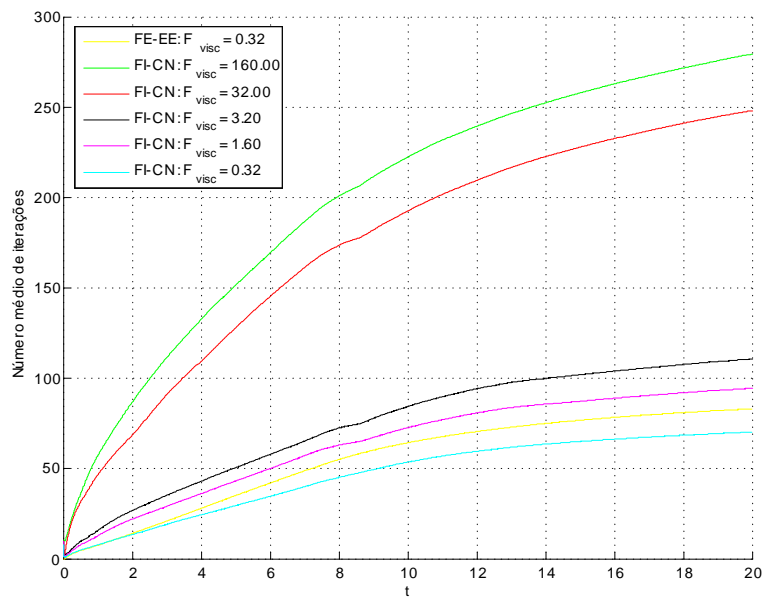

(b)

Figura 3.2.1: Número médio de iterações na resolução dos sistemas lineares envolvendo $\widetilde{\mathbf{u}}(\mathrm{a})$ e $\psi$ (b) presentes nas FE-EE e FI-CN

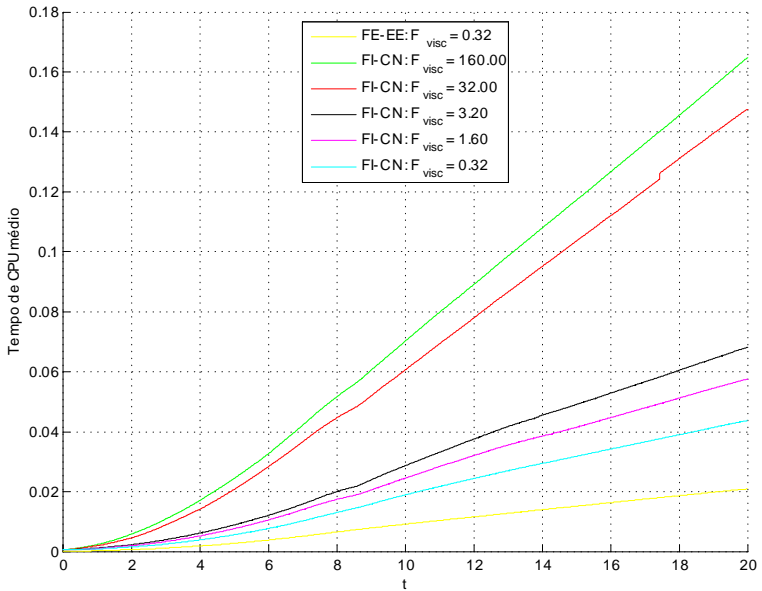

(a)

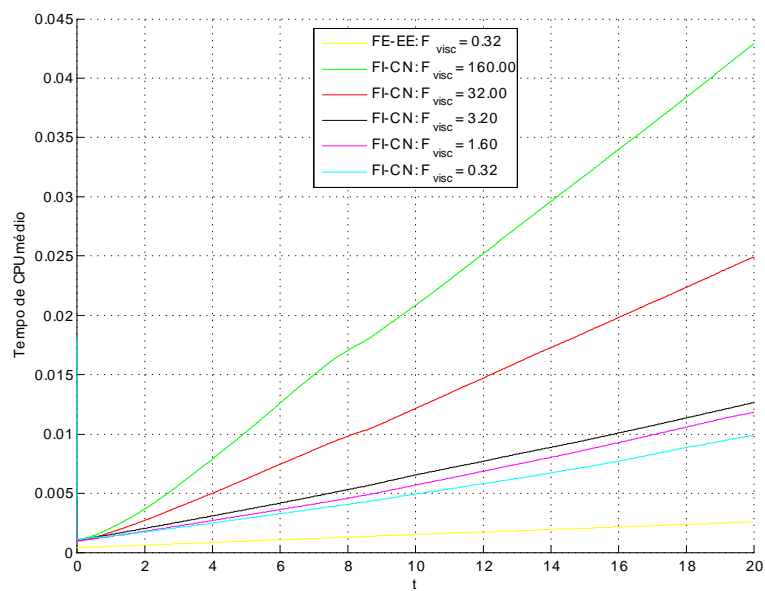

(b)

Figura 3.2.2: Tempo de CPU médio gasto na resolução dos sistemas lineares envolvendo $\widetilde{\mathbf{u}}$ (a) e $\psi$ (b) presentes nas FE-EE e FI-CN. Em (a), a curva associada à FE-EE corresponde ao tempo de CPU médio associado ao cálculo explícito de $\widetilde{\mathbf{u}}$. 


\subsubsection{Métodos de solução dos sistemas lineares}

Os argumentos apresentados no início desta seção, bem como os dados do Exemplo 1, atestam um fato muito importante: a eficiência de um ciclo computacional da FI deixa muito a desejar quando comparado ao da FE. Isto se deve, principalmente, às estratégias de solução dos sistemas lineares adotadas, as quais, conforme exemplificamos, se por um lado perdem muito desempenho quando aumentamos $\delta t$, por outro também deixam a desejar se usamos um passo temporal muito pequeno, tal como na FE. Neste contexto, o maior vilão é a etapa de solução de $\psi$, que consome a maior fração do tempo de CPU em um ciclo.

Assim, podemos concluir que existe uma boa margem para tornar a FI mais eficiente através de modificações nas estratégias de solução dessas etapas. Na verdade, as duas conclusões apontadas no início desta seção sugerem quais são essas alterações.

Com efeito, a adoção de métodos iterativos mais robustos (em substituição ao BiCG), que possam se adaptar melhor ao nosso tipo de problema, pode tornar menos relevante a questão da deterioração do chute inicial à medida que $\delta t$ aumenta. Embora exista uma grande variedade de métodos iterativos presentes na literatura, infelizmente, parece não haver consenso sobre qual a melhor escolha. Assim, neste trabalho, investigamos alguns dos mais citados, que são o BiCG (BiConjugate Gradient), o QMR (QuasiMinimal Residual), o TFQMR (Transpose-Free Quasi-Minimal Residual), o CGS (Conjugate Gradient Squared) e o BiCGstab (BiConjugate Gradient stabilized).

Da mesma forma, o emprego de outros pré-condicionadores pode ser algo muito útil, tendo em vista que o condicionamento dos sistemas lineares da FI piora à medida que aumentamos $\delta t$. Mais uma vez, existe uma grande quantidade de pré-condicionadores presentes na literatura, sem que exista consenso de qual é o melhor. Para a nossa aplicação de interesse, perceba que estamos resolvendo uma sequência de sistemas lineares cuja matriz coeficiente muda a cada ciclo computacional, dependendo da configuração do fluido no interior do domínio. Assim, é muito adequado que o pré-condicionador escolhido não exija armazenamento extra e pré-processamento.

Considere, em (3.2.1),

$$
A=L+D+U,
$$

onde $L, D$ e $U$ são, respectivamente, matrizes triangular inferior, diagonal e triangular superior, sendo as entradas de $D$ os elementos diagonais de $A$, com as matrizes $L$ e $D$ apresentando diagonal nula. Se $M$ é a matriz de pré-condicionamento a esquerda,

$$
\left(M^{-1} A\right) \mathbf{z}=M^{-1} \mathbf{b}
$$

é o sistema pré-condicionado, equivalente a (3.2.1), que é, efetivamente resolvido.

Portanto, atendendo aos requisitos anteriormente estipulados, empregamos três pré-condicionadores:

- Jacobi

$$
M=D
$$

- Gauss-Seidel

$$
M=(L+D)(U+D)
$$

- SOR

$$
M=\frac{1}{2-\omega}\left(L+\frac{1}{\omega} D\right)\left(\frac{1}{\omega} D\right)^{-1}\left(U+\frac{1}{\omega} D\right),
$$

onde $\omega \in] 0,2\left[\right.$ é um parâmetro capaz de minimizar o número de condição de $M^{-1} A$, e, consequentemente, o número de iterações do método iterativo.

As matrizes dos pré-condicionadores Gauss-Seidel e SOR fornecem uma fatoração aproximada de $A$ sem pré-processamento e sem a necessidade de armazenamento extra. Isto difere de outros précondicionadores que calculam uma fatoração aproximada de $A$, como o ILU, onde, além de armazenamento extra, existe a possibilidade de falha (se existir um pivô nulo) na fase de pré-processamento.

É muito importante citar que, neste trabalho, utilizamos os métodos iterativos e pré-condicionadores como ferramentas para a solução dos sistemas lineares de interesse, a fim de tornar a FI mais eficiente. 
Assim, detalhes sobre os métodos iterativos e pré-condicionadores, citados nesta seção e empregados no decorrer deste trabalho, podem ser encontrados em [GL89], [HK94] e [QT00], estando em [SD00] as versões destes procedimentos implementadas e utilizadas.

Portanto, no capítulo seguinte, investigamos os métodos citados ao longo desta seção, buscando responder a seguinte pergunta: "É possível tornar a FI mais eficiente pela adoção de outras estratégias de solução dos sistemas lineares para $\widetilde{\mathbf{u}} \mathrm{e}$, principalmente, para $\psi$ ?".

Exemplo 2 Voltando ao Exemplo 1, a primeira questão que surge a partir dos pré-condicionadores apresentados diz respeito a quanto eles podem diminuir o número de condição associado aos sistemas lineares para $\widetilde{\mathbf{u}}$ e para $\psi$.

Neste sentido, apresentamos a Tabela 3.2.3, onde podemos observar uma redução superior a 400 vezes no número de condição para o sistema linear envolvendo $\psi$ na FI-CN quando da aplicação do pré-condicionador Gauss-Seidel no caso em que $F_{v i s c}=160.0$. Além disso, o número de condição do sistema linear para $\widetilde{\mathbf{u}}$ tornou-se muito baixo quando do uso do pré-condicionador Gauss-Seidel, embora seja mantido quase inalterado pelo pré-condicionador de Jacobi.

Aliás, é interessante observar que embora o sistema linear para $\psi$ fique pior condicionado à medida que aumentamos $\delta t$, a aplicação do pré-condicionador Gauss-Seidel amortece consideravelmente essa dependência.

Sendo o pré-condicionador Gauss-Seidel bastante eficiente, é natural esperar que o SOR possa produzir resultados ainda melhores mediante a conveniente escolha de $\omega$. Surge, então, uma segunda questão relevante, que diz respeito ao parâmetro de relaxação $\omega$ ideal para cada sistema linear. Neste sentido, nenhum estudo teórico foi realizado, mas, os testes numéricos efetuados para a classe de problemas de interesse nos conduziu a duas respostas importantes.

Para o sistema linear associado a $\widetilde{\mathbf{u}}$ foi possivel perceber que o $\omega$ ótimo tende a 1.0 a medida que diminuímos $\delta t$, conforme a Figura 3.2.3 (a)-(b). Já no sistema linear para $\psi$ podemos observar que o $\omega$ ótimo praticamente independe de $\delta t$, conforme ilustra a Figura 3.2.3 (c)-(d).

No próximo capítulo, os testes realizados mostram uma tendência importante, pela qual $\omega \approx 1.8$ é uma escolha muito interessante para a resolução dos sistemas associados a $\psi$, ao passo que devemos usar bom senso na solução de $\widetilde{\mathbf{u}}$, tomando $\omega$ mais próximo de 1.0 à medida que o passo temporal é diminuído.

Tabela 3.2.3: Número de condição na norma $l_{2}$ para os sistemas lineares envolvendo $\widetilde{u}, \widetilde{v}$ e $\psi$ obtidos com as FE-EE e FI-CN, em $t=3.0$. Constam, também, os números de condição para tais sistemas com os pré-condicionadores Jacobi e Gauss-Seidel.

\begin{tabular}{|c|c|c|c|c|c|c|c|}
\hline \multicolumn{2}{|c|}{ método } & $F E-E E$ & \multicolumn{5}{|c|}{$F I-C N$} \\
\hline- & $\left(\times 10^{3}\right)$ & 4.99308 & 5.03336 & 5.13958 & 6.24802 & 57.68031 & 295.41117 \\
\hline Jacobi & $K_{2}^{\psi} \quad\left(\times 10^{3}\right)$ & 4.84811 & 4.88718 & 4.99106 & 5.11044 & 5.65706 & 5.80001 \\
\hline Gauss - Seidel & $\left(\times 10^{2}\right)$ & 6.66272 & 6.71612 & 6.85813 & 6.96316 & 7.27839 & 7.35232 \\
\hline- & & - & 1.31819 & 2.58345 & 4.14834 & 29.47840 & 101.00523 \\
\hline Jacobi & $K_{2}^{\tilde{u}}$ & - & 1.31740 & 2.57940 & 4.14019 & 29.40378 & 100.74331 \\
\hline Gauss - Seidel & & - & 1.00547 & 1.08788 & 1.24205 & 4.34974 & 13.34397 \\
\hline- & & - & 1.31739 & 2.57949 & 4.14043 & 29.40228 & 100.68822 \\
\hline Jacobi & $K_{2}^{\widetilde{v}}$ & - & 1.31725 & 2.57876 & 4.13898 & 29.38912 & 100.64220 \\
\hline Gauss - Seidel & & - & 1.00547 & 1.08785 & 1.24197 & 4.34836 & 13.33295 \\
\hline \multicolumn{2}{|c|}{$F_{\text {visc }}$} & 0.32 & 0.32 & 1.60 & 3.20 & 32.00 & 160.00 \\
\hline \multicolumn{2}{|c|}{ número de células $(\mathbf{F}: \mathbf{S})$} & $846: 28$ & $846: 28$ & $846: 28$ & $846: 28$ & $846: 28$ & $845: 28$ \\
\hline
\end{tabular}




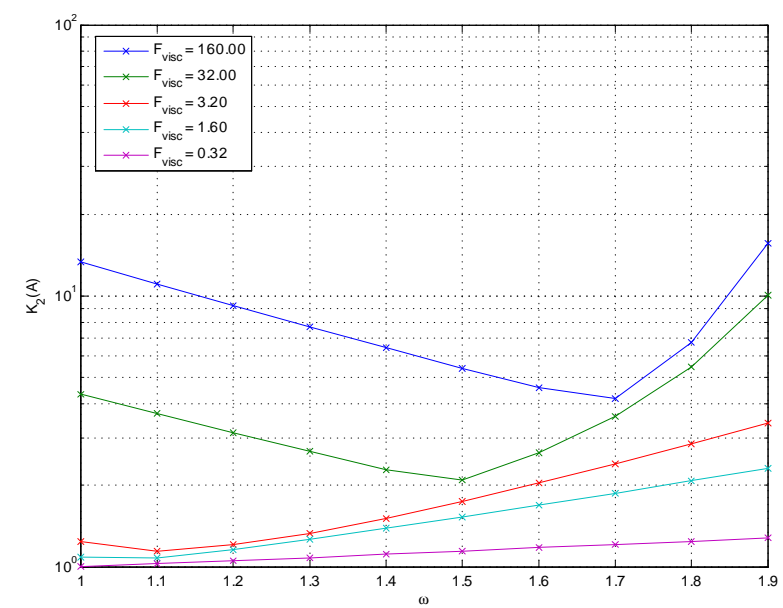

(a)

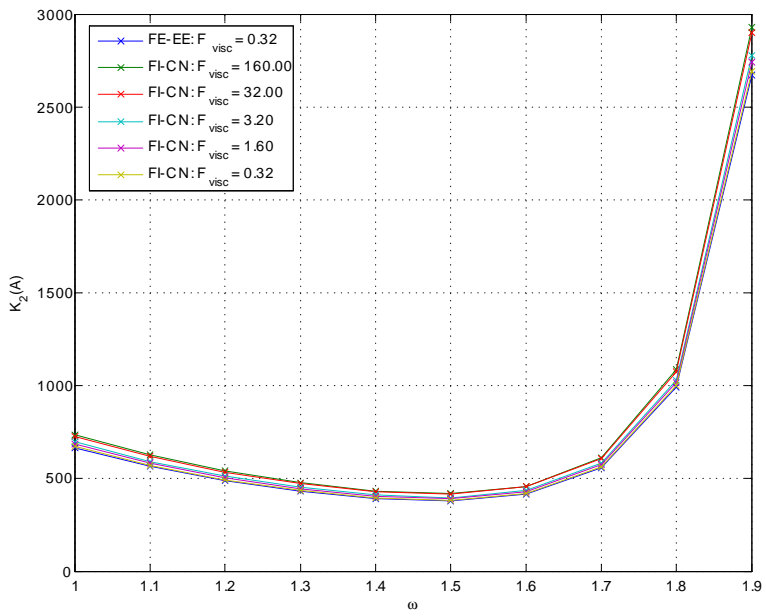

(c)

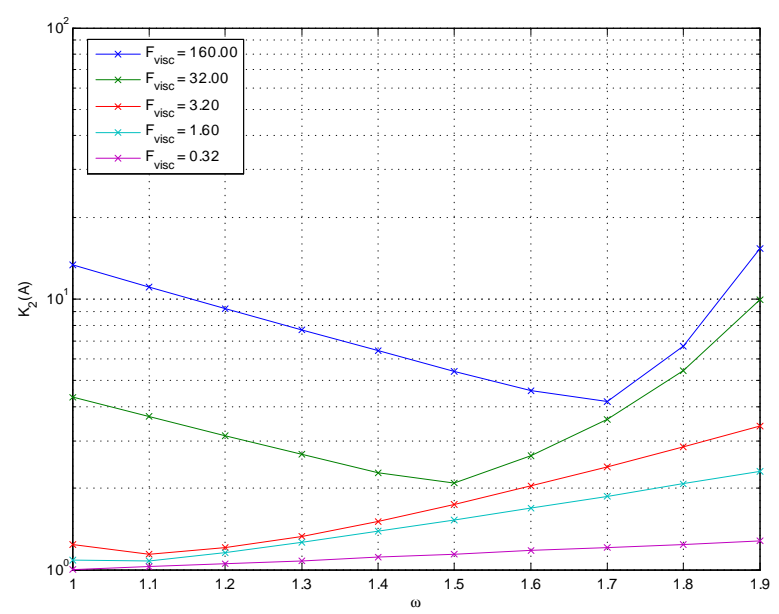

(b)

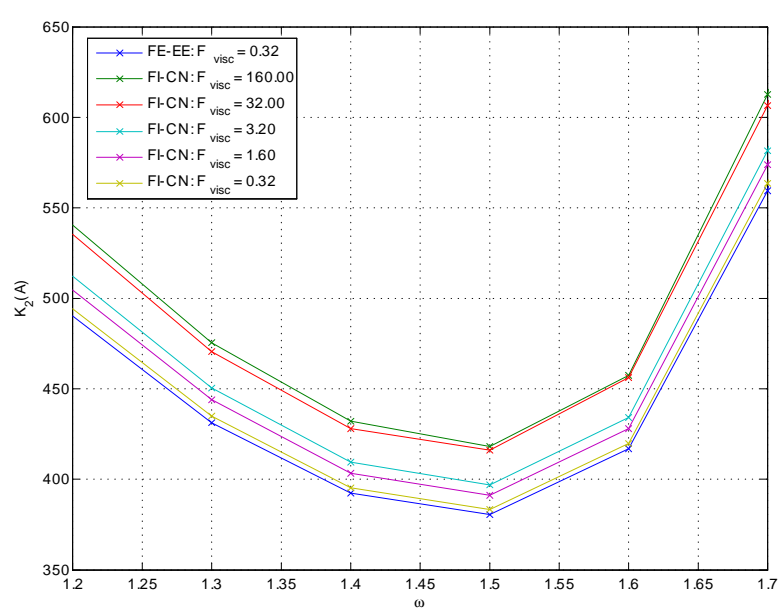

(d)

Figura 3.2.3: Comportamento do número de condição, na norma $l_{2}$, na aplicação do pré-condicionador SOR aos sistemas lineares obtidos com as FE-EE e FI-CN, em $t=3.0$. Analisamos o sistema linear para $\widetilde{u}$ em (a), $\widetilde{v}$ em (b) e $\psi$ em (c)-(d).

\subsection{A massa de fluido computacional}

\subsubsection{Introdução}

Conforme já dissemos, existem várias possibilidades na literatura para a representação computacional do fluido em escoamentos com superfície livre ([EM57], [GM88], [PI04], [OS01], [FD02], [LU05], [LI01], [YY05], [YT01]), nenhuma isenta de limitações.

É fundamental para um método numérico que simula escoamentos com superfície livre o correto posicionamento desta fronteira, pois, sua evolução ao longo do tempo determina o domínio variável onde as equações devem ser resolvidas. Assim, podemos considerar dois atributos importantes para a estratégia de representação da superfície livre, que são a qualidade com que a fronteira livre é aproximada e a manutenção da massa no interior do domínio, além, é claro, do custo computacional que isto acarreta.

Analisar o perfil transiente da superfície livre nem sempre é uma tarefa simples, pois, invariavelmente, soluções analíticas para esses tipos de escoamento são muito raras, dada a complexidade dos problemas.

No entanto, como a quantidade de fluido no interior do domínio é um dado próprio do problema, torna-se bastante simples analisar com que precisão o método numérico é capaz de aproximar sua correta 
evolução no tempo. Em outras palavras, se $m_{\text {exata }}=m_{\text {exata }}(t)$ é a massa exata em função do tempo,

$$
e_{m}(t)=\frac{m_{\text {numérica }}(t)-m_{\text {exata }}(t)}{m_{\text {exata }}(t)}
$$

define o erro na massa $m_{\text {numérica }}=m_{\text {numérica }}(t)$ calculada pelo procedimento numérico. Com efeito, sendo $e_{m}=e_{m}(t)$, para $e_{m}>0$ existe a criação indevida de massa no domínio (fonte numérica de massa), ao passo que, quando $e_{m}<0$, ocorre a perda da massa numérica (sorvedouro numérico de massa). A fim de sintetizar $e_{m}=e_{m}(t)$ em um único valor, estabelecendo o quanto a solução numérica se desvia da exata ao longo de toda simulação, definimos a função de perda total, dada por

$$
p_{m}(t)=\int_{0}^{t}\left|e_{m}(s)\right| d s
$$

Observação 6 Neste trabalho, aproximamos a função perda total por meio da Regra do Trapézio ([LB73], [BU03]), considerando o erro na massa em todos os ciclos computacionais, ou seja, em $t=t_{n}$,

$$
p_{m}\left(t_{n}\right) \approx \sum_{r=0}^{n-1}\left\{\frac{\delta t}{2}\left[\left|e_{m}\left(t_{r}\right)\right|+\left|e_{m}\left(t_{r+1}\right)\right|\right]\right\}
$$

Obviamente, uma margem pequena de erro na massa com a evolução da solução no tempo indica uma condição necessária para a qualidade da mesma. Consequentemente, esta é uma questão que merece ser investigada. Apesar disso, um estudo dessa natureza no contexto do GENSMAC praticamente inexiste na literatura, exceto por [TM01]. No trabalho citado, foi calculado o erro na massa em três simulações de enchimento de contêiner, sem ter sido feito, porém, nenhum estudo sobre seu comportamento mediante variação no passo temporal.

\subsubsection{Movimento da superfície livre}

Na metodologia GENSMAC, uma lista de partículas, que são coordenadas de pontos, define a superfície livre aproximada. Assim, o fluido é representado por um polígono cujas arestas são obtidas ligando duas posições coordenadas consecutivas presentes nessa lista por um segmento de reta.

Portanto, definimos a massa adimensional de fluido no interior do domínio pela área do polígono associado ao fluido. Sendo $\mathbf{x}_{0}^{n}, \mathbf{x}_{1}^{n}, \ldots, \mathbf{x}_{r}^{n}, \operatorname{com} \mathbf{x}_{0}^{n}=\mathbf{x}_{r}^{n}$, vértices consecutivos, em coordenadas adimensionais, desse polígono em $t=t_{n}$, temos a massa numérica adimensional dada por

$$
m_{\text {numérica }}\left(t_{n}\right)=\frac{1}{2}\left|\sum_{i=0}^{r}\left[\left(x_{i+1}^{n}-x_{i}^{n}\right)\left(y_{i+1}^{n}+y_{i}^{n}\right)\right]\right| .
$$

Como fontes para o erro na massa em uma simulação numérica temos os erros de truncamento na discretização das equações, as aproximações consideradas para a superfície livre (ilustradas na Figura 2.1.3 e na Figura 2.1.4) e a aplicação das condições de contorno nas células $\mathbf{S}$ (que são discretizadas considerando que tais células estão "cheias" de fluido mesmo na situação geral em que o fluido cobre apenas uma fração de sua área).

Além disso, a própria representação (linear por partes) e manutenção (pela inserção e remoção de partículas) da superfície livre ocasionam erro na massa numérica.

Outra fonte importante de erro na massa está na solução da equação (2.1.2), isto é,

$$
\frac{d \mathbf{x}}{d t}=\mathbf{u}(\mathbf{x}, t)=\mathbf{u}_{P}
$$

que é responsável pela atualização da posição x da partícula $P$ a cada instante de tempo. Neste processo, a velocidade $\mathbf{u}_{P}$ na posição em que $P$ se encontra é aproximada mediante interpolação bi-linear ([OL99], $[\mathrm{CF} 00])$. Portanto, como $\mathbf{u}_{P}$ é obtido por interpolação, a própria definição da equação (3.3.5) cria um erro que se reflete na massa numérica. 
Observação 7 O cálculo de $\mathbf{u}_{P}$ em (3.3.5) por interpolação bi-linear adotado neste trabalho, seguindo ([OL99], [CF00]), consiste em, inicialmente, localizar o quadrante da célula no qual a partícula $P$ se encontra, conforme Figura 3.3.1 (a). Determinado o quadrante, as velocidades mais próximas a $P$ definidas na malha fixa tornam-se conhecidas e serão utilizadas para a interpolação.

Supondo $P$ no quadrante $Q_{3}$, pela Figura 3.3.1 (b), com $R, P$ e L colineares, segue (pela aplicação de interpolação linear, primeiro ao longo da direção $x$, e, depois, ao longo da direção y):

$$
\begin{aligned}
u_{R} & =\frac{x_{P}-x_{i+\frac{1}{2}}}{x_{i-\frac{1}{2}}-x_{i+\frac{1}{2}}} u_{i-\frac{1}{2}, j-1}+\frac{x_{P}-x_{i-\frac{1}{2}}}{x_{i+\frac{1}{2}}-x_{i-\frac{1}{2}}-} u_{i+\frac{1}{2}, j-1}, \\
u_{L} & =\frac{x_{P}-x_{i+\frac{1}{2}}}{x_{i-\frac{1}{2}}-x_{i+\frac{1}{2}}} u_{i-\frac{1}{2}, j}+\frac{x_{P}-x_{i-\frac{1}{2}}}{x_{i+\frac{1}{2}}-x_{i-\frac{1}{2}}} u_{i+\frac{1}{2}, j}, \\
u_{p} & =\frac{y_{P}-y_{j-1}}{y_{j}-y_{j-1}} u_{L}+\frac{y_{P}-y_{j}}{y_{j-1}-y_{j}} u_{R} .
\end{aligned}
$$

Analogamente, considerando a Figura 3.3.1 (c), sendo $R, P$ e L colineares, segue (pela aplicação de interpolação linear, primeiro ao longo da direção $y$, e, depois, ao longo da direção $x$ ):

$$
\begin{aligned}
v_{R} & =\frac{y_{P}-y_{j+\frac{1}{2}}}{y_{j-\frac{1}{2}}-y_{j+\frac{1}{2}}} v_{i-1, j-\frac{1}{2}}+\frac{y_{P}-y_{j-\frac{1}{2}}}{y_{j+\frac{1}{2}}-y_{j-\frac{1}{2}}} v_{i-1, j+\frac{1}{2}} \\
v_{L} & =\frac{y_{P}-y_{j+\frac{1}{2}}}{y_{j-\frac{1}{2}}-y_{j+\frac{1}{2}}} v_{i, j-\frac{1}{2}}+\frac{y_{P}-y_{j-\frac{1}{2}}}{y_{j+\frac{1}{2}}-y_{j-\frac{1}{2}}} v_{i, j+\frac{1}{2}} \\
v_{p} & =\frac{x_{P}-x_{i-1}}{x_{i}-x_{i-1}} v_{L}+\frac{x_{P}-x_{i}}{x_{i-1}-x_{i}} v_{R} .
\end{aligned}
$$

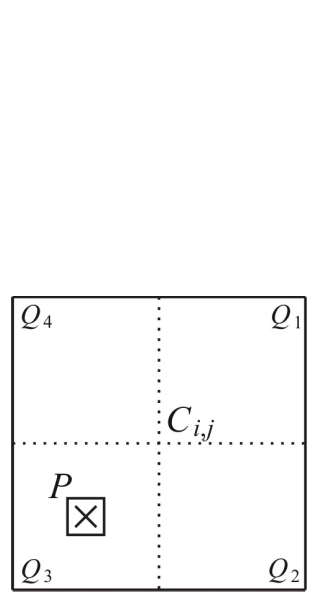

(a)

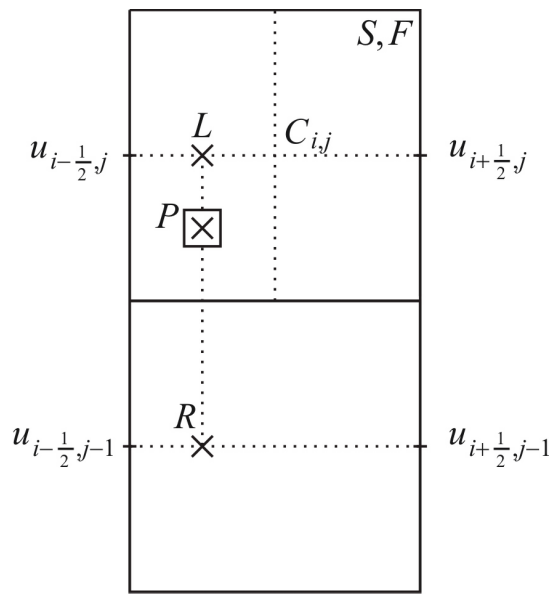

(b)

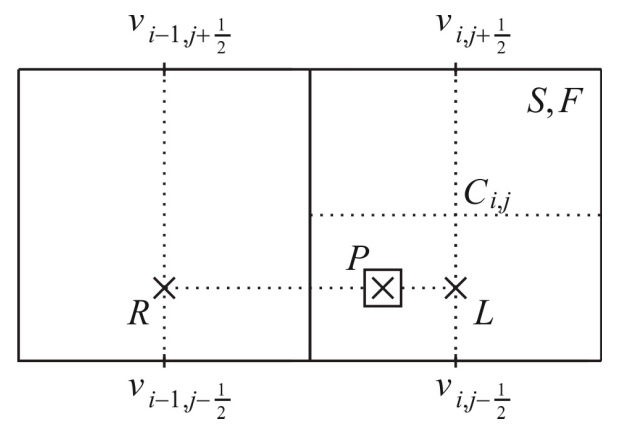

(c)

Figura 3.3.1: Partícula $P$ em uma célula $C_{i, j}$ (a) e esquema de interpolação das velocidades $u$ (b) e $v$ (c).

Entre todas as fontes de erro na massa citadas, nossa atenção se volta, especialmente, para o processo de integração temporal da equação (3.3.5), o qual afeta diretamente a quantidade de massa no interior do domínio por definir, em última instância, como a superfície livre evolui com o tempo.

Até então, a solução de (3.3.5) pelo GENSMAC faz-se por um procedimento semelhante a "Euler Explícito" (EE), no qual apenas o último campo de velocidade calculado é utilizado. Assim, a nova posição $\mathbf{x}^{n+1}$ de uma partícula originalmente $e m \mathbf{x}^{n}$ faz-se mediante a discretização de (3.3.5) por (2.3.14), isto é,

$$
\frac{\mathbf{x}^{n+1}-\mathbf{x}^{n}}{\delta t}=\mathbf{u}\left(\mathbf{x}^{n}, t_{n+1}\right)
$$


resultando em um procedimento de primeira ordem no tempo ([BU03]).

Perceba que, para a FE, em escoamentos a baixos Reynolds $(R e<<1)$, devido à restrição de estabilidade parabólica (2.3.18),

$$
\delta t=O\left(\delta t_{v i s c}\right)=O\left[\operatorname{Re}(\delta m)^{2}\right] .
$$

Assim, o fato da discretização (3.3.12) ser de primeira ordem torna-se aparentemente irrelevante, pois, o passo temporal empregado é sempre muito pequeno.

Para a FI, no entanto, a restrição (3.3.13) não mais serve de hipótese para tornar razoável o uso de (3.3.12). De fato, respeitando o compromisso entre precisão e custo computacional que deve sempre existir, a motivação para a FI é eliminar a restrição de estabilidade parabólica para marchar no tempo com passos temporais maiores que os utilizados pela FE, o que é imprescindível se desejamos efetuar simulações a baixos Reynolds ou em malhas mais refinadas.

Assim, o segundo dos três objetivos principais deste trabalho é investigar como $\delta t$ interfere no erro da massa mediante a precisão com que (3.3.5) é discretizada no tempo.

Para isso, propomos um novo procedimento para a solução de (3.3.5) através do esquema RK21, que é um método Runge-Kutta de segunda ordem no tempo, conforme [BU03]. Por este novo método, a discretização temporal de (3.3.5) passa a ser feita por

$$
\begin{aligned}
\frac{\overline{\mathbf{x}}-\mathbf{x}^{n}}{\delta t} & =\mathbf{u}\left(\mathbf{x}^{n}, t_{n}\right), \\
\frac{\mathbf{x}^{n+1}-\mathbf{x}^{n}}{\delta t} & =\frac{1}{2}\left[\mathbf{u}\left(\mathbf{x}^{n}, t_{n}\right)+\mathbf{u}\left(\overline{\mathbf{x}}, t_{n+1}\right)\right],
\end{aligned}
$$

onde $\overline{\mathbf{x}}$ é uma posição intermediária no processo de movimentação da partícula de sua posição $\mathbf{x}^{n}$, em $t=t_{n}$, a $\mathbf{x}^{n+1}$, em $t=t_{n+1}$.

Nestes termos, parte do próximo capítulo é dedicada a um exaustivo e sistemático estudo sobre o erro na massa a partir de quatro problemas estratégicos com superfície livre. Para cada um deles utilizamos os métodos FE-EE, FI-CN e FI-EI com os esquemas de movimentação EE (3.3.5) e RK21 (3.3.14)-(3.3.15) mediante uma variação no $\delta t$ desde o limite de estabilidade da FI $(\delta t=O(\delta m))$ até atingir valores abaixo do limite de estabilidade da FE $\left(\delta t=O\left[\operatorname{Re}(\delta m)^{2}\right]\right)$. 


\section{Resultados: modificações no GENSMAC}

\subsection{Introdução}

Apresentamos, no capítulo anterior, duas etapas da FI que precisavam ser aprimoradas. Em ambos os casos, isto se justificava na possibilidade de aumento do passo temporal, com relação à FE, que existe na FI.

Assim, realizamos, neste capítulo, uma série de simulações com problemas clássicos 2D que buscam demonstrar que as mudanças propostas podem, efetivamente, contribuir para maior eficiência e precisão da FI, respectivamente, nas etapas de solução dos sistemas lineares e de movimentação da superfície livre.

Para esta análise, sem perda de generalidade, será empregado o modelo newtoniano. Em última instância, isto se deve ao fato das etapas que estamos testando independerem do modelo de fluido.

Com efeito, para os sistemas lineares que calculam $\widetilde{\mathbf{u}}$ e $\psi$, apenas o termo independente é alterado na formulação com outros tipos de fluidos, sendo preservada a estrutura da matriz coeficiente. Da mesma forma, o procedimento de movimento da superfície livre também independe do modelo de fluido, estando ligado apenas à movimentação das partículas marcadoras via a solução numérica de uma Equação Diferencial Ordinária (EDO).

Outro motivo para o emprego do modelo newtoniano neste capítulo se dá, também, pelo fato de inexistir, até então, a extensão da FI para outros tipos de fluido em um domínio espacial 2D. Por outro lado, a versão 2D da FE já foi amplamente testada, servindo, portanto, como um bom referencial para avaliarmos os resultados das modificações na FI.

Antes de iniciarmos a apresentação dos resultados, é válido observar que expressamos a solução numérica, sempre, em variáveis adimensionais, sendo que dados dimensionais que caracterizam o problema são utilizados apenas na sua definição.

\subsection{Resolução dos sistemas lineares}

\subsubsection{Introdução}

Esta seção apresenta os resultados que envolvem testes na solução dos sistemas lineares para $\widetilde{\mathbf{u}}$ e $\psi$. Com esse propósito, empregamos cinco problemas clássicos 2D para os quais foram realizadas uma série de simulações com todas as possibilidades disponíveis de métodos iterativos e pré-condicionadores. Neste contexto, na análise da FI nos restringimos apenas à FI-CN, devido ao grande número de simulações envolvidas em cada teste realizado.

Ao longo desta seção será comum o emprego da notação "método $o_{1}$ método $o_{2}$ ", a qual indica que utilizamos o método 1 para a solução do sistema linear para $\widetilde{\mathbf{u}}$ e o método $o_{2}$ na solução do sistema linear para $\psi$. Além disso, sempre que apenas um método for indicado deve-se considerar que ele foi utilizado na solução de ambos os sistemas (para $\widetilde{\mathbf{u}}$ e para $\psi$ ). Novamente por simplicidade, sempre que apresentamos apenas 
o parâmetro de relaxação $\omega$ do pré-condicionador SOR fica subentendido ter sido utilizado o BiCGstabSOR. A fim de restringir o total de simulações, adotamos, sempre $\omega \in\{1.0,1.2,1.4,1.6,1.8,1.9\}$.

Portanto, após a apresentação dos resultados desta seção, esperamos responder, ao final deste capítulo:

- Entre os métodos testados, qual o mais adequado para ser utilizado na FI?

- Para o melhor método, qual o ganho médio, em tempo de CPU, com relação ao par CG/BiCGJacobi, empregado, até então, pela FI?

- Existe alguma possibilidade de superar o desempenho do CG na solução de $\widetilde{\mathbf{u}}$ pela FI?

- No caso do pré-condicionador SOR, como deve ser feita a escolha de $\omega$ para cada sistema ( $\widetilde{\mathbf{u}}$ e $\psi$ ) ?

- Para $\delta t$ pequeno e com o melhor método obtido, a FI continua altamente ineficiente se comparada à $\mathrm{FE}$ com o -/CG ?

\subsubsection{Problema 1: inchamento do extrudado}

O inchamento do extrudado consiste na extrusão do fluido a partir de um canal inicial de placas paralelas. Assim, o fluido é injetado na extremidade esquerda do canal, desenvolve-se durante sua extensão e é extrudado na extremidade direita do canal, a partir de onde existe certa distância até o posicionamento do ejetor continuado na extremidade direita do domínio. Os dados que definem o modelo empregado neste conjunto de simulações são resumidos abaixo:

- Domínio espacial: $x \in[0.0,15.15] m, y \in[0.0,3.0] \mathrm{m}$.

- Diâmetro do injetor: $L_{0}=1.0 \mathrm{~m}$.

- Velocidade no injetor: $U_{0}=1.0 \mathrm{~ms}^{-1}$, com perfil de injeção reto.

- Comprimento do canal inicial: $5.0 L_{0}$.

- Distância ente injetor e outflow: $15.0 L_{0}$.

- Diâmetro do outflow: $3.0 L_{0}$.

- Aceleração de campo: $g_{0}=9.81 \mathrm{~ms}^{-2}, g^{x}=0.0, g^{y}=0.0$.

- Dados do fluido: newtoniano, com $\mu_{0}\left(\rho_{0}\right)^{-1}=10.0 m^{2} s^{-1}$.

- Parâmetros adimensionais: $R e=0.1, F r \approx 0.319275, S t=1.0 t_{0}$.

- Discretização do domínio espacial: $606 \times 120$ células nas direções $x$ e $y$, respectivamente.

- Discretização do domínio temporal: $F_{o}=1.0, F_{C F L}=0.5, F_{v i s c}=64.0$.

- Passos adimensionais: $\delta m=0.025, \delta t_{v i s c}=1.5625 \times 10^{-5}, \delta t=\min \left\{1.0 \times 10^{-3}, F_{C F L} \delta t_{C F L}\right\}$.

- Refinamento da malha superficial: $c_{\min }=0.02 \delta m, c_{\text {máx }}=0.2 \delta m$.

- Tolerância para sistemas lineares: $\varepsilon=1.0 \times 10^{-12}$.

Desse modo, o uso da FI-CN com o par CG/BiCG-Jacobi é apresentado na Tabela 4.2.1. Esta serve de referência para a comparação com os demais métodos iterativos, cujos resultados estão expressos na Tabela 4.2.2, onde o pré-condicionador SOR utilizou $\omega=1.4$ e $\omega=1.8$ para, respectivamente, os sistemas lineares envolvendo $\widetilde{\mathbf{u}}$ e $\psi$.

Nesta, o método que mais desperta a atenção é o BiCGstab-SOR/BiCGstab-SOR, proporcionando um ganho de, aproximadamente, $70 \%$ em tempo de CPU total com relação ao CG/BiCG-Jacobi, resultando na simulação ilustrada na Figura 4.2.2. Assim, o BiCGstab-SOR teve ganhos em tempo de CPU médio, com relação à metodologia original, da ordem de $40 \%$ na solução da equação do momento e de $90 \%$ na solução de $\psi$, algo muito próximo ao desempenho do CGS-SOR. 
Tabela 4.2.1: Valores de referência para a solução dos sistemas lineares obtidos pela FI-CN no inchamento do extrudado de um fluido newtoniano $(R e=0.1, t=45.0)$ através do par CG/BiCG-Jacobi.

\begin{tabular}{|c|c|c|c|}
\hline \multicolumn{2}{|c|}{ método } & $C G$ & BiCG-Jacobi \\
\hline \multirow{2}{*}{ média } & iterações & 153.53682 & 596.50616 \\
& tempo de $C P U$ & $3.71048 \times 10^{-1}$ & $3.03300 \times 10^{-1}$ \\
\hline \hline \multirow{3}{*}{ total } & ciclos & \multicolumn{2}{|c|}{45000} \\
& células $(\mathbf{F}: \mathbf{S})$ & \multicolumn{2}{|c|}{$25524: 800$} \\
& tempo de CPU & \multicolumn{2}{|c|}{$50: 25: 20$} \\
\hline
\end{tabular}

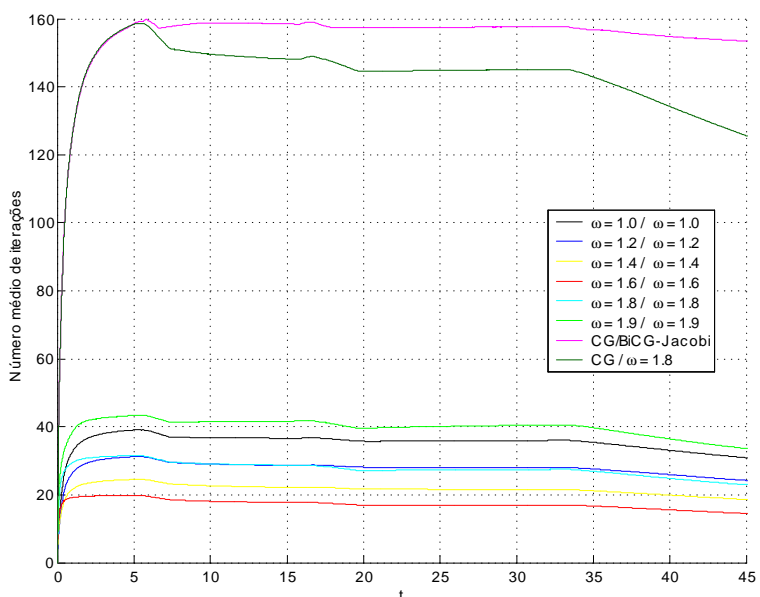

(a)

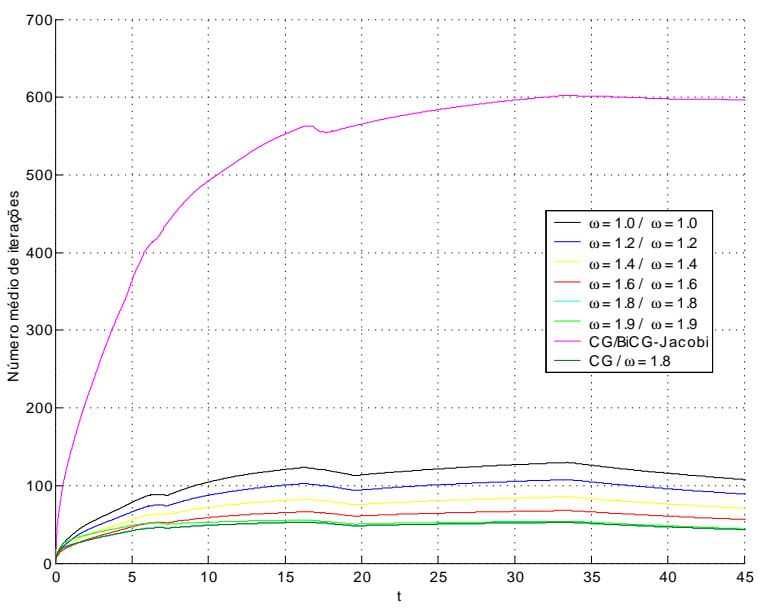

(c)

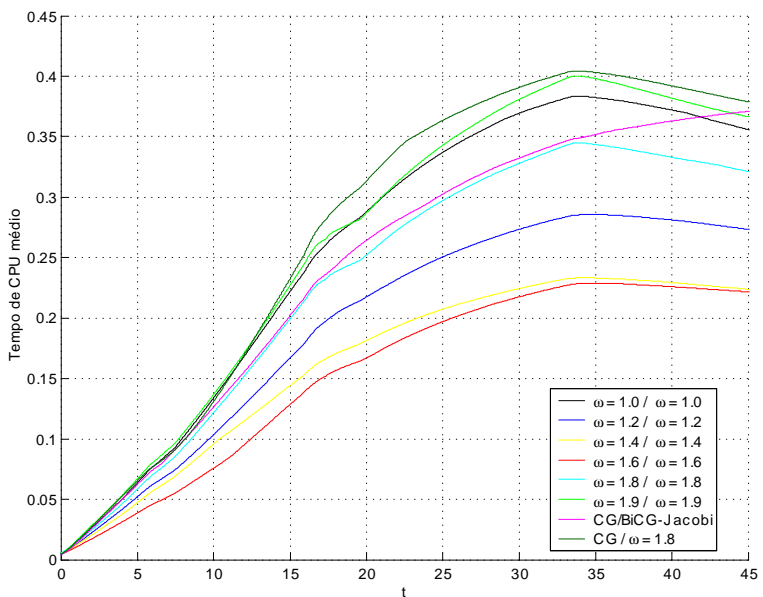

(b)

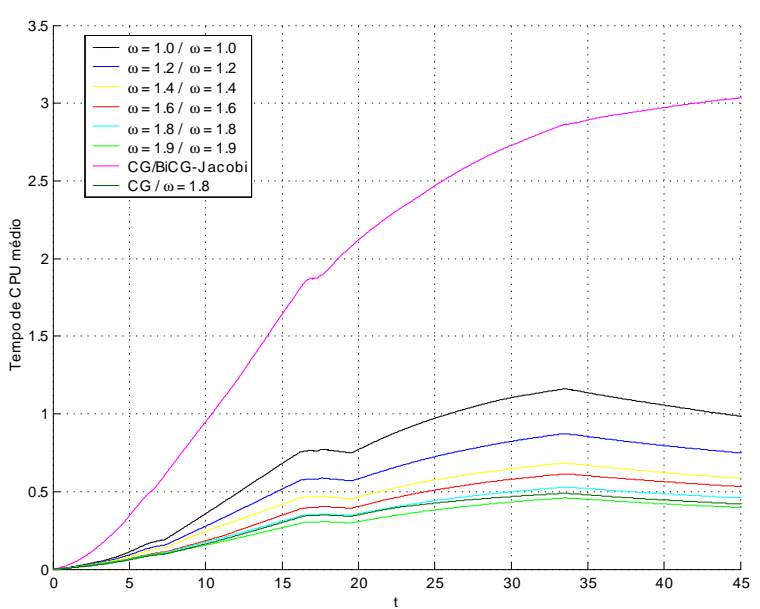

(d)

Figura 4.2.1: Análise comparativa, em função de $t$, do desempenho do pré-condicionador SOR (aplicado ao BiCGstab) com relação ao CG/BiCG-Jacobi, para a solução dos sistemas lineares obtidos pela FI$\mathrm{CN}$ no inchamento do extrudado de um fluido newtoniano $(R e=0.1)$ : número médio de iterações na resolução dos sistemas lineares para $\widetilde{\mathbf{u}}$ (a) e $\psi$ (c), com tempo de CPU médio, respectivamente, em (b) e (d). 


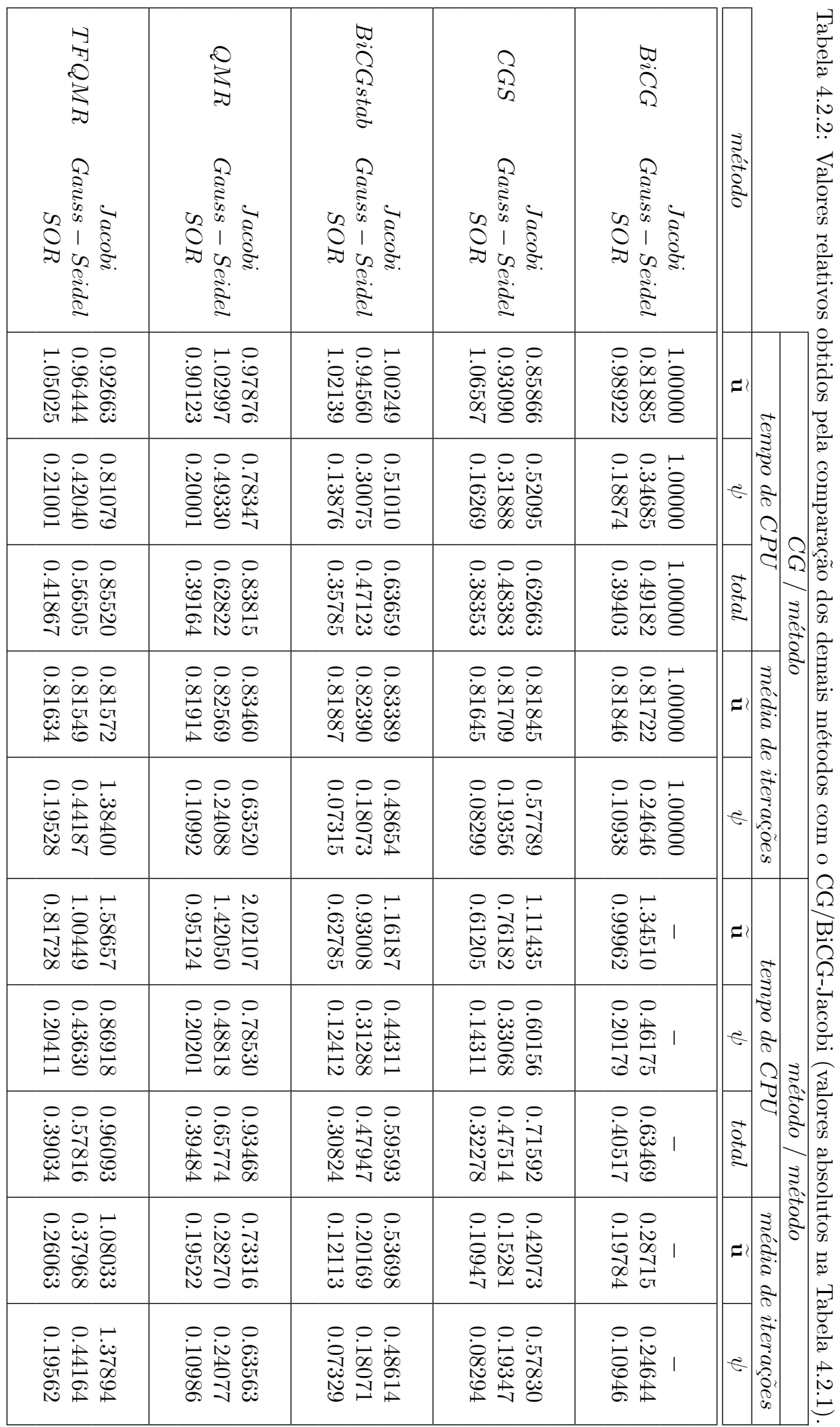




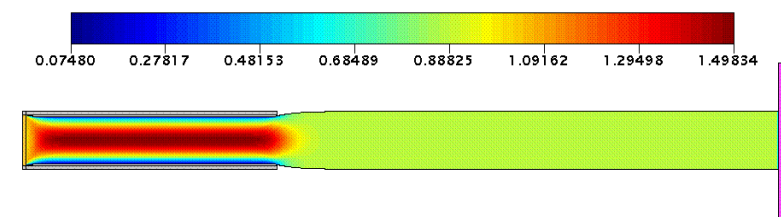

(a)

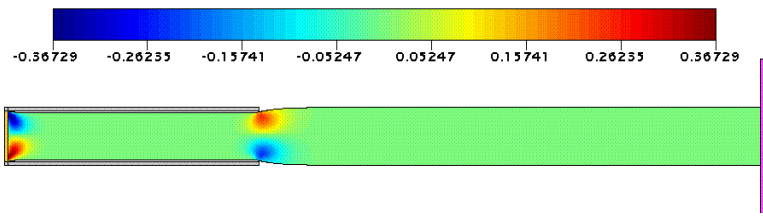

(b)

Figura 4.2.2: Solução numérica da FI-CN para $u$ (a) e $v$ (b) no inchamento do extrudado de um fluido newtoniano ( $R e=0.1, t=45.0)$ utilizando o BiCGstab-SOR na resolução dos sistemas lineares obtidos na discretização das equações de momento ( $\operatorname{com} \omega=1.4)$ e de Poisson (com $\omega=1.8)$.

Com isso, uma questão importante diz respeito à calibragem do parâmetro de relaxação $\omega$. Consideramos, então, o BiCGstab-SOR/BiCGstab-SOR com vários valores para $\omega$, bem como o CG/BiCG-Jacobi e o $\mathrm{CG} / \omega=1.8$, produzindo a Figura 4.2.1.

Inicialmente, indiferente à escolha para $\omega$, vemos, pela Figura 4.2.1, que o BiCGstab-SOR é mais eficiente (em número de iterações e em tempo de CPU médio) que o BiCG-Jacobi, sendo o caso ótimo quando $\omega \approx 1.8$.

Ainda na Figura 4.2.1, a solução da equação do momento pelo BiCGstab-SOR (independentemente de $\omega$ ) necessita de menos iterações que o CG. No entanto, para que isto se reflita em menor tempo de CPU médio, faz-se necessário que a escolha de $\omega$ seja adequada, caso contrário, o desempenho torna-se próximo daquele experimentado pelo CG. Neste problema, o caso ótimo para o cálculo de $\widetilde{\mathbf{u}}$ é obtido com $\omega \approx 1.4$.

\subsubsection{Problema 2: fountain flow}

Neste problema, temos um canal de placa paralelas, inicialmente vazio. Assim, o fluido é injetado na extremidade esquerda do canal, desenvolve-se em sua extensão e atinge o ejetor continuado na extremidade direita do canal, caracterizando o fountain flow. Dessa forma, consideramos o seguinte modelo:

- Domínio espacial: $x \in[0.0,5.15] m, y \in[0.0,1.15] m$.

- Diâmetro do injetor: $L_{0}=1.0 m$.

- Velocidade no injetor: $U_{0}=1.0 \mathrm{~ms}^{-1}$, com perfil de injeção parabólico.

- Comprimento do canal (distância ente injetor e outflow): $5.0 L_{0}$.

- Diâmetro do outflow: $L_{0}$.

- Aceleração de campo: $g_{0}=9.81 \mathrm{~ms}^{-2}, g^{x}=0.0, g^{y}=0.0$.

- Dados do fluido: newtoniano, com $\mu_{0}\left(\rho_{0}\right)^{-1}=10.0 m^{2} s^{-1}$.

- Parâmetros adimensionais: $R e=0.1, F r \approx 0.319275, S t=1.0 t_{0}$.

- Discretização do domínio espacial: $206 \times 46$ células nas direções $x$ e $y$, respectivamente.

- Discretização do domínio temporal: $F_{o}=1.0, F_{C F L}=0.5, F_{v i s c}=64.0$.

- Passos adimensionais: $\delta m=0.025, \delta t_{v i s c}=1.5625 \times 10^{-5}, \delta t=\min \left\{1.0 \times 10^{-3}, F_{C F L} \delta t_{C F L}\right\}$.

- Refinamento da malha superficial: $c_{\min }=0.02 \delta m, c_{m a ́ x}=0.2 \delta m$.

- Tolerância para sistemas lineares: $\varepsilon=1.0 \times 10^{-12}$.

Em um primeiro momento, utilizamos a FI-CN com o par CG/BiCG-Jacobi, produzindo os valores de referência expressos na Tabela 4.2.3. Assim, a utilização dos demais métodos iterativos e précondicionadores resultam na Tabela 4.2.4, onde empregamos $\omega=1.6$ e $\omega=1.8$ para, respectivamente, as equações de momento e de Poisson. 
Tabela 4.2.3: Valores de referência para a solução dos sistemas lineares obtidos pela FI-CN no fountain flow de um fluido newtoniano $(R e=0.1, t=100.0)$ através do par CG/BiCG-Jacobi.

\begin{tabular}{|c|c|c|c|}
\hline \multicolumn{2}{|c|}{ método } & $C G$ & BiCG-Jacobi \\
\hline \multirow{2}{*}{ média } & sistema & $\widetilde{\mathbf{u}}$ & $\psi$ \\
& iterações & 67.21991 & 186.85844 \\
& tempo de $C P U$ & $5.71678 \times 10^{-2}$ & $2.56379 \times 10^{-1}$ \\
\hline \hline \multirow{3}{*}{ total } & ciclos & \multicolumn{2}{|c|}{100028} \\
& células $(\mathbf{F}: \mathbf{S})$ & \multicolumn{2}{|c|}{$8000: 0$} \\
& tempo de CPU & \multicolumn{2}{|c|}{$09: 05: 14$} \\
\hline
\end{tabular}

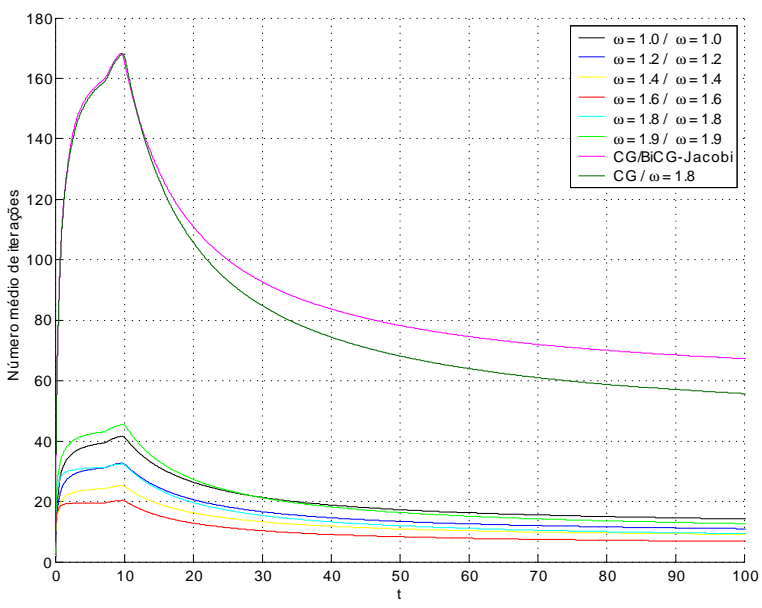

(a)

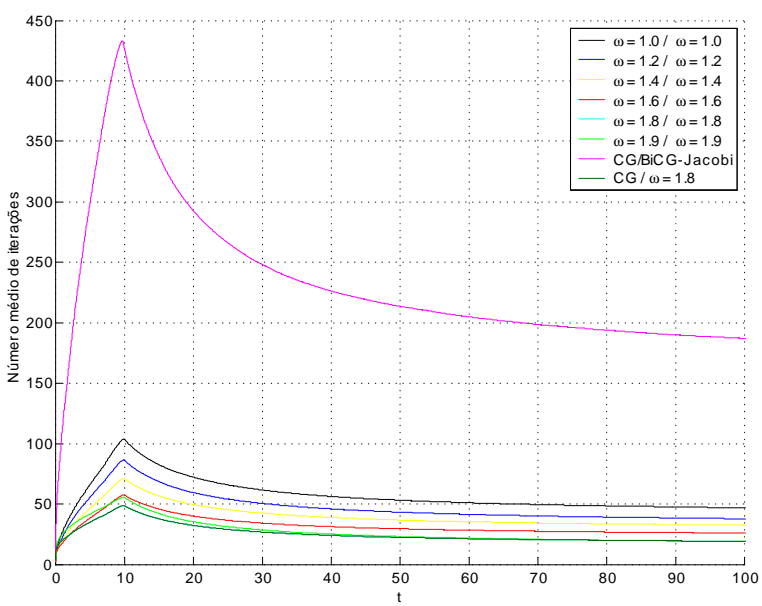

(c)

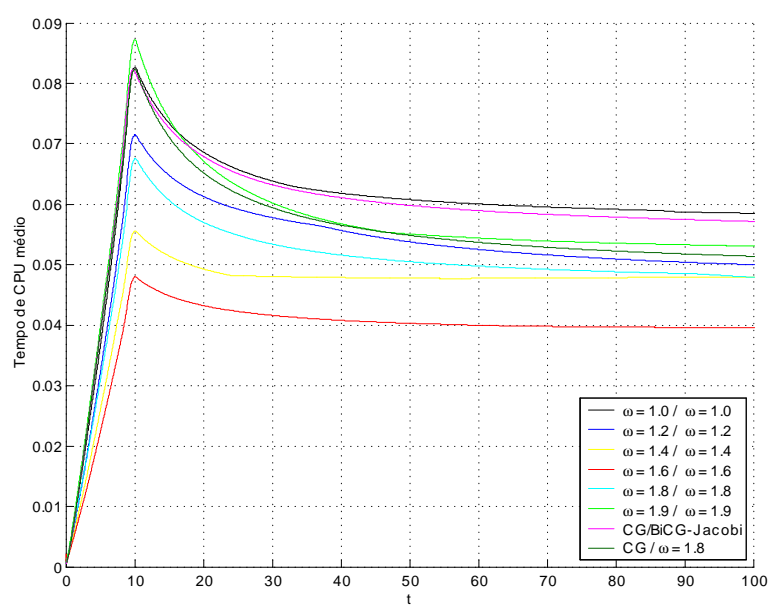

(b)

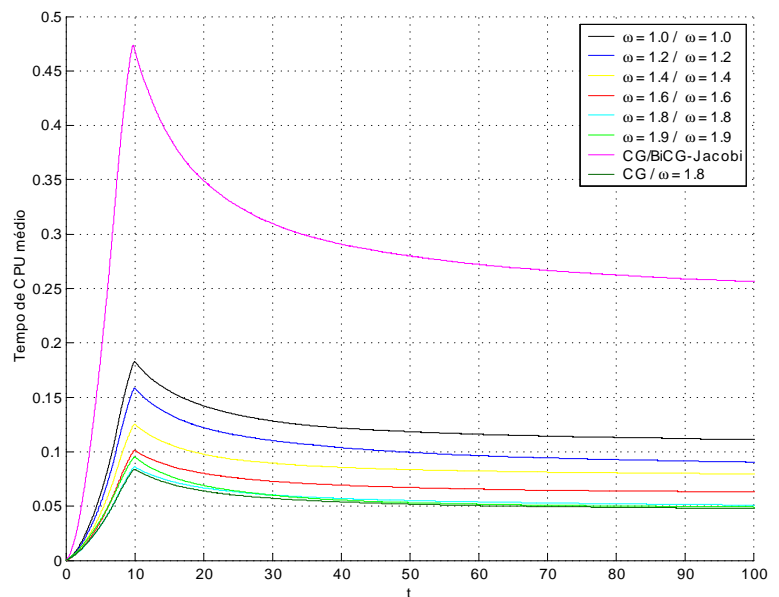

(d)

Figura 4.2.3: Análise comparativa, em função de $t$, do desempenho do pré-condicionador SOR (aplicado ao BiCGstab) com relação ao CG/BiCG-Jacobi, para a solução dos sistemas lineares obtidos pela FI-CN no fountain flow de um fluido newtoniano $(R e=0.1)$ : número médio de iterações na resolução dos sistemas lineares para $\widetilde{\mathbf{u}}(\mathrm{a})$ e $\psi(\mathrm{c})$, com tempo de CPU médio, respectivamente, em (b) e (d). 


\begin{tabular}{|c|c|c|c|c|c|}
\hline $\mid$ & 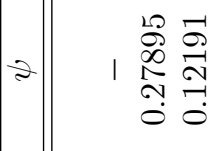 & 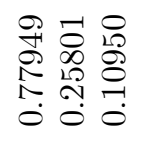 & 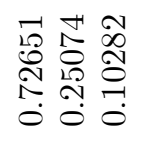 & 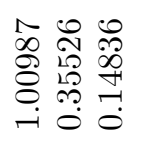 & 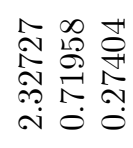 \\
\hline 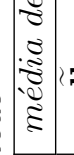 & 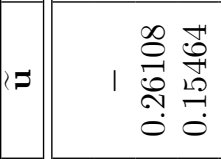 & 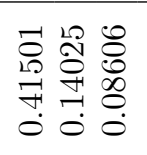 & 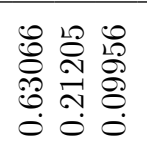 & 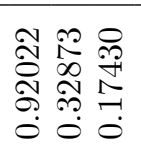 & 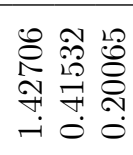 \\
\hline 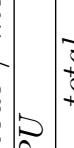 & 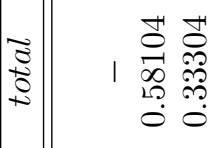 & 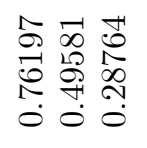 & 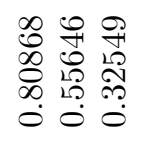 & 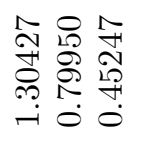 & 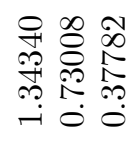 \\
\hline 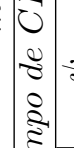 & 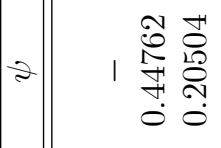 & 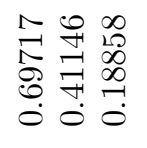 & 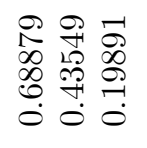 & 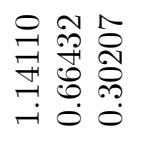 & 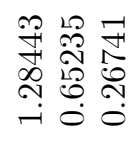 \\
\hline$\infty$ & 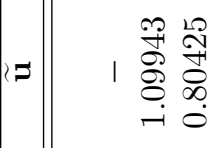 & 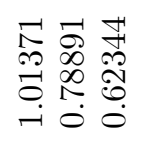 & 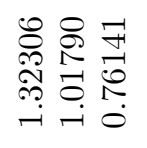 & 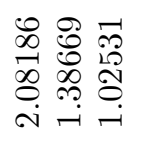 & 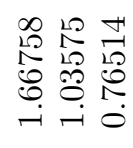 \\
\hline 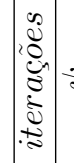 & 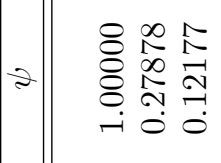 & 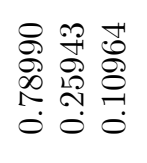 & 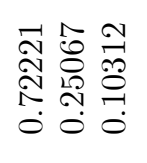 & 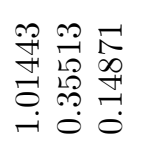 & 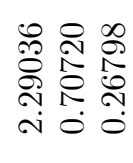 \\
\hline 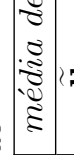 & 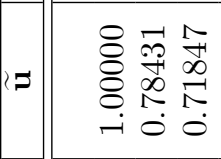 & 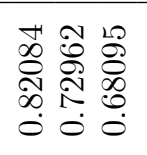 & 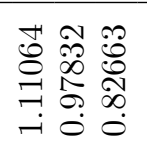 & 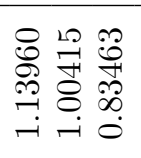 & 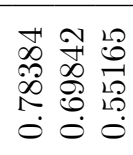 \\
\hline+ & 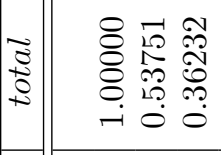 & 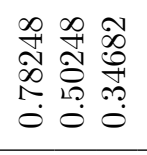 & 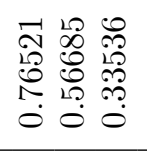 & 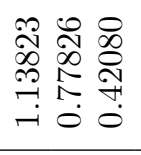 & 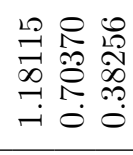 \\
\hline 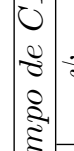 & 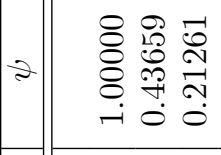 & 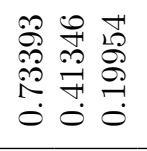 & 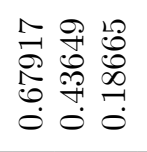 & 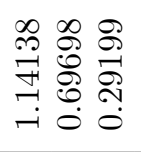 & 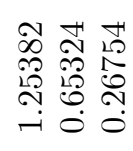 \\
\hline$\underset{\sim}{\Phi}$ & 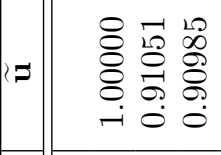 & 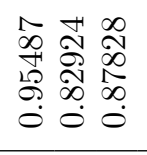 & 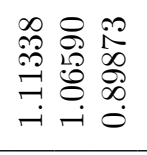 & 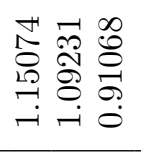 & 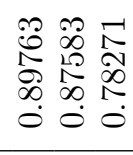 \\
\hline & 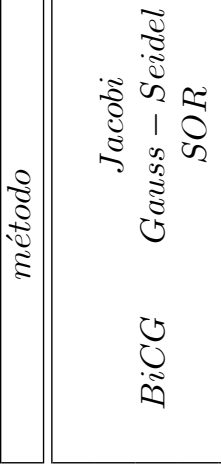 & 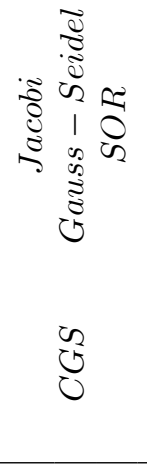 & 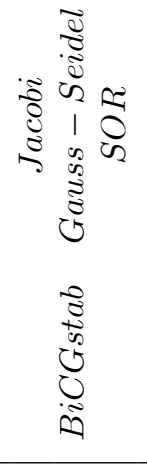 & 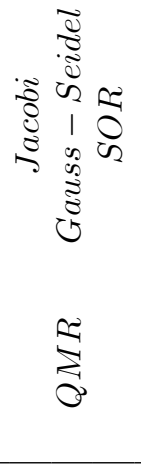 & 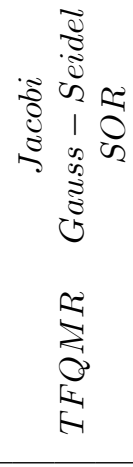 \\
\hline
\end{tabular}




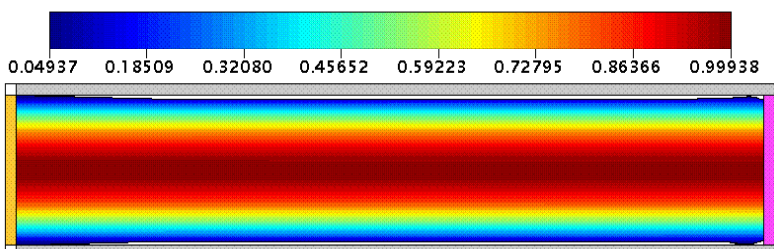

(a)

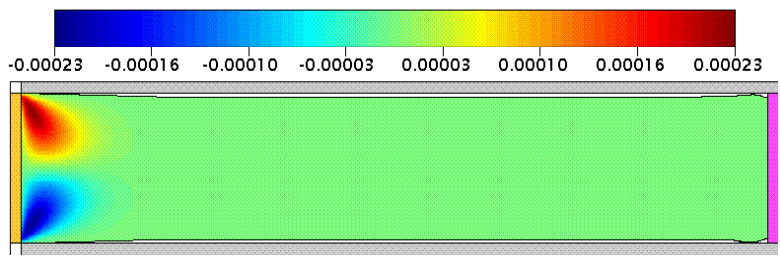

(b)

Figura 4.2.4: Solução numérica da FI-CN para $u(\mathrm{a})$ e $v$ (b) no fountain flow de um fluido newtoniano $(R e=0.1, t=100.0)$ utilizando o BiCGstab-SOR na resolução dos sistemas lineares obtidos na discretização das equações de momento $(\operatorname{com} \omega=1.6)$ e de Poisson $(\operatorname{com} \omega=1.8)$.

Mais uma vez, o BiCGstab-SOR/BiCGstab-SOR mostrou-se superior ao CG/BiCG-Jacobi, resultando na solução apresentada na Figura 4.2.4. Agora, porém, o melhor desempenho coube ao CGS-SOR/CGSSOR, com um ganho em relação ao CG/BiCG-Jacobi da ordem de $72 \%$ (aproximadamente $4 \%$ a mais que o BiCGstab-SOR/BiCGstab-SOR).

Assim, os desempenhos do CGS-SOR e do BiCGstab-SOR ficaram muito próximos, com uma economia de tempo de CPU médio de cerca de $80 \%$ na solução de $\psi$ com relação à abordagem original. Pelo mesmo critério, também houve ganho na etapa de solução de $\widetilde{\mathbf{u}}$ com relação ao CG/BiCG-Jacobi, que, neste caso, foram da ordem de $40 \%$ e $25 \%$ para, respectivamente, o CGS-SOR/CGS-SOR e o BiCGstabSOR/BiCGstab-SOR.

Quanto à escolha do parâmetro de relaxação ideal, temos o estudo realizado na Figura 4.2.3, onde voltamos a empregar o BiCGstab-SOR com diversos valores para $\omega$. Assim, percebe-se que, independentemente do $\omega$ escolhido, os sistemas lineares resolvidos pelo BiCGstab-SOR convergem em um menor número de iterações, tanto na solução de $\widetilde{\mathbf{u}}$ quanto de $\psi$. Ainda na Figura 4.2.3, todas escolhas para $\omega$ resultaram em menor tempo de CPU médio (com relação ao CG/BiCG-Jacobi) na solução de $\psi$, sendo $\omega \approx 1.8$ o valor ideal. Já para o sistema linear envolvendo $\widetilde{\mathbf{u}}$ faz-se necessária uma escolha mais criteriosa para o parâmetro de relaxação ótimo (que é $\omega \approx 1.6$ neste caso), sob pena de obtermos um desempenho semelhante ao CG.

\subsubsection{Problema 3: enchimento estável}

Consideramos, aqui, o problema de injeção de fluido no interior de um contêiner, descrito pelo modelo:

- Domínio espacial: $x \in[0.0,0.1] m, y \in[0.0,0.05] m$.

- Diâmetro do injetor: $L_{0}=4.0 \times 10^{-3} \mathrm{~m}$.

- Velocidade no injetor: $U_{0}=1.0 \mathrm{~ms}^{-1}$, com perfil de injeção reto.

- Altura (do injetor ao fundo da caixa): $h_{1}=0.047 \mathrm{~m}$.

- Largura (entre as paredes verticais da caixa): $h_{2}=0.997 \mathrm{~m}$.

- Aceleração de campo: $g_{0}=9.81 m s^{-2}, g^{x}=0.0, g^{y}=-1.0$.

- Dados do fluido: newtoniano, com $\mu_{0}\left(\rho_{0}\right)^{-1}=5.0 \times 10^{-3} \mathrm{~m}^{2} \mathrm{~s}^{-1}$.

- Parâmetros adimensionais: $R e=0.8, F r \approx 5.048188, S t=250.0 t_{0}$.

- Discretização do domínio espacial: $200 \times 100$ células nas direções $x$ e $y$, respectivamente.

- Discretização do domínio temporal: $F_{o}=1.0, F_{C F L}=0.5, F_{v i s c}=0.32$.

- Passos adimensionais: $\delta m=0.125, \delta t_{v i s c}=3.125 \times 10^{-3}, \delta t=\min \left\{1.0 \times 10^{-3}, F_{C F L} \delta t_{C F L}\right\}$.

- Refinamento da malha superficial: $c_{\min }=0.02 \delta m, c_{m a ́ x}=0.2 \delta m$.

- Tolerância para sistemas lineares: $\varepsilon=1.0 \times 10^{-12}$. 
Tabela 4.2.5: Valores de referência para a solução dos sistemas lineares obtidos pela FI-CN no enchimento estável de um fluido newtoniano ( $R e=0.8, t=250.0)$ através do par CG/BiCG-Jacobi.

\begin{tabular}{|c|c|c|c|}
\hline \multicolumn{2}{|c|}{ método } & $C G$ & BiCG-Jacobi \\
\hline \multirow{2}{*}{ média } & iterações & 16.14916 & 199.54803 \\
& tempo de CPU & $3.812608 \times 10^{-2}$ & $4.08451 \times 10^{-1}$ \\
\hline \hline \multirow{3}{*}{ total } & ciclos & \multicolumn{2}{|c|}{250000} \\
& células $(\mathbf{F}: \mathbf{S})$ & \multicolumn{2}{|c|}{$15790: 238$} \\
& tempo de CPU & \multicolumn{2}{|c|}{$36: 45: 50$} \\
\hline
\end{tabular}

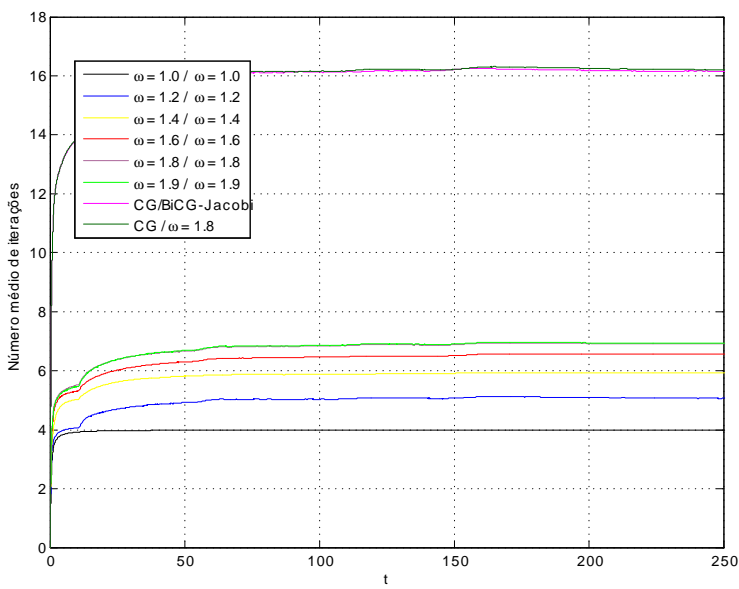

(a)

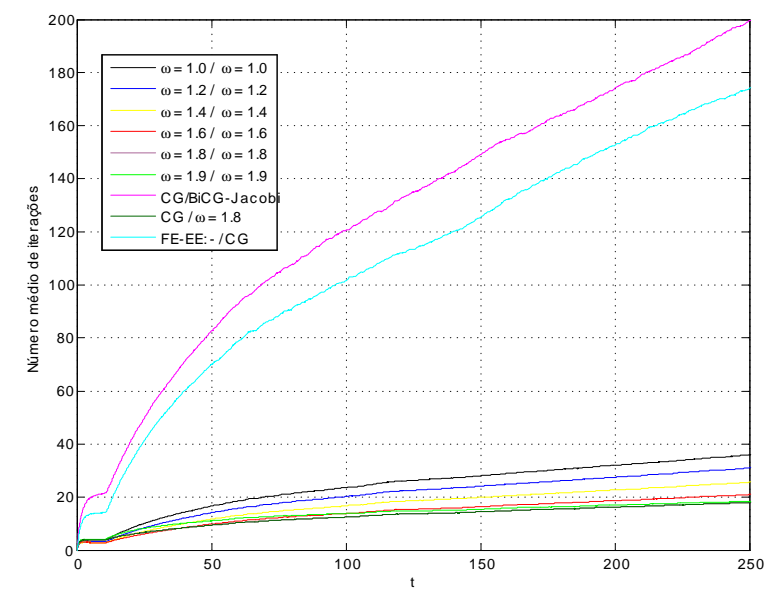

(c)

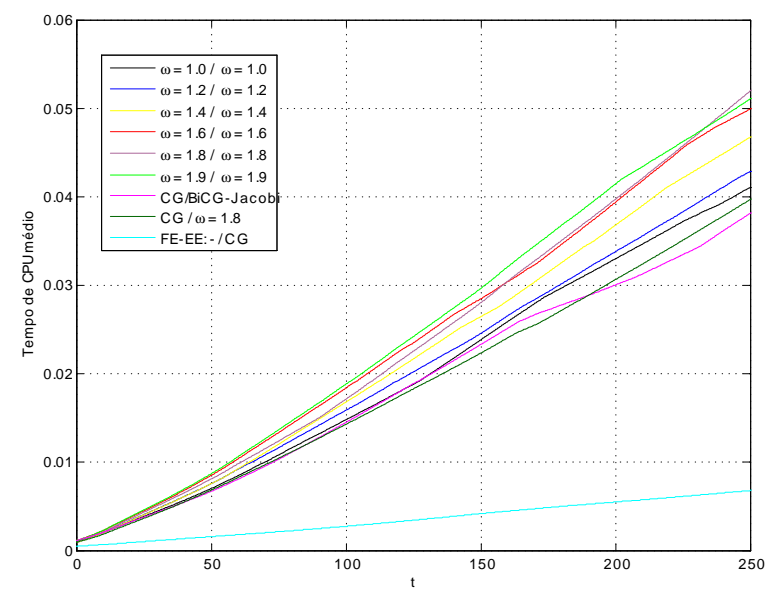

(b)

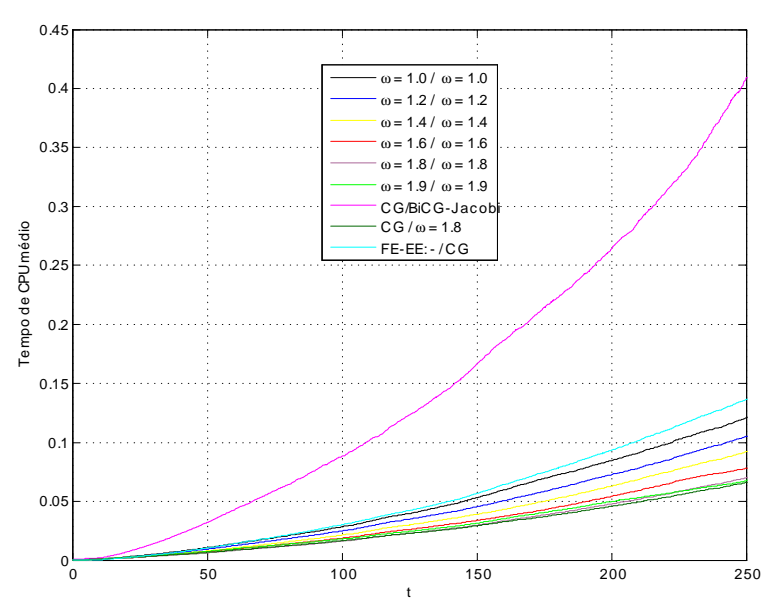

(d)

Figura 4.2.5: Análise comparativa, em função de $t$, do desempenho do pré-condicionador SOR (aplicado ao BiCGstab) com relação ao CG/BiCG-Jacobi, para a solução dos sistemas lineares obtidos pela FI-CN no enchimento estável de um fluido newtoniano $(R e=0.8)$ : número médio de iterações na resolução dos sistemas lineares para $\widetilde{\mathbf{u}}(\mathrm{a})$ e $\psi(\mathrm{c})$, com tempo de CPU médio, respectivamente, em (b) e (d). 


\begin{tabular}{|c|c|c|c|c|c|c|c|}
\hline 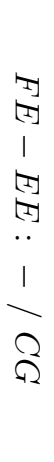 & 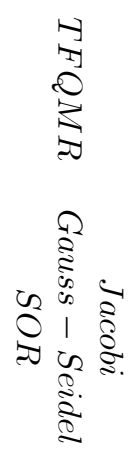 & 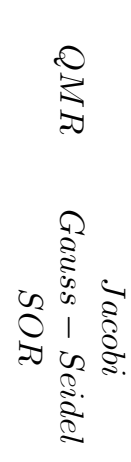 & 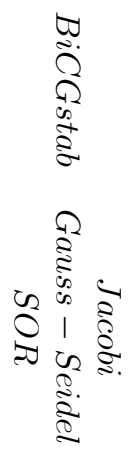 & 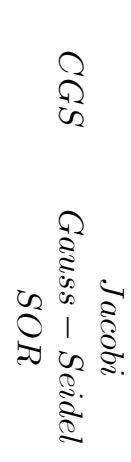 & 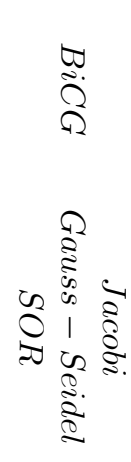 & 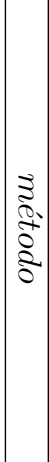 & \\
\hline $\begin{array}{l}0 \\
\stackrel{0}{=} \\
\infty \\
ٍ \\
O\end{array}$ & 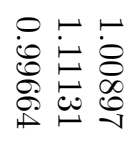 & 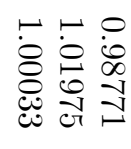 & 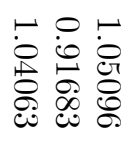 & 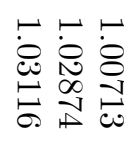 & 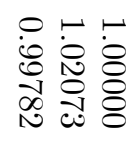 & $\boldsymbol{\Sigma}$ & \multirow{3}{*}{ 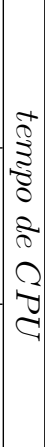 } \\
\hline 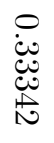 & 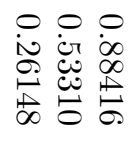 & 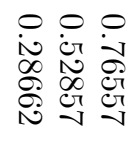 & 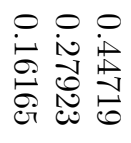 & 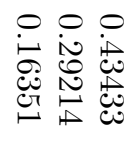 & 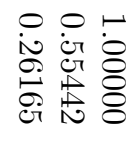 & $\varepsilon$ & \\
\hline 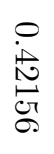 & 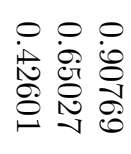 & 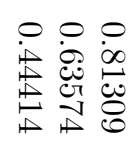 & 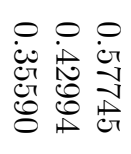 & 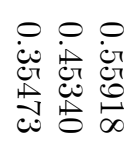 & $\begin{array}{l}0 \\
0\end{array}$ & $\frac{\tilde{2}}{2}$ & \\
\hline 1 & $\begin{array}{l} \\
0\end{array}$ & 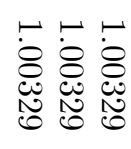 & 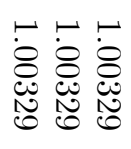 & 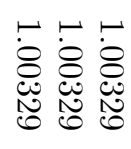 & 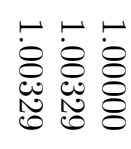 & $=$ & \multirow{2}{*}{ 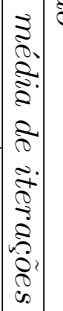 } \\
\hline 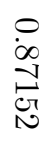 & 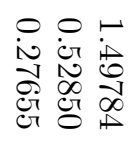 & 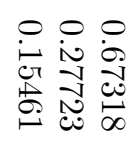 & 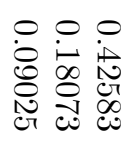 & 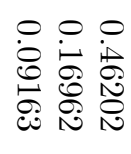 & 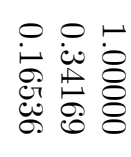 & $\epsilon$ & \\
\hline 1 & 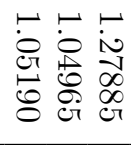 & 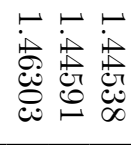 & 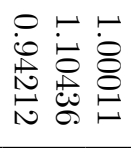 & 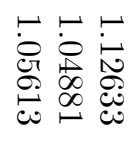 & 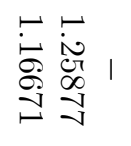 & $\approx$ & \multirow{3}{*}{ 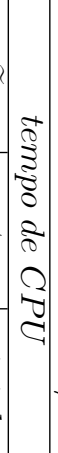 } \\
\hline 1 & 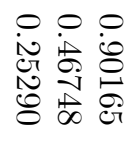 & 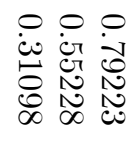 & 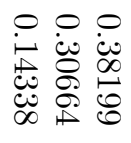 & 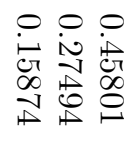 & 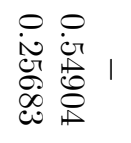 & $\epsilon$ & \\
\hline 1 & 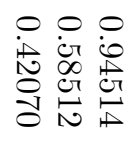 & 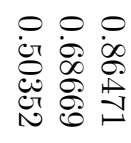 & 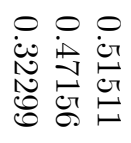 & 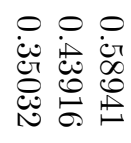 & 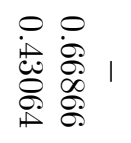 & $\frac{\widetilde{a}}{2}$ & \\
\hline 1 & 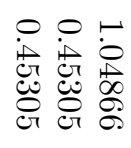 & 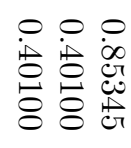 & 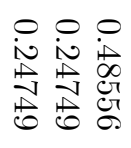 & 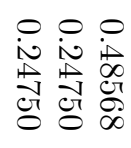 & 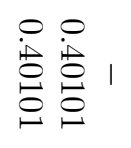 & $=$ & 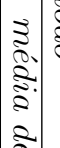 \\
\hline 1 & 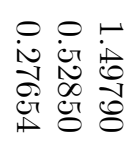 & 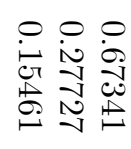 & 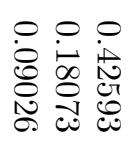 & 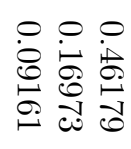 & 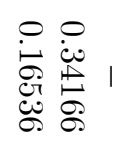 & $\epsilon$ & 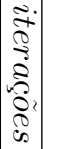 \\
\hline
\end{tabular}




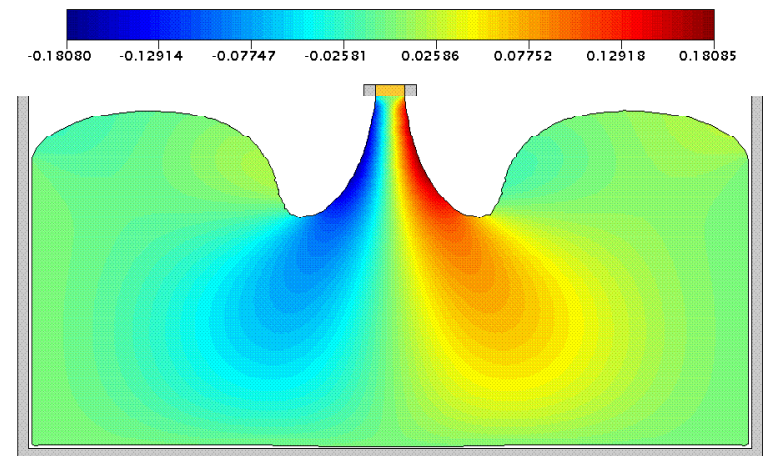

(a)

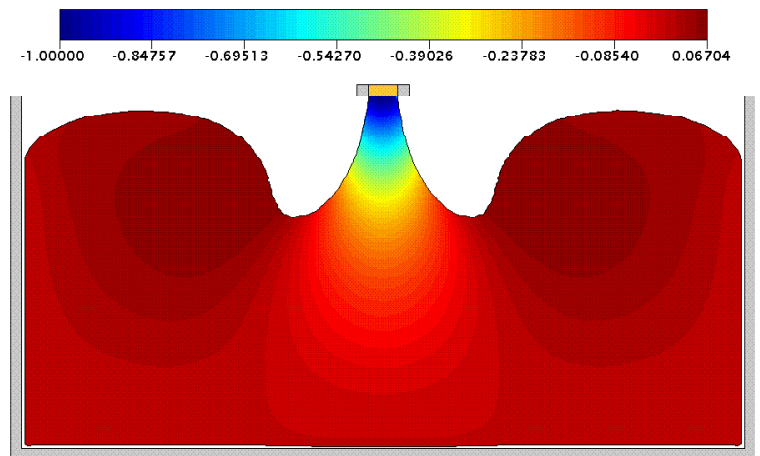

(b)

Figura 4.2.6: Solução numérica da FI-CN para $u$ (a) e $v$ (b) no enchimento estável de um fluido newtoniano $(R e=0.8, t=200.0)$ utilizando o BiCGstab-SOR na resolução dos sistemas lineares obtidos na discretização das equações de momento $(\operatorname{com} \omega=1.0)$ e de Poisson $(\operatorname{com} \omega=1.8)$.

Nos dois problemas anteriores, testamos a eficiência dos métodos iterativos e pré-condicionadores aplicados aos sistemas lineares da FI-CN mediante o uso de um $\delta t$ relativamente grande. Neste problema, no entanto, de porte intermediário, utilizamos um passo temporal pequeno a fim de tornar viável o uso da FE e, então, analisarmos se a FI continua relativamente ineficiente nesta circunstância.

Neste problema, a solução obtida pela FI-CN com os métodos iterativos originais está expressa na Tabela 4.2.5. Já na Tabela 4.2.6 apresentamos o desempenho dos novos métodos, com valores relativos à FI-CN com o CG/BiCG-Jacobi. Acrescentamos, porém, uma última linha, associada à solução obtida pela FE-EE com o par-/CG. Nesta tabela, utilizamos o pré-condicionador SOR com $\omega=1.0$ e $\omega=1.8$ na resolução dos sistemas lineares associados a $\widetilde{\mathbf{u}}$ e $\psi$, respectivamente.

Corroborando com o Exemplo 1, vemos que, novamente, a FI-CN com o par CG/BiCG-Jacobi perde desempenho para $\delta t$ pequeno se comparada à FE-EE com o par -/CG. Com efeito, a simulação obtida com a FE-EE foi realizada em, aproximadamente, $42 \%$ do tempo de CPU total gasto pela FI-CN original. Como causa para este pífio desempenho, temos os cálculos de $\widetilde{\mathbf{u}}$ e $\psi$ pela FE-EE realizados em, respectivamente, cerca de $18 \%$ e $33 \%$ do tempo de CPU médio gasto nas mesmas etapas da FI-CN com o par CG/BiCG-Jacobi.

Por outro lado, podemos observar que os métodos CGS-SOR e o BiCGstab-SOR voltam a apresentar uma eficiência muito boa, com ligeira vantagem para o BiCGstab-SOR/BiCGstab-SOR que proporcionou um ganho em tempo de CPU total de, aproximadamente, 70\%. Dessa forma, podemos ver que o desempenho da FI-CN com os pares CG/CGS-SOR, CGS-SOR/CGS-SOR, CG/BiCGstab-SOR e BiCGstab-SOR/BiCGstab-SOR superam, com boa margem, o desempenho da FE-EE.

Com efeito, embora o cálculo explícito de $\widetilde{\mathbf{u}}$ pela FE-EE não seja superado pela FI-CN (independente do método iterativo e pré-condicionador utilizado), tais métodos ultrapassam essa margem pelo ganho extra na etapa de solução de $\psi$.

Com isso, tanto o CGS-SOR quanto o BiCGstab-SOR tornaram o ciclo da FI mais eficiente que o da FE, resultando na solução apresentada na Figura 4.2.6. Com os demais métodos iterativos aplicados ao pré-condicionador SOR, mesmo quando não superaram o desempenho, conseguiram, ao menos, tornar o tempo de CPU da FI-CN mais próximo daquele da FE-EE.

Já a análise do parâmetro de relaxação ótimo para o pré-condicionador SOR é dividida em duas partes, conforme a Figura 4.2.5. Quanto à resolução do sistema linear associado a $\psi$, novamente, percebemos que o parâmetro ideal é $\omega \approx 1.8$. Já para a solução de $\widetilde{\mathbf{u}}$ temos que o ideal é $\omega \approx 1.0$, com tal escolha minimizando o número de iterações do BiCGstab-SOR a um nível médio muito baixo, de 4 iterações por ciclo. No entanto, com relação à solução de $\widetilde{\mathbf{u}}$ pelo CG, a margem para melhoria é muito pequena, pois, este gasta, em média, 16 iterações por ciclo. Com isto, para este problema, o tempo de CPU médio do CG e do BiCGstab-SOR para a solução de $\widetilde{\mathbf{u}}$ ficaram muito parecidos. 


\subsubsection{Problema 4: contração 4:1}

Neste conjunto de simulações foi considerado o problema da contração planar 4:1, descrito pelo modelo:

- Domínio espacial: $x \in[0.0,0.3075] m, y \in[0.0,0.0875] m$.

- Diâmetro do injetor: $8.0 L_{0}=8 \times 10^{-2} \mathrm{~m}$.

- Velocidade no injetor: $U_{0}=1.0 \mathrm{~ms}^{-1}$, com perfil de injeção parabólico.

- Comprimento do canal inicial: $15.0 L_{0}$.

- Comprimento do canal final: $15.0 L_{0}$.

- Distância ente injetor e outflow: $30.0 L_{0}$.

- Diâmetro do outflow: $2.0 L_{0}$.

- Aceleração de campo: $g_{0}=9.81 \mathrm{~ms}^{-2}, g^{x}=0.0, g^{y}=0.0$.

- Dados do fluido: newtoniano, com $\mu_{0}\left(\rho_{0}\right)^{-1}=0.2 m^{2} s^{-1}$.

- Parâmetros adimensionais: $R e=0.05, F r \approx 3.19275, S t=100.0 t_{0}$.

- Discretização do domínio espacial: $256 \times 70$ células nas direções $x$ e $y$, respectivamente.

- Discretização do domínio temporal: $F_{o}=1.0, F_{C F L}=0.5, F_{v i s c}=25.6$.

- Passos adimensionais: $\delta m=0.125, \delta t_{v i s c}=1.953125 \times 10^{-4}, \delta t=\min \left\{5.0 \times 10^{-3}, F_{C F L} \delta t_{C F L}\right\}$.

- Refinamento da malha superficial: $c_{\min }=0.02 \delta m, c_{m a ́ x}=0.2 \delta m$.

- Tolerância para sistemas lineares: $\varepsilon=1.0 \times 10^{-12}$.

A motivação para usarmos este modelo foi o fato dele constituir um problema confinado, sem superfície livre. Com isso, a equação de Poisson da FI resulta em um sistema linear cuja matriz coeficiente é simétrica. Consequentemente, podemos utilizar o CG na resolução do sistema que determina $\psi$. Dessa maneira, torna-se possível comparar o desempenho do BiCG-Jacobi com o CG na solução de $\psi$ pela FI usando um $\delta t$ relativamente grande para os padrões da FE.

Nestes termos, a solução do problema proposto via a FI-CN mediante o par CG/BiCG-Jacobi é resumida na Tabela 4.2.7. Já os demais métodos iterativos e pré-condicionadores utilizados têm seu desempenho relativo apresentado na Tabela 4.2.8, que emprega o pré-condicionador SOR com $\omega=1.4$ na solução de $\widetilde{\mathbf{u}}$ e $\omega=1.8$ na solução de $\psi$.

Assim, é possível perceber que o tempo de CPU médio gasto na solução de $\psi$ pelo CG/CG corresponde à cerca de $27 \%$ daquele utilizado pelo CG/BiCG-Jacobi na mesma etapa, demonstrando mais uma vez que a escolha do BiCG-Jacobi na solução do sistema associado a $\psi$ não é feliz.

Mesmo assim, as tentativas com BiCGstab-SOR e o CGS-SOR na solução de $\psi$ possibilitaram superar o desempenho do CG na solução de $\psi$. Da mesma forma, esses dois métodos voltaram a superar o CG na solução de $\widetilde{\mathbf{u}}$ com relação ao par CG/BiCG-Jacobi originalmente usado na FI. Desta vez, o BiCG-SOR também apresentou um desempenho muito bom, o qual não acreditamos ser válido em geral pelo fato do mesmo não ter sido observado em outros problemas.

Estando a solução obtida pelo BiCGstab-SOR ilustrada na Figura 4.2.8, resta efetuarmos uma análise do parâmetro de relaxação $\omega$ associado ao SOR.

Tal estudo produziu a Figura 4.2.7, onde observamos que o melhor desempenho na solução de $\psi$ (seja em número de iterações ou em tempo de CPU médio) foi obtido com $\omega \approx 1.8$. Já no sistema linear associado a $\widetilde{\mathbf{u}}$ vemos, novamente, que mediante uma escolha adequada (neste caso, $\omega \approx 1.4$ ) do parâmetro de relaxação é possível tornar o BiCGstab-SOR mais eficiente que o CG. 
Tabela 4.2.7: Valores de referência para a solução dos sistemas lineares obtidos pela FI-CN na contração 4:1 de um fluido newtoniano $(R e=0.05, t=450.0)$ através do par CG/BiCG-Jacobi.

\begin{tabular}{|c|c|c|c|}
\hline \multicolumn{2}{|c|}{ método } & $C G$ & BiCG-Jacobi \\
\hline \multirow{2}{*}{ média } & sistema & $\widetilde{\mathbf{u}}$ & $\psi$ \\
& iterações & 38.45973 & 187.06928 \\
& tempo de CPU & $5.43782 \times 10^{-2}$ & $3.44929 \times 10^{-1}$ \\
\hline \hline \multirow{3}{*}{ total } & ciclos & \multicolumn{2}{|c|}{90000} \\
& células $(\mathbf{F}: \mathbf{S})$ & \multicolumn{2}{|c|}{$9600: 0$} \\
& tempo de CPU & \multicolumn{2}{|c|}{$10: 14: 15$} \\
\hline
\end{tabular}

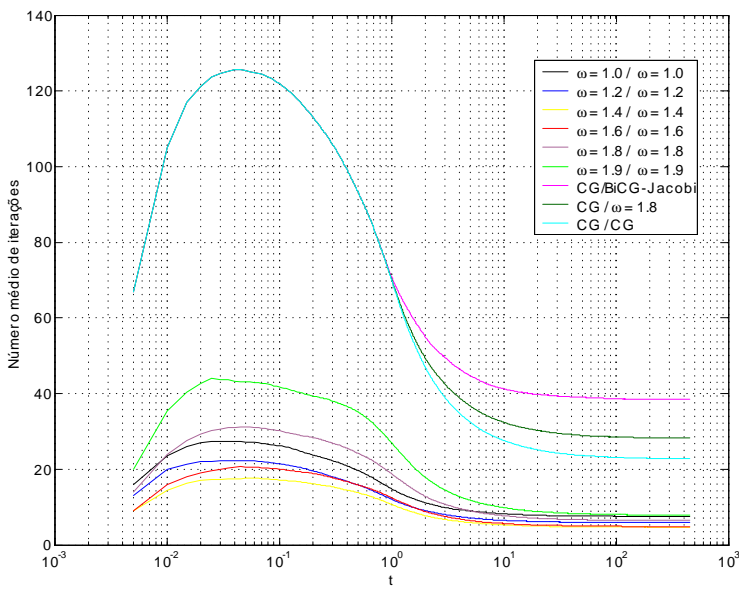

(a)

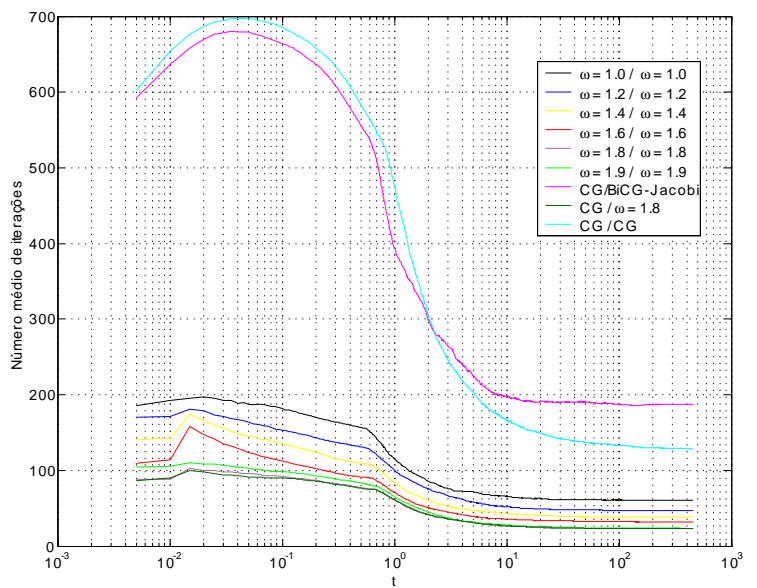

(c)

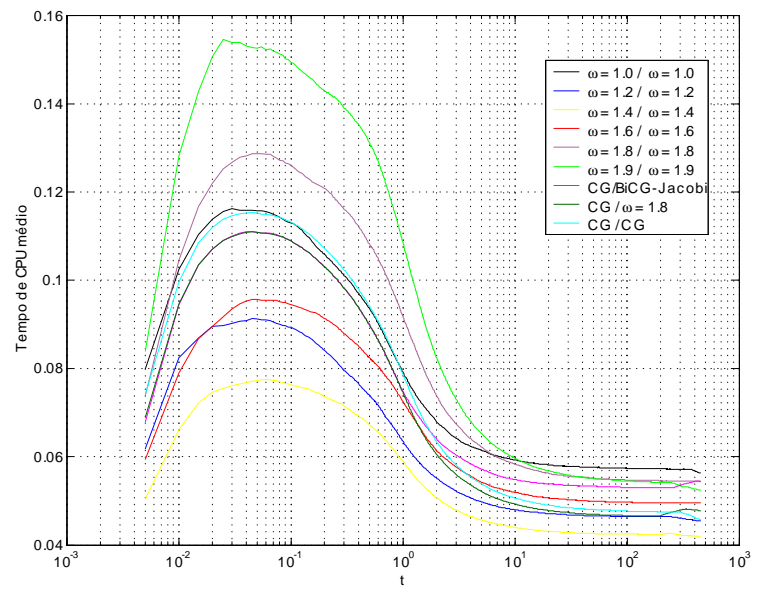

(b)

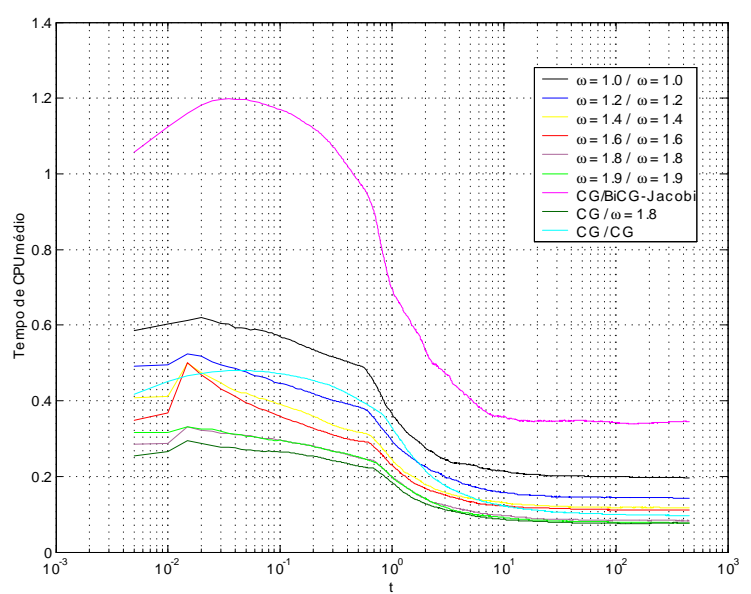

(d)

Figura 4.2.7: Análise comparativa, em função de $t$, do desempenho do pré-condicionador SOR (aplicado ao BiCGstab) com relação ao CG/BiCG-Jacobi, para a solução dos sistemas lineares obtidos pela FI-CN na contração 4:1 de um fluido newtoniano $(R e=0.05)$ : número médio de iterações na resolução dos sistemas lineares para $\widetilde{\mathbf{u}}(\mathrm{a})$ e $\psi(\mathrm{c})$, com tempo de CPU médio, respectivamente, em (b) e (d). 


\begin{tabular}{|c|c|c|c|c|c|c|c|}
\hline$\Omega$ & 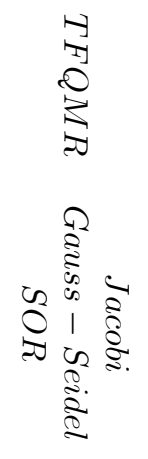 & 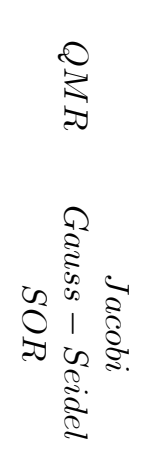 & 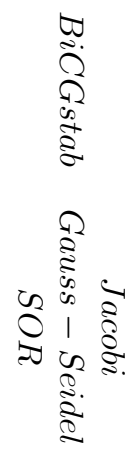 & 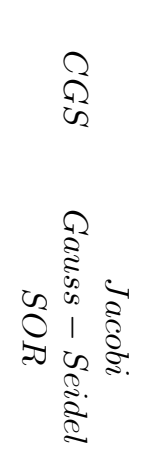 & 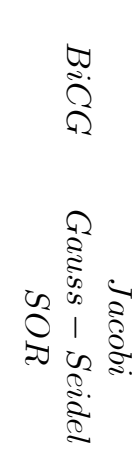 & $\begin{array}{l}8 \\
\vdots \\
0 \\
\vdots \\
\vdots \\
\vdots \\
3\end{array}$ & \\
\hline 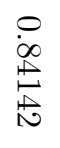 & 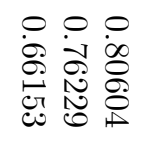 & 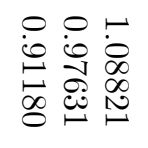 & 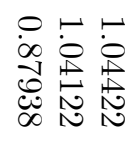 & 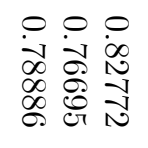 & 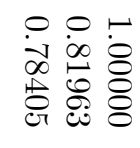 & $\approx$ & \\
\hline 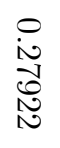 & 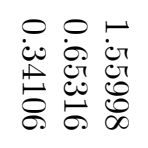 & 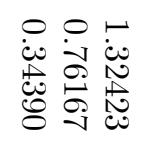 & 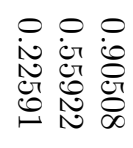 & 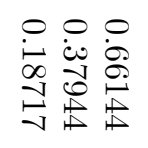 & 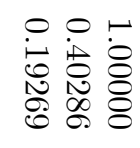 & $\epsilon$ & $\mid \begin{array}{l}3 \\
0 \\
0 \\
0 \\
0 \\
2 \\
2\end{array}$ \\
\hline $\begin{array}{l}0 \\
\dot{d} \\
\dot{d} \\
0 \\
0 \\
0\end{array}$ & 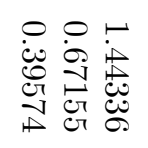 & 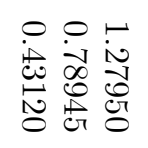 & 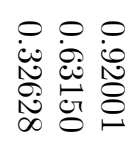 & 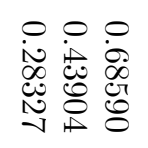 & 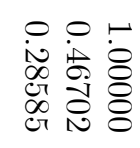 & के & $\sqrt{\frac{3}{3}}$ \\
\hline 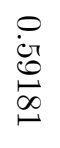 & 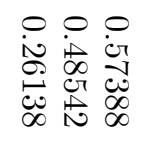 & 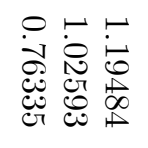 & 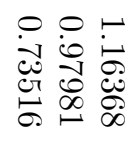 & 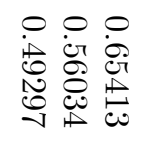 & 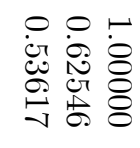 & $\approx$ & 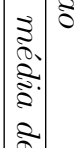 \\
\hline $\begin{array}{l}\stackrel{0}{0} \\
\dot{0} \\
0 \\
0 \\
0\end{array}$ & 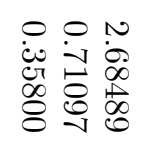 & 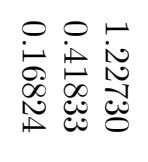 & 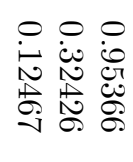 & 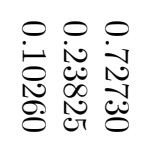 & 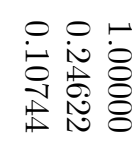 & $\varepsilon$ & 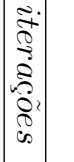 \\
\hline 1 & 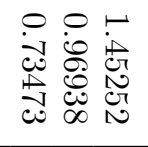 & 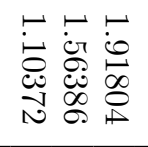 & 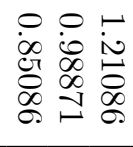 & 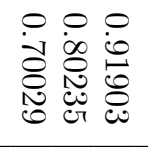 & 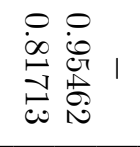 & $\approx$ & 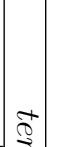 \\
\hline I & 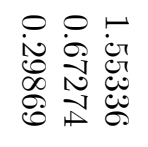 & 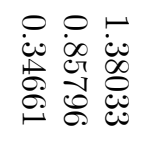 & 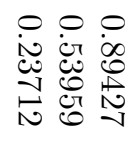 & 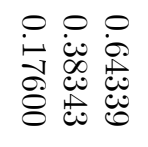 & 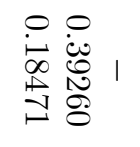 & $\epsilon$ & $\mid \begin{array}{l}3 \\
0 \\
0 \\
0 \\
2 \\
2\end{array}$ \\
\hline 1 & 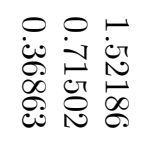 & 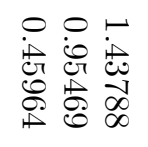 & 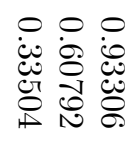 & 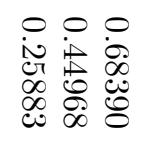 & 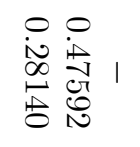 & $\begin{array}{l}\vdots \\
\vdots \\
\vdots\end{array}$ & (1) \\
\hline 1 & 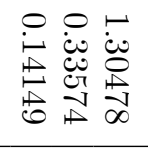 & 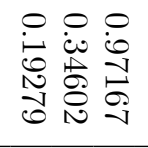 & 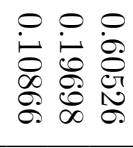 & 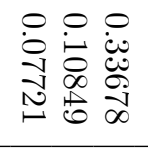 & 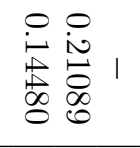 & $\approx$ & 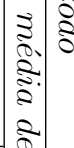 \\
\hline 1 & 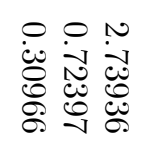 & 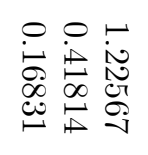 & 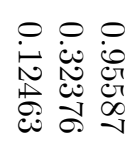 & 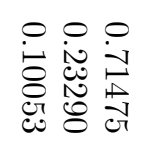 & 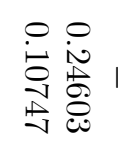 & $\epsilon$ & 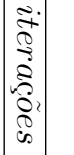 \\
\hline
\end{tabular}




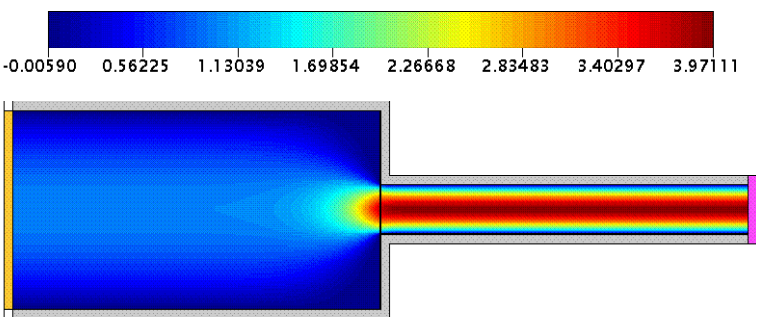

(a)

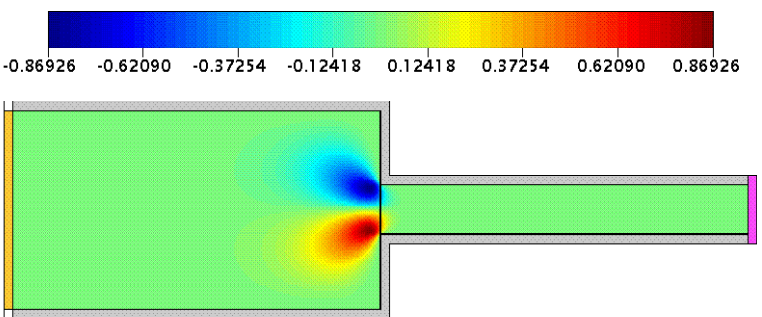

(b)

Figura 4.2.8: Solução numérica da FI-CN para $u(\mathrm{a})$ e $v$ (b) na contração 4:1 de um fluido newtoniano $(R e=0.05, t=450.0)$ utilizando o BiCGstab-SOR na resolução dos sistemas lineares obtidos na discretização das equações de momento ( $\operatorname{com} \omega=1.4)$ e de Poisson $(\operatorname{com} \omega=1.8)$.

\subsubsection{Problema 5: jet flow}

O jet flow consiste em uma piscina de fluido, inicialmente em repouso, que possui um injetor, do mesmo fluido, posicionado muito próximo a sua superfície livre. Assim, empregamos o seguinte modelo:

- Domínio espacial: $x \in[0.0,0.123] m, y \in[0.0,0.173] m$.

- Diâmetro do injetor: $L_{0}=4.0 \times 10^{-3} \mathrm{~m}$.

- Velocidade no injetor: $U_{0}=0.5 \mathrm{~ms}^{-1}$, com perfil de injeção reto.

- Altura (do injetor ao fundo da caixa): $h_{1}=0.17 \mathrm{~m}$.

- Largura (entre as duas paredes da caixa): $h_{2}=0.12 m$.

- Altura (da superfície livre do fluido inicial ao fundo da caixa): $h_{3}=0.16 \mathrm{~m}$.

- Aceleração de campo: $g_{0}=9.81 \mathrm{~ms}^{-2}, g^{x}=0.0, g^{y}=-1.0$.

- Dados do fluido: newtoniano, com $\mu_{0}\left(\rho_{0}\right)^{-1}=1.0 \times 10^{-2} m^{2} s^{-1}$.

- Parâmetros adimensionais: $R e=0.2, F r \approx 2.524094, S t=125.0 t_{0}$.

- Discretização do domínio espacial: $246 \times 346$ células nas direções $x$ e $y$, respectivamente.

- Discretização do domínio temporal: $F_{o}=1.0, F_{C F L}=0.5, F_{v i s c}=12.8$.

- Passos adimensionais: $\delta m=0.125, \delta t_{v i s c}=7.8125 \times 10^{-4}, \delta t=\min \left\{1.0 \times 10^{-2}, F_{C F L} \delta t_{C F L}\right\}$.

- Refinamento da malha superficial: $c_{\min }=0.02 \delta m, c_{m a ́ x}=0.2 \delta m$.

- Tolerância para sistemas lineares: $\varepsilon=1.0 \times 10^{-12}$.

A motivação para o estudo deste modelo é o fato dele resultar em um problema de grande porte em termos computacionais. Com efeito, a discretização que adotamos no domínio espacial resulta em mais células com fluido que nos quatro problemas anteriores, apresentados nesta seção, juntos. Obviamente, isto tem uma justificativa, pois, os problemas $2 \mathrm{D}$ comumente resolvidos possuem porte semelhante aos quatro primeiros modelos testados nesta seção. Este modelo, porém, embora exagerado em termos de simulações 2D, possui um número de células compatível com uma simulação 3D, conforme podemos observar em [TM01], [FR01] ou [OC08C]. Assim, enxergamos neste teste um bom indicador sobre a viabilidade dos resultados obtidos nesta seção para problemas em 3D. 
Tabela 4.2.9: Valores de referência para a solução dos sistemas lineares obtidos pela FI-CN no jet flow de um fluido newtoniano $(R e=0.2, t=70.0)$ através do par CG/BiCG-Jacobi.

\begin{tabular}{|c|c|c|c|}
\hline \multicolumn{2}{|c|}{ método } & $C G$ & BiCG-Jacobi \\
\hline \multirow{2}{*}{ média } & sistema & $\widetilde{\mathbf{u}}$ & $\psi$ \\
& iterações & 77.95957 & 1292.14886 \\
& tempo de $C P U$ & 1.01278 & 25.08609 \\
\hline \hline \multirow{3}{*}{ total } & ciclos & \multicolumn{2}{|c|}{7000} \\
& células $(\mathbf{F}: \mathbf{S})$ & \multicolumn{2}{c|}{$81061: 269$} \\
& tempo de $C P U$ & \multicolumn{2}{|c|}{$54: 51: 41$} \\
\hline
\end{tabular}

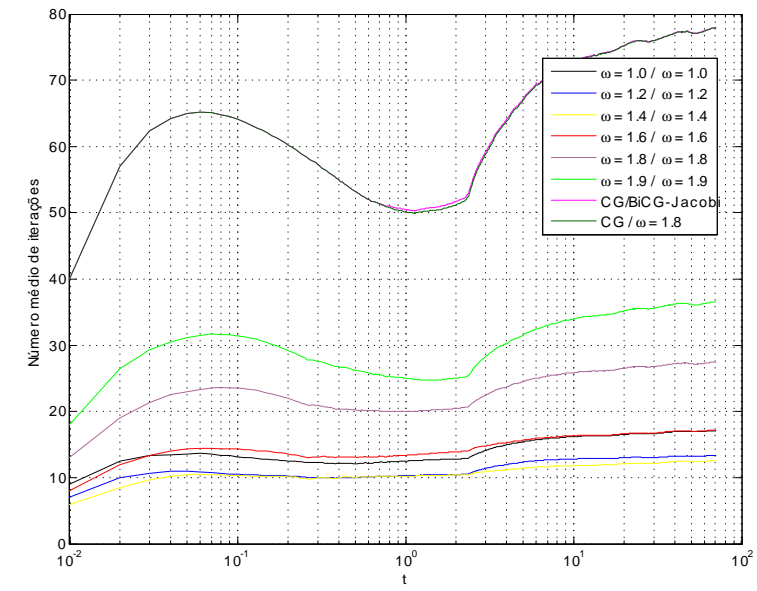

(a)

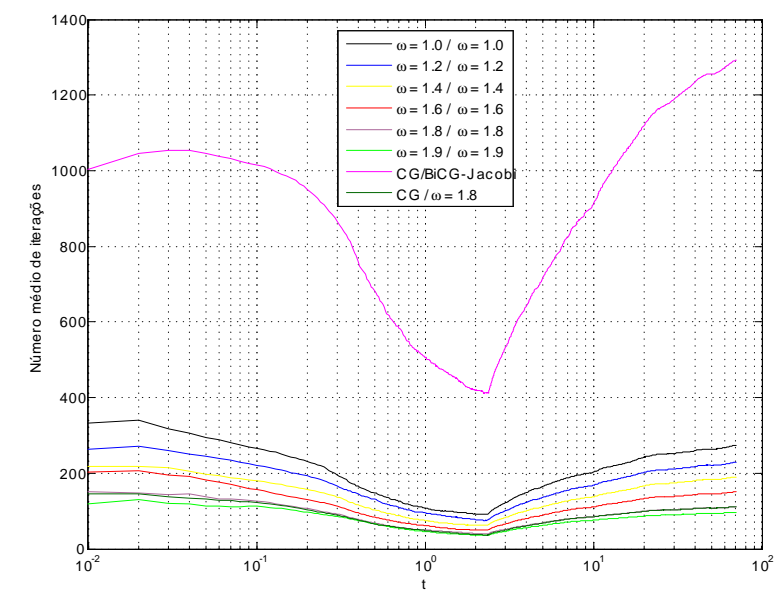

(c)

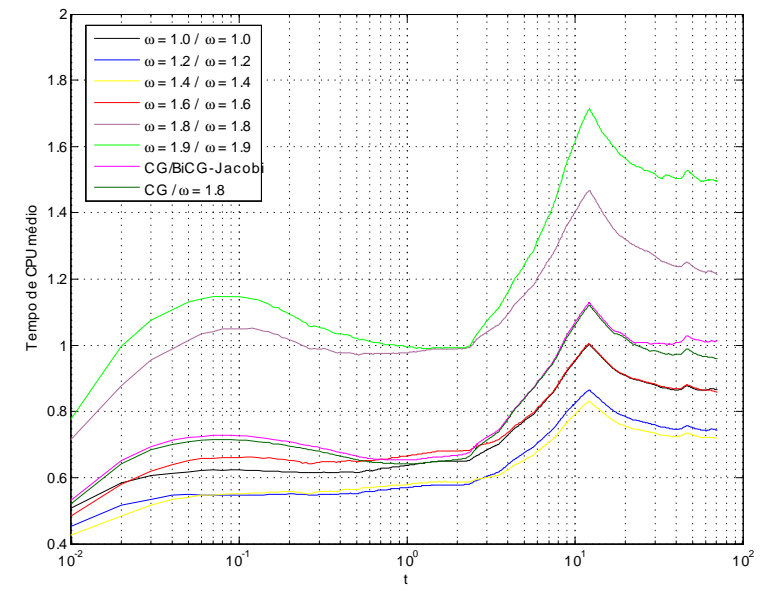

(b)

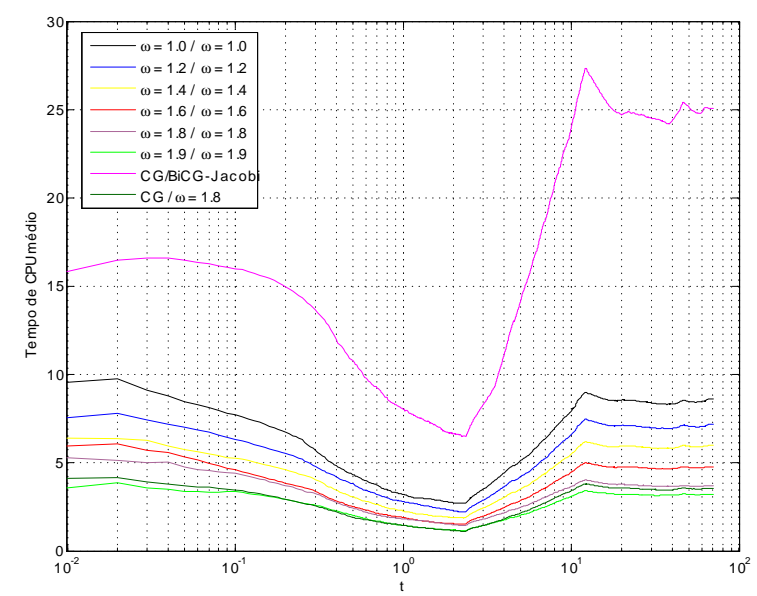

(d)

Figura 4.2.9: Análise comparativa, em função de $t$, do desempenho do pré-condicionador SOR (aplicado ao BiCGstab) com relação ao CG/BiCG-Jacobi, para a solução dos sistemas lineares obtidos pela FI-CN no jet flow de um fluido newtoniano $(R e=0.2)$ : número médio de iterações na resolução dos sistemas lineares para $\widetilde{\mathbf{u}}(\mathrm{a})$ e $\psi(\mathrm{c})$, com tempo de CPU médio, respectivamente, em (b) e (d). 


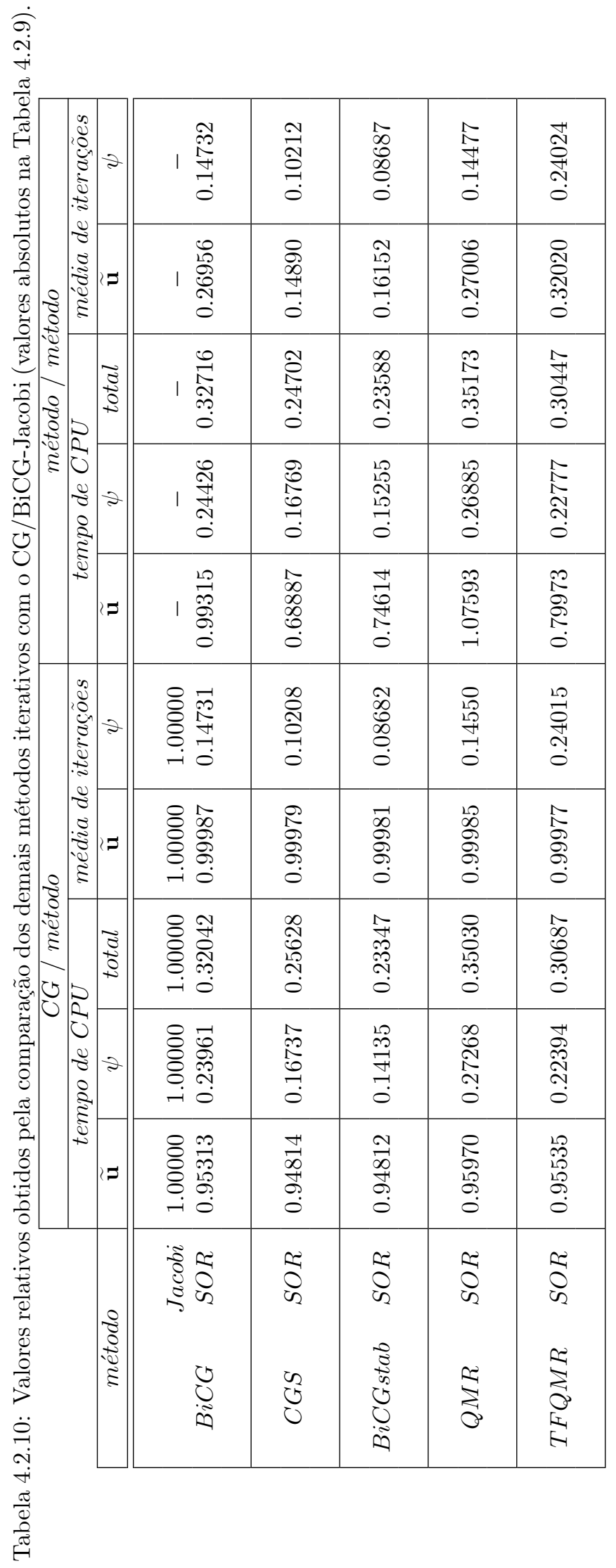




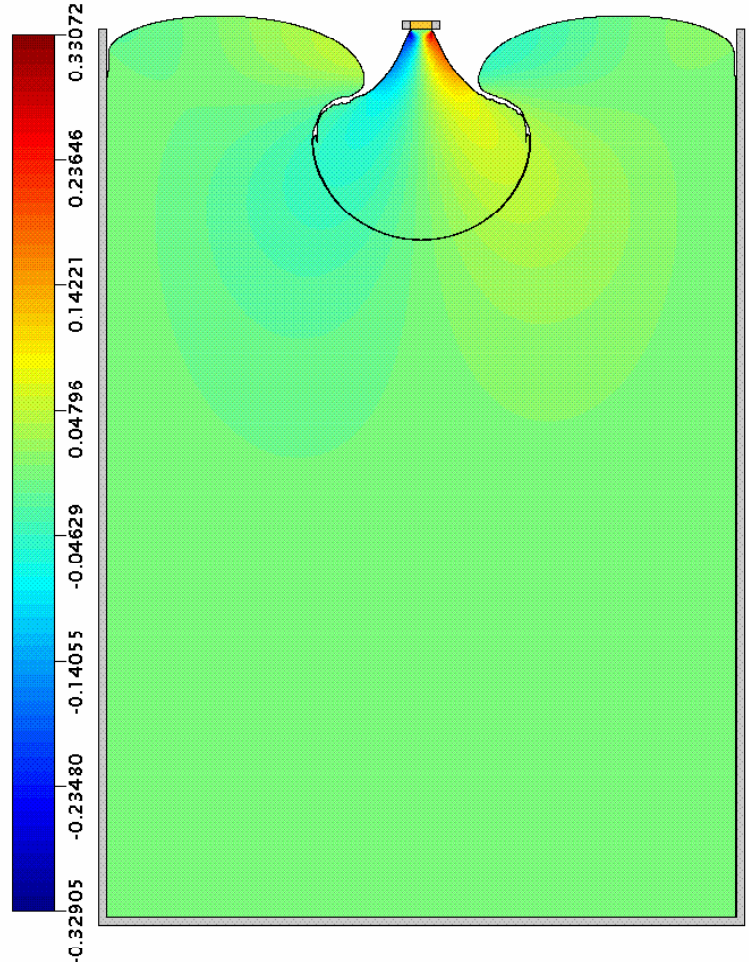

(a)

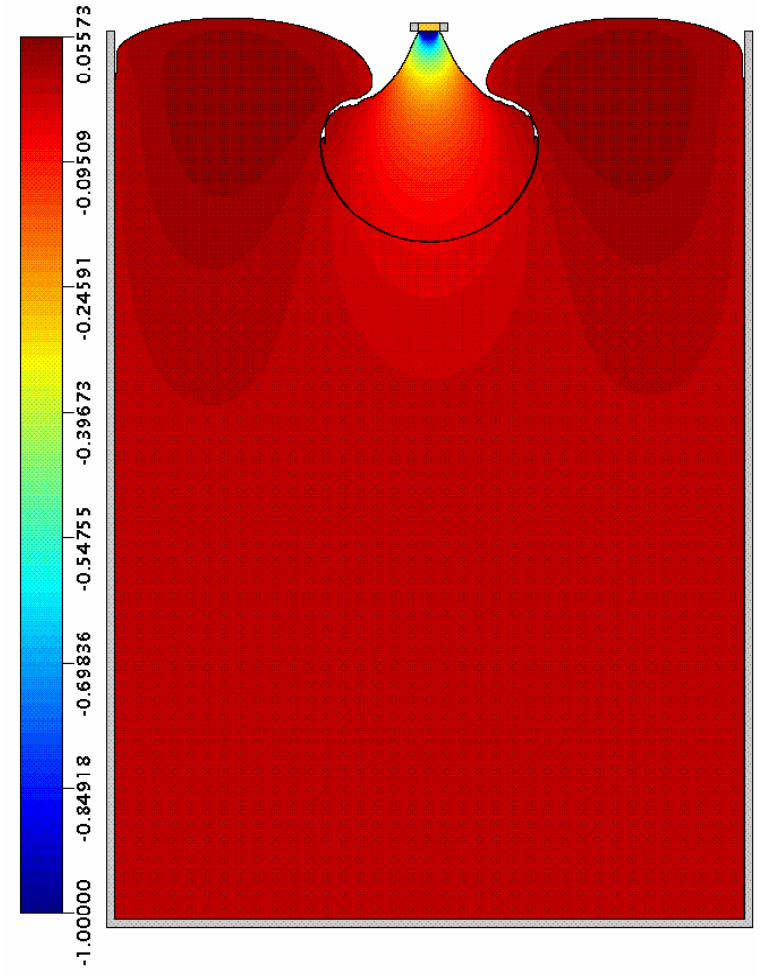

(b)

Figura 4.2.10: Solução numérica da FI-CN para $u(\mathrm{a})$ e $v(\mathrm{~b})$ no jet flow de um fluido newtoniano $(R e=$ $0.2, t=70.0)$ utilizando o BiCGstab-SOR na resolução dos sistemas lineares obtidos na discretização das equações de momento ( $\operatorname{com} \omega=1.4)$ e de Poisson $(\operatorname{com} \omega=1.8)$.

Assim, a solução do problema em questão pela FI-CN com o par CG/BiCG-Jacobi é apresentada na Tabela 4.2.9. Para os demais métodos iterativos, consideramos, neste problema, apenas o précondicionador SOR, onde sua aplicação com $\omega=1.4$ e $\omega=1.8$ para a resolução dos sistemas lineares associados a, respectivamente, $\widetilde{\mathbf{u}}$ e $\psi$, estão expressos na Tabela 4.2.10.

Mais uma vez o CGS-SOR e o BiCGstab-SOR apresentam-se como alternativas bastante robustas e eficientes, tanto em número de iterações quanto em tempo de CPU médio gasto na solução de $\widetilde{\mathbf{u}}$ e de $\psi$, com relação ao par CG/BiCG-Jacobi.

No que diz respeito à calibragem de $\omega$ a fim de minimizar o número de iterações, apresentamos a Figura 4.2.9. Nesta, podemos perceber que temos em $\omega \approx 1.8$ o valor ideal para a solução do sistema linear associado a $\psi$. Por sua vez, na solução de $\widetilde{\mathbf{u}}$, faz-se interessante tomar $\omega \approx 1.4$ no BiCGstab-SOR, o que proporciona um ganho considerável com relação ao CG no número médio tanto de iterações quanto de tempo de CPU.

Desta forma, constatamos que, justamente no problema de maior porte empregado nesta seção, foi obtida a maior margem de ganho em desempenho dos métodos CGS-SOR/CGS-SOR e BiCGstabSOR/BiCGstab-SOR com relação ao par original CG/BiCG-Jacobi, a qual é da ordem de 75\% em tempo de CPU total. 


\subsection{Esquemas de movimentação da superfície livre}

\subsubsection{Introdução}

O objetivo desta seção é investigar a influência da estratégia de solução da equação (3.3.5) no erro da massa numérica em função do $\delta t$ adotado. Com efeito, a utilização da FI permite passos temporais maiores e isto pode levar a uma pior conservação da massa, que, por sua vez, influencia a qualidade da solução numérica.

Para isso, testamos todos os métodos de solução do GENSMAC apresentados no Capítulo 2 (FE-EE, FI-CN e FI-EI) juntamente com os equemas de movimento das partículas marcadoras por EE (3.3.12) e RK21 (3.3.14)-(3.3.15). Combinando tais estratégias, obtemos seis métodos numéricos, denotados por: EE:FE-EE, EE:FI-CN, EE:FI-EI, RK21:FE-EE, RK21:FI-CN, RK21:FI-EI.

Em cada um dos quatro problemas apresentados nesta seção, a FE-EE, a FI-CN e a FI-EI são aplicadas sempre com parâmetros idênticos. Assim, em todos os casos, utilizamos o BiCGstab-SOR com $\omega=1.4$ e $\omega=1.8$ para, respectivamente, a solução dos sistemas lineares envolvendo $\widetilde{\mathbf{u}}$ e $\psi$. Com isso, qualquer diferença entre os métodos poderá ser atribuída, apenas, à estratégia de movimentação da superfície livre.

Assim, em cada problema e para cada método, analisamos o comportamento do erro na massa em função de um refinamento na discretização do tempo. Para isso, os menores passos temporais adotados são da ordem de $\delta t_{v i s c}$, possibilitando o uso da FE. Na outra extremidade, os maiores passos utilizados em cada problema são da ordem de $\delta t_{C F L}$, estando próximos do limite de estabilidade da FI.

Obviamente, a escolha de $\delta t$ não influi apenas no erro da massa computacional, afetando a precisão da solução numérica como um todo. No entanto, é (3.3.5) que define, em última instância, a evolução da massa numérica em função do tempo, e assim, é apenas nesta fonte de erro que concentramos o estudo mediante as estratégias de solução EE e RK21.

Portanto, após a apresentação dos testes que comparam o EE com o RK21 na solução de (3.3.5), esperamos responder, ao final do capítulo:

- Qual a estratégia que melhor preserva a massa numérica?

- Qual a estratégia que melhor representa o perfil da superfície livre?

- Sendo o RK21 a melhor estratégia, quanto ele possibilita aumentarmos $\delta t$ para um erro na massa equivalente à estratégia baseada em EE com passo temporal pequeno?

- Dada uma mesma estratégia para movimento das partículas, qual formulação do GENSMAC conserva melhor a massa?

\subsubsection{Problema 1: enchimento estável}

Consideramos, aqui, o seguinte problema de injeção de fluido em um contêiner:

- Domínio espacial: $x \in[0.0,0.0505] m, y \in[0.0,0.05] m$.

- Diâmetro do injetor: $L_{0}=5.5 \times 10^{-3} \mathrm{~m}$.

- Velocidade no injetor: $U_{0}=1.0 \mathrm{~ms}^{-1}$, com perfil de injeção reto.

- Altura (do injetor ao fundo da caixa): $h_{1}=0.047 m$.

- Largura (entre as paredes verticais da caixa): $h_{2}=0.0475 \mathrm{~m}$.

- Aceleração de campo: $g_{0}=9.81 \mathrm{~ms}^{-2}, g^{x}=0.0, g^{y}=-1.0$.

- Dados do fluido: newtoniano, com $\mu_{0}\left(\rho_{0}\right)^{-1}=5.5 \times 10^{-2} m^{2} s^{-1}$.

- Parâmetros adimensionais: $R e=0.1, F r \approx 4.305109, S t=U_{0}\left(L_{0}\right)^{-1} t_{0}$.

- Discretização do domínio espacial: $101 \times 100$ células nas direções $x$ e $y$, respectivamente. 
- Discretização do domínio temporal:

$$
\begin{aligned}
& -F_{o}=1.0, F_{C F L}=0.5 \\
& -F_{\text {visc }} \in\{48.4,24.2,12.1,6.05,3.872,1.936,0.968,0.484,0.3872,0.1936,0.121\} .
\end{aligned}
$$

- Passos adimensionais: $\delta m=0.0005\left(L_{0}\right)^{-1}, \delta t_{v i s c}=0.25 R e(\delta m)^{2}$.

- Instante final adimensional: $t_{F}=73.0$.

- Refinamento da malha superficial: $c_{\min }=0.02 \delta m, c_{m a ́ x}=0.2 \delta m$.

- Sistemas lineares:

- Tolerância: $\varepsilon=1.0 \times 10^{-10}$.

- Método para solução de $\widetilde{\mathbf{u}}$ : BiCGstab-SOR $(\omega=1.4)$.

- Método para solução de $\psi$ : BiCGstab-SOR $(\omega=1.8)$.

É interessante observar que, segundo [CJ81], o escoamento associado a este modelo deve resultar em um enchimento estável do contêiner (do inglês, steady filling), com o jato de fluido não apresentando "dobras" ou oscilações. O jato oscilante (do inglês, jet buckling) consiste em uma instabilidade física que proporciona a formação de dobras caóticas no jato de fluido à medida que ele se deposita no fundo do contêiner. Assim, segundo [CJ81], tal fenômeno deve ocorrer para um jato 2D de um fluido newtoniano quando, simultaneamente,

$$
\begin{aligned}
& R e<0.56, \\
& \frac{h_{1}}{L_{0}}>3 \pi,
\end{aligned}
$$

sendo $h_{1}$ a altura do injetor com relação ao fundo da caixa e $L_{0}$ o diâmetro do injetor. Para o problema em questão, no entanto, temos

$$
\begin{aligned}
& R e=0.1, \\
& \frac{h_{1}}{L_{0}} \approx 8.54<3 \pi,
\end{aligned}
$$

sugerindo um escoamento livre de oscilações.

Intencionalmente, porém, os parâmetros deste modelo foram escolhidos próximos da restrição apresentada, o que possibilita o estudo da influência do erro na massa na solução numérica. Com efeito, para a série de simulações realizadas com diferentes $\delta t$, nenhuma utilizando o RK21 resultou em oscilações, conforme ilustra a Figura 4.3.3.

Entretanto, nas simulações com EE, quando o $\delta t$ utilizado é pequeno, algumas soluções oscilam. De fato, as simulações com EE:FI-CN e com EE:FE-EE produziram esta instabilidade para o menor $\delta t$ utilizado $\left(F_{v i s c}=0.121\right)$. Já no caso do EE:FI-EI, os três menores passos temporais $\left(F_{v i s c} \in\right.$ $\{0.3872,0.1936,0.121\}$ ) produziram oscilações. Tal fato está ilustrado na Figura 4.3.2.

$\mathrm{Na}$ verdade, em [OC04], quando da primeira versão da FI, um problema semelhante a este (com parâmetros ainda mais próximos da restrição (4.3.1)-(4.3.2), mas, cuja solução também não deveria oscilar) foi resolvido com EE para o movimento das partículas. Na oportunidade, foi usado um passo temporal pequeno para a FE (devido à restrição de estabilidade parabólica) e, para a FI, um $\delta t 100$ vezes maior, aproximadamente. Nestes termos, foi possível perceber que a solução obtida pela FE (com um $\delta t$ pequeno) resultou em oscilações, enquanto que a solução calculada pela FI (com $\delta t$ grande) foi estável e não oscilou.

Acreditamos que o fenômeno observado em [OC04] é devido ao esquema de movimento das partículas. De fato, utilizando um modelo semelhante, reproduzimos aqui o mesmo fenômeno espúrio, o qual surge a medida que diminuímos o passo temporal. 
Uma possível explicação para esse fenômeno está no aumento do número de ciclos computacionais proporcionado pela redução no passo temporal: quanto mais ciclos, maior o número de vezes que a superfície livre é movimentada. Consequentemente, como o EE possui ordem de precisão inferior ao RK21, acumula mais erros, o que acaba refletindo na precisão e estabilidade da solução como um todo.

Quanto à conservação da massa, vemos na Figura 4.3.1 (a) que o erro final pelo RK21 é absolutamente inferior ao produzido pelo EE, independentemente do $\delta t$ adotado. Aliás, por meio da função perda total, vemos na Figura 4.3 .1 (c) que a precisão do RK21 para o maior $\delta t$ utilizado é próxima daquela obtida pelo EE usando o menor $\delta t$.

A Figura 4.3.1 (b) mostra o tempo de CPU total em cada simulação em função de $\delta t$, onde observamos que o RK21 utilizou um tempo de processamento semelhante ao do EE.

Por fim, na Figura 4.3.1 (d), temos, usando o maior $\delta t$ admitido pela FE $\left(F_{v i s c}=0.3872\right)$, a evolução do erro na massa em função do tempo. Essa figura é importante, pois, mostra que, independentemente da formulação do GENSMAC utilizada (explícita ou implícita), o principal fator que afeta o erro na massa é a estratégia de movimentação da superfície livre.

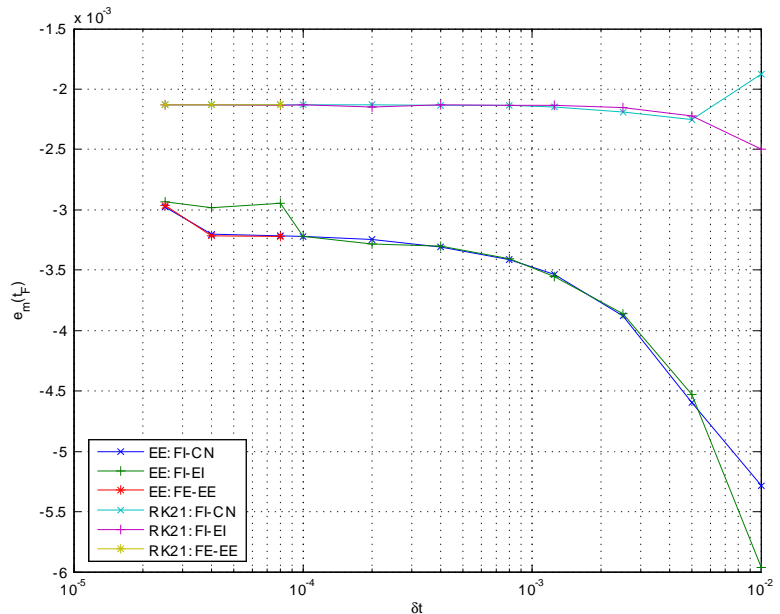

(a)

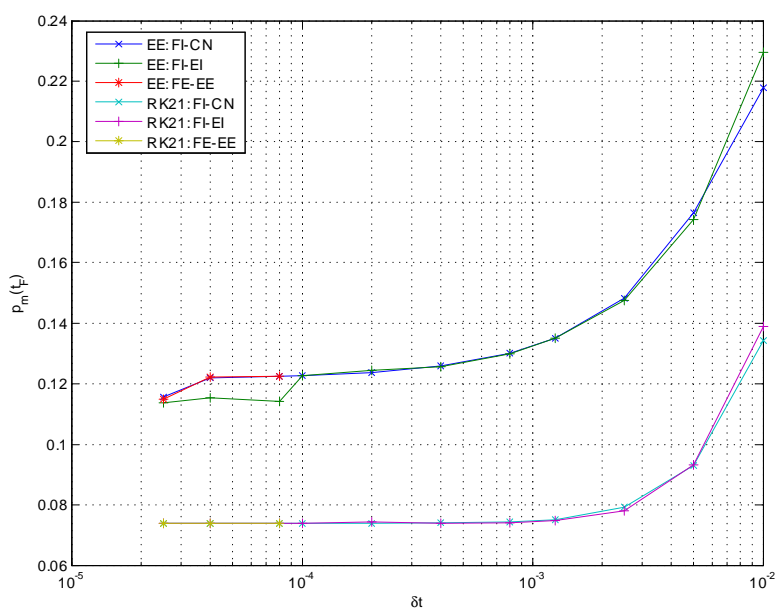

(c)

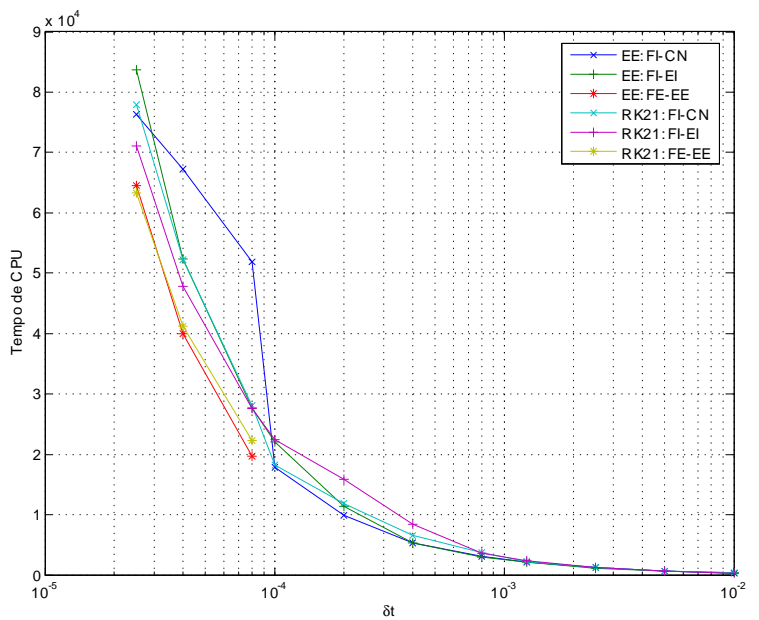

(b)

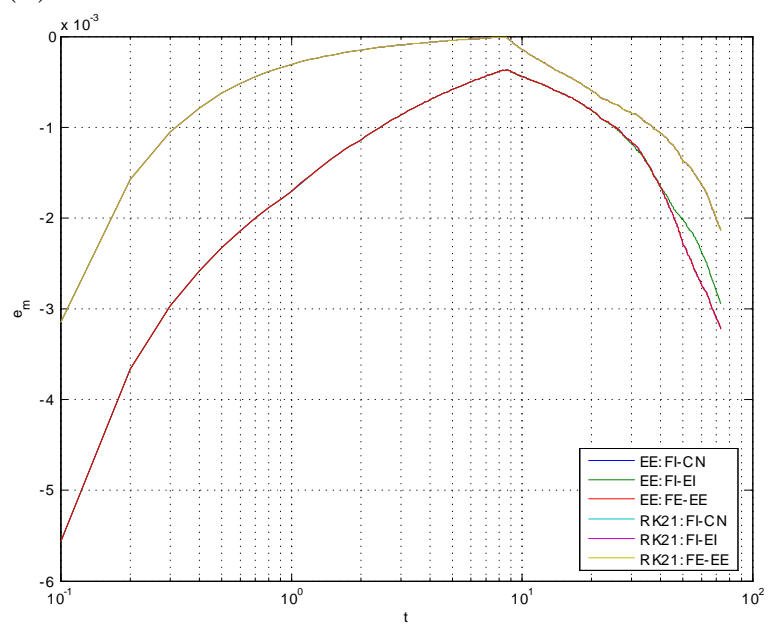

(d)

Figura 4.3.1: Erro na massa (a), tempo de CPU total (b) e função perda total (c), todos em função de $\delta t$, em $t=t_{F}$. Em (d) temos a evolução do erro na massa no tempo com os diferentes métodos usando o maior passo temporal admitido pela FE-EE $\left(F_{\text {visc }}=0.3872\right)$. 

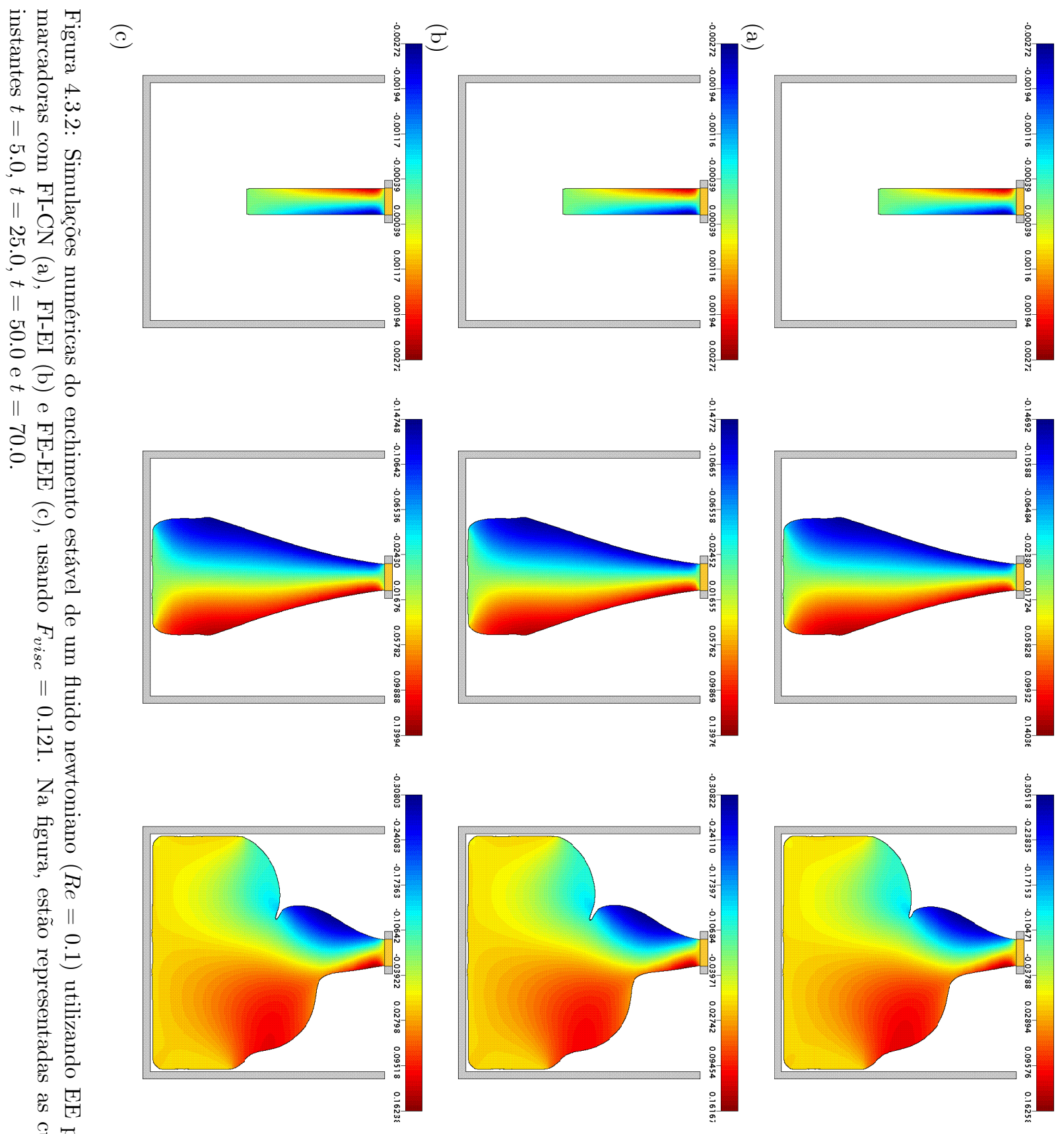

赵

กิ
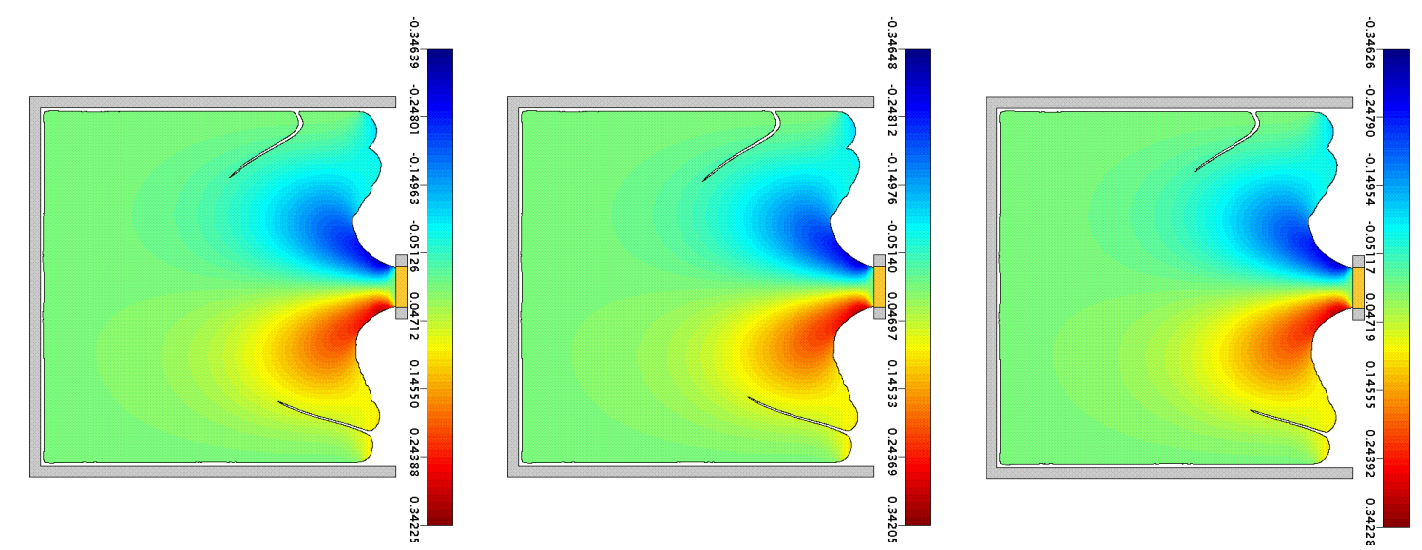
4.3. ESQUEMAS DE MOVIMENTAÇÃO DA SUPERFÍCIE LIVRE
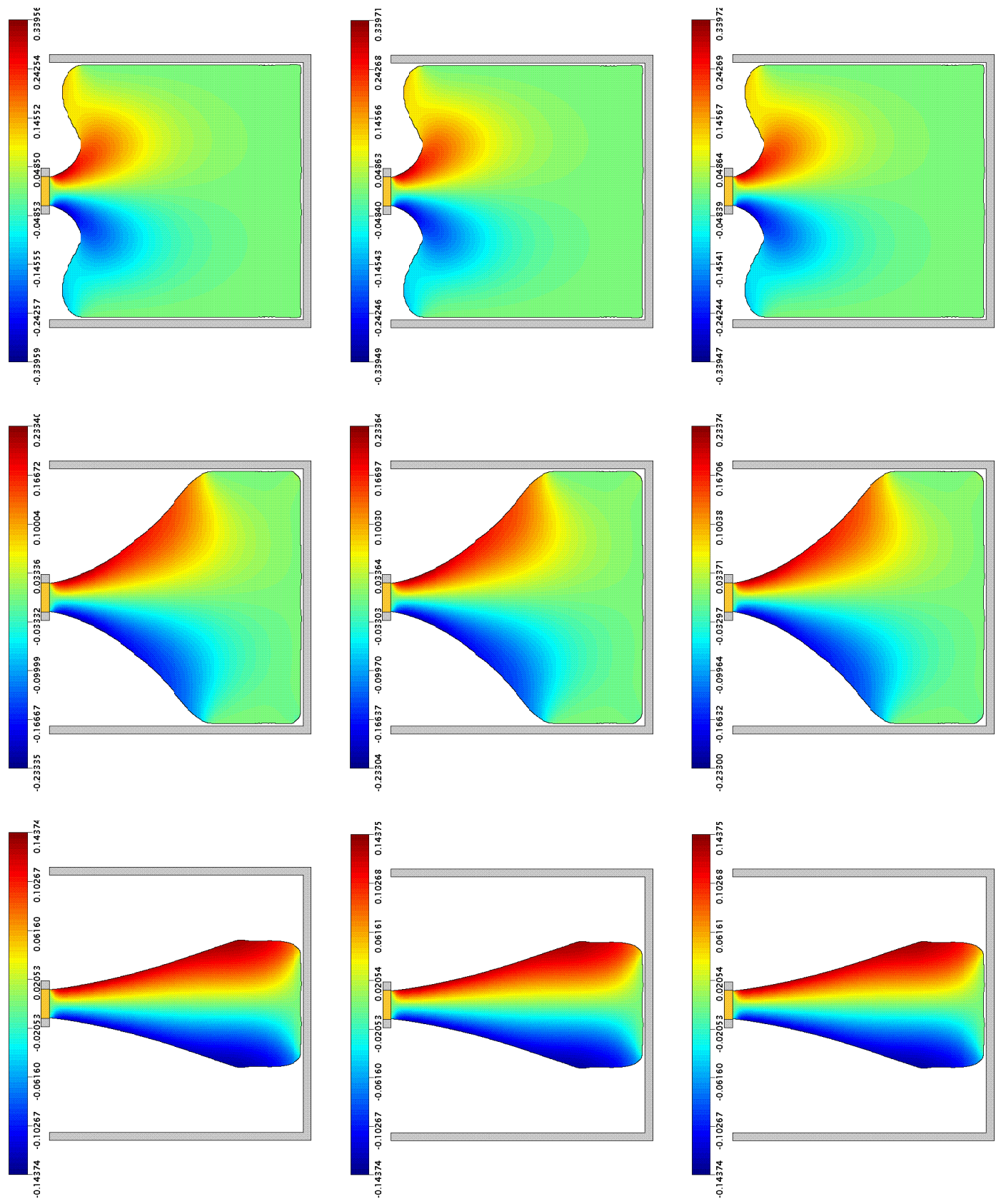

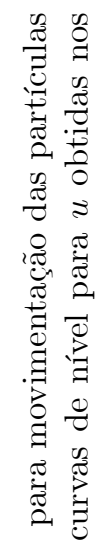

空

ح)

范

코

$\stackrel{2}{=}$

Э.

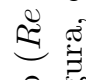

읟

沓 乙

금

융

录

छ

$\begin{array}{ll}0 & 0 \\ 0 & 0 \\ 0 & 0 \\ 0 & 0 \\ 0 & 0\end{array}$
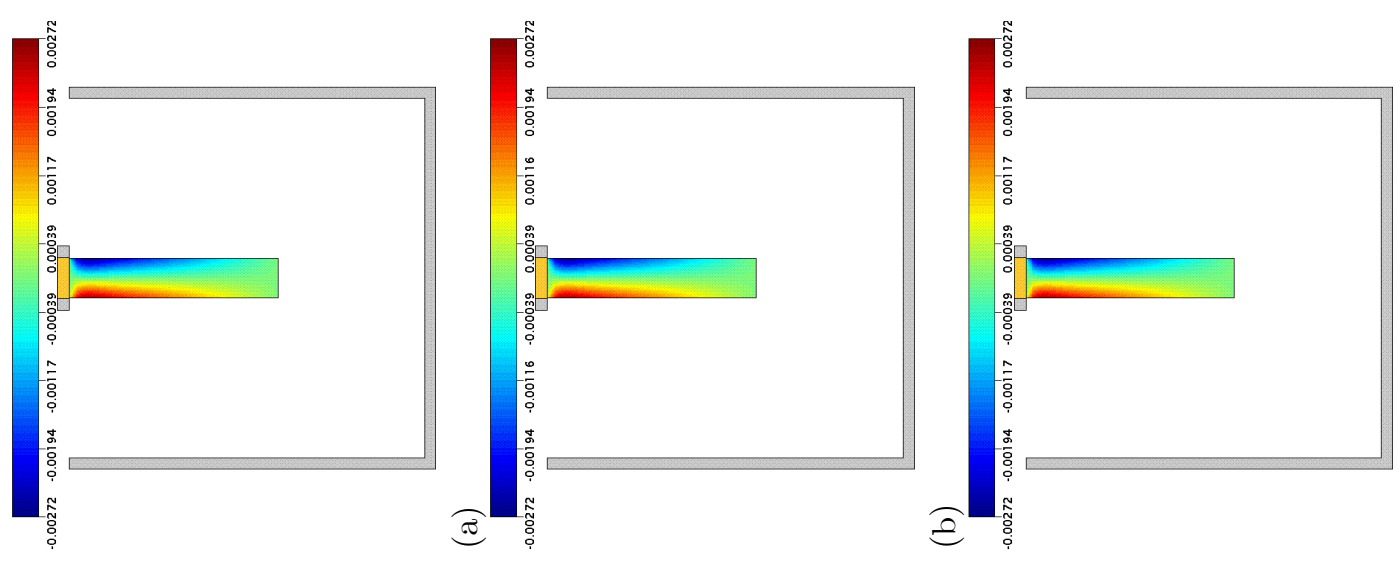

원

認国

$\frac{1}{1}$

当

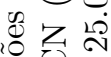

造 1

氙

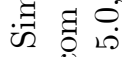

भें

$\stackrel{+}{\sim}$

(บ) 


\subsubsection{Problema 2: enchimento de uma caixa contendo fluido}

Neste problema, consideramos a injeção de fluido no interior de um contêiner, o qual, no instante inicial, possui depositada uma porção do mesmo fluido em repouso. Assim, temos o modelo:

- Domínio espacial: $x \in[0.0,0.05] m, y \in[0.0,0.07] m$.

- Diâmetro do injetor: $L_{0}=4.0 \times 10^{-3} \mathrm{~m}$.

- Velocidade no injetor: $U_{0}=1.0 \mathrm{~ms}^{-1}$, com perfil de injeção reto.

- Altura (do injetor ao fundo da caixa): $h_{1}=0.067 \mathrm{~m}$.

- Largura (entre as paredes verticais da caixa): $h_{2}=0.047 m$.

- Altura (da superfície livre do fluido inicial ao fundo da caixa): $h_{3}=0.02 m$.

- Aceleração de campo: $g_{0}=9.81 m s^{-2}, g^{x}=0.0, g^{y}=-1.0$.

- Dados do fluido: newtoniano, com $\mu_{0}\left(\rho_{0}\right)^{-1}=5.0 \times 10^{-3} m^{2} s^{-1}$.

- Parâmetros adimensionais: $R e=0.8, F r \approx 5.048188, S t=250.0 t_{0}$.

- Discretização do domínio espacial: $100 \times 140$ células nas direções $x$ e $y$, respectivamente.

- Discretização do domínio temporal:

$-F_{o}=1.0, F_{C F L}=0.5$

$-F_{v i s c} \in\{12.8,11.2,8.0,6.4,3.2,2.56,1.6,1.28,0.8,0.32,0.064,0.032\}$.

- Passos adimensionais: $\delta m=0.125, \delta t_{v i s c}=3.125 \times 10^{-3}$.

- Instante final adimensional: $t_{F}=73.0$.

- Refinamento da malha superficial: $c_{\min }=0.02 \delta m, c_{m a ́ x}=0.2 \delta m$.

- Sistemas lineares:

- Tolerância: $\varepsilon=1.0 \times 10^{-10}$.

- Método para solução de $\widetilde{\mathbf{u}}$ : BiCGstab-SOR $(\omega=1.4)$.

- Método para solução de $\psi$ : BiCGstab-SOR $(\omega=1.8)$.

A motivação para apresentarmos este problema está na interação que existe entre as duas porções de um mesmo fluido durante a simulação, criando uma situação bastante complexa. Com efeito, à medida que o jato atinge o fluido depositado na caixa, duas superfícies livres se juntam e é necessário que o esquema de movimentação das partículas se adapte a esta situação.

O limite de estabilidade da FE, neste problema, é próximo a $F_{v i s c}=0.32$. Já na FI, é possível usar um passo temporal próximo a $F_{v i s c}=12.8$, a partir de onde começamos a esbarrar na condição CFL. Todas as simulações realizadas produziram soluções, visualmente, muito parecidas, conforme ilustramos na Figura 4.3.4 para a FI-CN.

No entanto, quanto à conservação da massa, o RK21 foi superior ao EE mais uma vez, conforme mostram a Figura 4.3.5 (a) para o erro final e a Figura 4.3.5 (c) para a função perda total, ambos no instante final da simulação, quando a caixa está completamente cheia de fluido. Na Figura 4.3.5 (b) vemos que o tempo de CPU total praticamente não é afetado pela estratégia de movimentação da superfície livre, com o RK21 superando o EE em diversas oportunidades.

Por fim, a Figura 4.3.5 (d) mostra que, para o maior $\delta t$ admitido pela FE, o principal fator que afeta a evolução temporal do erro na massa é a estratégia de movimento das partículas, sendo o RK21 mais preciso que o EE. 


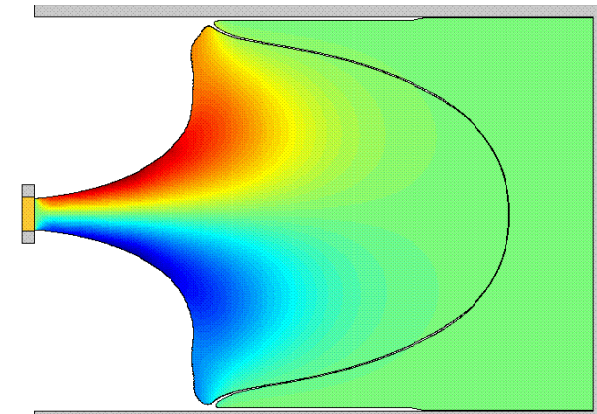

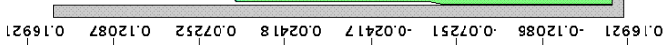
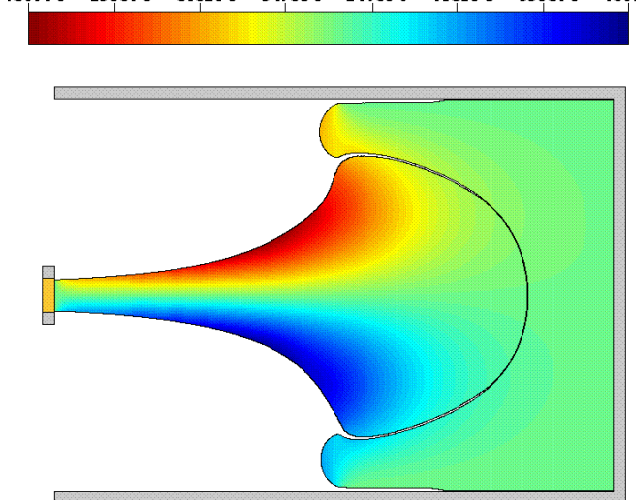

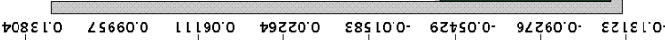
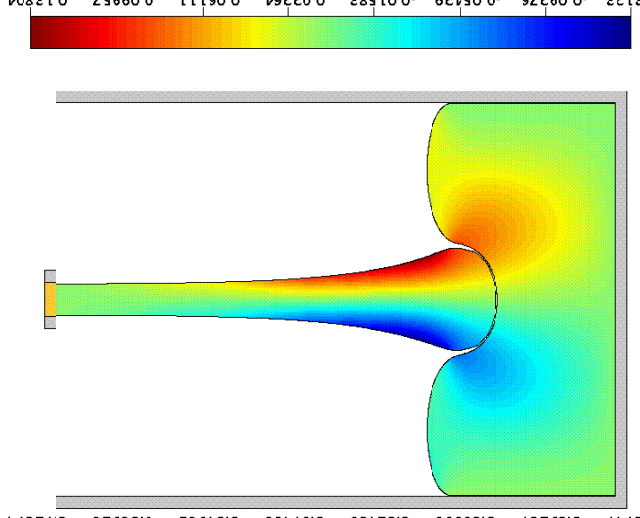

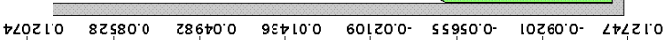
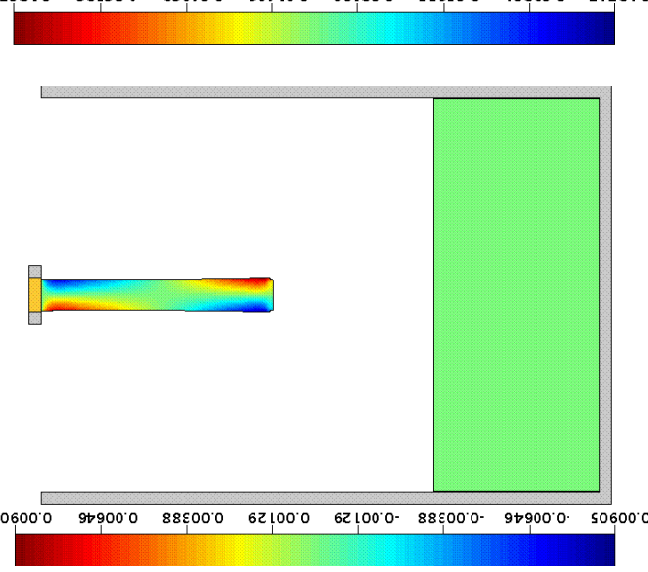
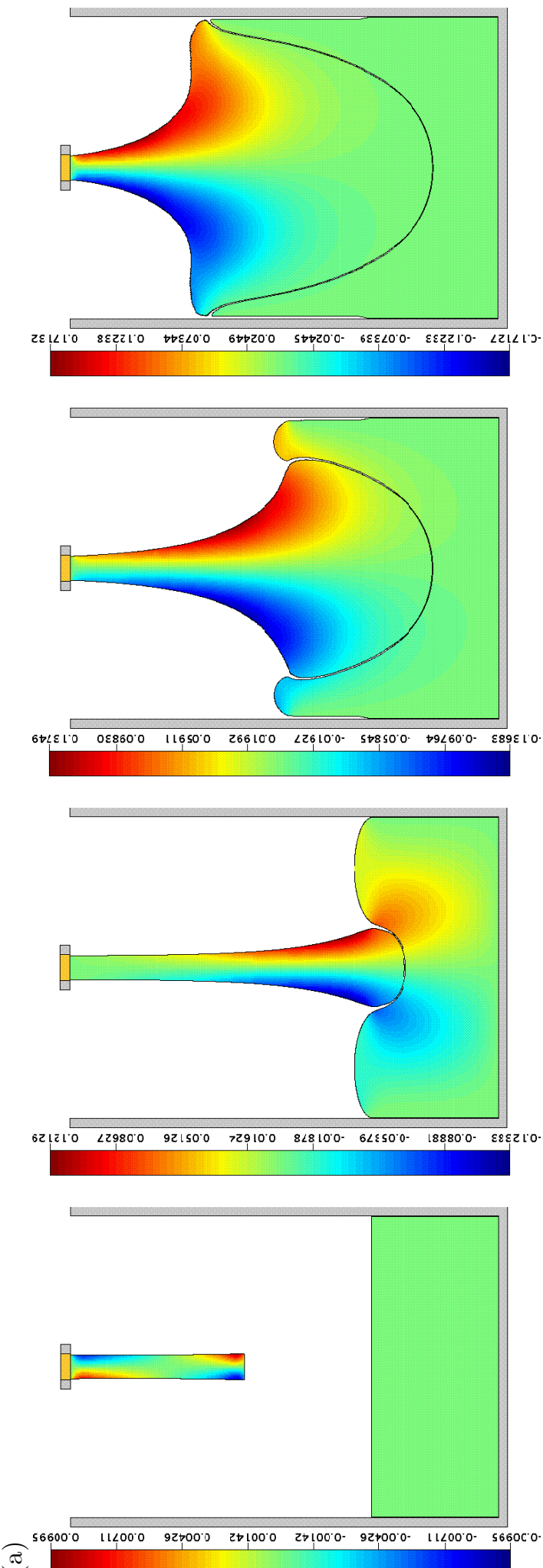

อิ

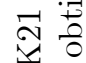

त्र

유

$\approx 0$

牙总

율

苂

寻

क人

0

||

류

임

긍

过

i

을

ส

8

ن

ㅇ

E

ฮ્.

8

ॠ

串

芯分

岸

읨요

of

马

过

$\circ$.

is

की

㐘

g

寻

की

8 叫

预

寻

व्.

क 苟

$\ddot{r}$ ठ

ॠे

$\forall$

$\dddot{\square}$

, 


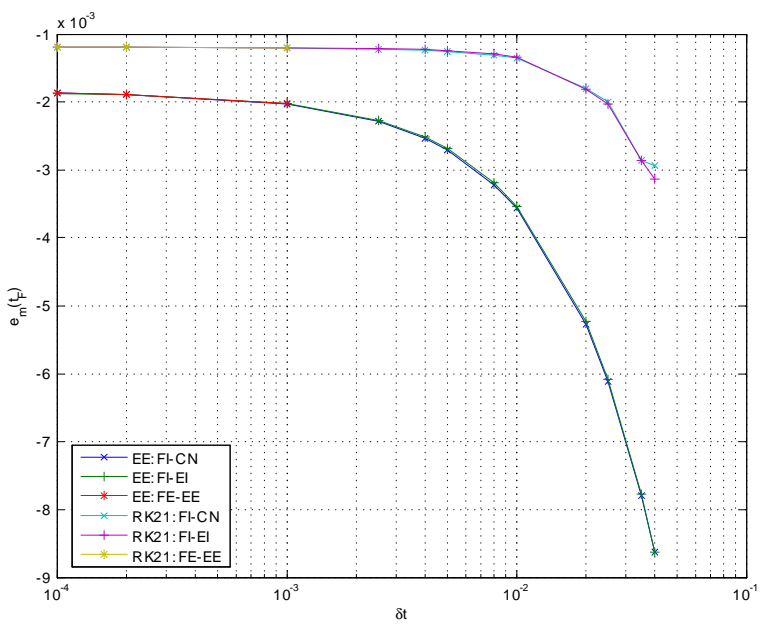

(a)

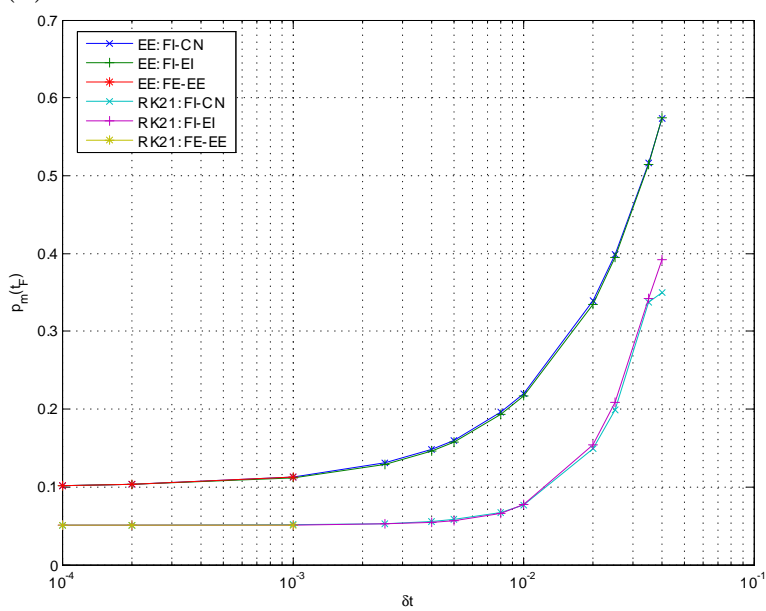

(c)

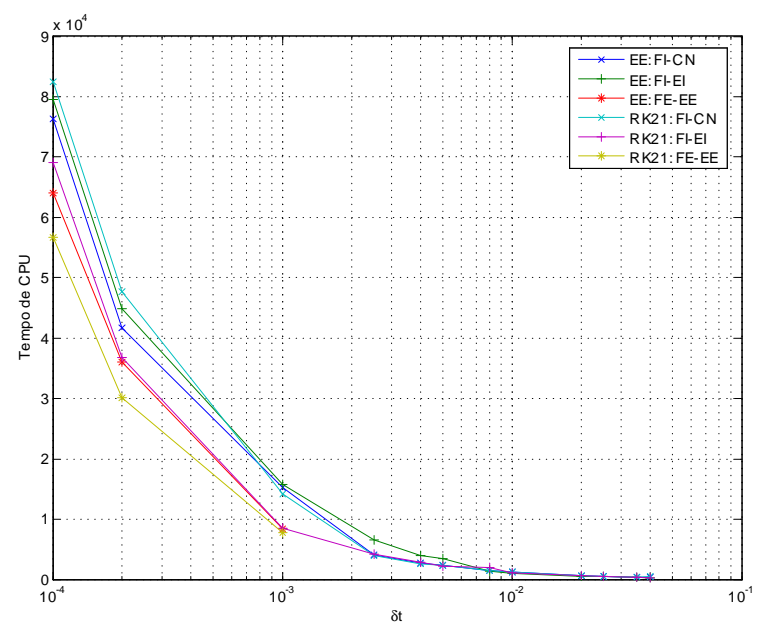

(b)

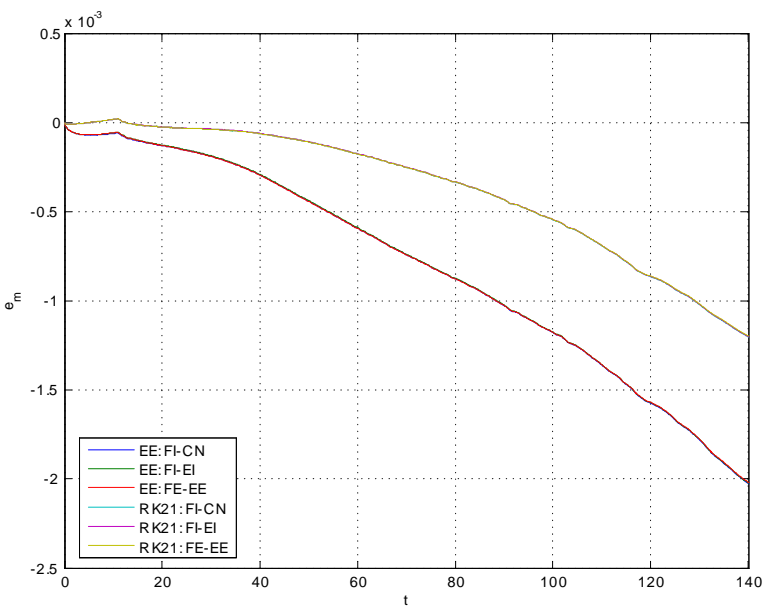

(d)

Figura 4.3.5: Erro na massa (a), tempo de CPU total (b) e função perda total (c), todos em função de $\delta t$, em $t=t_{F}$. Em (d) temos a evolução do erro na massa no tempo com os diferentes métodos usando o maior passo temporal admitido pela FE-EE $\left(F_{v i s c}=0.3\right)$.

\subsubsection{Problema 3: gota em impacto}

O problema da gota em impacto consiste no lançamento vertical de uma gota de fluido contra uma superfície rígida horizontal. Para simular tal problema, adotamos o modelo:

- Domínio espacial: $x \in[0.0,0.053] m, y \in[0.0,0.0515] m$.

- Diâmetro da gota (esférica): $L_{0}=2.0 \times 10^{-2} \mathrm{~m}$.

- Velocidade inicial da gota: $u^{0}=0.0 \mathrm{~ms}^{-1}, v^{0}=-1.0 \mathrm{~ms}^{-1}$.

- Escala de velocidade: $U_{0}=1.0 \mathrm{~ms}^{-1}$

- Altura inicial (entre o centro da gota e o fundo da caixa): $h_{1}=0.04 m$.

- Largura (entre as paredes verticais da caixa): $h_{1}=0.05 \mathrm{~m}$.

- Aceleração de campo: $g_{0}=9.81 m s^{-2}, g^{x}=0.0, g^{y}=-1.0$. 


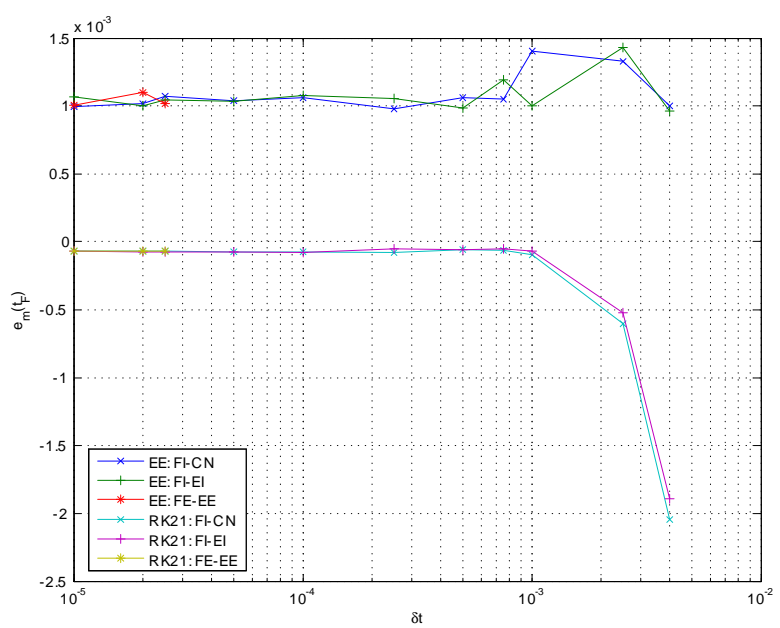

(a)

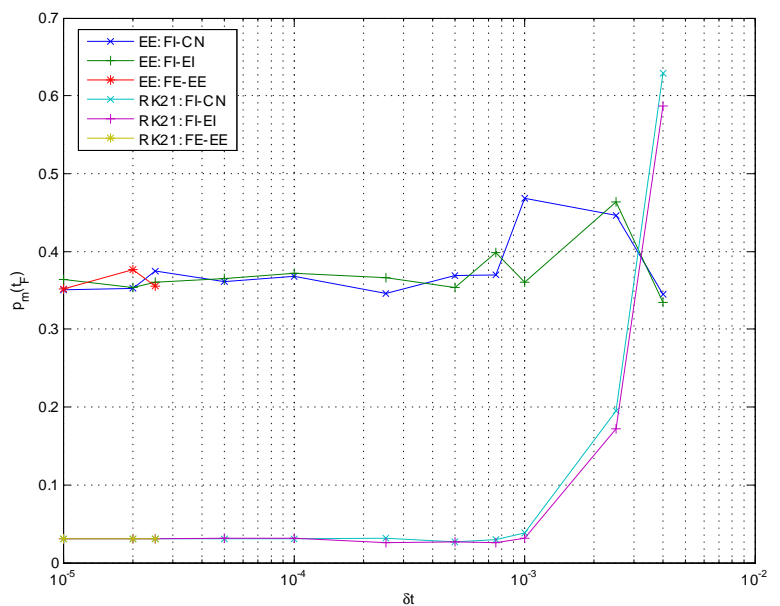

(c)

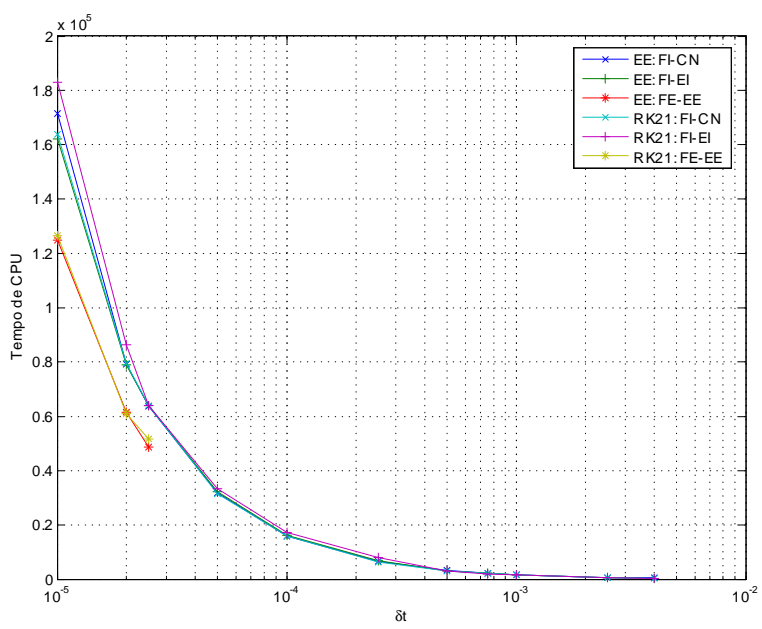

(b)

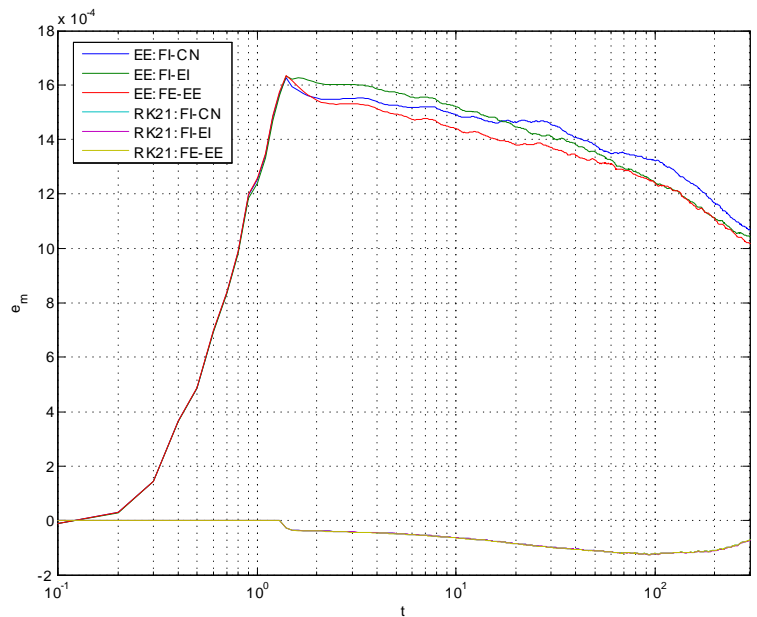

(d)

Figura 4.3.6: Erro na massa (a), tempo de CPU total (b) e função perda total (c), todos em função de $\delta t$, em $t=t_{F}$. Em (d) temos a evolução do erro na massa no tempo com os diferentes métodos usando o maior passo temporal admitido pela FE-EE $\left(F_{v i s c}=0.32\right)$.

- Dados do fluido: newtoniano, com $\mu_{0}\left(\rho_{0}\right)^{-1}=4.0 \times 10^{-2} m^{2} s^{-1}$.

- Parâmetros adimensionais: $R e=0.5, F r \approx 2.257618, S t=50.0 t_{0}$.

- Discretização do domínio espacial: $106 \times 103$ células nas direções $x$ e $y$, respectivamente.

- Discretização do domínio temporal:

$$
\begin{aligned}
& -F_{o}=1.0, F_{C F L}=0.5 . \\
& -F_{\text {visc }} \in\{51.2,32.0,12.8,9.6,6.4,3.2,1.28,0.64,0.32,0.256,0.128\} .
\end{aligned}
$$

- Passos adimensionais: $\delta m=0.025, \delta t_{v i s c}=7.8125 \times 10^{-5}$.

- Instante final adimensional: $t_{F}=73.0$.

- Refinamento da malha superficial: $c_{\min }=0.02 \delta m, c_{m a ́ x}=0.2 \delta m$. 

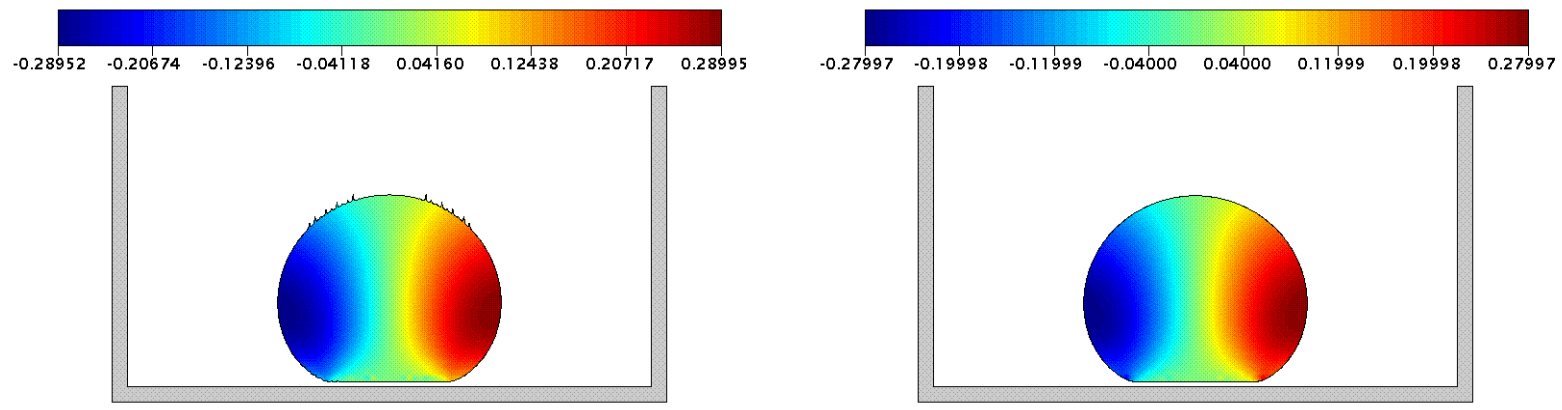

(a)
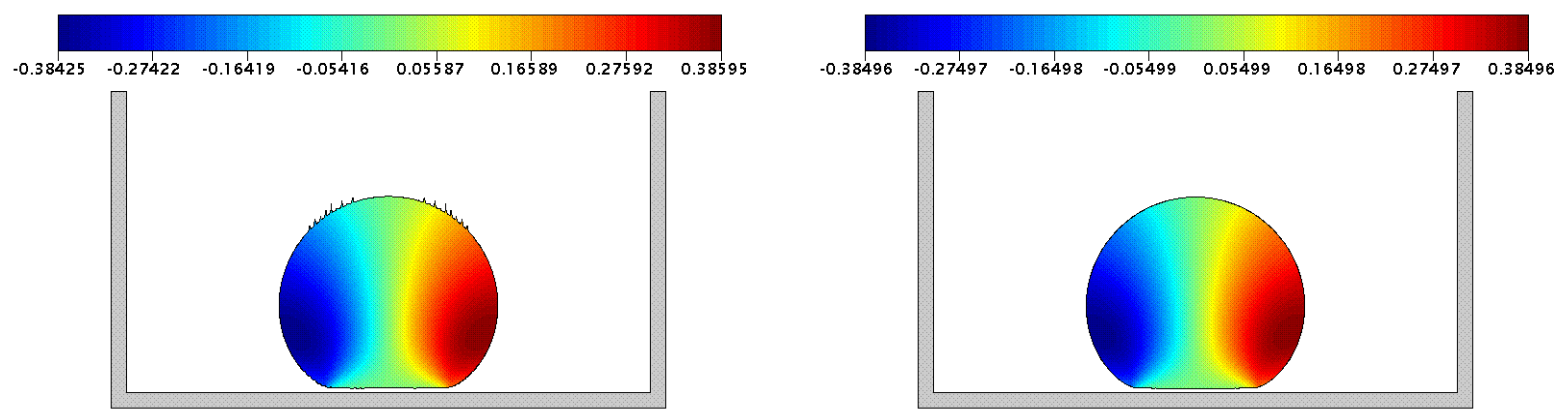

(b)
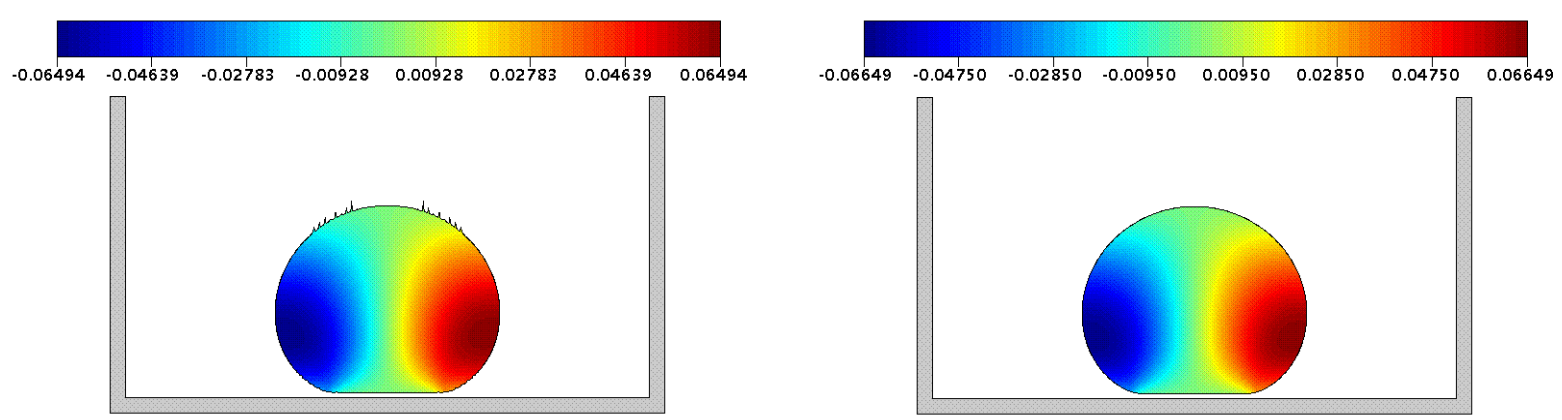

(c)

Figura 4.3.7: Simulações numéricas da gota em impacto de um fluido newtoniano $(R e=0.5)$ utilizando EE (coluna esquerda) e RK21 (coluna direita) com: FI-CN (a), FI-EI (b) e FE-EE (c), em $t=1.5$. Para a FI foi usado $F_{\text {visc }}=51.2 \mathrm{e}$, para a FE, $F_{\text {visc }}=0.128$.

- Sistemas lineares:

- Tolerância: $\varepsilon=1.0 \times 10^{-10}$.

- Método para solução de $\widetilde{\mathbf{u}}$ : BiCGstab-SOR $(\omega=1.4)$.

- Método para solução de $\psi$ : BiCGstab-SOR $(\omega=1.8)$.

A razão para o estudo deste problema está no fato de que a massa total de fluido durante o escoamento deve permanecer constante, apesar da deformação sofrida pela gota em seu impacto contra a superfície rígida. Desta forma, este é um problema interessante para testarmos os esquemas de movimento da superfície livre. 
Neste modelo, o maior $\delta t$ admitido, aproximadamente, pela FE resultou em $F_{v i s c}=0.32$. Já o limite de estabilidade da FI é atingido por volta de $F_{v i s c}=51.2$.

Para este problema, observamos um fenômeno espúrio quando usamos EE para movimentar as partículas marcadoras, o qual consiste na formação de pequenas oscilações em algumas partes da superfície livre desde o início da simulação, independentemente do $\delta t$. O emprego do RK21 conseguiu evitar o aparecimento dessas oscilações, mostrando agregar mais precisão e estabilidade à solução numérica. Tal situação está ilustrada na Figura 4.3.7, no instante logo após o impacto da gota com o fundo da caixa.

No que diz respeito ao erro na massa final, a Figura 4.3.6 (a) revela um comportamento mais adequado do RK21, com o erro se aproximando de zero a medida que o passo temporal é diminuído. Obviamente, o erro não se anula, pois, a malha espacial foi mantida constante em todas as simulações. Entretanto, as simulações que utilizaram o EE, além de resultarem em maior erro, não demonstraram sensibilidade à redução do passo temporal, com os erros na massa final oscilando, independentemente do $\delta t$ empregado, em torno de $1.0 \times 10^{-3}$.

A mesma situação também é observada quanto à função perda total, apresentada na Figura 4.3.6 (c). Nesta, se por um lado observamos um comportamento convergente do RK21 à medida que $\delta t$ tende a zero, a perda total associada ao EE é mantida sempre próxima de um mesmo patamar, maior que o do RK21 e insensível ao $\delta t$ utilizado.

Em termos de CPU total, porém, ambos os métodos demonstraram desempenho semelhante, conforme Figura 4.3.6 (b).

Apresentamos, na Figura 4.3.6 (d), a evolução do erro na massa durante a simulação, usando, para viabilizar a comparação de todos os esquemas, o maior passo temporal admitido pela FE-EE neste modelo. Assim, é nítida a superioridade do RK21, que mantém um erro muito próximo de zero durante toda simulação. Parece ficar claro, também, que o principal fator que interfere no erro da massa não é a formulação do GENSMAC utilizada para resolver Navier-Stokes, mas, o esquema de movimentação da superfície livre adotada.

\subsubsection{Problema 4: inchamento do extrudado}

Voltamos a utilizar, neste capítulo, o inchamento do extrudado, agora, porém, segundo o modelo:

- Domínio espacial: $x \in[0.0,15.30] m, y \in[0.0,3.0] m$.

- Diâmetro do injetor: $L_{0}=1.0 \mathrm{~m}$.

- Velocidade no injetor: $U_{0}=1.0 \mathrm{~ms}^{-1}$, com perfil de injeção parabólico.

- Comprimento do canal inicial: $5.0 L_{0}$.

- Distância ente injetor e outflow: $15.0 L_{0}$.

- Diâmetro do outflow: $3.0 L_{0}$.

- Aceleração de campo: $g_{0}=9.81 \mathrm{~ms}^{-2}, g^{x}=0.0, g^{y}=0.0$.

- Dados do fluido: newtoniano, $\operatorname{com} \mu_{0}\left(\rho_{0}\right)^{-1}=20.0 m^{2} s^{-1}$.

- Parâmetros adimensionais: $R e=0.05, F r \approx 0.319275, S t=1.0 t_{0}$.

- Discretização do domínio espacial: $306 \times 60$ células nas direções $x$ e $y$, respectivamente.

- Discretização do domínio temporal:

$$
\begin{aligned}
& -F_{o}=1.0, F_{C F L}=0.5 . \\
& -F_{\text {visc }} \in\{160.0,128.0,80.0,64.0,32.0,25.6,16.0,8.0,6.4,3.2,1.6,0.8,0.32\} .
\end{aligned}
$$

- Passos adimensionais: $\delta m=0.05, \delta t_{v i s c}=3.125 \times 10^{-5}$.

- Instante final adimensional: $t_{F}=23.0$. 
- Refinamento da malha superficial: $c_{\min }=0.02 \delta m, c_{m a ́ x}=0.2 \delta m$.

- Sistemas lineares:

- Tolerância: $\varepsilon=1.0 \times 10^{-10}$.

- Método para solução de $\widetilde{\mathbf{u}}$ : BiCGstab-SOR $(\omega=1.4)$.

- Método para solução de $\psi$ : BiCGstab-SOR $(\omega=1.8)$.

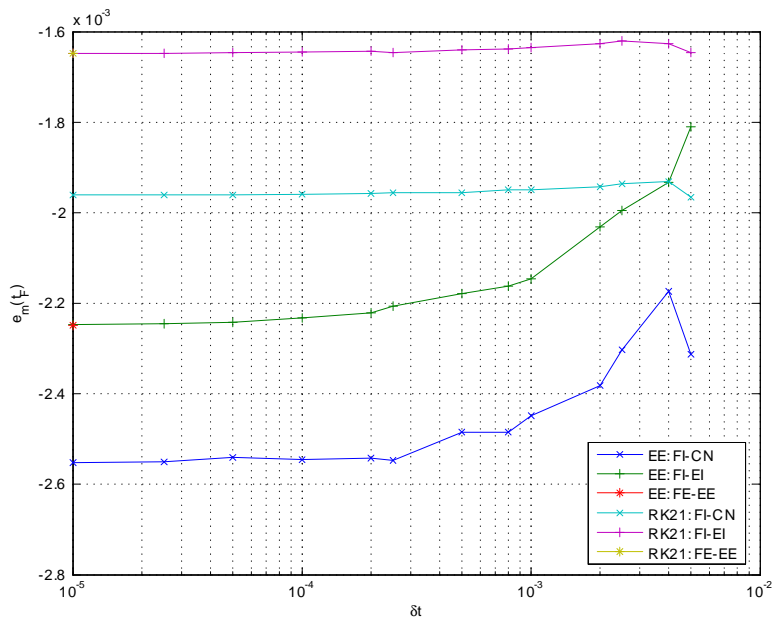

(a)

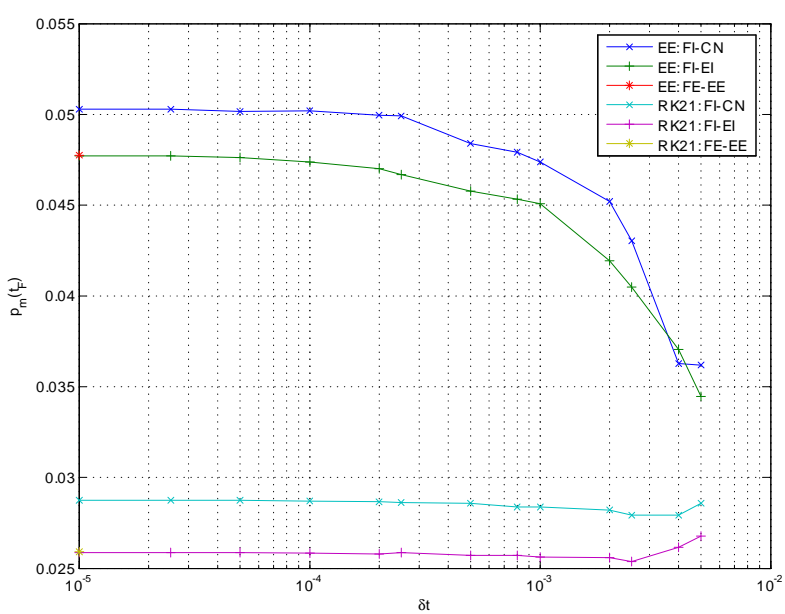

(c)

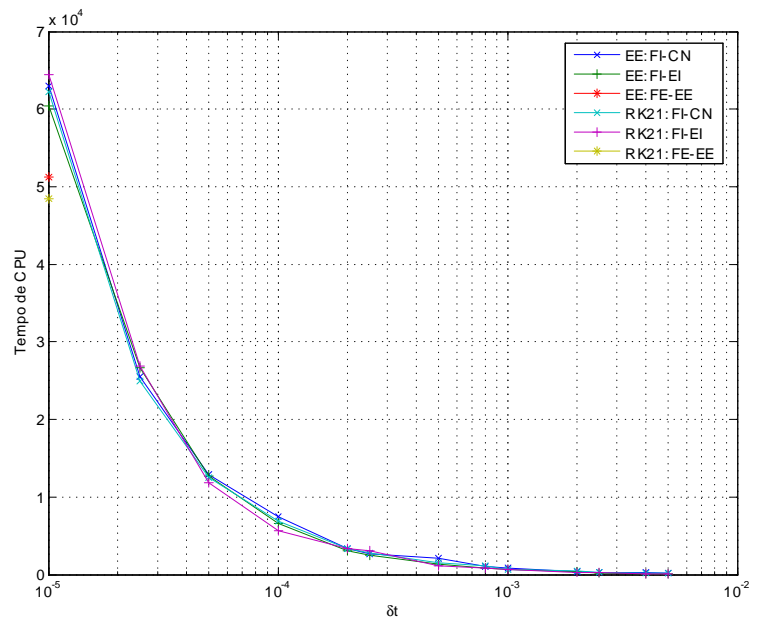

(b)

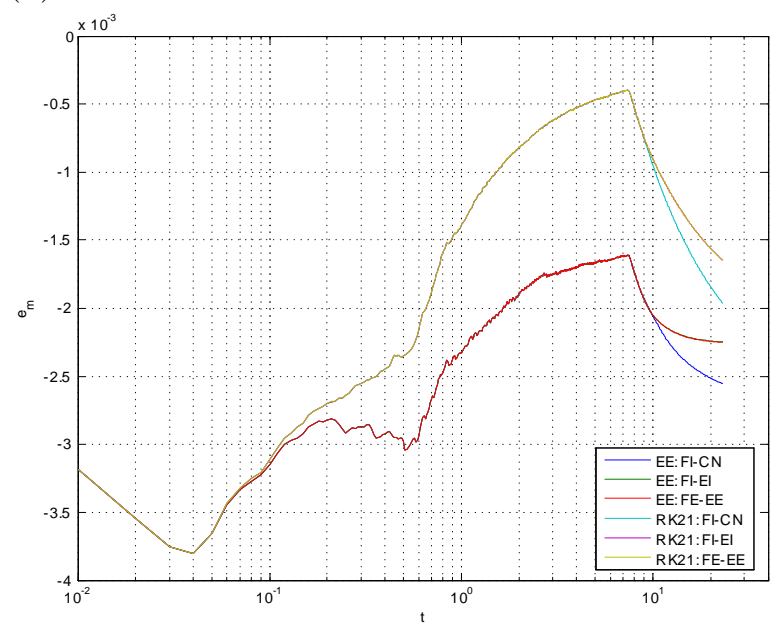

(d)

Figura 4.3.8: Erro na massa (a), tempo de CPU total (b) e função perda total (c), todos em função de $\delta t$, em $t=t_{F}$. Em (d) temos a evolução do erro na massa no tempo com os diferentes métodos usando o maior passo temporal admitido pela FE-EE $\left(F_{v i s c}=0.32\right)$. 

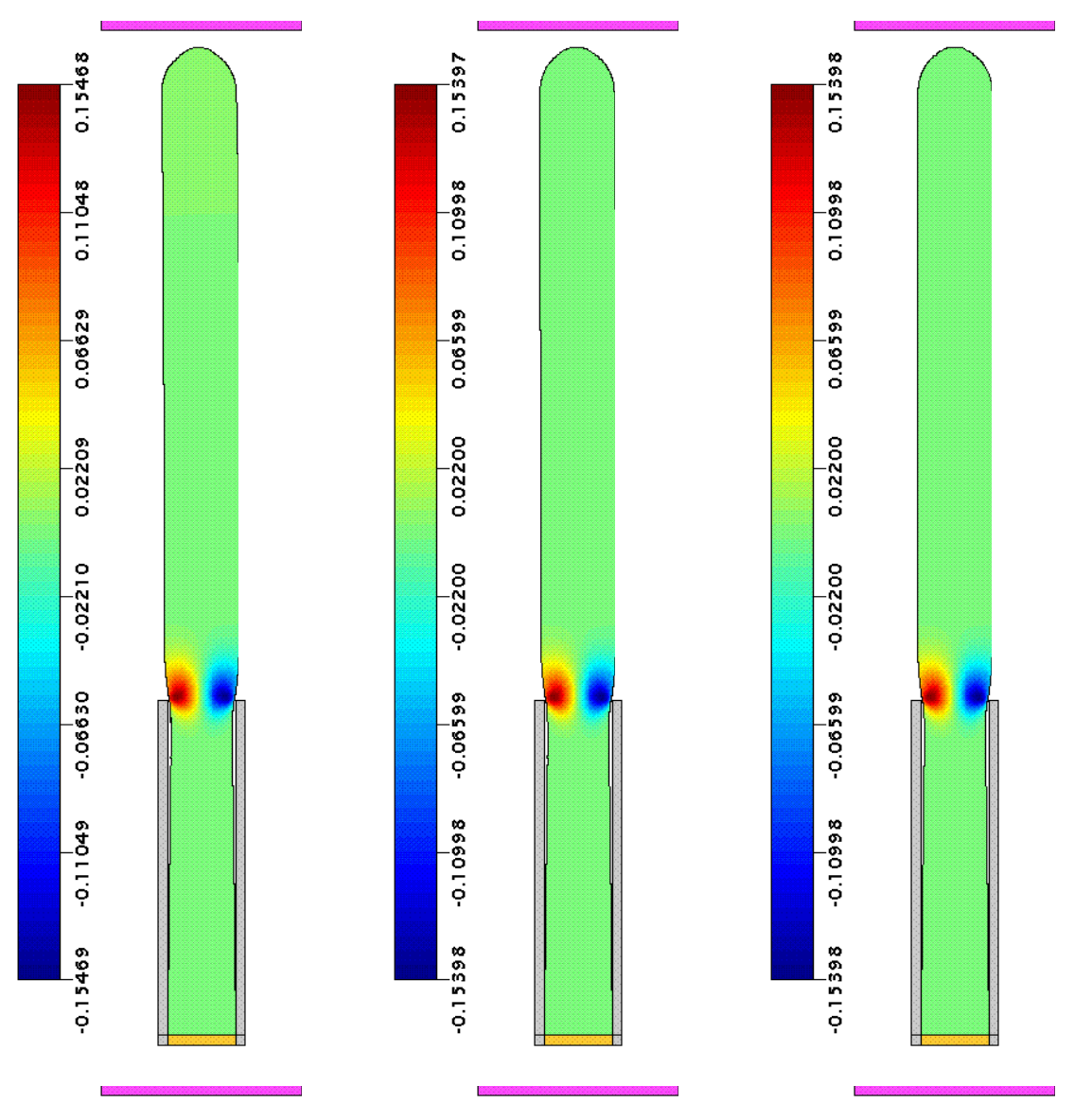

癸

1.: $\stackrel{\infty 0}{=}$

要 茫

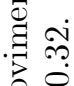

吾

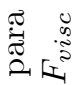

되잉

을

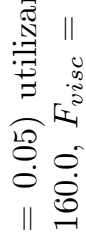

$\underset{\varepsilon}{2}$

䒕

胥

है

응.

皕 0
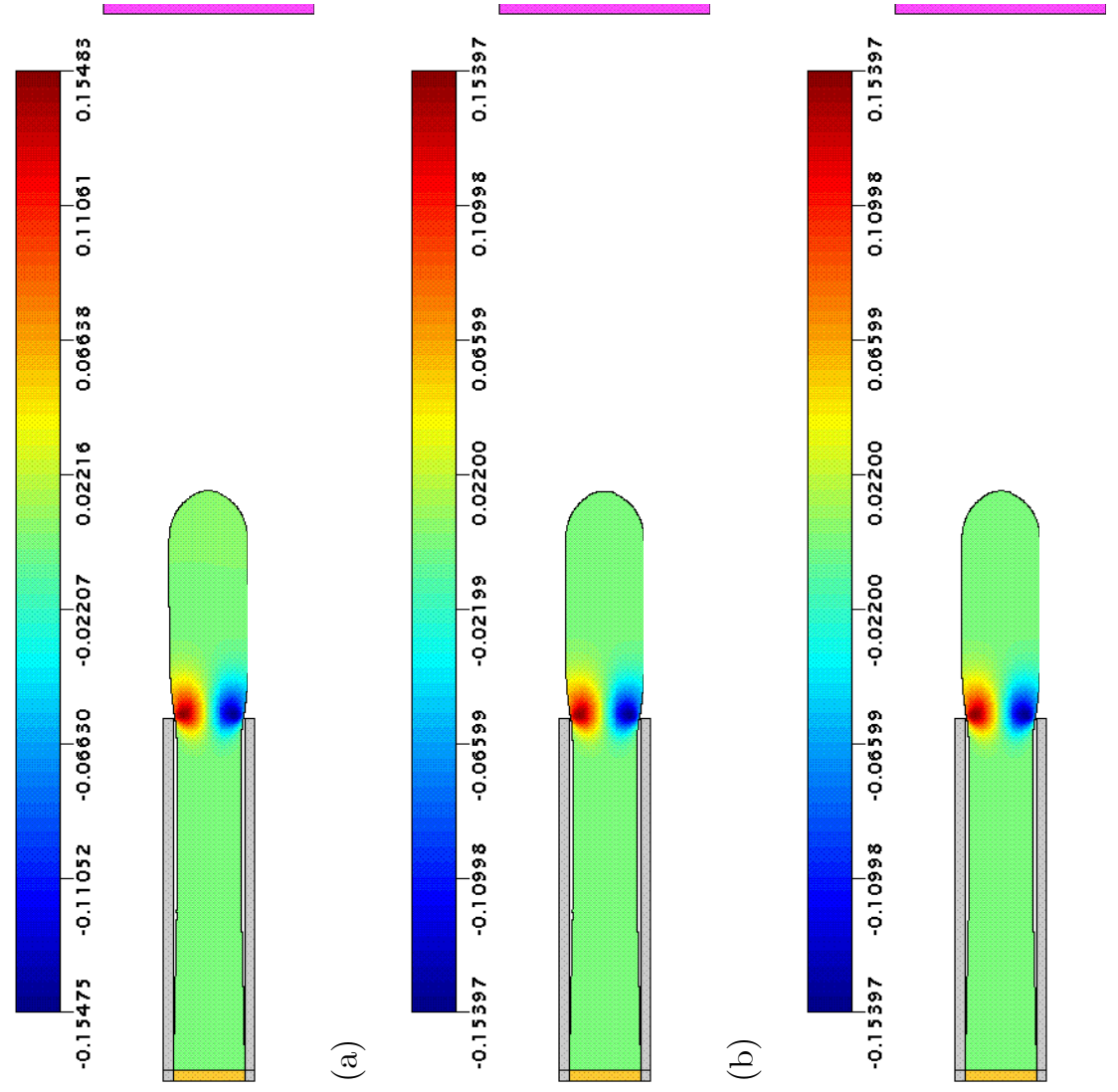

घ. ำ

율일

율

导

정

웜요

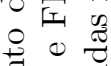

氖完获

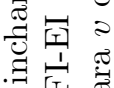

$\theta \stackrel{0}{\sigma}$

论 焉

包它

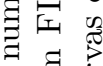

ช

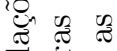

被

อ. ⿷匚

भ 蛋

อ

త్ర

昆要 


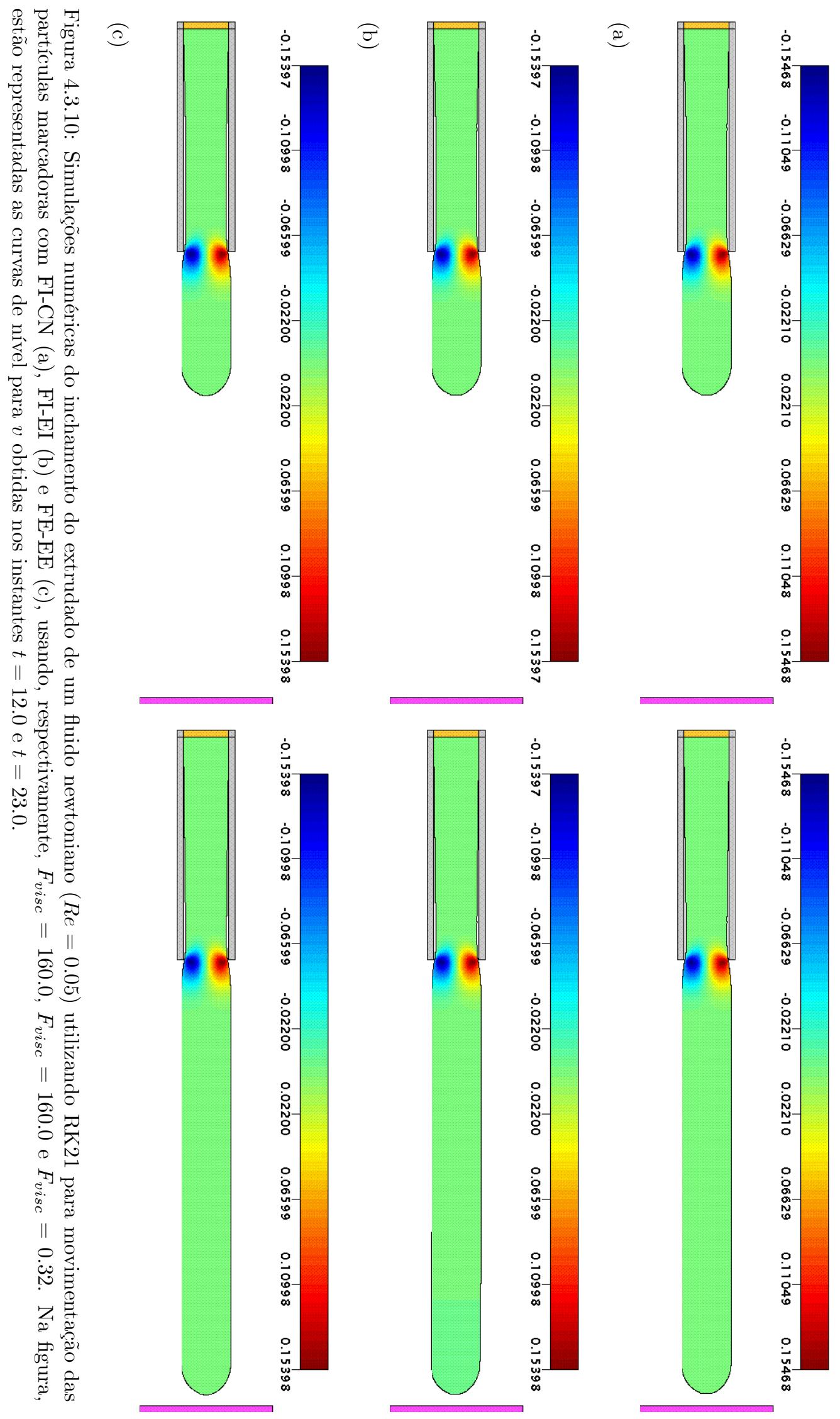


O inchamento do extrudado é um problema clássico no contexto de escoamentos com superfície livre, o que não implica, entretanto, menor complexidade. Logo, nada mais natural que utilizá-lo para testar os esquemas de movimentação da superfície.

Assim, apresentamos, na Figura 4.3.9, as simulações com o maior $\delta t$ obtidas com o EE. Por sua vez, na Figura 4.3.10 estão as respectivas simulações com o RK21. Como pode ser observado, mesmo utilizando um passo temporal 500 vezes maior que o da FE, a FI apresentou uma solução numérica bastante semelhante em termos visuais. Em todos os casos, a simetria da solução foi mantida pelos esquemas de movimentação das partículas, o que proporcionou boa representação do perfil da superfície livre.

Já no que diz respeito à conservação da massa numérica, tanto a Figura 4.3.8 (a) como a Figura 4.3.8 (c) revelam não apenas que o RK21 é mais preciso, mas, também, sua melhor convergência com relação ao EE. É nítido em ambas as figuras que o EE apresenta divergência para os maiores $\delta t$, estabilizando em um patamar de erro mais alto que o RK21 quando $\delta t$ tende a zero.

Neste problema, a Figuras 4.3.8 (a) e a Figura 4.3 .8 (c) revelam que a FI-CN, para um mesmo esquema de movimento das partículas, produziu um erro na massa ligeiramente maior que a FE-EE e a FI-EI. Apesar da diferença, no entanto, o erro ainda é menor àquele obtido com o EE. De fato, o RK21:FI-CN apresentou melhor precisão que as estratégias EE:FI-CN, EE:FI-EI e EE:FE-EE.

A Figura 4.3.8 (d), esboçando a evolução temporal do erro na massa quando empregado o maior passo temporal admitido pela FE-EE, mostra não só a superioridade do RK21, mas, também, que a deficiência da FI-CN neste problema ocorre na parte final da simulação.

Por fim, na Figura 4.3.8 (b), constatamos que, em termos de tempo de CPU total, o EE e o RK21 são, mais uma vez, bastante parecidos.

\subsection{Conclusões}

Testamos, neste capítulo, modificações que parecem melhorar a precisão e a eficiência da FI do GENSMAC. Para obter essas conclusões, um amplo e sistemático conjunto de testes foi executado. Os resultados finais apresentados neste capítulo envolveram 159 simulações na Seção 4.2 e 208 na Seção 4.3. Isto sem mencionar os vários testes preliminares que foram realizados durante o desenvolvimento das ferramentas utilizadas, o que faria esse total ser multiplicado algumas vezes.

Tal volume exigiu grande demanda por recurso computacional e só foi possível graças ao cluster do Laboratório de Computação de Alto Desempenho (LCAD) do ICMC-USP. Obviamente, apesar de numerosos e de apontarem sempre em uma direção favorável (conforme indicamos no capítulo anterior), sabemos que os resultados obtidos não esgotam o assunto. No entanto, é indiscutível que fornecem boa margem de segurança para que algumas decisões importantes sejam tomadas.

Inicialmente, quanto à questão da eficiência na solução dos sistemas lineares, o pré-condicionador SOR mostrou-se uma escolha bastante robusta. Aliás, para todos os métodos iterativos testados, o SOR, mediante a conveniente escolha de seu parâmetro de relaxação $\omega$, mostrou-se superior ao Gauss-Seidel e ao Jacobi.

Os resultados obtidos também apontam o CGS e o BiCGstab como os melhores métodos iterativos para a classe de problemas de interesse. Com efeito, o CGS-SOR e o BiCGstab-SOR apresentaram um desempenho muito homogêneo em todos os problemas testados, com ganhos da ordem de $70 \%$ em tempo de CPU total com relação ao par CG/BiCG-Jacobi, previamente usado pela FI.

$\mathrm{Na}$ literatura, o BiCGstab foi concebido para remediar eventuais problemas numéricos no caso de convergência irregular do CGS ([VS92], [SD00]). Portanto, consideramos o BiCGstab-SOR como a melhor opção para substituir o BiCG-Jacobi na resolução do sistema linear associado a $\psi$ na FI. Além disso, o BiCGstab-SOR também mostrou ganhos sobre o CG na solução de $\widetilde{\mathbf{u}}$. Mas, uma questão muito importante diz respeito à escolha do parâmetro de relaxação $\omega$ do pré-condicionador SOR.

Para o sistema linear associado a $\psi$ foi possível verificar que $\omega \approx 1.8$ é a escolha ótima, independentemente do $\delta t$ adotado. Diferentemente, na resolução do sistema linear para $\widetilde{\mathbf{u}}$ verificamos que o passo temporal influi no parâmetro de relaxação ótimo, com $\omega$ tendendo a 1.0 a medida que $\delta t$ é diminuído e se aproxima de $\delta t_{v i s c}$. Na verdade, essa influencia do passo temporal no $\omega$ ótimo já havia sido observada na Figura 3.2.3 em termos do condicionamento dos sistemas lineares e pôde ser percebida nos problemas deste capítulo através do número médio de iterações. 
Dessa forma, para $\delta t$ relativamente grande, foi possível observar ganhos do BiCGstab-SOR sobre o par CG/BiCG-Jacobi na solução de $\widetilde{\mathbf{u}}$ da ordem de $40 \%$ em tempo de CPU médio (Problema 1) mediante uma escolha adequada de $\omega$. No entanto, esta margem torna-se mais estreita à medida que $\delta t$ diminui, como no Problema 3, onde o BiCGstab-SOR e o CG/BiCG-Jacobi apresentam desempenhos semelhantes quanto ao tempo de CPU médio para a solução de $\widetilde{\mathbf{u}}$. Assim, com uma escolha adequada de $\omega$, podemos considerar que o BiCGstab-SOR apresenta um desempenho, no mínimo, semelhante ao CG/BiCG-Jacobi na solução de $\widetilde{\mathbf{u}}$.

Portanto, concluímos ser o BiCGstab-SOR uma escolha eficiente para a solução dos sistemas lineares da FI, conforme os problemas estratégicos utilizados demonstraram.

Com efeito, no Problema 1 e no Problema 2 utilizamos dois problemas clássicos com superfície livre, respectivamente, de grande e pequeno porte para uma simulação 2D. Em ambos os casos, a escolha do $\delta t$ foi próxima ao limite de estabilidade, que era uma situação, na qual, a FI-CN mostrava-se deficiente com o par CG/BiCG-Jacobi pela deterioração no chute inicial e no condicionamento dos sistemas. Assim, mesmo em situação adversa, o BiCGstab-SOR mostrou muito bom desempenho, com uma larga margem de vantagem sobre o par CG/BiCG-Jacobi.

No Problema 3 escolhemos um modelo de porte intermediário, atingindo, aproximadamente, 16000 células com fluido no final da simulação. Neste, utilizamos um passo temporal pequeno a fim de viabilizar o uso da FE. Mais uma vez a FI-CN com o par CG/BiCG-Jacobi demonstrou ter um ciclo computacional muito mais caro que a FE-EE e o par -/CG. No entanto, o BiCGstab-SOR aplicado com a FI mostrou ser possível alterar esta situação, tornando, inclusive a FI-CN mais eficiente que a FE-EE.

Por sua vez, o Problema 4 simulou um escoamento confinado para que fosse possível testar o CG na solução de $\psi$ pela FI com um $\delta t$ relativamente alto. Isto porque, com superfície livre, o sistema linear para $\psi$ na FI é não-simétrico e, consequentemente, não se pode utilizar o CG. Por outro lado, não existe na literatura método iterativo ou pré-condicionador considerado ideal para esse tipo de sistema linear. Com isso, tentamos estabelecer pelo par CG/CG, neste problema particular, um nível de desempenho supostamente ideal, que serviria como condição necessária para um método iterativo ser considerado eficiente para uso na FI. De fato, o CG/BiCG-Jacobi apresentou um desempenho muito inferior ao CG/CG, porém, o BiCGstab-SOR manteve um ganho da ordem de $70 \%$ em tempo de CPU total com relação ao par CG/BiCG-Jacobi. Assim, este teste foi muito importante, pois, exemplificou que, em comparação com o CG/CG, o BiCGstab-SOR pode ter um desempenho bastante parecido e, em alguns casos, levar até pequena vantagem.

O Problema 5 teve como objetivo verificar se o desempenho dos métodos iterativos e, em especial, do pré-condicionador SOR, seria mantido para situações de porte semelhante a um problema 3D. Com efeito, no início da simulação existem 76800 células com fluido, evoluindo para um total de quase 82000 no instante final. O bom desempenho ficou nítido nos resultados obtidos, com o SOR alcançando os melhores resultados de cada método iterativo justamente neste problema. Consequentemente, o BiCGstab-SOR apresentou seu melhor desempenho, conseguindo um ganho de mais de $75 \%$ em tempo de CPU total com relação ao par CG/BiCG-Jacobi.

Portanto, consideramos a questão da eficiência na FI nas etapas de solução de $\widetilde{\mathbf{u}}$ e $\psi$ resolvida, parecendo ser o BiCGstab-SOR a melhor alternativa, entre as testadas, para solução dos sistemas lineares.

Já no que diz respeito à estratégia de movimento da superfície livre, estudamos a influência do passo temporal na conservação da massa. Para a FE este estudo acaba sendo restrito devido à limitação parabólica no passo temporal. Na FI, entretanto, como a escolha do $\delta t$ é mais flexível, tal análise torna-se relevante.

Conforme já apresentamos, no GENSMAC, o que determina, em última instância, o erro na massa numérica é a estratégia de movimento da superfície livre. Para isso, confrontamos a estratégia original (baseada em Euler Explícito) com um novo esquema (o RK21), proposto neste trabalho.

Assim, escolhemos quatro problemas distintos e, para cada um deles, comparamos todos os métodos disponíveis (FE-EE, FI-EI e FI-CN) com os dois esquemas de movimento das partículas marcadoras (EE e RK21), originando 6 estratégias de solução. Nestes problemas, cada uma dessas estratégias foi empregada para diversos passos temporais, variando desde abaixo do limite de estabilidade da FE até próximo do limite de estabilidade da FI.

Dessa forma, podemos resumir os resultados obtidos na Seção 4.3 em uma única frase: o RK21 foi, 
nitidamente, superior. Isto, no entanto, não significa que o EE tenha uma má performance, mas, para a classe de problemas que analisamos (que supomos ser bastante representativa para o caso $2 \mathrm{D}$ a baixos Reynolds), o RK21 mostrou-se mais robusto.

De fato, o RK21 proporcionou um nível de erro na massa, em todos os problemas, menor que o EE. Em várias oportunidades observamos que, mesmo usando o maior $\delta t$ possível, o RK21 conservou melhor a massa que o EE usando o menor $\delta t$ da série de simulaçãoes. Além disso, o RK21 mostrou boa sensibilidade à variação no passo temporal, fazendo, efetivamente, o erro diminuir à medida que $\delta t$ é reduzido.

Por sua vez, com o EE para movimentar a superfície livre, nem sempre a redução do $\delta t$ agrega precisão à solução, o que fica nítido pela observação do erro na massa final e da função perda total. Com efeito, no Problema 3, a conservação da massa pelo EE é, praticamente, inalterada pela redução no passo temporal. Já no Problema 4 pôde ser observado um comportamento divergente, onde, à medida que $\delta t$ diminui, o erro e a perda total na massa aumentam em valor absoluto.

Quanto à questão da representação da superfície livre, consideramos que, mais uma vez, o RK21 foi superior ao EE. Na verdade, os perfis da superfície livre obtidos no Problema 2 foram, visualmente, muito parecidos, com o mesmo ocorrendo no Problema 4. Porém, no Problema 1, o EE causou um efeito espúrio na solução para passo temporal pequeno, produzindo uma solução numérica que sabemos não ser a solução física do problema. Já no Problema 3, o EE resultou no aparecimento de oscilações na superfície livre, as quais sequer são alteradas pela redução do passo temporal.

Assim, torna-se difícil dizer em quanto o RK21 possibilita um aumento no $\delta t$ para manter a perda de massa do EE com $\delta t$ pequeno, devido à superioridade do RK21. No Problema 1, a solução obtida com o menor $\delta t$ pelo EE não serve de referência, já que apresentou efeitos de natureza espúria (mesmo assim, qualquer passo no tempo para o RK21 é suficiente para bater o EE). No Problema 2, o terceiro maior $\delta t$ utilizado (ainda assim muito próximo ao limite de estabilidade da FI) já basta para erro final e perda total do RK21 inferiores aos níveis obtidos pelo EE com o menor $\delta t$. O Problema 3 também não serviria de referência, devido às oscilações na superfície livre ocasionadas pelo EE, mas, foi suficiente para o RK21 o maior passo temporal adotado para uma precisão semelhante à da estratégia original com qualquer $\delta t$. Por fim, no Problema 4, qualquer passo temporal é suficiente para superar o EE quanto à perda total.

Obviamente, a escolha para o passo temporal em uma simulação deve levar em conta o compromisso entre precisão desejada e custo computacional. Assim, mesmo com RK21 conservando melhor a massa, a escolha de $\delta t$ deve ser criteriosa.

Outra questão relevante diz respeito ao desempenho, em termos de tempo de CPU total, do RK21 em comparação ao EE. Neste sentido, os métodos apresentaram custos muito semelhantes, o que é justificado pelo fato da etapa de movimento das partículas representar apenas uma pequena fração de um ciclo computacional. Além disso, o emprego do RK21 exigiu um tratamento especial das extrapolações da velocidade na superfície quando ocorre o avanço dessa fronteira. Assim, esse tratamento distinto acaba compensando o maior custo computacional da fórmula (3.3.14)-(3.3.15) do RK21 em comparação à expressão (3.3.12), que define o EE. Detalhes sobre a extrapolação das velocidades na superfície livre são apresentados na Seção 5.3.7, dentro do contexto do GENSMAC-SXPP, uma vez que são idênticos ao efetuado para o GENSMAC.

Também é importante observar que os resultados apontaram como principal responsável pelo erro na massa numérica o esquema de movimento da superfície livre. De fato, para um mesmo passo temporal, as formulações explícita e implícita do GENSMAC apresentaram diferenças na evolução do erro na massa que são inferiores às ocasionadas pela escolha do esquema de movimentação das partículas.

Assim, embora tenha sido proposto para ser incorporado, apenas, à FI, os resultados apresentados pelo RK21 foram, surpreendentemente, satisfatórios até mesmo para a FE. Com isso, passamos a considerar o RK21 como uma alternativa bastante viável para o movimento da superfície livre em ambas formulações do GENSMAC.

Por fim, vale salientar que tanto a solução dos sistemas lineares quanto a estratégia de movimentação da superfície livre foram testadas em problemas a baixos Reynolds, que é onde a FI do GENSMAC faz-se mais promissora. Embora haja indícios de que tais resultados são mantidos para Reynolds maiores, seria necessário outro estudo minucioso para comprovar isso, o qual foge do escopo deste trabalho. 


\section{Capítulo \\ O GENSMAC-SXPP}

\subsection{Introdução}

Até aqui, tratamos da simulação numérica de escoamentos newtonianos com superfície livre. Para isso, apresentamos os principais conceitos do GENSMAC, bem como suas formulações explícita e implícita.

Neste trabalho, temos interesse na simulação numérica de escoamentos a baixos Reynolds, e, para isso, mudanças foram propostas à FI do GENSMAC com o objetivo de torná-la mais eficiente e precisa. Em outras palavras, tratamos até o momento do desenvolvimento da metodologia numérica implícita existente para que fosse possível, agora, generalizá-la para a simulação de escoamentos viscoelásticos a baixos Reynolds $(R e<<1)$ com superfície livre.

Uma primeira extensão da FI do GENSMAC para fluidos viscoelásticos foi realizada, no caso 3D, em [OC08B], usando os modelos UCM e Oldroyd-B ([BH87]). Em duas dimensões, entretanto, existe apenas a versão para fluidos newtonianos da FI.

Por outro lado, um modelo viscoelástico recente é o SXPP, proposto em [VB01], cuja equação constitutiva leva em conta a estrutura molecular do fluido e apresenta como caso particular o modelo Oldroyd-B $([\mathrm{BH} 87])$.

Modelos do tipo Pom-Pom ganharam respeito ao serem capazes de prever quantitativamente a reologia de soluções LDPE e HDPE, conforme apontam [VB01] e [AB05]. Entretanto, existem poucos relatos na literatura de métodos numéricos para simular escoamentos confinados de um fluido SXPP. Entre esses, temos [AJ04] e [AB05], que desenvolveram duas metodologias (uma baseada em elementos finitos e outra híbrida, do tipo volumes/elementos finitos) para resolver o modelo SXPP a partir de esquemas previamente propostos em [AB01] para o modelo Oldroyd-B. Assim, enquanto [AJ04] aplica tais metodologias em contrações planares, o trabalho [AB05] apresenta um estudo numérico do modelo SXPP no escoamento de Hagen-Poiseuille.

Além disso, são desconhecidos na literatura relatos de metodologias para a simulação numérica de escoamentos com superfície livre de um fluido SXPP, bem como a solução analítica para qualquer problema envolvendo este modelo de fluido.

Portanto, existe forte motivação para a extensão do GENSMAC para simular escoamentos viscoelásticos com o modelo SXPP. Em adição, como escoamentos viscoelásticos transcorrem, em sua maioria, a baixos Reynolds, a FI torna-se uma ferramenta indispensável.

Dessa forma, desenvolvemos, neste capítulo, o GENSMAC-SXPP, que é uma metodologia para a simulação numérica de escoamentos viscoelásticos com superfície livre segundo o modelo SXPP, estendendo a aplicabilidade do GENSMAC, em suas formulações explícita e implícita, para esse tipo de fluido.

Assim, o GENSMAC-SXPP herda as principais características do GENSMAC, como a estratégia de aproximação das equações por diferenças finitas em uma malha deslocada, a forma de representação da superfície livre e o esquema de classificação das células do domínio, bem como os demais detalhes apre- 
sentados no Capítulo 2. Além disso, incorporamos ao GENSMAC-SXPP os desenvolvimentos propostos no Capítulo 3 e testados no Capítulo 4 a respeito da solução dos sistemas lineares e da estratégia de movimentação da superfície livre. Em adição, o GENSMAC-SXPP apresenta uma nova abordagem para a integração temporal da contribuição não-newtoniana, evitando que valores defasados no tempo dessa grandeza sejam utilizados em partes do algoritmo computacional, conforme ocorre para a extensão 3D da FI apresentada em [OC08B] e em [OC08C] no caso dos modelos viscoelásticos UCM e Oldroyd-B.

Já que o Oldroyd-B é um caso especial do modelo SXPP, o GENSMAC-SXPP também é capaz de simular escoamentos desse fluido mediante a conveniente escolha dos parâmetros mencionada na Seção 1.2.3. Assim, surge também uma nova extensão do GENSMAC (com formulações explícita e implícita) para simular escoamentos 2D de fluidos Oldroyd-B.

Em tempo, formulações explícitas 2D que ampliam o GENSMAC para fluidos Oldroyd-B já haviam sido propostas em [TM02A], [TM02B] e [GF03]. Entretanto, a FE do GENSMAC-SXPP que permite simular escoamentos de um fluido Oldroyd-B difere das anteriores pela estratégia de aproximação da contribuição não-newtoniana no interior do domínio e, principalmente, nos contornos rígidos, conforme explicado na Seção 5.3.2.

Portanto, no que segue, apresentamos o GENSMAC-SXPP em suas formulações explícita e implícita, delineando o algoritmo computacional da metodologia. Em seguida, detalhamos o processo de discretização das equações por diferenças finitas em uma malha deslocada, bem como a aplicação das condições de contorno.

\subsection{Formulações explícita e implícita}

A formulação do GENSMAC-SXPP como método de projeção é análoga à do GENSMAC, apresentada na Seção 2.2. Isso porque a contribuição não-newtoniana é inserida na equação de conservação do momento, pela formulação EVSS, na forma de um termo fonte, que ao ser calculado explicitamente em nada altera a estratégia de projeção.

Com efeito, a equação (1.2.25) que define a contribuição não-newtoniana para o modelo SXPP pode ser rescrita na forma

$$
\frac{\partial T}{\partial t}=F(\mathbf{u}, T)
$$

onde, por (1.2.32),

$$
\begin{gathered}
F(\mathbf{u}, T)=2 \xi D-\frac{1}{W e}\left\{\nabla \cdot(\mathbf{u} T)-\left[L \cdot T+T \cdot L^{\dagger}\right]\right\}-\ldots \\
\ldots-\frac{1}{W e}\left\{f(\lambda, T) T+\xi(f(\lambda, T)-1) \mathbf{I}+\frac{\alpha}{\xi} T \cdot T\right\} .
\end{gathered}
$$

Sendo de caráter hiperbólico, integramos a equação (5.2.1) no tempo explicitamente usando o esquema RK21, que é um método de Runge-Kutta de segunda ordem de precisão ([LB73], [BU03]). Assim, o esquema RK21 é aplicado em duas etapas, sendo que, na primeira, por Euler Explícito, calculamos $\bar{T}^{n+1}$ por

$$
\frac{\bar{T}^{n+1}-T^{n}}{\delta t}=F\left(\mathbf{u}^{n}, T^{n}\right),
$$

e, na segunda etapa, calculamos $T^{n+1}$ de forma semelhante à Regra do Trapézio, por

$$
\frac{T^{n+1}-T^{n}}{\delta t}=\frac{1}{2}\left[F\left(\mathbf{u}^{n}, T^{n}\right)+F\left(\mathbf{u}^{n+1}, \bar{T}^{n+1}\right)\right] .
$$

Portanto, uma vez que os campos de velocidade sejam conhecidos nos devidos instantes, o cálculo da contribuição não-newtoniana é feito de forma independente da estratégia de projeção utilizada, apenas, para desacoplar os campos de velocidade e pressão.

Para isso, supondo que $\mathbf{u}^{n}, p^{n}$ e $T^{n}$ são conhecidos em $t=t_{n}$, apresentamos, no que segue, o ciclo computacional do GENSMAC-SXPP, permitindo o cálculo de $\mathbf{u}^{n+1}, p^{n+1}$ e $T^{n+1}$ em $t_{n+1}=t_{n}+\delta t$. 
Inicialmente, por (5.2.3), calculamos $\bar{T}^{n+1}$, que é uma aproximação por Euler Explícito para $T$ em $t=t_{n+1}$. Repare que, como $\mathbf{u}^{n+1}$ ainda é desconhecido, não podemos calcular, nesta etapa, $T^{n+1}$ por (5.2.4), o que será feito logo que seja possível.

Assim, sejam as aproximações discretas no tempo para (1.2.20) e (1.2.21), dadas por

$$
\frac{\mathbf{u}^{n+1}-\mathbf{u}^{n}}{\delta t}+\operatorname{conv}(\mathbf{u u})^{n+\zeta_{1}}+\nabla p^{n+1}=\frac{\beta}{R e}\left[(1-\nu) \nabla^{2} \mathbf{u}^{n}+\nu \nabla^{2} \mathbf{u}^{n+1}\right]+H(T)^{n+\zeta_{2}}+\frac{1}{F r^{2}} \mathbf{g}^{n+\zeta_{3}}
$$

$$
\nabla \cdot \mathbf{u}^{n+1}=0
$$

onde $\operatorname{conv}(\mathbf{u u})^{n+\zeta_{1}}, H(T)^{n+\zeta_{2}}$ e $\mathbf{g}^{n+\zeta_{3}}$ são aproximações explícitas para $\nabla \cdot(\mathbf{u u})$ em $t=t_{n+\zeta_{1}}, \nabla \cdot T$ em $t=t_{n+\zeta_{2}}$ e $\mathbf{g}$ em $t=t_{n+\zeta_{3}}$, respectivamente.

Três casos para $\nu \in[0,1]$ são considerados:

- FE-EE $(\nu=0)$ - método de Euler Explícito (EE), no qual $\zeta_{1}=\zeta_{2}=\zeta_{3}=0$ e

$$
H(T)^{n}=\nabla \cdot T^{n}
$$

- FI-EI $(\nu=1)$ - método de Euler Implícito (EI), onde tomamos $\zeta_{1}=0$ e $\zeta_{2}=\zeta_{3}=1$, sendo

$$
H(T)^{n+1}=\nabla \cdot \bar{T}^{n+1}
$$

- FI-CN $(\nu=0.5)$ - método de Crank-Nicholson $(\mathrm{CN})$, onde $\zeta_{1}=\zeta_{2}=\zeta_{3}=0.5$, sendo

$$
\operatorname{conv}(\mathbf{u u})^{n+\frac{1}{2}}=\frac{3}{2} \nabla \cdot(\mathbf{u u})^{n}-\frac{1}{2} \nabla \cdot(\mathbf{u u})^{n-1}
$$

uma aproximação obtida pelo método de Adams-Bashforth ([BU03]) inspirada em [AR97] e

$$
H(T)^{n+\frac{1}{2}}=\frac{1}{2}\left(\nabla \cdot \bar{T}^{n+1}+\nabla \cdot T^{n}\right)
$$

Definimos, então, a velocidade intermediária $\widetilde{\mathbf{u}}^{n+1}$ por

$$
\frac{\widetilde{\mathbf{u}}^{n+1}-\widetilde{\mathbf{u}}^{n}}{\delta t}+\operatorname{conv}(\mathbf{u u})^{n+\zeta_{1}}+\nabla \widetilde{p}^{n+1}=\frac{\beta}{R e}\left[(1-\nu) \nabla^{2} \widetilde{\mathbf{u}}^{n}+\nu \nabla^{2} \widetilde{\mathbf{u}}^{n+1}\right]+H(T)^{n+\zeta_{2}}+\frac{1}{F r^{2}} \mathbf{g}^{n+\zeta_{3}},
$$

$\operatorname{com} \widetilde{\mathbf{u}}^{n}=\mathbf{u}^{n}$ e $\widetilde{p}^{n+1}$ uma aproximação para $p^{n+1}$ que é escolhida como

$$
\widetilde{p}^{n+1}=p^{n}
$$

De (2.2.3), consideramos

$$
\mathbf{u}^{n+1}=\widetilde{\mathbf{u}}^{n+1}-\nabla \psi^{n+1},
$$

que, juntamente com $(5.2 .6)$, resulta em

$$
\nabla^{2} \psi^{n+1}=\nabla \cdot \widetilde{\mathbf{u}}^{n+1},
$$

cujas condições de contorno, segundo [TM93], em fronteiras rígidas e injetores é

$$
\frac{\partial \psi^{n+1}}{\partial \vec{n}}=0
$$

e, em ejetores continuados,

$$
\psi^{n+1}=0 .
$$

Uma vez calculado $\widetilde{\mathbf{u}}^{n+1}$ e $\psi^{n+1}$ resolvendo, respectivamente, (5.2.11) e (5.2.14), calculamos $\mathbf{u}^{n+1}$ por (5.2.13). Resta, porém, obtermos uma expressão para o cálculo de $p^{n+1}$, o que pode ser feito ([BR01]) 
substituindo (5.2.13) em (5.2.11) e comparando a expressão resultante com (5.2.5), de onde obtemos, então,

$$
p^{n+1}=\widetilde{p}^{n+1}+\frac{\psi^{n+1}}{\delta t}-\frac{\nu \beta}{R e} \nabla^{2} \psi^{n+1} .
$$

Finalmente, uma vez calculado $\mathbf{u}^{n+1}$ utilizamos (5.2.4) para obtermos $T^{n+1}$, encerrando um ciclo do GENSMAC-SXPP.

No entanto, com base em [GF03], utilizamos também uma segunda estratégia para a FE do GENSMACSXPP, que denominamos FE-RK22. Esta consiste em tomar $\nu=0$ em (5.2.17) e definir $\widetilde{\mathbf{u}}^{n+1}$ discretizando

$$
\frac{\partial \widetilde{\mathbf{u}}}{\partial t}=G(\mathbf{u}, T, \mathbf{g})
$$

com

$$
G(\mathbf{u}, T, \mathbf{g})=\frac{\beta}{R e} \nabla^{2} \mathbf{u}+H(T)+\frac{1}{F r^{2}} \mathbf{g}-\operatorname{conv}(\mathbf{u u})-\nabla \widetilde{p}^{n+1},
$$

pelo esquema Runge-Kutta de segunda ordem de precisão RK22 ([LB73], [BU03]), isto é,

$$
\begin{aligned}
& \frac{\widetilde{\mathbf{u}}^{n+\frac{1}{2}}-\widetilde{\mathbf{u}}^{n}}{\delta t}=G\left(\widetilde{\mathbf{u}}^{n}, T^{n}, \mathbf{g}^{n}\right), \\
& \frac{\widetilde{\mathbf{u}}^{n+1}-\widetilde{\mathbf{u}}^{n}}{\delta t}=G\left(\widetilde{\mathbf{u}}^{n+\frac{1}{2}}, T^{n+\frac{1}{2}}, \mathbf{g}^{n+\frac{1}{2}}\right),
\end{aligned}
$$

sendo

$$
H(T)^{n+\frac{1}{2}}=\frac{1}{2}\left(\nabla \cdot \bar{T}^{n+1}+\nabla \cdot T^{n}\right)
$$

e $\widetilde{\mathbf{u}}^{n}=\mathbf{u}^{n}$. Uma vez determinado $\widetilde{\mathbf{u}}^{n+1}$, o ciclo da FE-RK22 segue como nas demais estratégias apresentadas, calculando $\psi^{n+1}$ por (5.2.14) e, $\mathbf{u}^{n+1}$ por (5.2.13) e, por fim, $T^{n+1}$ por (5.2.4).

Portanto, supondo que os campos de velocidade solenoidal e de pressão, bem como a contribuição não-newtoniana e a posição da superfície livre sejam conhecidos em $t=t_{n}$, o ciclo computacional a ser executado pela FE do GENSMAC-SXPP a fim de calcular a solução em $t=t_{n}+\delta t=t_{n+1}$ é dado, simplificadamente, por:

Passo 1: cálculo de $\bar{T}^{n+1}$ através de (5.2.3).

Passo 2: cálculo de $\widetilde{\mathbf{u}}^{n+1}$ por (5.2.11) com $\nu=0$ para a FE-EE ou por (5.2.20)-(5.2.21) para a FE-RK22.

Passo 3: cálculo de $\psi^{n+1}$ pela solução da equação de Poisson (5.2.14) com a condição de contorno do tipo Dirichlet homogênea

$$
\psi^{n+1}=0
$$

ao longo da superfície livre, isto é, em células $\mathbf{S}$.

Passo 4: cálculo da velocidade $\mathbf{u}^{n+1}$ usando (5.2.13) no interior do domínio e as equações (1.3.16) e (5.2.6), discretizadas em $t=t_{n+1}$, como condição de contorno na superfície livre, isto é,

$$
\begin{aligned}
\frac{\beta}{R e}\left[2 n_{x} n_{y}\left(\frac{\partial v^{n+1}}{\partial y}-\frac{\partial u^{n+1}}{\partial x}\right)\right. & \left.+\left(n_{x}^{2}-n_{y}^{2}\right)\left(\frac{\partial u^{n+1}}{\partial y}+\frac{\partial v^{n+1}}{\partial x}\right)\right]=\ldots \\
\ldots & =n_{x} n_{y}\left(\left.\bar{T}^{x x}\right|^{n+1}-\left.\bar{T}^{y y}\right|^{n+1}\right)+\left.\left(n_{y}^{2}-n_{x}^{2}\right) \bar{T}^{x y}\right|^{n+1}
\end{aligned}
$$

e

$$
\frac{\partial u^{n+1}}{\partial x}+\frac{\partial v^{n+1}}{\partial y}=0
$$

Passo 5: cálculo de $T^{n+1}$ através de (5.2.4). 
Passo 6: cálculo da pressão $p^{n+1}$ usando (5.2.17) no interior do domínio, isto é,

$$
p^{n+1}=\widetilde{p}^{n+1}+\frac{\psi^{n+1}}{\delta t}
$$

em células $\mathbf{F}$, e a equação (1.3.15) na superfície livre, isto é,

$$
\begin{aligned}
& p^{n+1}=\frac{2 \beta}{R e}\left[n_{x}^{2} \frac{\partial u^{n+1}}{\partial x}+n_{x} n_{y}\left(\frac{\partial u^{n+1}}{\partial y}\right.\right.\left.\left.+\frac{\partial v^{n+1}}{\partial x}\right)+n_{y}^{2} \frac{\partial v^{n+1}}{\partial y}\right]+\ldots \\
& \ldots+\left.n_{x}^{2} T^{x x}\right|^{n+1}+\left.2 n_{x} n_{y} T^{x y}\right|^{n+1}+\left.n_{y}^{2} T^{y y}\right|^{n+1}
\end{aligned}
$$

em células $\mathbf{S}$.

Passo 7: movimento da superfície livre pelo cálculo da nova posição $\mathbf{x}^{n+1}$, a partir da posição anterior $\mathrm{x}^{n}$ das partículas que definem essa fronteira, resolvendo (2.1.2), ou seja,

$$
\frac{d \mathbf{x}}{d t}=\mathbf{u}(\mathbf{x}, t)
$$

por "Euler Explícito",

$$
\frac{\mathbf{x}^{n+1}-\mathbf{x}^{n}}{\delta t}=\mathbf{u}\left(\mathbf{x}^{n}, t_{n+1}\right)
$$

ou pelo RK21,

$$
\begin{aligned}
\frac{\overline{\mathbf{x}}-\mathbf{x}^{n}}{\delta t} & =\mathbf{u}\left(\mathbf{x}^{n}, t_{n}\right) \\
\frac{\mathbf{x}^{n+1}-\mathbf{x}^{n}}{\delta t} & =\frac{1}{2}\left[\mathbf{u}\left(\mathbf{x}^{n}, t_{n}\right)+\mathbf{u}\left(\overline{\mathbf{x}}, t_{n+1}\right)\right]
\end{aligned}
$$

No Passo 7, a velocidade na posição das partículas é aproximada no espaço por interpolação bi-linear, conforme a Observação 7 .

Analogamente ao GENSMAC, o GENSMAC-SXPP utiliza um passo temporal variável, de acordo com a restrição de estabilidade da $\mathrm{FE}$, gerando duas limitações para $\delta t$.

A primeira, imposta pelo tratamento dispensado ao termo não-linear da equação de conservação do momento, resulta no chamado $\delta t_{C F L}$, dado por

$$
\delta t_{C F L}=\min \left\{\delta t_{C F L x}, \delta t_{C F L y}\right\},
$$

onde

$$
\begin{aligned}
& \frac{\delta t_{C F L x} \operatorname{má} x\left\{\left|u^{n}\right|\right\}}{\delta x} \leqslant 1 \\
& \frac{\delta t_{C F L y} m a ́ x\left\{\left|v^{n}\right|\right\}}{\delta y} \leqslant 1
\end{aligned}
$$

expressam a condição CFL ([FL91]) ao longo das direções $x$ e $y$, respectivamente. Por outro lado, o caráter explícito de integração temporal da equação do momento faz com que seu termo viscoso ocasione a chamada restrição de estabilidade parabólica, determinando $\delta t_{v i s c}$, que definimos por

$$
\delta t_{v i s c}=\beta \frac{R e}{2}\left[\frac{1}{(\delta x)^{2}}+\frac{1}{(\delta y)^{2}}\right]^{-1}=\beta \frac{R e}{4}(\delta m)^{2} .
$$

Dessa forma, o passo temporal da FE é calculado como

$$
\delta t=F_{o} \min \left\{F_{v i s c} \delta t_{v i s c}, F_{C F L} \delta t_{C F L}\right\},
$$


onde $F_{o}, F_{v i s c}, F_{C F L}$ são fatores de controle do passo no tempo, tomados no intervalo ]0,1] para haver estabilidade.

Novamente, pelas equações (5.2.32) e (5.2.35), para $R e<<1$ ou para uma malha muito refinada,

$$
\delta t_{v i s c}=O\left[\operatorname{Re}(\delta m)^{2}\right]<<\delta t_{C F L}=O(\delta m),
$$

fazendo com que a FE exija passo temporal muito pequeno, escolhido por questão de estabilidade e não de precisão. Com isso, é natural esperar um elevado número de ciclos computacionais da FE nessas situações, acarretando um elevado tempo de processamento.

Assim, torna-se fundamental para o GENSMAC-SXPP o desenvolvimento de uma formulação implícita que elimine a restrição de estabilidade parabólica. Neste contexto, a primeira mudança com relação à $\mathrm{FE}$ ocorre na integração temporal da equação do momento, fazendo com que o Passo 2 seja alterado para:

Passo 2-FI: cálculo de $\widetilde{\mathbf{u}}^{n+1}$ de forma semi-implícita por (5.2.11), definindo a FI-EI $(\nu=1)$ ou a FI-CN $(\nu=0.5)$.

Na FI do GENSMAC-SXPP, o cálculo da pressão na superfície livre faz-se de forma implícita, com base na estratégia utilizada na FI do GENSMAC. Assim, para a FI, o Passo 3 e o Passo 6 da FE do GENSMAC-SXPP são alterados em:

Passo 3-FI: solução da equação de Poisson (5.2.14) com a condição de contorno para $\psi^{n+1}$ ao longo da superfície livre dada por (5.2.13), (1.3.15) e (5.2.17), isto é,

$$
\begin{gathered}
\mathbf{u}^{n+1}=\widetilde{\mathbf{u}}^{n+1}-\nabla \psi^{n+1}, \\
p^{n+1}=\left.n_{x}^{2} T^{x x}\right|^{n+1}+\left.2 n_{x} n_{y} T^{x y}\right|^{n+1}+\left.n_{y}^{2} T^{y y}\right|^{n+1}+\ldots \\
\ldots+\frac{2 \beta}{R e}\left[n_{x}^{2} \frac{\partial u^{n+1}}{\partial x}+n_{x} n_{y}\left(\frac{\partial u^{n+1}}{\partial y}+\frac{\partial v^{n+1}}{\partial x}\right)+n_{y}^{2} \frac{\partial v^{n+1}}{\partial y}\right],
\end{gathered}
$$

$\mathrm{e}$

$$
p^{n+1}=\widetilde{p}^{n+1}+\frac{\psi^{n+1}}{\delta t}-\frac{\nu \beta}{R e} \nabla^{2} \psi^{n+1}
$$

ou melhor,

$$
\begin{aligned}
& \widetilde{p}^{n+1}+\frac{\psi^{n+1}}{\delta t}- \frac{\nu \beta}{R e} \nabla^{2} \psi^{n+1}=\ldots \\
& \ldots=\frac{2 \beta}{R e}\left\{\frac{\partial}{\partial x}\left(\widetilde{u}^{n+1}-\frac{\partial \psi^{n+1}}{\partial x}\right) n_{x}^{2}+\frac{\partial}{\partial y}\left(\widetilde{v}^{n+1}-\frac{\partial \psi^{n+1}}{\partial y}\right) n_{y}^{2}+\ldots\right. \\
&\left.\ldots+\left[\frac{\partial}{\partial y}\left(\widetilde{u}^{n+1}-\frac{\partial \psi^{n+1}}{\partial x}\right)+\frac{\partial}{\partial x}\left(\widetilde{v}^{n+1}-\frac{\partial \psi^{n+1}}{\partial y}\right)\right] n_{x} n_{y}\right\}+\ldots \\
& \ldots+\left.n_{x}^{2} \bar{T}^{x x}\right|^{n+1}+\left.2 n_{x} n_{y} \bar{T}^{x y}\right|^{n+1}+\left.n_{y}^{2} \bar{T}^{y y}\right|^{n+1} .
\end{aligned}
$$

Passo 6-FI: cálculo da pressão $p^{n+1}$ por (5.2.17) tanto no interior do domínio quanto na superfície livre, isto é, em células $\mathbf{F}$ e $\mathbf{S}$; em células $\mathbf{E}$ adotamos, como condição de contorno,

$$
\psi^{n+1}=0 .
$$

Portanto, na FE calculamos explicitamente a pressão na superfície livre por (5.2.27), usando (5.2.23) como condição de contorno para a solução da equação de Poisson (5.2.14). Já na FI, é a própria condição de contorno para a pressão na superfície livre que é usada como condição de contorno na solução da equação de Poisson, resultando na equação (5.2.41) para $\psi^{n+1}$ em células $\mathbf{S}$. 
Dessa maneira, a FI do GENSMAC-SXPP é formada pelos Passos 1, 2-FI, 3-FI, 4, 5, 6-FI e 7, constituindo um algoritmo que elimina a restrição de estabilidade parabólica em escoamentos com superfície livre, possibilitando um aumento considerável no passo temporal e uma redução significativa do custo computacional, especialmente em escoamentos a baixos Reynolds.

Assim, torna-se possível usar $F_{v i s c}>1$ em (5.2.36), restando, em termos de estabilidade, apenas a restrição CFL para a FI. Portanto, devido à boa condição de estabilidade, a escolha de $\delta t$ na FI deve ser guiada, principalmente, pelo compromisso entre precisão e custo computacional.

No GENSMAC-SXPP, os Passos 2-FI, 3 e 3-FI envolvem sistemas lineares esparsos e de grande porte, os quais são resolvidos pelo BiCGstab-SOR ([SD00]).

Observação 8 A estratégia de integração temporal adotada para $T$ tem como objetivo eliminar seu uso com valores defasados no tempo em outras partes do ciclo computacional. Assim, se fosse adotada a mesma estratégia de cálculo de $T^{n+1}$ a partir de $T^{n}$ utilizada em [OC08B], teríamos esse problema nos Passos 2-FI e 3-FI. Da mesma forma, se na FE-RK22 fosse utilizada a mesma estratégia de marcha no tempo para $T$ feita em [GF03], tal problema surgiria no Passo 2.

Com efeito, consideramos $H(T)^{n+\zeta_{2}}$ uma aproximação explícita para $\nabla \cdot T$ em $t=t_{n+\zeta_{2}}$, onde $\zeta_{2}$ é escolhido de forma coerente com a discretização temporal da equação resolvida nos Passos 2 e 2-FI. Já nas estratégias utilizadas em [GF03] e [OCO8B] adota-se $\zeta_{2}=0$ no cálculo da velocidade intermediária pelo fato de, em seus ciclos computacionais, não existir ainda nenhuma aproximação para $T$ em $t=t_{n+1}$ quando do cálculo de $\widetilde{\mathbf{u}}^{n+1}$.

Pelo mesmo motivo, o termo envolvendo $T^{n+1}$ em (5.2.39) é aproximado com defasagem no tempo utilizando $T^{n}$, enquanto que, neste trabalho, utilizamos $\bar{T}^{n+1}$, resultando em (5.2.41) no Passo 3-FI.

O mesmo acontece no Passo 4, onde, sendo $T^{n+1}$ ainda desconhecido, utilizamos $\bar{T}^{n+1}$ em (5.2.24).

\subsection{Discretização das equações governantes}

\subsubsection{Introdução}

Uma vez delineada a estratégia de solução adotada pelo GENSMAC-SXPP, cuidamos, agora, da discretização espacial das equações discretas no tempo apresentadas na seção anterior, bem como da aplicação de suas condições de contorno.

Para isso, a exemplo do GENSMAC, consideramos uma malha espacial estruturada, cartesiana e deslocada, a qual é composta por células conforme ilustra a Figura 2.1.1. Assim, sendo $q=q(x, y, t)$ uma variável genérica que representa a solução exata da EDP considerada, denotamos por $q_{i_{j}}^{n}$ a solução aproximada, obtida pelo método numérico, para o valor exato $q\left(x_{i}, y_{j}, t_{n}\right)$.

Um aspecto importante na discretização das equações diz respeito ao tratamento espacial dispensado ao termo convectivo não-linear da equação de conservação do momento e da contribuição não-newtoniana. Por ser responsável por fenômenos importantes na solução, se não tratado corretamente pode ocasionar efeitos espúrios de natureza intrinsecamente numérica, como a dissipação e a dispersão ([FL91], [CM02], [FR01], [TO99], [SM85], [LE02]). Existe, na literatura, uma enorme variedade de esquemas convectivos, sem que haja consenso de qual o melhor. Assim, neste trabalho, consideramos os termos convectivos não-lineares discretizados no espaço pelo esquema CUBISTA ([AP03]).

Desse modo, adotaremos um tratamento genérico a estes termos, os quais não serão discretizados, mas, apenas, denotados por convx () e por convy (), conforme remetam, respectivamente, a derivadas parciais ao longo das direções $x$ e $y$, sendo o parâmetro dessas duas funções o mesmo que o da derivada parcial associada que define o termo convectivo.

Em escoamentos com superfícies livres é importante salientar que a aplicação das condições de contorno é feita duas vezes por ciclo computacional. Com efeito, o ciclo computacional que atualiza a solução de $t=t_{n}$ para $t=t_{n+1}$ pode ser particionado em três fases. Sem perda de generalidade, detalhes sobre essa questão no contexto do GENSMAC podem ser encontrados em [OL99].

Assim, em um primeiro momento, a solução e a posição da superfície livre estão definidas em $t=t_{n}$. Em um segundo momento, após resolver as equações nessa configuração de fluido, obtemos a solução das equações em $t=t_{n+1}$, mas, a fronteira livre ainda permanece na posição em $t=t_{n}$. Ocorre, 
então, a primeira aplicação das condições de contorno (baseada na solução calculada em $t=t_{n+1}$ ) e a movimentação da fronteira livre no domínio (que adquire seu perfil em $t=t_{n+1}$ ). Finalmente, no terceiro momento, após o movimento da superfície livre, temos um novo posicionamento do fluido no interior do domínio. Consequentemente, as células que não mais apresentam fluido têm as grandezas nelas definidas eliminadas e as condições de fronteira voltam a ser aplicadas para estabelecer a solução na fronteira espacial da nova configuração de fluido, permitindo que o próximo ciclo seja iniciado.

Com isso, nesta seção, sempre que não houver ambiguidade omitimos o índice temporal da variável na aplicação das condições de contorno, devendo ficar subentendido que as discretizações espaciais envolvem grandezas em um mesmo instante de tempo.

\subsubsection{Equação para a contribuição não-newtoniana}

Conforme a Figura 2.1.1 e mantendo a abordagem de trabalhos anteriores ([TM02A], [TM02B], [GF03], [DO03], [GR03], [SL05], [PL06], [SV07]), a contribuição não-newtoniana é avaliada no centro das células na malha deslocada. Portanto, a equação (5.2.1) deve ser discretizada no centro de células tipo $\mathbf{F}$ e $\mathbf{S}$. Considerando coordenadas cartesianas bidimensionais, segue de (1.2.25) que, em (5.2.1),

$$
\begin{aligned}
& F^{x x}(\mathbf{u}, T)=F^{x x}= 2\left(\frac{\partial u}{\partial x} T^{x x}+\frac{\partial u}{\partial y} T^{x y}\right)-\left[\frac{\partial\left(u T^{x x}\right)}{\partial x}+\frac{\partial\left(v T^{x x}\right)}{\partial y}\right]+\ldots \\
& \ldots+2 \xi \frac{\partial u}{\partial x}-\frac{1}{W e}\left\{f(\lambda, T) T^{x x}+\xi(f(\lambda, T)-1)+\frac{\alpha}{\xi}\left[\left(T^{x x}\right)^{2}+\left(T^{x y}\right)^{2}\right]\right\}, \\
& F^{y y}(\mathbf{u}, T)=F^{y y}= 2\left(\frac{\partial v}{\partial x} T^{x y}+\frac{\partial v}{\partial y} T^{y y}\right)-\left[\frac{\partial\left(u T^{y y}\right)}{\partial x}+\frac{\partial\left(v T^{y y}\right)}{\partial y}\right]+\ldots \\
& \ldots+2 \xi \frac{\partial v}{\partial y}-\frac{1}{W e}\left\{f(\lambda, T) T^{y y}+\xi(f(\lambda, T)-1)+\frac{\alpha}{\xi}\left[\left(T^{y y}\right)^{2}+\left(T^{x y}\right)^{2}\right]\right\}, \\
& F^{x y}(\mathbf{u}, T)=F^{x y}=\left(\frac{\partial v}{\partial x} T^{x x}+\frac{\partial u}{\partial y} T^{y y}\right)-\left[\frac{\partial\left(u T^{x y}\right)}{\partial x}+\frac{\partial\left(v T^{x y}\right)}{\partial y}\right]+\ldots \\
& \ldots+\xi\left(\frac{\partial u}{\partial y}+\frac{\partial v}{\partial x}\right)-\frac{1}{W e}\left\{f(\lambda, T) T^{x y}+\frac{\alpha}{\xi}\left[T^{x y}\left(T^{x x}+T^{y y}\right)\right]\right\},
\end{aligned}
$$

com, por (1.2.26),

$$
f(\lambda, T)=\frac{2}{\gamma}\left(1-\frac{1}{\lambda}\right) e^{Q_{o}(\lambda-1)}+\frac{1}{\lambda^{2}}\left\{1-\frac{\alpha}{3 \xi^{2}}\left[\left(T^{x x}\right)^{2}+2 T^{x y}+\left(T^{y y}\right)^{2}\right]\right\},
$$

e, por (1.2.27),

$$
\lambda=\sqrt{1+\frac{1}{3 \xi}\left|T^{x x}+T^{y y}\right|} .
$$

Portanto, as equações acima apresentadas são discretizadas no espaço por

$$
\begin{aligned}
f_{i, j} & =f\left(\lambda_{i, j}, T_{i, j}\right), \\
\lambda_{i, j} & =\sqrt{1+\frac{1}{3 \xi}\left|T^{x x}\right|_{i, j}+\left.T^{y y}\right|_{i, j} \mid},
\end{aligned}
$$

e

$$
\begin{aligned}
\left.F^{x x}\right|_{i, j}=2 & \left(\left.\left.\frac{\partial u}{\partial x}\right|_{i, j} T^{x x}\right|_{i, j}+\left.\left.\frac{\partial u}{\partial y}\right|_{i, j} T^{x y}\right|_{i, j}\right)-\left[\left.\operatorname{convx}\left(u T^{x x}\right)\right|_{i, j}+\left.\operatorname{convy}\left(v T^{x x}\right)\right|_{i, j}\right]+\ldots \\
& \ldots+\left.2 \xi \frac{\partial u}{\partial x}\right|_{i, j}-\frac{1}{W e}\left\{\left.f_{i, j} T^{x x}\right|_{i, j}+\xi\left(f_{i, j}-1\right)+\frac{\alpha}{\xi}\left[\left(\left.T^{x x}\right|_{i, j}\right)^{2}+\left(\left.T^{x y}\right|_{i, j}\right)^{2}\right]\right\},
\end{aligned}
$$




$$
\begin{aligned}
\left.F^{y y}\right|_{i, j}= & 2\left(\left.\left.\frac{\partial v}{\partial x}\right|_{i, j} T^{x y}\right|_{i, j}+\left.\left.\frac{\partial v}{\partial y}\right|_{i, j} T^{y y}\right|_{i, j}\right)-\left[\left.\operatorname{convx}\left(u T^{y y}\right)\right|_{i, j}+\left.\operatorname{convy}\left(v T^{y y}\right)\right|_{i, j}\right]+\ldots \\
& \ldots+\left.2 \xi \frac{\partial v}{\partial y}\right|_{i, j}-\frac{1}{W e}\left\{\left.f_{i, j} T^{y y}\right|_{i, j}+\xi\left(f_{i, j}-1\right)+\frac{\alpha}{\xi}\left[\left(\left.T^{y y}\right|_{i, j}\right)^{2}+\left(\left.T^{x y}\right|_{i, j}\right)^{2}\right]\right\}, \\
\left.F^{x y}\right|_{i, j}= & \left(\left.\left.\frac{\partial v}{\partial x}\right|_{i, j} T^{x x}\right|_{i, j}+\left.\left.\frac{\partial u}{\partial y}\right|_{i, j} T^{y y}\right|_{i, j}\right)-\left[\left.\operatorname{convx}\left(u T^{x y}\right)\right|_{i, j}+\left.\operatorname{convy}\left(v T^{x y}\right)\right|_{i, j}\right]+\ldots \\
& \ldots+\xi\left(\left.\frac{\partial u}{\partial y}\right|_{i, j}+\left.\frac{\partial v}{\partial x}\right|_{i, j}\right)-\frac{1}{W e}\left\{\left.f_{i, j} T^{x y}\right|_{i, j}+\frac{\alpha}{\xi}\left[\left.T^{x y}\right|_{i, j}\left(\left.T^{x x}\right|_{i, j}+\left.T^{y y}\right|_{i, j}\right)\right]\right\} .
\end{aligned}
$$

onde, mediante emprego de diferenças centradas de segunda ordem para as derivadas espaciais,

$$
\left.\frac{\partial u}{\partial x}\right|_{i, j}=\frac{u_{i+\frac{1}{2}, j}-u_{i-\frac{1}{2}, j}}{\delta x},\left.\frac{\partial v}{\partial y}\right|_{i, j}=\frac{v_{i, j+\frac{1}{2}}-v_{i, j-\frac{1}{2}}}{\delta y},
$$

e

$$
\left.\frac{\partial u}{\partial y}\right|_{i, j}=\frac{u_{i, j+\frac{1}{2}}-u_{i, j-\frac{1}{2}}}{\delta y},\left.\frac{\partial v}{\partial x}\right|_{i, j}=\frac{v_{i+\frac{1}{2}, j}-v_{i-\frac{1}{2}, j}}{\delta x},
$$

com as velocidades não definidas na malha obtidas via interpolação a partir dos valores mais próximos presentes na malha. Assim, seguindo [TM02A], utilizamos média aritmética para definir

$$
\begin{aligned}
& u_{i, j+\frac{1}{2}}=\frac{1}{4}\left(u_{i+\frac{1}{2}, j}+u_{i-\frac{1}{2}, j}+u_{i+\frac{1}{2}, j+1}+u_{i-\frac{1}{2}, j+1}\right), \\
& v_{i+\frac{1}{2}, j}=\frac{1}{4}\left(v_{i, j+\frac{1}{2}}+v_{i, j-\frac{1}{2}}+v_{i+1, j+\frac{1}{2}}+v_{i+1, j-\frac{1}{2}}\right) .
\end{aligned}
$$

Desse modo, esboçamos o procedimento numérico que aproxima a equação (5.2.1) em células $\mathbf{F}$ e $\mathbf{S}$. Porém, quando calculamos a equação do momento ou mesmo a equação que define $T$, faz-se necessário que a contribuição não-newtoniana esteja também definida em células $\mathbf{I}, \mathbf{O}, \mathbf{E}$, e $\mathbf{B}$. Em células $\mathbf{I}, \mathbf{O}$ e $\mathbf{E}$ seguimos a estratégia apresentada em [TM02A], discretizando convenientemente as condições de contorno para $T$ descritas na Seção 1.3.

Já nas células $\mathbf{B}$, o procedimento adotado consiste em aproximar $T$ na fronteira rígida, isto é, na face de $\mathbf{B}$ em contato com células $\mathbf{F}$ ou $\mathbf{S}$. A seguir, $T$ é definido no centro da célula $\mathbf{B}$ via extrapolação quadrática envolvendo o valor calculado na face e nas duas células vizinhas no interior do domínio.

Para isso, utilizamos as equações simplificadas para $T$ dadas na Observação 1 da Seção 1.3. Nestas, empregamos a estratégia de integração temporal pelo RK21 (5.2.3)-(5.2.4) análoga à aplicada em (5.2.1).

Assim, para uma fronteira rígida paralela ao eixo y e escrevendo (1.3.28)-(1.3.30) na forma (5.2.1),

$$
\begin{aligned}
F^{x x} & =-\frac{1}{W e}\left\{f(\lambda, T) T^{x x}+\xi(f(\lambda, T)-1)+\frac{\alpha}{\xi}\left[\left(T^{x x}\right)^{2}+\left(T^{x y}\right)^{2}\right]\right\}, \\
F^{y y} & =2 \frac{\partial v}{\partial x} T^{x y}-\frac{1}{W e}\left\{f(\lambda, T) T^{y y}+\xi(f(\lambda, T)-1)+\frac{\alpha}{\xi}\left[\left(T^{y y}\right)^{2}+\left(T^{x y}\right)^{2}\right]\right\}, \\
F^{x y} & =\frac{\partial v}{\partial x}\left(T^{x x}+\xi\right)-\frac{1}{W e}\left\{f(\lambda, T) T^{x y}+\frac{\alpha}{\xi}\left[T^{x y}\left(T^{x x}+T^{y y}\right)\right]\right\} .
\end{aligned}
$$

Pela Figura 5.3.1 (a), discretizando essas equações na face comum das células $C_{i, j}$ e $C_{i+1, j}$, obtemos

$$
\begin{aligned}
\left.F^{x x}\right|_{i+\frac{1}{2}, j} & =\ldots \\
\ldots & =-\frac{1}{W e}\left\{\left.f_{i+\frac{1}{2}, j} T^{x x}\right|_{i+\frac{1}{2}, j}+\xi\left(f_{i+\frac{1}{2}, j}-1\right)+\frac{\alpha}{\xi}\left[\left(\left.T^{x x}\right|_{i+\frac{1}{2}, j}\right)^{2}+\left(\left.T^{x y}\right|_{i+\frac{1}{2}, j}\right)^{2}\right]\right\}
\end{aligned}
$$


76

CAPÍTULO 5. O GENSMAC-SXPP

$$
\begin{aligned}
\left.F^{y y}\right|_{i+\frac{1}{2}, j} & =\left.\left.2 \frac{\partial v}{\partial x}\right|_{i+\frac{1}{2}, j} T^{x y}\right|_{i+\frac{1}{2}, j}-\ldots \\
\ldots & -\frac{1}{W e}\left\{\left.f_{i+\frac{1}{2}, j} T^{y y}\right|_{i+\frac{1}{2}, j}+\xi\left(f_{i+\frac{1}{2}, j}-1\right)+\frac{\alpha}{\xi}\left[\left(\left.T^{y y}\right|_{i+\frac{1}{2}, j}\right)^{2}+\left(\left.T^{x y}\right|_{i+\frac{1}{2}, j}\right)^{2}\right]\right\}, \\
\left.F^{x y}\right|_{i+\frac{1}{2}, j} & =\left.\frac{\partial v}{\partial x}\right|_{i+\frac{1}{2}, j}\left(\left.T^{x x}\right|_{i+\frac{1}{2}, j}+\xi\right)-\ldots \\
\ldots- & \frac{1}{W e}\left\{\left.f_{i+\frac{1}{2}, j} T^{x y}\right|_{i+\frac{1}{2}, j}+\frac{\alpha}{\xi}\left[\left.T^{x y}\right|_{i+\frac{1}{2}, j}\left(\left.T^{x x}\right|_{i+\frac{1}{2}, j}+\left.T^{y y}\right|_{i+\frac{1}{2}, j}\right)\right]\right\},
\end{aligned}
$$

com, por diferenças centrais de segunda ordem,

$$
\left.\frac{\partial v}{\partial x}\right|_{i+\frac{1}{2}, j}=\frac{v_{i+1, j}-v_{i, j}}{\delta x},
$$

onde,

$$
v_{i, j}=\frac{1}{2}\left(v_{i, j+\frac{1}{2}}+v_{i, j-\frac{1}{2}}\right) .
$$

Portanto, após o cálculo de $T_{i+\frac{1}{2}, j}$ na face da célula $\mathbf{B}$, definimos

$$
T_{i, j}=\frac{8}{3} T_{i+\frac{1}{2}, j}-2 T_{i+1, j}+\frac{1}{3} T_{i+2, j} .
$$

Da mesma forma, no caso de uma fronteira rígida paralela ao eixo $x$, colocando (1.3.22)-(1.3.24) na forma (5.2.1), obtemos

$$
\begin{aligned}
F^{x x} & =2 \frac{\partial u}{\partial y} T^{x y}-\frac{1}{W e}\left\{f(\lambda, T) T^{x x}+\xi(f(\lambda, T)-1)+\frac{\alpha}{\xi}\left[\left(T^{x x}\right)^{2}+\left(T^{x y}\right)^{2}\right]\right\}, \\
F^{y y} & =-\frac{1}{W e}\left\{f(\lambda, T) T^{y y}+\xi(f(\lambda, T)-1)+\frac{\alpha}{\xi}\left[\left(T^{y y}\right)^{2}+\left(T^{x y}\right)^{2}\right]\right\}, \\
F^{x y} & =\frac{\partial u}{\partial y}\left(T^{y y}+\xi\right)-\frac{1}{W e}\left\{f(\lambda, T) T^{x y}+\frac{\alpha}{\xi}\left[T^{x y}\left(T^{x x}+T^{y y}\right)\right]\right\} .
\end{aligned}
$$

Considerando a situação ilustrada na Figura 5.3.1 (b) e discretizando as equações acima na face comum das células $C_{i, j}$ e $C_{i, j-1}$,

$$
\begin{aligned}
&\left.F^{x x}\right|_{i, j-\frac{1}{2}}=\left.\left.2 \frac{\partial u}{\partial y}\right|_{i, j-\frac{1}{2}} T^{x y}\right|_{i, j-\frac{1}{2}}-\ldots \\
& \ldots-\frac{1}{W e}\left\{\left.f_{i, j-\frac{1}{2}} T^{x x}\right|_{i, j-\frac{1}{2}}+\xi\left(f_{i, j-\frac{1}{2}}-1\right)+\frac{\alpha}{\xi}\left[\left(\left.T^{x x}\right|_{i, j-\frac{1}{2}}\right)^{2}+\left(\left.T^{x y}\right|_{i, j-\frac{1}{2}}\right)^{2}\right]\right\}, \\
&\left.F^{y y}\right|_{i, j-\frac{1}{2}}=\ldots \ldots \\
& \ldots=-\frac{1}{W e}\left\{\left.f_{i, j-\frac{1}{2}} T^{y y}\right|_{i, j-\frac{1}{2}}+\xi\left(f_{i, j-\frac{1}{2}}-1\right)+\frac{\alpha}{\xi}\left[\left(\left.T^{y y}\right|_{i, j-\frac{1}{2}}\right)^{2}+\left(\left.T^{x y}\right|_{i, j-\frac{1}{2}}\right)^{2}\right]\right\}, \\
&\left.F^{x y}\right|_{i, j-\frac{1}{2}}=\left.\frac{\partial u}{\partial y}\right|_{i, j-\frac{1}{2}}\left(\left.T^{y y}\right|_{i, j-\frac{1}{2}}+\xi\right)-\ldots \\
& \quad \ldots-\frac{1}{W e}\left\{\left.f_{i, j-\frac{1}{2}} T^{x y}\right|_{i, j-\frac{1}{2}}+\frac{\alpha}{\xi}\left[\left.T^{x y}\right|_{i, j-\frac{1}{2}}\left(\left.T^{x x}\right|_{i, j-\frac{1}{2}}+\left.T^{y y}\right|_{i, j-\frac{1}{2}}\right)\right]\right\},
\end{aligned}
$$


com, por diferenças centrais de segunda ordem,

$$
\left.\frac{\partial u}{\partial y}\right|_{i, j-\frac{1}{2}}=\frac{u_{i, j}-u_{i, j-1}}{\delta y}
$$

onde,

$$
u_{i, j}=\frac{1}{2}\left(u_{i+\frac{1}{2}, j}+u_{i-\frac{1}{2}, j}\right) .
$$

Assim, calculado $T_{i, j-\frac{1}{2}}$ na face da célula $\mathbf{B}$, definimos

$$
T_{i, j}=\frac{8}{3} T_{i, j-\frac{1}{2}}-2 T_{i, j-1}+\frac{1}{3} T_{i, j-2} .
$$

Por outro lado, na Figura 5.3.1 (c), temos uma quina na fronteira rígida, a qual causa uma mudança brusca na orientação do contorno. Neste caso, calculamos $T$ nas duas faces de $\mathbf{B}$ em contato com célula $\mathbf{F}$ ou $\mathbf{S}$ independentemente e, usando interpolação bidirecional, obtemos a aproximação

$$
T_{i, j}=\frac{1}{2}\left[\left(\frac{8}{3} T_{i+\frac{1}{2}, j}-2 T_{i+1, j}+\frac{1}{3} T_{i+2, j}\right)+\left(\frac{8}{3} T_{i, j-\frac{1}{2}}-2 T_{i, j-1}+\frac{1}{3} T_{i, j-2}\right)\right] .
$$

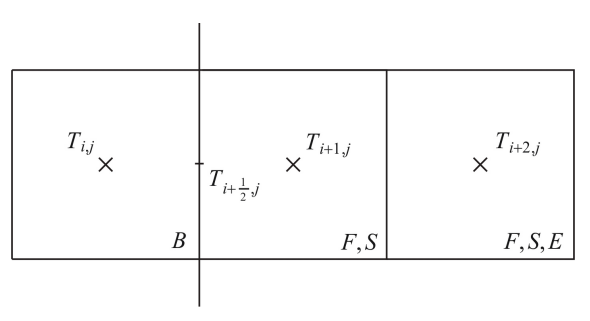

(a)

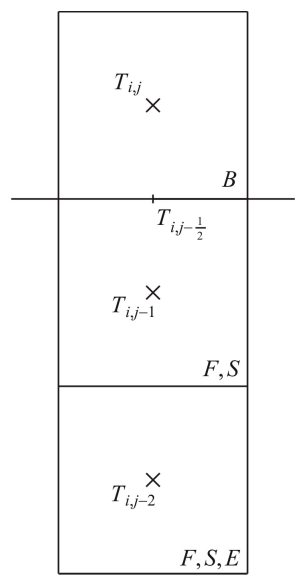

(b)

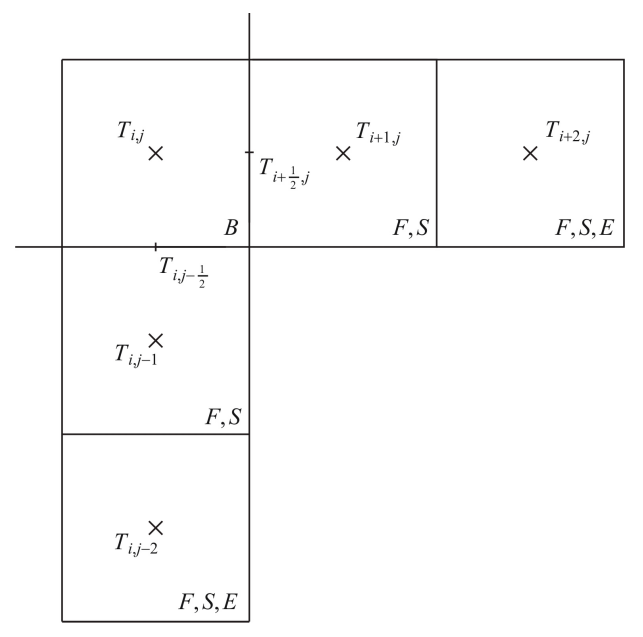

(c)

Figura 5.3.1: Ilustração de fronteira rígida paralela ao eixo $y$ (a), paralela ao eixo $x$ (b) e com mudança brusca na orientação (c).

Assim, os casos base para o cálculo de $T$ no contorno rígido foram apresentados considerando a Figura 5.3.1, sendo as demais situações análogas.

Por fim, é válido observar que a simulação de um escoamento envolvendo um fluido Oldroyd-B pelo GENSMAC-SXPP utiliza uma estratégia diferente da utilizada em [TM02A], [TM02B] e [GF03] para o cálculo da contribuição não-newtoniana em células $\mathbf{F}, \mathbf{S}$ e $\mathbf{B}$. Isto se dá, basicamente, por três motivos:

- Enquanto utilizamos o RK21 para a integração temporal da contribuição não-newtoniana, [TM02A] e [TM02B] utilizam Euler Explícito e, [GF03], o RK22.

- Em [TM02A], [TM02B] e [GF03] é realizada uma mudança de variável nas equações simplificadas que definem a contribuição não-newtoniana do modelo Oldroyd-B nos contornos rígidos, tornandoas mais simples. Para o modelo SXPP, as não-linearidades que não estão presentes no modelo Oldroyd-B tornam tal tratamento inviável. Assim, o GENSMAC-SXPP resolve diretamente as equações (1.3.22)-(1.3.24) e (1.3.28)-(1.3.30) simplificadas para $T$ nos contornos rígidos.

- O GENSMAC-SXPP utiliza extrapolação quadrática para definir $T$ em células $\mathbf{B}$, enquanto os trabalhos anteriores usam extrapolação linear. 


\subsubsection{Equações de conservação}

Pelo conceito de malha deslocada, as componentes da velocidade são discretizadas nas faces das células. Isso possibilita o uso de diferenças centradas de segunda ordem para aproximar as derivadas espaciais presentes nas equações de conservação (exceto o termo convectivo, que precisa de tratamento especial).

Assim, a equação da continuidade (1.2.21) discretizada no centro de uma célula $C_{i, j}$ resulta em

$$
\frac{u_{i+\frac{1}{2}, j}-u_{i-\frac{1}{2}, j}}{\delta x}+\frac{v_{i, j+\frac{1}{2}}-v_{i, j-\frac{1}{2}}}{\delta y}=0 .
$$

Por sua vez, pela estratégia de solução utilizada no GENSMAC-SXPP, a equação de conservação do momento é utilizada para calcular um campo de velocidade intermediário $\widetilde{\mathbf{u}}$. Neste processo, apesar das quatro estratégias de integração temporal discutidas na Seção 5.2, a discretização espacial das derivadas espaciais não muda, seguindo as idéias apresentadas em [TM02A], [TM02B] e [GF03].

Assim, sem perda de generalidade, segue que as componentes de (5.2.19) ao longo das direções $x$ e $y$ são, respectivamente, $G^{x}$ e $G^{y}$, com

$$
\begin{array}{r}
G^{x}=\frac{\beta}{R e}\left(\frac{\partial^{2} u}{\partial x^{2}}+\frac{\partial^{2} u}{\partial y^{2}}\right)+\left(\frac{\partial T^{x x}}{\partial x}+\frac{\partial T^{x y}}{\partial y}\right)+\ldots \\
\ldots+\frac{1}{F r^{2}} g^{x}-\left[\operatorname{convx}\left(u^{2}\right)+\operatorname{convy}(v u)+\frac{\partial \widetilde{p}^{n+1}}{\partial x}\right], \\
G^{y}=\frac{\beta}{R e}\left(\frac{\partial^{2} v}{\partial x^{2}}+\frac{\partial^{2} v}{\partial y^{2}}\right)+\left(\frac{\partial T^{x y}}{\partial x}+\frac{\partial T^{y y}}{\partial y}\right)+\ldots \\
\ldots+\frac{1}{F r^{2}} g^{y}-\left[\operatorname{conv} x(u v)+\operatorname{convy}\left(v^{2}\right)+\frac{\partial \widetilde{p}^{n+1}}{\partial y}\right] .
\end{array}
$$

Como o GENSMAC, o GENSMAC-SXPP aplica a equação do momento nas faces comuns de células com fluido, isto é, em faces de células $\mathbf{F}$ ou $\mathbf{S}$ com células $\mathbf{F}$ ou $\mathbf{S}$ (ou, equivalentemente, nas interfaces $\mathbf{F}|\mathbf{F}, \mathbf{F}| \mathbf{S}$ e $\mathbf{S} \mid \mathbf{S})$. Portanto, discretizando a equação (5.3.35) na face comum de $C_{i, j} \operatorname{com} C_{i+1, j}$, obtemos

$$
\begin{aligned}
\left.G^{x}\right|_{i+\frac{1}{2}, j}=\frac{\beta}{R e}\left[\frac{u_{i+\frac{3}{2}, j}-2 u_{i+\frac{1}{2}, j}+u_{i-\frac{1}{2}, j}}{(\delta x)^{2}}+\frac{u_{i+\frac{1}{2}, j+1}-2 u_{i+\frac{1}{2}, j}+u_{i+\frac{1}{2}, j-1}}{(\delta y)^{2}}\right]+\ldots \\
\quad \ldots+\left(\frac{\left.T^{x x}\right|_{i+1, j}-\left.T^{x x}\right|_{i, j}}{\delta x}+\frac{\left.T^{x y}\right|_{i+\frac{1}{2}, j+\frac{1}{2}}-\left.T^{x y}\right|_{i+\frac{1}{2}, j-\frac{1}{2}}}{\delta y}\right)+\ldots \\
\ldots+\left.\frac{1}{F r^{2}} g^{x}\right|_{i+\frac{1}{2}, j}+-\left[\left.\operatorname{convx}\left(u^{2}\right)\right|_{i+\frac{1}{2}, j}+\left.\operatorname{convy}(v u)\right|_{i+\frac{1}{2}, j}+\frac{\widetilde{p}_{i+1, j}^{n+1}-\widetilde{p}_{i, j}^{n+1}}{\delta x}\right] .
\end{aligned}
$$

Do mesmo modo, discretizando (5.3.36) na face comum entre as células $C_{i, j}$ e $C_{i, j+1}$,

$$
\begin{aligned}
\left.G^{y}\right|_{i, j+\frac{1}{2}}=\frac{\beta}{R e}[ & \left.\frac{v_{i+1, j+\frac{1}{2}}-2 v_{i, j+\frac{1}{2}}+v_{i-1, j+\frac{1}{2}}}{(\delta x)^{2}}+\frac{v_{i, j+\frac{3}{2}}-2 v_{i, j+\frac{1}{2}}+v_{i, j-\frac{1}{2}}}{(\delta y)^{2}}\right]+\ldots \\
& \ldots+\left(\frac{\left.T^{x y}\right|_{i+\frac{1}{2}, j+\frac{1}{2}}-\left.T^{x y}\right|_{i-\frac{1}{2}, j+\frac{1}{2}}}{\delta x}+\frac{\left.T^{y y}\right|_{i, j+1}-\left.T^{y y}\right|_{i, j}}{\delta y}\right)+\ldots \\
& \ldots+\left.\frac{1}{F r^{2}} g^{y}\right|_{i, j+\frac{1}{2}}-\left[\left.\operatorname{convx}(u v)\right|_{i, j+\frac{1}{2}}+\left.\operatorname{convy}\left(v^{2}\right)\right|_{i, j+\frac{1}{2}}+\frac{\widetilde{p}_{i, j+1}^{n+1}-\widetilde{p}_{i, j}^{n+1}}{\delta y}\right] .
\end{aligned}
$$

Em ambos os casos, precisamos de uma aproximação para $T^{x y}$ nos extremos da face onde a equação é discretizada. Não estando $T$ definido nestas posições, mas, apenas no centro de cada célula, tais valores 
são obtidos, conforme [TM02A], através de média aritmética dos valores de $T^{x y}$ associados às quatro células que compartilham o mesmo vértice, ou seja,

$$
\left.T^{x y}\right|_{i+\frac{1}{2}, j+\frac{1}{2}}=\frac{1}{4}\left(\left.T^{x y}\right|_{i+1, j+1}+\left.T^{x y}\right|_{i+1, j}+\left.T^{x y}\right|_{i, j}+\left.T^{x y}\right|_{i, j+1}\right) .
$$

\subsubsection{Condições de fronteira para a velocidade intermediária}

A solução da equação do momento para a velocidade intermediária necessita que condições de contorno sejam especificadas para $\widetilde{\mathbf{u}}$, o que tem influência direta na precisão e na estabilidade da solução obtida, conforme concluem [BR01] e [OC08A], respectivamente.

Assim, para a FE-EE, o cálculo de $\widetilde{\mathbf{u}}^{n+1}$ por (5.2.11) necessita apenas da condição auxiliar

$$
\widetilde{\mathbf{u}}^{n}=\mathbf{u}^{n},
$$

conforme apresentam [TM94], [TM02A] e [TM02B]. Perceba que (5.3.40) não cria maiores dificuldades, uma vez que a solução em $t=t_{n}$ é conhecida desde o início do ciclo computacional.

Já na FE-RK22, além de (5.3.40), considera-se, conforme [GF03],

$$
\widetilde{\mathbf{u}}^{n+\frac{1}{2}}=\mathbf{u}^{n}
$$

nas extremidades do domínio, onde a equação (5.2.20)-(5.2.21) não pode ser aplicada.

Por sua vez, a FI merece atenção especial na aplicação das condições de contorno, justificando estudo apresentado em [OC08B] no contexto do GENSMAC. Isto se deve, basicamente, ao fato da resolução semi-implícita de (5.2.11) utilizar não apenas (5.3.40), mas, também necessitar de $\widetilde{\mathbf{u}}^{n+1}$ como condição de contorno na fronteira do domínio. Em fronteiras rígidas no-slip e em injetores, onde a condição de contorno para a velocidade é do tipo Dirichlet, isto é contornado por

$$
\widetilde{\mathbf{u}}^{n+1}=\mathbf{u}^{n+1},
$$

dando origem às chamadas condições de contorno implícitas, discutidas em [OC08B] e utilizadas no GENSMAC-SXPP. Por outro lado, a discretização semi-implícita de (5.2.11) próxima à superfície livre exige $\widetilde{\mathbf{u}}^{n+1}$ em interfaces $\mathbf{E} \mid \mathbf{E}$ (adjacente a alguma interface $\mathbf{S} \mid \mathbf{S}$ ) e em interfaces $\mathbf{S} \mid \mathbf{E}$. Neste caso, conforme [OC06], tomamos

$$
\widetilde{\mathbf{u}}^{n+1}=\mathbf{u}^{n}
$$

como condição de fronteira na superfície livre, sendo a condição de contorno para $\mathbf{u}$ calculada conforme apresentamos na Seção 5.3.7. Da mesma forma, sempre que (5.2.11) exige $\widetilde{\mathbf{u}}^{n+1}$ em alguma face de célula tipo $\mathbf{O}$, empregamos

$$
\widetilde{\mathbf{u}}^{n+1}=\mathbf{u}^{n} .
$$

Por fim, independentemente da formulação adotada, uma vez calculado $\widetilde{\mathbf{u}}^{n+1}$ nas interfaces $\mathbf{F}|\mathbf{F}, \mathbf{F}| \mathbf{S}$ e $\mathbf{S} \mid \mathbf{S}$ com as condições de contorno acima exibidas, faz-se necessário definir a componente normal de $\widetilde{\mathbf{u}}^{n+1}$ nas faces de célula tipo $\mathbf{O}$ em contato com fluido (isto é, nas interfaces $\mathbf{O} \mid \mathbf{F}$ e $\mathbf{O} \mid \mathbf{S}$ ) através da equação (1.3.9). O GENSMAC, entretanto, considera tal condição na forma

$$
\frac{\partial \widetilde{\mathbf{u}}_{\vec{n}}}{\partial \vec{n}}=0
$$

conforme detalhado em [OL99]. Portanto, sem perda de generalidade, existem duas situações a considerar, conforme ilustra a Figura 5.3.2, onde, admitindo que $\vec{n}$ aponta "para fora" da porção de fluido, discretizamos (5.3.45) por diferença regressiva de primeira ordem, conforme [OL99].

Assim, na Figura 5.3.2 (a) temos uma interface de ejeção continuada paralela ao eixo $y$, onde, tomando

$$
\frac{\widetilde{u}_{i-\frac{1}{2}, j}^{n+1}-\widetilde{u}_{i+\frac{1}{2}, j}^{n+1}}{\delta x}=0
$$

obtemos

$$
\widetilde{u}_{i-\frac{1}{2}, j}^{n+1}=\widetilde{u}_{i+\frac{1}{2}, j}^{n+1}
$$


Da mesma forma, na Figura 5.3.2 (b) a interface de ejeção continuada é paralela ao eixo $x$. Assim, a partir de

$$
\frac{\tilde{v}_{i, j-\frac{1}{2}}^{n+1}-\tilde{v}_{i, j+\frac{1}{2}}^{n+1}}{\delta y}=0
$$

conseguimos

$$
\tilde{v}_{i, j-\frac{1}{2}}^{n+1}=\tilde{v}_{i, j+\frac{1}{2}}^{n+1}
$$

(a)

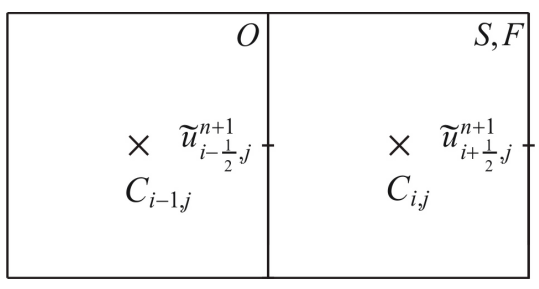

(b)

\begin{tabular}{|ll|}
\hline$S, F$ & $\widetilde{v}_{i, j+\frac{1}{2}}^{n+1}$ \\
& $\times$ \\
& $C_{i, j}$ \\
& \\
\hline$O$ & $\widetilde{v}_{i, j-\frac{1}{2}}^{n+1}$ \\
& \\
& $\times$ \\
& $C_{i, j-1}$ \\
\end{tabular}

Figura 5.3.2: Ilustração de uma célula de ejetores continuados paralelos ao eixo $y$ (a) e ao eixo $x$ (b).

\subsubsection{Cálculo do campo de pressão e da velocidade solenoidal}

Inicialmente, consideramos em (5.2.12)

$$
\widetilde{p}_{i, j}^{n+1}=p_{i, j}^{n}
$$

para toda célula $C_{i, j}$ classificada em $t=t_{n}$ como $\mathbf{F}$ ou $\mathbf{S}$. Como o campo de pressão tentativo não necessariamente é o correto campo de pressão, isto é, em geral

$$
\widetilde{p}_{i, j}^{n+1} \neq p_{i, j}^{n+1},
$$

segue que a velocidade intermediária $\widetilde{\mathbf{u}}$ calculada não necessariamente é solenoidal. Assim, a correção do campo de velocidade intermediário para o campo de velocidade solenoidal dá-se fazendo

$$
\begin{aligned}
& u_{i+\frac{1}{2}, j}^{n+1}=\widetilde{u}_{i+\frac{1}{2}, j}^{n+1}-\frac{\psi_{i+1, j}^{n+1}-\psi_{i, j}^{n+1}}{\delta x} \\
& v_{i, j+\frac{1}{2}}^{n+1}=\widetilde{v}_{i, j+\frac{1}{2}}^{n+1}-\frac{\psi_{i, j+1}^{n+1}-\psi_{i, j}^{n+1}}{\delta x}
\end{aligned}
$$

em toda interface $\mathbf{F}|\mathbf{F}, \mathbf{F}| \mathbf{S}, \mathbf{S}|\mathbf{S}, \mathbf{O}| \mathbf{F}$ e $\mathbf{O} \mid \mathbf{S}$. Novamente, empregou-se diferença centrada de segunda ordem para a aproximação do gradiente de $\psi$, possibilitando obter (5.3.52) e (5.3.53) a partir de (5.2.13).

Por sua vez, o cálculo do campo de pressão atualizado faz-se a partir de (5.2.17), onde utilizamos o laplaciano discreto de cinco pontos para obter

$$
p_{i, j}^{n+1}=\widetilde{p}_{i, j}^{n+1}+\frac{\psi_{i, j}^{n+1}}{\delta t}-\frac{\nu \beta}{R e}\left[\frac{\psi_{i+1, j}^{n+1}-2 \psi_{i, j}^{n+1}+\psi_{i-1, j}^{n+1}}{(\delta x)^{2}}+\frac{\psi_{i, j+1}^{n+1}-2 \psi_{i, j}^{n+1}+\psi_{i, j-1}^{n+1}}{(\delta y)^{2}}\right] .
$$

Conforme já mencionado, a equação (5.3.54) é empregada de forma distinta por cada formulação do GENSMAC-SXPP. Assim, a FI utiliza (5.3.54) em toda célula $C_{i, j}$ tipo $\mathbf{F}$ ou $\mathbf{S}$, pois, a condição de contorno (1.3.16) para a pressão na superfície livre já foi aplicada de forma implícita no cálculo de $\psi$ nas células S. Por outro lado, a FE calcula explicitamente a pressão na superfície livre (conforme mostrado na Seção 5.3.8), e assim, avalia (5.3.54) apenas em células $C_{i, j}$ tipo $\mathbf{F}$. 


\subsubsection{Equação de Poisson para o potencial auxiliar}

A estratégia de solução adotada pelo GENSMAC-SXPP exige o cálculo do potencial auxiliar $\psi$, que força a conservação da massa no campo de velocidade calculado por (5.3.52) e (5.3.53).

Assim, sendo $C_{i, j}$ uma célula $\mathbf{F}$ e discretizando as derivadas espaciais de (5.2.14) por diferenças centradas de segunda ordem, obtemos

$$
\frac{\psi_{i+1, j}^{n+1}-2 \psi_{i, j}^{n+1}+\psi_{i-1, j}^{n+1}}{(\delta x)^{2}}+\frac{\psi_{i, j+1}^{n+1}-2 \psi_{i, j}^{n+1}+\psi_{i, j-1}^{n+1}}{(\delta y)^{2}}=\frac{\widetilde{u}_{i+\frac{1}{2}, j}^{n+1}-\widetilde{u}_{i-\frac{1}{2}, j}^{n+1}}{\delta x}+\frac{\widetilde{v}_{i, j+\frac{1}{2}}^{n+1}-\widetilde{v}_{i, j-\frac{1}{2}}^{n+1}}{\delta y} .
$$

Por outro lado, a condição de contorno (5.2.15) deve ser aplicada nas faces de células $\mathbf{F}$ ou $\mathbf{S}$ com células $\mathbf{B}$ ou $\mathbf{I}$, (isto é, nas interfaces $\mathbf{F}|\mathbf{B}, \mathbf{F}| \mathbf{I}, \mathbf{S}|\mathbf{B}, \mathbf{S}| \mathbf{I}$ ). Assim, supondo que a célula $C_{i, j}$ seja tipo $\mathbf{F}$ ou $\mathbf{S}$ e usando diferença centrada de segunda ordem para discretizar (5.2.15), devemos considerar quatro casos (supondo $\vec{n}$ orientado "para fora" do fluido):

- $C_{i+1, j}$ é tipo $\mathbf{B}$ ou $\mathbf{I}$ : então, tomamos

$$
\frac{\psi_{i+1, j}^{n+1}-\psi_{i, j}^{n+1}}{\delta x}=0 \Rightarrow \psi_{i, j}^{n+1}=\psi_{i+1, j}^{n+1}
$$

- $C_{i-1, j}$ é tipo B ou I: então, tomamos

$$
\frac{\psi_{i-1, j}^{n+1}-\psi_{i, j}^{n+1}}{\delta x}=0 \Rightarrow \psi_{i, j}^{n+1}=\psi_{i-1, j}^{n+1}
$$

- $C_{i, j+1}$ é tipo $\mathbf{B}$ ou $\mathbf{I}$ : então, tomamos

$$
\frac{\psi_{i, j}^{n+1}-\psi_{i, j+1}^{n+1}}{\delta y}=0 \Rightarrow \psi_{i, j}^{n+1}=\psi_{i, j+1}^{n+1}
$$

- $C_{i, j-1}$ é tipo $\mathbf{B}$ ou $\mathbf{I}$ : então, tomamos

$$
\frac{\psi_{i, j-1}^{n+1}-\psi_{i, j}^{n+1}}{\delta y}=0 \Rightarrow \psi_{i, j}^{n+1}=\psi_{i, j-1}^{n+1} .
$$

Em ejetores continuados, a condição de contorno do tipo Dirichlet homogênea, dada em (5.2.16), consiste em tomar, para cada célula $C_{i, j}$ tipo $\mathbf{O}$,

$$
\psi_{i, j}^{n+1}=0 .
$$

Portanto, para células $\mathbf{F}, \mathbf{B}, \mathbf{I}$ e $\mathbf{O}$, o cálculo de $\psi$ é idêntico para ambas as formulações do GENSMACSXPP. No entanto, para células $\mathbf{S}$, existe distinção no procedimento de cálculo de $\psi$ pelas formulações explícita e implícita, conforme observado na Seção 5.2.

Assim, a FE do GENSMAC-SXPP utiliza condição de Dirichlet homogênea para $\psi$, isto é,

$$
\psi_{i, j}^{n+1}=0
$$

em cada célula $C_{i, j}$ tipo $\mathbf{S}$, não sendo necessário definir $\psi$ em células do tipo $\mathbf{E}$.

Por outro lado, a FI não impõe, mas, calcula $\psi$ na superfície livre aplicando a equação (5.2.41) em cada célula $C_{i, j}$ tipo $\mathbf{S}$, definindo $\vec{n}$ como ilustrado nas Figuras 2.1.3 e 2.1.4. Nas células $\mathbf{S}$ onde não é possível definir $\vec{n}$, adotamos

$$
\psi_{i, j}^{n+1}=0
$$

bem como nas células tipo $\mathbf{E}$.

Para ilustrar como (5.2.41) é construída, discretizada e aplicada, consideraremos dois casos para $\vec{n}$, sendo as demais situações análogas. 
Desse modo, sendo

$$
\vec{n}=\left[\begin{array}{l}
0 \\
1
\end{array}\right]
$$

a equação (5.2.39) reduz-se a

$$
p^{n+1}=\frac{2 \beta}{R e} \frac{\partial v^{n+1}}{\partial y}+\left.\bar{T}^{y y}\right|^{n+1},
$$

de onde, usando (5.2.6) e (5.2.17), obtemos

$$
\widetilde{p}^{n+1}+\frac{\psi^{n+1}}{\delta t}-\frac{\nu \beta}{R e} \nabla^{2} \psi^{n+1}=-\frac{2 \beta}{R e} \frac{\partial u^{n+1}}{\partial x}+\left.\bar{T}^{y y}\right|^{n+1} .
$$

Por fim, segue de (5.2.13) que

$$
\widetilde{p}^{n+1}+\frac{\psi^{n+1}}{\delta t}-\frac{\nu \beta}{R e} \nabla^{2} \psi^{n+1}=-\frac{2 \beta}{R e}\left(\frac{\partial \widetilde{u}^{n+1}}{\partial x}-\frac{\partial^{2} \psi^{n+1}}{\partial x^{2}}\right)+\left.\bar{T}^{y y}\right|^{n+1},
$$

cuja discretização espacial, por diferenças centradas de segunda ordem,

$$
\begin{gathered}
\widetilde{p}_{i, j}^{n+1}+\frac{\psi_{i, j}^{n+1}}{\delta t}-\frac{\nu \beta}{R e}\left[\frac{\psi_{i-1, j}^{n+1}-2 \psi_{i, j}^{n+1}+\psi_{i+1, j}^{n+1}}{(\delta x)^{2}}+\frac{\psi_{i, j-1}^{n+1}-2 \psi_{i, j}^{n+1}+\psi_{i, j+1}^{n+1}}{(\delta y)^{2}}\right]=\ldots \\
\ldots=-\frac{2 \beta}{R e}\left[\frac{\widetilde{u}_{i+\frac{1}{2}, j}^{n+1}-\widetilde{u}_{i-\frac{1}{2}, j}^{n+1}}{\delta x}-\frac{\psi_{i-1, j}^{n+1}-2 \psi_{i, j}^{n+1}+\psi_{i+1, j}^{n+1}}{(\delta x)^{2}}\right]+\left.\bar{T}^{y y}\right|_{i, j} ^{n+1} .
\end{gathered}
$$

De modo semelhante, quando

$$
\vec{n}=\left[\begin{array}{c}
\frac{\sqrt{2}}{2} \\
\frac{\sqrt{2}}{2}
\end{array}\right]
$$

segue de (5.2.39), usando (5.2.6),

$$
p^{n+1}=\frac{\beta}{R e}\left(\frac{\partial u^{n+1}}{\partial y}+\frac{\partial v^{n+1}}{\partial x}\right)+\frac{1}{2}\left(\left.\bar{T}^{x x}\right|^{n+1}+\left.2 \bar{T}^{x y}\right|^{n+1}+\left.\bar{T}^{y y}\right|^{n+1}\right),
$$

que, através de (5.2.17) e de (5.2.13), resulta em

$$
\begin{aligned}
\widetilde{p}^{n+1}+\frac{\psi^{n+1}}{\delta t} & -\frac{\nu \beta}{R e} \nabla^{2} \psi^{n+1}=\ldots \\
\ldots & =\frac{\beta}{R e}\left(\frac{\partial \widetilde{u}^{n+1}}{\partial y}+\frac{\partial \widetilde{v}^{n+1}}{\partial x}-2 \frac{\partial^{2} \psi^{n+1}}{\partial y \partial x}\right)+\frac{1}{2}\left(\left.\bar{T}^{x x}\right|^{n+1}+\left.2 \bar{T}^{x y}\right|^{n+1}+\left.\bar{T}^{y y}\right|^{n+1}\right)
\end{aligned}
$$

cuja discretização espacial é dada por

$$
\begin{gathered}
\widetilde{p}_{i, j}^{n+1}+\frac{\psi_{i, j}^{n+1}}{\delta t}-\frac{\nu \beta}{R e}\left[\frac{\psi_{i-1, j}^{n+1}-2 \psi_{i, j}^{n+1}+\psi_{i+1, j}^{n+1}}{(\delta x)^{2}}+\frac{\psi_{i, j-1}^{n+1}-2 \psi_{i, j}^{n+1}+\psi_{i, j+1}^{n+1}}{(\delta y)^{2}}\right]=\ldots \\
\ldots=\frac{\beta}{R e}\left(\left.\frac{\partial \widetilde{u}}{\partial y}\right|_{i, j-\frac{1}{2}} ^{n+1}+\left.\frac{\partial \widetilde{v}}{\partial x}\right|_{i-\frac{1}{2}, j} ^{n+1}-\left.2 \frac{\partial^{2} \psi}{\partial x \partial y}\right|_{i-\frac{1}{2}, j-\frac{1}{2}} ^{n+1}\right)+\ldots \\
\ldots+\frac{1}{2}\left(\left.\bar{T}^{x x}\right|_{i, j} ^{n+1}+\left.2 \bar{T}^{x y}\right|_{i, j} ^{n+1}+\left.\bar{T}^{y y}\right|_{i, j} ^{n+1}\right),
\end{gathered}
$$


onde, por diferenças centradas de segunda ordem, obtemos

$$
\begin{aligned}
&\left.\frac{\partial^{2} \psi}{\partial x \partial y}\right|_{i-\frac{1}{2}, j-\frac{1}{2}} ^{n+1}=\frac{\left(\psi_{i, j}^{n+1}-\psi_{i-1, j}^{n+1}\right)-\left(\psi_{i, j-1}^{n+1}-\psi_{i-1, j-1}^{n+1}\right)}{(\delta x)(\delta y)}, \\
&\left.\frac{\partial \widetilde{u}}{\partial y}\right|_{i, j-\frac{1}{2}} ^{n+1}= \frac{\widetilde{u}_{i, j}^{n+1}-\widetilde{u}_{i, j-1}^{n+1}}{\delta y}, \widetilde{u}_{i, j}^{n+1}=\frac{1}{2}\left(\widetilde{u}_{i+\frac{1}{2}, j}^{n+1}+\widetilde{u}_{i-\frac{1}{2}, j}^{n+1}\right), \\
&\left.\frac{\partial \widetilde{v}}{\partial x}\right|_{i-\frac{1}{2}, j} ^{n+1}=\frac{\widetilde{v}_{i, j}^{n+1}-\widetilde{v}_{i-1, j}^{n+1}}{\delta x}, \widetilde{v}_{i, j}^{n+1}=\frac{1}{2}\left(\widetilde{v}_{i, j+\frac{1}{2}}^{n+1}+\widetilde{v}_{i, j-\frac{1}{2}}^{n+1}\right) .
\end{aligned}
$$

Portanto, as equações apresentadas formam um sistema linear para $\psi$, o qual é simétrico na FE e não simétrico na FI, devido às condições de contorno na superfície livre.

\subsubsection{Condições de fronteira na superfície livre}

A solução numérica das equações do momento e da contribuição não-newtoniana exigem condições de contorno para u e $T$ na superfície livre. Além disso, precisamos definir u e $T$ ao longo da superfície livre para que não sejam criados "buracos" sem solução no domínio quando da sua movimentação.

Assim, as condições de contorno são calculadas aplicando as equações (1.2.21), (1.3.16) e (1.3.18) nas configurações da superfície livre representadas nas Figuras 2.1.3 e 2.1.4. Sem perda de generalidade, no que segue, utilizamos a situação esboçada na Figura 5.3.3 para aplicar as condições de contorno para $\mathbf{u}$ e $T$ na superfície livre.

(a)

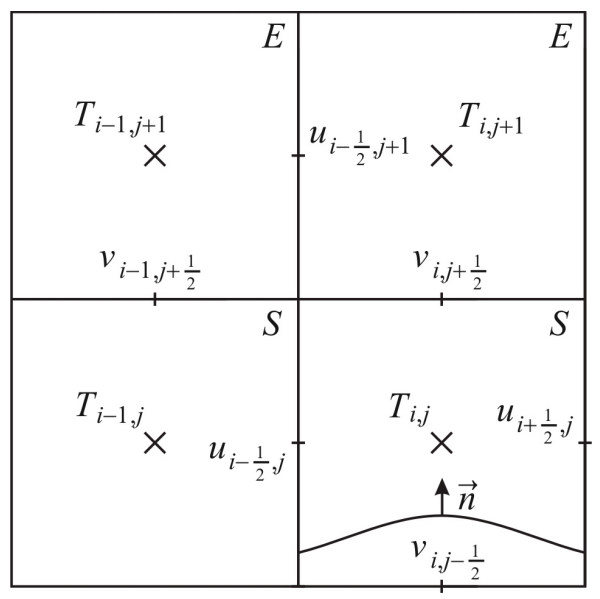

(b)

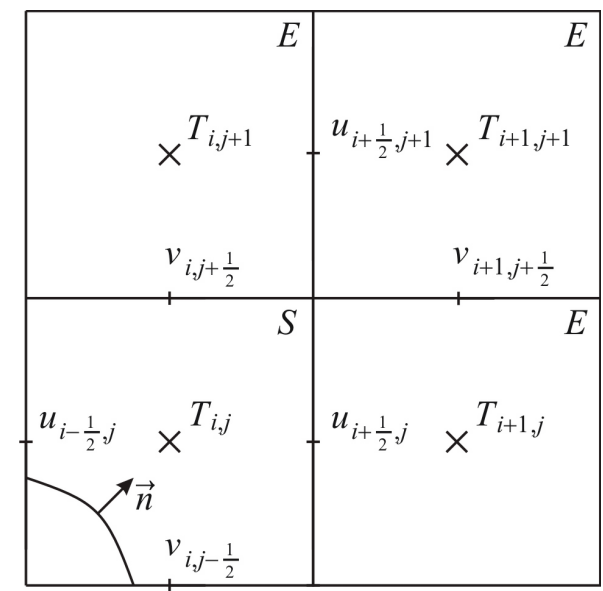

Figura 5.3.3: Superfície livre interceptando duas faces opostas (a) e adjacentes (b) de uma célula $C_{i, j}$ tipo S. Em (a), admitimos $\vec{n}$ paralelo ao eixo $y$ e, em (b), supomos que $\vec{n}$ forma um ângulo de $45^{\circ}$ com os eixos coordenados.

Inicialmente, discretizando a equação da continuidade (1.2.21), como em (5.3.34), no centro da célula $C_{i, j}$ representada na Figura 5.3.3 (a), segue que a velocidade normal à superfície livre é dada por

$$
v_{i, j+\frac{1}{2}}=v_{i, j-\frac{1}{2}}-\frac{\delta y}{\delta x}\left(u_{i+\frac{1}{2}, j}-u_{i-\frac{1}{2}, j}\right)
$$

Ainda neste caso, a equação (1.3.16) reduz-se a

$$
\frac{\partial u}{\partial y}+\frac{\partial v}{\partial x}+\frac{R e}{\beta} T^{x y}=0
$$

onde, discretizando as derivadas parciais por diferença centrada de segunda ordem no ponto $\left(x_{i-\frac{1}{2}}, y_{j+\frac{1}{2}}\right)$, obtemos a velocidade tangencial à superfície livre

$$
u_{i-\frac{1}{2}, j+1}=u_{i-\frac{1}{2}, j}-\delta y\left(\frac{v_{i, j+\frac{1}{2}}-v_{i-1, j+\frac{1}{2}}}{\delta x}+\left.\frac{R e}{\beta} T^{x y}\right|_{i-\frac{1}{2}, j+\frac{1}{2}}\right),
$$


na qual a componente da contribuição não-newtoniana é obtida pela extrapolação linear

$$
\left.T^{x y}\right|_{i-\frac{1}{2}, j+\frac{1}{2}}=\left.\frac{3}{2} T^{x y}\right|_{i-\frac{1}{2}, j}-\left.\frac{1}{2} T^{x y}\right|_{i-\frac{1}{2}, j-1},\left.T^{x y}\right|_{i-\frac{1}{2}, j}=\frac{1}{2}\left(\left.T^{x y}\right|_{i, j}+\left.T^{x y}\right|_{i-1, j}\right),
$$

seguindo a mesma estratégia usada em [PL06].

Finalmente, discretizando (1.3.18) por diferença centrada de segunda ordem na face comum às células $C_{i, j}$ e $C_{i, j+1}$, obtemos

$$
T_{i, j+1}=T_{i, j}
$$

Já na configuração esboçada na Figura 5.3.3 (b), as equações (1.2.21) e (1.3.16) resultam em, respectivamente,

$$
\begin{aligned}
& \frac{\partial u}{\partial x}+\frac{\partial v}{\partial y}=0 \\
& \frac{\partial v}{\partial y}-\frac{\partial u}{\partial x}=\frac{R e}{2 \beta}\left(T^{x x}-T^{y y}\right)
\end{aligned}
$$

onde, após a discretização das derivadas por diferenças centradas de segunda ordem no centro da célula $C_{i, j}$, obtemos

$$
\begin{aligned}
& v_{i, j+\frac{1}{2}}=v_{i, j-\frac{1}{2}}+\delta y \frac{R e}{4 \beta}\left(\left.T^{x x}\right|_{i, j}-\left.T^{y y}\right|_{i, j}\right), \\
& u_{i+\frac{1}{2}, j}=u_{i-\frac{1}{2}, j}-\frac{\delta x}{\delta y}\left(v_{i, j+\frac{1}{2}}-v_{i, j-\frac{1}{2}}\right) .
\end{aligned}
$$

Por fim, utilizando a condição de contorno (1.3.16) discretizada por diferença centrada de segunda ordem, definimos

$$
T_{i+1, j}=T_{i+1, j+1}=T_{i, j+1}=T_{i, j} .
$$

Observação 9 Ao se empregar EE para o movimento da superfície livre, sempre que uma partícula marcadora entra em uma célula $\boldsymbol{E}$, tal célula se torna $\boldsymbol{S}$ (de acordo com o algoritmo de classificação das células apresentado na Seção 2.1) e a velocidade em suas faces comum com alguma célula tipo $\boldsymbol{E}$ são inicializadas com a velocidade definida na respectiva face da célula de onde veio a partícula. Assim, os trabalhos anteriores (como [TM94], [TM02A] e [OC06], entre outros) empregam extrapolação de ordem zero para a definição de $u_{i+\frac{1}{2}, j+1}$ e $v_{i+1, j+\frac{1}{2}}$ na Figura 5.3.3 (b), mas, não nesta etapa, e sim, de forma dinâmica durante o movimento da superfície livre. Com o RK21, entretanto, tal procedimento torna-se confuso, devido à posição intermediária da partícula marcadora, que pode situar-se em uma célula distinta de sua posição final. Assim, quando o RK21 é utilizado para movimentar as partículas marcadoras, definimos previamente as velocidades $u_{i+\frac{1}{2}, j+1}$ e $v_{i+1, j+\frac{1}{2}}$ via extrapolação linear, isto é,

$$
\begin{aligned}
& u_{i+\frac{1}{2}, j+1}=2 u_{i+\frac{1}{2}, j}-u_{i+\frac{1}{2}, j-1}, \\
& v_{i+1, j+\frac{1}{2}}=2 v_{i, j+\frac{1}{2}}-v_{i-1, j+\frac{1}{2}},
\end{aligned}
$$

de modo que o campo de velocidade deixa de ser manipulado durante a movimentação da superfície livre.

\subsubsection{Cálculo da pressão na superfície livre}

Conforme já abordado, a FI do GENSMAC-SXPP utiliza uma estratégia implícita para o cálculo da pressão na superfície livre, baseada na condição de contorno (5.2.41) e na equação (5.2.17).

Por outro lado, a FE do GENSMAC-SXPP faz esse cálculo de forma direta, explícita, avaliando a equação (1.3.15) nas células tipo $\mathbf{S}$ ilustradas nas Figuras 2.1.3 e 2.1.4. Assim, sem perda de generalidade, utilizamos os dois casos representados na Figura 5.3.3 para ilustrar como é feito o cálculo da pressão na superfície livre pela FE do GENSMAC-SXPP, o qual segue a mesma estratégia utilizada em [TM02A].

Na Figura 5.3.3 (a), a aproximação adotada para $\vec{n}$ faz com que a equação (5.2.27) se reduza a

$$
p^{n+1}=\frac{2 \beta}{R e}\left(\frac{\partial v^{n+1}}{\partial y}\right)+\left.T^{y y}\right|^{n+1} .
$$


Logo, usando diferença centrada de segunda ordem para a derivada parcial, obtemos, discretizando (5.3.87) no centro da célula $C_{i, j}$,

$$
p_{i, j}^{n+1}=\frac{2 \beta}{R e}\left(\frac{v_{i, j+\frac{1}{2}}^{n+1}-v_{i, j-\frac{1}{2}}^{n+1}}{\delta y}\right)+\left.T^{y y}\right|_{i, j} ^{n+1}
$$

Do mesmo modo, quando a configuração da superfície livre é dada pela Figura 5.3.3 (b), a aproximação adotada para $\vec{n}$ e a equação da continuidade fazem com que (5.2.27) resulte em

$$
p^{n+1}=\frac{\beta}{R e}\left(\frac{\partial u^{n+1}}{\partial y}+\frac{\partial v^{n+1}}{\partial x}\right)+\frac{1}{2}\left(\left.T^{x x}\right|^{n+1}+\left.2 T^{x y}\right|^{n+1}+\left.T^{y y}\right|^{n+1}\right) .
$$

Assim, avaliando a pressão e a contribuição não-newtoniana no centro da célula $C_{i, j}$ e utilizando diferenças regressivas de primeira ordem para discretizar as derivadas espaciais, "descentradas para o lado que possui fluido", a fim de "envolver" a superfície livre, obtemos, a partir de (5.3.89),

$$
p_{i, j}^{n+1}=\frac{\beta}{R e}\left(\frac{u_{i, j}^{n+1}-u_{i, j-1}^{n+1}}{\delta y}+\frac{v_{i, j}^{n+1}-v_{i-1, j}^{n+1}}{\delta x}\right)+\frac{1}{2}\left(\left.T^{x x}\right|_{i, j} ^{n+1}+\left.2 T^{x y}\right|_{i, j} ^{n+1}+\left.T^{y y}\right|_{i, j} ^{n+1}\right),
$$

onde

$$
\begin{aligned}
u_{i, j}^{n+1} & =\frac{1}{2}\left(u_{i+\frac{1}{2}, j}^{n+1}+u_{i-\frac{1}{2}, j}^{n+1}\right) \\
v_{i, j}^{n+1} & =\frac{1}{2}\left(v_{i, j+\frac{1}{2}}^{n+1}+v_{i, j-\frac{1}{2}}^{n+1}\right) .
\end{aligned}
$$

Por fim, para uma célula $C_{i, j}$ tipo $\mathbf{S}$ degenerada, na qual uma aproximação plausível para $\vec{n}$ não pode ser obtida (e, consequentemente, a equação (5.2.27) não pode ser aplicada), tomamos

$$
p_{i, j}^{n+1}=0 \text {. }
$$

\subsubsection{Condição de contorno rígido}

Durante o processo de solução do GENSMAC-SXPP faz-se necessário impor condições de fronteira para a velocidade em células do tipo $\mathbf{B}$ que possuem contato com fluido, isto é, com células $\mathbf{F}$ ou $\mathbf{S}$. Para isso, seguindo [PL06], devemos aplicar as condições de impermeabilidade e de aderência completa nas fronteiras rígidas, descritas pelas equações (1.3.1) e (1.3.2), respectivamente.

Neste trabalho, consideramos que a fronteira rígida está em repouso e coincide com a face da célula B. Assim, existem duas situações principais a serem consideradas, esboçadas na Figura 5.3.4.

Na Figura 5.3.4 (a), a fronteira rígida é paralela ao eixo y. Com isso, segue da condição de impermeabilidade na fronteira que a componente da velocidade normal à mesma deve ser nula, isto é,

$$
u_{i+\frac{1}{2}, j}=0 \text {. }
$$

Por outro lado, segue da condição no-slip que a componente tangencial da velocidade sobre a fronteira rígida deve ser nula, ou seja,

$$
v_{i+\frac{1}{2}, j+\frac{1}{2}}=v_{i+\frac{1}{2}, j-\frac{1}{2}}=0 .
$$

Assim, via extrapolação linear, estabelecemos

$$
\begin{aligned}
& v_{i, j+\frac{1}{2}}=2 v_{i+\frac{1}{2}, j+\frac{1}{2}}-v_{i+1, j+\frac{1}{2}}, \\
& v_{i, j-\frac{1}{2}}=2 v_{i+\frac{1}{2}, j-\frac{1}{2}}-v_{i+1, j-\frac{1}{2}},
\end{aligned}
$$

e, portanto,

$$
\begin{aligned}
& v_{i, j+\frac{1}{2}}=-v_{i+1, j+\frac{1}{2}}, \\
& v_{i, j-\frac{1}{2}}=-v_{i+1, j-\frac{1}{2}} .
\end{aligned}
$$




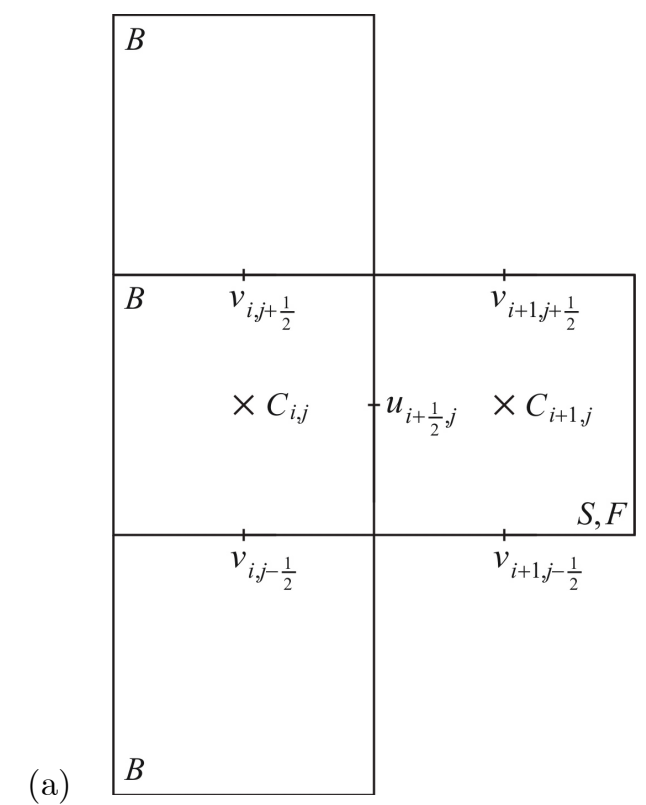

(b)

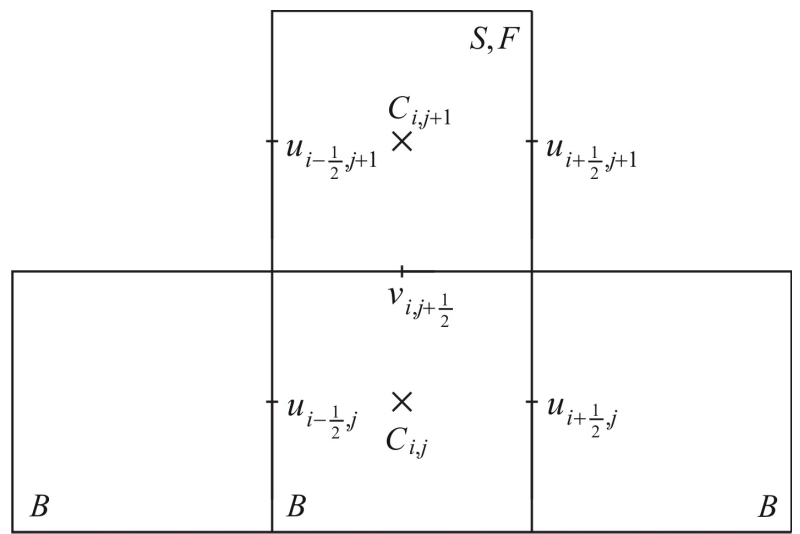

Figura 5.3.4: Ilustração de uma célula $C_{i, j}$ em fronteiras rígidas e impermeáveis paralelas ao eixo $y$ (a) e ao eixo $x(\mathrm{~b})$.

Já no caso apresentado na Figura 5.3.4 (b), a fronteira rígida é paralela ao eixo $x$. Logo, da condição de impermeabilidade segue que

$$
v_{i, j+\frac{1}{2}}=0 .
$$

Quanto à condição no-slip, exigimos que a componente tangencial da velocidade sobre a fronteira deve ser nula, isto é,

$$
u_{i-\frac{1}{2}, j+\frac{1}{2}}=u_{i+\frac{1}{2}, j+\frac{1}{2}}=0 .
$$

Assim, via extrapolação linear, estabelecemos

$$
\begin{aligned}
& u_{i-\frac{1}{2}, j}=2 u_{i-\frac{1}{2}, j+\frac{1}{2}}-u_{i-\frac{1}{2}, j+1}, \\
& u_{i+\frac{1}{2}, j}=2 u_{i+\frac{1}{2}, j+\frac{1}{2}}-u_{i+\frac{1}{2}, j+1},
\end{aligned}
$$

e, portanto,

$$
\begin{aligned}
& u_{i-\frac{1}{2}, j}=-u_{i-\frac{1}{2}, j+1}, \\
& u_{i+\frac{1}{2}, j}=-u_{i+\frac{1}{2}, j+1} .
\end{aligned}
$$

\subsubsection{Condição de contorno em injetores}

As fronteiras de injeção prescrita são responsáveis pela entrada de fluido no domínio a uma dada vazão. Assim, a solução deve ser especificada nesta fronteira de acordo com o problema modelado. No GENSMAC-SXPP, os injetores são representados por células tipo I, sendo a interface de injeção coincidente a alguma face dessas células.

Sem perda de generalidade, conforme [PL06], existem duas situações básicas a serem consideradas, conforme ilustra a Figura 5.3.5, sendo os demais casos análogos e, por isso, omitidos.

Desse modo, na Figura 5.3.5 (a) temos uma interface de injeção paralela ao eixo y. Assim, para alcançar a vazão desejada, devemos especificar a velocidade $u_{i+\frac{1}{2}, j}$, normal ao injetor, de forma arbitrária e fisicamente plausível em função do tempo e do espaço em cada célula $C_{i, j}$ que o compõe. Já a velocidade tangencial, por (1.3.6), deve ser considerada nula. Com isso, temos

$$
v_{i+\frac{1}{2}, j+\frac{1}{2}}=v_{i+\frac{1}{2}, j-\frac{1}{2}}=0
$$


(a)

\begin{tabular}{|lll|ll|}
\hline$I$ & $v_{i, j+\frac{1}{2}}$ & $S, F$ & $v_{i+1, j+\frac{1}{2}}$ \\
& $T_{i, j} \times$ & $u_{i+\frac{1}{2}, j}$ & & \\
& & $T_{i+\frac{1}{2}, j}$ & $\times T_{i+1, j}$ \\
& $v_{i, j-\frac{1}{2}}$ & & $v_{i+1, j-\frac{1}{2}}$ \\
\hline
\end{tabular}

(b)

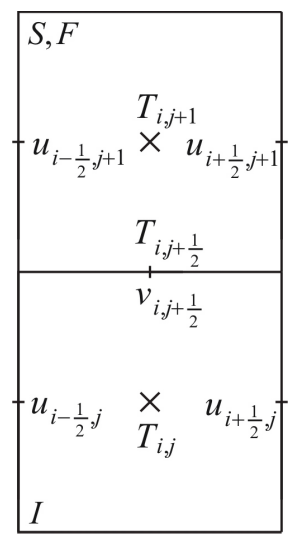

Figura 5.3.5: Ilustração da célula $C_{i, j}$ de injetores paralelos ao eixo $y$ (a) e ao eixo $x$ (b).

e, por extrapolação linear,

$$
\begin{aligned}
& v_{i, j+\frac{1}{2}}=2 v_{i+\frac{1}{2}, j+\frac{1}{2}}-v_{i+1, j+\frac{1}{2}}, \\
& v_{i, j-\frac{1}{2}}=2 v_{i+\frac{1}{2}, j-\frac{1}{2}}-v_{i+1, j-\frac{1}{2}} .
\end{aligned}
$$

Portanto,

$$
\begin{aligned}
& v_{i, j+\frac{1}{2}}=-v_{i+1, j+\frac{1}{2}}, \\
& v_{i, j-\frac{1}{2}}=-v_{i+1, j-\frac{1}{2}} .
\end{aligned}
$$

Quanto à definição do tensor $T$ na célula $\mathbf{I}$, uma vez especificado $T_{i+\frac{1}{2}, j}$ como dado do problema, utilizamos extrapolação linear para obter

$$
T_{i, j}=2 T_{i+\frac{1}{2}, j}-T_{i+1, j}
$$

Já para a situação esboçada na Figura 5.3.5 (b), onde a interface de injeção é paralela ao eixo $x$, especifica-se $v_{i, j+\frac{1}{2}}$ de forma arbitrária, de acordo com o problema considerado. Para a componente da velocidade tangencial no injetor, novamente por (1.3.6), temos

$$
u_{i-\frac{1}{2}, j+\frac{1}{2}}=u_{i+\frac{1}{2}, j+\frac{1}{2}}=0
$$

e, via extrapolação linear,

$$
\begin{aligned}
& u_{i-\frac{1}{2}, j}=2 u_{i-\frac{1}{2}, j+\frac{1}{2}}-u_{i-\frac{1}{2}, j+1}, \\
& u_{i+\frac{1}{2}, j}=2 u_{i+\frac{1}{2}, j+\frac{1}{2}}-u_{i+\frac{1}{2}, j+1} .
\end{aligned}
$$

Logo,

$$
\begin{aligned}
& u_{i-\frac{1}{2}, j}=-u_{i-\frac{1}{2}, j+1}, \\
& u_{i+\frac{1}{2}, j}=-u_{i+\frac{1}{2}, j+1},
\end{aligned}
$$

e, especificando $T_{i, j+\frac{1}{2}}$ como dado do problema, definimos $T$ na célula $C_{i, j}$ pela extrapolação linear

$$
T_{i, j}=2 T_{i, j+\frac{1}{2}}-T_{i, j+1}
$$

\subsubsection{Condição de contorno em ejetores continuados}

Uma vez que as equações (5.3.52) e (5.3.53) determinam a componente normal da velocidade em interfaces $\mathbf{O} \mid \mathbf{S}$ ou $\mathbf{O} \mid \mathbf{F}$, buscamos, agora, estabelecer a componente tangencial $\mathbf{u}_{\vec{t}}$ da velocidade e o tensor $T$ em 
células tipo O. Para isso, devemos considerar as condições de contorno (1.3.8) e (1.3.10), isto é, aplicar

$$
\begin{aligned}
& \frac{\partial \mathbf{u}_{\vec{t}}}{\partial \vec{n}}=0 \\
& \frac{\partial T}{\partial \vec{n}}=0
\end{aligned}
$$

nas interfaces de ejeção continuada. Novamente, sem perda de generalidade, conforme [PL06], consideramos as situações ilustradas na Figura 5.3.6, supondo $\vec{n}$ orientado "para fora" da região com fluido.

(a)

\begin{tabular}{|lcl|ll|}
\hline$O$ & $v_{i, j+\frac{1}{2}}$ & $S, F$ & $v_{i+1, j+\frac{1}{2}}$ \\
& $T_{i, j} \times$ & $u_{i+\frac{1}{2}, j}$ & & $\times T_{i+1, j}$ \\
& $v_{i, j-\frac{1}{2}}$ & & $v_{i+1, j-\frac{1}{2}}$ \\
\hline
\end{tabular}

(b)

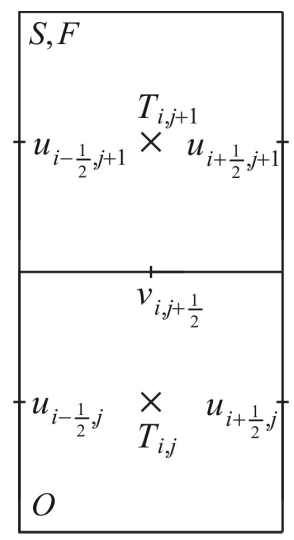

Figura 5.3.6: Ilustração da célula $C_{i, j}$ de ejetores continuados paralelos ao eixo $y$ (a) e ao eixo $x$ (b).

Assim, na Figura 5.3.6 (a) temos uma célula $\mathbf{O}$ de um ejetor continuado paralelo ao eixo $y$. Neste, discretizando as derivadas espaciais das equações (5.3.118) e (5.3.119) por diferenças centradas de segunda ordem na face da célula $\mathbf{O}$ em contato com fluido, obtemos

$$
\begin{aligned}
\frac{v_{i, j+\frac{1}{2}}-v_{i+1, j+\frac{1}{2}}}{\delta x} & =0, \\
\frac{v_{i, j-\frac{1}{2}}-v_{i+1, j-\frac{1}{2}}}{\delta x} & =0, \\
\frac{T_{i, j}-T_{i+1, j}}{\delta x} & =0 .
\end{aligned}
$$

Consequentemente,

$$
\begin{aligned}
v_{i, j+\frac{1}{2}} & =v_{i+1, j+\frac{1}{2}}, \\
v_{i, j-\frac{1}{2}} & =v_{i+1, j-\frac{1}{2}}, \\
T_{i, j} & =T_{i+1, j} .
\end{aligned}
$$

Semelhantemente, na Figura 5.3.6 (b) temos uma célula $\mathbf{O}$ de um ejetor continuado paralelo ao eixo $x$, onde a discretização das condições de fronteira por diferenças centradas de segunda ordem resulta em

$$
\begin{aligned}
\frac{u_{i-\frac{1}{2}, j}-u_{i-\frac{1}{2}, j+1}}{\delta y}=0, & 0, \\
\frac{u_{i+\frac{1}{2}, j}-u_{i+\frac{1}{2}, j+1}}{\delta y}=0, & 0,
\end{aligned}
$$

e, portanto,

$$
\begin{aligned}
u_{i-\frac{1}{2}, j} & =u_{i-\frac{1}{2}, j+1} \\
u_{i+\frac{1}{2}, j} & =u_{i+\frac{1}{2}, j+1} \\
T_{i, j} & =T_{i, j+1} .
\end{aligned}
$$




\section{CAPÍtulo}

\section{Resultados: GENSMAC-SXPP}

\subsection{Introdução}

No capítulo anterior foi apresentada a metodologia numérica denominada GENSMAC-SXPP, que permite a simulação de escoamentos viscoelásticos com superfícies livres de fluidos modelados pela equação constitutiva SXPP.

Neste capítulo, temos como objetivo apresentar os testes com a nova metodologia e suas formulações, evidenciando sua precisão e robustez. Para isso, uma série de problemas complexos (comumente empregados em trabalhos que abordam a simulação numérica de escoamentos viscoelásticos, como observamos em [TM02A], [TM02B], [GF03], [DO03], [GR03], [AB05], [SL05], [PL06] e [SV07], entre outros) são resolvidos.

No entanto, boa parte dos problemas com superfície livre considerados não apresentam solução exata. Também, não são conhecidas soluções analíticas para problemas envolvendo o modelo SXPP.

Dessa forma, na Seção 6.2, consideramos o escoamento em um canal de placas paralelas para verificar a implementação do GENSMAC-SXPP ajustando os parâmetros de modo a obter um fluido Oldroyd-B, que possui solução analítica conhecida. Isto se constitui uma estratégia comum na literatura e vem sendo utilizada no contexto do modelo SXPP, como podemos observar em [AB01] e [AB05]. Por fim, ainda na Seção 6.2, realizamos um estudo de convergência da solução numérica em várias malhas espaciais, utilizando, efetivamente, o modelo SXPP.

Em seguida, na Seção 6.3, utilizamos o GENSMAC-SXPP e os dois esquemas de movimento da superfície livre, citados ao longo deste trabalho, para simular o inchamento do extrudado de um fluido viscoelástico. Um estudo semelhante é realizado na Seção 6.4 para o problema da gota em impacto. Nestes dois problemas, como os resultados obtidos por cada formulação distinta do GENSMAC-SXPP são semelhantes e coerentes entre si, acabamos produzindo uma validação qualitativa da metodologia numérica proposta. Tal procedimento também é bastante comum na literatura, podendo ser observado em [AJ04] e [AB05], onde, ao serem propostas duas estratégias de solução para o modelo SXPP, os autores argumentam a correção da implementação e da metodologia numérica a partir da semelhança dos resultados obtidos pelos dois métodos distintos.

Finalmente, na Seção 6.5, utilizamos a FI do GENSMAC-SXPP para simular o jato oscilante de fluidos Oldroyd-B e SXPP a baixo número de Reynolds. Neste caso, comparações entre as metodologias numéricas não são justificáveis, uma vez que se aborda um problema que se caracteriza por ser fisicamente instável ([CJ81]). Mesmo assim, utilizamos este problema com o intuito de mostrar que o GENSMAC-SXPP pode simulá-lo, produzindo resultados coerentes. Além disso, a simulação numérica do jato oscilante serve, também, para demonstrar a robustez numérica e computacional do GENSMACSXPP, dada a elevada deformação e complexidade geométrica do fluido no domínio durante o escoamento. 


\subsection{Escoamento em um canal}

\subsubsection{Introdução}

Nesta seção, simulamos o escoamento no interior de um canal de placas paralelas, inicialmente vazio (caracterizando o fountain flow), descrito pelo seguinte modelo:

- Domínio espacial:

$$
\begin{aligned}
& \text { - M1: } x \in[0.0,6.2] m, y \in[0.0,2.2] m . \\
& \text { - M2: } x \in[0.0,5.6] m, y \in[0.0,1.6] m \\
& \text { - M3: } x \in[0.0,5.3] m, y \in[0.0,1.3] m \\
& \text { - M4: } x \in[0.0,5.15] m, y \in[0.0,1.15] m
\end{aligned}
$$

- Diâmetro do injetor: $L_{0}=1.0 \mathrm{~m}$.

- Velocidade no injetor: $U_{0}=1.0 \mathrm{~ms}^{-1}$, com perfil de injeção parabólico.

- Comprimento do canal: $5.0 L_{0}$.

- Distância ente injetor e outflow: $5.0 L_{0}$.

- Diâmetro do outflow: $1.0 L_{0}$.

- Aceleração de campo: $g_{0}=9.81 \mathrm{~ms}^{-2}, g^{x}=0.0, g^{y}=0.0$.

- Parâmetros adimensionais: $F r \approx 0.319275, S t=1.0 t_{0}$.

- Discretização do domínio temporal: $F_{o}=1.0, F_{C F L}=0.5$.

- Passo adimensional:

- M1: $\delta m=0.2$.

- M2: $\delta m=0.1$.

- M3: $\delta m=0.05$.

$-\mathrm{M} 4: \delta m=0.025$.

- Malha superficial: $c_{m i n}=0.02 \delta m, c_{m a ́ x}=0.2 \delta m$, movimentada pelo RK21.

- Sistemas lineares:

- Tolerância: $\varepsilon=1.0 \times 10^{-10}$.

- Método para solução de $\widetilde{\mathbf{u}}$ : BiCGstab-SOR $(\omega=1.6)$.

- Método para solução de $\psi$ : BiCGstab-SOR $(\omega=1.8)$.

Assim, são adotadas quatro malhas espaciais que preservam uma mesma área interna para o canal, as quais possibilitam analisar a convergência das soluções numéricas obtidas por cada formulação do GENSMAC-SXPP. Vale observar que as diferenças de dimensão no domínio espacial se devem, apenas, às espessuras das paredes que formam o canal, que, no Sistema Freeflow 2D ([OL99], [CF00]) devem possuir espessura mínima de três células. Além disso, a escolha de $F_{v i s c}$ para a determinação do passo temporal foi realizada, em cada caso, a fim de que $\delta t$ ficasse próximo ao limite de estabilidade.

Um perfil parabólico, que corresponde ao da solução totalmente desenvolvida, é imposto no injetor. Para o modelo Oldroyd-B, esse perfil é dado, segundo [BH87], por

$$
\begin{gathered}
u(y)=4 U_{0}\left(\frac{y}{L_{0}}\right)\left(1-\frac{y}{L_{0}}\right), v(y)=0 \\
T^{x x}(y)=\frac{2 W e}{R e}\left(1-\frac{\lambda_{2}}{\lambda_{1}}\right)\left(\frac{\partial u}{\partial y}\right)^{2}, T^{x y}(y)=\frac{1}{R e}\left(1-\frac{\lambda_{2}}{\lambda_{1}}\right)\left(\frac{\partial u}{\partial y}\right), T^{y y}(y)=0,
\end{gathered}
$$


onde é assumido que, na parede inferior do canal $y=0$ e, na superior, $y=L_{0}$.

Assim, o canal inicialmente vazio é preenchido a partir de sua extremidade esquerda, onde se localiza o injetor. Por volta do instante $t=8.0$ o canal está completamente cheio e, a partir de então, a solução aproxima-se do estado estacionário. Consideramos, nesse conjunto de simulações, o instante final em $t=100.0$, quando um perfil da solução (transversal ao canal e paralelo ao injetor) é extraído para análise.

Para o modelo Oldroyd-B, o perfil transversal é extraído em $2.5 L_{0}$ do injetor, isto é, na metade do canal. No caso do modelo SXPP, entretanto, o perfil da solução totalmente desenvolvida não é conhecido. Assim, seguimos a mesma abordagem presente em [AJ04] e em [AB05], impondo no injetor o perfil desenvolvido do modelo Oldroyd-B e justificando que o mesmo evolui ao longo do canal e, próximo ao ejetor, é obtido o perfil próprio do modelo SXPP. Dessa forma, para o modelo SXPP, capturamos o perfil transversal da solução em $4.8 L_{0}$ do injetor (ou seja, a uma célula do ejetor na malha M1).

\subsubsection{Verificação do método numérico: modelo Oldroyd-B}

No caso do modelo Oldroyd-B, é possível comparar a solução numérica com a solução analítica (6.2.1)(6.2.2). Além disso, em complemento ao problema inicialmente apresentado, definimos os demais parâmetros dimensionais a fim de que:

- Parâmetros adimensionais: $W e=2.0, \beta=0.5, \operatorname{Re} \in\{0.1,0.01\}$.

Assim, dois valores para o número de Reynolds foram considerados, ambos satisfazendo $R e<<1$. Na Figura 6.2.1 apresentamos a simulação obtida pela FI-EI com $R e=0.01$ na malha M4, sendo os demais casos omitidos por serem semelhantes.

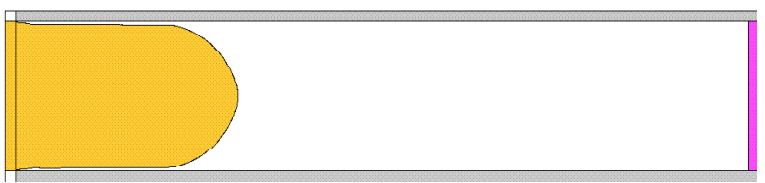

(a)

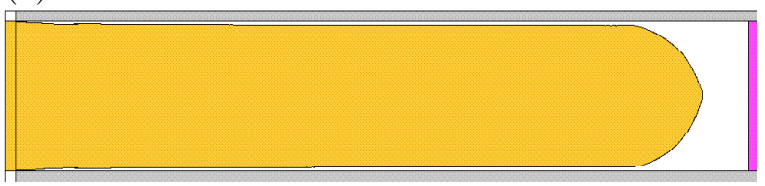

(c)

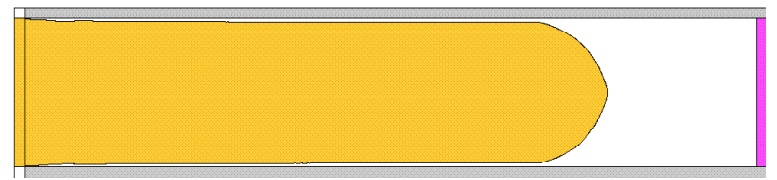

(b)

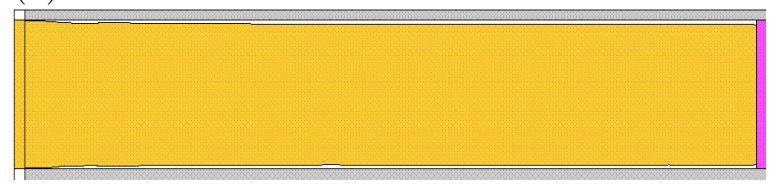

(d)

Figura 6.2.1: Simulação numérica da FI-EI para o fountain flow de um fluido Oldroyd-B $(R e=0.01$, $W e=2.0, \beta=0.5) \mathrm{em}: t=2.0(\mathrm{a}) ; t=5.5(\mathrm{~b}) ; t=6.5(\mathrm{c}) ; t=8.0(\mathrm{~d})$.

Para uma variável genérica $\eta$, calculamos os erros relativos na norma $l_{2}$ por

$$
\left\|e_{\eta}\right\|_{2}=\sqrt{\frac{\sum\left(\eta_{\text {exato }}-\eta_{\text {numérico }}\right)^{2}}{\sum\left(\eta_{\text {exato }}\right)^{2}}}
$$

e utilizamos, para aproximar a ordem de convergência,

$$
n_{i}=\frac{\ln \left(\frac{\left\|e_{M_{j}}\right\|_{2}}{\left\|e_{M_{i}}\right\|_{2}}\right)}{\ln \left(\frac{\delta m_{M_{j}}}{\delta m_{M_{i}}}\right)},
$$

com $i \in\{1,2,3\}, j=i+1$, onde definimos $n_{\text {total }}$ como a ordem de convergência total obtida usando a malha mais grossa para definir $i$ e a mais fina para definir $j$ em (6.2.4).

Nestes termos, a Tabela 6.2.1 resume os resultados com $R e=0.1$. Já na Tabela 6.2.2 estão os resultados obtidos para $R e=0.01$. 
Pela FI-CN com $R e=0.01$, na Figura 6.2.2 apresentamos as soluções numéricas obtidas e a distribuição espacial do erro relativo em cada malha empregada. As figuras análogas para os demais métodos foram omitidas por serem semelhantes.

Assim, observamos que todos os métodos mostraram-se convergentes, com o erro diminuindo à medida que a malha espacial é refinada. Além disso, a ordem de convergência aproximada confirma a segunda ordem de precisão espacial dos métodos, o que é coerente com a precisão das discretizações espaciais realizadas. Por outro lado, pelo fato da solução considerada representar um estado estacionário, o passo temporal exerceu pouca influencia nos erros calculados.

Tabela 6.2.1: Resultados para o fountain flow de um fluido Oldroyd-B ( $R e=0.1, W e=2.0, \beta=0.5)$.

\begin{tabular}{|c|c|c|c|c|c|c|c|}
\hline método & malha & $F_{v i s c}$ & tempo & $C P U$ & $\left\|e_{u}\right\|_{2}$ & $\left\|e_{T^{x x}}\right\|_{2}$ & $\mid e_{T^{x y}} \|_{2}$ \\
\hline \multirow{8}{*}{$F I-C N$} & $M 1$ & 20.0 & \multicolumn{2}{|c|}{$00: 00: 06$} & $5.4590 \times 10^{-2}$ & $9.4301 \times 10^{-2}$ & $5.8505 \times 10^{-2}$ \\
\hline & $M 2$ & 16.0 & \multicolumn{2}{|c|}{ 00:01:59 } & $1.4993 \times 10^{-2}$ & $1.5132 \times 10^{-2}$ & $5.1749 \times 10^{-3}$ \\
\hline & M3 & 32.0 & \multicolumn{2}{|c|}{$00: 17: 10$} & $3.6056 \times 10^{-3}$ & $6.6333 \times 10^{-3}$ & $2.4510 \times 10^{-3}$ \\
\hline & $M 4$ & 64.0 & \multicolumn{2}{|c|}{ 02:59:03 } & $9.1260 \times 10^{-4}$ & $1.9486 \times 10^{-3}$ & $1.3495 \times 10^{-3}$ \\
\hline & \multirow{4}{*}{\multicolumn{3}{|c|}{ ordem de convergência }} & $n_{1}$ & 1.8644 & 2.6397 & 3.4989 \\
\hline & & & & $n_{2}$ & 2.0560 & 1.1898 & 1.0781 \\
\hline & & & & $n_{3}$ & 1.9822 & 1.7673 & 0.8609 \\
\hline & & & & $n_{\text {total }}$ & 1.9675 & 1.8656 & 1.8127 \\
\hline \multirow{8}{*}{$F I-E I$} & $M 1$ & 20.0 & \multicolumn{2}{|c|}{ 00:00:06 } & $5.4590 \times 10^{-2}$ & $9.4301 \times 10^{-2}$ & $5.8504 \times 10^{-2}$ \\
\hline & $M 2$ & 40.0 & \multicolumn{2}{|c|}{ 00:00:45 } & $1.4993 \times 10^{-2}$ & $1.5132 \times 10^{-2}$ & $5.1752 \times 10^{-3}$ \\
\hline & M3 & 80.0 & \multicolumn{2}{|c|}{ 00:06:51 } & $3.6055 \times 10^{-3}$ & $6.6333 \times 10^{-3}$ & $2.4510 \times 10^{-3}$ \\
\hline & $M 4$ & 160.0 & \multicolumn{2}{|c|}{$01: 19: 54$} & $9.1264 \times 10^{-4}$ & $1.9486 \times 10^{-3}$ & $1.3496 \times 10^{-3}$ \\
\hline & \multirow{4}{*}{\multicolumn{3}{|c|}{ ordem de convergência }} & $n_{1}$ & 1.8644 & 2.6397 & 3.4988 \\
\hline & & & & $n_{2}$ & 2.0560 & 1.1898 & 1.0782 \\
\hline & & & & $n_{3}$ & 1.9821 & 1.7673 & 0.8609 \\
\hline & & & & $n_{\text {total }}$ & 1.9675 & 1.8656 & 1.8127 \\
\hline \multirow{6}{*}{$F E-E E$} & $M 1$ & 1.0 & \multicolumn{2}{|c|}{$00: 01: 31$} & $5.4590 \times 10^{-2}$ & $9.4296 \times 10^{-2}$ & $5.8502 \times 10^{-2}$ \\
\hline & $M 2$ & 1.0 & \multicolumn{2}{|c|}{$00: 24: 38$} & $1.4993 \times 10^{-2}$ & $1.5132 \times 10^{-2}$ & $5.1750 \times 10^{-3}$ \\
\hline & M3 & 1.0 & \multicolumn{2}{|c|}{$06: 29: 35$} & $3.6055 \times 10^{-3}$ & $6.6333 \times 10^{-3}$ & $2.4510 \times 10^{-3}$ \\
\hline & \multirow{3}{*}{\multicolumn{3}{|c|}{ ordem de convergência }} & & 1.8644 & 2.6396 & 3.4988 \\
\hline & & & & $n_{2}$ & 2.0560 & 1.1898 & 1.0782 \\
\hline & & & & $n_{\text {total }}$ & 1.9602 & 1.9147 & 2.2885 \\
\hline \multirow{6}{*}{$F E-R K 22$} & $M 1$ & 1.0 & \multicolumn{2}{|c|}{$00: 01: 46$} & $5.4590 \times 10^{-2}$ & $9.4302 \times 10^{-2}$ & $5.8507 \times 10^{-2}$ \\
\hline & $M 2$ & 1.0 & \multicolumn{2}{|c|}{$00: 29: 33$} & $1.4993 \times 10^{-2}$ & $1.5132 \times 10^{-2}$ & $5.1749 \times 10^{-3}$ \\
\hline & M3 & 1.0 & \multicolumn{2}{|c|}{ 08:09:11 } & $3.6055 \times 10^{-3}$ & $6.6333 \times 10^{-3}$ & $2.4510 \times 10^{-3}$ \\
\hline & \multirow{3}{*}{\multicolumn{3}{|c|}{ ordem de convergência }} & $n_{1}$ & 1.8644 & 2.6397 & 3.4990 \\
\hline & & & & $n_{2}$ & 2.0560 & 1.1898 & 1.0782 \\
\hline & & & & $n_{\text {total }}$ & 1.9602 & 1.9148 & 2.2886 \\
\hline
\end{tabular}


O passo temporal necessário para a estabilidade da FE no caso da malha M4 tornou seu uso proibitivo. Este fato é ilustrado observando que a solução obtida pela FI na malha M4 exige um tempo de processamento muito menor que o gasto pela FE na malha M3. Nesse contexto, é interessante observar que a FI consegue, em todos os casos, superar com larga margem a restrição de estabilidade parabólica.

Assim, para a FE foi necessário adotar $F_{\text {visc }}=1.0$ enquanto que a FI foi estável com $F_{\text {visc }}>>1$, resultando em um tempo de CPU consideravelmente menor. O passo temporal permitido pela FI-CN foi 64 vezes maior que o da FE na malha M4, sendo, porém, ligeiramente inferior ao empregado pela FI-EI, que ficou muito próximo da condição CFL.

Essa pequena margem de diferença nos passos temporais existente entre a FI-EI e a FI-CN pode ser atribuída ao limite de oscilação que existe para o método de Crank-Nicholson, conforme apresentado em [FL91] e [SM85] para a equação de difusão. Tais valores para o passo temporal da FI-CN do GENSMACSXPP, entretanto, são coerentes com o limite de estabilidade da FI-CN do GENSMAC, conforme podemos observar em [OC08A].

Tabela 6.2.2: Resultados para o fountain flow de um fluido Oldroyd-B $(R e=0.01, W e=2.0, \beta=0.5)$.

\begin{tabular}{|c|c|c|c|c|c|c|c|}
\hline método & malha & $F_{\text {visc }}$ & tempo & e $C P U$ & $\left\|e_{u}\right\|_{2}$ & $\left\|e_{T^{x x}}\right\|_{2}$ & $\left\|e_{T^{x y}}\right\|_{2}$ \\
\hline \multirow{8}{*}{$F I-C N$} & $M 1$ & 20.0 & \multicolumn{2}{|c|}{ 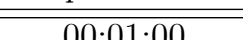 } & $5.4592 \times 10^{-2}$ & $9.4264 \times 10^{-2}$ & $5.8487 \times 10^{-2}$ \\
\hline & $M 2$ & 16.0 & \multicolumn{2}{|c|}{$00: 18: 48$} & $1.4992 \times 10^{-2}$ & $1.5129 \times 10^{-2}$ & $5.1707 \times 10^{-3}$ \\
\hline & M3 & 32.0 & \multicolumn{2}{|c|}{$02: 43: 28$} & $3.6056 \times 10^{-3}$ & $6.6299 \times 10^{-3}$ & $2.4499 \times 10^{-3}$ \\
\hline & $M 4$ & 64.0 & \multicolumn{2}{|c|}{$31: 48: 43$} & $9.1291 \times 10^{-4}$ & $1.9383 \times 10^{-3}$ & $1.3579 \times 10^{-3}$ \\
\hline & \multirow{4}{*}{\multicolumn{3}{|c|}{ ordem de convergência }} & $n_{1}$ & 1.8645 & 2.6394 & 3.4997 \\
\hline & & & & $n_{2}$ & 2.0559 & 1.1902 & 1.0777 \\
\hline & & & & $n_{3}$ & 1.9817 & 1.7742 & 0.8513 \\
\hline & & & & $n_{\text {total }}$ & 1.9674 & 1.8680 & 1.8095 \\
\hline \multirow{8}{*}{$F I-E I$} & $M 1$ & 200.0 & \multicolumn{2}{|c|}{$0 \cdot 00 \cdot 0 \cdot 07$} & $5.4594 \times 10^{-2}$ & $9.4253 \times 10^{-2}$ & $5.8475 \times 10^{-2}$ \\
\hline & $M 2$ & 400.0 & \multicolumn{2}{|c|}{$00: 00: 46$} & $1.4992 \times 10^{-2}$ & $1.5129 \times 10^{-2}$ & $5.1706 \times 10^{-3}$ \\
\hline & M3 & 800.0 & \multicolumn{2}{|c|}{$00: 06: 56$} & $3.6056 \times 10^{-3}$ & $6.6299 \times 10^{-3}$ & $2.4499 \times 10^{-3}$ \\
\hline & $M 4$ & 1600.0 & \multicolumn{2}{|c|}{$01: 22: 37$} & $9.1271 \times 10^{-4}$ & $1.9470 \times 10^{-3}$ & $1.3491 \times 10^{-3}$ \\
\hline & \multirow{4}{*}{\multicolumn{3}{|c|}{ ordem de convergência }} & $n_{1}$ & 1.8645 & 2.6393 & 3.4994 \\
\hline & & & & $n_{2}$ & 2.0559 & 1.1902 & 1.0776 \\
\hline & & & & $n_{3}$ & 1.9820 & 1.7677 & 0.8607 \\
\hline & & & & $n_{\text {total }}$ & 1.9675 & 1.8657 & 1.8126 \\
\hline \multirow{6}{*}{$F E-E E$} & $M 1$ & 1.0 & \multicolumn{2}{|c|}{$00: 14: 52$} & $5.4592 \times 10^{-2}$ & $9.4263 \times 10^{-2}$ & $5.8486 \times 10^{-2}$ \\
\hline & $M 2$ & 1.0 & \multicolumn{2}{|c|}{ 03:49:05 } & $1.4992 \times 10^{-2}$ & $1.5129 \times 10^{-2}$ & $5.1707 \times 10^{-3}$ \\
\hline & M3 & 1.0 & \multicolumn{2}{|c|}{$68: 51: 55$} & $3.6056 \times 10^{-3}$ & $6.6299 \times 10^{-3}$ & $2.4499 \times 10^{-3}$ \\
\hline & \multirow{3}{*}{\multicolumn{3}{|c|}{ ordem de convergência }} & $n_{1}$ & 1.8645 & 2.6394 & 3.4997 \\
\hline & & & & $n_{2}$ & 2.0559 & 1.1902 & 1.0776 \\
\hline & & & & $n_{\text {total }}$ & 1.9602 & 1.9148 & 2.2887 \\
\hline \multirow{6}{*}{$F E-R K 22$} & $M 1$ & 1.0 & \multicolumn{2}{|c|}{$00: 17: 39$} & $5.4592 \times 10^{-2}$ & $9.4265 \times 10^{-2}$ & $5.8487 \times 10^{-2}$ \\
\hline & $M 2$ & 1.0 & \multicolumn{2}{|c|}{$04: 33: 26$} & $1.4992 \times 10^{-2}$ & $1.5129 \times 10^{-2}$ & $5.1707 \times 10^{-3}$ \\
\hline & M3 & 1.0 & \multicolumn{2}{|c|}{$81: 51: 14$} & $3.6056 \times 10^{-3}$ & $6.6299 \times 10^{-3}$ & $2.4499 \times 10^{-3}$ \\
\hline & \multirow{3}{*}{\multicolumn{3}{|c|}{ ordem de convergência }} & & 1.8645 & 2.6394 & 3.4997 \\
\hline & & & & $n_{2}$ & 2.0559 & 1.1902 & 1.0777 \\
\hline & & & & $n_{\text {total }}$ & 1.9602 & 1.9148 & 2.2887 \\
\hline
\end{tabular}



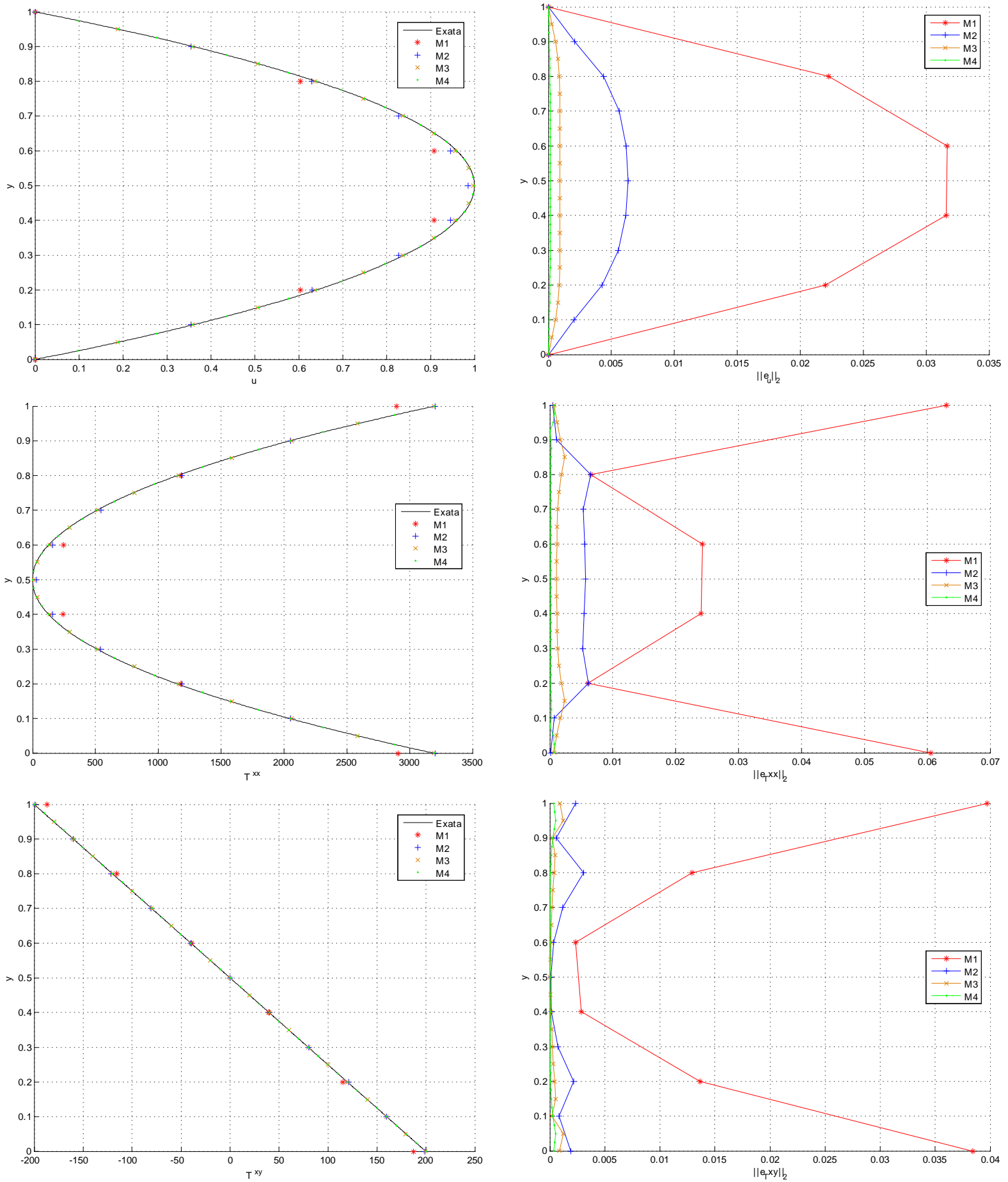

Figura 6.2.2: Comparação das soluções numéricas obtidas pela FI-CN com a solução analítica (coluna esquerda) e comportamento do erro (coluna direita) em cada malha empregada para simular o escoamento totalmente desenvolvido em um canal $(t=100.0)$ de um fluido Oldroyd-B $(R e=0.01, W e=2.0, \beta=0.5)$. 


\subsubsection{Análise de convergência do método numérico: modelo SXPP}

Para o modelo SXPP, além do problema apresentado inicialmente, consideramos:

- Parâmetros adimensionais: $W e=2.0, \beta=0.5, \gamma=0.5, \alpha=0.2, Q=2.0, R e \in\{0.1,0.01\}$.

Na Figura 6.2.3 temos a simulação numérica obtida pela FI-CN com $R e=0.01$, sendo as demais figuras análogas omitidas por serem semelhantes.

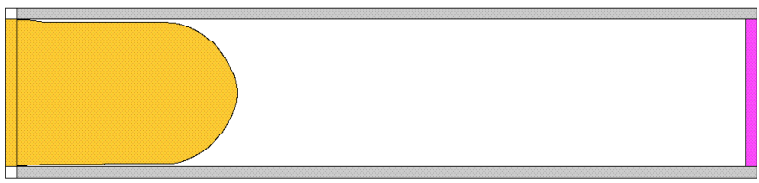

(a)

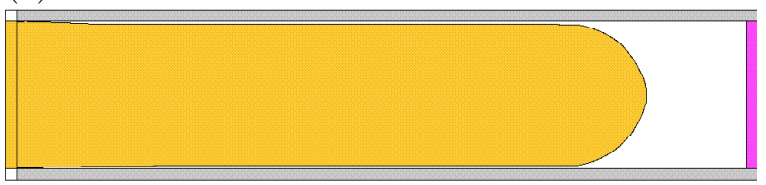

(c)

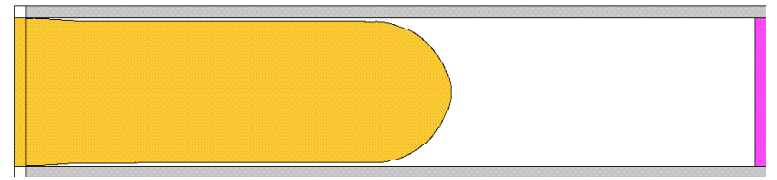

(b)

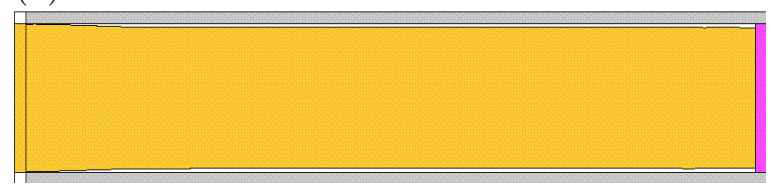

(d)

Figura 6.2.3: Simulação numérica da FI-CN para o fountain flow de um fluido SXPP $(R e=0.01$, $W e=2.0, \alpha=0.2, \beta=0.5, \gamma=0.5, Q=2.0) \mathrm{em}: t=2.0(\mathrm{a}) ; t=4.0(\mathrm{~b}) ; t=6.0(\mathrm{c}) ; t=8.0(\mathrm{~d})$.

Entretanto, como a solução analítica não é conhecida, limitamo-nos a aproximar a taxa de convergência de uma variável genérica $\eta$ por

$$
r_{i}=\frac{\ln \left(\frac{\left\|\eta_{M_{i+2}}-\eta_{M_{i+1}}\right\|_{2}}{\left\|\eta_{M_{i+1}}-\eta_{M_{i}}\right\|_{2}}\right)}{\ln \left(\frac{1}{2}\right)}, i \in\{1,2\},
$$

onde

$$
\frac{\delta m_{M_{j+1}}}{\delta m_{M j}}=\frac{1}{2}, j \in\{1,2,3\} .
$$

Tabela 6.2.3: Taxa de convergência para o fountain flow de um fluido $\operatorname{SXPP}(W e=2.0, \beta=0.5, \gamma=0.5$, $\alpha=0.2, Q=2.0)$.

\begin{tabular}{|c|c|c|c|c|c|c|c|c|}
\hline Re & método & taxa & $u$ & $v$ & $T^{x x}$ & $T^{x y}$ & $T^{y y}$ & $\lambda$ \\
\hline \hline \multirow{4}{*}{0.1} & $F I-C N$ & $r_{1}$ & 1.8682 & 2.2152 & 0.4505 & 3.5240 & -0.3773 & 0.3668 \\
& & $r_{2}$ & 2.2650 & 3.5051 & 2.3764 & 1.8760 & 2.4369 & 2.3670 \\
\cline { 2 - 9 } & $F I-E I$ & $r_{1}$ & 1.8682 & 2.2154 & 0.4505 & 3.5240 & -0.3773 & 0.3668 \\
& & $r_{2}$ & 2.2650 & 3.5038 & 2.3764 & 1.8760 & 2.4369 & 2.3670 \\
\cline { 2 - 9 } & $F E-E E$ & $r_{1}$ & 1.8682 & 2.2150 & 0.4505 & 3.5240 & -0.3773 & 0.3668 \\
\cline { 2 - 9 } & $F E-R K 22$ & $r_{1}$ & 1.8682 & 2.2153 & 0.4505 & 3.5240 & -0.3773 & 0.3668 \\
\hline \hline \multirow{4}{*}{0.01} & $F I-C N$ & $r_{1}$ & 1.8682 & 2.2229 & 0.4507 & 3.5245 & -0.3778 & 0.3668 \\
& & $r_{2}$ & 2.2650 & 3.5067 & 2.3764 & 1.8755 & 2.4367 & 2.3671 \\
\cline { 2 - 8 } & $F I-E I$ & $r_{1}$ & 1.8682 & 2.2230 & 0.4507 & 3.5245 & -0.3778 & 0.3668 \\
& & $r_{2}$ & 2.2650 & 3.5074 & 2.3764 & 1.8755 & 2.4367 & 2.3671 \\
\cline { 2 - 8 } & $F E-E E$ & $r_{1}$ & 1.8682 & 2.2230 & 0.4507 & 3.5245 & -0.3778 & 0.3668 \\
\cline { 2 - 8 } & $F E-R K 22$ & $r_{1}$ & 1.8682 & 2.2231 & 0.4507 & 3.5245 & -0.3778 & 0.3668 \\
\hline
\end{tabular}

Na Tabela 6.2.3 apresentamos as taxas de convergência obtidas por cada uma das formulações do GENSMAC-SXPP neste problema, estando na Tabela 6.2.4 o tempo de CPU dispensado e o fator de controle para o passo temporal empregado pelos métodos em cada malha. 

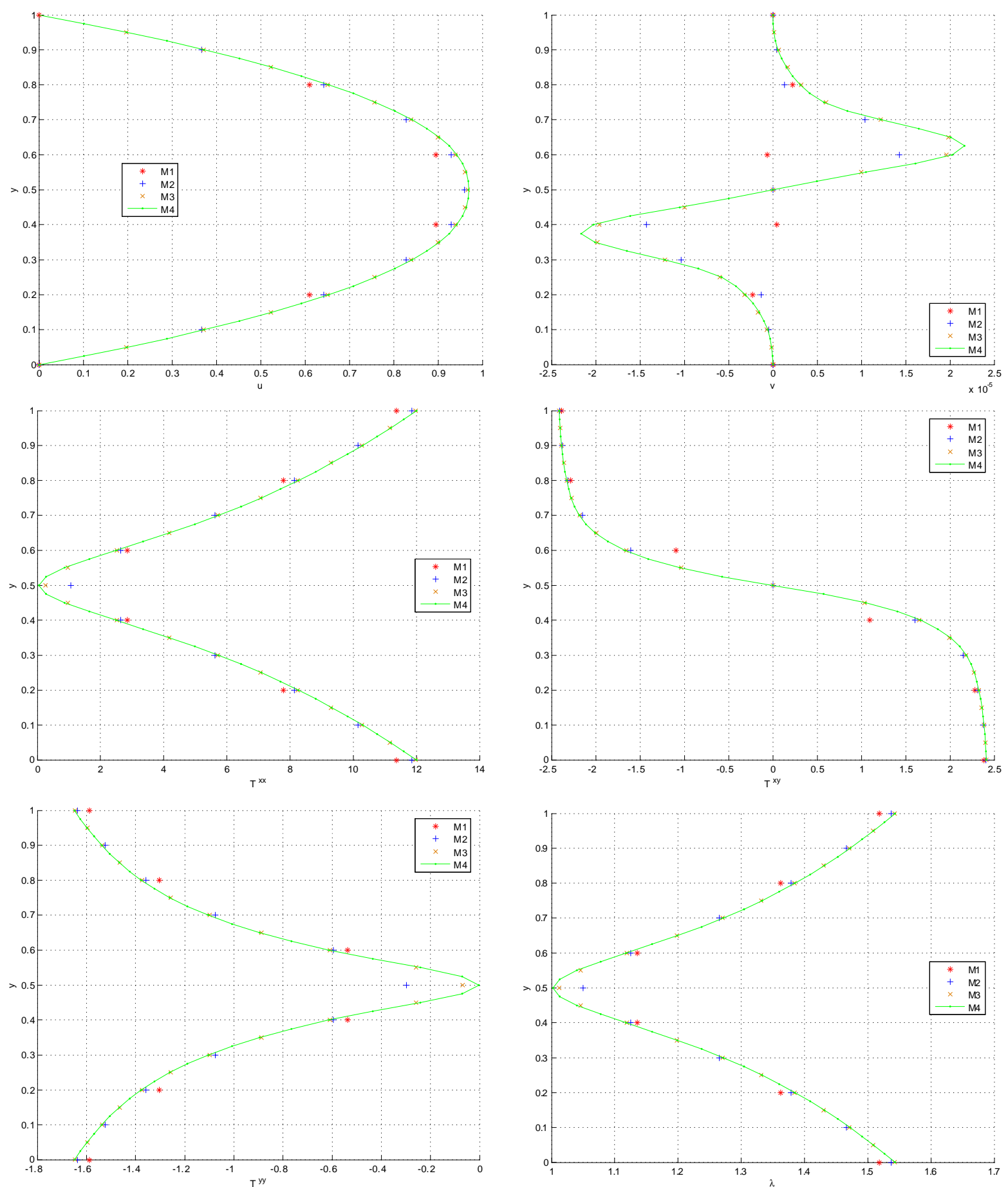

Figura 6.2.4: Soluções numéricas obtidas pela FI-EI em cada malha empregada para simular o escoamento totalmente desenvolvido em um canal $(t=100.0)$ de um fluido $\operatorname{SXPP}(\operatorname{Re}=0.1, W e=2.0, \alpha=0.2$, $\beta=0.5, \gamma=0.5, Q=2.0)$. 

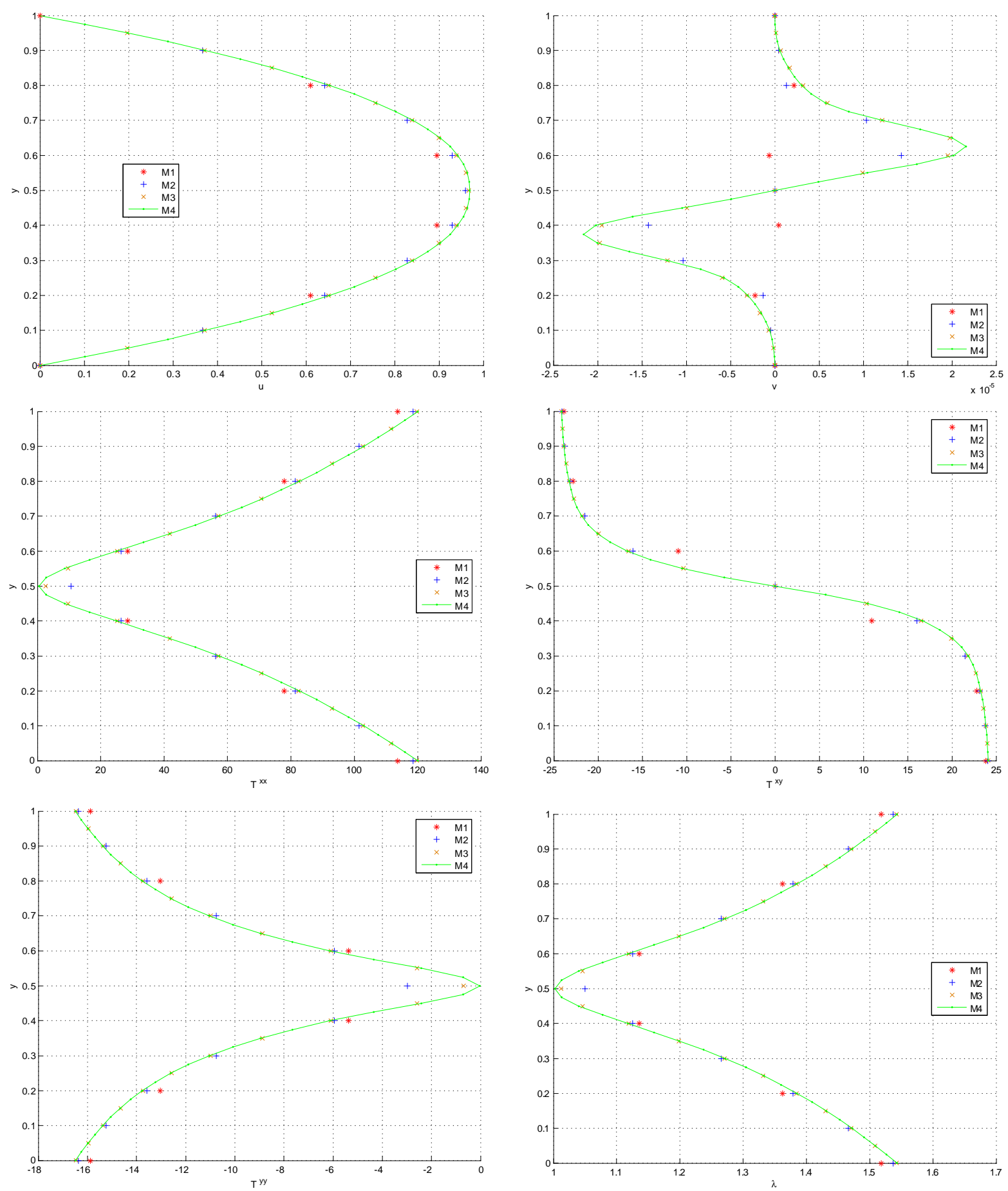

Figura 6.2.5: Soluções numéricas obtidas pela FI-CN em cada malha empregada para simular o escoamento totalmente desenvolvido em um canal $(t=100.0)$ de um fluido $\operatorname{SXPP}(R e=0.01, W e=2.0$, $\alpha=0.2, \beta=0.5, \gamma=0.5, Q=2.0)$. 
Tabela 6.2.4: Tempos de CPU para o fountain flow de um fluido $\operatorname{SXPP}(W e=2.0, \beta=0.5, \gamma=0.5$, $\alpha=0.2, Q=2.0)$.

\begin{tabular}{|c|c|c|c|c|c|}
\cline { 3 - 6 } \multicolumn{2}{c|}{} & \multicolumn{2}{c|}{ Re=0.1 } & \multicolumn{2}{c|}{ Re=0.01 } \\
\hline método & malha & $F_{\text {visc }}$ & tempo de CPU & $F_{\text {visc }}$ & tempo de CPU \\
\hline \hline \multirow{3}{*}{$F I-C N$} & $M 1$ & 20.0 & $00: 00: 06$ & 20.0 & $00: 01: 02$ \\
& $M 2$ & 16.0 & $00: 02: 04$ & 16.0 & $00: 20: 52$ \\
& $M 3$ & 32.0 & $00: 18: 53$ & 32.0 & $03: 31: 11$ \\
& $M 4$ & 64.0 & $05: 17: 49$ & 64.0 & $42: 09: 23$ \\
\hline \hline \multirow{3}{*}{$F I-E I$} & $M 1$ & 20.0 & $00: 00: 06$ & 200.0 & $00: 00: 06$ \\
& $M 2$ & 40.0 & $00: 00: 48$ & 400.0 & $00: 00: 45$ \\
& $M 3$ & 80.0 & $00: 07: 24$ & 800.0 & $00: 06: 24$ \\
& $M 4$ & 160.0 & $02: 22: 31$ & 1600.0 & $02: 24: 41$ \\
\hline \hline \multirow{3}{*}{$F E-E E$} & $M 1$ & 1.0 & $00: 01: 38$ & 1.0 & $00: 17: 04$ \\
& $M 2$ & 1.0 & $00: 26: 58$ & 1.0 & $04: 15: 28$ \\
& $M 3$ & 1.0 & $08: 11: 34$ & 1.0 & $82: 50: 18$ \\
\hline \hline \multirow{3}{*}{$F E-R K 22$} & $M 1$ & 1.0 & $00: 01: 55$ & 1.0 & $00: 18: 57$ \\
& $M 2$ & 1.0 & $00: 31: 00$ & 1.0 & $05: 01: 34$ \\
& $M 3$ & 1.0 & $10: 23: 21$ & 1.0 & $107: 16: 47$ \\
\hline
\end{tabular}

O perfil desenvolvido obtido pelas formulações do GENSMAC-SXPP foram muito semelhantes entre si, e assim, exibimos na Figura 6.2.4 a solução pela FI-EI com $R e=0.1$ e, na Figura 6.2 .5 o perfil análogo obtido pela FI-CN para $R e=0.01$.

Nestas figuras, como a solução analítica é desconhecida, algumas observações são pertinentes. Inicialmente, em todos os casos, é visível que à medida que a malha espacial é refinada a solução numérica está convergindo para algo que supomos ser a solução exata do problema. Quanto à componente $v$ da velocidade, podemos perceber que a solução numérica está muito próxima de zero, com, numericamente, $v=O\left(10^{-5}\right)$, o que é compatível com o fato de tratar-se do perfil de solução totalmente desenvolvido (pelo qual, é suposto $v=0$ ).

Quanto à influência do número de Reynolds na solução, percebemos que $u, v$ e $\lambda$ não são afetados por esse parâmetro. Já o tensor $T$, embora mantenha um mesmo perfil, teve sua escala de valores multiplicada por 10 quando passamos de $R e=0.1$ para $R e=0.01$. Logo, embora a solução analítica para $T$ seja desconhecida, percebe-se no modelo SXPP que $T$ é inversamente proporcional ao número de Reynolds, analogamente ao observado para o Oldroyd-B em (6.2.2).

Na Tabela 6.2.3 podemos observar que as diferentes formulações produziram resultados muito semelhantes, o que é coerente com o fato das discretizações espaciais serem as mesmas em todas formulações do GENSMAC-SXPP, em que se pese a estratégia de integração no tempo. Assim, observando $r_{2}$ (cujo cálculo despreza a malha M1, que é bastante grossa), vemos que a metodologia numérica é, aproximadamente, de segunda ordem de precisão.

Para a FE, no entanto, não foi possível calcular $r_{2}$, pois, para a malha M4, o $\delta t$ requerido na restrição de estabilidade parabólica tornou o uso da FE proibitivo. Conforme era esperado, na Tabela 6.2.4 pode-se notar que a FI elimina a restrição de estabilidade parabólica, permitindo $F_{v i s c}>>1$, o que se reflete em uma grande discrepância entre os tempos de processamento da FE e da FI.

\subsection{0 inchamento do extrudado}

Nesta seção, consideramos o inchamento do extrudado de fluidos viscoelásticos. Um dado importante relativo a este problema diz respeito à razão de inchamento do extrudado $S_{r}([\mathrm{BH} 87])$, dada por

$$
S_{r}=\frac{L_{e}}{L_{0}}
$$

onde $L_{e}$ é o diâmetro máximo da porção de fluido extrudada do canal inicial, que possui diâmetro $L_{0}$. Em outras palavras, após emergir do canal inicial a porção de fluido sofre uma variação na largura radial, 
contabilizada por (6.3.1).

No caso de um fluido newtoniano (em que ambas diferenças de tensões normais são nulas), temos $S_{r} \approx 1.13$ na situação limite para baixo Reynolds, conforme [BH87]. Para o GENSMAC, a razão de inchamento observada no caso de um fluido newtoniano varia de 108\% (com perfil de velocidade parabólico no injetor) até $113 \%$ (com perfil de velocidade reto no injetor), aproximadamente, conforme podemos observar nas simulações apresentadas no Capítulo 4 para baixos números de Reynolds.

Já para fluidos viscoelásticos ocorre o chamado efeito Barus, sendo $L_{e}$ apreciavelmente maior que $L_{0}$, conforme [BH87]. Assim, para simular tal situação, adotamos o modelo:

- Domínio espacial: $x \in[0.0,10.30] m, y \in[0.0,3.0] m$.

- Diâmetro do injetor: $L_{0}=1.0 \mathrm{~m}$.

- Velocidade no injetor: $U_{0}=1.0 \mathrm{~ms}^{-1}$, com perfil de injeção parabólico.

- Comprimento do canal inicial: $4.0 L_{0}$.

- Distância ente injetor e outflow: $10.0 L_{0}$.

- Diâmetro do outflow: $3.0 L_{0}$.

- Aceleração de campo: $g_{0}=9.81 \mathrm{~ms}^{-2}, g^{x}=0.0, g^{y}=0.0$.

- Parâmetros adimensionais: $F r \approx 0.319275, S t=1.0 t_{0}$.

- Discretização do domínio espacial: $206 \times 60$ células nas direções $x$ e $y$, respectivamente.

- Discretização do domínio temporal: $F_{o}=1.0, F_{C F L}=0.5$.

- Passo adimensional: $\delta m=0.05$.

- Refinamento da malha superficial: $c_{\min }=0.02 \delta m, c_{m a ́ x}=0.2 \delta m$.

- Sistemas lineares:

- Tolerância: $\varepsilon=1.0 \times 10^{-10}$.

- Método para solução de $\widetilde{\mathbf{u}}$ : BiCGstab-SOR $(\omega=1.4)$.

- Método para solução de $\psi$ : BiCGstab-SOR $(\omega=1.8)$.

O modelo acima descrito foi utilizado por todas as formulações do GENSMAC-SXPP, com ambos os esquemas de movimento das partículas marcadoras e, adicionalmente, os seguintes parâmetros adimensionais:

- Oldroyd-B: $R e=0.05, W e=2.0, \beta=0.5$.

- SXPP: $R e=0.05, W e=20.0, \alpha=0.01, \beta=0.5, \gamma=0.8, Q=8.0$.

Em ambos os fluidos, foi considerado no injetor o perfil (6.2.1)-(6.2.2), sendo que os dados de cada modelo foram escolhidos experimentalmente a fim de que os resultados obtidos fossem, visualmente, próximos.

Assim, no caso do modelo Oldroyd-B as simulações numéricas obtidas estão ilustradas na Figura 6.3.1 e detalhadas na Tabela 6.3.1. Por sua vez, os resultados obtidos com o modelo SXPP estão ilustrados na Figura 6.3.2 e detalhados na Tabela 6.3.2.

Para a FE escolhemos $F_{\text {visc }}$ de modo que $\delta t$ ficasse próximo do limite de estabilidade do método neste problema. Já para a FI, escolhemos $F_{\text {visc }}$ com base no compromisso existente entre estabilidade e precisão, fazendo com que seu passo temporal fosse cerca de 50 vezes maior que o empregado pela FE. Como consequência imediata, o tempo de CPU exigido na FI é, aproximadamente, 34 vezes menor que o gasto pela FE, proporcionando soluções numéricas, ainda assim, semelhantes. 
Com efeito, para cada fluido, podemos observar que os resultados obtidos pelas formulações do GENSMAC-SXPP foram visualmente muito parecidos entre si, independentemente do esquema de movimento das partículas. A Tabela 6.3.1 e a Tabela 6.3.2, entretanto, mostram que o RK21 volta a proporcionar um erro menor na massa numérica do fluido, relativamente ao EE, a um custo computacional bastante competitivo.

De forma geral, podemos concluir que a taxa de inchamento calculada para o escoamento envolvendo o fluido Oldroyd-B é de, aproximadamente, $240 \%$. Já no caso do fluido SXPP, os resultados apontam uma taxa de inchamento da ordem de $215 \%$. Em ambos os casos, as diferenças obtidas entre as formulações giram em torno de $1 \%$, constituindo uma margem de diferença bastante pequena.

No Apêndice 2 voltamos a considerar o inchamento do extrudado de fluidos Oldroyd-B e SXPP, onde realizamos, com o GENSMAC-SXPP, um estudo numérico relativo à taxa de inchamento obtida em função dos parâmetros envolvidos em cada modelo. Em especial, os resultados obtidos com o modelo Oldroyd-B são comparados com resultados teóricos e numéricos disponíveis na literatura.
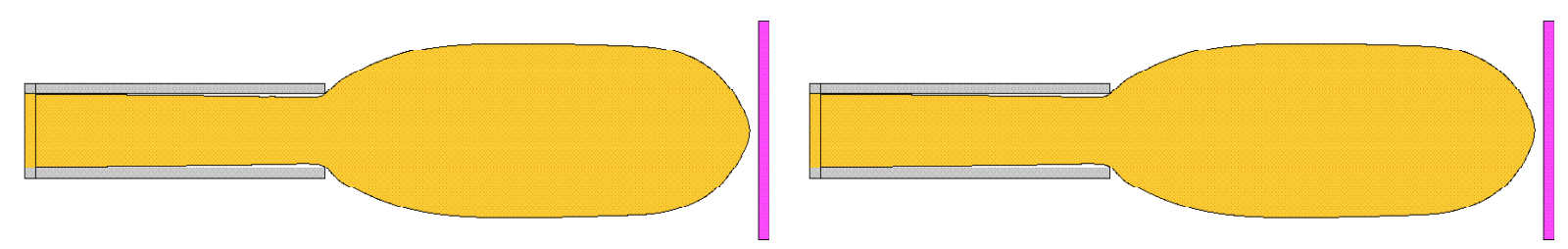

(a)
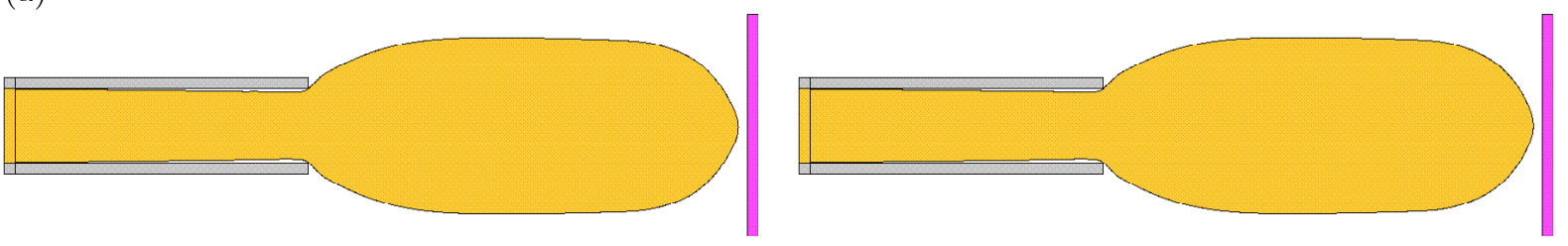

(b)
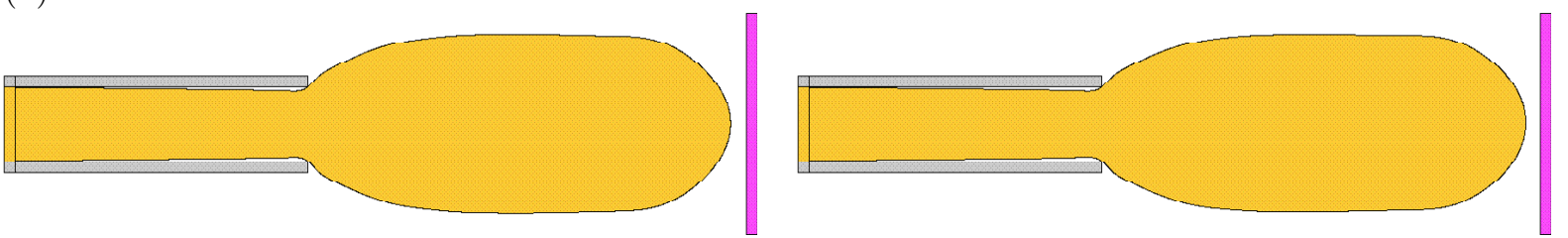

(c)
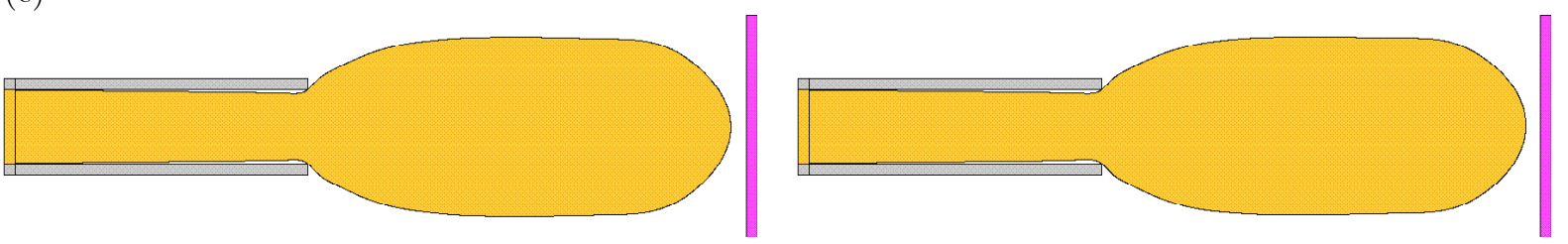

(d)

Figura 6.3.1: Simulações numéricas para o inchamento do extrudado $(t=24.0)$ de um fluido Oldroyd-B $(R e=0.05, W e=2.0, \beta=0.5)$ com: FI-CN (a); FI-EI (b); FE-RK22 (c); FE-EE (d). Na coluna esquerda foi usado EE para movimentar as partículas marcadoras, enquanto que, na coluna direita, adotou-se o RK21. 
Tabela 6.3.1: Comparação dos métodos usados nas simulações numéricas apresentadas na Figura 6.3.1.

\begin{tabular}{|c|c|c|c|c|c|c|c|c|}
\cline { 2 - 9 } \multicolumn{1}{c|}{} & \multicolumn{2}{c|}{$F E-E E$} & \multicolumn{2}{c|}{$F E-R K 22$} & \multicolumn{2}{c|}{$F I-E I$} & \multicolumn{2}{c|}{$F I-C N$} \\
\hline$F_{\text {visc }}$ & \multicolumn{2}{c|}{0.64} & \multicolumn{2}{c|}{0.64} & \multicolumn{2}{c|}{32.0} & \multicolumn{2}{c|}{32.0} \\
ciclos & \multicolumn{2}{c|}{2400000} & \multicolumn{2}{c|}{2400000} & \multicolumn{2}{c|}{48000} & \multicolumn{2}{c|}{48000} \\
\hline \hline particulas & $E E$ & $R K 21$ & $E E$ & $R K 21$ & $E E$ & $R K 21$ & $E E$ & $R K 21$ \\
\hline \hline tempo de $C P U$ & $24: 50: 40$ & $18: 13: 05$ & $27: 32: 21$ & $19: 36: 54$ & $00: 32: 42$ & $00: 32: 08$ & $00: 36: 56$ & $00: 35: 08$ \\
$e_{m}\left(\times 10^{-3}\right)$ & -1.4885 & -1.4133 & -1.4456 & -1.4159 & -3.4006 & -1.8549 & -3.4100 & -1.8660 \\
$S_{r}$ & 2.4211 & 2.4017 & 2.4234 & 2.4014 & 2.3849 & 2.3848 & 2.3846 & 2.3849 \\
\hline
\end{tabular}

Tabela 6.3.2: Comparação dos métodos usados nas simulações numéricas apresentadas na Figura 6.3.2.

\begin{tabular}{|c|c|c|c|c|c|c|c|c|}
\cline { 2 - 8 } \multicolumn{1}{c|}{} & \multicolumn{2}{c|}{$F E-E E$} & \multicolumn{2}{c|}{$F E-R K 22$} & \multicolumn{2}{c|}{$F I-E I$} & \multicolumn{2}{c|}{$F I-C N$} \\
\hline$F_{\text {visc }}$ & \multicolumn{2}{c|}{0.64} & \multicolumn{2}{c|}{0.64} & \multicolumn{2}{c|}{32.0} & \multicolumn{2}{c|}{32.0} \\
ciclos & \multicolumn{2}{c|}{2200000} & \multicolumn{2}{c|}{2200000} & 44000 & 44000 \\
\hline \hline partículas & $E E$ & $R K 21$ & $E E$ & $R K 21$ & $E E$ & $R K 21$ & $E E$ & $R K 21$ \\
\hline \hline tempo de CPU & $17: 00: 01$ & $15: 33: 01$ & $18: 23: 33$ & $17: 16: 54$ & $00: 28: 40$ & $00: 28: 49$ & $00: 35: 32$ & $00: 30: 33$ \\
$e_{m}\left(\times 10^{-4}\right)$ & -22.5404 & -8.7210 & -22.2848 & -8.7286 & -29.3994 & -14.1789 & -29.3766 & -14.1553 \\
$S_{r}$ & 2.1589 & 2.1528 & 2.1522 & 2.1587 & 2.1387 & 2.1416 & 2.1387 & 2.1411 \\
\hline
\end{tabular}
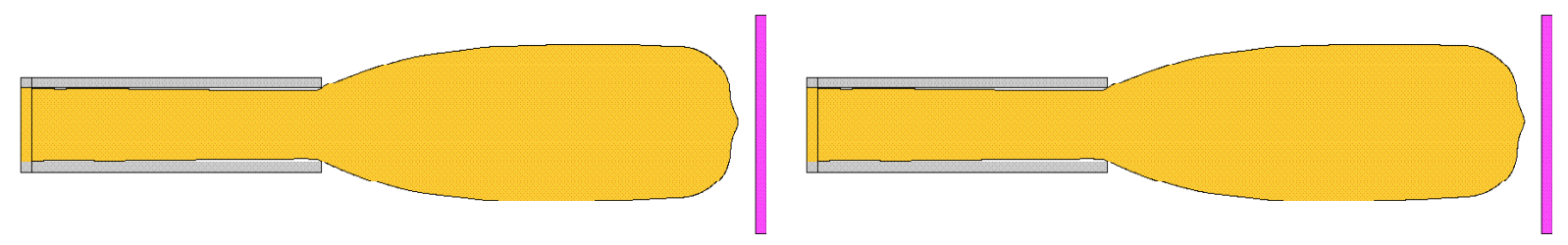

(a)
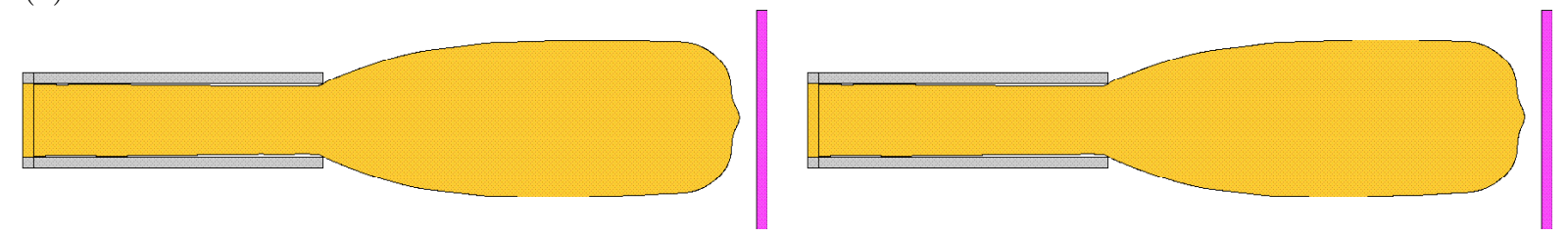

(b)
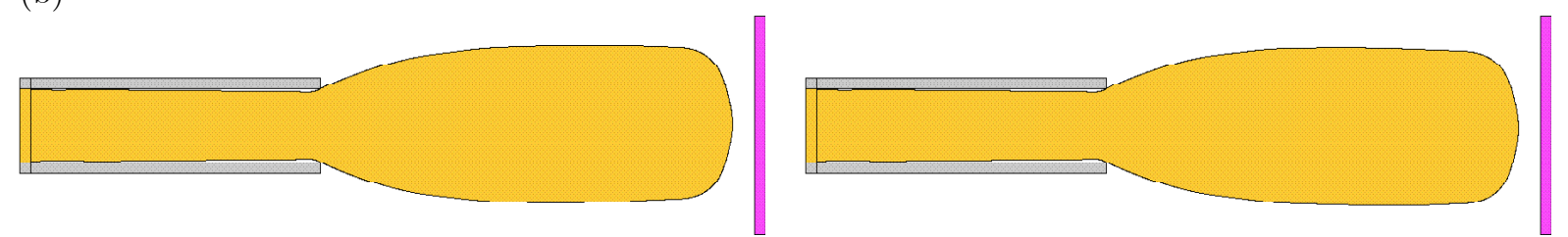

(c)
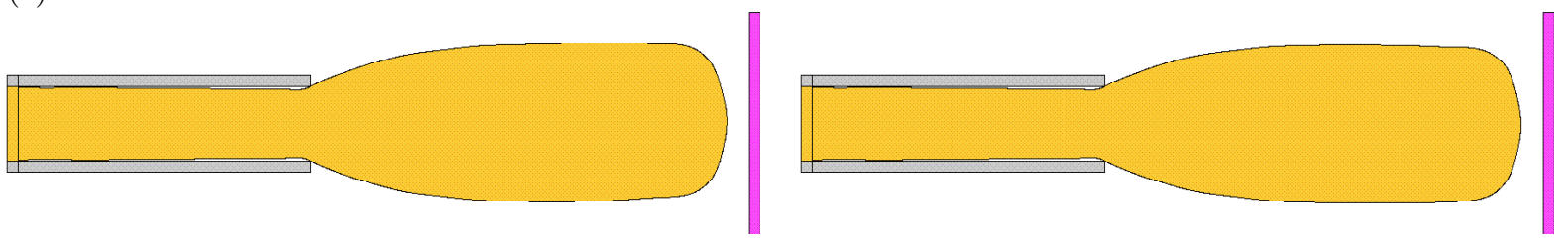

(d)

Figura 6.3.2: Simulações numéricas para o inchamento do extrudado $(t=22.0)$ de um fluido SXPP $(R e=0.05, W e=20.0, \alpha=0.01, \beta=0.5, \gamma=0.8, Q=8.0)$ com: FI-CN (a); FI-EI (b); FE-RK22 (c); FE-EE (d). Na coluna esquerda foi usado EE para movimentar as partículas marcadoras, enquanto que, na coluna direita, adotou-se o RK21. 


\subsection{A gota em impacto}

Consideramos, nesta seção, o problema da gota em impacto de fluido viscoelástico, segundo o modelo:

- Domínio espacial: $x \in[0.0,0.053] m, y \in[0.0,0.0515] m$.

- Diâmetro da gota (esférica): $L_{0}=2.0 \times 10^{-2} \mathrm{~m}$.

- Velocidade inicial da gota: $u^{0}=0.0 \mathrm{~ms}^{-1}, v^{0}=-1.0 \mathrm{~ms}^{-1}$.

- Escala de velocidade: $U_{0}=1.0 \mathrm{~ms}^{-1}$

- Altura inicial (entre o centro da gota e o fundo da caixa): $h_{1}=0.04 \mathrm{~m}$.

- Largura (entre as paredes verticais da caixa): $h_{1}=0.05 \mathrm{~m}$.

- Aceleração de campo: $g_{0}=9.81 m s^{-2}, g^{x}=0.0, g^{y}=-1.0$.

- Parâmetros adimensionais: $F r \approx 2.257618, S t=50.0 t_{0}$.

- Discretização do domínio espacial: $106 \times 103$ células nas direções $x$ e $y$, respectivamente.

- Discretização do domínio temporal: $F_{o}=1.0, F_{C F L}=0.5$.

- Passo adimensional: $\delta m=0.025$.

- Refinamento da malha superficial: $c_{\min }=0.02 \delta m, c_{m a ́ x}=0.2 \delta m$.

- Sistemas lineares:

- Tolerância: $\varepsilon=1.0 \times 10^{-10}$.

- Método para solução de $\widetilde{\mathbf{u}}$ : BiCGstab-SOR $(\omega=1.4)$.

- Método para solução de $\psi$ : BiCGstab-SOR $(\omega=1.8)$.

Neste problema, uma gota de fluido é lançada verticalmente contra uma superfície rígida horizontal. No caso de um fluido newtoniano, após o impacto, a porção de fluido, simplesmente, escoa lateralmente. Para fluidos viscoelásticos, entretanto, a viscoelasticidade faz com que a gota apresente contrações após o impacto, caracterizadas por sucessivos movimentos de retração e expansão, conforme descrito por [TM02A], [GF03] e [PL06], entre outros. Detalhes sobre esse fenômeno são apresentados no Apêndice 3.

Utilizando todas as formulações do GENSMAC-SXPP e os dois esquemas de movimento da superfície livre, o modelo inicialmente apresentado é resolvido com os seguintes parâmetros adimensionais:

- Oldroyd-B: $R e=5.0, W e=1.0, \beta=0.1$.

- SXPP: $R e=5.0, W e=1.0, \alpha=0.01, \beta=0.1, \gamma=0.8, Q=4.0$.

Assim, a Figura 6.4.1 e a Figura 6.4.2 apresentam os resultados obtidos, respectivamente, com os fluidos Oldroyd-B e SXPP. Em ambos os casos, as figuras retratam o instante em que a última contração da gota está sendo desfeita e a mesma volta a escoar lateralmente. Desse modo, detalhes sobre as figuras são apresentados, respectivamente, na Tabela 6.4.1 e na Tabela 6.4.2.

Novamente, para a FE escolhemos $F_{v i s c}$ a fim de que o passo temporal ficasse próximo do limite de estabilidade observado para o problema. Já para a FI, foi adotado um passo temporal 80 vezes maior, possibilitando, ainda assim, resultados visualmente semelhantes.

Podemos observar, então, que os resultados obtidos com o GENSMAC-SXPP e o esquema RK21 para movimentar as partículas da superfície livre são, para cada fluido, muito coerentes entre si, preservando muito bem a simetria do problema. Já para o EE, assim como ocorreu no caso newtoniano, tratado na Seção 4.3.4, temos o aparecimento de pequenas oscilações na superfície livre que deterioram a simetria da solução. 

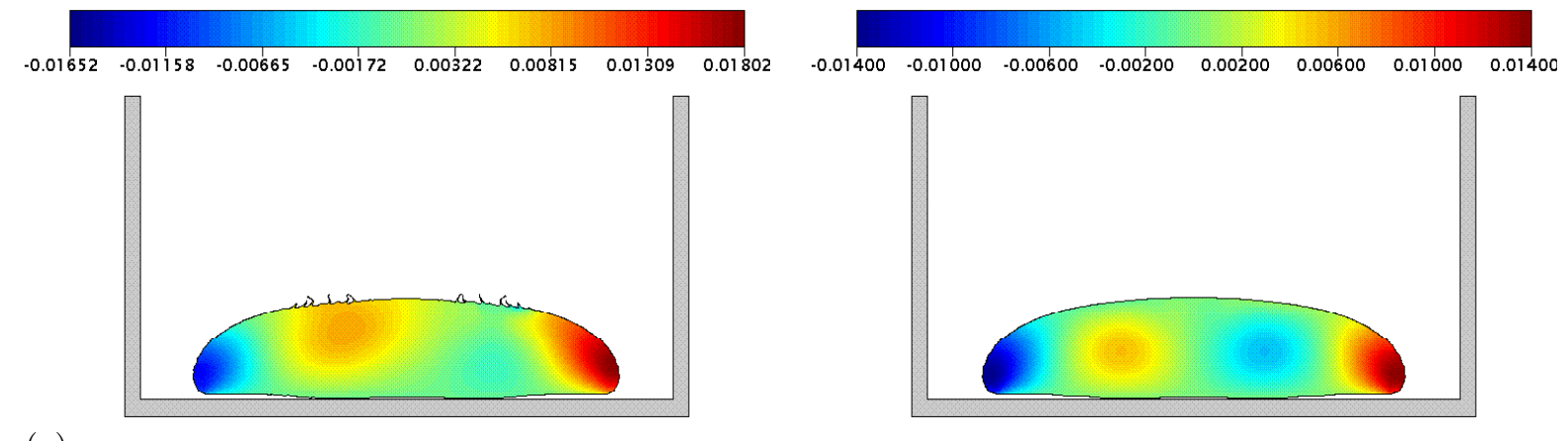

(a)
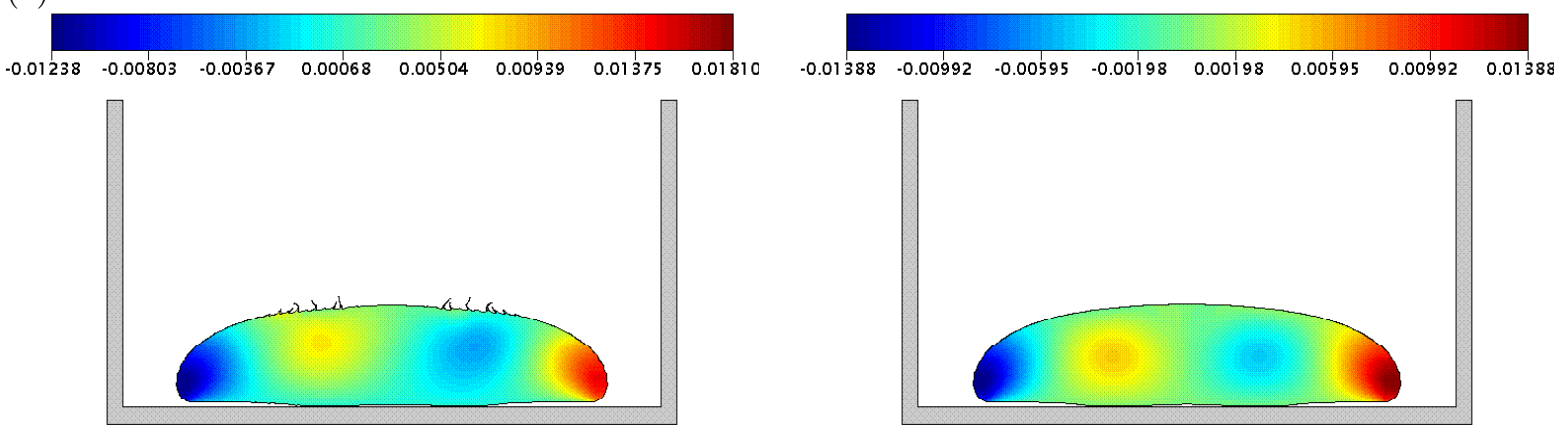

(b)
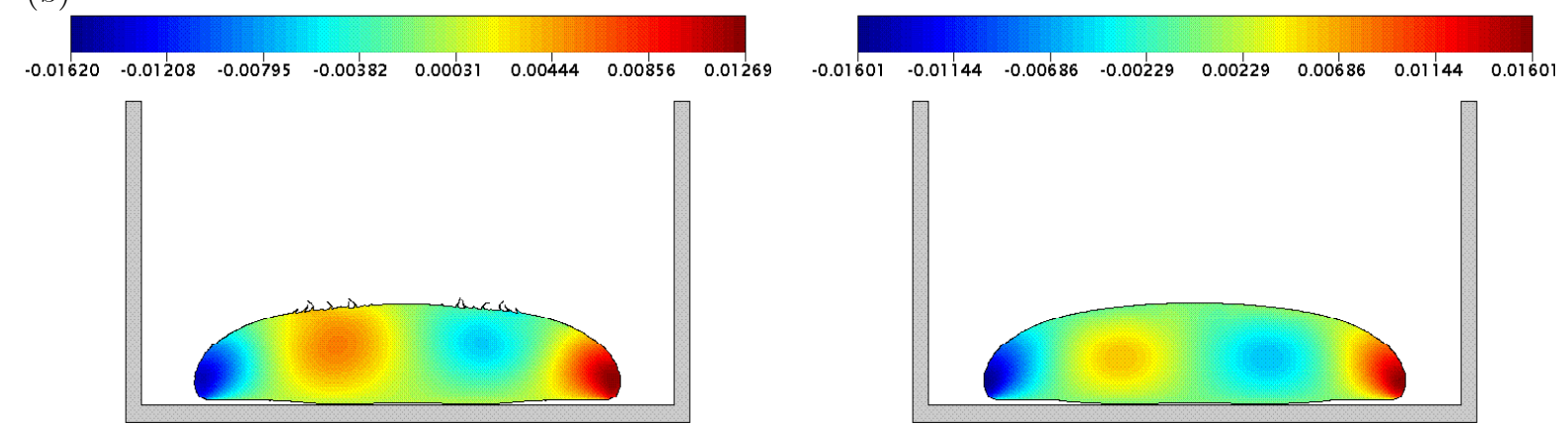

(c)
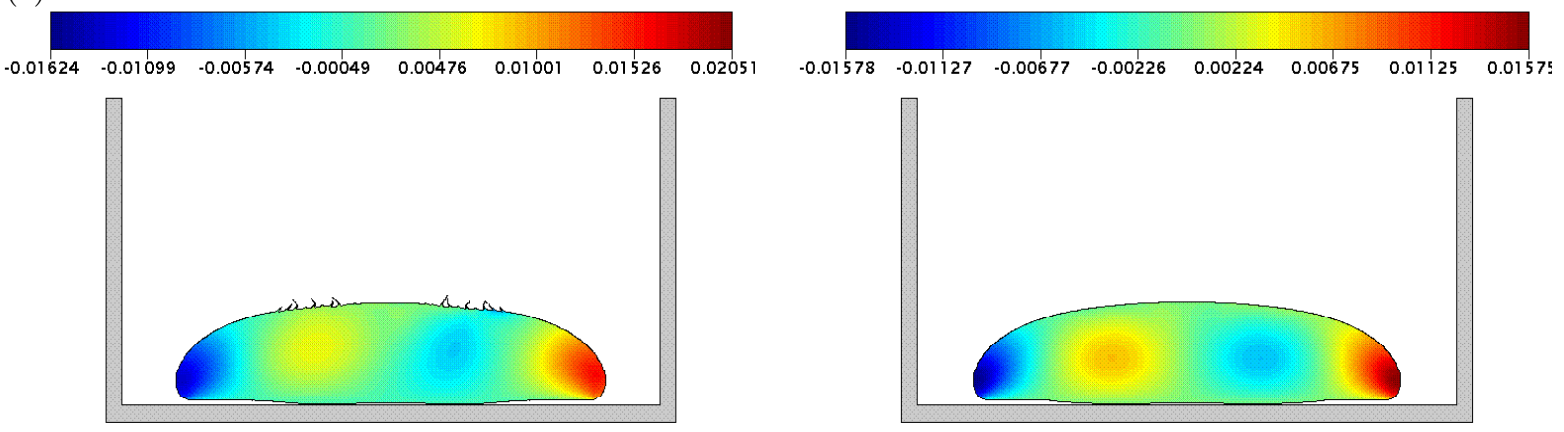

(d)

Figura 6.4.1: Solução numérica para $u$ no problema da gota em impacto $(t=7.0)$ de um fluido Oldroyd-B $(R e=5.0, W e=1.0, \beta=0.1)$ com: FI-CN (a); FI-EI (b); FE-RK22 (c); FE-EE (d). Na coluna esquerda foi usado EE para movimentar as partículas marcadoras, enquanto que, na coluna direita, adotou-se o RK21. 

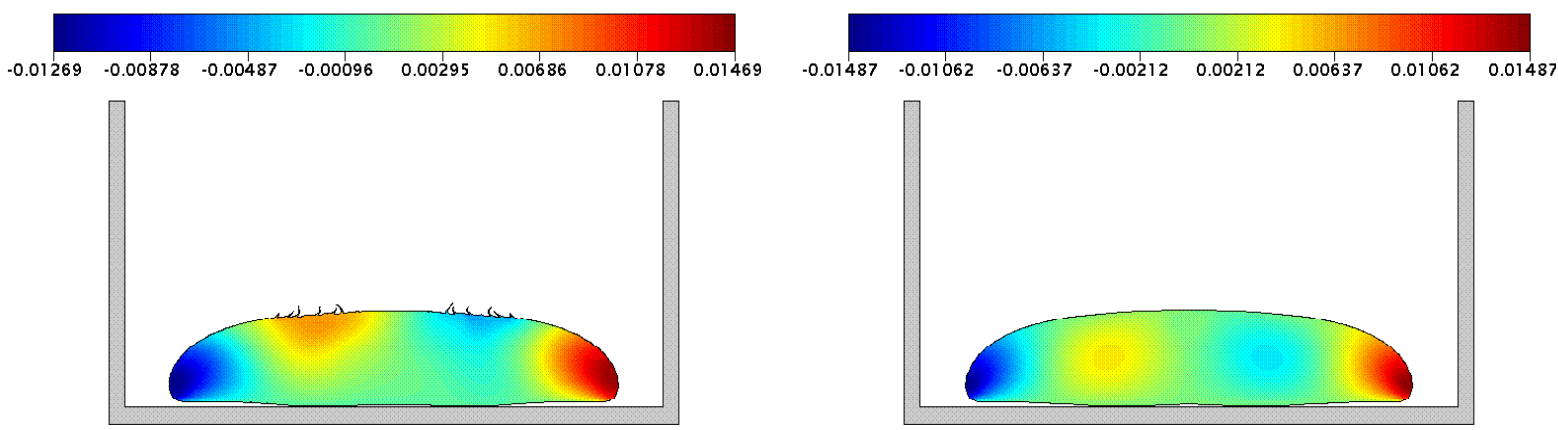

(a)
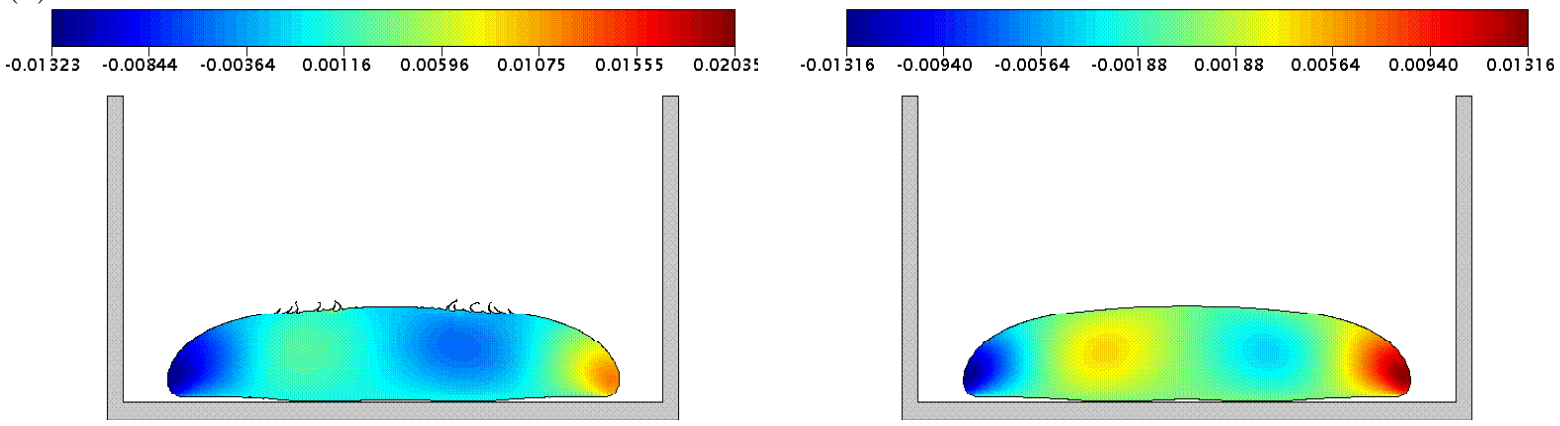

(b)
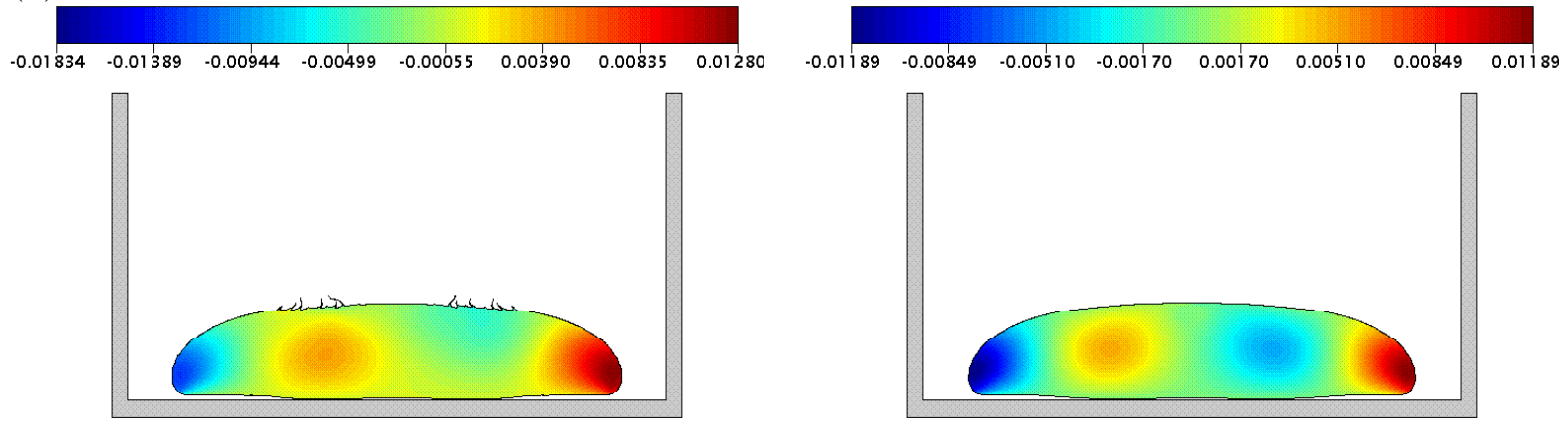

(c)
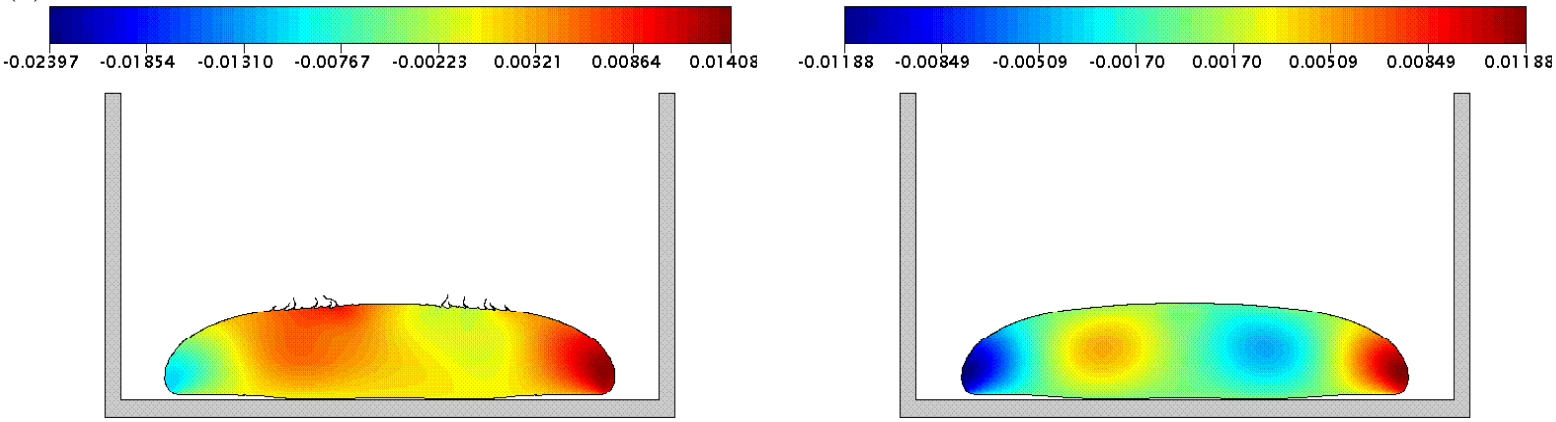

(d)

Figura 6.4.2: Solução numérica para $u$ no problema da gota em impacto $(t=7.0)$ de um fluido SXPP $(R e=5.0, W e=1.0, \alpha=0.01, \beta=0.1, \gamma=0.8, Q=4.0)$ com: FI-CN (a); FI-EI (b); FE-RK22 (c); FE-EE (d). Na coluna esquerda foi usado EE para movimentar as partículas marcadoras, enquanto que, na coluna direita, adotou-se o RK21. 
Tabela 6.4.1: Comparação dos métodos usados nas simulações numéricas apresentadas na Figura 6.4.1.

\begin{tabular}{|c|c|c|c|c|c|c|c|c|}
\multicolumn{1}{c|}{} & \multicolumn{2}{c|}{$F E-E E$} & \multicolumn{2}{c|}{$F E-R K 22$} & \multicolumn{2}{c|}{$F I-E I$} & \multicolumn{2}{c|}{$F I-C N$} \\
\hline$F_{\text {visc }}$ & \multicolumn{2}{c|}{0.32} & \multicolumn{2}{c|}{0.32} & \multicolumn{2}{|c|}{25.6} & \multicolumn{2}{c|}{25.6} \\
ciclos & 280000 & \multicolumn{2}{c|}{280000} & \multicolumn{2}{c|}{3514} & \multicolumn{2}{c|}{3514} \\
\hline \hline partículas & $E E$ & $R K 21$ & $E E$ & $R K 21$ & $E E$ & $R K 21$ & $E E$ & $R K 21$ \\
\hline \hline tempo de CPU & $00: 33: 50$ & $00: 32: 49$ & $00: 41: 08$ & $00: 38: 34$ & $00: 00: 42$ & $00: 00: 46$ & $00: 00: 40$ & $00: 00: 49$ \\
$e_{m}\left(\times 10^{-4}\right)$ & 10.3318 & 1.2940 & 10.0852 & 1.2785 & -11.8026 & -7.3384 & -11.5618 & -7.0820 \\
\hline
\end{tabular}

Tabela 6.4.2: Comparação dos métodos usados nas simulações numéricas apresentadas na Figura 6.4.2.

\begin{tabular}{|c|c|c|c|c|c|c|c|c|}
\cline { 2 - 9 } \multicolumn{1}{c|}{} & \multicolumn{2}{c|}{$F E-E E$} & \multicolumn{2}{c|}{$F E-R K 22$} & \multicolumn{2}{c|}{$F I-E I$} & \multicolumn{2}{c|}{$F I-C N$} \\
\hline ciclos & \multicolumn{2}{c|}{0.32} & \multicolumn{2}{c|}{0.32} & \multicolumn{2}{|c|}{25.6} & \multicolumn{2}{c|}{25.6} \\
visc & 280000 & \multicolumn{2}{c|}{280000} & \multicolumn{2}{c|}{3514} & \multicolumn{2}{c|}{3514} \\
\hline \hline partículas & $E E$ & $R K 21$ & $E E$ & $R K 21$ & $E E$ & $R K 21$ & $E E$ & $R K 21$ \\
\hline \hline tempo de CPU & $00: 45: 42$ & $00: 38: 21$ & $00: 46: 38$ & $00: 52: 30$ & $00: 00: 37$ & $00: 00: 41$ & $00: 00: 48$ & $00: 00: 48$ \\
$e_{m}\left(\times 10^{-4}\right)$ & 12.3370 & 2.0563 & 12.4622 & 2.0170 & -9.4853 & -6.5450 & -8.9719 & -6.3544 \\
\hline
\end{tabular}

\subsection{O jato oscilante}

Nesta seção, consideramos o jato oscilante de fluidos viscoelásticos, adotando o modelo:

- Domínio espacial: $x \in[0.0,0.05] m, y \in[0.0,0.078] m$.

- Diâmetro do injetor: $L_{0}=4.0 \times 10^{-3} \mathrm{~m}$.

- Velocidade no injetor: $U_{0}=0.5 \mathrm{~ms}^{-1}$, com perfil de injeção reto.

- Altura (do injetor ao fundo da caixa): $h_{1}=0.075 \mathrm{~m}$.

- Largura (entre as paredes verticais da caixa): $h_{2}=0.047 \mathrm{~m}$.

- Aceleração de campo: $g_{0}=9.81 \mathrm{~ms}^{-2}, g^{x}=0.0, g^{y}=-1.0$.

- Dados do fluido: $\rho_{0}=1.0 \mathrm{kgm}^{-3}, \mu_{0}=0.2 \mathrm{Nm}^{-2} \mathrm{~s}$.

- Parâmetros adimensionais: $R e=0.01, F r \approx 2.524094, S t=125.0 t_{0}$.

- Discretização do domínio espacial: $100 \times 156$ células nas direções $x$ e $y$, respectivamente.

- Discretização do domínio temporal: $F_{o}=1.0, F_{C F L}=0.5, F_{v i s c}=51.2$.

- Passo adimensional: $\delta m=0.125$.

- Malha superficial: $c_{m i n}=0.02 \delta m, c_{m a ́ x}=0.2 \delta m$, movimentada pelo RK21.

- Sistemas lineares:

- Tolerância: $\varepsilon=1.0 \times 10^{-10}$.

- Método para solução de $\widetilde{\mathbf{u}}$ : BiCGstab-SOR $(\omega=1.4)$.

- Método para solução de $\psi$ : BiCGstab-SOR $(\omega=1.8)$. 

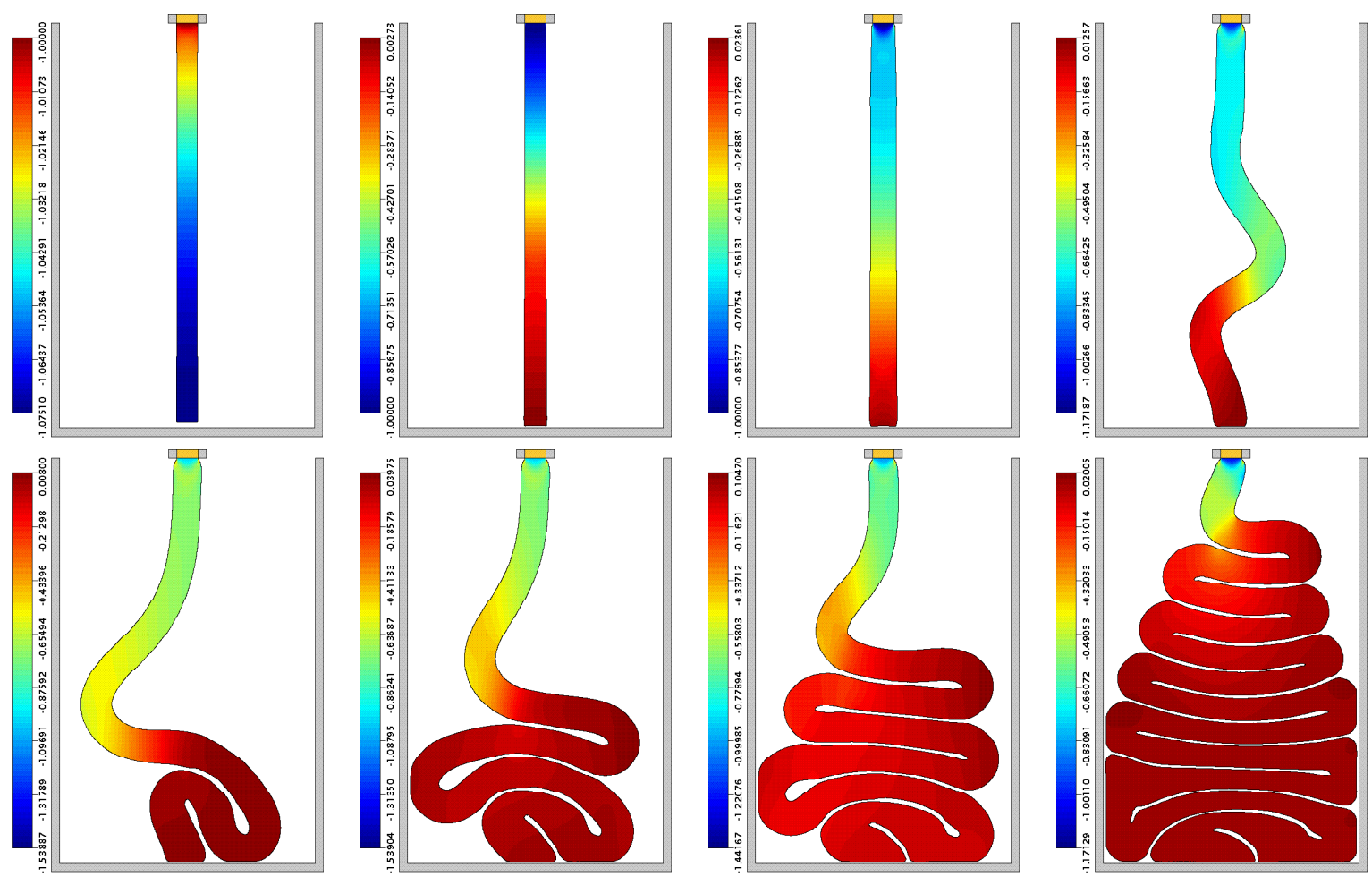

(a)
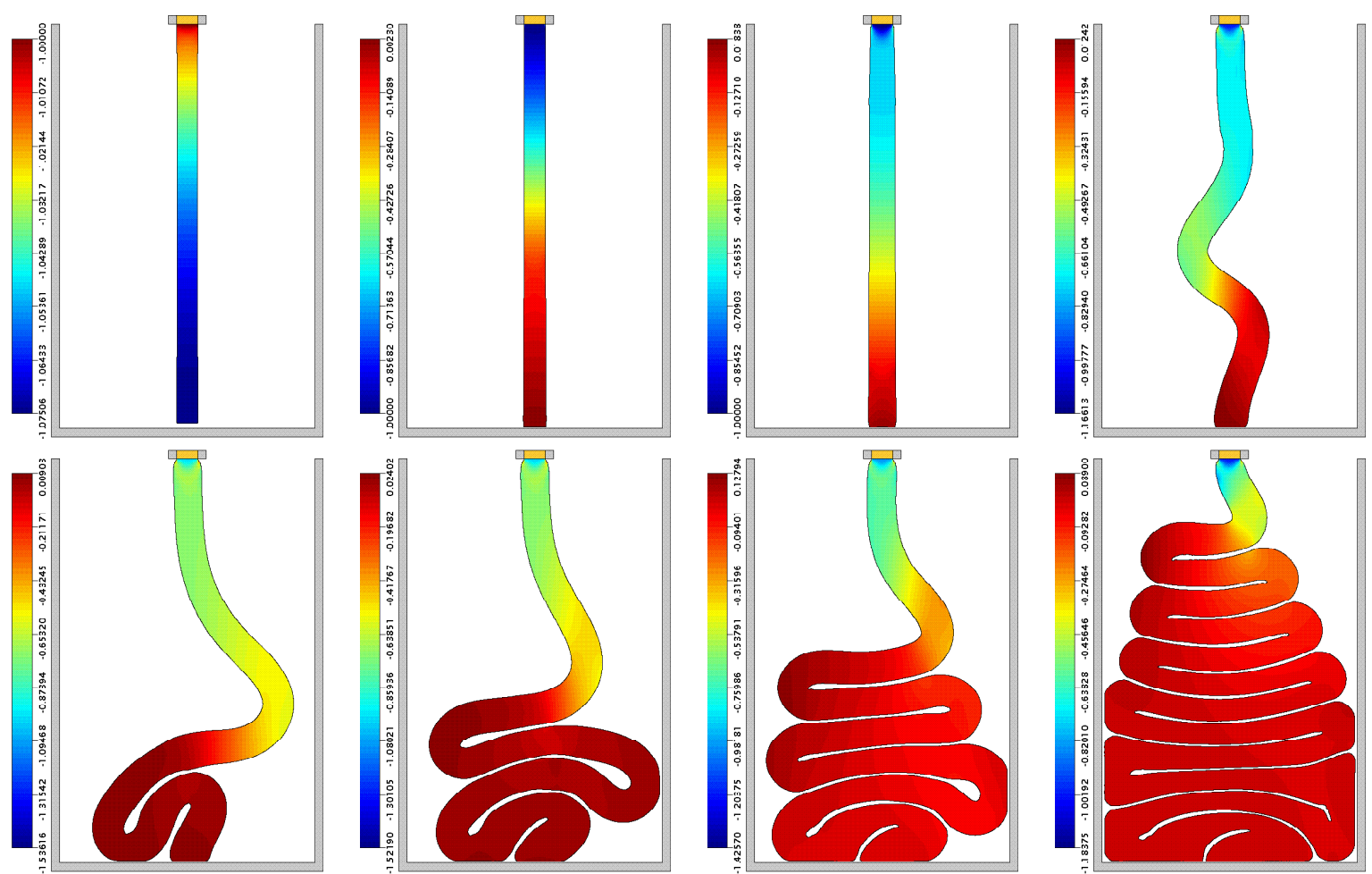

(b)

Figura 6.5.1: Solução numérica para $v$ no jato oscilante de um fluido Oldroyd-B $(R e=0.01, W e=2.0$, $\beta=0.1$ ) com: FI-CN (a); FI-EI (b). Visualização nos instantes $t=18.0, t=19.0, t=23.0, t=30.0$, $t=50.0, t=75.0, t=100.0, t=150.0$. 

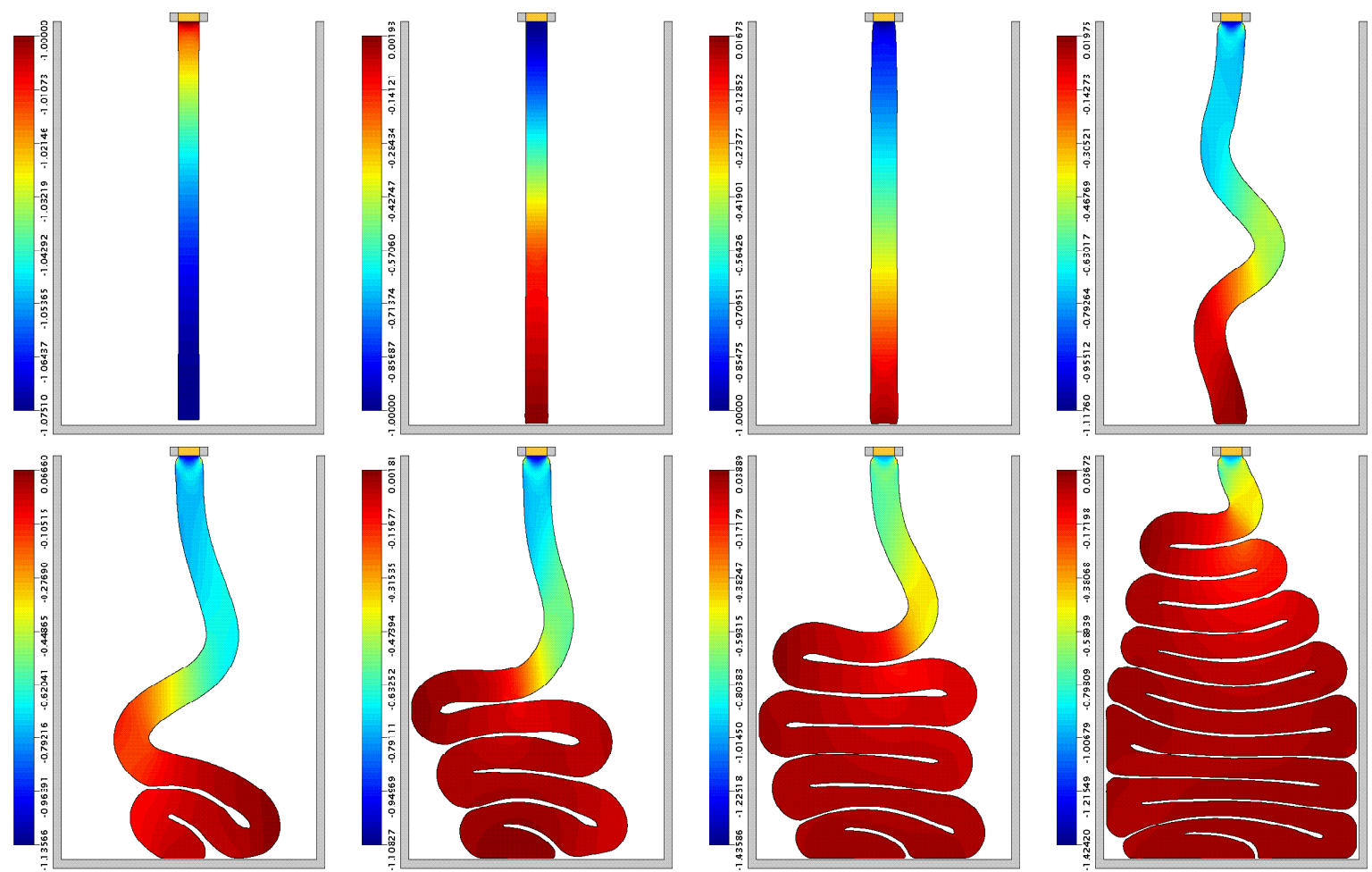

(a)
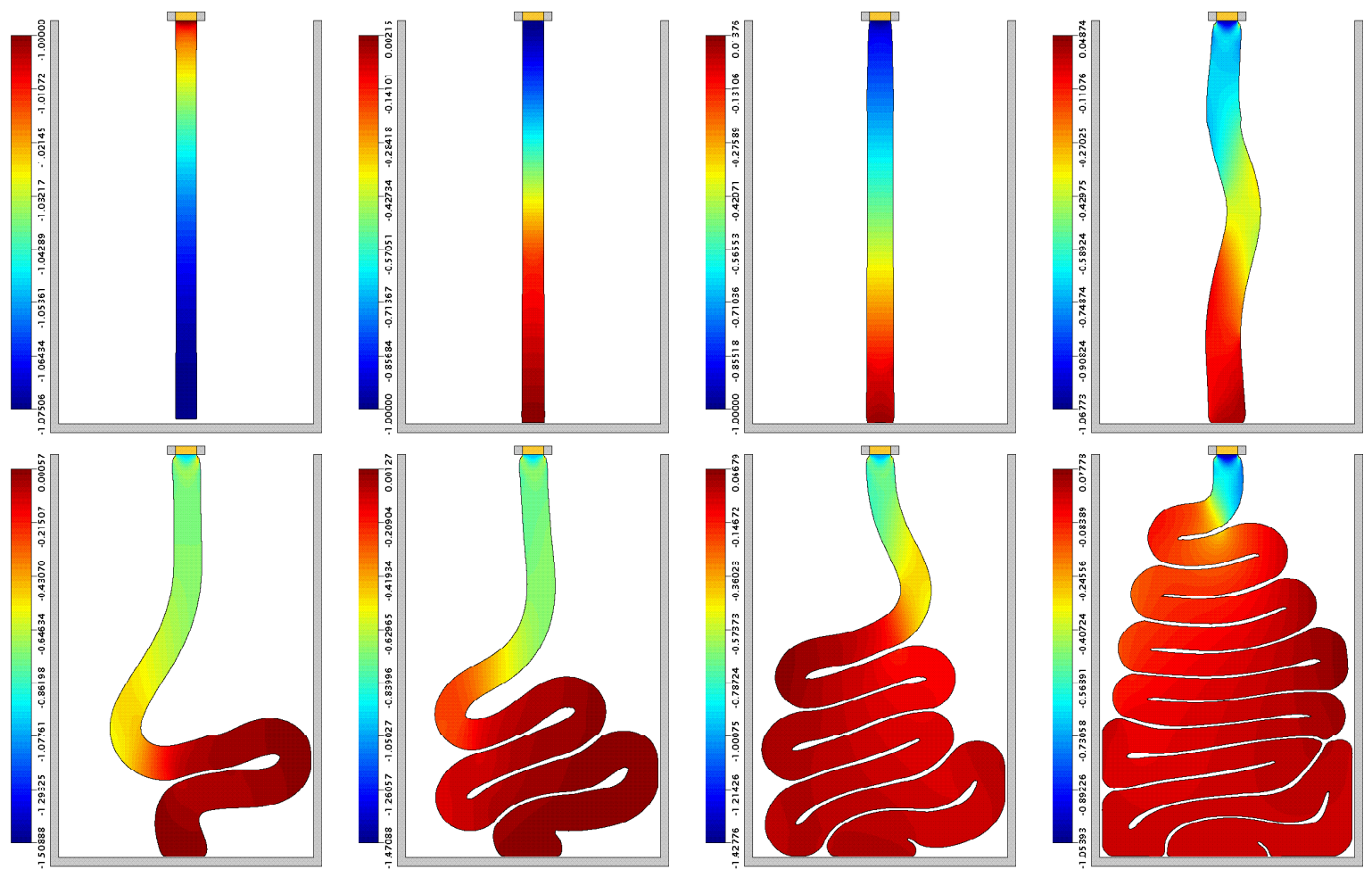

(b)

Figura 6.5.2: Solução numérica para $v$ no jato oscilante de um fluido $\operatorname{SXPP}(R e=0.01, W e=2.0$, $\alpha=0.1, \beta=0.1, \gamma=0.8, Q=4.0)$ com: FI-CN (a); FI-EI (b). Visualização nos instantes $t=18.0$, $t=19.0, t=23.0, t=30.0, t=50.0, t=75.0, t=100.0, t=150.0$. 
O jato oscilante constitui-se um problema, fisicamente, instável. Para fluidos newtonianos, [CJ81] estabelece condições para que ocorra a oscilação do jato de fluido. Em [PL06], realiza-se um estudo numérico que mostra que a viscoelasticidade também influência a ocorrência ou não das "dobras" que caracterizam esse problema.

Aqui, entretanto, não pretendemos investigar o problema em si e nos limitamos a mostrar que o GENSMAC-SXPP consegue simular esse complexo fenômeno. Para utilizarmos um número de Reynolds bastante pequeno, empregamos apenas a FI, produzindo os resultados ilustrados na Figura 6.5.1 e na Figura 6.5.2 para os fluidos Oldroyd-B e SXPP, respectivamente. Nestas simulações, adicionalmente ao modelo apresentado, foram considerados os seguintes parâmetros adimensionais:

- Oldroyd-B: $R e=0.01, W e=2.0, \beta=0.1$.

- SXPP: $R e=0.01, W e=2.0, \alpha=0.1, \beta=0.1, \gamma=0.8, Q=4.0$.

Assim, ligeiras discrepâncias podem ser observadas entre as soluções numéricas produzidas pela FI-EI e pela FI-CN, o que é perfeitamente justificável pela instabilidade física associada ao problema. Entretanto, é interessante observar que, em todos os casos, imediatamente após a colisão da coluna de fluido com o fundo da caixa, ocorre a inversão da velocidade vertical. Tal fenômeno pode ser atribuído à viscoelasticidade do jato, conforme aponta [TM02A].

\subsection{Conclusões}

Utilizamos, neste capítulo, alguns problemas que permitiram mostrar a precisão e robustez do GENSMACSXPP na simulação de escoamentos viscoelásticos com superfícies livre.

Inicialmente, o método numérico foi verificado no escoamento em um canal formado por placas paralelas, demonstrando tratar-se de um método numérico convergente e de segunda ordem de precisão no espaço. Em seguida, foram considerados outros problemas bastante conhecidos no contexto de escoamentos viscoelásticos, onde, apesar da inexistência de solução analítica ou dados experimentais para comparação, o GENSMAC-SXPP voltou a apresentar resultados bastante coerentes, compatíveis com os obtidos com outros modelos viscoelásticos em trabalhos correlatos citados.

Conforme ocorre com o GENSMAC, a FI do GENSMAC-SXPP também elimina a restrição de estabilidade parabólica, possibilitando passos temporais maiores que os admitidos pela FE. Apesar disso, deve-se cuidar para que o aumento em $\delta t$ não prejudique excessivamente a precisão da solução numérica, devendo ser escolhido com base no compromisso entre precisão desejada e poder de processamento disponível.

Portanto, os resultados obtidos neste capítulo apontam que o GENSMAC-SXPP pode simular precisamente escoamentos com superfícies livres. Por outro lado, conforme já citamos, o modelo SXPP é bastante recente, e não são conhecidas na literatura nem soluções analíticas e nem simulações numéricas para escoamentos com superfícies livres desse fluido. Assim, nos apêndices, apresentamos um estudo numérico do modelo SXPP, por meio do GENSMAC-SXPP, evidenciando como os parâmetros do modelo influenciam na solução obtida. Para isso, dois problemas clássicos foram considerados: o escoamento de Hagen-Poiseuille (Apêndice 1) e o inchamento do extrudado (Apêndice 2). Além disso, o Apêndice 3 detalha a solução do problema da gota em impacto dos fluidos Oldroyd-B e SXPP, complementando as informações apresentadas neste capítulo. 


\section{Considerações finais}

O foco deste trabalho foi o estudo de métodos numéricos para a simulação de escoamentos com superfícies livres. Em especial, foi interesse o caso de escoamentos viscosos, a baixos Reynolds.

Para isso, após a apresentação da formulação matemática (equações e condições de contorno) no Capítulo 1, realizamos, no Capítulo 2, uma revisão bibliográfica do GENSMAC e suas formulações. Nesta oportunidade, foi apresentada a formulação implícita, recentemente proposta para a metodologia GENSMAC, a qual se faz importante, principalmente, na simulação de escoamentos a baixos Reynolds, uma vez que elimina a restrição de estabilidade parabólica no passo temporal em escoamentos com superfícies livres.

A partir de então, o restante do trabalho pôde ser dividido em duas partes. Na primeira delas, analisamos algumas dificuldades ainda inerentes à FI do GENSMAC, em termos de eficiência (solução dos sistemas lineares) e precisão (movimento da superfície livre). Essas duas situações críticas, conforme apontamos no Capítulo 3, eram originadas pela possibilidade de aumento no passo temporal, com relação à $\mathrm{FE}$, existente na FI do GENSMAC. Assim, novas estratégias para contornar essas dificuldades foram propostas no Capítulo 3 e testadas no Capítulo 4, produzindo resultados bastante promissores. Nesses testes, aplicamos as modificações propostas também à FE do GENSMAC. A título de experimento de controle, uma versão do método numérico sem a modificação testada sempre foi resolvida, a fim de servir como referência.

Especificamente, a questão da eficiência na FI foi melhorada pela adoção de métodos iterativos e pré-condicionadores mais eficientes. Nesse sentido, o BiCGstab-SOR mostrou-se bastante adequado, produzindo um ganho em eficiência para a FI da ordem de $70 \%$ em tempo total de processamento com relação aos métodos iterativos e pré-condicionadores empregados anteriormente. Embora nenhum estudo teórico tenha sido realizado, os experimentos deixaram claro que, usando bom-senso, é possível efetuar boas escolhas para o parâmetro de relaxação $\omega$ do pré-condicionador SOR, tornando-as próximas do valor ótimo. Com efeito, na solução do sistema linear associado a $\psi$, a escolha $\omega \approx 1.8$ foi, invariavelmente, a mais adequada. Para o sistema ligado a $\widetilde{\mathbf{u}}$, torna-se necessário tomar $\omega$ cada vez mais próximo de 1.0 à medida que $\delta t$ se aproxima de $\delta t_{v i s c}$.

Por sua vez, a questão da precisão na FI foi aprimorada utilizando um novo esquema para o movimento da superfície livre. Tal esquema, denominado RK21, é um método Runge-Kutta de segunda ordem de precisão e mostrou-se, ao longo deste trabalho, mais adequado que o esquema de primeira ordem de precisão anteriormente utilizado (Euler Explícito). De fato, o RK21 mostrou-se não apenas mais preciso que o EE, mas, em diversas oportunidades, agregou mais estabilidade à solução obtida, representando melhor o perfil da superfície livre.

Assim, é importante salientar que os resultados obtidos nesta primeira parte do trabalho, embora relativamente simples, ainda não haviam sido testados. Logo, têm sua importância ao tornarem a FI do GENSMAC ainda mais eficiente e precisa. Aliás, os ganhos com tais resultados foram além do esperado, 
mostrando que podem contribuir, também, com a FE do GENSMAC.

Já na segunda parte do trabalho, a partir dos desenvolvimentos realizados, ampliamos o GENSMAC para simular escoamentos viscoelásticos modelados pela equação constitutiva SXPP, originando o GENSMAC-SXPP. Por ser um modelo recente, são escassos na literatura relatos de resultados numéricos envolvendo o SXPP para escoamentos confinados, sendo inexistentes no caso de superfícies livres. Assim, no Capítulo 5, apresentamos uma metodologia que permite a simulação numérica de escoamentos com superfície livre desse tipo de fluido. No Capítulo 6, a metodologia é verificada e resultados numéricos que apontam a robustez do método proposto são apresentados. Adicionalmente, nos apêndices, são exibidos alguns estudos numéricos realizados por meio da FI do GENSMAC-SXPP.

Portanto, a contribuição inerente a este trabalho pode ser sintetizada em duas partes. Na primeira, propomos e testamos modificações no GENSMAC, no contexto de um fluido newtoniano. Na segunda, ampliamos a metodologia existente para a simulação de escoamentos viscoelásticos modelados pela equação SXPP, originando o GENSMAC-SXPP.

Dessa forma, os resultados obtidos com este trabalho serão comunicados à comunidade na forma de artigos. Resumos abordando algumas partes especíicas do trabalho já foram submetidos e apresentados em congressos da área, como no DINCON 2008, no Congreso de Métodos Numéricos en Ingeniería 2009 e no CNMAC 2009, bem como em seminário periódico local (Seminários do LCAD).

O presente trabalho, entretanto, não esgota o assunto. Assim, possíveis temas para trabalhos futuros dizem respeito à:

- implementação paralela e teste de novos métodos iterativos e pré-condicionadores para a solução dos sistemas lineares;

- teste de outros esquemas para movimento da superfície livre;

- repetir e analisar os testes realizados na primeira parte do trabalho (solução dos sistemas lineares e movimentação da superfície livre) para o caso 3D e em situações nas quais $R e>>1$;

- ampliar o GENSMAC-SXPP para a simulação de escoamentos tridimensionais;

- ampliar o GENSMAC para outros modelos viscoelásticos da família Pom-Pom, tanto para o caso $2 \mathrm{D}$ quanto $3 \mathrm{D}$. 
APÊNDICE

A

\section{Análise do GENSMAC-SXPP: o escoamento de Hagen-Poiseuille}

\section{A.1 Introdução}

No escoamento de Hagen-Poiseuille, voltamos a considerar um canal de placas paralelas, porém, inicialmente cheio de fluido em repouso, com um injetor na extremidade esquerda e, na direita, um ejetor continuado. No instante inicial, o injetor faz com que o fluido passe a escoar e, após algum tempo, o estado estacionário é atingido.

Aqui, estamos interessados no perfil (transversal ao canal) de solução totalmente desenvolvido. Em geral, este problema costuma apresentar solução analítica para, praticamente, todos os modelos de fluido. O SXPP, entretanto, é uma das exceções a essa regra. No caso do modelo newtoniano, o perfil de solução desenvolvida obtido neste problema é dado pela equação (6.2.1). Para o modelo Oldroyd-B, ele é dado por (6.2.1)-(6.2.2).

Assim, como o perfil analítico da solução do modelo SXPP é desconhecido, torna-se interessante aproxima-lo numericamente. Para isso, consideramos a malha M4 do problema apresentado na Seção 6.2.1 e utilizamos a FI-EI e a FI-CN do GENSMAC-SXPP, sempre com $F_{\text {visc }}=64.0$. Impomos, no injetor, o perfil desenvolvido do modelo Oldroyd-B e mantemos a mesma abordagem para a obtenção do perfil transversal da solução descrita quando verificamos o método numérico.

Dessa forma, o objetivo deste apêndice é mostrar como cada parâmetro do modelo influi no perfil de solução desenvolvida. Um estudo semelhante a este foi realizado em [AB05], onde, entretanto, discordamos de um detalhe na abordagem seguida. Isto se deve à condição de simetria imposta na linha central do canal (paralela a suas paredes), a qual é utilizada com o argumento de que a solução é simétrica com relação a esta linha. No entanto, o próprio perfil do Oldroyd-B (6.2.1)-(6.2.2), imposto no injetor, não é simétrico (devido a componente $T^{x y}$ da contribuição não newtoniana). Adicionalmente, conforme fica claro nos resultados obtidos, o mesmo se verifica para os perfis obtidos com o modelo SXPP. Logo, como durante todo este trabalho, nenhuma condição de simetria é usada na obtenção dos resultados apresentados a seguir.

Portanto, no que segue, limitamo-nos apresentar os resultados obtidos com o estudo mencionado, especificando, no início de cada seção, os parâmetros adimensionais utilizados. Por fim, apenas chamamos a atenção para a grande coerência entre os resultados obtidos com a FI-EI e com a FI-CN, o que reforça, em termos qualitativos, a precisão e robustez do GENSMAC-SXPP. 


\section{A.2 Influência de $\alpha$}

Adicionalmente ao modelo considerado, adotamos:

- Parâmetros adimensionais:

- Re $=0.05, W e=3.0, \beta=0.12, \gamma=0.35, Q=2.0$.

- $\alpha \in\{0.0,0.05,0.1,0.2,0.3,0.4,0.5,0.6,0.7,0.8\}$.
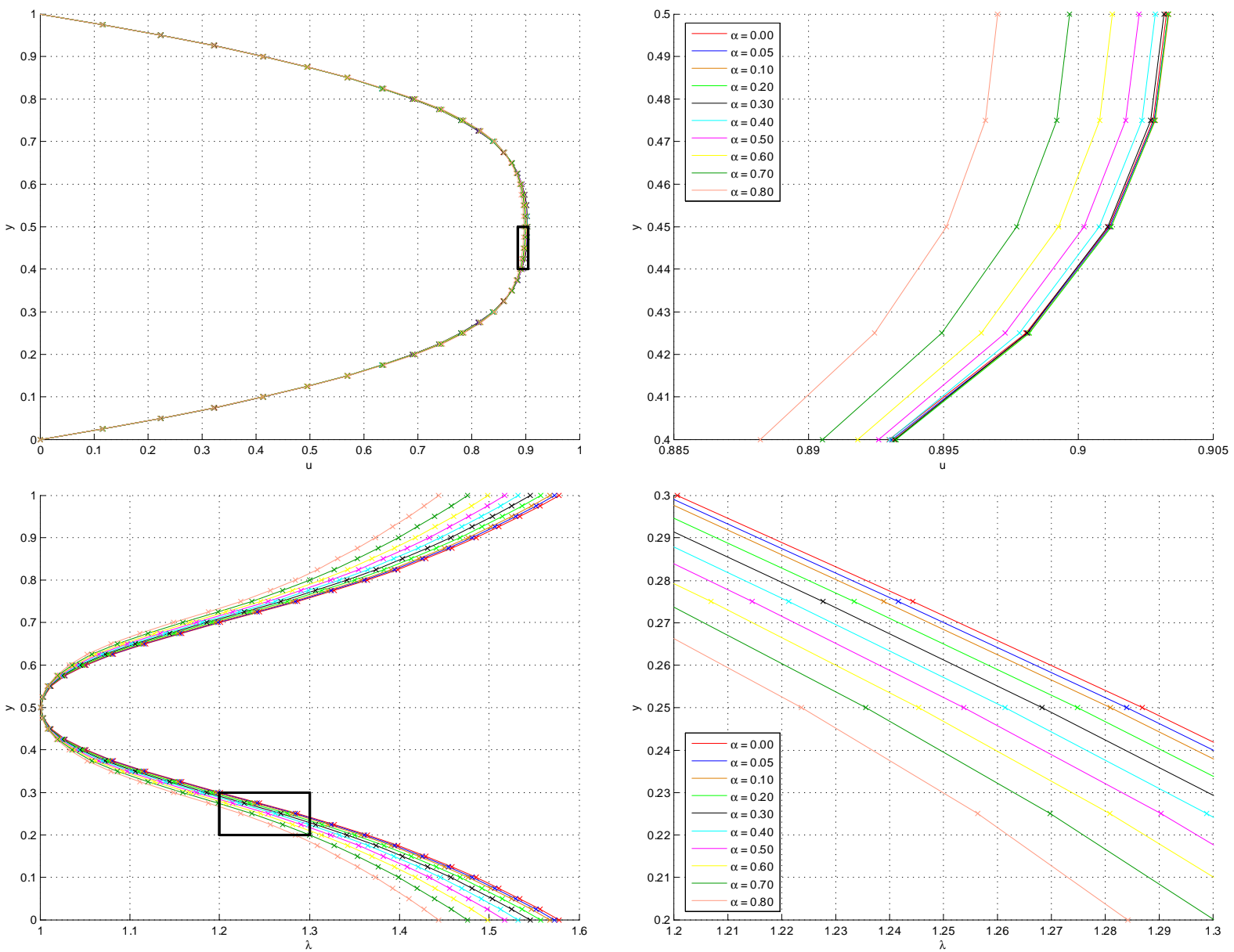

Figura A.2.1: Simulações numéricas para o escoamento de Hagen - Poiseuille de um fluido SXPP $(R e=0.05, W e=3.0, \beta=0.12, \gamma=0.35, Q=2.0)$ : perfis de solução totalmente desenvolvida $(t=100.0)$ de $u$ e $\lambda$, em função de $\alpha$, obtidos com a FI-CN (-) e com a FI-EI (x). 

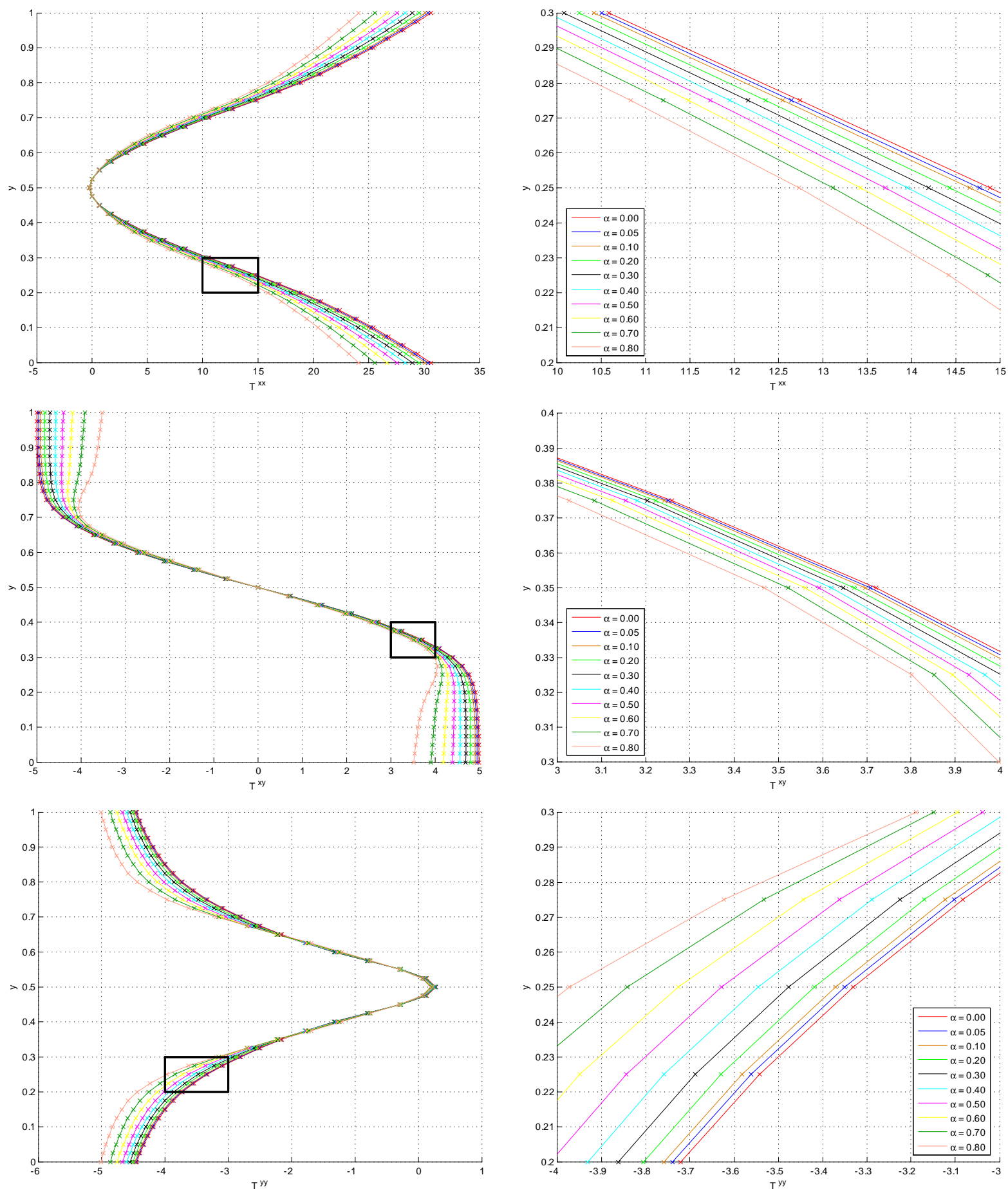

Figura A.2.2: Simulações numéricas para o escoamento de Hagen - Poiseuille de um fluido SXPP $(R e=0.05, W e=3.0, \beta=0.12, \gamma=0.35, Q=2.0)$ : perfis de solução totalmente desenvolvida $(t=100.0)$ de $T$, em função de $\alpha$, obtidos com a FI-CN $(-)$ e com a FI-EI (x). 


\section{A.3 Influência de $\beta$}

Adicionalmente ao modelo considerado, adotamos:

- Parâmetros adimensionais:

$-R e=0.05, W e=3.0, \alpha=0.15, \gamma=0.35, Q=2.0$.

$-\beta \in\{0.1,0.2,0.3,0.4,0.5,0.6,0.7,0.8,0.9\}$.
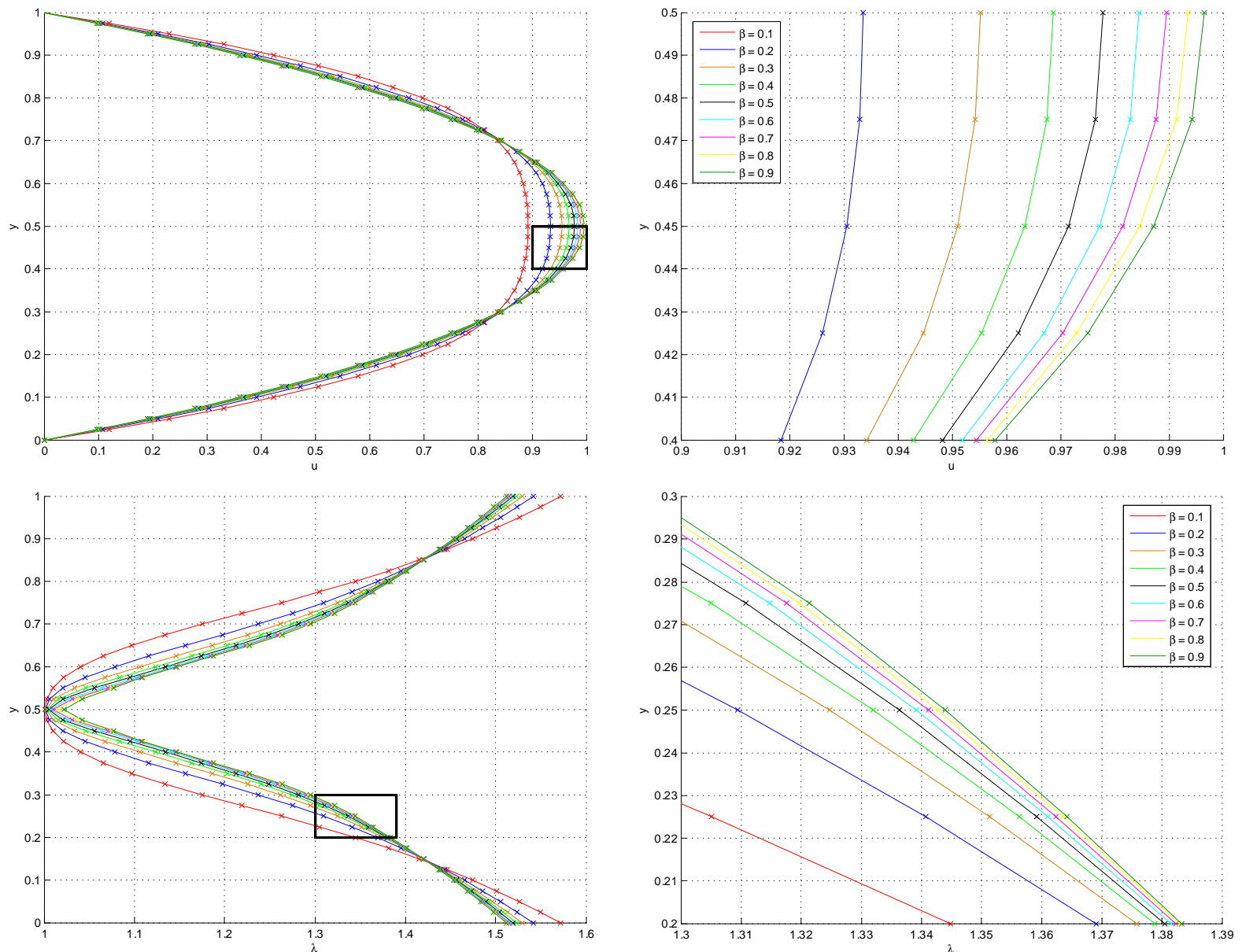

Figura A.3.1: Simulações numéricas para o escoamento de Hagen - Poiseuille de um fluido SXPP $(R e=0.05, W e=3.0, \alpha=0.15, \gamma=0.35, Q=2.0)$ : perfis de solução totalmente desenvolvida $(t=100.0)$ de $u$ e $\lambda$, em função de $\beta$, obtidos com a FI-CN (-) e com a FI-EI (x). 

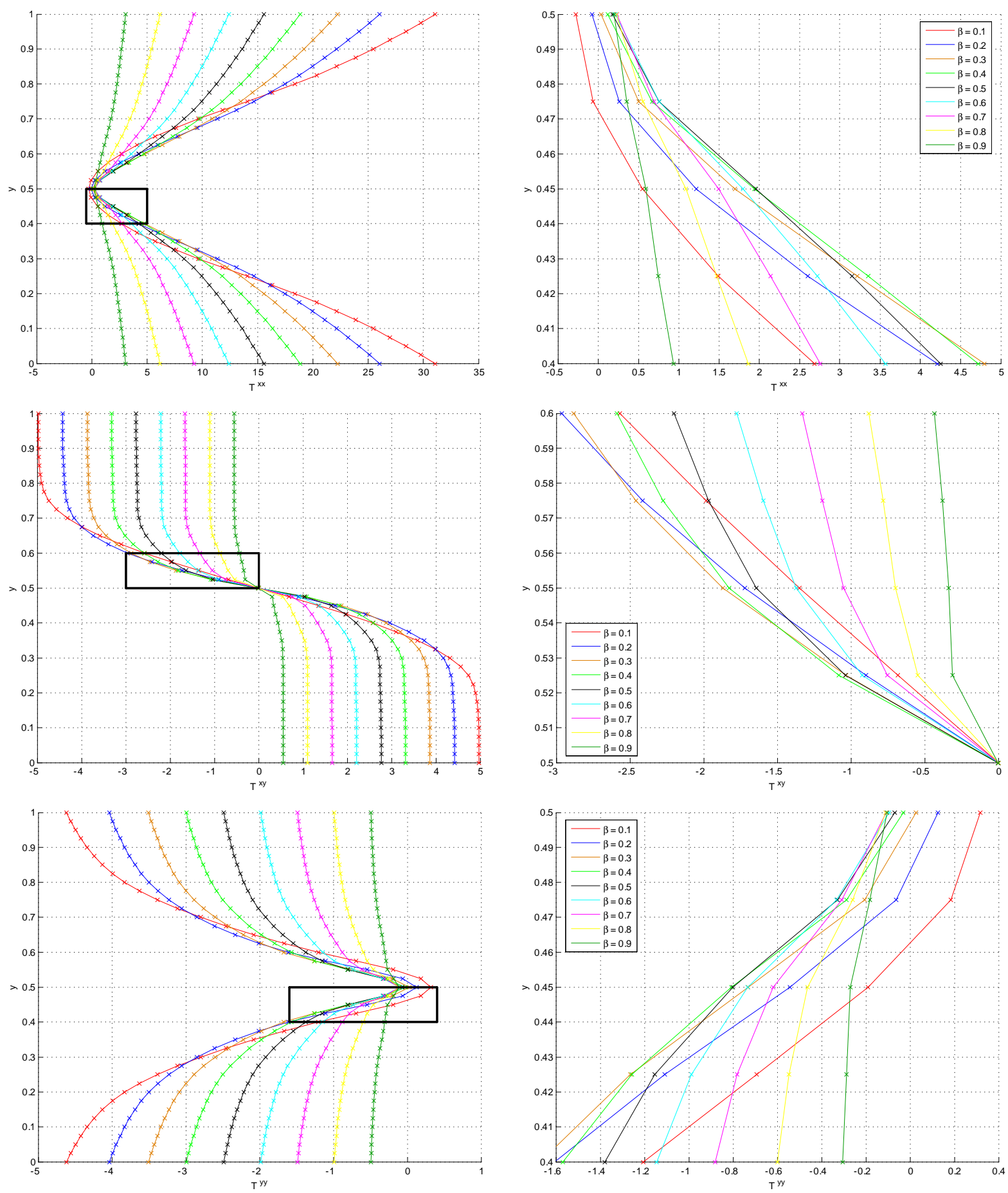

Figura A.3.2: Simulações numéricas para o escoamento de Hagen - Poiseuille de um fluido SXPP $(R e=0.05, W e=3.0, \alpha=0.15, \gamma=0.35, Q=2.0)$ : perfis de solução totalmente desenvolvida $(t=100.0)$ de $T$, em função de $\beta$, obtidos com a FI-CN (-) e com a FI-EI (x). 


\section{A.4 Influência de $\gamma$}

Adicionalmente ao modelo considerado, adotamos:

- Parâmetros adimensionais:

$-R e=0.05, W e=3.0, \alpha=0.15, \beta=0.12, Q=2.0$.

$-\gamma \in\{0.2,0.3,0.4,0.5,0.6,0.7,0.8,0.9\}$.
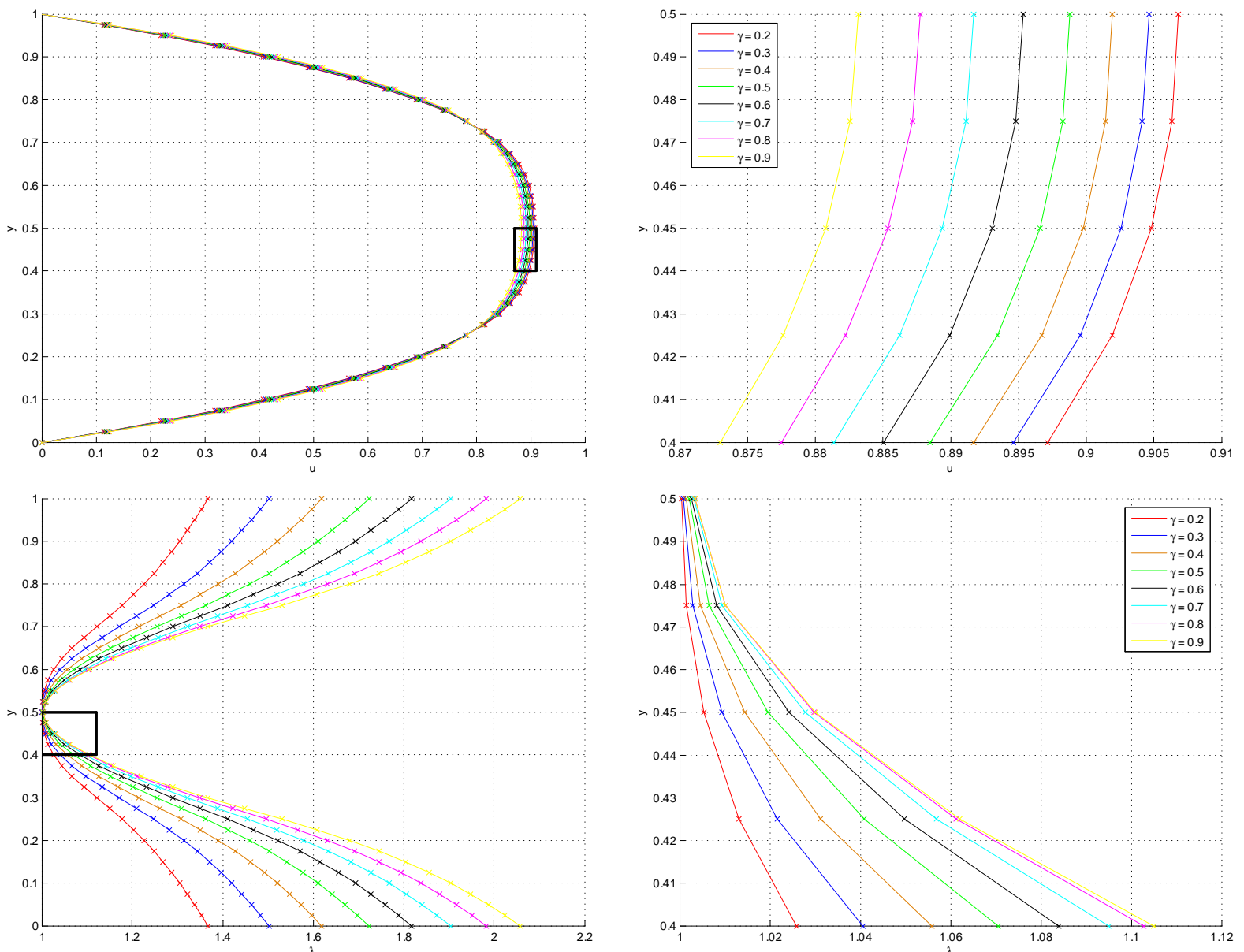

Figura A.4.1: Simulações numéricas para o escoamento de Hagen - Poiseuille de um fluido SXPP $(R e=0.05, W e=3.0, \alpha=0.15, \beta=0.12, Q=2.0)$ : perfis de solução totalmente desenvolvida $(t=100.0)$ de $u$ e $\lambda$, em função de $\gamma$, obtidos com a FI-CN (-) e com a FI-EI (x). 

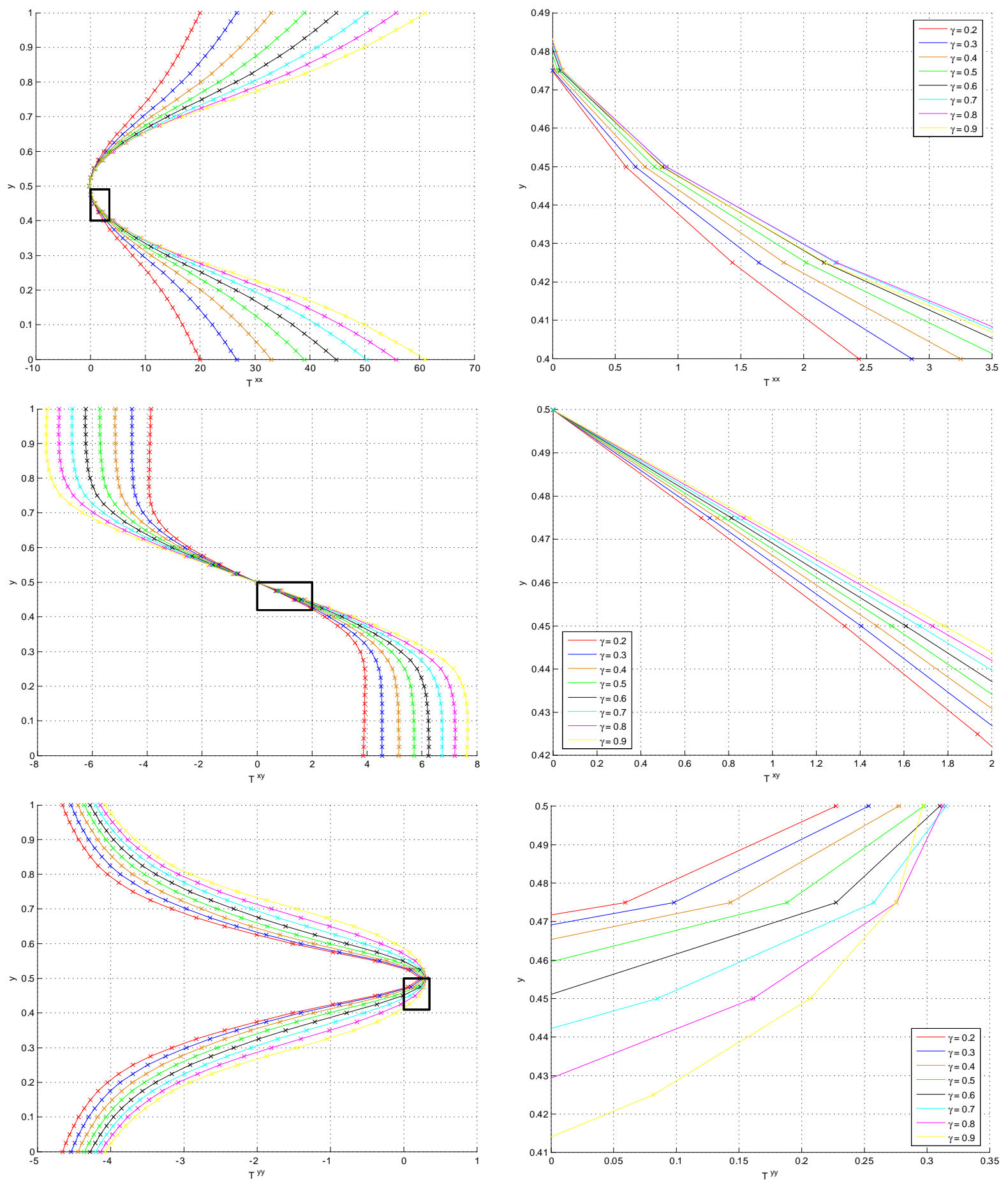

Figura A.4.2: Simulações numéricas para o escoamento de Hagen - Poiseuille de um fluido SXPP $(R e=0.05, W e=3.0, \alpha=0.15, \beta=0.12, Q=2.0)$ : perfis de solução totalmente desenvolvida $(t=100.0)$ de $T$, em função de $\gamma$, obtidos com a FI-CN $(-)$ e com a FI-EI $(\mathrm{x})$. 


\section{A.5 Influência de $W e$}

Adicionalmente ao modelo considerado, adotamos:

- Parâmetros adimensionais:

- Re $=0.05, \alpha=0.15, \beta=0.12, \gamma=0.35, Q=2.0$.

- We $\in\{0.5,1.0,2.0,4.0,6.0,8.0,10.0,12.0,14.0,16.0,20.0\}$.
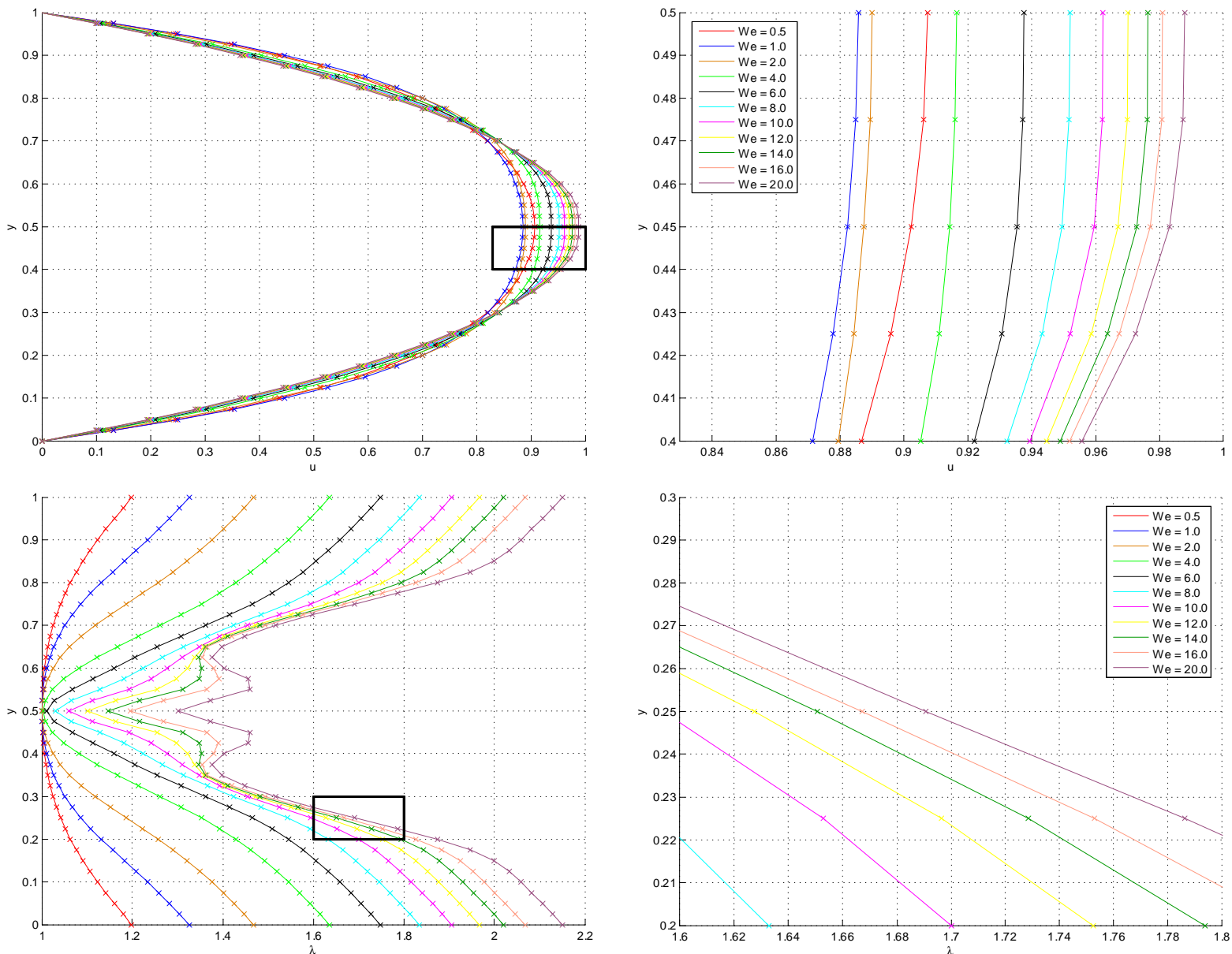

Figura A.5.1: Simulações numéricas para o escoamento de Hagen - Poiseuille de um fluido SXPP $(R e=0.05, \alpha=0.15, \beta=0.12, \gamma=0.35, Q=2.0)$ : perfis de solução totalmente desenvolvida $(t=100.0)$ de $u$ e $\lambda$, em função de $W e$, obtidos com a FI-CN (-) e com a FI-EI (x). 

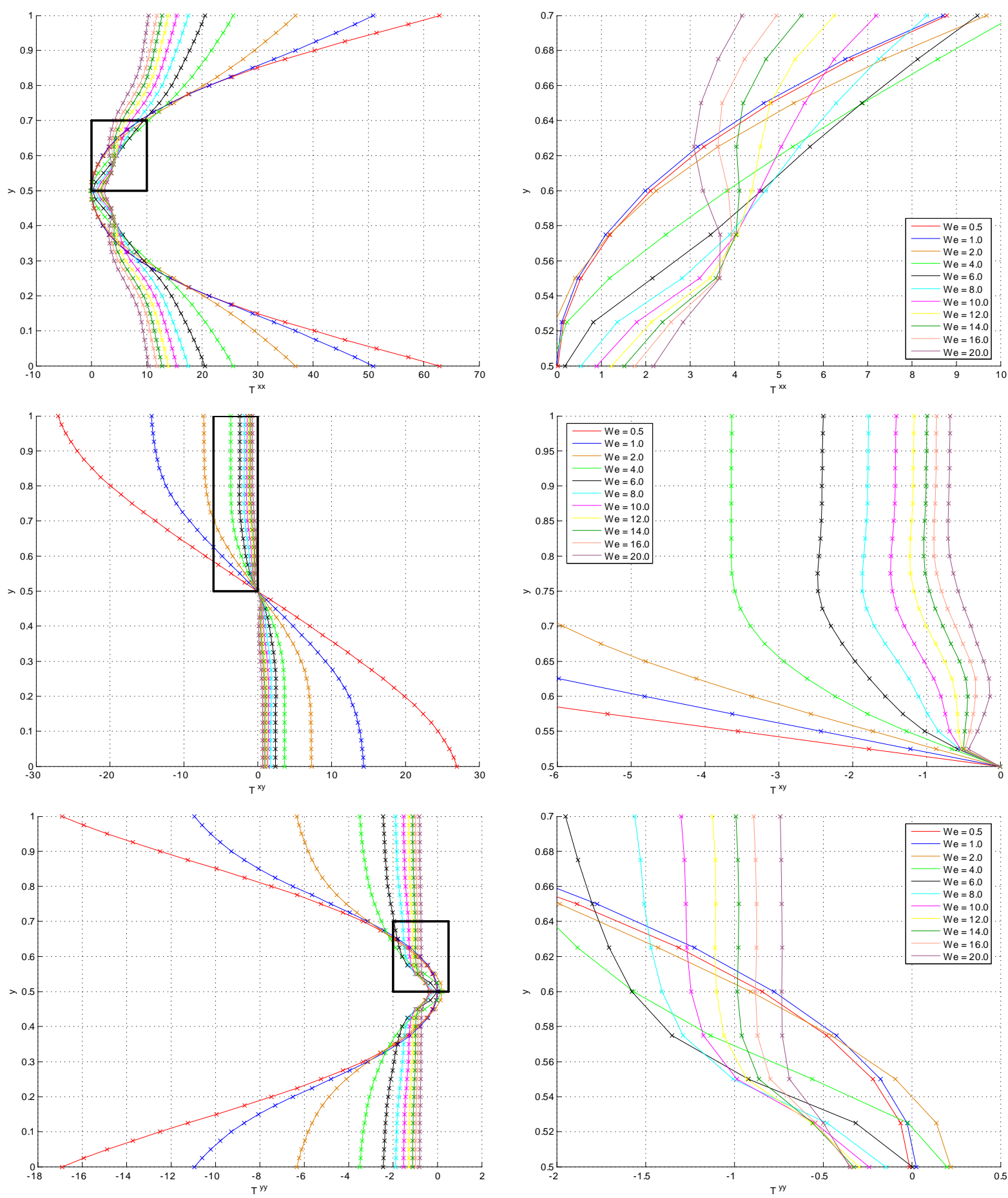

Figura A.5.2: Simulações numéricas para o escoamento de Hagen - Poiseuille de um fluido SXPP $(R e=0.05, \alpha=0.15, \beta=0.12, \gamma=0.35, Q=2.0)$ : perfis de solução totalmente desenvolvida $(t=100.0)$ de $T$, em função de $W e$, obtidos com a FI-CN (-) e com a FI-EI (x). 


\section{A.6 Influência de $Q$}

Adicionalmente ao modelo considerado, adotamos:

- Parâmetros adimensionais:

$-R e=0.05, W e=3.0, \alpha=0.15, \beta=0.12, \gamma=0.35$.

$-Q \in\{1.0,2.0,3.0,5.0,7.0,9.0,12.0,16.0,20.0\}$.
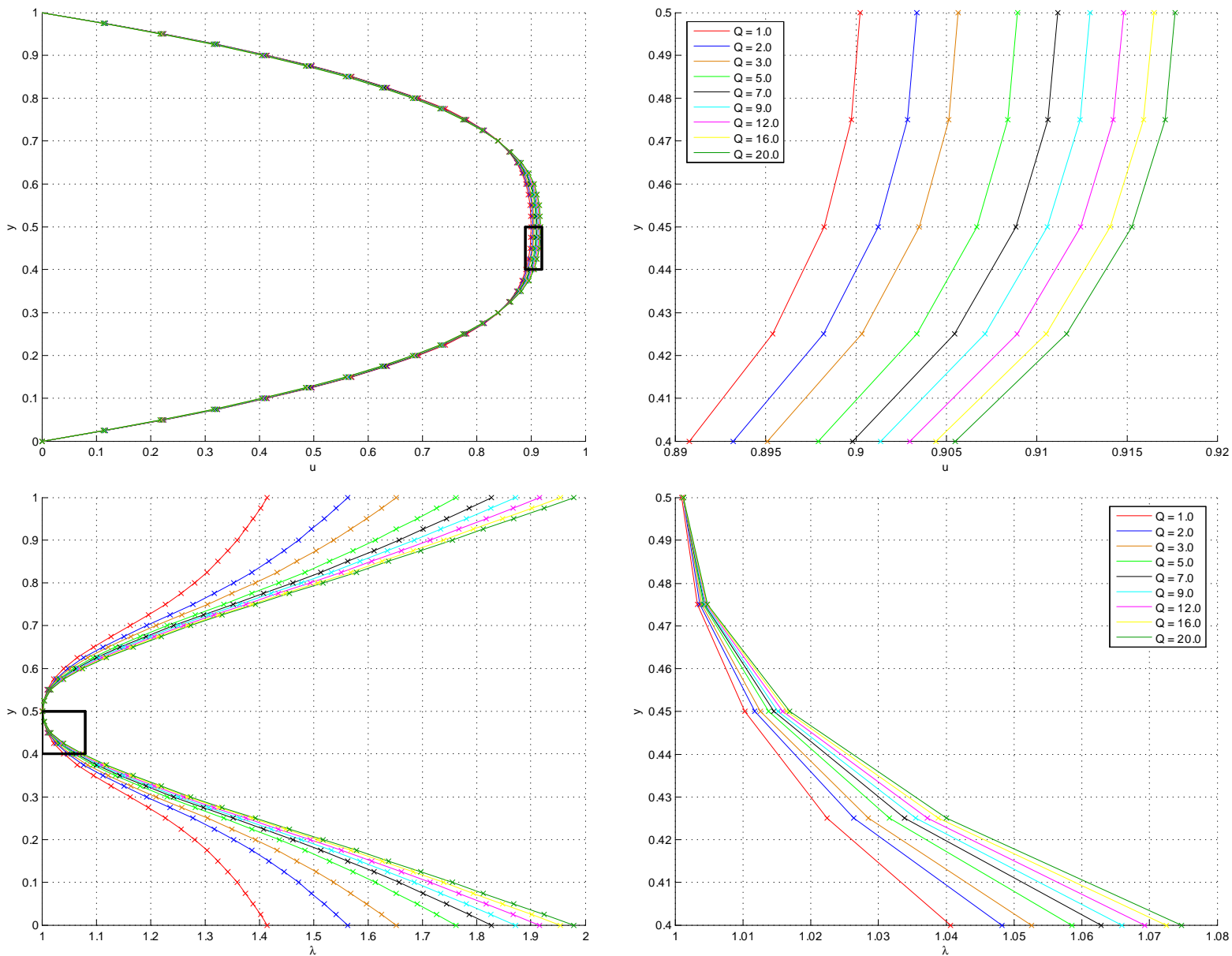

Figura A.6.1: Simulações numéricas para o escoamento de Hagen - Poiseuille de um fluido SXPP $(R e=0.05, W e=3.0, \alpha=0.15, \beta=0.12, \gamma=0.35)$ : perfis de solução totalmente desenvolvida $(t=100.0)$ de $u$ e $\lambda$, em função de $Q$, obtidos com a FI-CN (-) e com a FI-EI (x). 

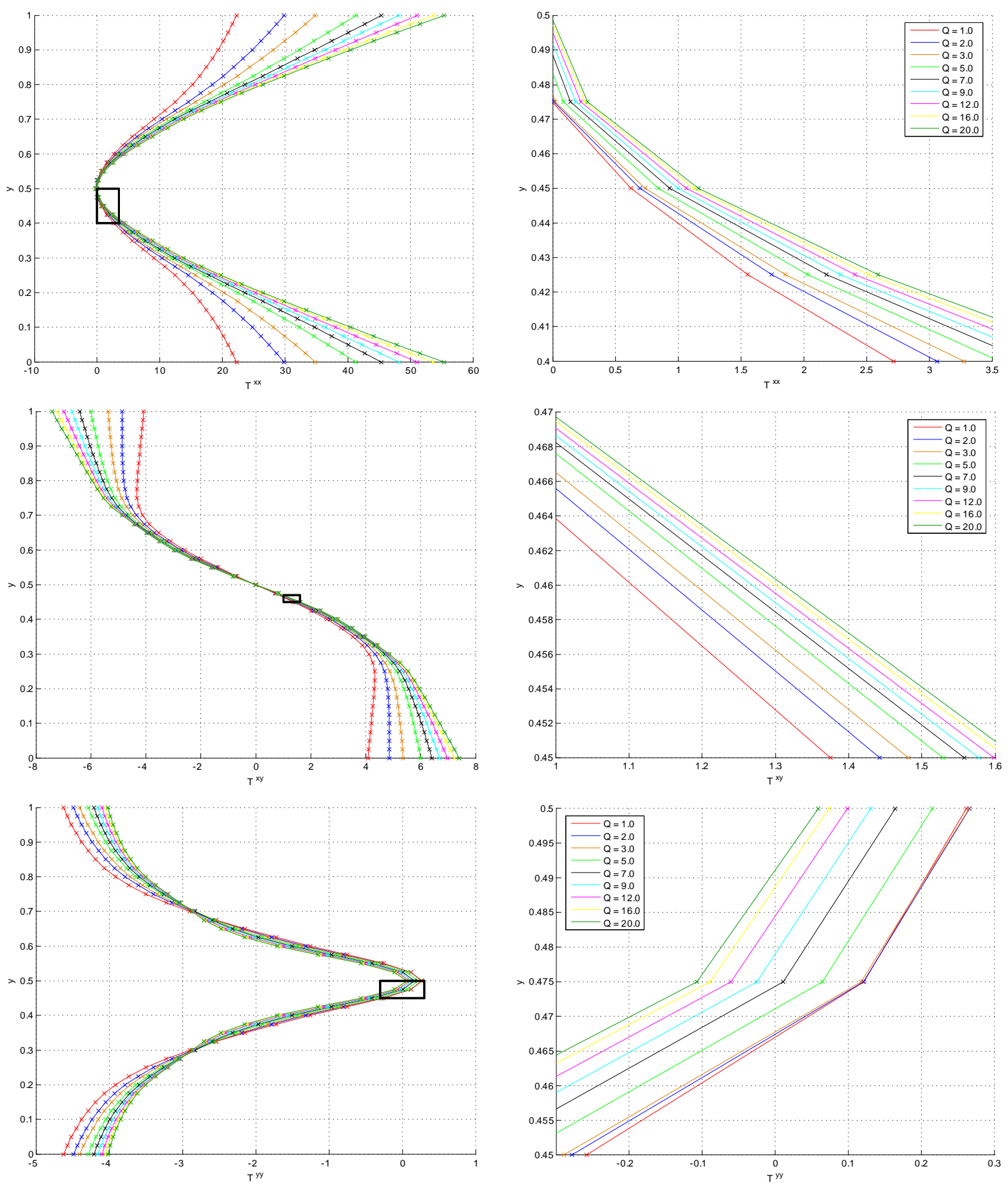

Figura A.6.2: Simulações numéricas para o escoamento de Hagen - Poiseuille de um fluido SXPP $(R e=0.05, W e=3.0, \alpha=0.15, \beta=0.12, \gamma=0.35)$ : perfis de solução totalmente desenvolvida $(t=100.0)$ de $T$, em função de $Q$, obtidos com a FI-CN $(-)$ e com a FI-EI $(\mathrm{x})$. 
122 APENNDICE A. ANÁLISE DO GENSMAC-SXPP: O ESCOAMENTO DE HAGEN-POISEUILLE 
APÊNDICE

$\mathrm{B}$

\section{Análise do GENSMAC-SXPP: o inchamento do extrudado}

\section{B.1 Introdução}

Consideramos, neste apêndice, o inchamento do extrudado de fluidos Oldroyd-B e SXPP. Para isso, utilizamos o problema apresentado na Seção 6.3, o qual foi resolvido pela FI-EI e pela FI-CN do GENSMACSXPP, utilizando o RK21 para movimentar a superfície livre.

Nosso interesse consiste em analisar o comportamento da taxa de inchamento $S_{r}$, definida em (6.3.1), como função de cada parâmetro dos fluidos considerados. Em outras palavras, desejamos saber como se comporta $S_{r}$ quando variamos um dado parâmetro do modelo, mantendo os demais fixos.

No caso do modelo Oldroyd-B existem alguns resultados experimentais e aproximações analíticas, que fornecem indícios do comportamento de $S_{r}$, conforme mostramos a seguir. Quanto ao modelo SXPP, entretanto, não são conhecidos relatos na literatura de sua aplicação neste problema, e assim, o estudo aqui realizado torna-se pertinente e inédito.

Nas seções seguintes, as figuras e tabelas são auto-explicativas. Os dados adicionais ao problema da Seção 6.3 são citados oportunamente, para cada conjunto de simulações apresentado. Em todos os casos, medimos a taxa de inchamento $S_{r}$ em três instantes distintos: imediatamente após a extrusão do canal inicial, antes do ejetor continuado ser atingido e, por fim, depois que o estado estacionário foi alcançado. Tais dados são apresentados em tabelas e gráficos, evidenciando a influência do parâmetro analisado na taxa $S_{r}$ de inchamento do extrudado.

Conforme fica nítido no decorrer do apêndice, os resultados obtidos pela FI-EI e pela FI-CN são coerentes entre si, apontando, qualitativamente, a robustez do GENSMAC-SXPP. Assim, para dois casos extremos do parâmetro analisado, exibimos as curvas de nível das soluções obtidas, alternadamente, por cada um dos métodos empregados. Além disso, algumas simulações numéricas são exibidas em instantes de tempo distintos, ilustrando a influência do parâmetro considerado na evolução da taxa de inchamento.

\section{B.2 Modelo Oldroyd-B}

\section{B.2.1 Influência de $W e$}

Adicionalmente ao modelo considerado, adotamos:

- Parâmetros adimensionais:

$$
\begin{aligned}
& -R e=0.05, \beta=0.5 . \\
& -W e \in\{0.2,0.4,0.6,0.8,1.0,1.2,1.4,1.6,1.8,2.0\} .
\end{aligned}
$$


- Fator de controle do passo temporal: $F_{\text {visc }}=32.0$.

Para este caso, faz-se possível comparar os resultados obtidos com a aproximação analítica proposta por Tanner. Segundo esta e de acordo com [BH87], temos

$$
S_{r}=0.1+\left[1+\frac{1}{2}\left(\frac{T^{x x}-T^{y y}}{2 T^{x y}}\right)^{2}\right]^{\frac{1}{6}}=0.1+\left[1+\frac{1}{2}\left(S_{R}\right)^{2}\right]^{\frac{1}{6}},
$$

devendo $S_{R}$ ser avaliado na parede do canal. Por [TM02A], no modelo Oldroyd-B,

$$
S_{R}=6 W e .
$$

Tabela B.2.1: Taxas de inchamento de um fluido Oldroyd-B $(R e=0.05, \beta=0.5)$ em função de $W e$.

\begin{tabular}{|c|c|c|c|c|c|c|c|}
\cline { 2 - 8 } \multicolumn{1}{c|}{} & \multicolumn{3}{c|}{$F I-E I$} & \multicolumn{3}{c|}{$F I-C N$} & Tanner \\
\hline$W e$ & $t=10.0$ & $t=15.0$ & $t=30.0$ & $t=10.0$ & $t=15.0$ & $t=30.0$ & equação (B.2.1) \\
\hline \hline 0.2 & 1.1247 & 1.1458 & 1.3097 & 1.1251 & 1.1465 & 1.3065 & 1.1946 \\
0.4 & 1.1866 & 1.2280 & 1.3833 & 1.1866 & 1.2291 & 1.3835 & 1.3535 \\
0.6 & 1.3827 & 1.4426 & 1.4811 & 1.3836 & 1.4426 & 1.4814 & 1.4985 \\
0.8 & 1.5021 & 1.5623 & 1.6076 & 1.5022 & 1.5625 & 1.6085 & 1.6238 \\
1.0 & 1.5907 & 1.7034 & 1.7378 & 1.5915 & 1.7042 & 1.7381 & 1.7335 \\
1.2 & 1.6584 & 1.8382 & 1.8676 & 1.6587 & 1.8383 & 1.8678 & 1.8312 \\
1.4 & 1.7121 & 1.9410 & 1.9967 & 1.7126 & 1.9410 & 1.9979 & 1.9195 \\
1.6 & 1.7635 & 2.0766 & 2.1470 & 1.7641 & 2.0763 & 2.1475 & 2.0002 \\
1.8 & 1.8108 & 2.1871 & 2.2790 & 1.8108 & 2.1870 & 2.2790 & 2.0748 \\
2.0 & 1.8492 & 2.2832 & 2.4065 & 1.8496 & 2.2831 & 2.4064 & 2.1443 \\
\hline
\end{tabular}

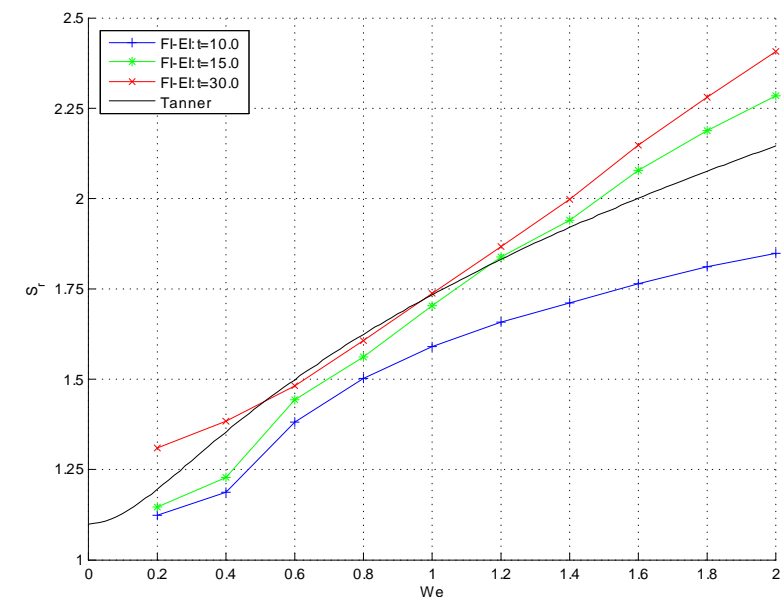

(a)

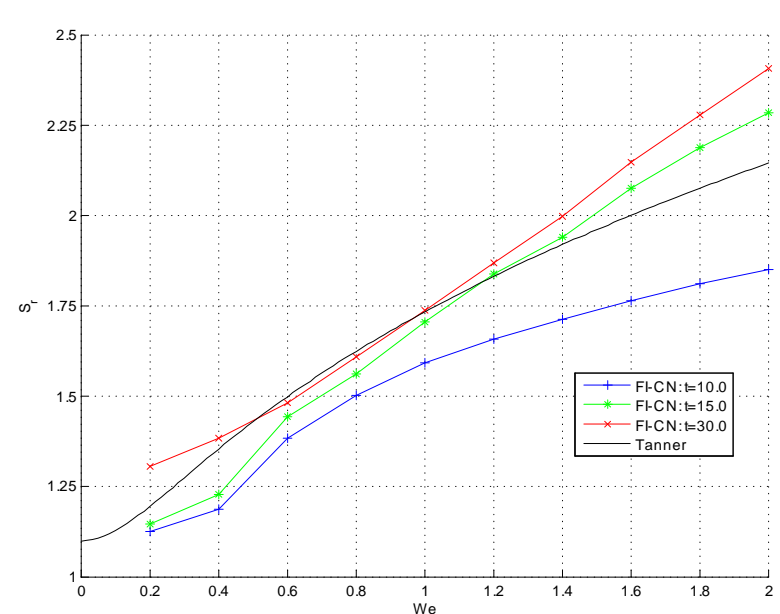

(b)

Figura B.2.1: Taxa de inchamento $S_{r}$ de um fluido Oldroyd-B $(R e=0.05, \beta=0.5)$ em função de $W e$ para as soluções numéricas obtidas com: FI-EI (a); FI-CN (b).

Portanto, conforme observamos na Tabela B.2.1 ou na Figura B.2.1, existe boa concordância entre os resultados numéricos do GENSMAC-SXPP e a aproximação analítica (B.2.1). As discrepâncias existentes podem ser justificadas pelo fato de (B.2.1) ser apenas uma aproximação para $S_{r}$. Com efeito, para o Oldroyd-B, as equações (B.2.1) e (B.2.2) desprezam a influência de $\beta$ e de $R e$ em $S_{r}$, o que sabemos não ser adequado, conforme podemos perceber pelos resultados apresentados na Seção B.2.2. 

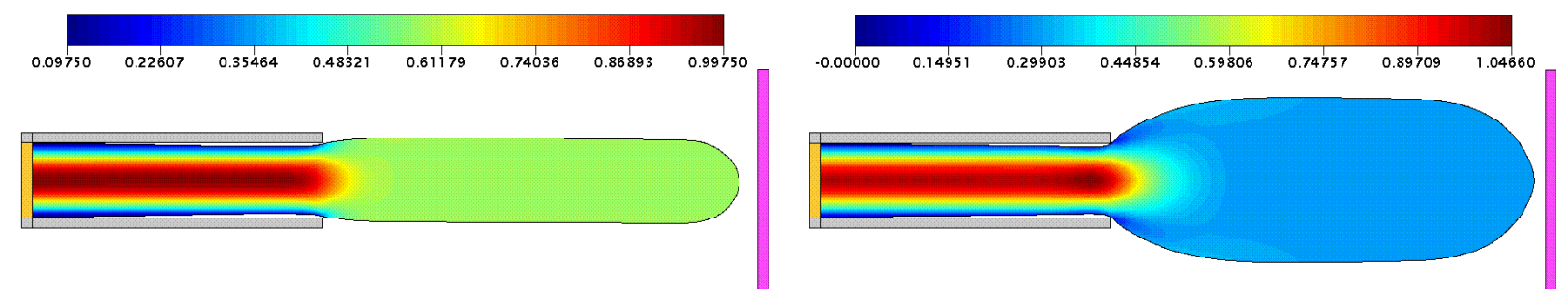

(a)
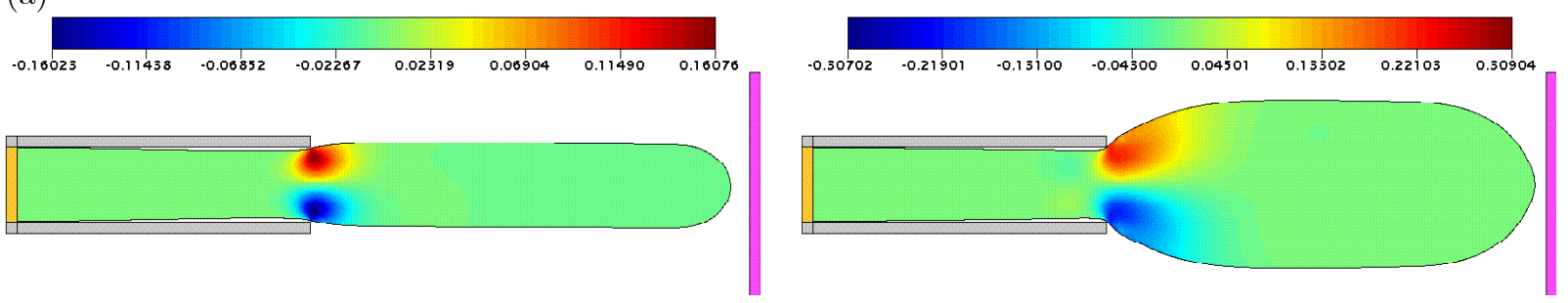

(b)
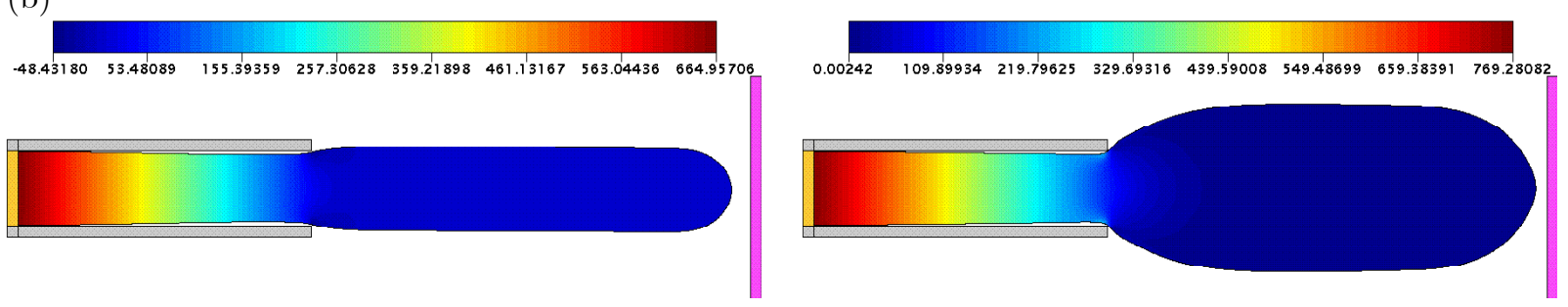

(c)
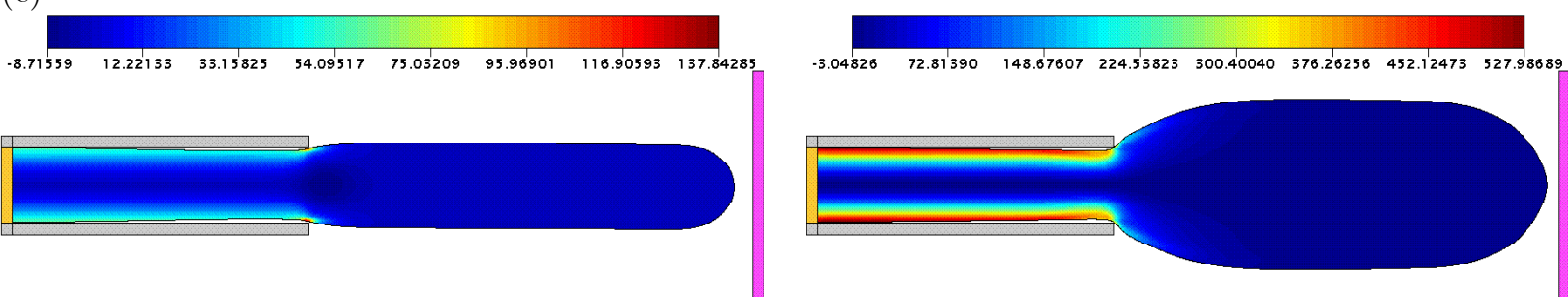

(d)
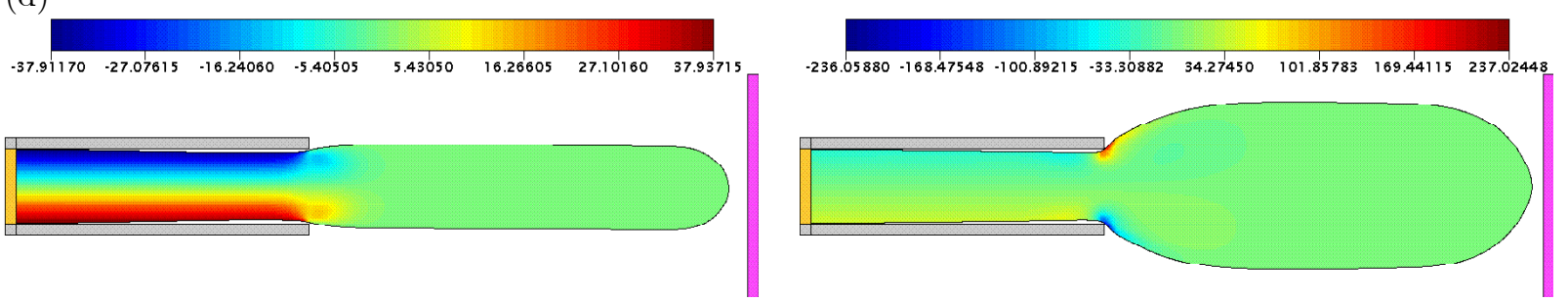

(e)
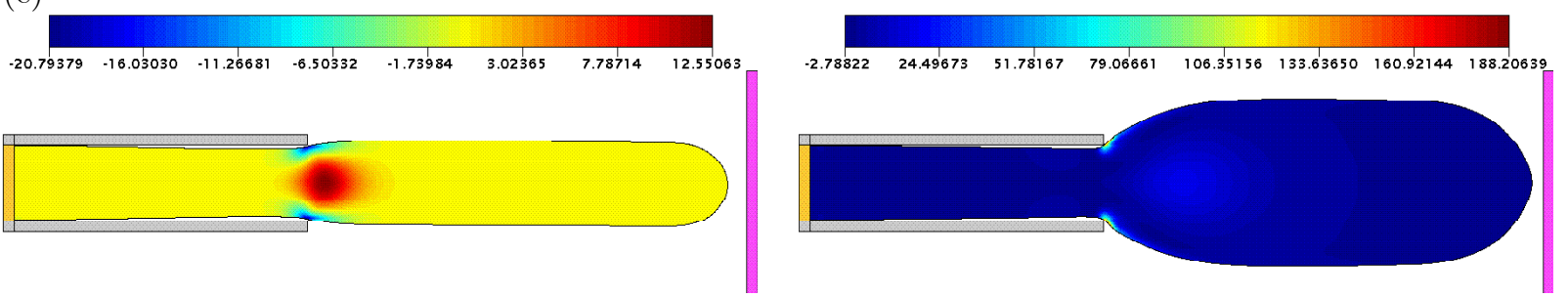

(f)

Figura B.2.2: Soluções numéricas (curvas de nível para $u$ (a), $v$ (b), $p$ (c), $T^{x x}$ (d), $T^{x y}$ (e) e $T^{y y}$ (f)) da FI-EI para o inchamento do extrudado de um fluido Oldroyd-B ( $R e=0.05, \beta=0.5)$ com $W e=0.2$ (coluna esquerda) e $W e=1.8$ (coluna direita), respectivamente, nos instantes $t=15.0$ e $t=23.0$. 


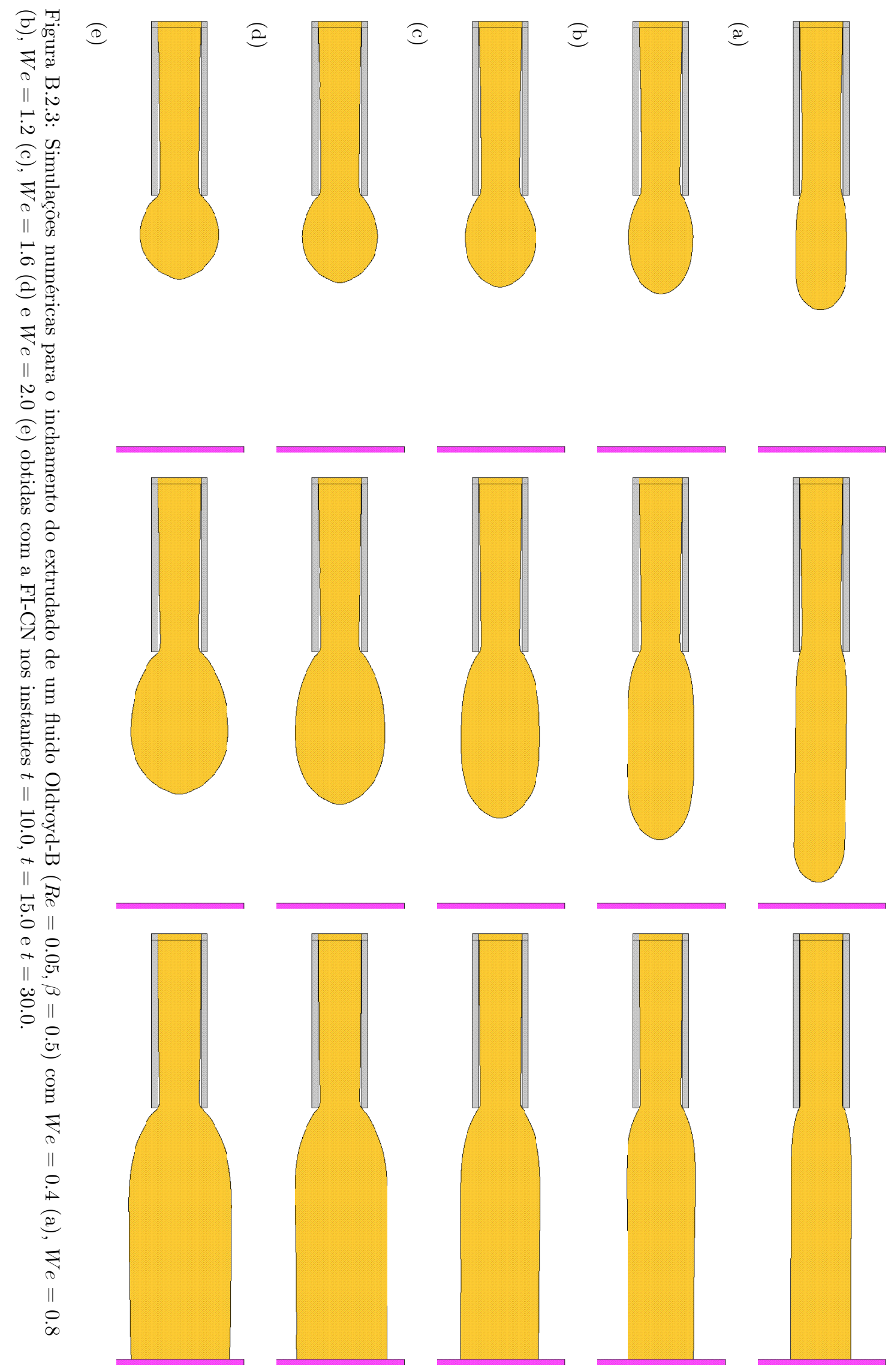




\section{B.2.2 Influência de $\beta$}

Adicionalmente ao modelo considerado, adotamos:

- Parâmetros adimensionais:

$$
\begin{aligned}
& -R e=1.0, W e=1.0 . \\
& -\beta \in\{0.1,0.3,0.5,0.7,0.9\} .
\end{aligned}
$$

- Fator de controle do passo temporal: $F_{v i s c}=4.0$.

Neste caso, os parâmetros adimensionais do modelo foram escolhidos para tornar possível a comparação com os resultados apresentados em [GF03], obtidos com a versão anterior do GENSMAC para o modelo Oldroyd-B. Em [GF03], considerou-se $R e=1.0, W e=1.0$ e $\beta \in\{0.1,0.5,0.9\}$.

Portanto, podemos observar na Tabela B.2.2 que existe boa semelhança entre os resultados obtidos pelo GENSMAC-SXPP e os apresentados em [GF03], embora estes estejam disponíveis apenas para três valores de $\beta$, dificultando uma análise mais precisa.

Tabela B.2.2: Taxas de inchamento de um fluido Oldroyd-B $(R e=1.0, W e=1.0)$ em função de $\beta$.

\begin{tabular}{|c|c|c|c|c|c|c|c|}
\cline { 2 - 8 } \multicolumn{1}{c|}{} & \multicolumn{3}{|c|}{$F I-E I$} & \multicolumn{3}{c|}{$F I-C N$} & $F E$ \\
\hline$\beta$ & $t=10.0$ & $t=15.0$ & $t=30.0$ & $t=10.0$ & $t=15.0$ & $t=30.0$ & {$[\mathrm{GF} 03]$} \\
\hline \hline 0.1 & 1.6882 & 1.7701 & 1.8662 & 1.6912 & 1.7713 & 1.8662 & 1.89 \\
0.3 & 1.6415 & 1.7339 & 1.8477 & 1.6416 & 1.7338 & 1.8486 & - \\
0.5 & 1.5748 & 1.7026 & 1.7714 & 1.5782 & 1.7099 & 1.7728 & 1.76 \\
0.7 & 1.3772 & 1.4388 & 1.6624 & 1.3775 & 1.4393 & 1.6632 & - \\
0.9 & 1.2250 & 1.2730 & 1.5592 & 1.2266 & 1.2738 & 1.5599 & 1.25 \\
\hline
\end{tabular}

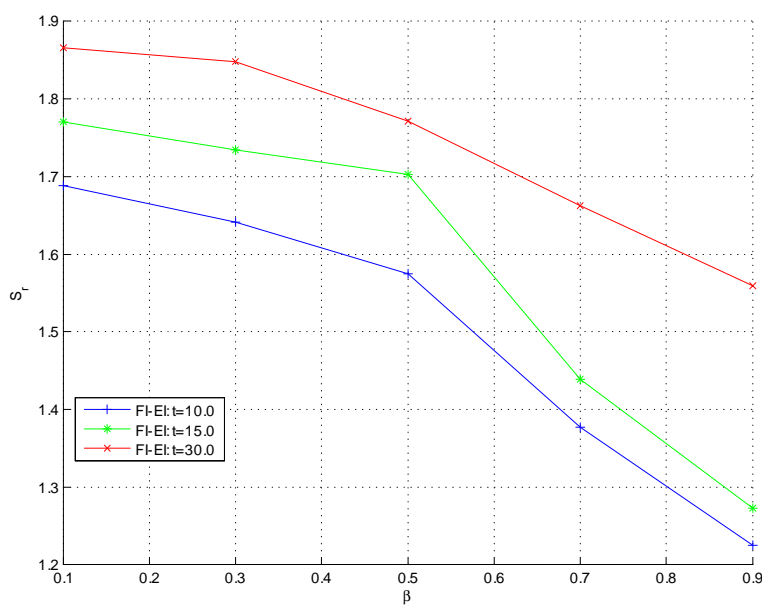

(a)

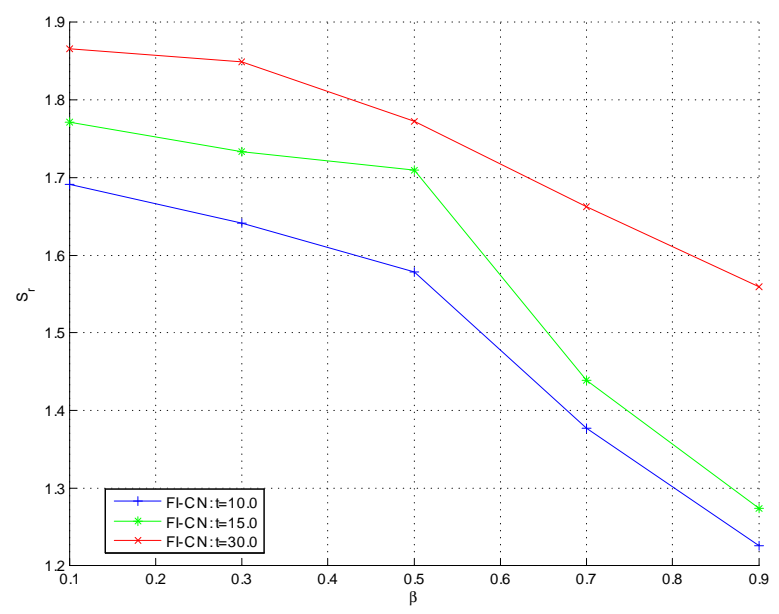

(b)

Figura B.2.4: Taxa de inchamento $S_{r}$ de um fluido Oldroyd-B ( $\left.R e=1.0, W e=1.0\right)$ em função de $\beta$ para as soluções numéricas obtidas com: FI-EI (a); FI-CN (b). 

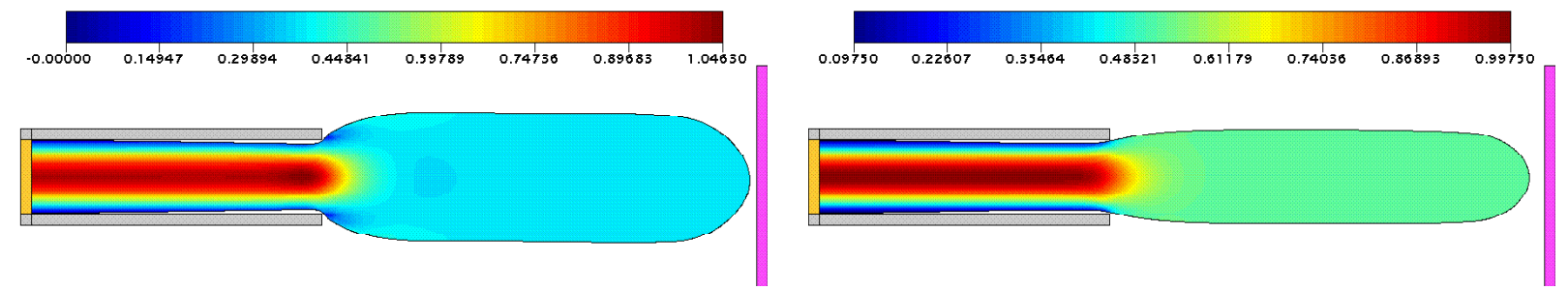

(a)
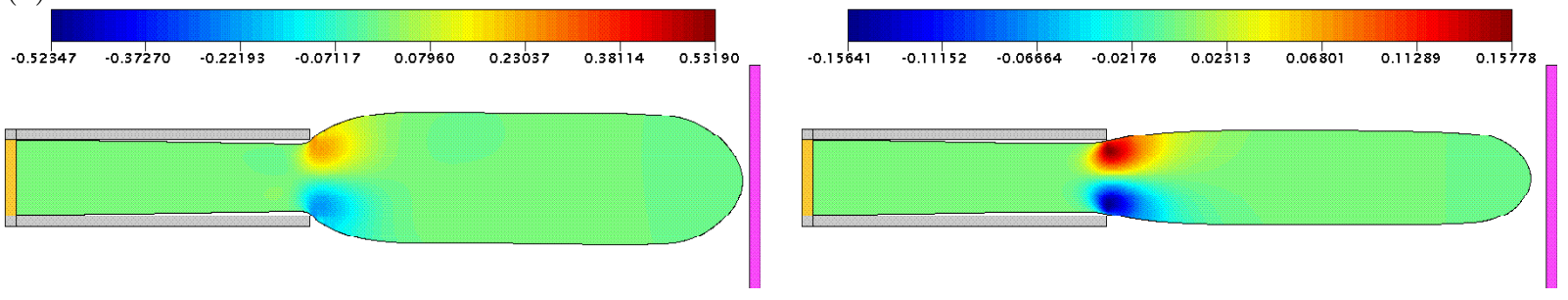

(b)
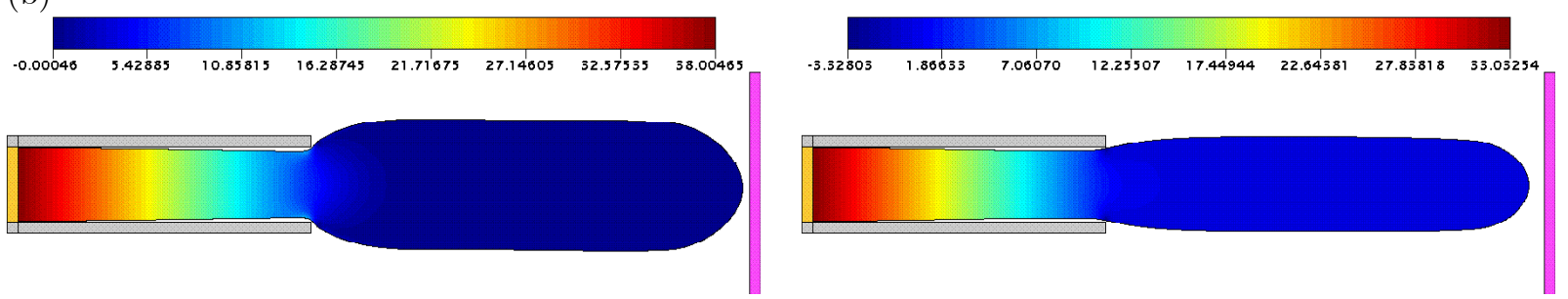

(c)
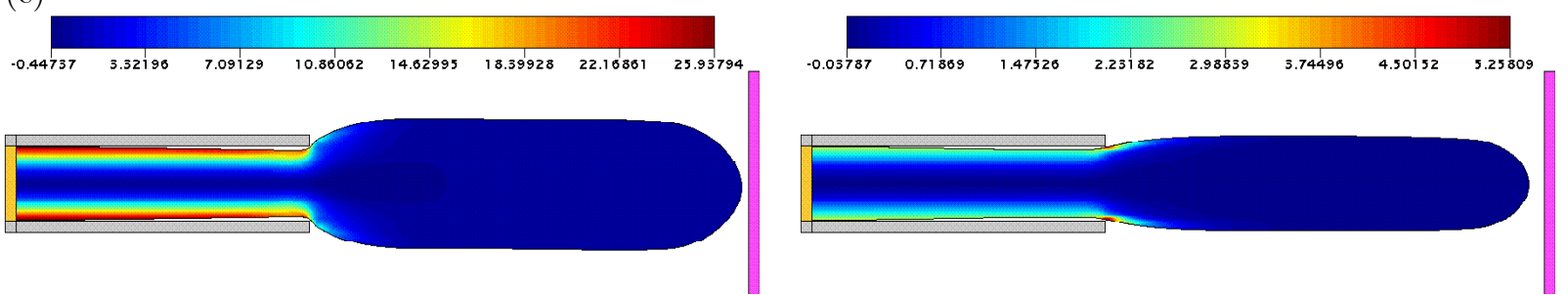

(d)
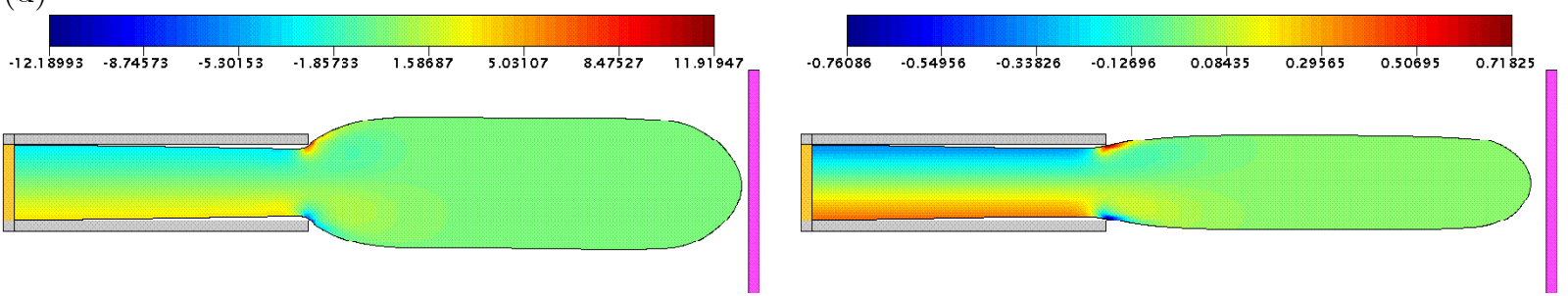

(e)
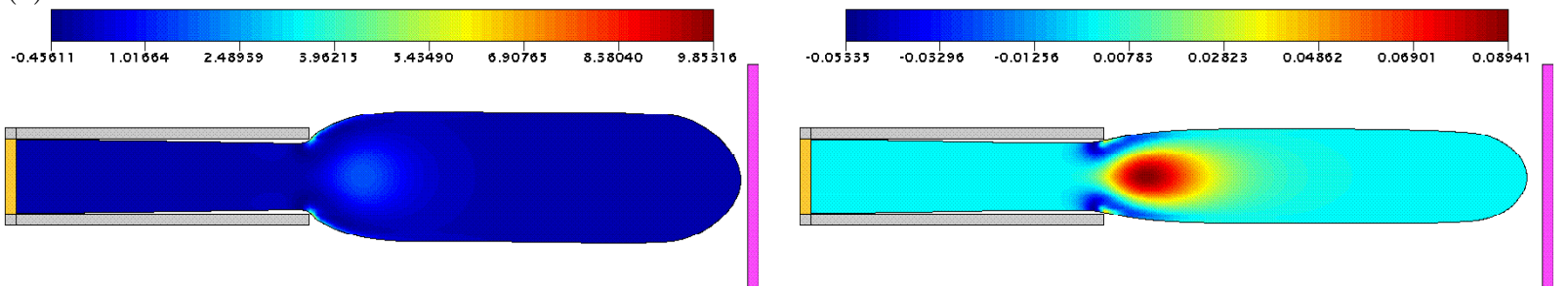

(f)

Figura B.2.5: Soluções numéricas (curvas de nível para $u$ (a), $v$ (b), $p$ (c), $T^{x x}$ (d), $T^{x y}$ (e) e $T^{y y}$ (f)) da FI-CN para o inchamento do extrudado de um fluido Oldroyd-B $(R e=1.0, W e=1.0)$ com $\beta=0.1$ (coluna esquerda) e $\beta=0.9$ (coluna direita), respectivamente, nos instantes $t=20.0$ e $t=16.0$. 


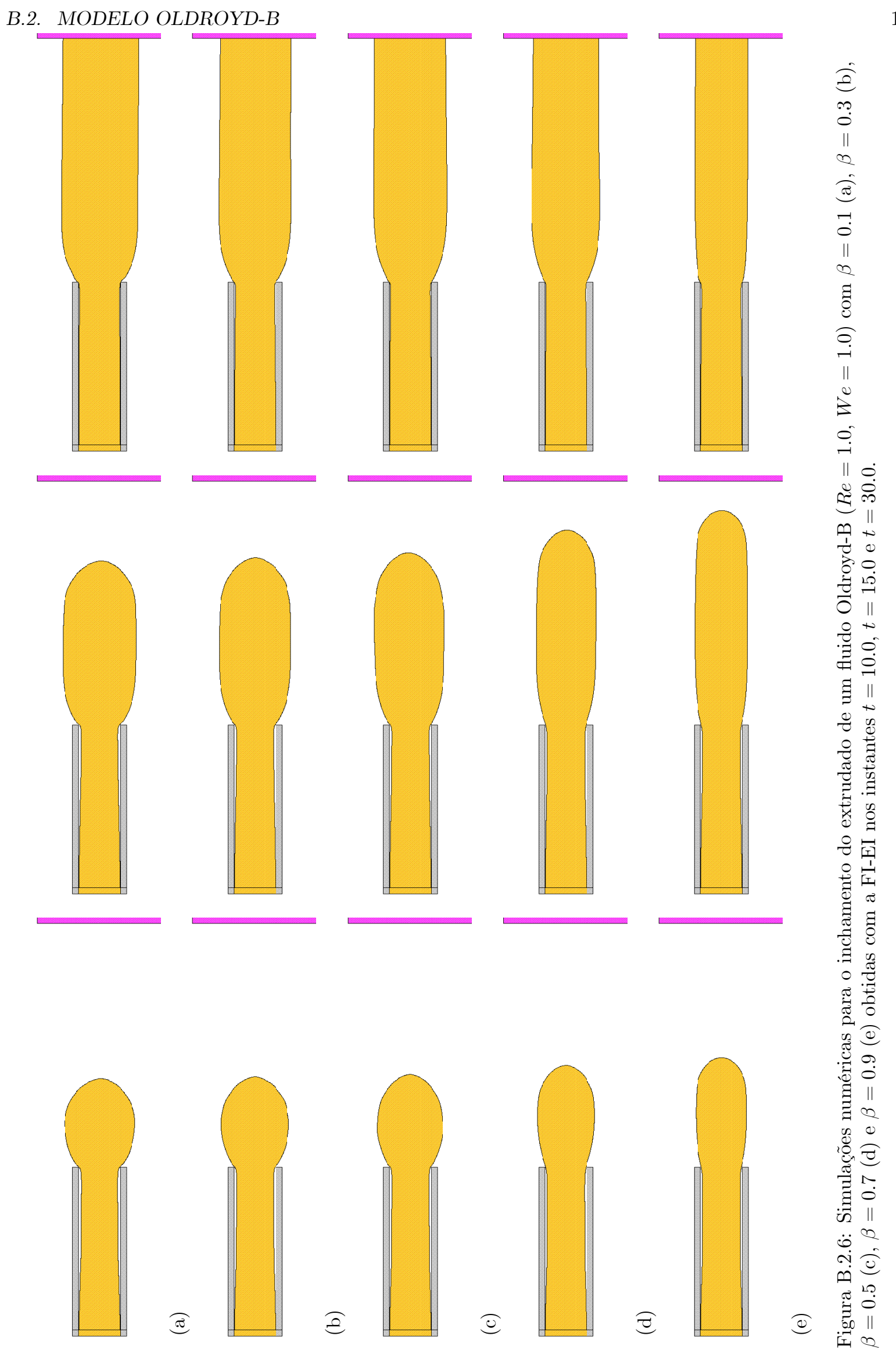




\section{B.3 Modelo SXPP}

\section{B.3.1 Influência de $\alpha$}

Adicionalmente ao modelo considerado, adotamos:

- Parâmetros adimensionais:

$$
\begin{aligned}
& -R e=0.05, W e=10.0, \beta=0.5, \gamma=0.8, Q=8.0 . \\
& -\alpha \in\{0.0,0.05,0.10,0.20,0.40,0.60,0.80\} .
\end{aligned}
$$

- Fator de controle do passo temporal: $F_{v i s c}=16.0$.

Portanto, os resultados apontam que a taxa de inchamento $S_{r}$ é diminuída quando aumentamos o valor de $\alpha$, havendo boa concordância entre os resultados obtidos pela FI-EI e pela FI-CN.

Tabela B.3.1: Taxas de inchamento de um fluido $\operatorname{SXPP}(R e=0.05, W e=10.0, \beta=0.5, \gamma=0.8$, $Q=8.0)$ em função de $\alpha$.

\begin{tabular}{|c|c|c|c|c|c|c|}
\cline { 2 - 7 } \multicolumn{1}{c|}{} & \multicolumn{3}{c|}{$F I-E I$} & \multicolumn{3}{c|}{$F I-C N$} \\
\hline$\alpha$ & $t=10.0$ & $t=15.0$ & $t=65.0$ & $t=10.0$ & $t=15.0$ & $t=65.0$ \\
\hline \hline 0.00 & 1.7567 & 2.0633 & 2.1486 & 1.7548 & 2.0623 & 2.1478 \\
0.05 & 1.7910 & 2.0114 & 2.0945 & 1.7908 & 2.0118 & 2.0940 \\
0.10 & 1.7878 & 1.8812 & 1.9951 & 1.7881 & 1.8773 & 1.9905 \\
0.20 & 1.6192 & 1.6562 & 1.6735 & 1.6212 & 1.6557 & 1.6742 \\
0.40 & 1.4092 & 1.4070 & 1.4251 & 1.4091 & 1.4070 & 1.4252 \\
0.60 & 1.3395 & 1.3376 & 1.3732 & 1.3396 & 1.3377 & 1.3733 \\
0.80 & 1.1618 & 1.2465 & 1.3451 & 1.1619 & 1.2088 & 1.3451 \\
\hline
\end{tabular}

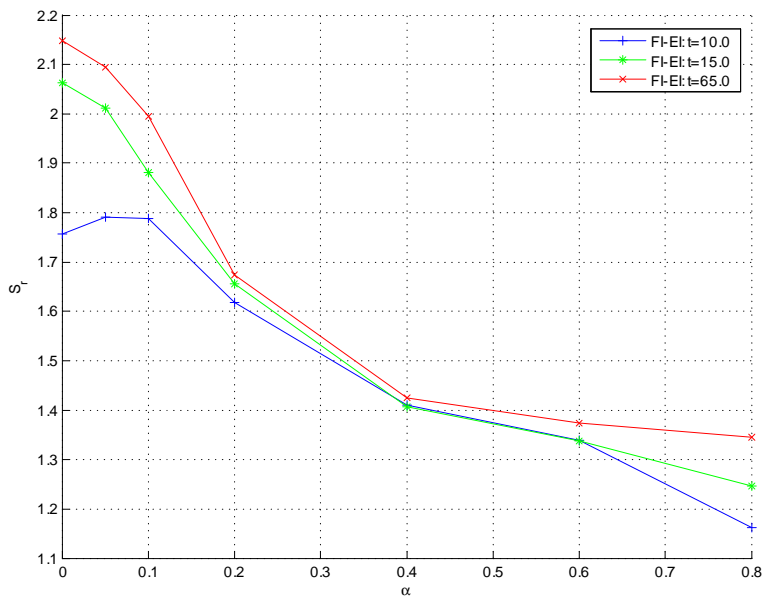

(a)

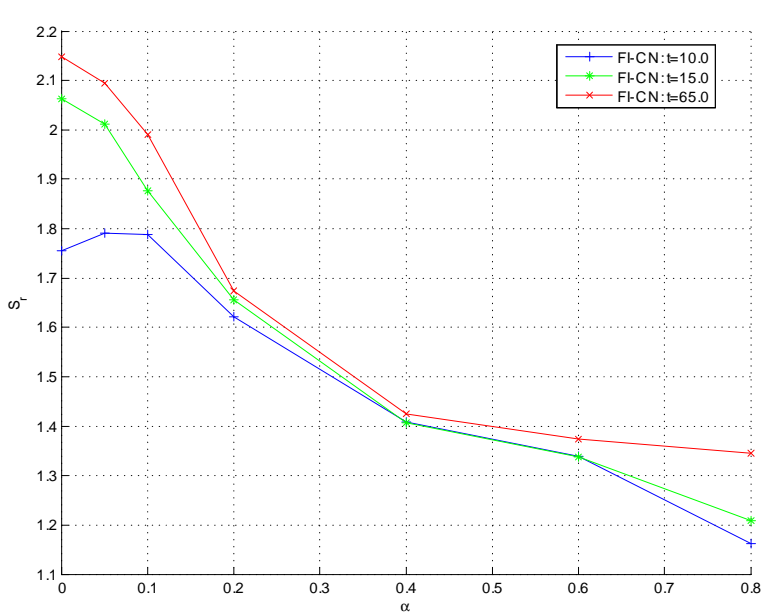

(b)

Figura B.3.1: Taxa de inchamento $S_{r}$ de um fluido $\operatorname{SXPP}(R e=0.05, W e=10.0, \beta=0.5, \gamma=0.8$, $Q=8.0$ ) em função de $\alpha$ para as soluções numéricas obtidas com: FI-EI (a); FI-CN (b). 
(a)
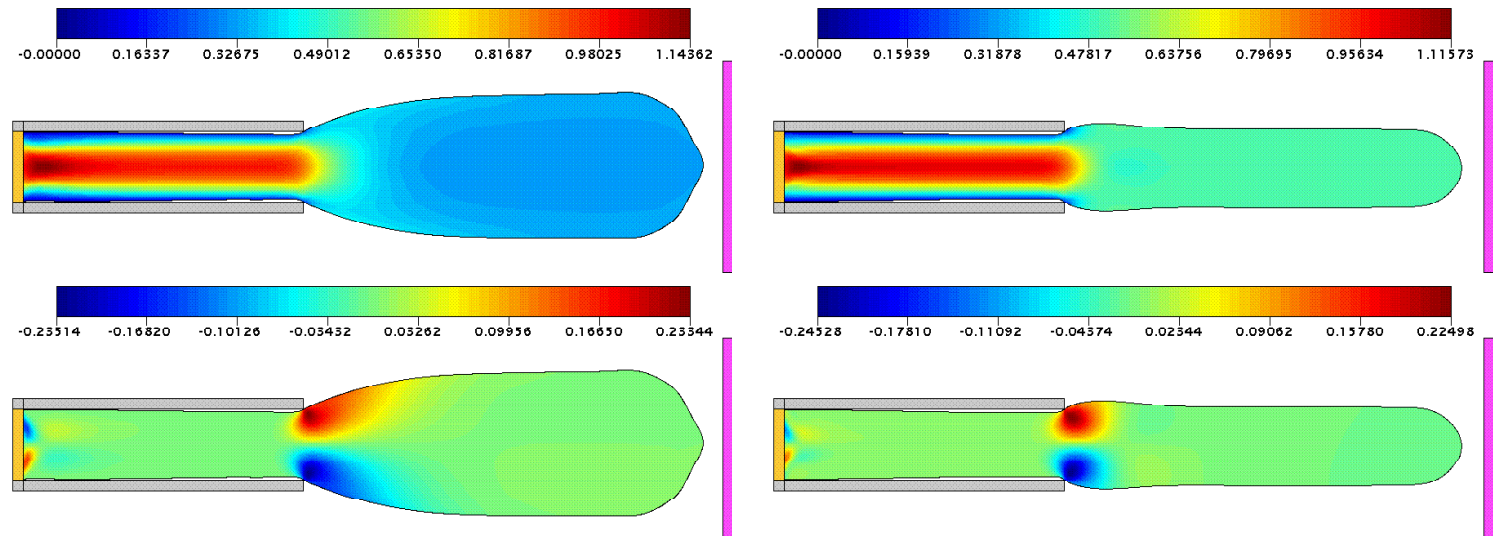

(b)
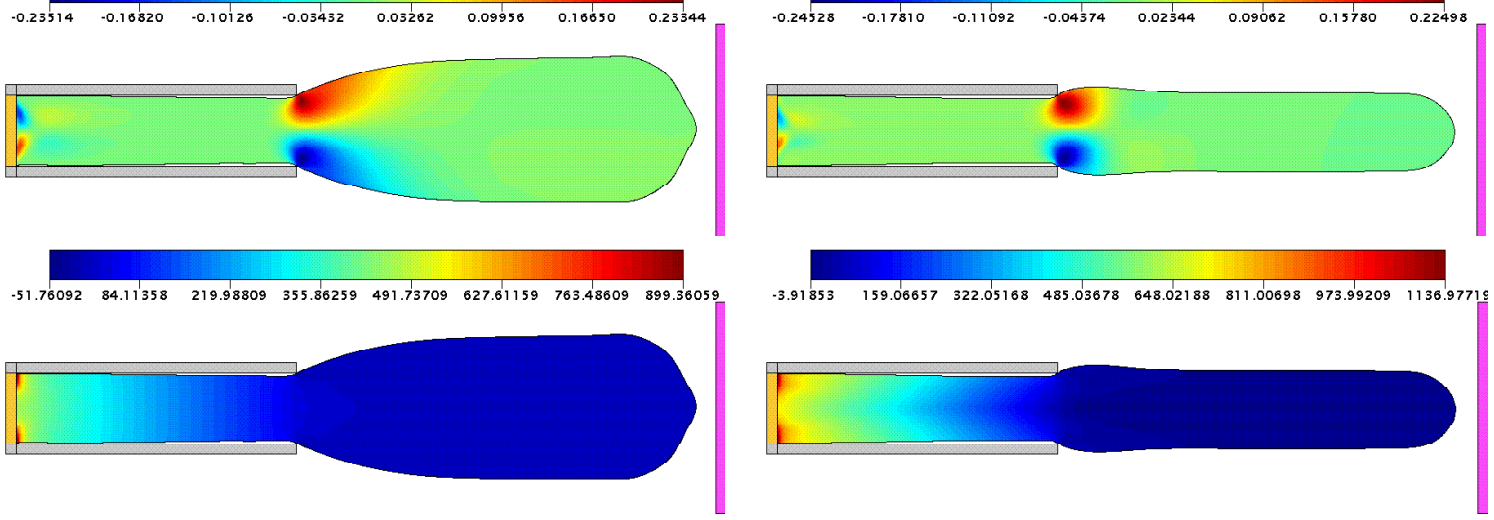

(c)

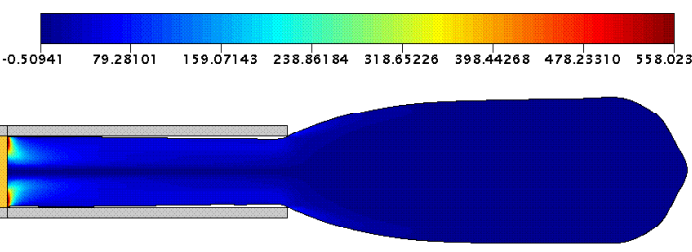

(d)
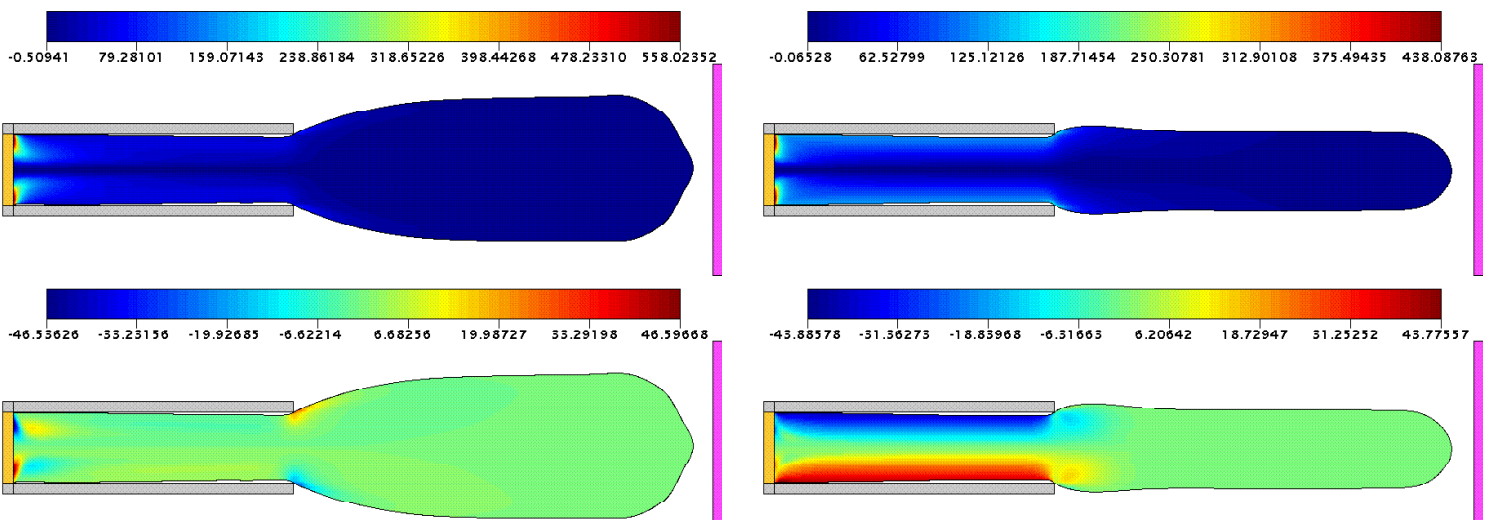

(e)
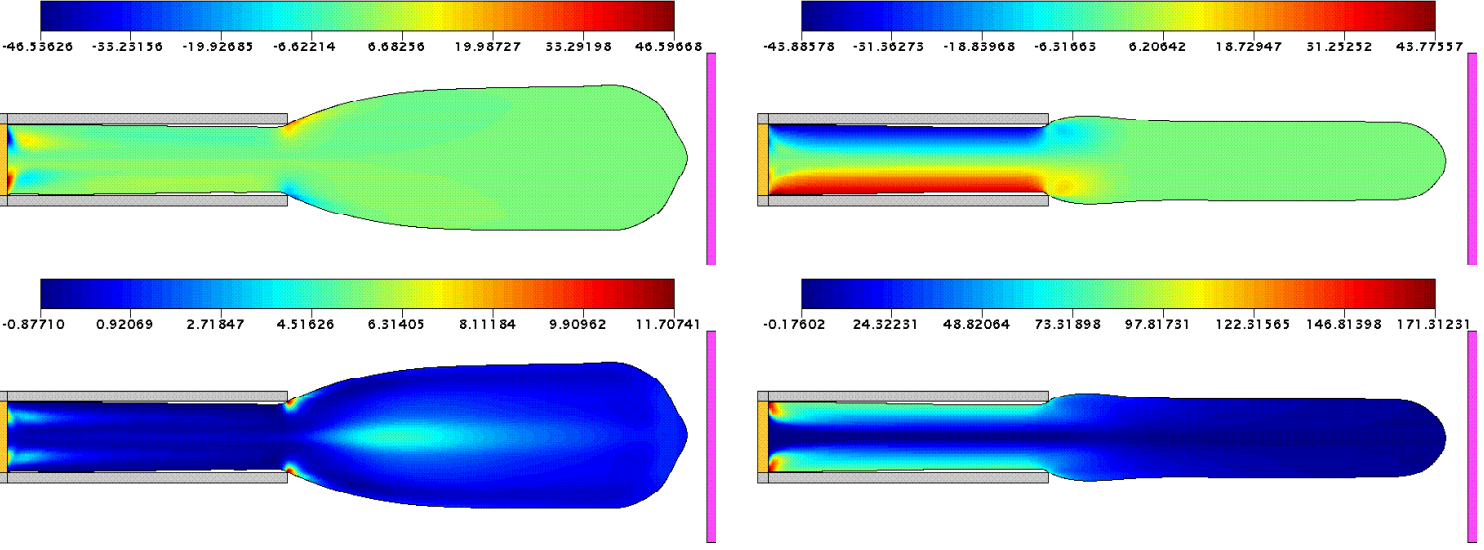

(f)
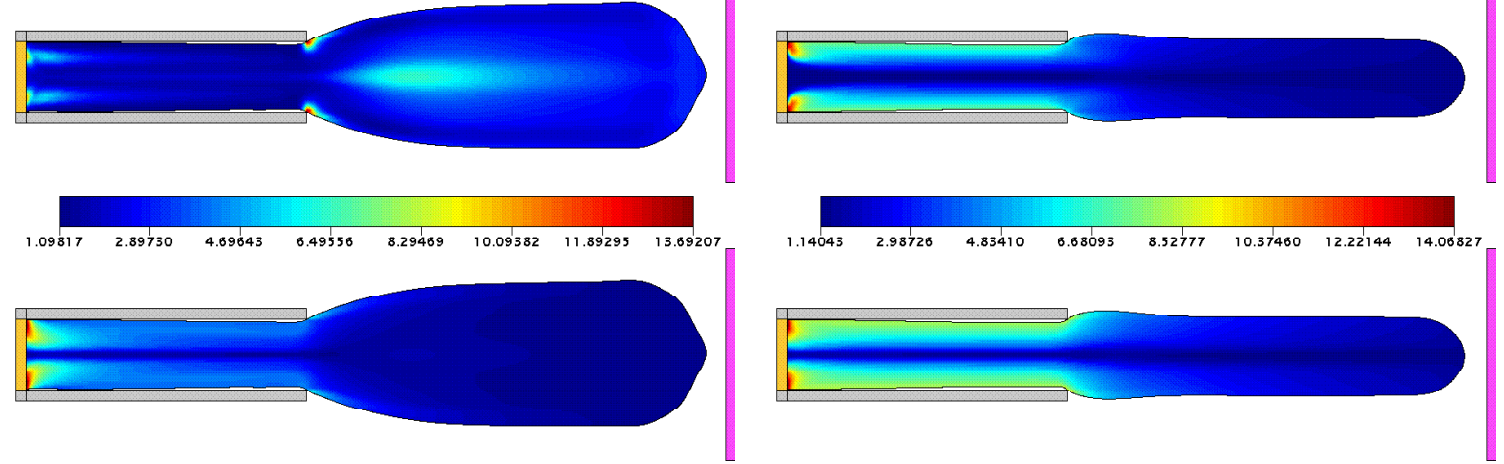

(g)

Figura B.3.2: Soluções numéricas (curvas de nível para $u$ (a), $v$ (b), $p(\mathrm{c}), T^{x x}$ (d), $T^{x y}$ (e), $T^{y y}$ (f) e $\lambda(\mathrm{g}))$ da FI-EI para o inchamento do extrudado de um fluido $\operatorname{SXPP}(R e=0.05, W e=10.0, \beta=0.5$, $\gamma=0.8, Q=8.0$ ) com $\alpha=0.0$ (coluna esquerda) e $\alpha=0.8$ (coluna direita), respectivamente, nos instantes $t=21.0$ e $t=15.0$. 


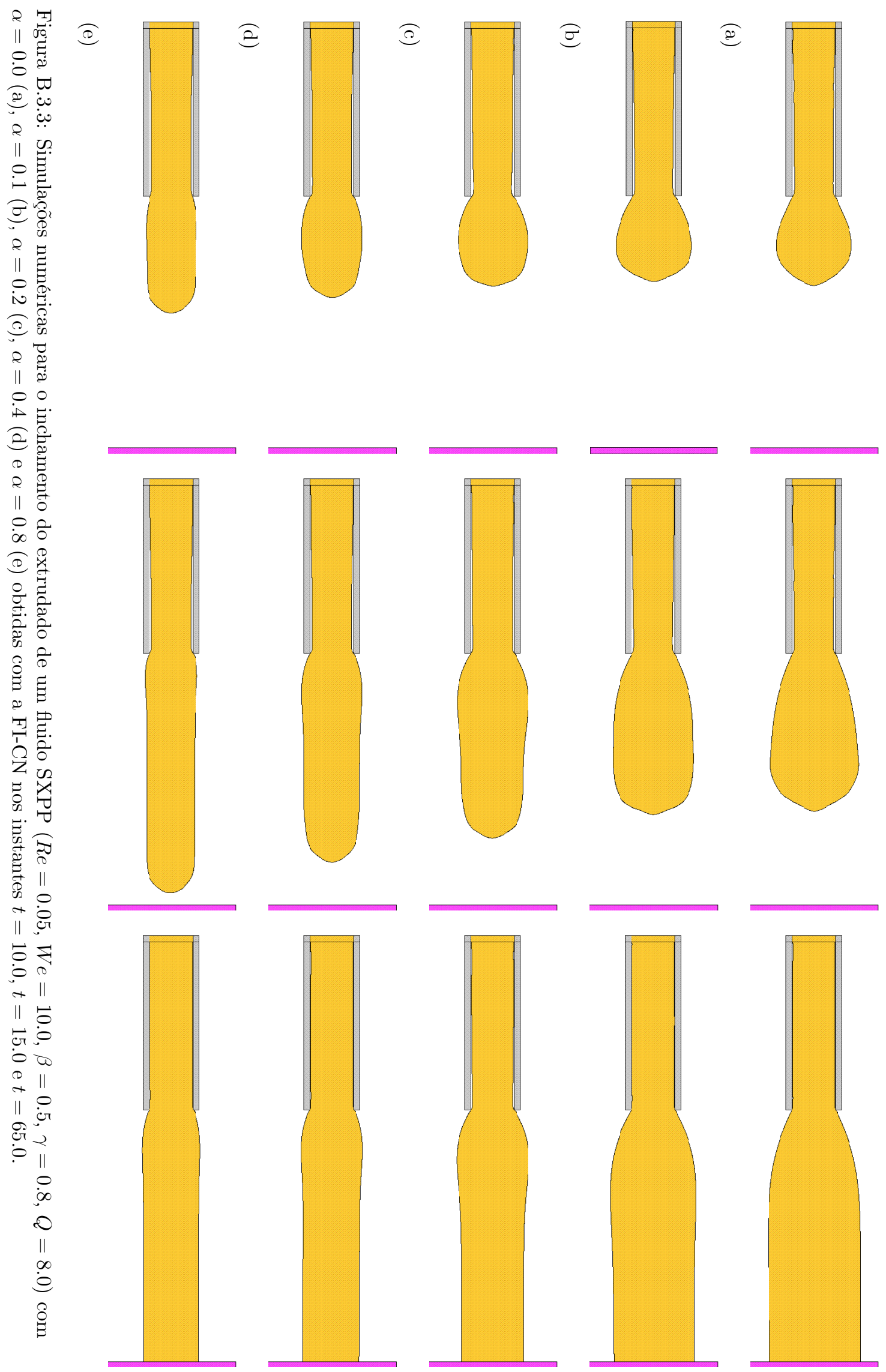




\section{B.3.2 Influência de $\beta$}

Adicionalmente ao modelo considerado, adotamos:

- Parâmetros adimensionais:

$$
\begin{aligned}
& -R e=0.05, W e=10.0, \alpha=0.01, \gamma=0.3, Q=8.0 . \\
& -\beta \in\{0.2,0.4,0.6,0.8,0.9\} .
\end{aligned}
$$

- Fator de controle do passo temporal: $F_{v i s c}=16.0$.

Portanto, os resultados apontam que a taxa de inchamento $S_{r}$ é incrementada quando aumentamos o valor de $\beta$. Com efeito, quando $\beta$ tende a 1, o comportamento newtoniano do fluido é reforçado, justificando os baixos valores obtidos para $S_{r}$ quando $\beta=0.9$. Novamente, existe boa concordância entre os resultados obtidos pela FI-EI e pela FI-CN.

Tabela B.3.2: Taxas de inchamento de um fluido $\operatorname{SXPP}(R e=0.05, W e=10.0, \alpha=0.01, \gamma=0.3$, $Q=8.0)$ em função de $\beta$.

\begin{tabular}{|c|c|c|c|c|c|c|}
\cline { 2 - 7 } \multicolumn{1}{c|}{} & \multicolumn{3}{c|}{$F I-E I$} & \multicolumn{3}{c|}{$F I-C N$} \\
\hline$\beta$ & $t=10.0$ & $t=15.0$ & $t=30.0$ & $t=10.0$ & $t=15.0$ & $t=30.0$ \\
\hline \hline 0.2 & 1.7177 & 1.7388 & 1.7208 & 1.7171 & 1.7386 & 1.7204 \\
0.4 & 1.4731 & 1.6088 & 1.6451 & 1.4732 & 1.6074 & 1.6443 \\
0.6 & 1.3209 & 1.4391 & 1.4409 & 1.3209 & 1.4391 & 1.4408 \\
0.8 & 1.1990 & 1.2600 & 1.2406 & 1.1992 & 1.2603 & 1.2405 \\
0.9 & 1.1482 & 1.1784 & 1.1665 & 1.1482 & 1.1786 & 1.1666 \\
\hline
\end{tabular}

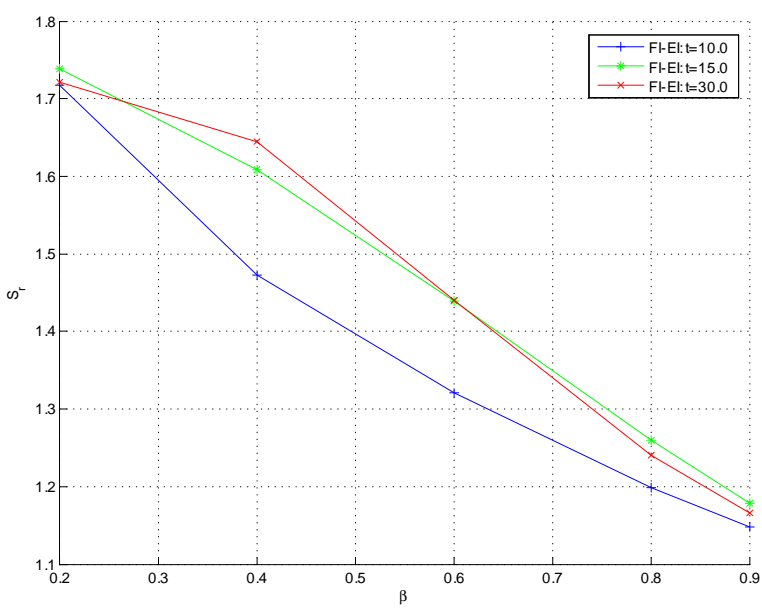

(a)

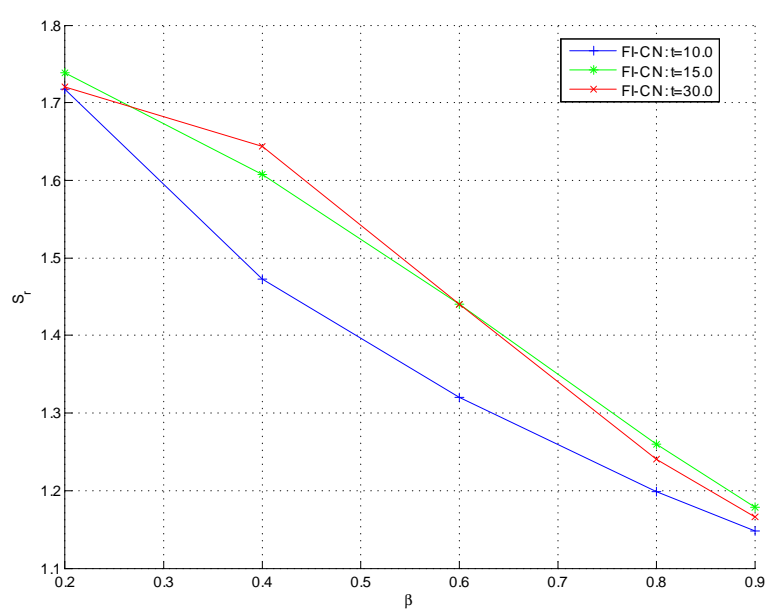

(b)

Figura B.3.4: Taxa de inchamento $S_{r}$ de um fluido $\operatorname{SXPP}(R e=0.05, W e=10.0, \alpha=0.01, \gamma=0.3$, $Q=8.0$ ) em função de $\beta$ para as soluções numéricas obtidas com: FI-EI (a); FI-CN (b). 
(a)
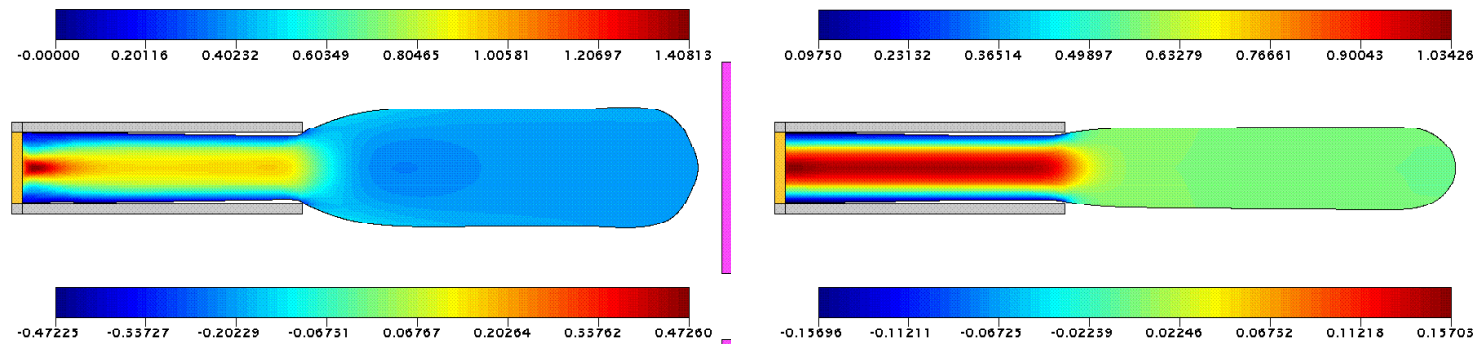

$\begin{array}{llllllll}-0.47225 & -0.33727 & -0.20229 & -0.06731 & 0.06767 & 0.20284 & 0.33762 & 0.47260\end{array}$
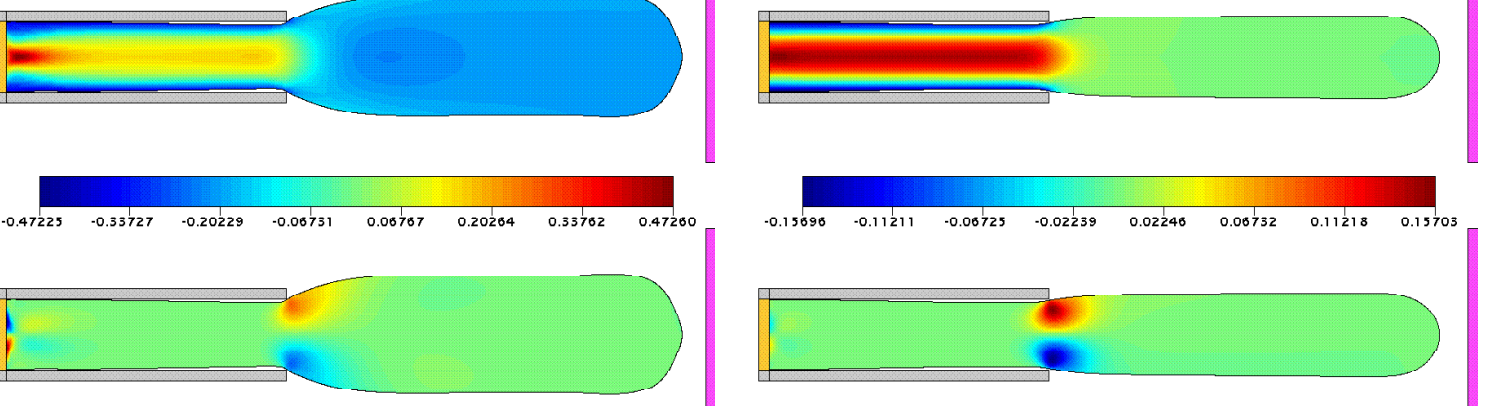

(b)
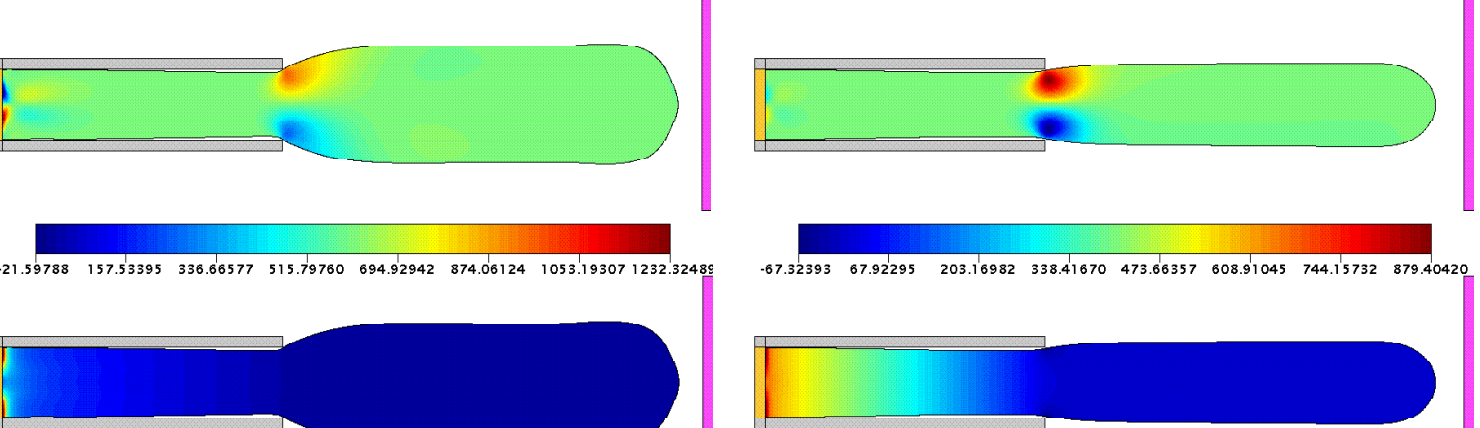

(c)
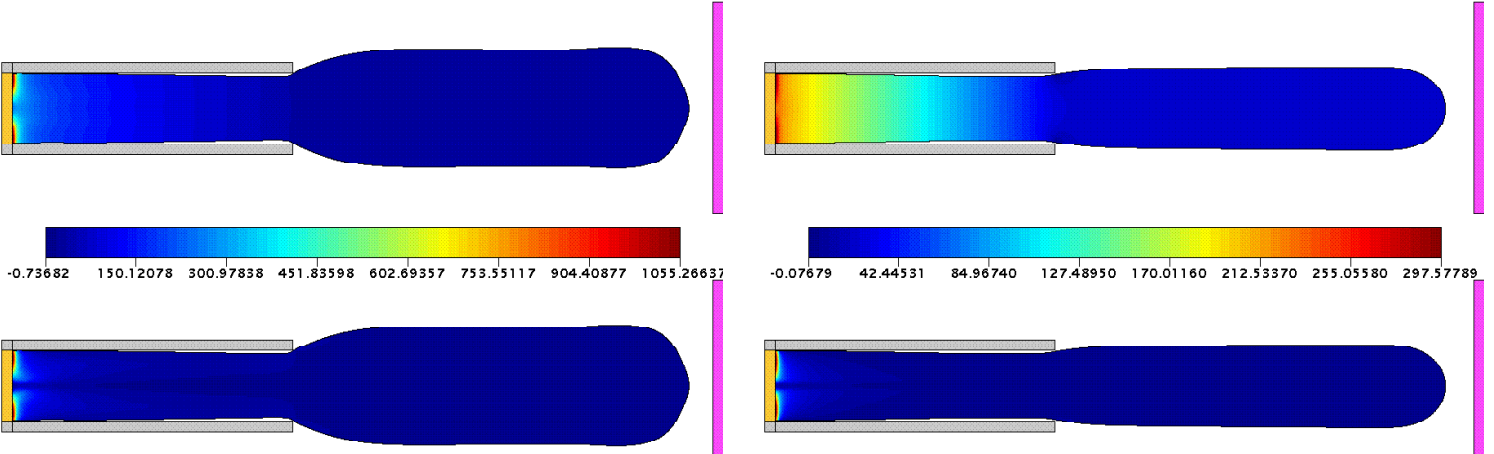

(d)

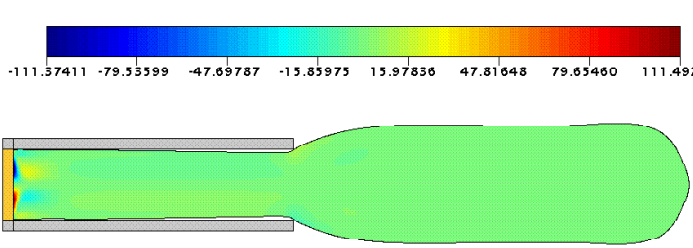

(e)
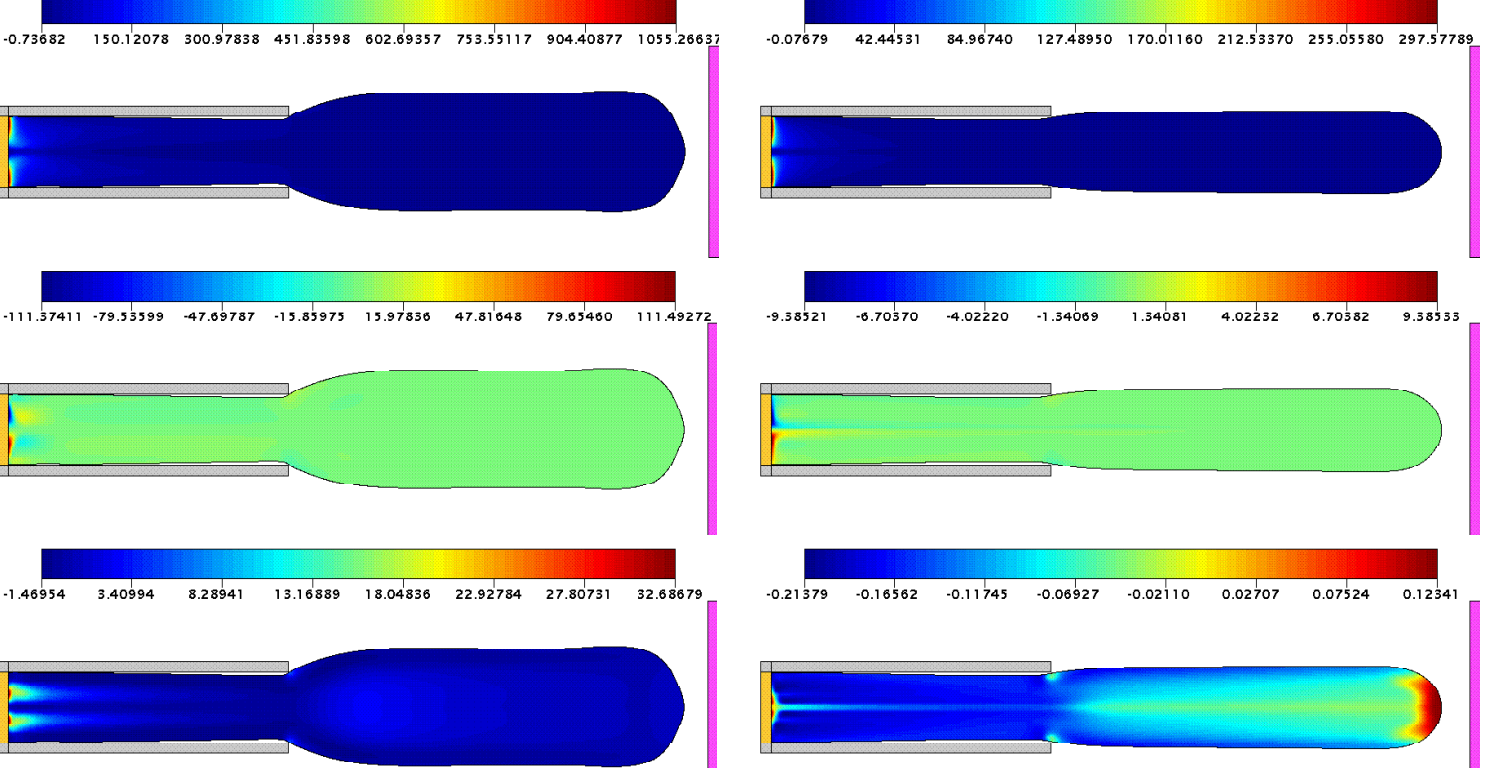

(f)
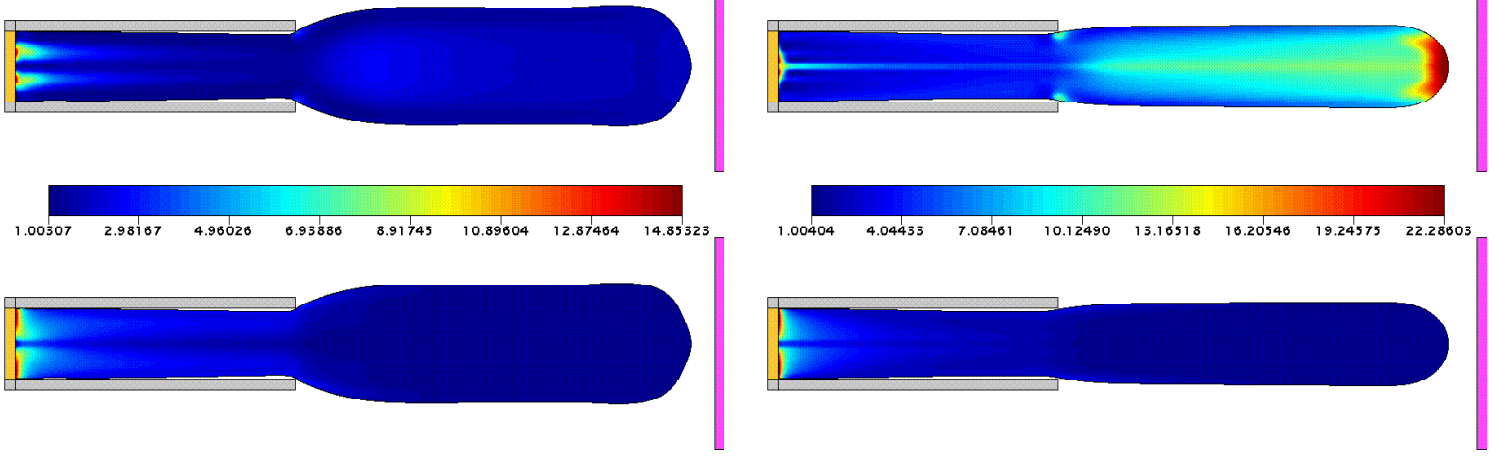

(g)

Figura B.3.5: Soluções numéricas (curvas de nível para $u$ (a), $v$ (b), $p$ (c), $T^{x x}$ (d), $T^{x y}$ (e), $T^{y y}$ (f) e $\lambda$ (g)) da FI-CN para o inchamento do extrudado de um fluido SXPP $(R e=0.05, W e=10.0, \alpha=0.01$, $\gamma=0.3, Q=8.0$ ) com $\beta=0.2$ (coluna esquerda) e $\beta=0.9$ (coluna direita), respectivamente, nos instantes $t=19.0$ e $t=15.0$. 


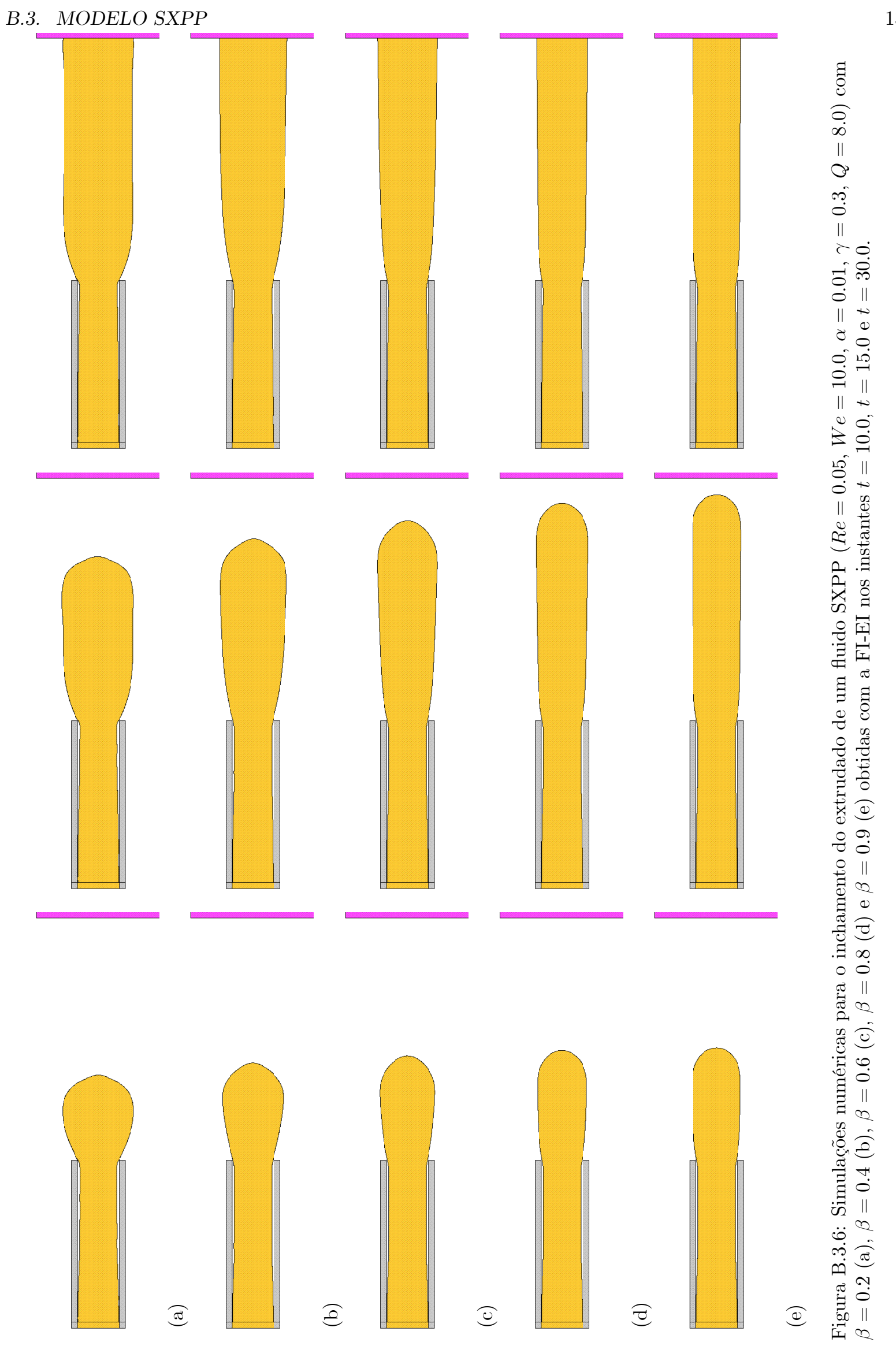




\section{B.3.3 Influência de $\gamma$}

Adicionalmente ao modelo considerado, adotamos:

- Parâmetros adimensionais:

$$
\begin{aligned}
& -R e=0.05, W e=10.0, \alpha=0.01, \beta=0.5, Q=8.0 . \\
& -\gamma \in\{0.2,0.25,0.4,0.6,0.8,0.9\} .
\end{aligned}
$$

- Fator de controle do passo temporal: $F_{v i s c}=16.0$.

Portanto, os resultados apontam que a taxa de inchamento $S_{r}$ é incrementada quando aumentamos o valor de $\gamma$. Mais uma vez, existe boa concordância entre os resultados obtidos pela FI-EI e pela FI-CN.

Tabela B.3.3: Taxas de inchamento de um fluido $\operatorname{SXPP}(R e=0.05, W e=10.0, \alpha=0.01, \beta=0.5$, $Q=8.0)$ em função de $\gamma$.

\begin{tabular}{|c|c|c|c|c|c|c|}
\cline { 2 - 7 } \multicolumn{1}{c|}{} & \multicolumn{3}{c|}{$F I-E I$} & \multicolumn{3}{c|}{$F I-C N$} \\
\hline$\gamma$ & $t=10.0$ & $t=15.0$ & $t=30.0$ & $t=10.0$ & $t=15.0$ & $t=30.0$ \\
\hline \hline 0.20 & 1.3007 & 1.4009 & 1.3775 & 1.3007 & 1.4017 & 1.3773 \\
0.25 & 1.3491 & 1.4668 & 1.4530 & 1.3492 & 1.4670 & 1.4529 \\
0.40 & 1.4791 & 1.6417 & 1.6566 & 1.4791 & 1.6419 & 1.6558 \\
0.60 & 1.6621 & 1.9260 & 1.9711 & 1.6625 & 1.9255 & 1.9712 \\
0.80 & 1.7626 & 2.0610 & 2.0612 & 1.7633 & 2.0613 & 2.0616 \\
0.90 & 1.7550 & 2.0863 & 2.0859 & 1.7557 & 2.0869 & 2.0866 \\
\hline
\end{tabular}

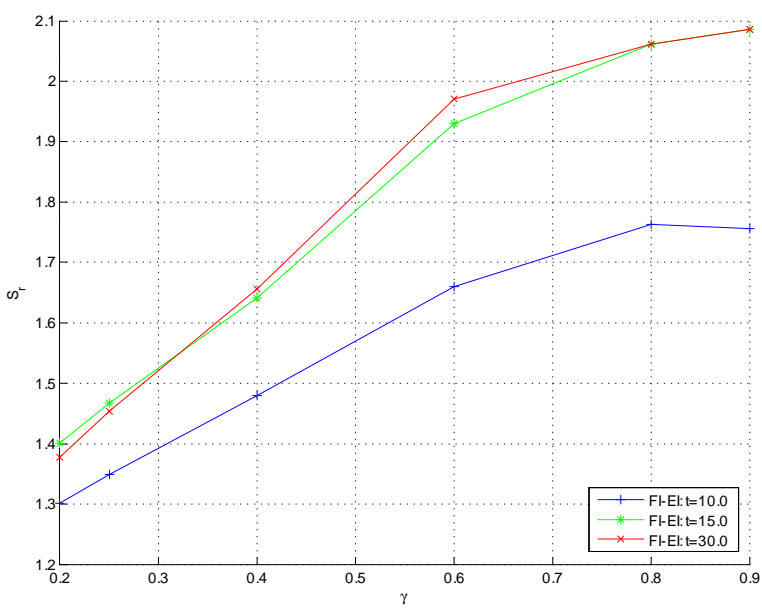

(a)

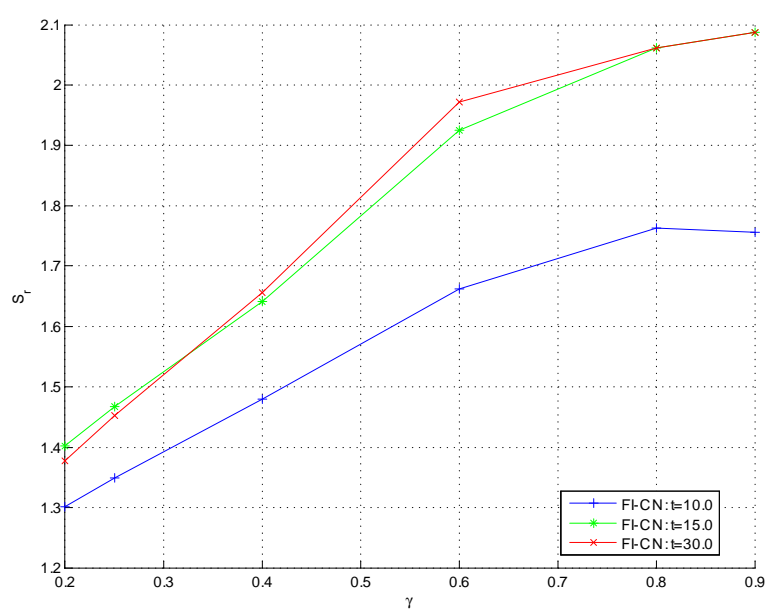

(b)

Figura B.3.7: Taxa de inchamento $S_{r}$ de um fluido SXPP $(R e=0.05, W e=10.0, \alpha=0.01, \beta=0.5$, $Q=8.0$ ) em função de $\gamma$ para as soluções numéricas obtidas com: FI-EI (a); FI-CN (b). 
(a)
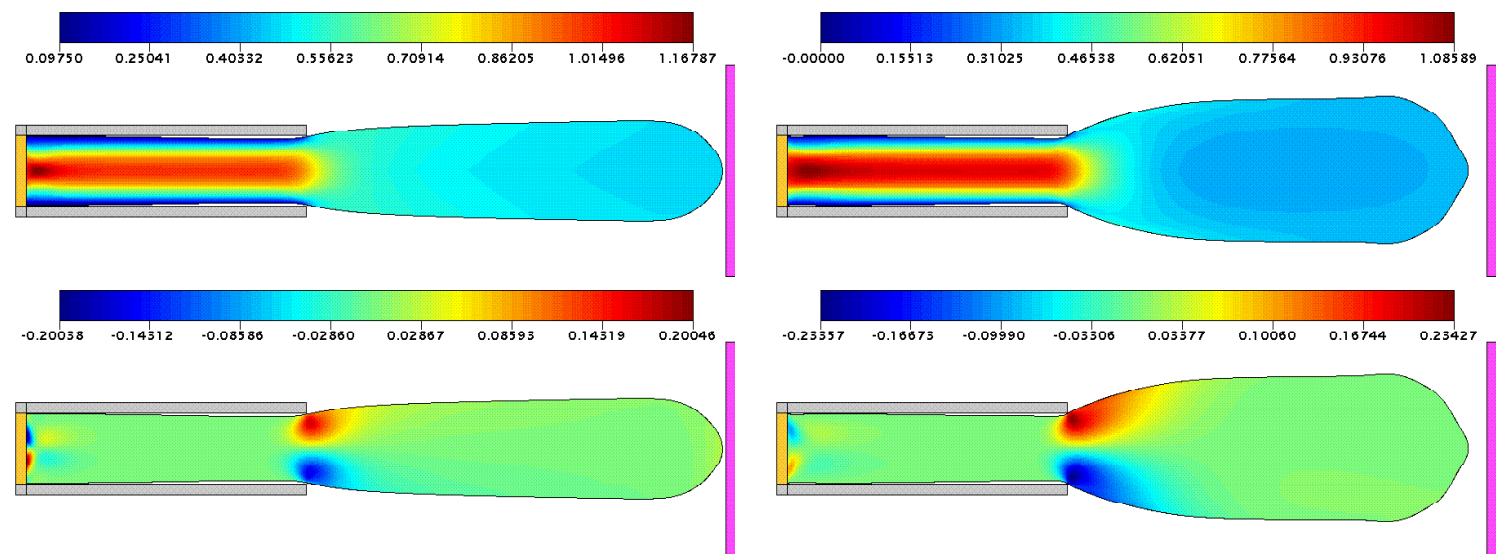

(b)
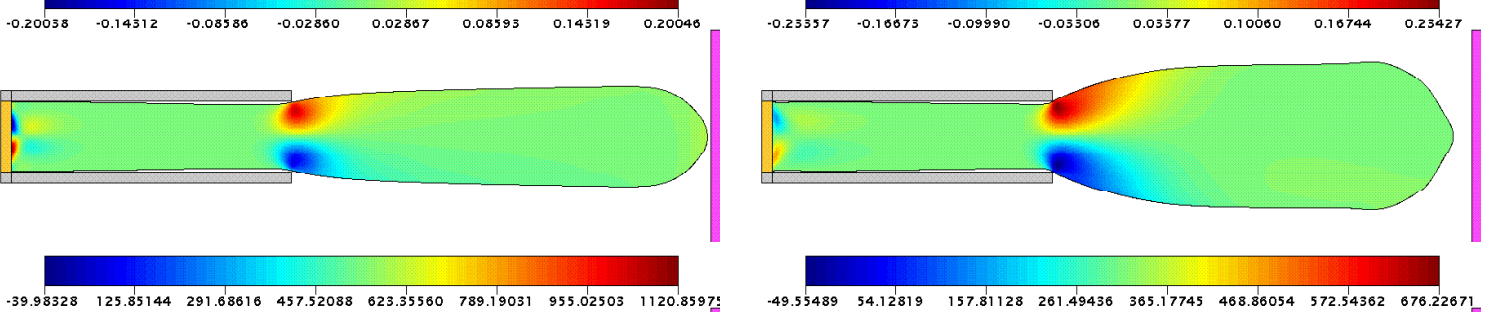

$\begin{array}{llllllllll}-39.98328 & 125.85144 & 291.88818 & 457.52083 & 825.55580 & 789.19031 & 955.02503 & 1120.859\end{array}$
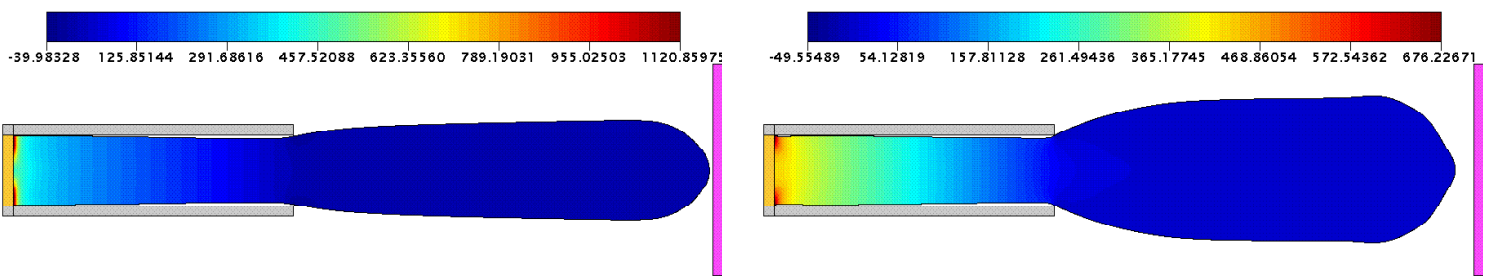

(c)
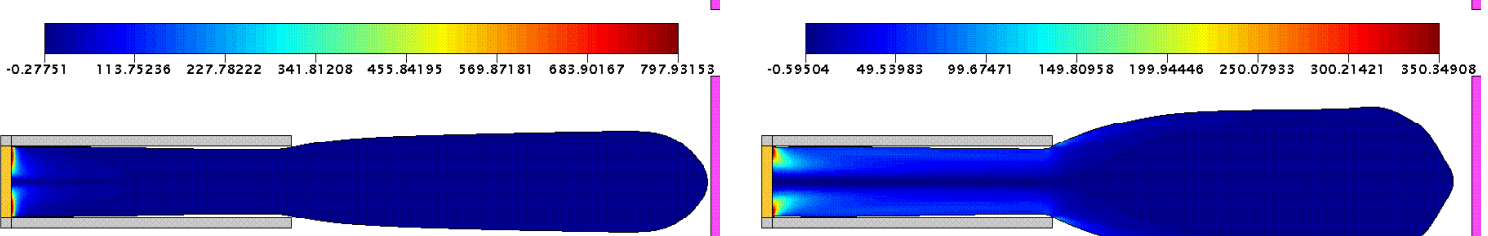

(d)
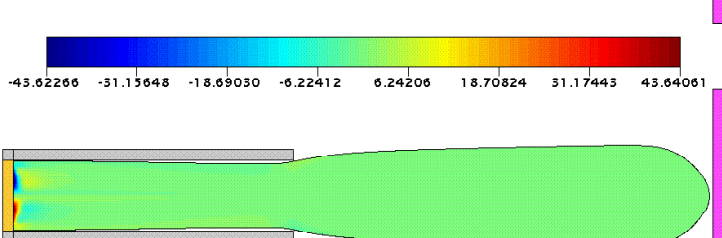

(e)
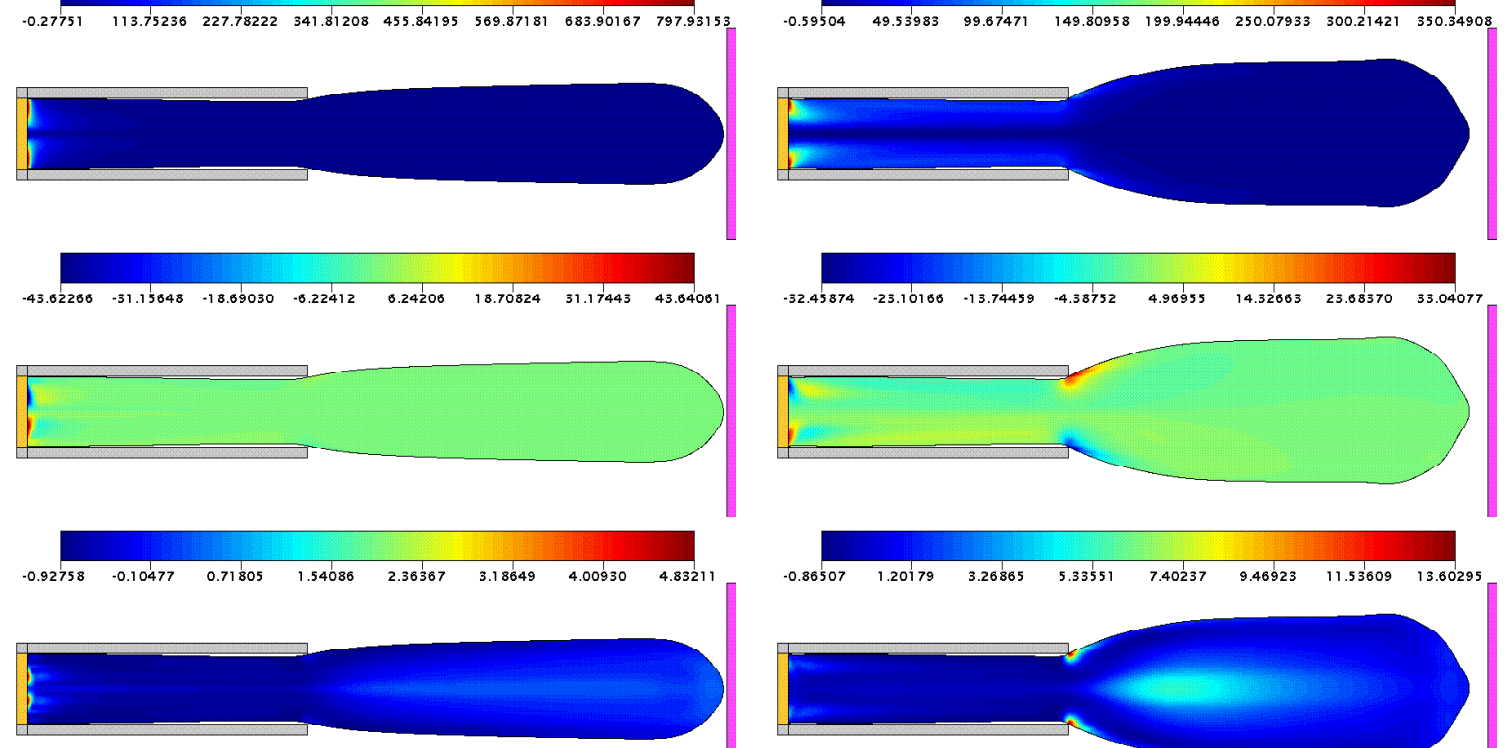

(f)
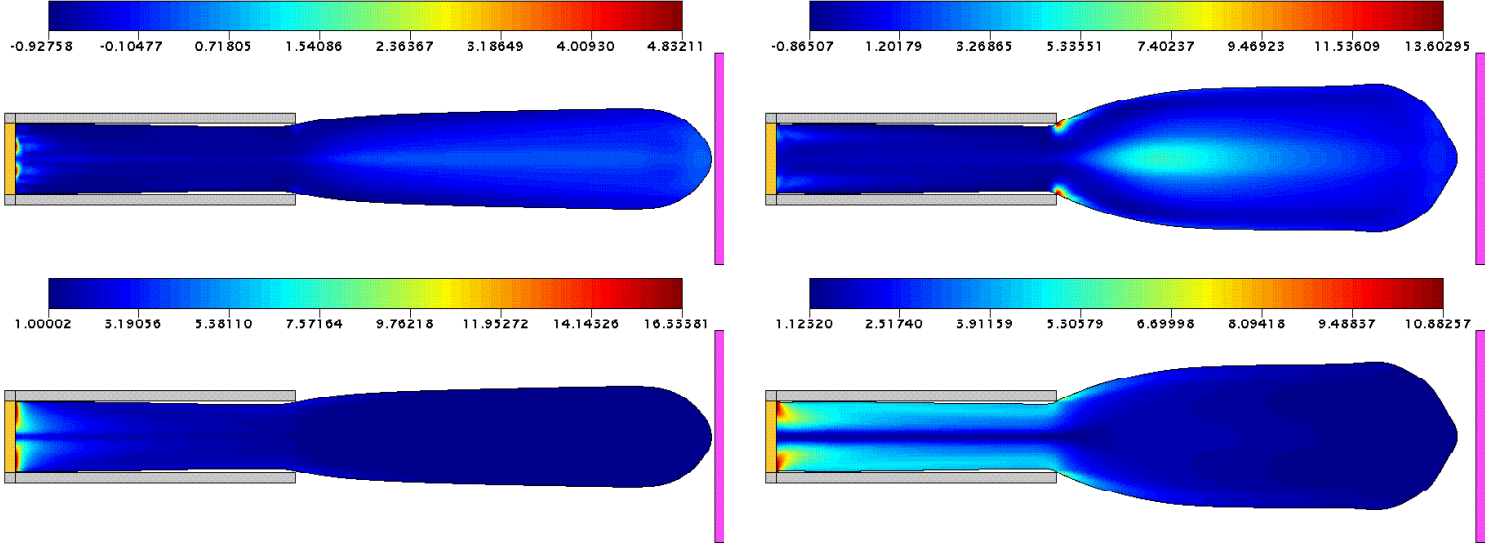

(g)

Figura B.3.8: Soluções numéricas (curvas de nível para $u$ (a), $v(\mathrm{~b}), p(\mathrm{c}), T^{x x}$ (d), $T^{x y}$ (e), $T^{y y}$ (f) e $\lambda(\mathrm{g}))$ da FI-EI para o inchamento do extrudado de um fluido $\operatorname{SXPP}(R e=0.05, W e=10.0, \alpha=0.01$, $\beta=0.5, Q=8.0$ ) com $\gamma=0.2$ (coluna esquerda) e $\gamma=0.9$ (coluna direita), respectivamente, nos instantes $t=17.0$ e $t=21.0$. 


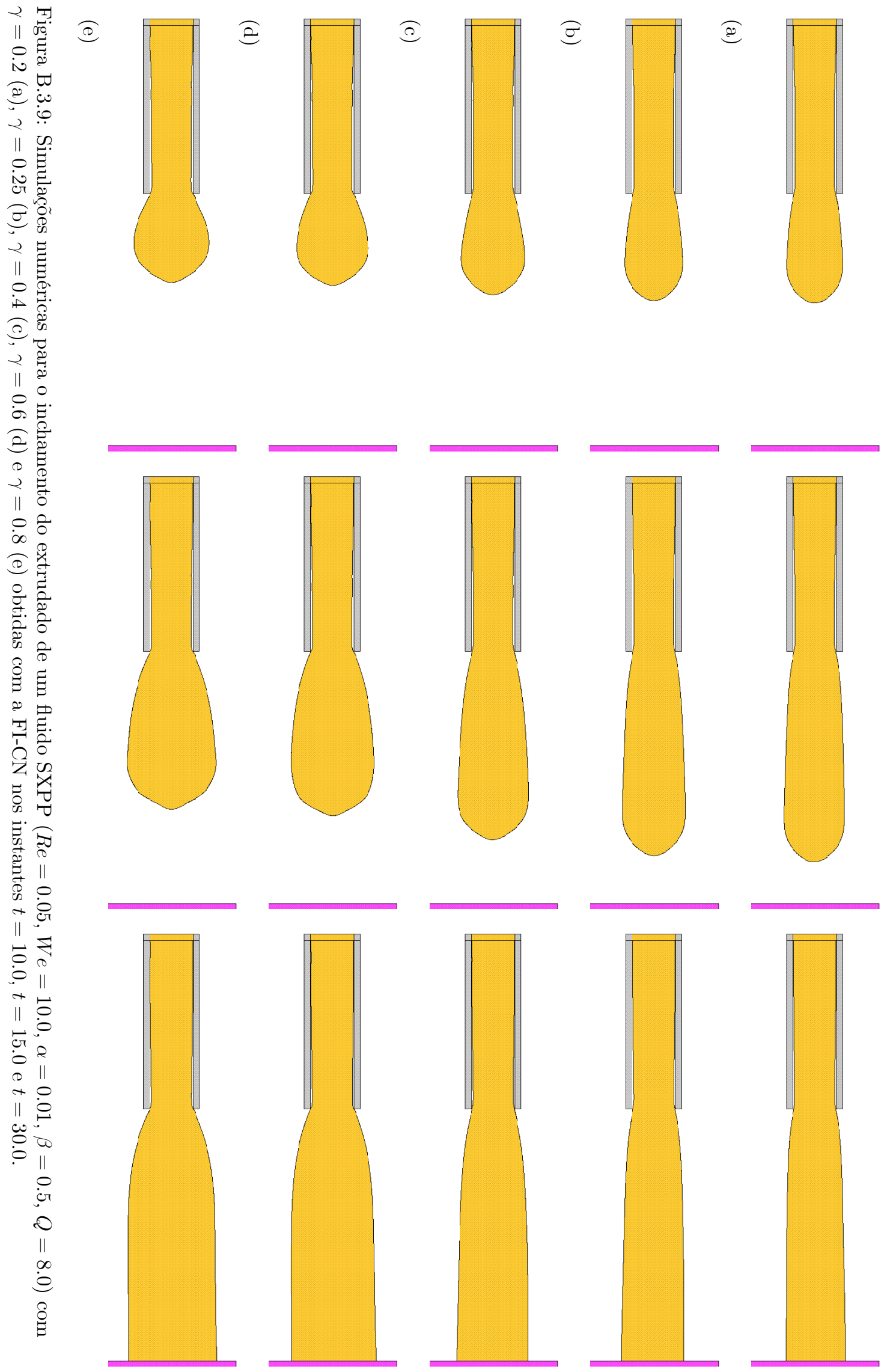




\section{B.3.4 Influência de $W e$}

Adicionalmente ao modelo considerado, adotamos:

- Parâmetros adimensionais:

$$
\begin{aligned}
& -R e=0.05, \alpha=0.01, \beta=0.5, \gamma=0.8, Q=8.0 . \\
& -W e \in\{0.5,1.0,2.0,4.0,7.0,11.0,15.0,20.0\}
\end{aligned}
$$

- Fator de controle do passo temporal: $F_{v i s c}=16.0$.

Portanto, os resultados apontam que a taxa de inchamento $S_{r}$ é incrementada quando aumentamos o valor de $W e$. Novamente, existe boa concordância entre os resultados obtidos pela FI-EI e pela FI-CN.

Tabela B.3.4: Taxas de inchamento de um fluido $\operatorname{SXPP}(R e=0.05, \alpha=0.01, \beta=0.5, \gamma=0.8, Q=8.0)$ em função de $W e$.

\begin{tabular}{|c|c|c|c|c|c|c|}
\cline { 2 - 7 } \multicolumn{1}{c|}{} & \multicolumn{3}{c|}{$F I-E I$} & \multicolumn{3}{c|}{$F I-C N$} \\
\hline$W e$ & $t=10.0$ & $t=15.0$ & $t=65.0$ & $t=10.0$ & $t=15.0$ & $t=65.0$ \\
\hline \hline 0.5 & 1.1435 & 1.1634 & 1.3503 & 1.1433 & 1.1631 & 1.3519 \\
1.0 & 1.2170 & 1.2458 & 1.4101 & 1.2168 & 1.2435 & 1.4095 \\
2.0 & 1.3423 & 1.3830 & 1.5406 & 1.3424 & 1.3424 & 1.5410 \\
4.0 & 1.4849 & 1.5649 & 1.7355 & 1.4851 & 1.5685 & 1.7353 \\
7.0 & 1.6668 & 1.8885 & 1.9778 & 1.6653 & 1.8886 & 1.9785 \\
11.0 & 1.7851 & 2.0949 & 2.1842 & 1.7855 & 2.0945 & 2.1775 \\
15.0 & 1.8357 & 2.1540 & 2.2661 & 1.8356 & 2.1548 & 2.2662 \\
20.0 & 1.8542 & 2.1474 & 2.2972 & 1.8546 & 2.1472 & 2.2983 \\
\hline
\end{tabular}

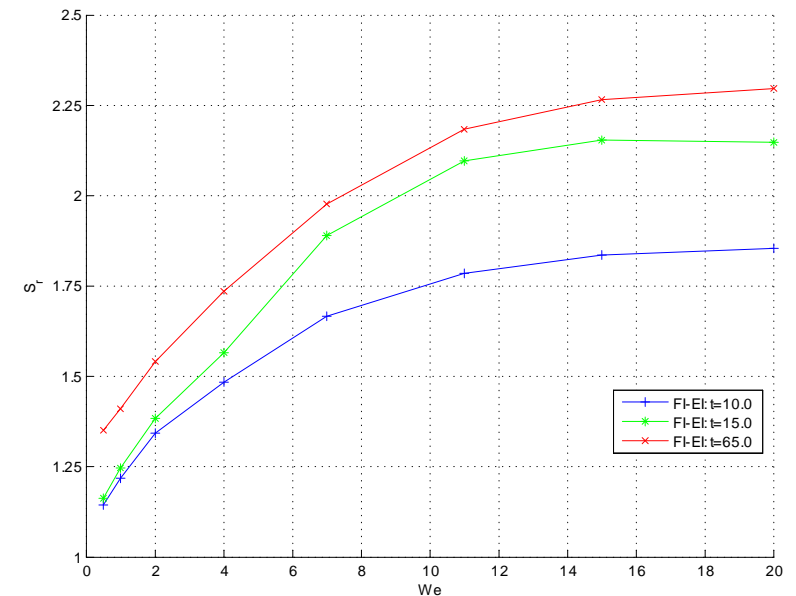

(a)

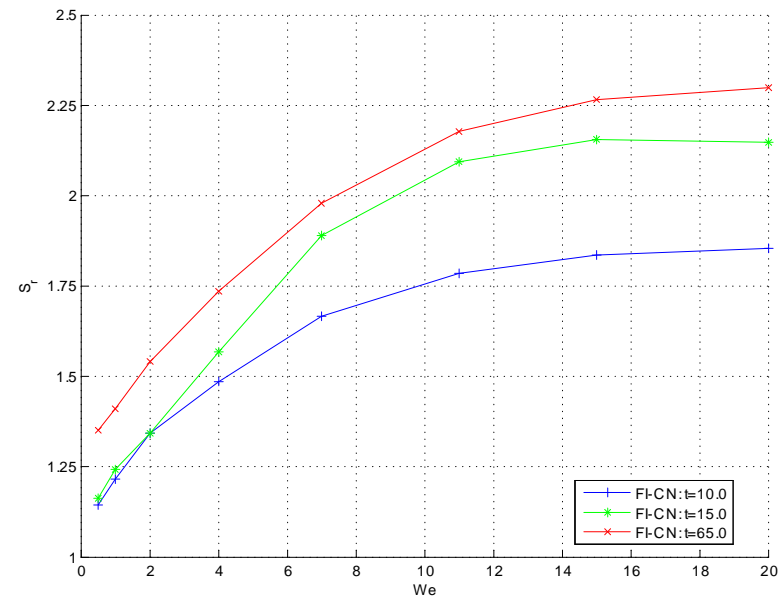

(b)

Figura B.3.10: Taxa de inchamento $S_{r}$ de um fluido $\operatorname{SXPP}(R e=0.05, \alpha=0.01, \beta=0.5, \gamma=0.8$, $Q=8.0$ ) em função de $W e$ para as soluções numéricas obtidas com: FI-EI (a); FI-CN (b). 

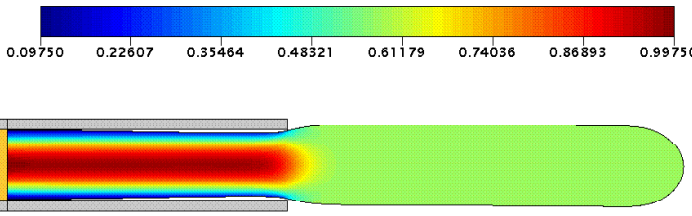

(a)
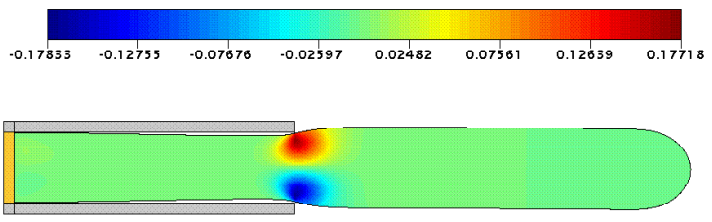

(b)
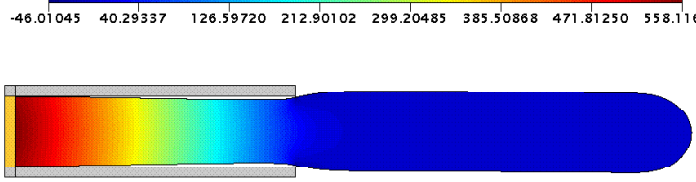

(c)
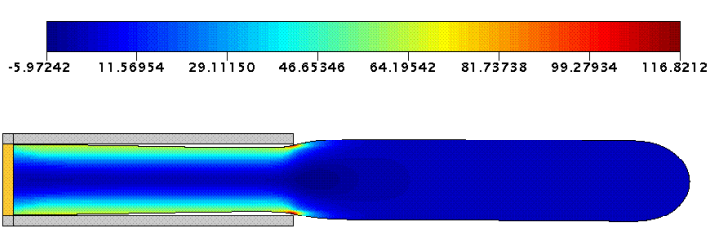

(d)

(e)
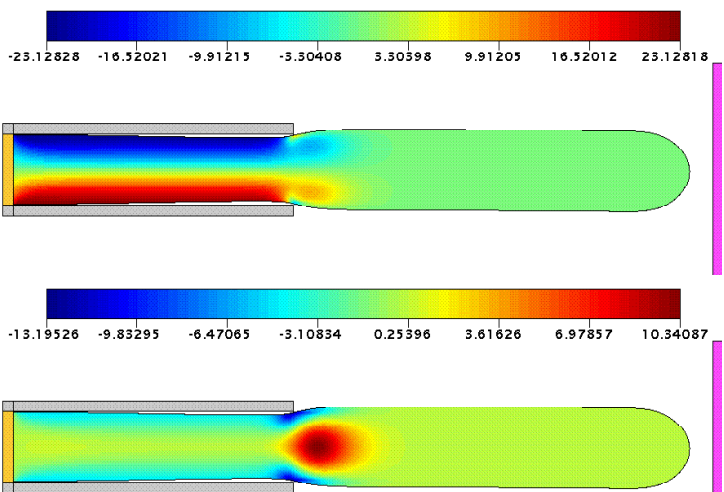

(f)
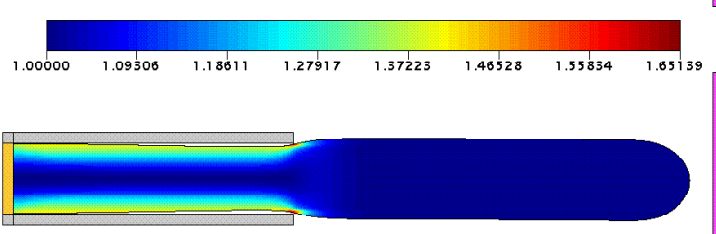

$(\mathrm{g})$
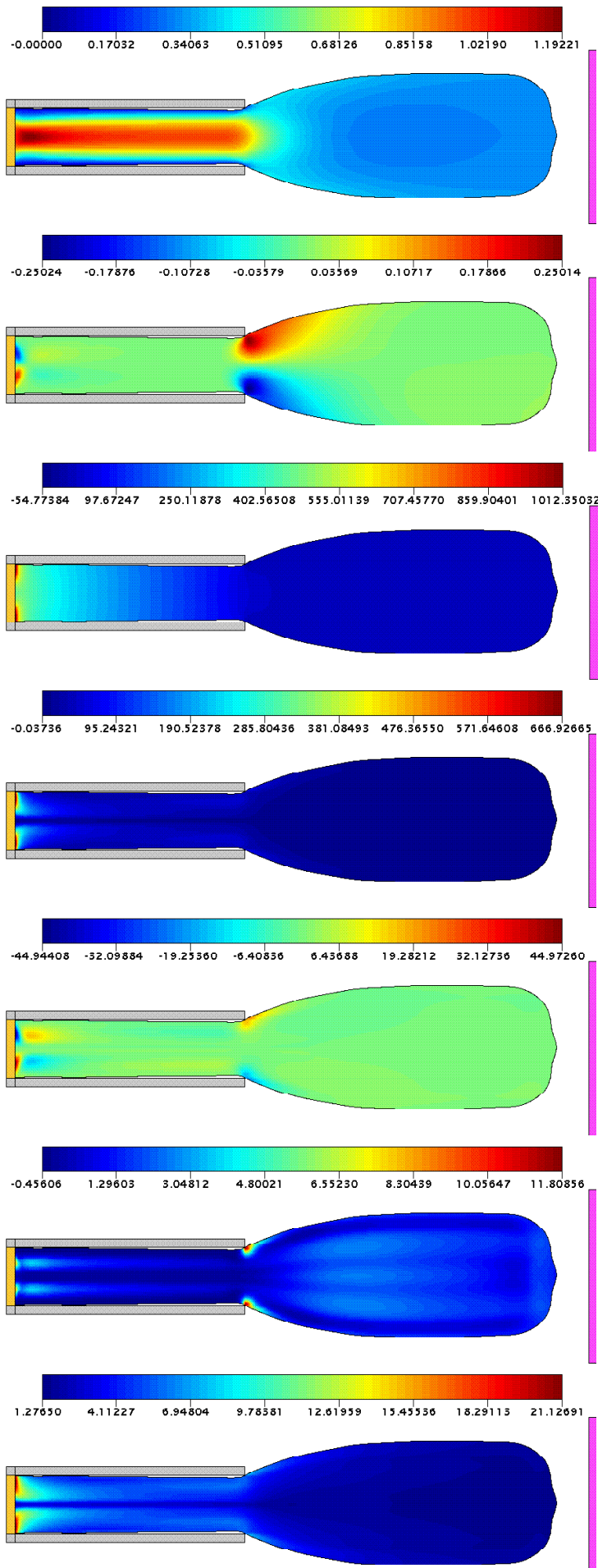

Figura B.3.11: Soluções numéricas (curvas de nível para $u$ (a), $v$ (b), $p$ (c), $T^{x x}$ (d), $T^{x y}$ (e), $T^{y y}$ (f) e $\lambda(\mathrm{g}))$ da FI-EI para o inchamento do extrudado de um fluido SXPP $(R e=0.05, \alpha=0.01, \beta=0.5$, $\gamma=0.8, Q=8.0$ ) com $W e=0.5$ (coluna esquerda) e $W e=15.0$ (coluna direita), respectivamente, nos instantes $t=15.0$ e $t=21.0$. 


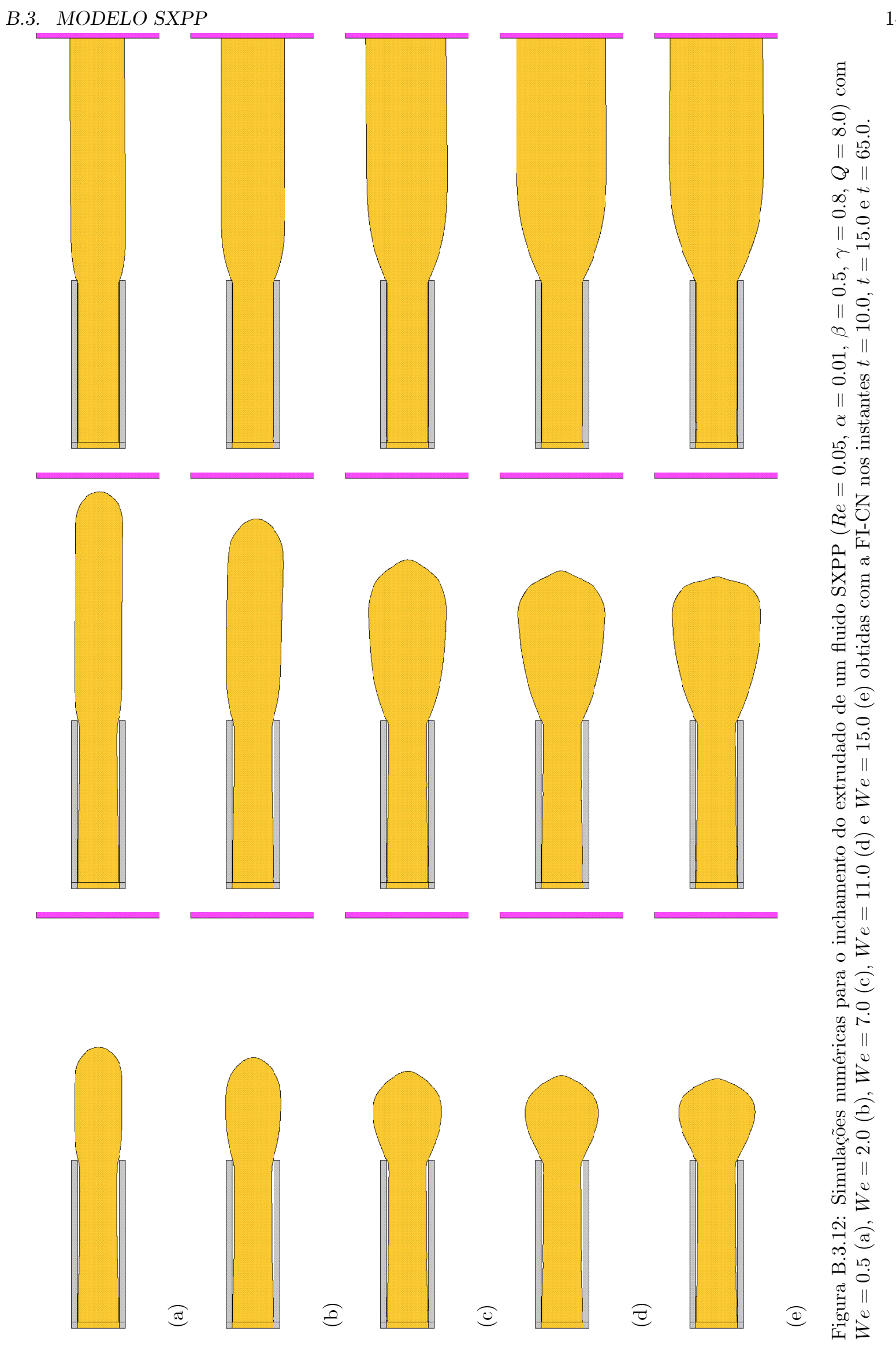




\section{B.3.5 Influência de $Q$}

Adicionalmente ao modelo considerado, adotamos:

- Parâmetros adimensionais:

- Re $=0.05, W e=10.0, \alpha=0.01, \beta=0.5, \gamma=0.3$.

$-Q \in\{1.0,2.0,4.0,7.0,11.0,15.0,20.0\}$.

- Fator de controle do passo temporal: $F_{v i s c}=16.0$.

Portanto, os resultados apontam que a taxa de inchamento $S_{r}$ é incrementada quando aumentamos o valor de $Q$. Mais uma vez, existe boa concordância entre os resultados obtidos pela FI-EI e pela FI-CN.

Tabela B.3.5: Taxas de inchamento de um fluido $\operatorname{SXPP}(R e=0.05, W e=10.0, \alpha=0.01, \beta=0.5$, $\gamma=0.3)$ em função de $Q$.

\begin{tabular}{|c|c|c|c|c|c|c|}
\cline { 2 - 7 } \multicolumn{1}{c|}{} & \multicolumn{3}{c|}{$F I-E I$} & \multicolumn{3}{c|}{$F I-C N$} \\
\hline$Q$ & $t=10.0$ & $t=15.0$ & $t=65.0$ & $t=10.0$ & $t=15.0$ & $t=65.0$ \\
\hline \hline 1.0 & 1.2364 & 1.3161 & 1.4527 & 1.2366 & 1.3167 & 1.4524 \\
2.0 & 1.2740 & 1.3696 & 1.5228 & 1.2738 & 1.3696 & 1.5217 \\
4.0 & 1.3240 & 1.4371 & 1.5853 & 1.3246 & 1.4374 & 1.5842 \\
7.0 & 1.3775 & 1.5090 & 1.6551 & 1.3777 & 1.5091 & 1.6699 \\
11.0 & 1.4507 & 1.5890 & 1.7946 & 1.4506 & 1.5891 & 1.7951 \\
15.0 & 1.5153 & 1.6554 & 1.8956 & 1.5160 & 1.6558 & 1.8929 \\
20.0 & 1.5823 & 1.7279 & 1.9793 & 1.5825 & 1.7283 & 1.9797 \\
\hline
\end{tabular}

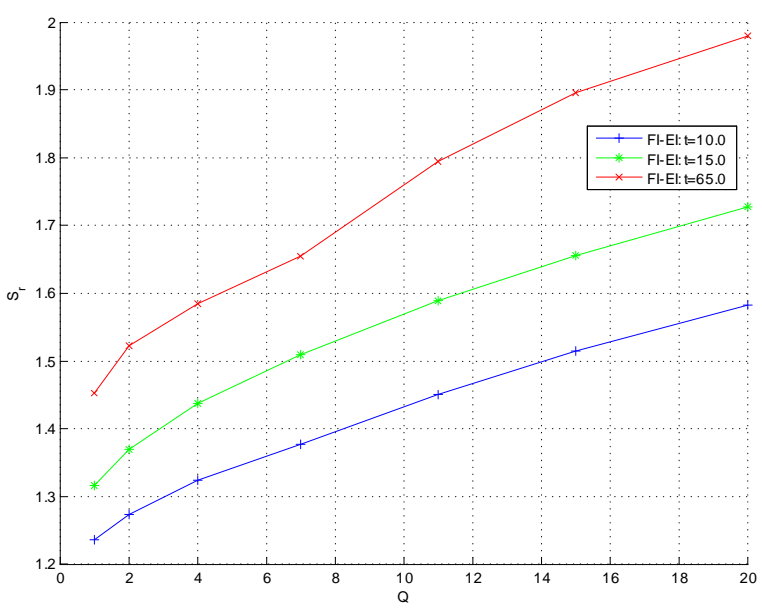

(a)

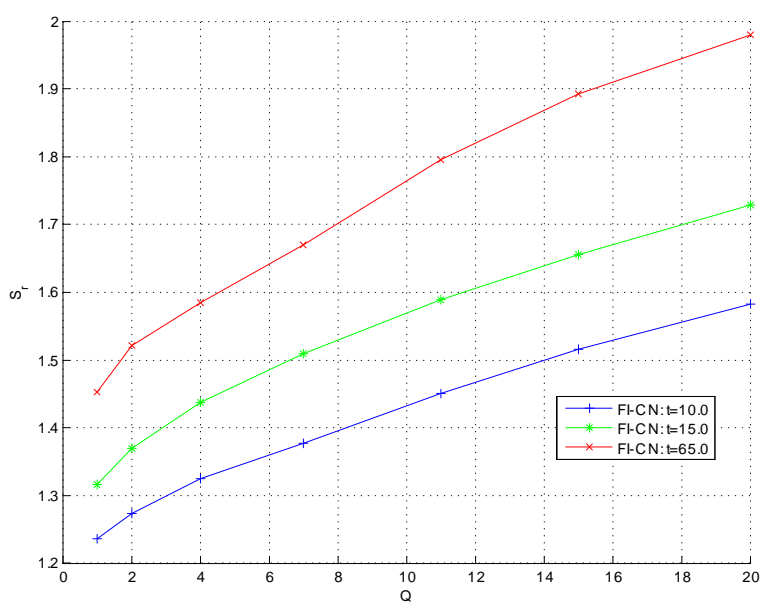

(b)

Figura B.3.13: Taxa de inchamento $S_{r}$ de um fluido $\operatorname{SXPP}(R e=0.05, W e=10.0, \alpha=0.01, \beta=0.5$, $\gamma=0.3)$ em função de $Q$ para as soluções numéricas obtidas com: FI-EI (a); FI-CN (b). 

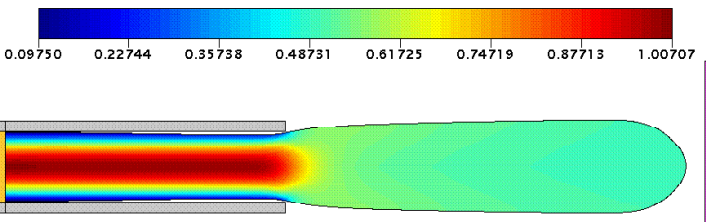

(a)
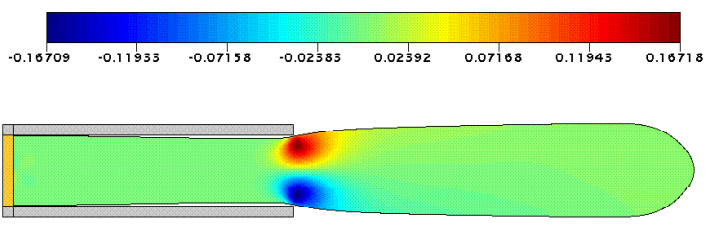

(b)
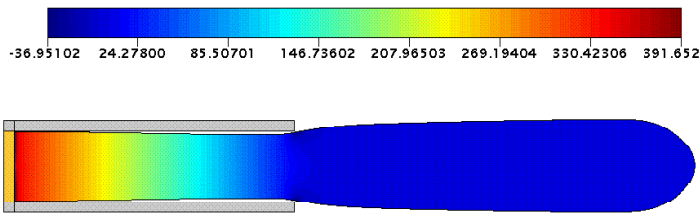

(c)
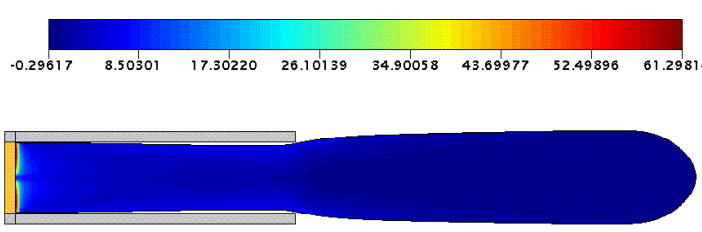

(d)
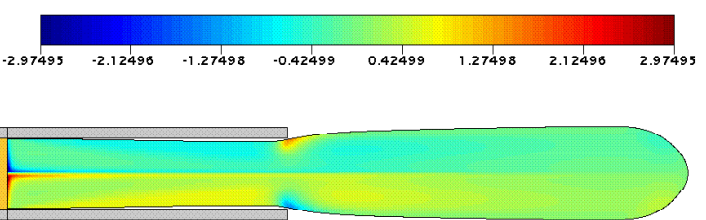

(e)
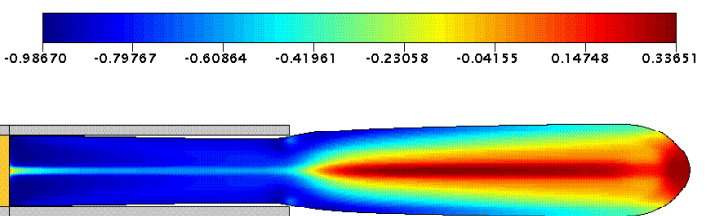

(f)
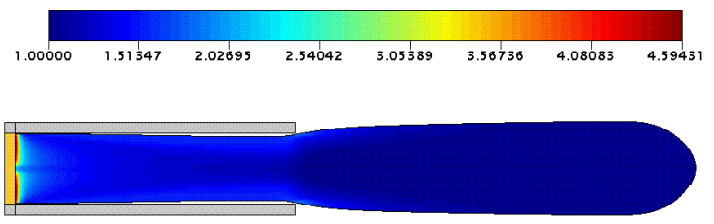

(g)
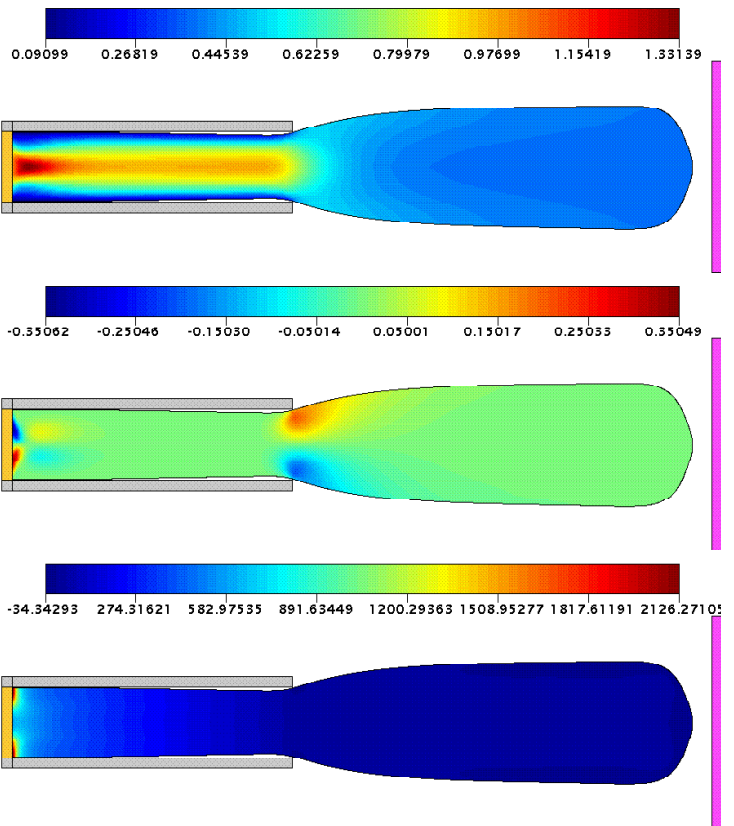

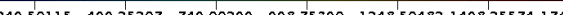
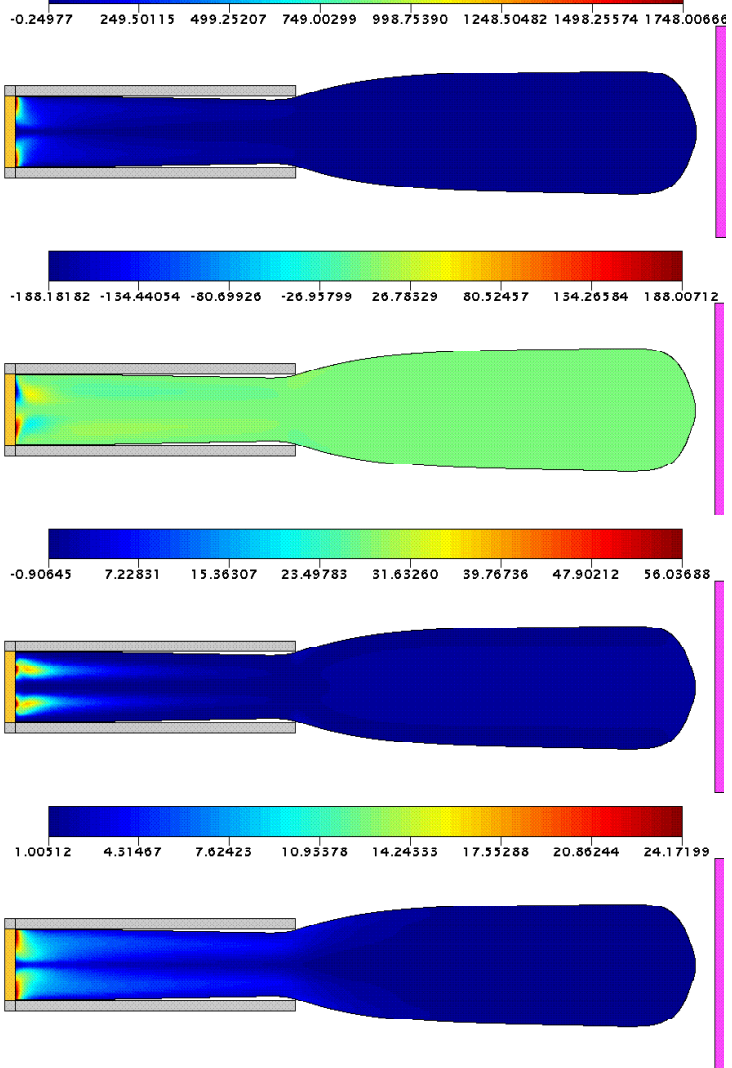

Figura B.3.14: Soluções numéricas (curvas de nível para $u$ (a), $v(\mathrm{~b}), p(\mathrm{c}), T^{x x}$ (d), $T^{x y}$ (e), $T^{y y}$ (f) e $\lambda$ (g)) da FI-CN para o inchamento do extrudado de um fluido SXPP $(R e=0.05, W e=10.0, \alpha=0.01$, $\beta=0.5, \gamma=0.3$ ) com $Q=1.0$ (coluna esquerda) e $Q=20.0$ (coluna direita), respectivamente, nos instantes $t=16.0$ e $t=19.0$. 


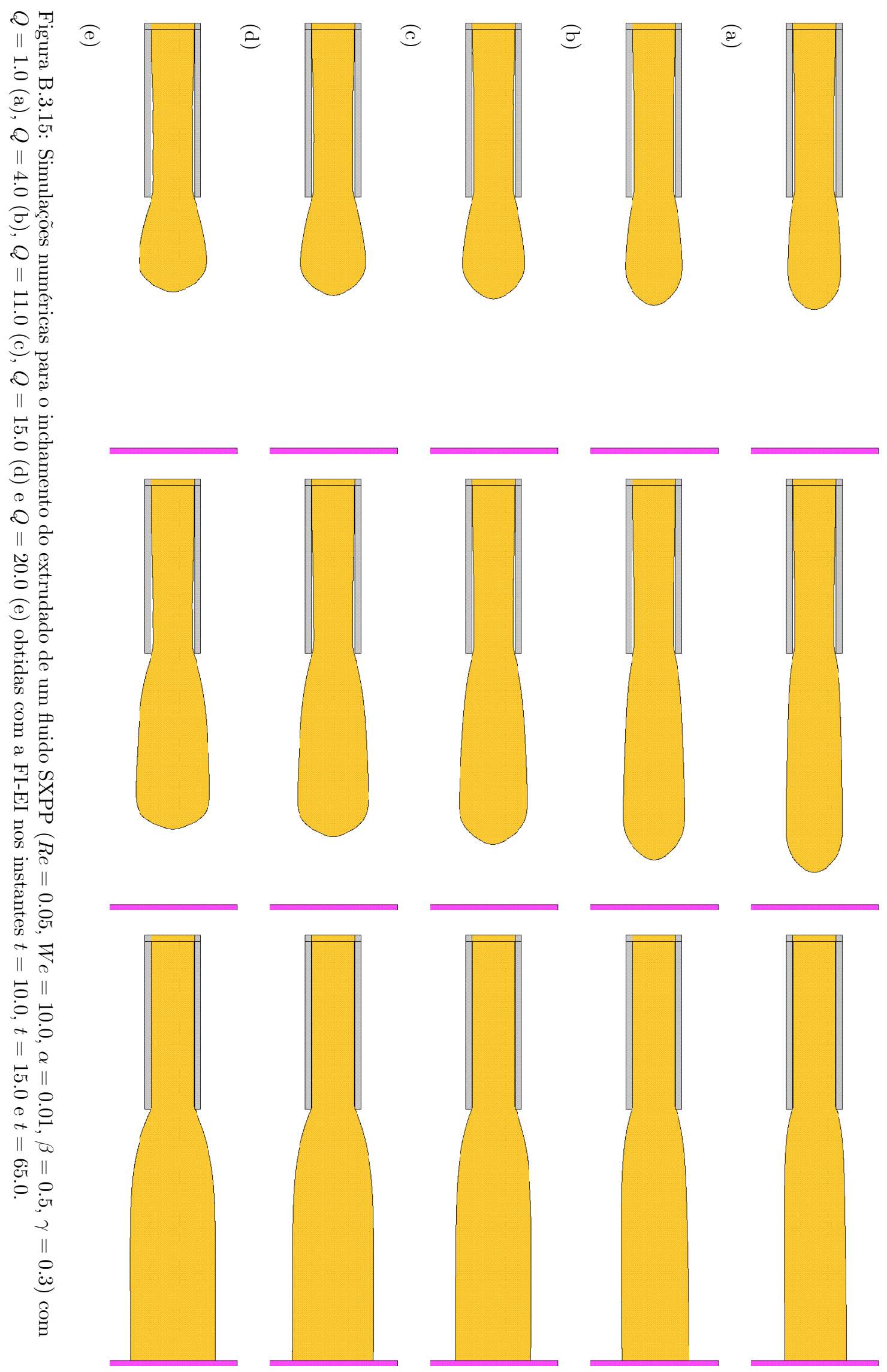




\section{APÊNDICE}

\section{Simulações numéricas para o problema da gota em impacto}

\section{C.1 Introdução}

Nos dois apêndices anteriores, foi realizado um exaustivo estudo com objetivo de mostrar, para cada problema considerado, a influência dos parâmetros da equação constitutiva na solução numérica obtida. Neste apêndice, nossas pretensões se resumem a detalhar o problema da gota em impacto, no entanto, sem repetir um estudo nos moldes do Apêndice 1 e do Apêndice 2.

Este problema foi utilizado várias vezes neste trabalho e faz-se interessante pelo fato de suas soluções para fluidos newtoniano e viscoelástico apresentarem comportamentos muito diferentes, possibilitando que a viscoelasticidade seja, efetivamente, "enxergada". Assim, o objetivo deste apêndice é detalhar visualmente a evolução temporal da solução do problema da gota em impacto considerando um fluido newtoniano e fluidos viscoelásticos dos tipos Oldroyd-B e SXPP. Para isso, voltamos a considerar o modelo principal utilizado na Seção 6.4 e utilizamos a FI do GENSMAC e do GENSMAC-SXPP, ambas com o esquema RK21 para movimentar a superfície livre.

Novamente, a FI-EI e a FI-CN produziram resultados muito semelhantes. Assim, nas figuras apresentadas, escolhemos alternadamente o método numérico, a fim de evitar repetições desnecessárias. Portanto, na Seção C.2 detalhamos o comportamento da solução para uma gota de fluido newtoniano, enquanto na Seção C.3 abordamos o comportamento de gotas viscoelásticas do tipo Oldroyd-B e SXPP.

\section{C.2 Modelo newtoniano}

Neste caso, o modelo inicialmente apresentado é complementado com:

- Parâmetro adimensional: $R e=5.0$.

- Fator de controle do passo temporal: $F_{v i s c}=25.6$.

Assim, na Figura C.2.1, é apresentada a evolução temporal da componente $u$ da velocidade, obtida com a FI-EI do GENSMAC. Analisando a sequência, podemos notar que, após o impacto contra a superfície rígida, a gota passa a se espalhar lateralmente no fundo da caixa. Entretanto, a medida que a energia mecânica é dissipada pelas forças viscosas, internas ao fluido, percebe-se que a velocidade horizontal da gota diminui, em módulo, até praticamente estacionar no funda da caixa. Portanto, após o impacto, o movimento da gota newtoniana consiste, apenas, em um escoamento lateral, no qual a velocidade diminui de forma monótona até a gota ocupar todo fundo da caixa. 
146 APÊNDICE C. SIMULAÇÕES NUMÉRICAS PARA O PROBLEMA DA GOTA EM IMPACTO

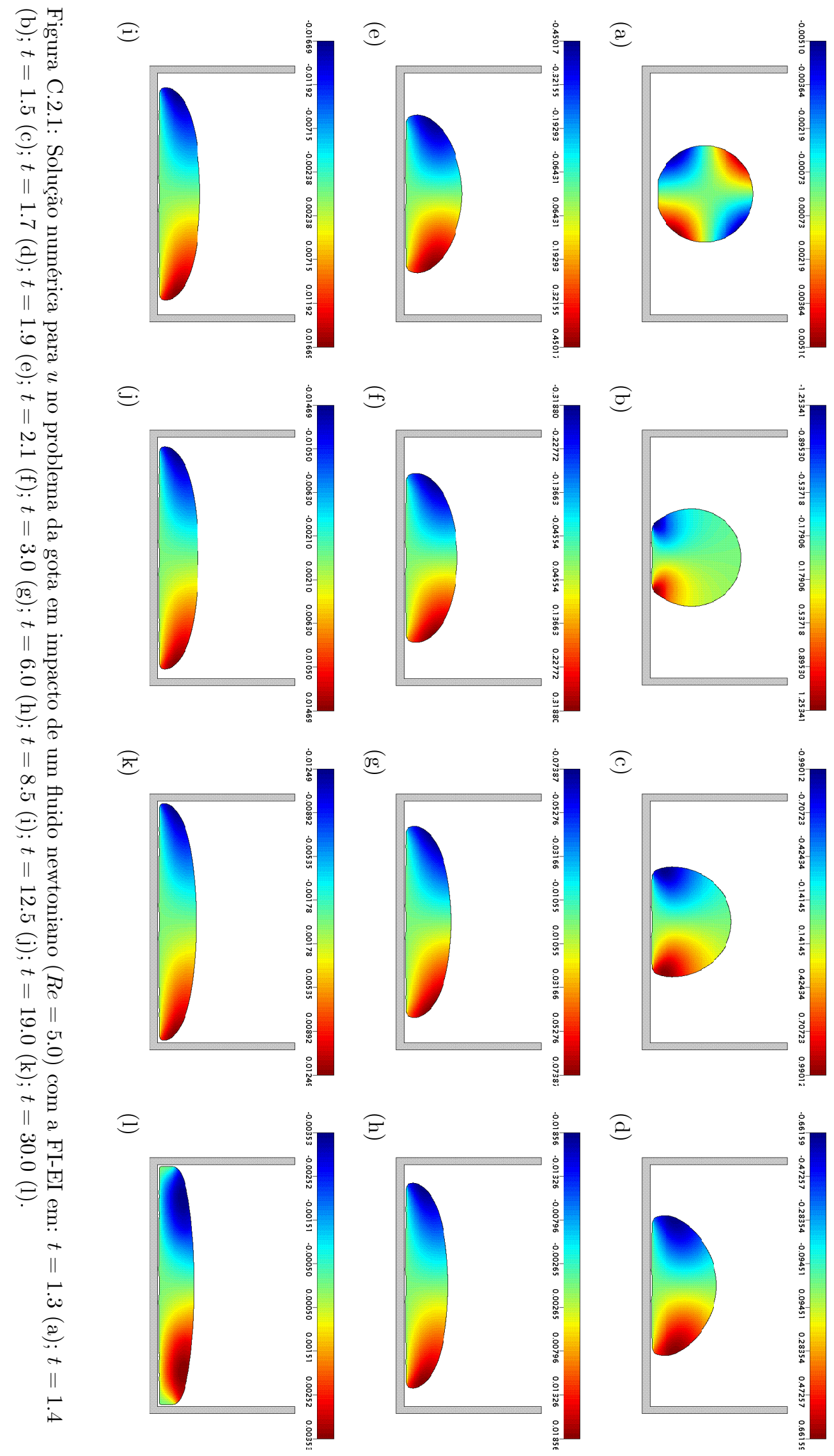




\section{C.3 Modelos viscoelásticos (Oldroyd-B e SXPP)}

O modelo inicialmente citado é complementado com os seguintes dados:

- Parâmetros adimensionais:

- Oldroyd-B: $R e=5.0, \beta=0.1, W e \in\{1.0,2.5\}$.

- SXPP: Re $=5.0, \alpha=0.01, \beta=0.1, \gamma=0.8, Q=4.0, W e \in\{1.0,2.5\}$.

- Fator de controle do passo temporal: $F_{\text {visc }}=25.6$.

Assim, a Figura C.3.1 e a Figura C.3.2 mostram as soluções obtidas para o fluido Oldroyd-B com $W e=1.0$ e $W e=2.5$, respectivamente. Da mesma forma, a Figura C.3.3 e a Figura C.3.4 apresentam a solução do modelo SXPP com $W e=1.0$ e $W e=2.5$, respectivamente. É importante citar que as soluções de ambos os fluidos para um mesmo $W e$ são bastante semelhantes, o que foi obtido propositadamente pela escolha efetuada nos demais parâmetros.

Nessas figuras, para ambos $W e$, percebe-se que o escoamento da gota difere do padrão newtoniano. Com efeito, após o impacto em (a), a gota inicia o processo de espalhamento lateral diminuindo sua velocidade de (b) para (c). Entretanto, a partir de um dado momento, a gota estaciona e começa a se contrair em (d), adquirindo, novamente, parte da forma inicial em (g). Em seguida, tal fenômeno começa a ser desfeito, com, a partir de $(\mathrm{j})$, as velocidades completamente desinvertidas. Próximo a (k), novamente, a gota estaciona e começa a se contrair, em um processo finalizado em (o). A partir de então e até (s), as velocidades se desinvertem novamente, e o escoamento da gota passa a consistir em um simples espalhamento lateral. Nesta parte final, conforme observamos em (t), (u) e (v), a velocidade de espalhamento da gota não é monótona, mas, podemos detectar certa vibração na superfície livre. De fato, a velocidade atinge, em módulo, um máximo local próximo a (t); em (u) esses valores extremos diminuem e, em (y), a velocidade de espalhamento da gota voltou a aumentar.

Em outras palavras, após o impacto inicial, o escoamento da gota viscoelástica pode ser dividido em fases de contração (onde a gota se retrai e, depois, volta a expandir) e, finalmente, em uma fase vibratória, onde a gota não mais se contrai, mas, expande-se com uma velocidade de espalhamento não monótona.

Assim, tais fenômenos podem ser atribuídos à viscoelasticidade da gota, responsável por armazenar energia para, em seguida, devolvê-la ao escoamento e causar os efeitos observados. Com isso, a gota viscoelástica possui uma memória de passado recente, responsável por fazê-la retornar a uma configuração próxima à alcançada em um passado próximo. Essa proximidade do passado pode ser regulada, entre outros, pelo número de Weissemberg. Com isso, quanto maior $W e$, maior a viscoelasticidade e, consequentemente, a memória do fluido. Com efeito, isto fica nítido observando as simulações nas figuras (d) e (g), pois, as soluções com $W e=2.5$ apresentam um espalhamento lateral maior que com $W e=1.0$ em (d), mas, conseguem retrair e recuperar, em ambos os casos, praticamente a mesma forma em (g). Logo, o fluido apresentou mais memória com $W e=2.5$ do que com $W e=1.0$, o que é coerente com os argumentos apresentados.

Portanto, enquanto o comportamento da gota newtoniana resume-se a uma expansão lateral, a gota viscoelástica resulta em um escoamento mais complexo. 
148 APÊNDICE C. SIMULAÇÕES NUMÉRICAS PARA O PROBLEMA DA GOTA EM IMPACTO
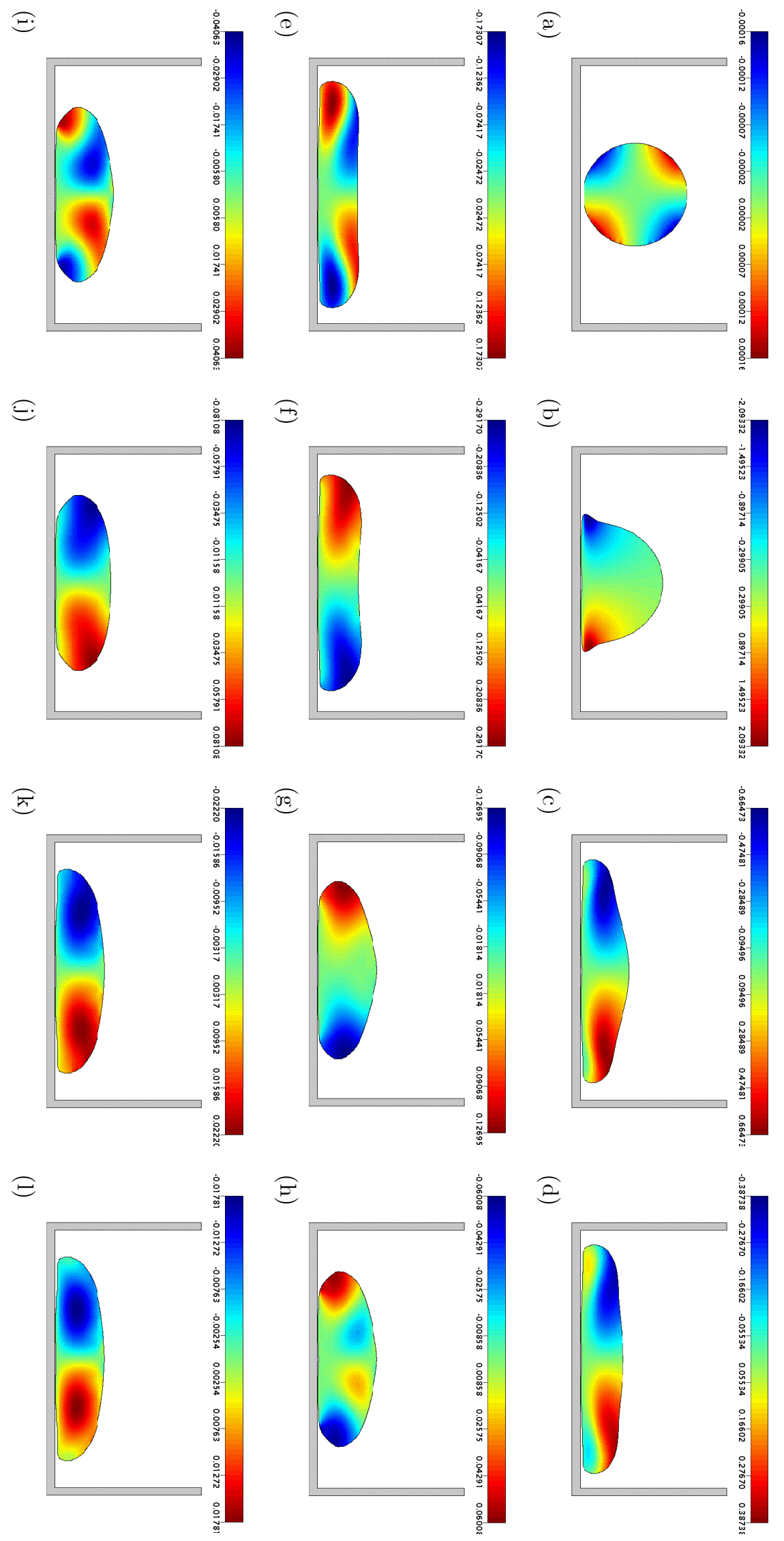

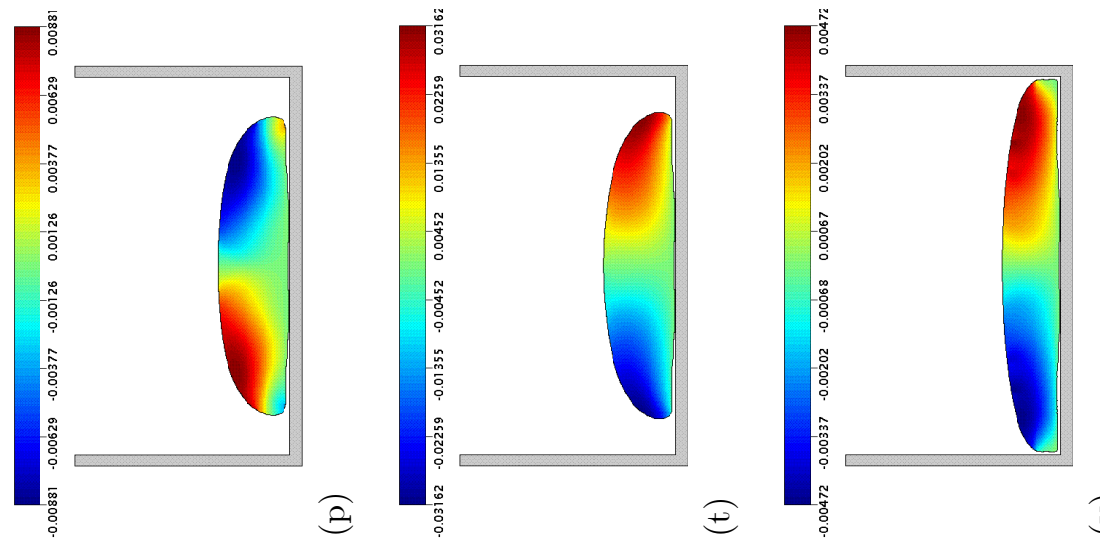

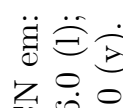
$\begin{array}{lll}Z & 0 & 0 \\ 0 & 0 & 0 \\ 1 & \| 1 & 0\end{array}$ I $+\|$

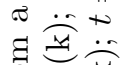

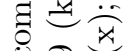
$\rightarrow 10 \mathrm{~m}$

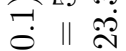
$\|+\|$

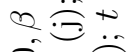
$\stackrel{\rightarrow}{\rightarrow} \underset{\sim}{\stackrel{2}{P}}$
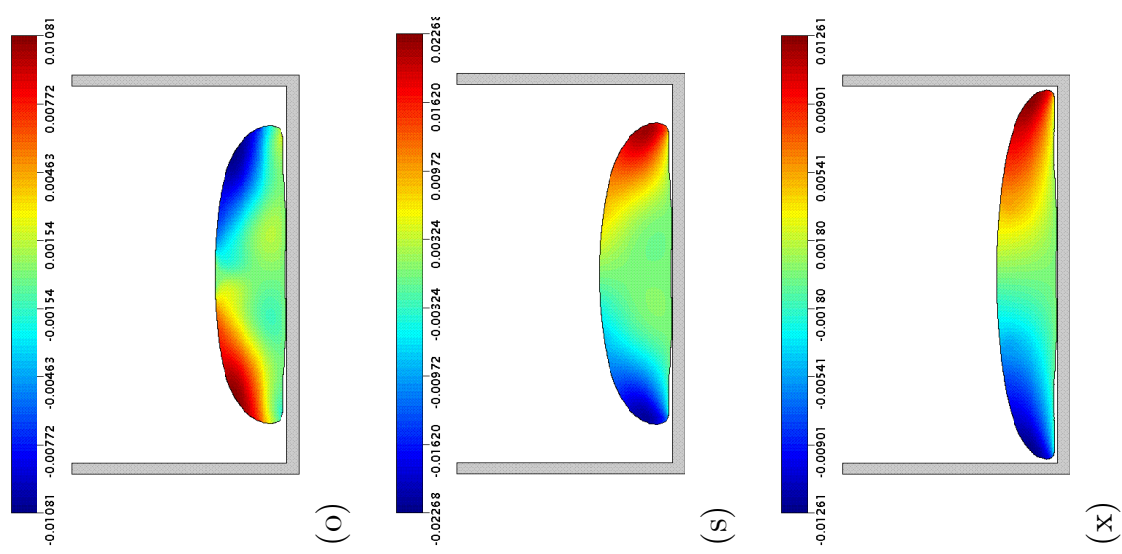

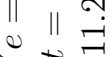

$B \ldots$

0.

$11+\mathcal{F}$

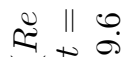

ต $\stackrel{1}{I}+$

क人

矛 $\| \stackrel{\infty}{\infty}$

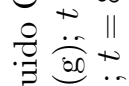

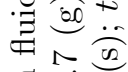
घ o $N$
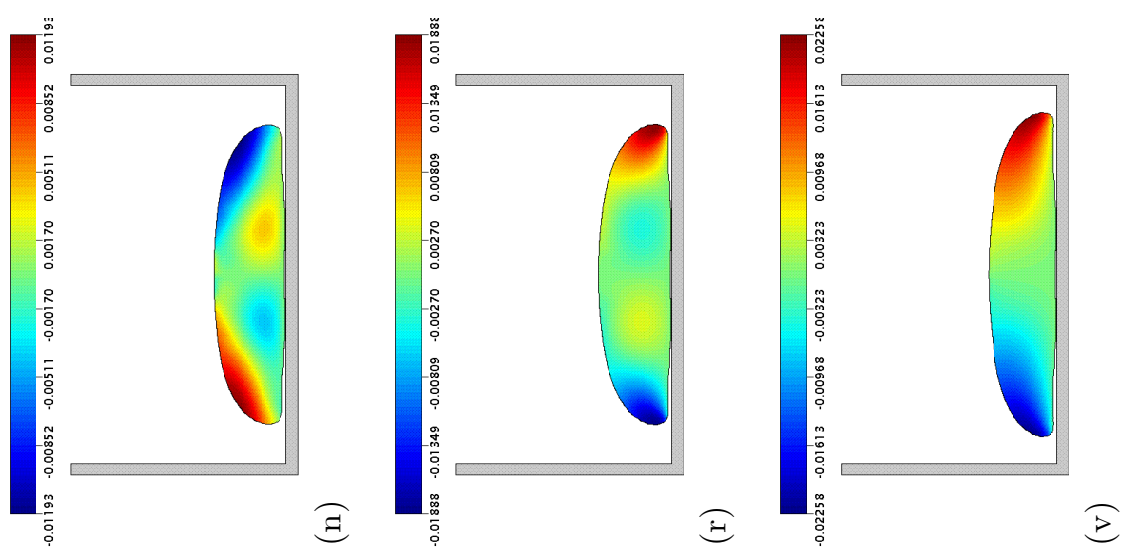

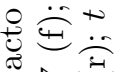

记 ํํ है। $\|$ त) $\infty$ म $\infty$ 이 $\sim$

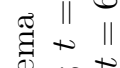

유

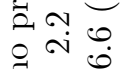

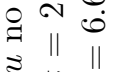
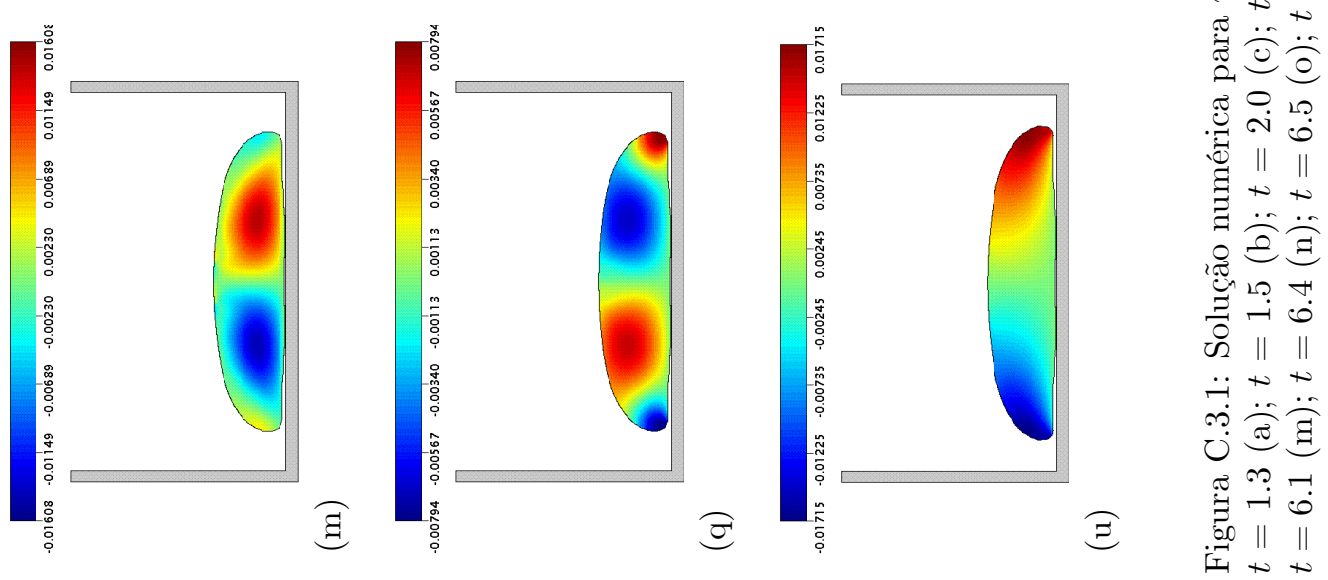
150 APÊNDICE C. SIMULAÇÕES NUMÉRICAS PARA O PROBLEMA DA GOTA EM IMPACTO
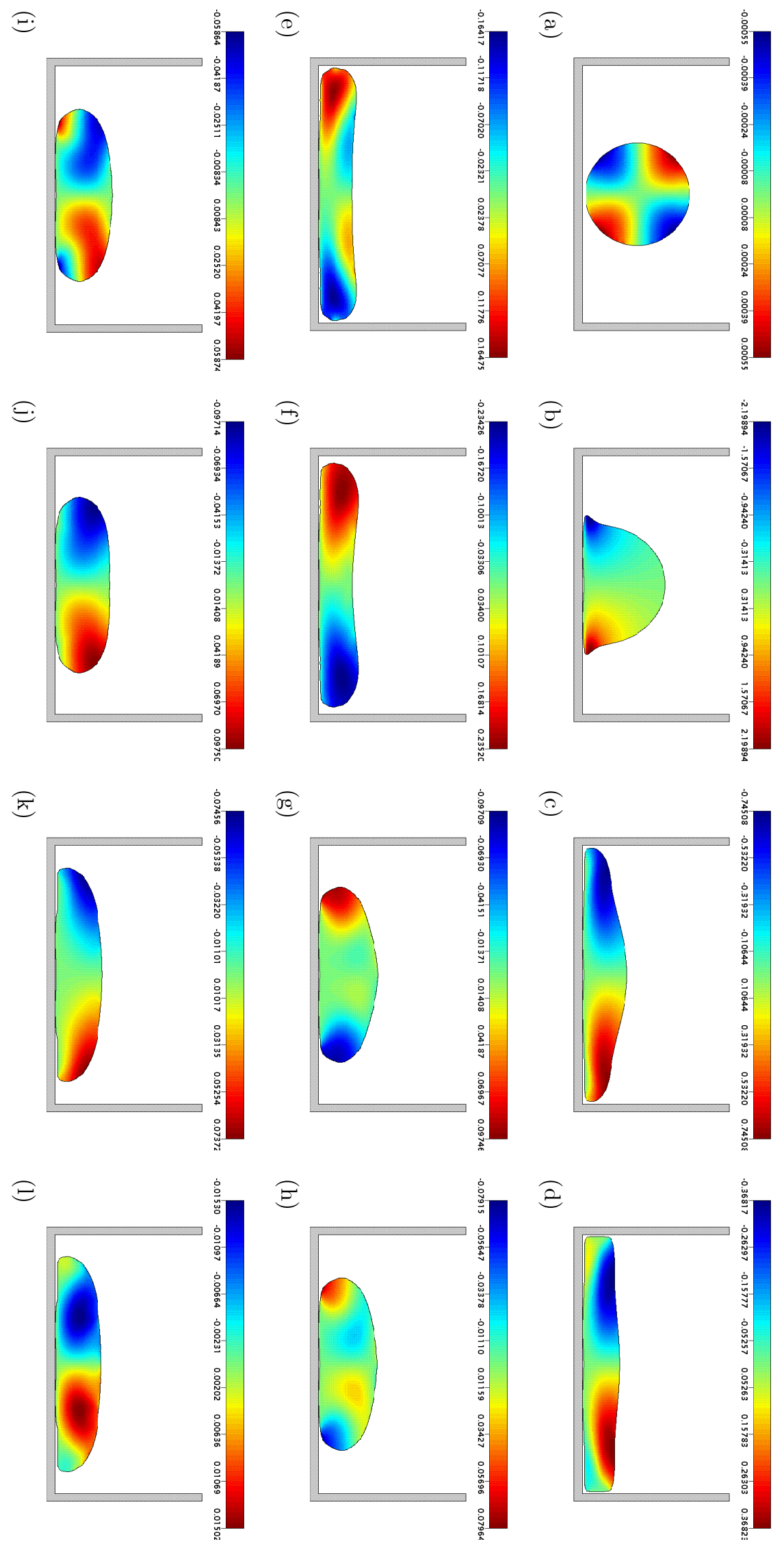

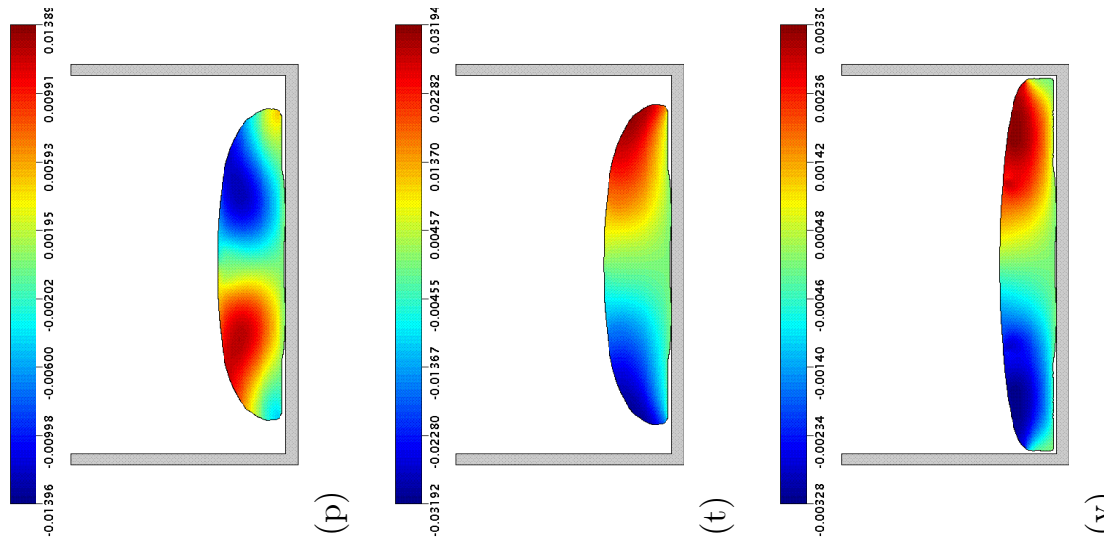

$\ddot{g} \ddot{\ominus} \dot{\ominus}$

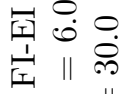
$\approx \stackrel{\infty}{\cdots}+\infty$

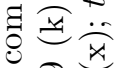
I 100 อ $\| \stackrel{2}{2}$ $\|+\|$

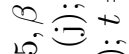
ค่

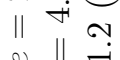
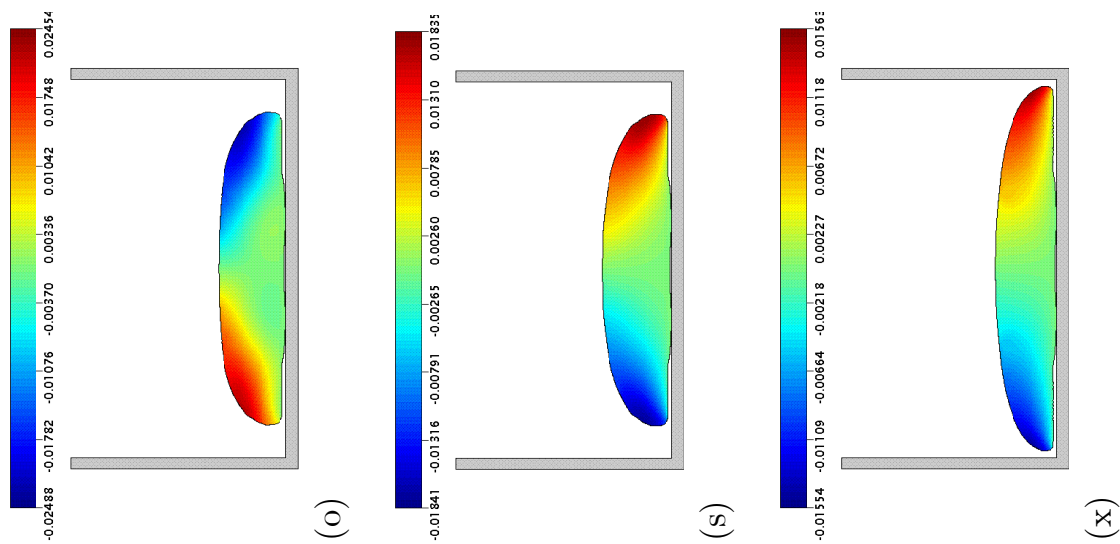

$\stackrel{0}{1}=$ $0 . \ddot{\Theta}$

$10 \stackrel{\circ}{\circ}$

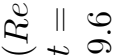

ต $\stackrel{1}{I}+$

b

@ ○ $\| \stackrel{\infty}{0}$

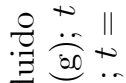
井离 $\exists$ ฉ 0 $\|^{\infty} N$
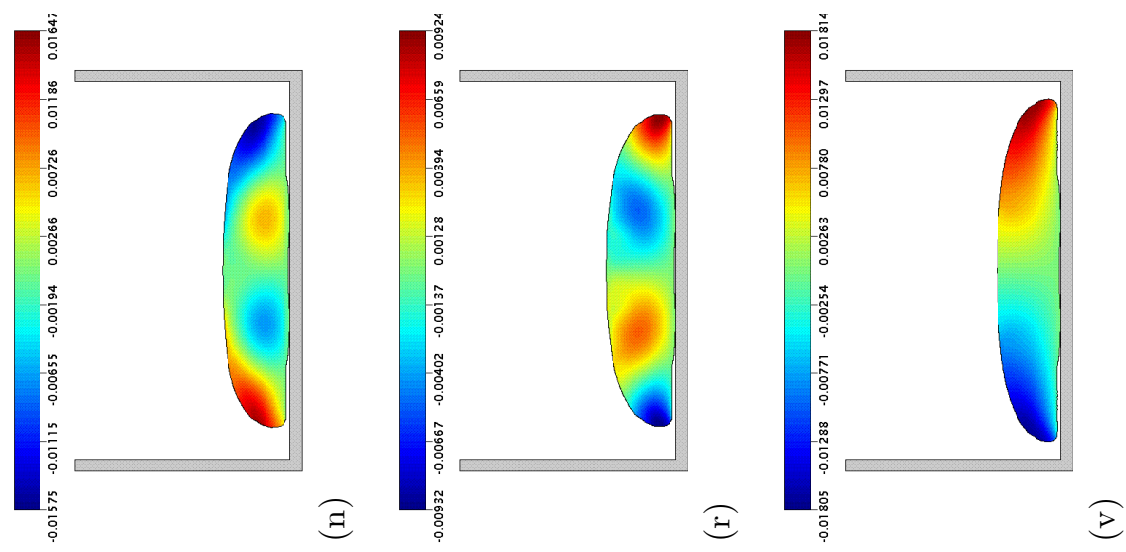

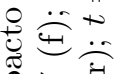
手 저 द्व $\infty$ 莟光 ণ 当 $\|{ }^{0}$ 0항 $\begin{array}{lll}a & 0 \\ \circ & \sim & 0\end{array}$

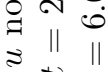
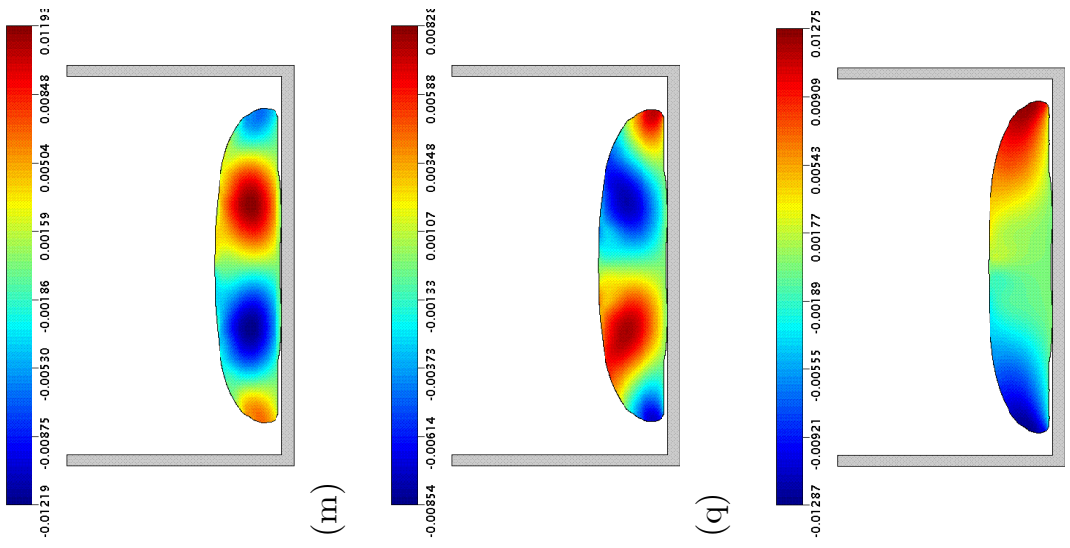

ڤั) ن ฮै। || $\exists+\infty$ 을 نّ ऊ || || $\ddot{\sim}+\ldots$

भ่ ๑ 造 $\|$ 
152 APÊNDICE C. SIMULAÇÕES NUMÉRICAS PARA O PROBLEMA DA GOTA EM IMPACTO
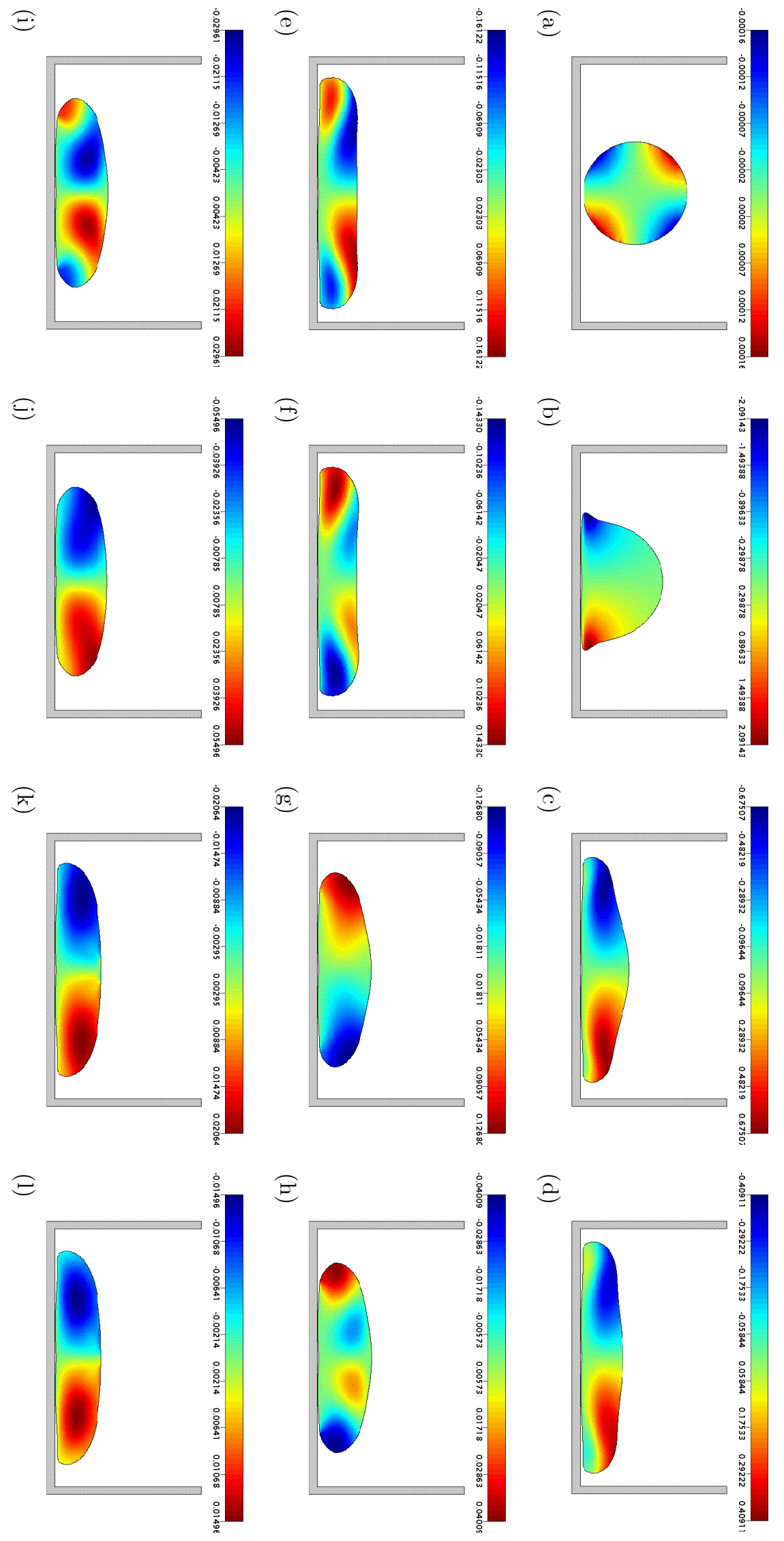
C.3. MODELOS VISCOELÁSTICOS (OLDROYD-B E SXPP)
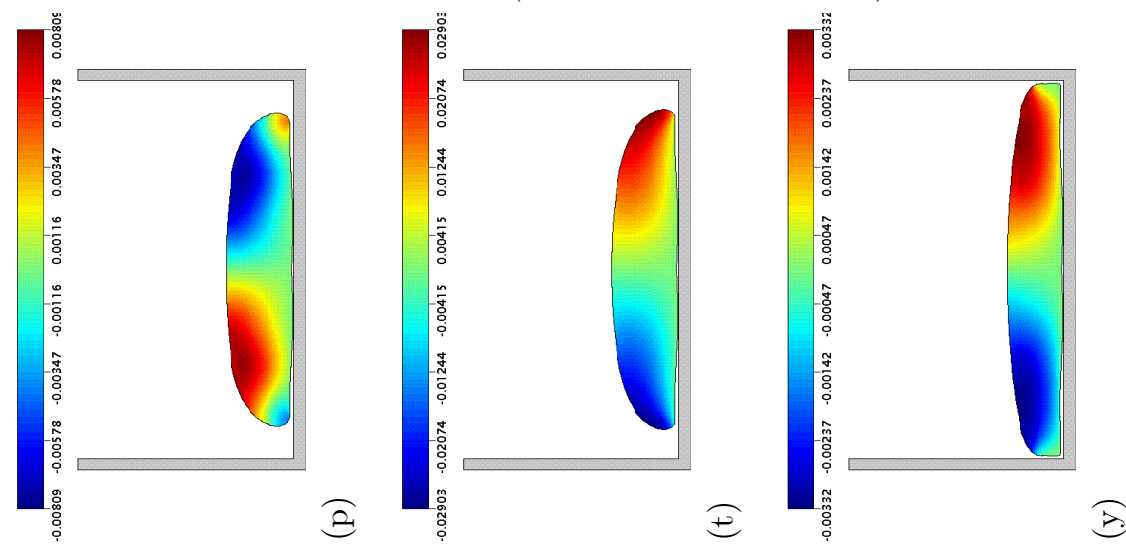

Оิำ

\|\|$\|$

$\infty \div$

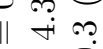

$\tau \| \stackrel{\circ}{\circ}$

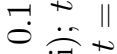

$\| \stackrel{-1}{*}$

i.

$\ddot{0} \| \stackrel{N}{0}$
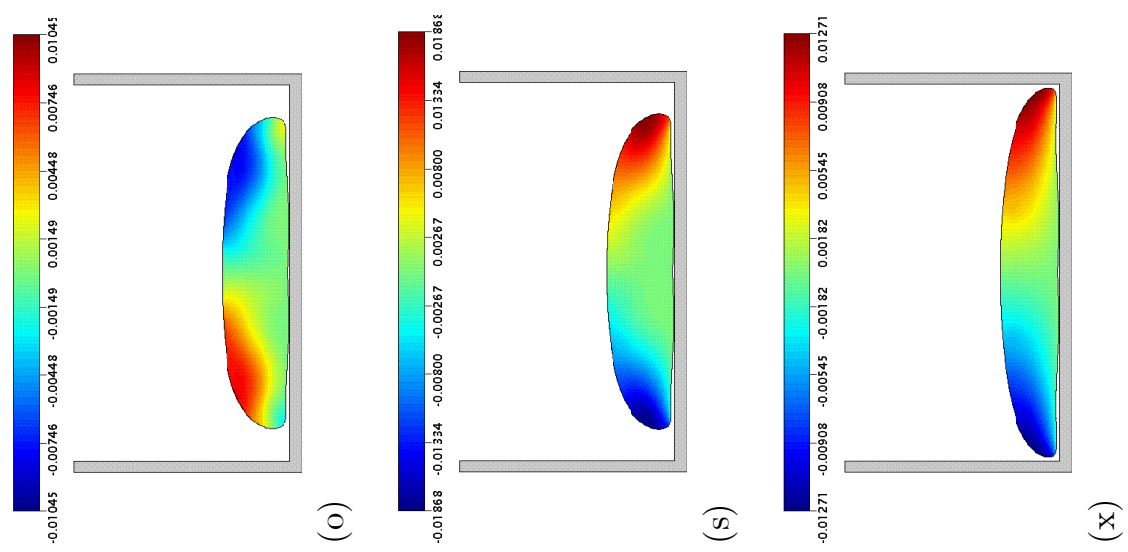

$\|$ है

웅

\|\|$\underset{\infty}{\infty}$

$\sum \stackrel{+}{30}$

$0 . \sqrt{3}$

$\operatorname{lin} 2$ is

(1)

$\pm+11$

苗芎

$x$ is

인

$\exists+\|$

䒠苍

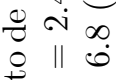
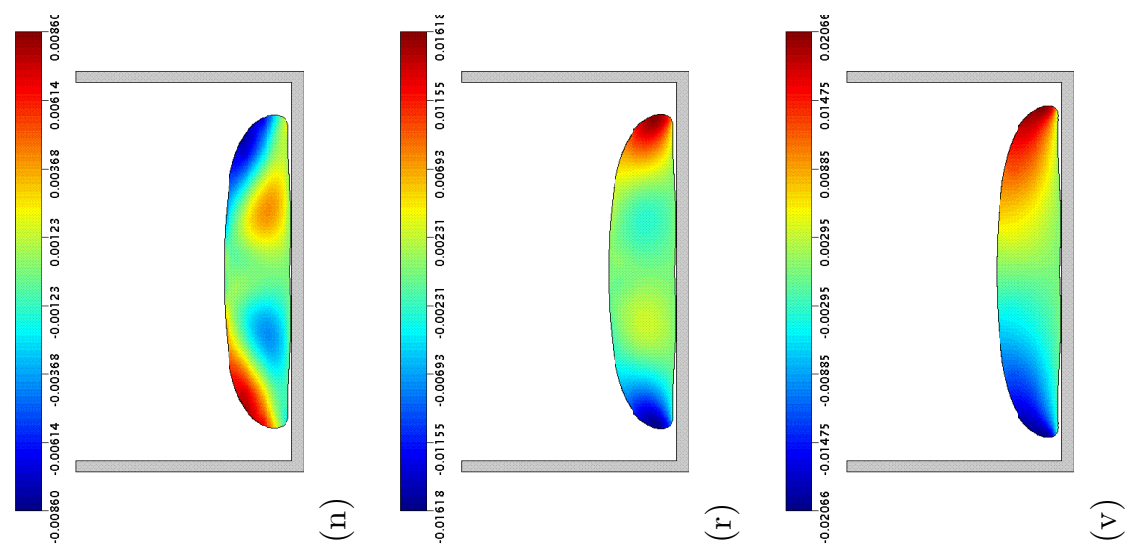

苛产㐫

象

घี 구

ॠ $\|$ i

$8+11$

कृ

๑ 0

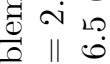

ㄴ

商前

更
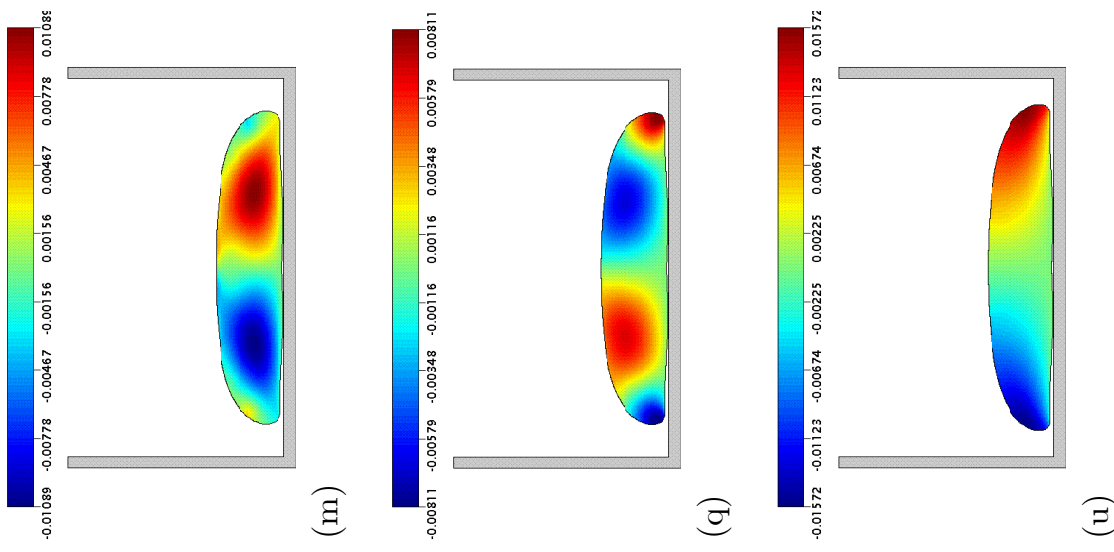

व

สี || 0

赵

范

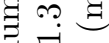

० $\|$

象

当

थ

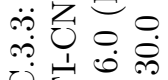

ن | $\|$

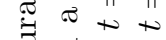

总 
154 APÊNDICE C. SIMULAÇÕES NUMÉRICAS PARA O PROBLEMA DA GOTA EM IMPACTO
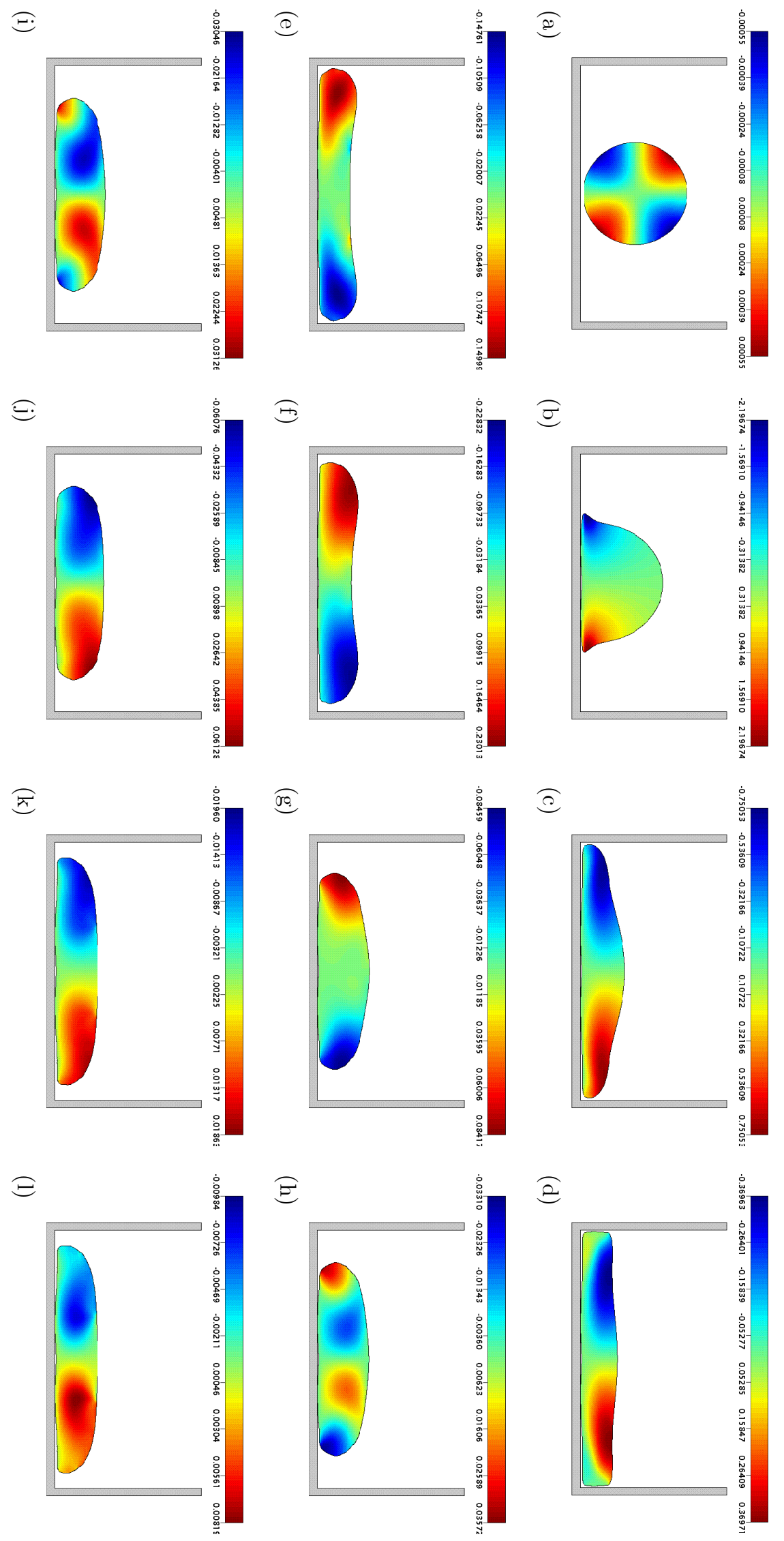
C.3. MODELOS VISCOELÁSTICOS (OLDROYD-B E SXPP)
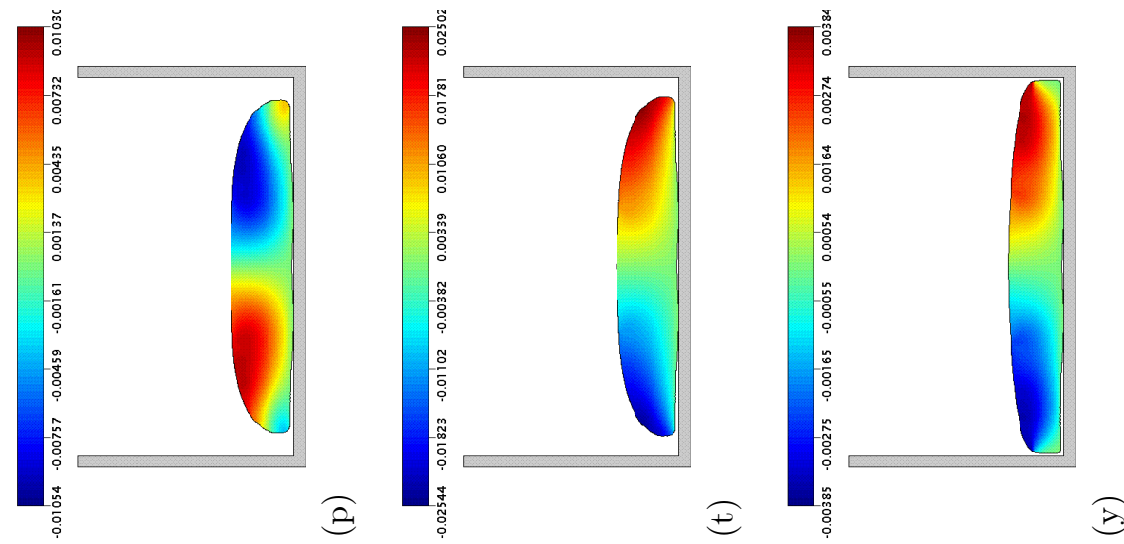

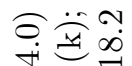

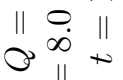

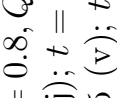

\|I $\cong$

CN $\stackrel{10}{-1}$

$\because 20$

$\| \rightarrow+$

a $\stackrel{\cdots}{\Xi}$

它谓

$0+\infty$
$0+\infty$
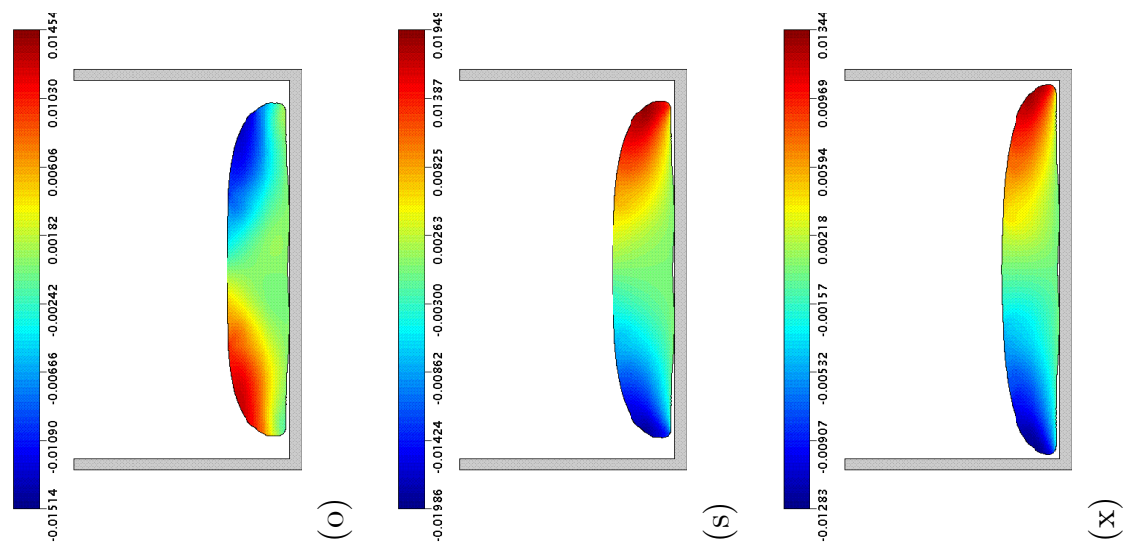

งิ

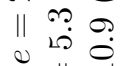

$\stackrel{1}{\infty} \stackrel{1}{1}$

$3+11$

is

$11=\frac{10}{0}$

¿ 110

$\hat{\mathrm{A}} \stackrel{\cdots}{\cdots}$

$x_{0 \rightarrow+\infty}^{\infty}$

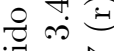

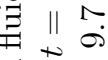

苛苍
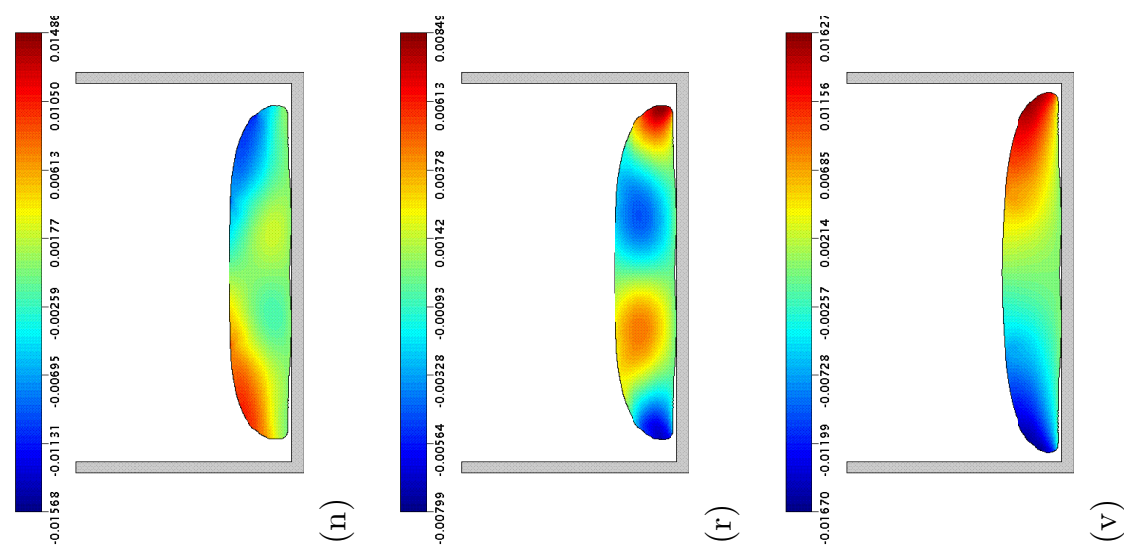

o

范 11 L

:

$\exists+$

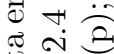

$811 m$

$\approx+\infty$

$\approx \stackrel{0}{ }$

름

○

$\vec{\Delta}+\infty$

产
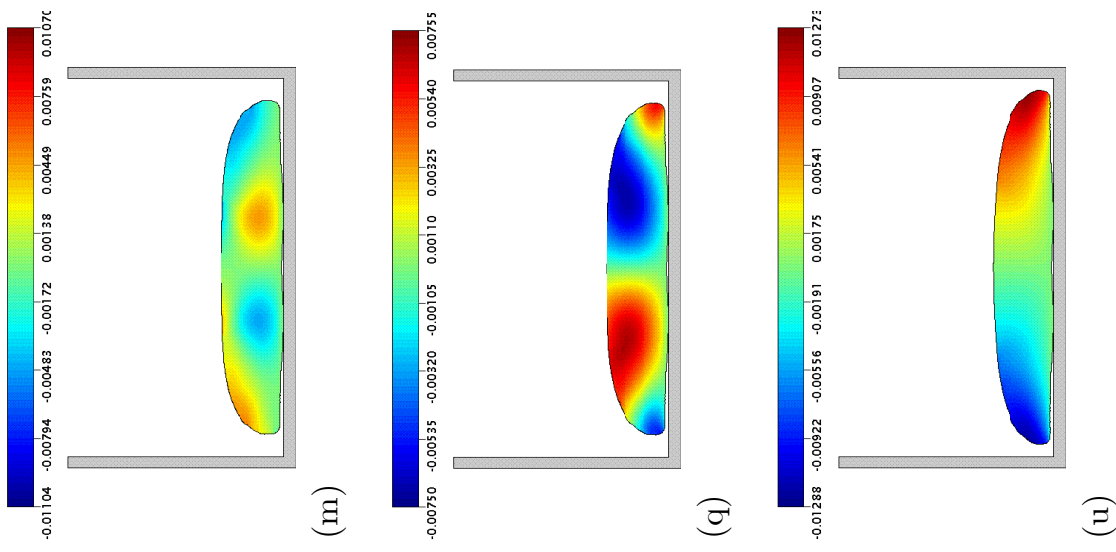

药

2 11

$\mathscr{0}+\infty$

焉

寻

\& 기

$\exists+\neg$

官 $\ddot{g}^{\infty} \dot{B}$

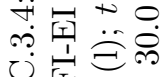

ช $\approx \|$

$\Xi$

总 
156 APÊNDICE C. SIMULAÇÕES NUMÉRICAS PARA O PROBLEMA DA GOTA EM IMPACTO 


\section{Bibliografia}

[AB01] Aboubacar, M.; Webster, M.F.; "A cell-vertex finite volume/element method on triangles for abrupt contraction viscoelastic flows". J. Non-Newtonian Fluid Mech., 98:83-106, 2001.

[AB05] Aboubacar, M.; Aguayo, J.P.; Phillips, P.M.; Phillips, T.N.; Tammadon-Jahromi, H.R.; Snigerev, B.A.; Webster, M.F.; "Modelling pom-pom type models with-high order finite volume schemes". J. Non-Newtonian Fluid Mech., 126:207-220, 2005.

[AH70] Amsden, A.; Harlow, F.H.; "A simplified MAC technique for incompressible fluid flow calculations". J. Comp. Physics, 6:332-335, 1970.

[AJ04] Aguayo, J.P.; Phillips, P.M.; Phillips, T.N.; Snigerev, B.A., Tamaddon-Jahromi, H.R; Webster, M.F.; "The numerical prediction of viscoelastic flows using the Pom-Pom model and high-order finite volume schemes". Proc. XIVth Int. Congr. on Rheology, Seoul, Korea, 2004.

[AP03] Alves, M.; Oliveira, P.; Pinho, F.; "A convergent and universally bounded interpolation scheme for the treatment of advection". Int. J. Numer. Meth. Fluids, 41:47-75, 2003.

[AR97] Armenio, V.; "An improved MAC method (SIMAC) for unsteady high-reynolds free surface flows". Int. J. Numer. Meth. Fluids, 24:185-214, 1997.

[BA02] Bijl, H.; Ampenter, M.H.; Vatsa, V.; Kennedy, C.; "Implicit time integration schemes for the unsteady compressible Navier-Stokes equations: laminar flows". J. Comp. Physics, 179:313-329, 2002.

[BC89] Bell, J.B.; Colella, P.; Glaz, H.M.; "A second order projection method for the incompressible Navier-Stokes equations". J. Comp. Physics, 85:257-283, 1989.

[BH87] Bird, R.B.; Armstrong, R.C.; Hassager, O.; "Dynamics of polymeric liquids". Vol I, John Willey ES Sons, 1987.

[BR01] Brown, D.L.; Cortez, R.; Minion, M.L.; "Accurate projection methods for the incompressible Navier-Stokes equations". J. Comp. Physics, 168:464-499, 2001.

[BT67] Batchelor, G.K.; "An Introduction to Fluid Dynamics". Cambridge University Press, 1967.

[BU03] Butcher, J.; "Numerical Methods for Ordinary Differential Equations". Wiley, 2003.

[CF99] Castelo, A.F.; Tomé, M.F.; Cuminato, J.A.; Oliveira, J.; Mangiavacchi, N.; Fortuna, A.O.; McKee, S.; "Surface tension implemention for GENSMAC2D". XV Congresso Brasileiro de Engenharia Mecânica - COBEM, 1999.

[CF00] Castelo, A.F.; Tomé, M.F.; Cesar, M.L.; Cuminato, J.A.; Mckee, S.; "Freeflow: an integrated simulation system for three-dimensional free surface flows". Comput. Vis. Sci., 2:199-210, 2000. 
[CH67] Chorin, A.; "A numerical method for solving incompressible viscous flow problems". J. Comp. Physics, 2:12-26, 1967.

[CH68] Chorin, A.; "Numerical solution of the Navier-Stokes". Mathematics of Computation, 2:745-762, 1968.

[CH00] Chorin, A.; Marsden, J.E.; "A mathematical introduction to fluid mechanics". Springer, 2000.

[CJ81] Cruickshank, J.O.; Munson, B.R.; "Viscous-fluid buckling of plane and axisymmetric jets". J. Non-Newtonian Fluid Mech., 113:221-239, 1981.

[CM02] Cuminato, J.A.; Meneguette, M.; "Discretização de Equações Diferenciais Parciais: Técnicas de Diferenças Finitas". Notas de Aula, ICMC/USP - São Carlos, 2000.

[CR62] Courant, R.; Hilbert, D.; "Methods of mathematical physics". Wiley, 1962.

[DO03] Doricio, J.L.; "GENSMAC-SOF: um método numérico para simular escoamentos incompressíveis de fluidos de segunda ordem". Dissertação de Mestrado, ICMC/USP - São Carlos, 2003

[DE03] Denaro, F.M.; "On the applications of the Helmoltz-Hodge decomposition in projection methods for incompressible flows with general boundary conditions". Int. J. Numer. Meth. Fluids, 43:4369, 2003.

[DR05] Drikakis, D.; Rider, W.; "High-resolution methods for incompressible and low-speed flows". Springer, 2005.

[EM57] Evans, M.W.; Harlow, F.H.; "The particles-in-cell method for hydrodynamic calculations". Los Alamos National Laboratory Report, LA-2139, 1957.

[FD02] Fedkiw, R.; "Coupling an Eulerian fluid calculation to a Lagrangian solid calculation with the ghost fluid method". J. Comp. Physics, 175:200-224, 2002.

[FI06] Freire, A.P.S.; Ilha, A.; Colaço, M.J.; "Turbulência". Coleção Cadernos de Turbulência, Vol. $V, 5^{a}$ Escola de Primavera em Transição e Turbulência, Instituto Militar de Engenharia - Rio de Janeiro, 2006.

[FL91] Fletcher, C.A.J.; "Computational techniques for fluid dynamics". Vol. I e II., Springer, 1991.

[FR01] Ferreira, V.G.; "Análise e implementação de esquemas de convecção e modelos de turbulência para simulação de escoamentos incompressíveis envolvendo superfícies livres". Tese de Doutorado, ICMC/USP - São Carlos, 2001.

[FT00] Fortuna, A.O.; "Técnicas computacionais para dinâmica dos fluidos: conceitos básicos e aplicações". EDUSP, 2000.

[FX01] Fox, R.W.; McDonald, A.T.; "Introdução à Mecânica dos Fluidos". LTC, 2001.

[FZ97] Ferziger, J.H.; Perić, M.; "Computational Methods for Fluid Dynamics". Springer-Verlag, 1997.

[GF03] Silva, G.F.; "Simulação numérica de escoamentos viscoelástico com superfície livre usando o ambiente Freeflow2D". Dissertação de Mestrado, ICMC/USP - São Carlos, 2003.

[GM88] Glimm, J.; Grove, J.; Lindquist, B.; McBryan, O; Tryggvason, G.; "The bifurcation of tracked scalar waves". SIAM J. Scientific and Statistical Computing, 9:61-79, 1988.

[GL89] Golub, G.H.; Loan, C.F.V.; "Matrix Computations". Jonhs Hopkins University Press, 1989.

[GR03] Grossi, L.; "Desenvolvimento de métodos numéricos para a simulação de escoamentos nãonewtonianos e viscoelástico com superfície livre". Tese de Doutorado, ICMC/USP - São Carlos, 2003. 
[HK94] Hackbush, W.; "Iterative Solution of Large Sparse System of Equations". Springer, 1994.

[HC72] Hirt, C.W.; Cook, J.L.; "Calculating three-dimensional flows around structures and over rough terrain". J. Comp. Physics, 10:324-340, 1972.

[HN71] Hirt, C.; Nichols, B.; "Improved free surface boundary-conditions for numerical incompressibleflow calculations". J. Comp. Physics, 8:434-448, 1971.

[HN81] Hirt, C.; Nichols, B.; "Volume of fluid (VOF) method for the dynamics of free boundaries". J. Comp. Physics, 39(1):201-225, 1981.

[HW65] Harlow, F.; Welch, J.; "Numerical calculation of time-dependent viscous incompressible flow of fluid with free surface". Physics of Fluids, 8:2182-2189, 1965.

[KM85] Kim, J.; Moin P.; "Application of a fractional-step method to incompressible Navier-Stokes equations". J. Comp. Physics, 59:308, 1985.

[KN86] Kan, J.V.; "A second-order accurate pressure-correction schemes for viscous incompressible flow". SIAM J. Scientific Computing, 7:870-891, 1986.

[LB73] Lambert, J.D.; "Computational methods in ordinary diferential equations". A Wiley Series, 1973.

[LE02] LeVeque,R.J.; "Finite volume methods for hyperbolic problems". Cambridge University Press, 2002.

[LI01] Li Z.; Lai, M.C.; "The immersed interface method for the Navier-Stokes equation with singular forces". J. Comp. Physics, 171:822-842, 2001.

[LU05] Liu, T.G.; Khoo, B.C.; Wang, C.W.; "The ghost fluid method for compressible gas-water simulation". J. Comp. Physics, 204:193-221, 2005.

[LM96] Lemos, C.; "Higher-order schemes for free surface flows with arbitrary configurations". Int. J. Numer. Meth. Fluids, 23:545-566, 1996.

[LK01] Lee, M.; Oh, D.; Kim, Y.; "Canonical fractional-step methods and consistent boundary conditions for the incompressible Navier-Stokes equations". J. Comp. Physics, 168:73-100, 2001.

[LX99] Lomax, H.; Pulliam, T.H.; Zingg, D.W.; "Fundamentals of computational fluid dynamics". NASA Ames Research Center, 1999.

[MD97] Mompean, G.; Deville, M.; "Unsteady finite volume of Oldroyd-B fluid through a threedimensional planar contraction". J. Non-Newtonian Fluid Mech., 72:253-279, 1997.

[MI96] Minion, M.; "A projection method for locally refined grids". J. Comp. Physics, 127:158-177, 1996.

[ML98] McLeish, T.C.B.; Larson, R.G.; "Molecular constitutive equations for a class of branched polymers: The pom-pom polymer". J. Rheol., 42:101-110, 1998.

[MY88] Mäntylä, M.; "An introduction to solid modeling". Computer Science Press, Rockville, MD, 1988.

[OC04] Oishi, C.; "Análise e implementação de métodos implícitos no sistema Freeflow2D". Dissertação de Mestrado, ICMC/USP - São Carlos, 2004.

[OC06] Oishi, C.; Cuminato, J.A.; Ferreira, V.; Tomé, M.F.; Castelo, A.F.; Mangiavacchi, N.; McKee, S.; "A stable semi-implicit method for free surface flows". J. of Applied Mech., 73:940-947, 2006. 
[OC08A] Oishi, C.; Cuminato, J.A.; Yuan, J.Y.; McKee, S.; "Stability of numerical schemes on staggered grids". Numerical Linear Algebra with Applications, aceito para publicação, 2008, DOI: $10.1002 /$ nla.597.

[OC08B] Oishi, C.; "Análise e implementação de métodos implícitos e de projeção para escoamentos com superfície livre". Tese de Doutorado, ICMC/USP - São Carlos, 2008.

[OC08C] Oishi, C.; Tomé, M.F.; Cuminato, J.A.; McKee, S.; "An implicit technique for solving 3D low Reynolds number moving free surface flows". J. Comp. Physics, 227:7446-7468, 2008.

[OL99] Oliveira, J.; "Desenvolvimento de um sistema de simulacão de escoamentos de fluidos com superfícies livres bidimensionais". Dissertação de Mestrado, ICMC/USP - São Carlos, 1999.

[OS01] Osher, S.; Fedkiw, R.; "Level set methods: an overview and some recent results". J. Comp. Physics, 169:463-502, 2001.

[PI04] Pilliod, J.E.; Pucket, E.G.; "Second-order accurate Volume-Of-Fluid algorithms for tracking material interface". J. Comp. Physics, 199:465-502, 2004.

[PL06] de Paulo, G.S.; "Solução numérica do modelo PTT para escoamentos viscoelásticos com superfícies livres". Tese de Doutorado, ICMC/USP - São Carlos, 2006.

[RJ90] Rajagopalan, D.; Amstrong, R.; Brown, R.; "Finite element methods for calculation of steady viscoelastic flow using constitutive equations with newtonian viscosity". J. Non-Newtonian Fluid Mech., 36:159-192, 1990.

[PR71] Pracht, W.E.; "A numerical method for calculating transient creep flows". J. Comp. Physics, 7:46-60, 1971.

[PT93] Perot, J.B.; "An analysis of the fractional step method". J. Comp. Physics, 108:51-58, 1993.

[QT00] Quarteroni, A.; Sacco, R.; Saleri, F.; "Numerical mathematics". Springer, 2000.

[SC78] Schlichting, H.; "Boundary-layer theory". Mcgraw-Hill, 1979.

[SD00] Saad, Y.; "Iterative methods for sparse linear systems". 2ed., 2000.

[SL05] da Silva, M.A.; "Desenvolvimento de um método numérico para simular escoamentos viscoelásticos axissimétricos com superfícies livres". Dissertação de Mestrado, ICMC/USP - São Carlos, 2005 .

[SM85] Smith, G.D.; "Numerical solution partial differential equations". Clarendron Press, 1985.

[SO02] Souza, F.S.; "Simulação numérica de escoamentos multifásicos usando o ambiente Freeflow3D". Dissertação de Mestrado, ICMC/USP - São Carlos, 2002.

[SV07] Silva, R.A.P.; "Solução numérica do modelo de Maxwell para escoamentos tridimensionais com superfícies livres". Dissertação de Mestrado, ICMC/USP - São Carlos, 2005.

[ST99] Strikwerda, J.C.; Lee, Y.; "The accuracy of the fractional step method". SIAM J. Numerical and Analitical, 37:37-47, 1999.

[TK85] Takemitsu, N.; "Finite difference method to solve incompressible fluid flow". J. Comp. Physics, 61:499-518, 1985.

[TM93] Tome, M.F.; "GENSMAC: a multiple free surface fluid flow solver". Tese de Doutorado, Department of Mathematics / University of Atrathclyde, 1993.

[TM94] Tome, M.F.; McKee, S.; "GENSMAC: a computational marker-and-cell method for free surface flows in general domains". J. Comp. Physics, 110:171-186, 1994. 
[TM96] Tome, M.F.; Duffy, B.; McKee, S.; "A numerical technique for solving unsteady non-newtonian free surface flows". J. Non-Newtonian Fluid Mech., 62:9-34, 1996.

[TM99] Tome, M.F.; McKee, S.; Barratt, L.; Jarvis, D.A.; Patrick, A.J; "An experimental and numerical investigations of container filling whith viscous liquids". Int. J. Numer. Meth. Fluids, 31:1333$1353,1999$.

[TM00] Tomé, M.F.; Castelo, A.F.; Murakami, J.; Cuminato, J.A.; Minghim, R.; Oliveira, C.; Mangiavacchi, N.; McKee, S.; "Numerical simulation of axisymmetric free surface flows". J. Comp. Physics, 157:441-472, 2000.

[TM01] Tome, M.F.; Castelo, A.F.; Cuminato, J.A.; Mangiavacchi, N.; McKee, S.; "GENSMAC3D: a numerical technique for solving three-dimensional free surface flows". Int. J. Numer. Meth. Fluids, 37:747-796, 2001.

[TM02A] Tomé, M.F.; Mangiavacchi, N.; Cuminato, J.; Castelo, A.; McKee, S.; "A finite difference technique for simulating unsteady viscoelastic free surface flows". J. Non-Newtonian Fluid Mech., 106:61-106, 2002.

[TM02B] Tomé, M.F.; "GENSMACVISCO: um método numérico para resolver escoamentos viscoelásticos não-estacionários com fronteiras livres". Tese de Livre Docência, ICMC/USP - São Carlos, 2001.

[TO99] Toro, E.F.; "Riemann solvers and numerical methods for fluid dynamics: a practical introduction". Springer, 1999.

[VB01] Verbeeten, W.M.H.; Peters, G.W.M.; Baaijens, F.T.P.; "Differential constitutive equations for polymer melts: the extended Pom-Pom model". J. Rheol., 45:823-843, 2001.

[VK86] Vanka, S.P.; "Block-implicit multigrid solution of Navier-Stokes equations in primitive variable". J. Comp. Physics, 65:138-158, 1986.

[VN98] Varonos, A.; Bergeles, G.; "Development and assessment of a variable-order nonoscillatory for convection term discretization". Int. J. Numer. Meth. Fluids, 26:1-16, 1998.

[VS92] Van Der Vorst, H.A.; "BI-CGSTAB: a fast and smoothly converging variant of BI-CG for the solution of nonsymmetric linear systems". SIAM J. Scientific and Statistical Computing, 13(2):631-644, 1992.

[WL95] Weinan, E.; Liu, J.; "Projection method I: convergence and numerical boundary layers. SIAM J. Numerical and Analitical, 32:1017, 1995.

[XP98] Xue, S.; Phan-Thien, N.; Tanner, R.; "Three-dimensional numerical simulation of viscoelastic flows through planar contractions". J. Non-Newtonian Fluid Mech., 74:195-245, 1998.

[YY05] Yang, Y.; Udaykumar, H.S.; "Sharp interface cartesian grid method ii: solidification of pure materials and binary solutions". J. Comp. Physics, 210:55-74, 2005.

[YT01] Ye, T.; Shyy, W.; Chung, J.; "A fixed-grid, shap interface method for bubble dynamics and phase change". J. Comp. Physics, 174:781-815, 2001. 